\title{
Physiological and genetic dissection of rice tolerance to water-deficit stress
}

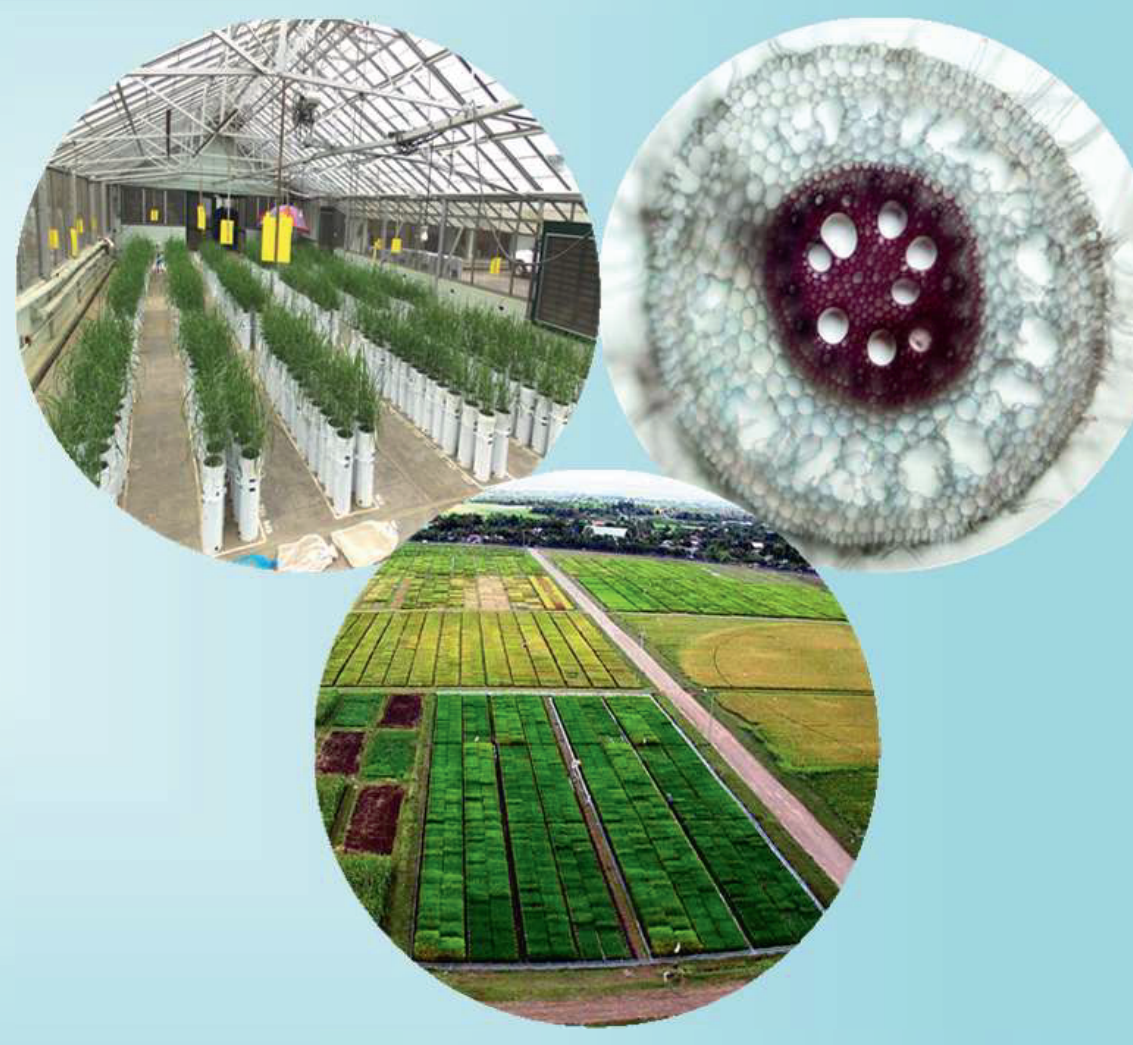

\section{Niteen Narharirao Kadam}




\section{Propositions}

1. Phenotypic plasticity of above and below ground organs is less synergistic to tolerate water-deficit in rice than in wheat.

(this thesis)

2. The development of a crop ideotype through QTL-based ecophysiological modeling is still in its infancy.

(this thesis)

3. The natural evolution of any stress tolerance mechanism is a random phenomenon and requires extensive scientific endeavors to detect and use in crop improvements.

4. Science should always be both theory and problem oriented.

5. Communication between plant breeders and ecophysiologists is complicated.

6. Maintaining a proper balance between professional work and personal life is extremely challenging in the sandwich $\mathrm{PhD}$ programme of Wageningen University \& Research.

7. No matter how intelligent an early career researcher is, (s)he needs some mental support during the initial scientific journey.

Propositions belonging to the thesis, entitled

"Physiological and genetic dissection of rice tolerance to water-deficit stress"

Niteen Narharirao Kadam

Wageningen, $20^{\text {th }}$ February 2018 


\section{Physiological and genetic dissection of rice tolerance to water-deficit stress}




\section{Thesis committee}

\section{Promotor}

Prof. Dr Paul C. Struik

Professor of Crop Physiology

Wageningen University \& Research

\section{Co-promotors}

Dr Xinyou Yin

Senior Scientist, Centre for Crop Systems Analysis

Wageningen University \& Research

Dr Krishna S.V. Jagadish

Associate Professor, Department of Agronomy

Kansas State University, Manhattan, Kansas, USA

\section{Other members}

Prof. Dr L.F.M. Marcelis, Wageningen University and Research Prof. Dr H. Stützel, Leibniz University of Hannover, Germany Prof. Dr C.S. Testerink, Wageningen University and Research Prof. Dr E. Jacobsen, Wageningen University and Research

This research was conducted under the auspices of the C.T. de Wit Graduate School for Production Ecology and Resource Conservation (PE \& RC). 


\section{Physiological and genetic dissection of rice tolerance to water-deficit stress}

\section{Niteen Narharirao Kadam}

\section{Thesis}

submitted in fulfilment of the requirements for the degree of doctor at Wageningen University by the authority of the rector Magnificus, Prof. Dr A.P.J. Mol, in the presence of the

Thesis Committee appointed by the Academic Board to be defended in public on Tuesday 20 February 2018 at 1.30 p.m. in the Aula. 


\section{Niteen Narharirao Kadam}

Physiological and genetic dissection of rice tolerance to water-deficit stress, 242 pages.

$\mathrm{PhD}$ thesis, Wageningen University, Wageningen, the Netherlands (2018)

With references, with summary in English

ISBN: 978-94-6343-740-0

DOI: $10.18174 / 431721$ 


\begin{abstract}
Niteen N. Kadam (2018) "Physiological and genetic dissection of rice tolerance to water-deficit stress". PhD thesis, Wageningen University \& Research, Wageningen, The Netherlands. 242 pp.
\end{abstract}

Rice (Oryza sativa L.) is the world's most important staple food crop, especially in Asia. As a semi-aquatic crop species, water-scarcity and increasing severity of water-deficit stress owing to climate change, are a major threat to sustaining irrigated rice production. Improving the rice adaptation to water-deficit is, therefore, a primary breeding target. The main goal of this dissertation is to study the morphological, anatomical, physiological and genetic basis for responses of a rice plant to water-deficit stress.

To give leads into how water-deficit tolerant rice should behave, a comparative study was conducted, whereby representative rice genotypes was compared at the same moisture stress during the vegetative stage with genotypes of wheat, a dryland cereal wheat (Triticum aestivum L.) known to be more tolerant to water-deficit than rice. Under-water-deficit, rice genotypes (IR64 \& Apo) developed thinner roots allowing rapid water-acquisition, whereas wheat followed a water-conserving strategy through developing thicker leaves and roots, and moderate tillering. Root anatomy such as root diameter, xylem and stele diameter and xylem number were more plastic in wheat than in rice under-water-deficit.

The methodology and findings from those representative genotypes were then projected to a diverse panel of nearly 300 rice genotypes. Such a panel was previously constructed by the International Rice Research Institute as a potential means of discovery of novel beneficial alleles for diverse phenotypic traits and their plasticity, with $46 \mathrm{~K}$ high-quality single nucleotide polymorphisms (SNPs). A genome-wide association study (GWAS) was undertaken to identify the genomic regions regulating the morphological, physiological and root anatomical traits in rice, based on a large-scale greenhouse phenotyping of these traits. The genetic basis of these traits was different in control and water-deficit stress (strong quantitative trait loci [QTL] $\times$ environment interaction), in line with novel loci detected for the plasticity of traits. Key a priori candidate genes near to these genetic loci were also identified.

Rice grain yield is strongly affected by water-deficit stress coinciding with sensitive reproductive stage. Strong genotypic variability for grain yield as well as yield components and related traits were observed in the same rice indica diversity panel, under control and reproductive stage water-deficit stress in field conditions across two years. The GWAS analysis 
identified the core loci of rice genome governing the grain yield and related traits. Most of the genomic loci were specific to treatment and year, indicating strong QTL $\times$ environment interactions.

To enable GWAS findings to be used for better designing of genotypes by breeding, an existing process-based crop model GECROS was used in a case study, where grain yield of the same indica diversity panel (267 rice genotypes) from the control treatment in one season was dissected into eight physiological parameters. Some parameters had a stronger effect on grain yield than other parameters. Using these parameters, the model showed the ability to predict the genotypic variation of rice diversity panel for grain yield under different field conditions. Further, the GWAS analysis was extended to model-input parameters on randomly chosen 213 genotypes as a training dataset. The SNP-based estimates of parameter values calculated from the additive allelic effect of the loci were used as input to the crop model GECROS. Although the SNP-based modelling approach demonstrated the ability to predict the genotypic variation in training datasets under different environments, the prediction accuracy was lower in the remaining 54 genotypes used as a testing dataset. In addition, the prediction accuracy of grain yield was also lower using either parameter or SNP-based GECROS model in completely new season. However, the model-based sensitivity analysis effectively identified the different SNPs between control and water-deficit environments. Virtual ideotypes designed based on pyramiding the SNPs identified by modelling had a higher yield than those based on SNPs for yield per se.

Keywords: water-deficit stress, rice, wheat, root anatomy, root diameter, stele diameter, Oryza sativa L., Triticum aestivum L., eco-physiological crop modelling, GECROS, single nucleotide polymorphism, genome-wide association study quantitative trait loci, training dataset, testing dataset. 


\section{Table of Contents}

\begin{tabular}{|c|c|c|}
\hline & Abstract & \\
\hline Chapter 1 & General introduction & 1 \\
\hline Chapter 2 & $\begin{array}{l}\text { Does morphological and anatomical plasticity during the vegetative } \\
\text { stage make wheat more tolerant of water-deficit stress than rice? }\end{array}$ & 15 \\
\hline Chapter 3 & $\begin{array}{l}\text { Genetic control of plasticity in root morphology and anatomy of rice } \\
\text { in response to water-deficit }\end{array}$ & 47 \\
\hline Chapter 4 & $\begin{array}{l}\text { Genome wide association reveals genetic basis of rice grain yield and } \\
\text { its component traits under water-deficit stress during the reproductive } \\
\text { stage }\end{array}$ & 111 \\
\hline Chapter 5 & $\begin{array}{l}\text { Linking eco-physiological modelling with genome wide association } \\
\text { mapping to design crop ideotypes: A case study on rice under water- } \\
\text { deficit conditions }\end{array}$ & 157 \\
\hline Chapter 6 & General discussion & 189 \\
\hline & References & 203 \\
\hline & Summary & 229 \\
\hline & Acknowledgements & 233 \\
\hline & List of publications & 237 \\
\hline & PE\&RC Training and Education statement & 239 \\
\hline & Curriculum vitae & 241 \\
\hline & Funding & 242 \\
\hline
\end{tabular}



CHAPTER 1

General introduction 


\section{Background on general response of cereals to abiotic stress}

During the 1960s, breeding efforts to enhance grain yield created improved cultivars responsive to increased input resources and improved agronomic practices that resulted in the Green Revolution in rice and wheat. Breeding and agronomic improvement during the Green Revolution had a tremendous impact on grain production. This yield increase allowed maintaining better balance between food supply and demand to feed the global population. However, there is serious challenge to maintain the same balance with steady increases in global population, which will reach 8.5-10 billion in 2050 (Tester and Langridge, 2010). To meet the needs of this projected future population, grain production needs to be increased at least by $50 \%$ compared to current production, and by $110 \%$ relative to that of the production obtained during 2006 (Tilman et al., 2011). The current crop breeding or genetic gain (Fischer and Edmeades, 2010) and agronomic interventions (Zorrilla et al., 2012 ) have not been able to increase the production enough to keep pace with the growing population. Hence, in the future, achieving sustainable increases in grain production would need substantial changes in crop breeding and cultural practices. Further, there is continuous reduction in availability of water resources for agriculture due to the growing population, increasing industrial demand and climate change effect (Kang et al., 2009). Therefore, achieving increased production would be challenging in stable environments, but undoubtedly even more challenging under changing global climate conditions. The current global climate change has already displayed damaging effects on crop yield, and is expected to have a more severe impact on grain production in future climate scenarios (IPCC, 2013).

The gradual increase in atmospheric carbon dioxide concentration $\left[\mathrm{CO}_{2}\right]$ is the major driver of global climate change. The $\mathrm{CO}_{2}$ level has increased approximately from the preindustrial level of 280 to the current average of $405 \mu \mathrm{mol} \mathrm{mol}{ }^{-1}$ (August 2017; https://www.co2.earth/annual-co2; last visited October 10, 2017), and is projected to reach 700 $\mu \mathrm{mol} \mathrm{mol}{ }^{-1}$ by the end of the twenty-first century (Pearson and Palmer, 2000; Prentice, 2001; Leakey et al., 2009). The potential effects of gradually rising $\left[\mathrm{CO}_{2}\right]$ include occurrence of abiotic stresses such as high temperature, water-deficit and flooding stress (Wassmann et al., 2009). Current models predict a mean increase in temperature of $1.0-3.7^{\circ} \mathrm{C}$, and a wide spread of severe water-deficit stress by the end of the twenty-first century (IPCC, 2013). The impact of increasing temperature and water-deficit stress, owing to climate change, is region specific, severely affecting crop productivity and socio-economic conditions (Figure 1). The individual effects of high temperatures and water-deficit stress were extensively studied in cereals. 
However, under real field conditions these two major abiotic stresses strongly interact with each other (Jagadish et al., 2012) and are often considered as a commonly occurring companion stress (Mittler, 2006). In addition, recent studies demonstrated the occurrence of high temperature and water-deficit stress in many parts of the world (Asia, Europe, the USA and Africa), which threatens crop productivity (Ciais et al., 2005; Wassmann et al., 2009; Olesen et al., 2011; Lobell et al., 2011; Bindi and Olesen, 2011; Zhang and Huang, 2012). Wassman et al. (2009) demonstrated the occurrence of water-deficit and high temperature stress during critical flowering and early grain-filling stages in major rice growing areas of Asia. Crop plants display both unique and similar responses to a combined effect of water-deficit and high temperature stress at whole plant to cellular level. In addition, combined effects strongly depend upon many factors such as intensity and duration of stress, crop species (Araus et al., 2002), crop genotype (Ristic and Cass, 1992; Acevedo et al., 1999), developmental stage of the organ, and cellular compartments (Ristic and Cass, 1992; Barnabás et al., 2008). Together, such complex responses make water-deficit and high-temperature stresses complex traits. The individual and combined effects of water-deficit and high-temperature stress in interaction with elevated $\mathrm{CO}_{2}$ on agronomic and physiological traits in major cereals were systematically summarized in detail by my review paper published as Kadam et al. (2014).

\section{Water scarcity: a growing concern for rice productivity}

Water scarcity is one of the major threats to rice (Oryza sativa L.) production because rice has a semi-aquatic origin and is mostly cultivated in lowland conditions. Rice alone consumes $30 \%$ of water used for agriculture, while other dryland cereals require 2-3 times less water than rice (Peng et al., 2006). However, approximately 15-20 million hectares of rice growing area will most likely become affected by water scarcity by the year 2025 (http://irri.org/ourwork/research/rice-and-the-environment/coping-with-water-scarcity). Exploring alternative solutions to reduce water requirement of rice cultivation will, therefore, have a greater significant impact on sustaining rice production in a water scarce world (Molden et al., 2010). To reduce rice water requirement, several water saving technologies have been developed such as alternate wetting and drying (Yao et al., 2012), the system of rice intensification (Stoop et al., 2002) and aerobic rice (Bouman et al., 2005). While these new technologies each have their own benefits, there are several associated risk factors including a strong yield penalty. The latter might be overcome by breeding for stress tolerant cultivars. Hence, combining these water saving technologies with breeding genotypes for a better tolerance to water-deficit stress would 


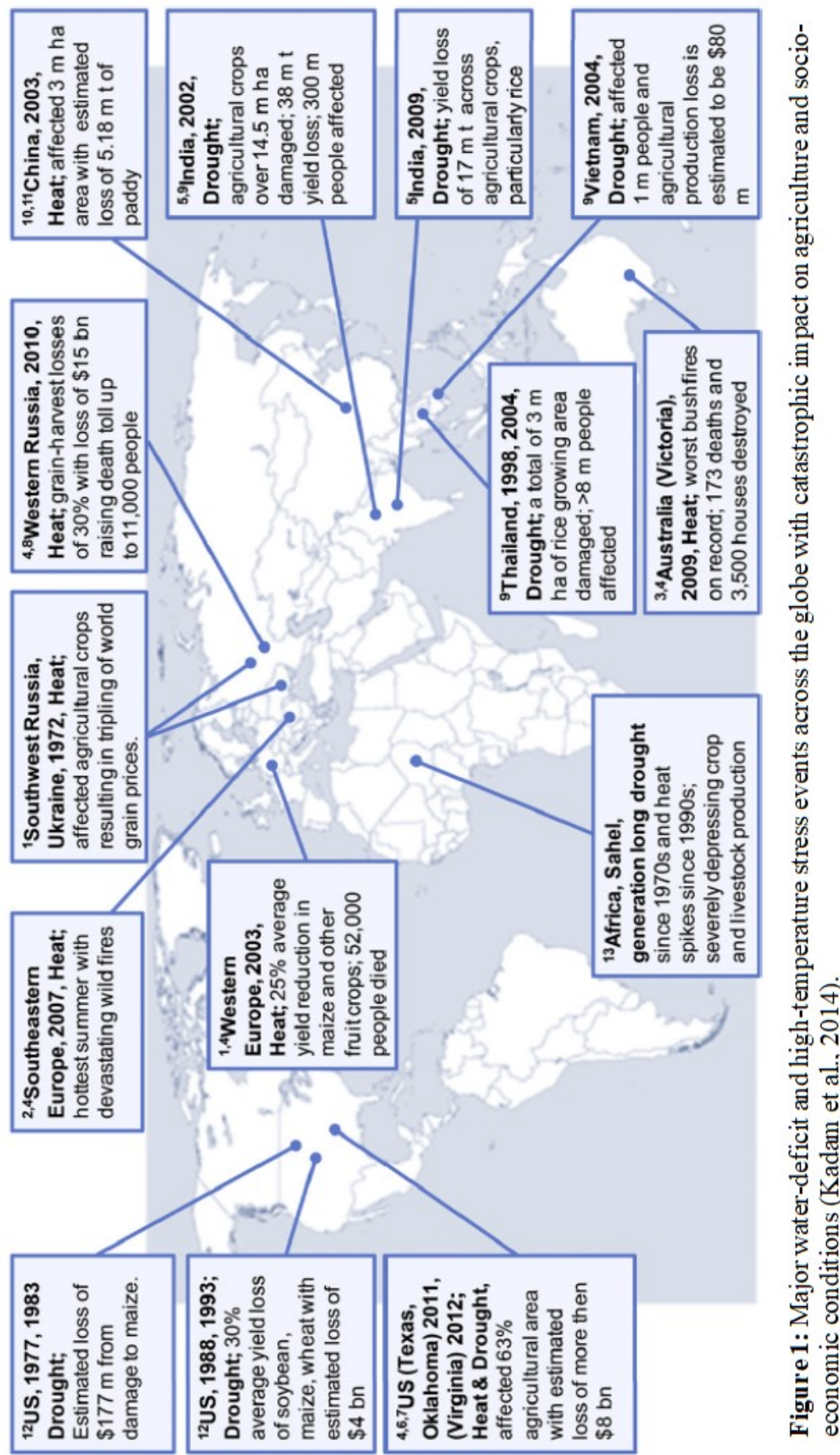


be a more viable approach to address water scarcity in rice production (Bouman et al., 2006).

There are clear morphological differences between rice varieties adapted to upland and flooded conditions. Upland rice varieties generally have more roots and probably less shoot biomass under dry conditions, which could be one of the main reasons for overcoming waterdeficit stress (De Datta et al. 1975). Adachi et al. (2010) documented that high hydraulic conductance due to large root surface area had significantly helped to maintain a higher rate of leaf photosynthesis in rice. However, precise information on physiological and morphological plasticity of root and shoot traits in response to water-deficit conditions is limited. Rice is highly sensitive to water-deficit during its reproductive stage and efforts have been devoted in understanding and improving rice stress resilience (Lanceras et al., 2004; Yue et al., 2006; Bernier et al., 2007; Vikram et al., 2011; Swamy et al., 2011). Nevertheless, water-deficit stress also occurs during the vegetative stage in major rainfed rice growing areas. For instance, vegetative stage water-deficit stress is more common in the Mekong region of Cambodia, where rainfall follows a bimodal pattern-decreasing during the early-middle part of the rainy season (Kamoshita et al., 2008).

Water-deficit stress striking during the vegetative stage reduces shoot elongation rate, leaf area and tillering due to decreased $\mathrm{CO}_{2}$ assimilation because of lower stomatal conductance, transpiration and lower relative water content (Barnabás et al., 2008; Lipiec et al., 2013; Aslam et al., 2013). In contrast, stress occurring during young microspore development and anthesis (reproductive stage) leads to pollen and spikelet abortion, induces poor anther dehiscence, and restricts panicle exertion, thereby strongly reducing grain yield in major cereals (Kato et al., 2008; Lobell et al., 2011), including rice. A mild water-deficit stress during the pre-anthesis period showed $70 \%$ reduction in secondary branches of panicles and $45 \%$ decrease in spikelets per panicle in rice (Kato et al., 2008). The higher pollen abortion in rice and other cereals under water-deficit stress was mostly associated with accumulation of abscisic acid (ABA) that supresses the supply of sucrose (Powell et al., 2012). In addition, stress coinciding during the grain filling (terminal water-deficit stress) in rice and other cereals results in early senescence with shorter grain-filling period that strongly reduces the 1000-grain weight and total grain yield (Samarah, 2005; Foulkes et al., 2007). For these reasons, rice genotypes should possess a range of characteristics to become adapted to varying levels of stress intensity at any given time during the growing period. Therefore, a fundamental understanding of the physiological and morphological traits contributing to stress tolerance with underlying genetic control is essential for designing rice-breeding strategies under water-deficit stress. 


\section{General response and adaptive strategies of plants to tolerate water-deficit stress}

In general, plants have developed or acquired several strategies to mitigate water-deficit stress such as escape (phenological plasticity, i.e. early maturing), avoidance (maintaining high tissue water status), tolerance (physiologically active at low leaf tissue water status), and recovery (ability of plants to recover completely after water-deficit stress). These strategies are not mutually exclusive and can operate in combination under water-deficit stress (Ludlow and Muchow, 1990). Water-deficit stress induces a complex network of interactions between different morphological, physiological and biochemical processes at the whole plant as well as organ and cellular level. Roots are capable to first perceive stress and synthesize the chemical compound (phytohormone ABA) that communicates the stress signal to the shoot. This indeed allows the shoot and root to respond rapidly to the stress conditions. The molecular mechanism underlying these processes consists of stress signalling genes that trigger downstream genes leading to the activation of the stress tolerance pathway (Chaves et al., 2003). In the longer term, these responses can lead to fine-tuning of root biomass (increased root to shoot ratio), and alteration of morphology and anatomy of root and shoot. These adjustments help plants to avoid and tolerate stress conditions. Together, such a network, involving both short-term responses and long-term adaptive adjustments, makes water-deficit stress tolerance complex; identifying the key determinants is therefore always challenging.

To better understand the plant responses to water-deficit stress, Kamoshita et al. (2008) classified water-deficit responsive traits into primary, secondary, integrative, and phenology and plant type traits (Table 1). Primary traits are further grouped into constitutive traits (i.e. also expressed under non-stress conditions, e.g. root depth, cuticle thickness) and induced traits (i.e. expressed under stress, e.g. osmotic adjustment). These traits are highly interactive, for example, constitutive root traits under water-deficit stress may help to extract water from deeper layers of soil. This influences the expression of induced and secondary traits such as maintenance of plant water status and canopy temperature. Better performance of these secondary traits strongly influences the grain yield component traits (also called integrative traits; Figure 2). For instance, better water uptake by roots maintains cooler canopy, which reduces water-deficit induced spikelet sterility and ultimately grain yield (Kobata et al., 1994). Phenology (flowering time) and plant-type (plant height and tillering) traits play a major role in stress adaptation, and strongly affect the expression of secondary and integrative traits and thereby grain yield. Further, compared to other traits, plant-type and phenology traits are 
genetically less complex with higher heritability; hence they have extensively been used in traditional crop breeding (Cooper et al., 1999a; Cooper et al., 1999b). In contrast, grain yield and its component traits are genetically more complex traits controlled by many genes/quantitative trait loci (QTL) compared to the traits belonging to other categories in Table 1.

\section{Rice root development and function: Present status and future challenges}

Roots, because of their primary role in water and nutrient uptake, have historically gained much more attention than shoot development in improving water-deficit stress tolerance. Rice has a well-described fibrous root system, and mainly exhibits nodal and lateral roots although it also has active seminal roots during the first two weeks of seedling growth (Yoshida and Hasegawa, 1982). Significant genotypic variation for root morphological and anatomical traits, and their functional relevance in stress tolerance have been reported in rice [for details see the review by Gowda et al. (2011)]. A deeper root system is generally viewed as a desirable trait (Gowda et al., 2011), which improves rice grain yield in water-deficit stress (Uga et al., 2013). In addition, direct selection of grain yield as a criterion under water-deficit stress resulted in the tolerant genotypes (Lanceras et al., 2004; Yue et al., 2006; Bernier et al., 2007; Vikram et al., 2011). Further studies also proved the role of root morphology and anatomy in improving grain yield under stress (Henry et al., 2012). In summary, rice root research is already in an advanced stage, and much of the knowledge is generated allowing to design the root ideotype to improve stress adaptation. Despite this fundamental progress and knowledge in root research, rice cultivars are more stress susceptible than genotypes of other dryland species (wheat, maize and sorghum). Rice developed several different root characteristics compared to dryland species, such as root aerenchyma that allows adapting to lowland/flooded conditions. In addition, we hypothesize that rice possesses a narrower xylem diameter than dryland cereals. Thus overall water transport in rice will be slower because the transport rate depends on the overall cross-sectional area of the xylem (Niklas, 1985). Although such differences are obvious, the direct comparison of physiology and rooting plasticity between cereals under stress conditions is lacking. A recent study demonstrated that rice and wheat responses can be compared at the same moisture conditions (Praba et al., 2009). We assume that such direct comparison of rice with other dryland species will help to understand why rice cannot perform like other cereals in terms of its ability to tolerate stress. It will also allow identifying the key root mechanisms / traits that can be prioritized in genetic mapping to understand the molecular pathway (potential QTLs/ 


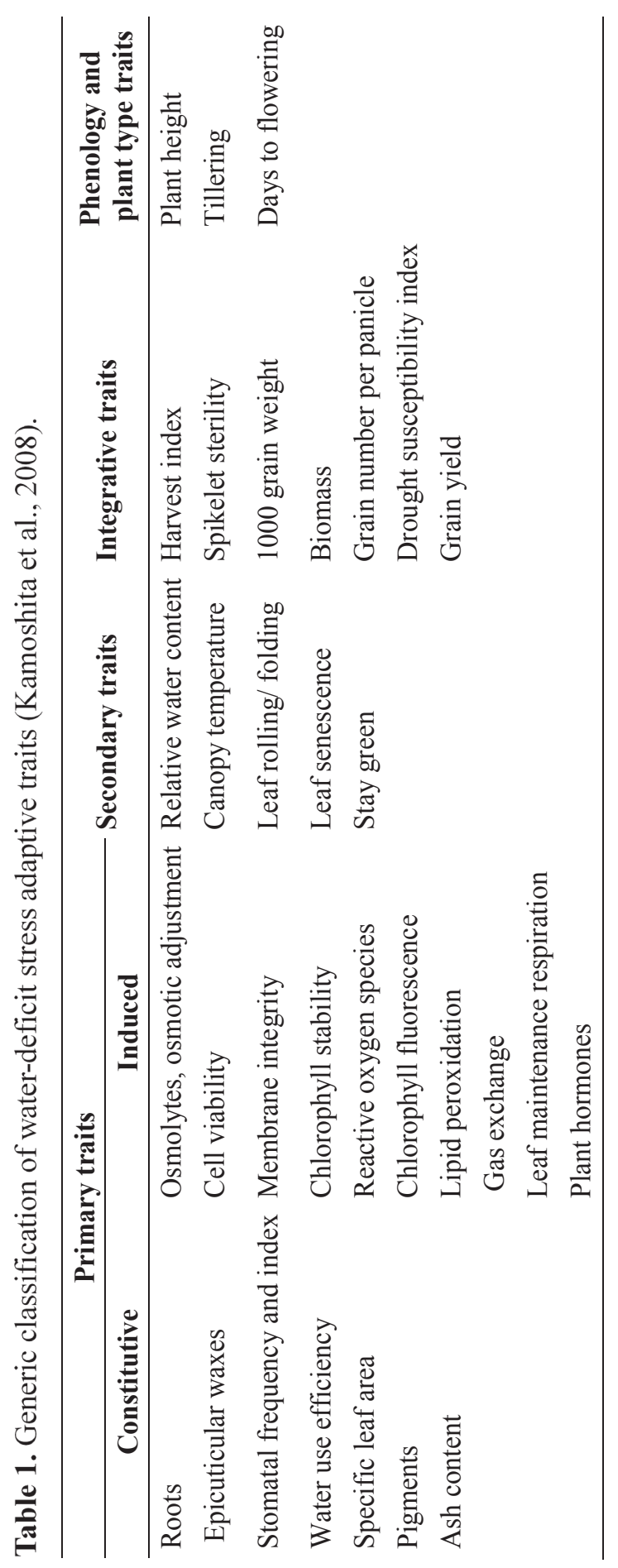


genes) underlying rice adaptation to water-deficit conditions.

\section{Genetic dissection of complex water-deficit stress tolerance in rice through a} genome-wide association study

Water-deficit stress tolerance is a complex trait controlled by many genes/QTLs (Fleury et al., 2010; Ravi et al., 2011). Recent advances in genotyping coupled with precise phenotyping can potentially help to dissect the genetic architecture and regulatory pathways that confer adaptation of rice to water-deficit stress. So far, the use of genotypic variation to identify markers/genes for water-deficit traits has been typically conducted using a population resulting from a bi-parental cross. Gu et al. (2012), for example, recently identified QTLs for highly environmentally sensitive and difficult-to-measure photosynthetic parameters in rice under field water-deficit condition. In addition, several QTLs were identified for water-deficit stress tolerant traits such as root traits [for more details see the review by Mai et al. (2014)], grain yield and yield component traits (Lanceras et al., 2004; Bernier et al., 2007; Kumar et al., 2007; Venuprasad et al., 2009b; Vikram et al., 2011). Although the bi-parental QTL approach has been extensively used in rice for mining QTLs linked to several traits (Mir et al., 2012), one major limitation is the use of only two parents and hence limited genetic variation. It is unlikely that a wide range of traits that can potentially confer water-deficit stress tolerance (Table 1) can be identified in bi-parental populations since the two parents used are unlikely to substantially contrast for so many traits of interest. Further, a bi-parental population includes insufficient recombination events, hence identified QTLs often localize in a large genomic segment. Thus, a bi-parental linkage approach lacks the potential to exploit the vast genomic variability and tremendous phenotypic plasticity housed within the 120,000 rice accessions in public germplasm repositories (Zhao et al., 2011).

To overcome the constraints of conventional QTL mapping, genome wide association studies (GWAS) or linkage disequilibrium (LD) mapping, in which statistical associations between genotype and phenotype are assessed in large panels of germplasm, are now emerging as a viable strategy to identify QTLs/genes underlying quantitative variation of traits (Rafalski, 2010). In general, a GWAS consists of six steps namely: (1) germplasm selection with wide coverage of genetic diversity, (2) genotyping a population with available markers, (3) phenotypic measurement of traits of interest, (4) quantification of extent of LD in the population using marker data, (5) determining population structure (level of genetic differentiation among groups within the population) and kinship (coefficient of relatedness between pairs of each 


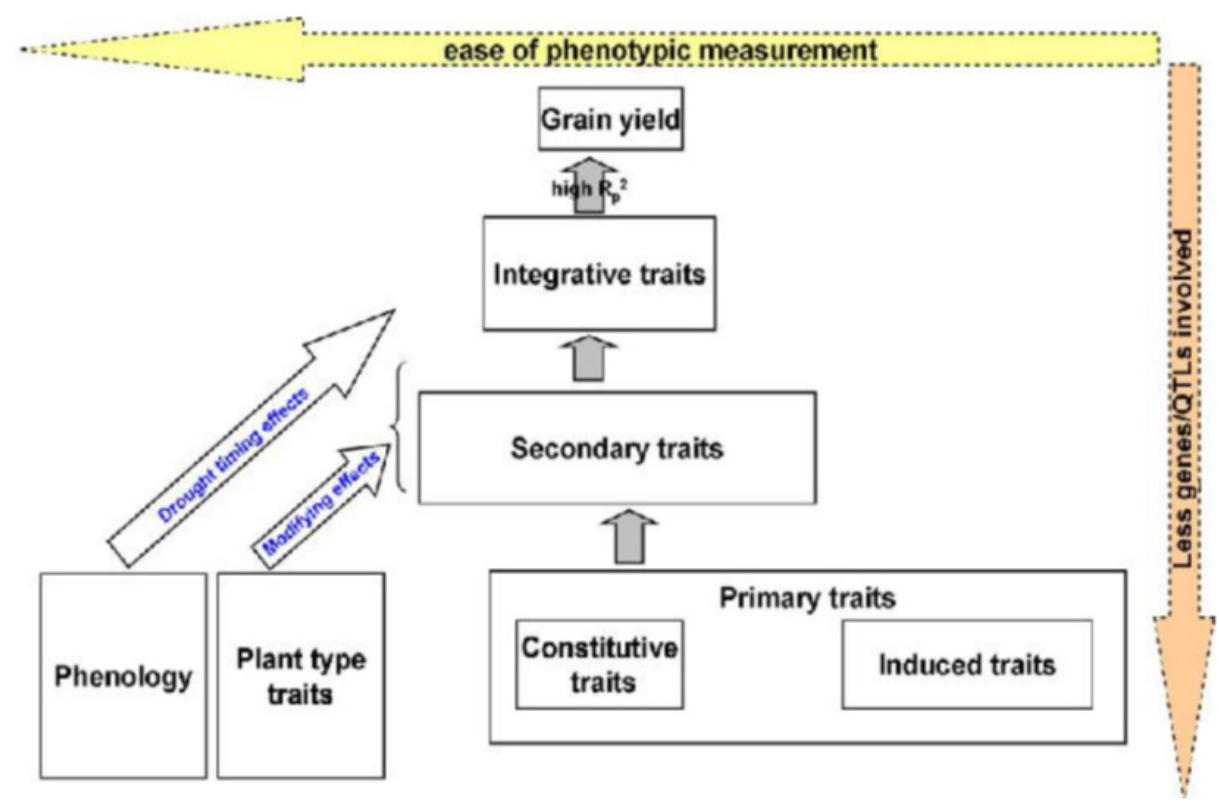

Figure 2: Relationship between water-deficit stress tolerance traits classified by Kamoshita et al. (2008). High $\mathrm{R}_{\mathrm{P}}{ }^{2}$ indicates the strength of correlation between grain yield and integrative traits.

individual within the population), and (6) construction of a high-density haplotype map of the genome and association of phenotypic traits (Abdurakhmonov and Abdukarimov, 2008). This approach fits perfectly with rice due to the availability of a reference genome sequence and genome wide single nucleotide polymorphisms (SNPs) dataset for a large number of accessions at the International Rice Research Institute (IRRI), which will enhance the analytical power of the GWAS.

\section{Linking eco-physiological crop modelling with genome-wide association mapping to design ideotypes for improved grain yield}

A common challenge for both geneticists and physiologists is to predict the effect of genomic regions/genes identified in one environment on trait phenotypes in another environment. Crop modelling provides an option in this regard. Recent case studies on integration of crop ecophysiological models with traditional genetic mapping (QTL-based eco-physiological modelling) extended the scope of modelling to determine the QTL-trait phenotype relationships and genotype-environment interactions, in which QTL-based parameter values are used to calibrate the eco-physiological model. The QTL-based modelling was first used in barley to 
predict grain yield by (Yin et al., 1999; Yin et al., 2000) and subsequently, with more success, applied to simpler crop traits such as leaf elongation rate in maize (Reymond et al., 2003), flowering time in barley (Xu et al., 2005; Yin et al., 2005) and in rice (Nakagawa et al., 2005). There is evidence to demonstrate that such a linking of genetic mapping and eco-physiological modelling can help to transform QTL mapping into more efficient marker-assisted breeding strategies (Hammer et al., 2006). Recently, it was shown that a rice ideotype designed using this approach by pyramiding the alleles of yield component traits shows higher grain yield than the ideotype based on alleles for grain yield per se (Gu et al., 2014). However, it remains to be seen whether this approach can be extended to link the process-based eco-physiological model with QTLs identified by GWAS analysis for design of the virtual grain yield ideotype.

\section{Major objectives of this study}

Although there have been several independent studies conducted to understand the water-deficit stress tolerance in rice, studies integrating various aspects to better understand the water-deficit stress tolerance are limited. In this study, I tried to use a multidisciplinary approach, covering physiology, genetics and crop modelling, to understand the rice water-deficit stress tolerance. This study has multiple key objectives:

1) Quantify physiological, morphological and root anatomical traits plasticity of rice and wheat to vegetative stage water-deficit conditions;

2) Investigate the genetic control of physiological, morphological and root anatomical traits plasticity through GWAS under vegetative stage water-deficit stress;

3) Investigate the genetic control of grain yield and yield components variation through GWAS under water-deficit stress during the sensitive reproductive stage;

4) Examine the ability of an existing process-based eco-physiological "GECROS" model to quantify the grain yield differences in rice association mapping panel;

5) Model the proportional contribution of the identified SNPs or QTLs to grain yield increase under water-deficit conditions;

6) Pyramiding the genetic effects of the SNPs or QTLs to design the virtual grain yield ideotype using modelling approach.

I surmise that addressing these above key objectives will help to establish a new platform for identification and selection of key traits for breeding stable high-yielding rice varieties for water 


\section{Chapter 1}

-deficit stress.

\section{Methodological framework}

To achieve the above objectives, I studied underlying mechanisms of rice water-deficit stress tolerance through a multidisciplinary approach of integrating physiology, genetics and crop growth modelling. The general methodological framework is summarized in four steps. In the first step, I characterized the physiological, morphological (shoot and root) and root anatomical response of rice and wheat under well watered and water-deficit stress conditions to create meaningful knowledge and insight useful for designing the genetic mapping study. In the second step, I scaled down the finding of the first study on a large set of rice genotypes to identify the phenotypic and genetic variation in physiological, morphological and anatomical traits. In the third step, I screened the same set of genotypes under field conditions to quantify the variation in grain yield and yield components under reproductive stage water-deficit stress. Further, I conducted a genetic analysis to link these phenotypic variations with genomic regions (high-density SNP markers) to identify the QTLs/genes with their possible environmental interaction. In the last step, I tried to incorporate the effect of QTLs detected for grain yieldinfluencing parameters into a process-based "GECROS" crop model to design the virtual grain yield ideotype, with the hope to assist the traditional breeding for improved rice stress tolerance. I hypothesize that such an integrated multidisciplinary approach can lead to new insights into water-deficit stress tolerance, providing the breeder with more knowledge that can improve the selection efficiency to increase the grain yield under changing climate.

\section{Outline of this thesis}

This dissertation consists of six chapters including this chapter as general introduction (Chapter 1). The general content and key message of Chapters 2-6 are summarized below.

In Chapter 2, I describe the key findings from an experiment conducted to test the physiological, morphological and root anatomical response of rice and wheat to vegetativestage water-deficit stress. The results show that wheat has stronger morphological and root anatomical plasticity in response to water-deficit stress than rice.

In Chapter 3, I scale the key findings from Chapter 2 up to a rice association mapping panel and quantify the quantitative genotypic variation of phenotypic plasticity for physiological, morphological and root anatomical responses under vegetative stage water-deficit stress. 
Significant genotypic variation is found for phenotypic plasticity. Genome-wide association analysis identifies several genomic regions associated with phenotypic plasticity that upon validation can be used for marker-assisted selection (MAS). In addition, key a priori candidate genes are identified near to these genomic regions, which can be used for further molecular validation.

In Chapter 4, I describe the field experiment to investigate the variation in grain yield as well as in yield components under control and reproductive-stage water-deficit stress conditions across two independent growing seasons. Significant genotypic variation and genotype-byenvironment interaction are observed for grain yield and yield components. Genome-wide association analysis identifies genomic regions regulating the grain yield, yield components, and demonstrates strong interactions with environment. Key a priori candidate genes are identified.

In Chapter 5, I describe a case study to link the process-based "GECROS" model with genomewide association mapping to design a virtual grain yield ideotype. Using the model input parameters derived from an experiment as described in Chapter 4, I calibrate the "GECROS" model to quantify the grain yield differences in a rice association mapping panel under control. I also extrapolate this calibrated "GECROS" model to predict the grain yield difference under water-deficit stress and in completely new environments. A model based-sensitivity analysis identifies the key grain yield determining SNPs marker that breeder can prioritize. By pyramiding the alleles of SNPs detected for model input traits, a virtual grain yield ideotype is designed.

In Chapter 6, I summarize the key conclusions of this thesis; discuss their potential implications and limitations in improving the rice water-deficit stress tolerance. In addition, I also discuss future potential avenues to improve rice adaption to water-deficit stress. 



\section{Does morphological and anatomical plasticity during the vegetative stage make wheat more tolerant of water-deficit stress than rice?} Niteen N. Kadam ${ }^{1,2}$, Xinyou Yin ${ }^{2}$, Prem S. Bindraban ${ }^{3}$, Paul C. Struik $^{2}$,
Krishna S.V. Jagadish

\footnotetext{
${ }^{1}$ International Rice Research Institute, DAPO Box 7777, Metro Manila, Philippines ${ }^{2}$ Centre for Crop Systems Analysis, Wageningen University and Research Centre, PO Box 430, 6700 AK Wageningen, The Netherlands

${ }^{3}$ Virtual Fertilizer Research Center, Washington, D.C. 20005, USA
} 


\begin{abstract}
Water scarcity and the increasing severity of water-deficit stress are major challenges to sustaining irrigated rice (Oryza sativa L.) production. Despite the technologies developed to reduce the water requirement, rice growth is seriously constrained under water-deficit stress compared with other dryland cereals such as wheat (Triticum aestivum L.). We exposed rice cultivars with contrasting responses to water-deficit stress and wheat cultivars well adapted to water-limited conditions, to the same moisture stress during vegetative growth to unravel the whole-plant (shoot and root morphology) and organ/tissue (root anatomy) responses. Wheat cultivars followed a water-conserving strategy by reducing specific leaf area and developing thicker roots and moderate tillering. In contrast, rice 'IR64' and 'Apo' adopted a rapid water acquisition strategy through thinner roots under water-deficit stress. Root diameter, stele and xylem diameter, and xylem number were more responsive and varied with different positions along the nodal root under water-deficit stress in wheat, whereas they were relatively conserved in rice cultivars. Increased metaxylem diameter and lower metaxylem number near the root tips and exactly the opposite phenomena at the root-shoot junction facilitated the efficient use of available soil moisture in wheat. Tolerant rice 'Nagina 22' had an advantage in root morphological and anatomical attributes over cultivars IR64 and Apo but lacked plasticity, unlike wheat cultivars exposed to water-deficit stress. The key traits determining the adaptation of wheat to dryland conditions have been summarized and discussed.
\end{abstract}

Keywords: rice, nodal root anatomy, root diameter, stele diameter, water-deficit stress, water use efficiency, wheat, root-shoot junction.

\begin{abstract}
Abbreviations
$\Delta^{13} \mathrm{C}$ : carbon isotope discrimination, $\mathrm{g}_{\mathrm{s}}$ : stomatal conductance, LMXD: late metaxylem diameter, LMXN: late metaxylem number, LWR: leaf weight ratio, LPr: radial hydraulic conductance, MRL: maximum root length, RA: root apex, RB: root biomass, RLD: root length density, RSJ: root-shoot junction, RV: root volume, RWR: root weight ratio, SD:RD: stele diameter in proportion to root diameter, SLA: specific leaf area, SRL: specific root length, SWR: stem weight ratio, TRL: total root length, WUE: water use efficiency.
\end{abstract}




\section{Introduction}

Among cereals, rice (Oryza sativa L.) and wheat (Triticum aestivum L.) are the most important staple food crops and they belong to the family Poaceae. These two cereals share a common ancestor and diverged about 65 million years ago (Sorrells et al., 2003). Rice eventually developed strong adaptation potential for fully flooded conditions across tropical to temperate environments, while wheat became well adapted to aerobic conditions mostly restricted to temperate environments. Rice, with a semi-aquatic behavior, consumes about $30 \%$ of the total fresh water available for agricultural crops worldwide, which equates to a 2-3-fold higher consumption than other cereals such as wheat and maize [Zea mays L.] (Peng et al., 2006). Despite significantly lower water requirement, the potential yield of wheat in a favorable environment $\left(9 \mathrm{t} \mathrm{ha}^{-1}\right)$ is comparable with the yield of fully flooded rice $\left(9 \mathrm{t} \mathrm{ha}^{-1}\right)$ in the dry season at the International Rice Research Institute (IRRI; Fischer and Edmeades, 2010). Hence, rice records very low water productivity compared with wheat and other dry-land cereals. Because of growing concerns about water scarcity and increased frequency and magnitude of water-deficit stress events under current and future climates, increasing or even sustaining rice yield under fully flooded conditions is highly challenging. To minimize the total water requirement for cultivating rice, several water-saving technologies have been developed such as direct-seeded aerobic rice cultivation (Bindraban et al., 2006). These water-saving technologies increased water productivity substantially compared with flooded conditions, but were invariably associated with a yield penalty. A major challenge that water-saving technologies including aerobic rice currently face is the lack of mechanistic understanding for further genetic improvement.

Rice, by virtue of its wider adaptation to a range of edaphic conditions, is considered to possess the diversity to adapt to upland or aerobic scenarios extending into water-deficit conditions (Khush et al., 1997). Genetic differences in rice root biomass and rooting depth and variation in root morphology with water-deficit stress exposure are well documented (Kato et al., 2006, 2007; Henry et al., 2011; Kano et al., 2011). But, the underlying mechanisms differing across diverse germplasm that influence water uptake under water-deficit stress are not fully understood (Gowda et al., 2011). A recent report has documented water-deficit-tolerant genotypes recording a lower bleeding rate and narrow xylem diameter under stress (Henry et al., 2012). Contrastingly, a higher root hydraulic conductivity helped to maintain a higher photosynthetic rate (Adachi et al., 2010), with tolerant cultivars maintaining greater root hydraulic conductivity than susceptible cultivars (Matsuo et al., 2009). Further, upland rice 


\section{Chapter 2}

cultivars with deeper roots outperformed lowland cultivars possessing a shallow root system when encountering water-deficit stress (Uga et al., 2013). Additionally, major-effect grain yield MQTL (meta-quantitative trait loci) under water-deficit stress identified in rice were found to co-localize on the genomes of other dry-land cereals such as wheat, maize, and pearl millet [Pennisetum glaucum L.] (Swamy et al., 2011), indicating a possible common evolutionary pathway for water-deficit adaptation across cereals. Despite these achievements and the relatedness among cereals, rice does not respond in a way like other dry-land cereals to waterdeficit stress conditions. To bring in a revolutionary change in future breeding strategies for upland/aerobic and water-deficit tolerance in rice, there is a need for a fundamental understanding and identification of the key traits that determine water-deficit stress response in well-adapted dry-land cereals. Hence, comparing whole-plant responses (shoot and root) of rice with those of other dry-land cereals such as wheat is essential. A comparative study between two $\mathrm{C}_{3}$ cereals (rice and wheat) will help identify the core adaptive mechanisms and/or a suite of traits that render wheat to grow with less water and more tolerant of water-deficit stress. Such comparative analysis should target key morphological, physiological, anatomical and agronomic traits throughout the crop growth cycle, as water-deficit stress occurs at both early (vegetative stage) and late (reproductive stage) season in rice (Pandey et al., 2007). Extensive research efforts are currently ongoing to reduce the impact of water-deficit stress during the reproductive stage in rice (Venuprasad et al., 2008; Verulkar et al., 2010; Vikram et al., 2011; Kumar et al., 2014) and in wheat (Oliveras-Villegas et al., 2007; Lopes and Reynolds, 2010; Pinto et al., 2010). Therefore, our study focused on stress during the vegetative stage, to identify key checkpoints that determine whole-plant responses of representative rice cultivars adapted to lowland, upland/aerobic, or water-deficit conditions and of wheat cultivars with moderate to high water-deficit tolerance. Cultivars from both species, were exposed to moisture levels that resembles aerobic conditions and water-deficit stress during the vegetative stage. Our study follows a previous report that has successfully demonstrated the approach to expose rice and wheat to the same moisture stress conditions (Praba et al., 2009) and is designed to address the following specific objectives-(1) to quantify the adaptive plasticity in shoot and root morphology and biomass partitioning among different plant parts (leaves, stem, and root); (2) to estimate the key supportive physiological mechanisms such as whole-plant water use efficiency and leaf-level carbon isotope discrimination; and (3) to dissect root anatomical plasticity across different key zones in both rice and wheat roots exposed to water-deficit stress. Finally, novel traits that benefit dry-land adaptation in wheat compared with rice cultivars 
requiring more water are highlighted.

\section{Materials and Methods}

\section{Plant materials and growth conditions}

Greenhouse and controlled-environment experiments were conducted to compare the vegetative-stage water-deficit stress response of rice and wheat with emphasis on root morphological and anatomical plasticity. Three rice cultivars, rice 'IR64' (susceptible to waterdeficit stress), rice 'Apo' (aerobic/water-deficit tolerant), and rice 'N22' (water-deficit and high-temperature tolerant), were chosen for our study based on previous reports (Liu et al., 2006; Jagadish et al., 2011; Rang et al., 2011; Venuprasad et al., 2012). The two wheat cultivars selected were wheat 'SeriM82,' which is moderately susceptible (Pfeiffer, 1988) to tolerant of water-limited conditions (Villareal et al., 1995), and wheat 'Weebill4,' a highly droughttolerant check cultivar (Reynolds et al., 2007; Praba et al., 2009). Dormancy of rice seeds was broken after exposure to $50^{\circ} \mathrm{C}$ for 3 days, and pre-germinated seeds were sown in white-painted pots (55 cm long and $15 \mathrm{~cm}$ diameter) as recommended by Poorter et al. (2012) to minimize the confounding effects of increasing temperature of pot surface and soil. The pots were filled with $11 \mathrm{~kg}$ of clay loam soil and maintained under natural greenhouse conditions at the IRRI during the 2012 wet season (i.e. during the season when temperature in the greenhouse and pot can be controlled best). Each pot was drilled with holes on either side at the bottom for imposing controlled water-deficit stress and lined with polythene covers to facilitate easier separation of roots from soil at the end of the treatment. Simultaneously, wheat seeds were directly sown in pots with the same dimensions and maintained in controlled-environment large walk-in chambers (10.6- $\mathrm{m}^{2}$ area), built as an extension to the greenhouse where the rice plants were maintained. The chambers were maintained at day/night temperatures of $21^{\circ} \mathrm{C} / 18^{\circ} \mathrm{C}, 60 \%$ to $70 \%$ relative humidity, $16 \mathrm{~h} / 8 \mathrm{~h}$ light/dark cycle, and light at $650 \mathrm{mmol} \mathrm{m}^{-2} \mathrm{~s}^{-1}$, following Praba et al. (2009). Across both cereals and the treatments imposed, three replications were maintained and placed in a completely randomized design.

\section{Water-deficit stress imposition, cumulative water transpiration, and whole-plant WUE}

Both rice and wheat plants were maintained at two moisture regimes: control at $100 \%$ field capacity (FC) that is the maximum soil moisture content after drainage of excess waterresembling an aerobic condition and water-deficit stress at 55 to $60 \%$ FC. Water-deficit stress was imposed after seedling establishment, that is, 15 days after seedling emergence, before 
which all the pots were maintained uniformly at $100 \%$ FC. Pots with the control treatment were maintained at $100 \%$ FC throughout the experiment while water-deficit stress was imposed by unplugging the stoppers at the bottom of the pots. A standardized gravimetric approach of daily pot weighing (Raju et al., 2014) was followed to gradually attain 55 to $60 \%$ FC and thereafter maintained at the same level until the end of the experiment (for details, see Supplementary Figure S1). Once the target stress level was reached, daily consumed water due to transpiration was replenished by adding an exact amount of water to bring back the moisture content to the desired target in each pot. The soil surface was covered with a circular polythene sheet to protect from direct evaporative loss of water and a slit across the radius of the polythene sheet prevented heat buildup on the soil surface. In addition, a set of filled pots without a plant was also maintained to correct for evaporative loss of water from the opening created by the slit in the circular-shaped polythene sheet. Daily pot weights recorded for 30 consecutive days of stress period were used to calculate the daily evapo-transpiration. After correcting for evaporative loss from empty pots, actual transpiration was calculated. Finally, daily actual transpiration was summed for the 30-day period to calculate cumulative water transpired. Whole-plant water use efficiency $\left(\mathrm{g} \mathrm{kg}^{-1}\right)$ was calculated as the ratio of total biomass (root and shoot) to cumulative water transpired.

\section{Shoot morphology and leaf $\Delta^{13} \mathrm{C}$}

Following 30 days of stress, plants were harvested 45 days after sowing and tiller numbers were counted, and total leaf area was estimated by a leaf area meter (LI-3000, LI-COR, Lincoln, NE, USA). Leaves and stems were separately oven-dried at $70{ }^{\circ} \mathrm{C}$ for $72 \mathrm{~h}$ to compute specific leaf area and shoot biomass. Top-most fully expanded leaves from 4-5 tillers per plant were collected from control and from water-deficit-stressed pots immediately before relieving stress separately for three replications and oven-dried and ground to fine powder. Samples were analyzed for carbon isotope composition $\left(\delta^{13} \mathrm{C}\right)$ by a stable isotope ratio mass spectrometer (IRMS) facility available in the analytical service laboratory of IRRI (http://asl.irri.org/lims/). The analytical precision of the samples was within $0.1 \%$. Further, carbon isotope discrimination $\left(\Delta^{13} \mathrm{C}\right)$ value was calculated relative to the atmospheric ${ }^{13} \mathrm{C}$ isotopic composition $\left(\delta^{13} \mathrm{C}\right)$ as follows (Farquhar et al., 1989).

$$
\Delta^{13} \mathrm{C}=\frac{\delta^{13} C_{\mathrm{a}}-\delta^{13} C_{\mathrm{p}}}{1+\delta^{13} C_{\mathrm{p}} / 1000}
$$

where $\delta^{13} C_{\mathrm{a}}$ and $\delta^{13} C_{\mathrm{p}}$ denote the carbon isotope compositions of atmosphere (-8\%) and leaf 
sample, respectively.

\section{Root sample processing}

The entire column of soil along with the roots was placed on a $1 \mathrm{~mm}$ sieve and meticulously washed using a gentle stream of water to minimize the loss of small roots and root hairs. Rice root system is mainly composed of nodal roots and only one radicle or seminal root (primary root), with the latter growing to a maximum length of $15 \mathrm{~cm}$ and being viable until the 7-leaf stage. On the contrary, wheat develops and maintains several seminal roots until maturity (Yoshida and Hasegawa, 1982). To make meaningful comparison between rice and wheat, nodal root was investigated in our study. Across both rice and wheat cultivars, three replicate root sections $(2-3 \mathrm{~cm})$ were collected from three different positions along the nodal root for root anatomy study: (1) near the root-shoot junction (RSJ), (2) $\sim 15 \mathrm{~cm}$ from the root apex (RA) from water-deficit-stressed samples and $\sim 10 \mathrm{~cm}$ from RA on control samples following Henry et al. (2012), and (3) at $6 \mathrm{~cm}$ from RA in both treatments (Fig. 1A). Collected samples were stored in $40 \%$ alcohol to study root anatomy. The remaining whole-plant root samples were placed in $20 \%$ alcohol and stored at $4{ }^{\circ} \mathrm{C}$ for root scanning and image analysis.

\section{Root image acquisition and root morphology traits}

Root samples stored in $20 \%$ alcohol were cut meticulously to fit the scanner tray and aligned vertically in plates to avoid overlapping. An 8-bit gray-scale image was acquired by scanning with an EPSON perfection 7000 scanner at 600 dots-per-inch resolution next to a ruler. After capturing the image, root samples were oven-dried at $70^{\circ} \mathrm{C}$ for $72 \mathrm{~h}$ to record the total root biomass. Morphological attributes such as total root length, average root thickness, and root volume were computed by analyzing images with WinRHIZO Reg 2012b software (http://www.regent.qc.ca/assets/winrhizo_software.html). To avoid underestimation of fine root lengths during image processing, the threshold pixel was adjusted to automatic mode (Kato et al., 2010; Kato and Okami, 2011).

\section{Derived shoot and root growth parameters}

Leaf weight ratio (LWR), stem weight ratio (SWR), and root weight ratio (RWR) were calculated as a ratio of leaf, stem, and root weight to total biomass. Average specific leaf area was calculated as the ratio of total leaf area to leaf dry weight. Root length density was calculated as the ratio of total root length to the volume of soil in the pot, and total root weight 
density was calculated as the ratio of root length density to root biomass. Specific root length was calculated as the ratio of total root length to root biomass.

\section{Root anatomy and theoretically calculated axial conductance}

To investigate root anatomical features, samples stored in $40 \%$ ethanol obtained from three different positions along the root (Fig. 1A) were hand-sectioned with a razor blade under a dissecting microscope. Root sections were stained with $0.5 \% \mathrm{w} / \mathrm{w}$ phloroglucinol in water followed by $20 \%(\mathrm{~V} / \mathrm{V})$ hydrochloric acid (Jensen, 1962) for lignin staining. Images of the root sections were acquired with a Zeiss axioplan 2 compound microscope (Zeiss, Germany) with $50 \times$ and $100 \times$ magnification. At least 3-5 root images per replicate and tissue position were considered for measuring anatomical traits such as root cross-section diameter, stele diameter, late metaxylem diameter, and sclerenchyma with image J software (for details, see Abramoff et al., 2004). A schematic sketch of the different root anatomical traits measured using image $J$ is provided in Fig. 1B.

If the number of xylem vessels is $n$, their overall theoretical axial conductance $\left(\mathrm{K}_{\mathrm{h}} ; \mathrm{mg}\right.$ $\mathrm{m} \mathrm{MPa}^{-1} \mathrm{~s}^{-1}$ ) was calculated with the modified Hagen-Poisseuille's law described by Tyree and Ewers (1991) and Tombesi et al. (2010).

$$
K_{\mathrm{h}}=\frac{\pi \rho}{128 \eta} \sum_{\mathrm{i}=1}^{n} d_{\mathrm{i}}^{4}
$$

where $d_{\mathrm{i}}$ is the radius of the $i^{\text {th }}$ vessel in meters, $\rho$ is the fluid density (assumed to be $1 \times 10^{9} \mathrm{mg}$ $\mathrm{m}^{-3}$ ), and $\eta$ is the viscosity (assumed to be $1 \times 10^{-9} \mathrm{MPa} \cdot \mathrm{s}$ ).

\section{Statistical analysis}

The shoot and root morphological data were analyzed to check the significance level through analysis of variance (ANOVA) in Genstat release13 (https://www.vsni.co.uk/genstat), with cultivar and treatment as a main factor. But for root anatomy data, root tissue position was included in analysis as a factor along with cultivar and treatment.

\section{Results}

\section{Shoot morphology and whole-plant and leaf-level WUE}

A significant reduction in total leaf area, total biomass, and cumulative water transpiration was recorded under water-deficit stress in rice cultivars with a stronger reduction in the tolerant N22 $(P<0.001)$ and in both wheat cultivars $(P<0.01$ to $P<0.001$; Supplementary Table S1). Specific 
leaf area and tiller number decreased under water-deficit stress only in wheat cultivars $(P<0.001$; Fig. 2A and Supplementary Table S1). Whole-plant water use efficiency (WUE) increased in response to water-deficit stress in two out of three rice cultivars (IR64: 32\%; Apo: $16 \%)$ and in both wheat cultivars ( 40\%). The tolerant rice cultivar N22 recorded higher WUE than the other two cultivars in the absence of stress and was not altered by water-deficit stress; hence, significant cultivar and treatment interaction $(P<0.01$; Fig. $2 \mathrm{~B})$ was observed. Carbon isotope discrimination $\left(\Delta^{13} \mathrm{C}\right)$ of leaf is often used as a proxy to measure WUE (lower $\Delta^{13} \mathrm{C}$ means higher WUE; Impa et al., 2005). In both species, water-deficit stress had a strong effect on $\Delta^{13} \mathrm{C}(P<0.001)$, but there were no cultivar differences $(P>0.05)$. On average, $\Delta^{13} \mathrm{C}$ decreased by $6.3 \%$ in rice cultivars and by $8 \%$ in wheat cultivars. The absolute value of $\Delta^{13} \mathrm{C}$ was higher in wheat cultivars than in rice cultivars (Fig. 2C).

\section{Biomass partitioning among leaf, stem, and root}

In general, both species recorded higher biomass partitioning to leaf (LWR) and stem (SWR) than to root (RWR), with a higher proportion of biomass partitioned to roots in wheat than in rice (Supplementary Table S1). Leaf weight ratio (LWR) and stem weight ratio (SWR) varied only in rice cultivars $(P<0.01)$, with a significant effect of water-deficit stress $(P<0.05$ to $P<0.01)$. The susceptible IR64 had 16\% lower LWR and 24\% higher SWR with water-deficit stress. The tolerant N22 had lower LWR and higher SWR than other cultivars and was not altered by water-deficit stress. In both species, root weight ratio (RWR) did not differ significantly among cultivars and treatments $(P>0.05)$, but an increasing trend was observed with tolerant rice cultivar N22 and both wheat cultivars in water-deficit stress.

\section{Root morphology}

Root morphological traits such as maximum root length (MRL), total root length (TRL) and root length density (RLD) did not differ with either cultivars or stress treatments in rice $(P>0.05)$, but root volume (RV) and root biomass (RB) differed with both cultivars and treatments $(P<0.05$ to $P<0.01$; Supplementary Table S2). Conversely, in both wheat cultivars, the water-deficit stress treatment effect was highly significant for all the above-mentioned traits $(P<0.05$ to $P<0.001)$, but there were no cultivar differences. The MRL of the two wheat cultivars was increased in response to water-deficit stress compared with control conditions (Supplementary Table S2). 


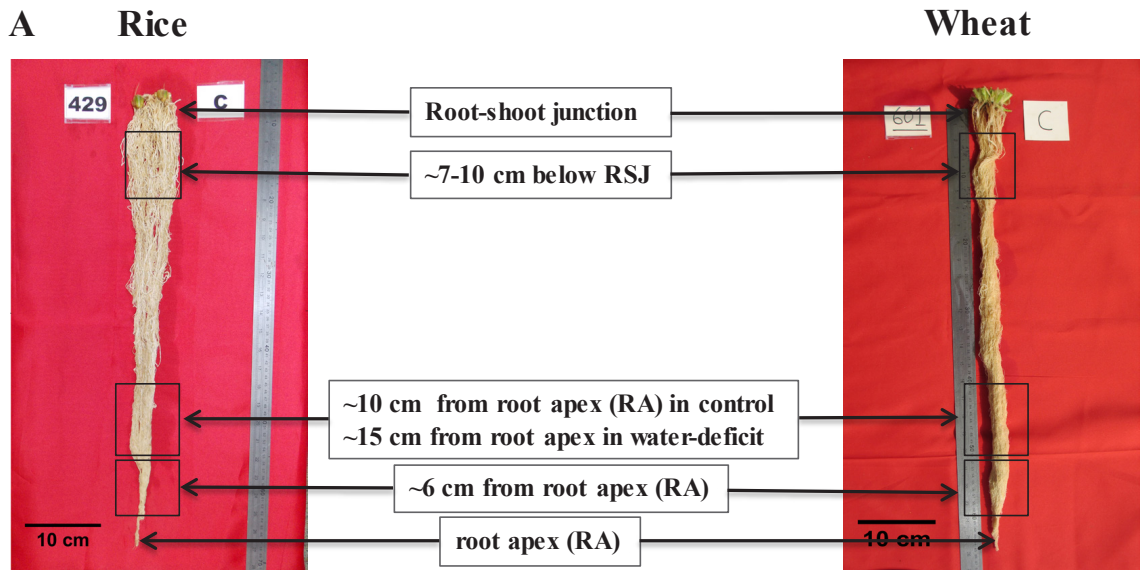

\section{B Rice Wheat}

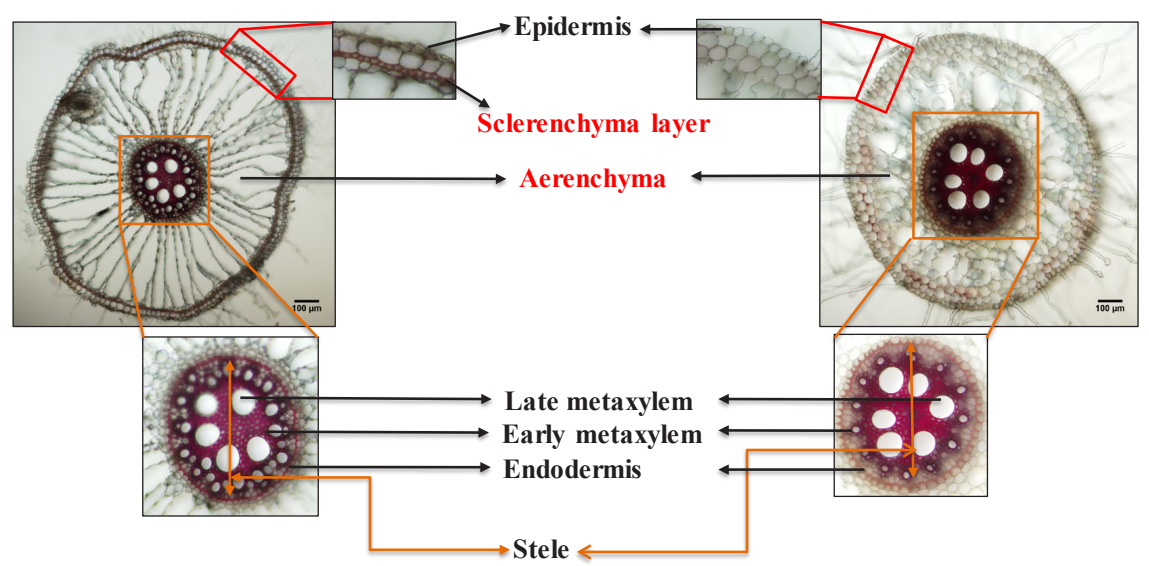

Figure 1. Root samples were collected in three different zones on nodal roots for anatomy study (Panel A). Radial root cross sections showing anatomical variation in rice and wheat (Panel B). Scale bars on root image $=10 \mathrm{~cm}($ Panel A) and $100 \mu \mathrm{m}($ Panel B).

\section{Specific root length, average root thickness, and total root weight density}

Specific root length (SRL), expressed as the ratio of root length to root biomass, is a key indicator of root thickness. In response to water-deficit stress, SRL increased significantly in two of the three rice cultivars (IR64: 59\%; Apo: 28\%), but decreased in both wheat cultivars by $\sim 40 \%$ (Fig. 3A). The SRL is independently controlled by two other components, root thickness and root weight density (Ostonen et al., 2007). Our results support this, with an increased SRL in rice cultivar IR64 determined mainly by reduced total root weight density (42\%), while in Apo this was due to a reduction in both average root thickness (15\%) and total root weight density (18\%). On average, the lower SRL in the wheat cultivars was due to a 
greater increase in total root weight density (68.5\%) than average root thickness (29\%; Fig. 3BC).

\section{Radial root anatomy}

To further confirm the observed variation in root morphology, we investigated root anatomical variables at three different locations along the root length (Fig. 1A-B). Note that root crosssections were stained with phloroglucinol to assess the secondary cell-wall thickening and lignin deposition under water-deficit stress. While it appears that there were changes in the staining pattern (e.g. Weebill4 under water-deficit treatment; Fig. 4), results were not consistent across replications in both the species and thus we will avoid discussing such changes.

\section{Root diameter}

Variation in root diameter is due to change in number and size/width of cortical cells and in stele diameter. In both species, root diameter varied significantly with cultivar $(P<0.05$ to $<0.001)$ and position along the root $(P<0.001)$. A significant effect of water-deficit stress on root diameter was documented with rice cultivars $(P<0.01$; Supplementary Table S3). Root diameter at the root-shoot junction (RSJ; see Fig. 1A) decreased with stress exposure in rice cultivar IR64 (25\%), with no change in the aerobic Apo and tolerant N22 (Fig. 4A). A clear pattern was not observed at 10 to $15 \mathrm{~cm}$ from the root apex (RA; Fig. 4B). However, an opposite response was observed at $6 \mathrm{~cm}$ from the RA, where the tolerant cultivars showed lower root diameter (N22: 19\%; Apo: 20\%), with no change in IR64 (Fig. 4C). Unlike in rice cultivars, root diameter at the RSJ increased significantly in both wheat cultivars (SeriM82: 42\%; Weebill4: 30\%), but at other two positions a decreasing trend was observed (i.e. 10 to $15 \mathrm{~cm}$ from RA and $6 \mathrm{~cm}$ from RA; Fig. 4).

\section{Stele diameter and stele diameter in proportion to root diameter (SD:RD)}

Stele is the central part of the root system that contains vascular tissue (i.e. xylem and phloem; Fig. 1B). Both cereals recorded a strong cultivar and spatial (different positions along the root) variation $(P<0.001)$ for stele diameter (Supplementary Table S3). Stele diameter at the RSJ did not differ in any of the rice cultivars (Fig. 5A), but the tolerant N22 maintained a higher stele diameter at 10 to $15 \mathrm{~cm}$ from RA and at $6 \mathrm{~cm}$ from RA (Fig. 5B-C). Additionally, stele diameter was more stable and was not affected by water-deficit stress in rice $(P>0.05)$. Unlike in rice, stele diameter increased significantly under water-deficit stress at the RSJ in wheat cultivars, 


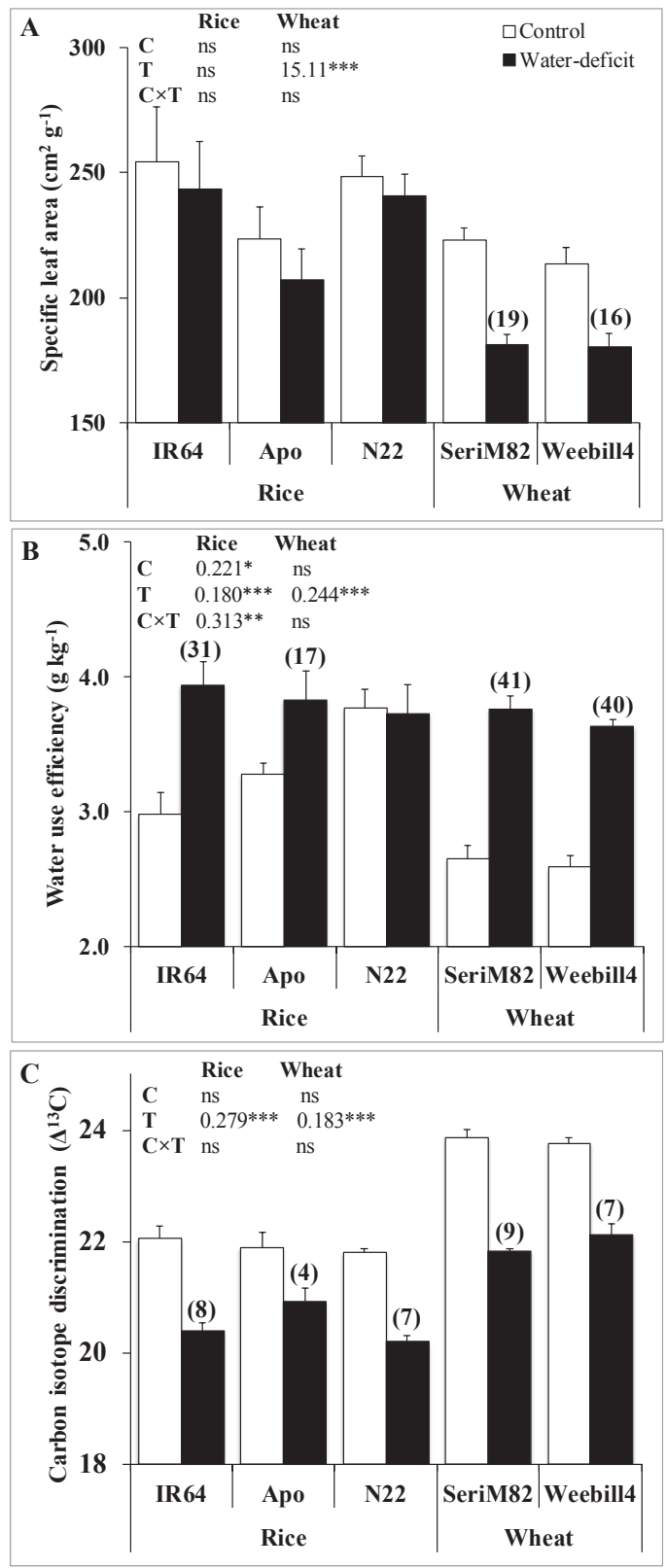

Figure 2: Specific leaf area (Panel A), whole-plant water use efficiency (Panel B), and carbon isotope discrimination $\left(\Delta^{13} \mathrm{C}\right.$; Panel $\left.\mathbf{C}\right)$ of rice and wheat. In the figure, the white column represents control and dark water-deficit stress. Values in parentheses represent the significant percentage change (increase or decrease) over the control. The analysis of variance results with least significant difference (LSD) value are given on panel for cultivar (C), treatment (T), and $\mathrm{C} \times \mathrm{T}$ interaction. Significance level: $* P<0.05$, $* * P<0.01, * * * P<0.001$. 

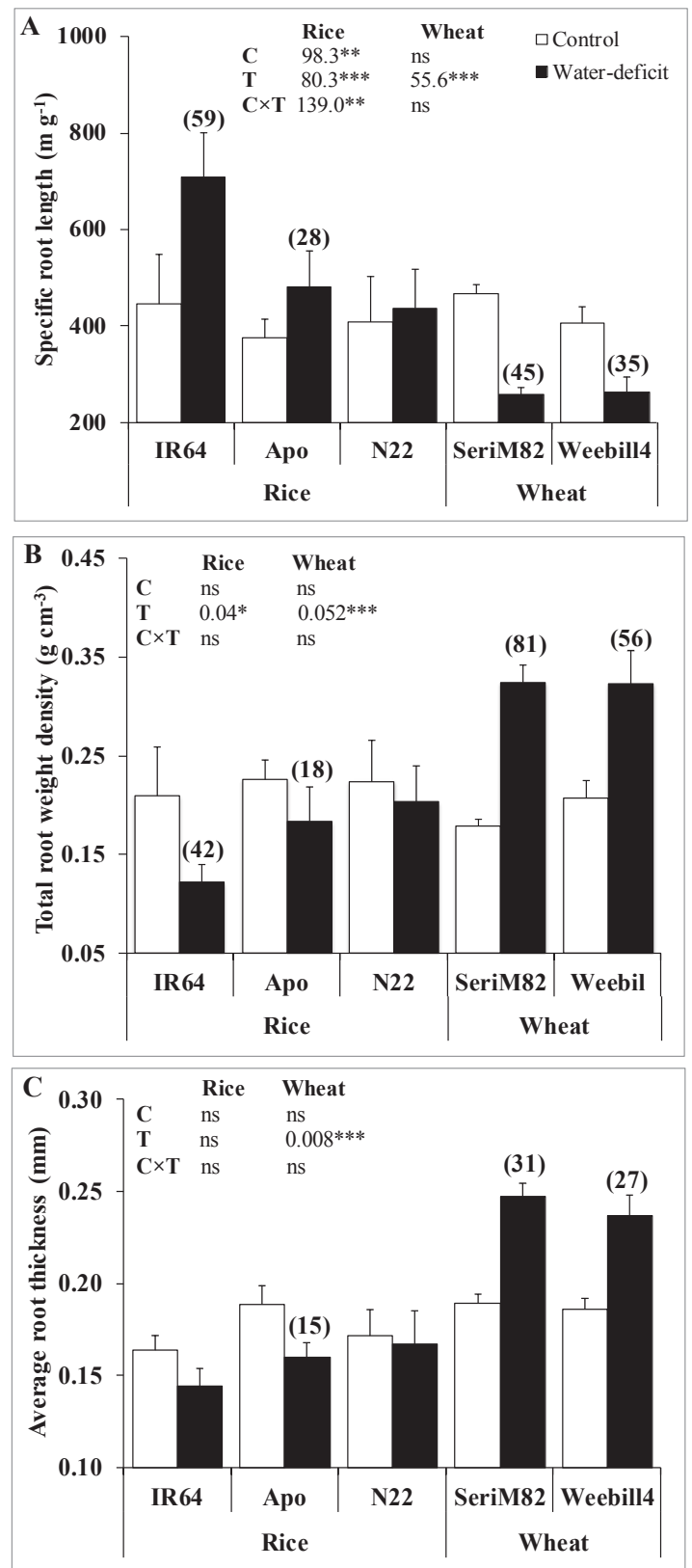

Figure 3: Specific root length (Panel A), total root weight density (Panel B), and average root thickness (Panel C) of rice and wheat. In the figure, the white column represents control and dark water-deficit stress. Values in parentheses represent the significant percentage change (increase or decrease) over the control. The analysis of variance results with least significant difference (LSD) value are given on panel for cultivar (C), treatment (T), and $\mathrm{C} \times \mathrm{T}$ interaction. Significance level: $* P<0.05, * * P<0.01, * * * P<0.001$. 

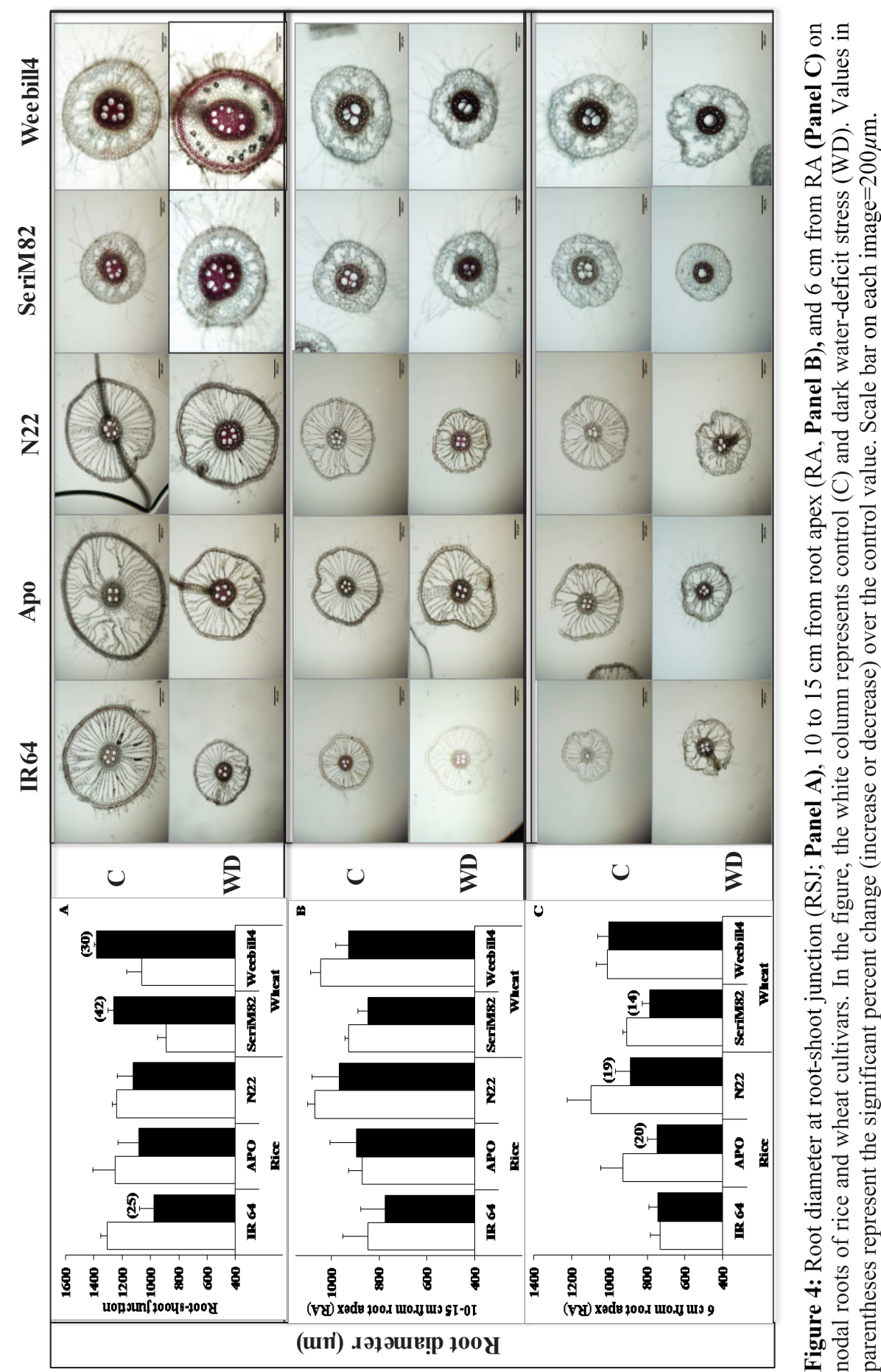
with SeriM82 (52\%) showing a greater increase than Weebill4 (33\%; Fig. 5A). Stele diameter responded in an opposite manner with a strong reduction at two other positions on roots (10 to $15 \mathrm{~cm}$ and $6 \mathrm{~cm}$ from the root apex) in SeriM82 and at 10 to $15 \mathrm{~cm}$ from RA only for Weebill4 (Fig. 5B-C).

Stele diameter in proportion to root diameter (SD:RD) was strongly affected by waterdeficit stress in rice $(P<0.001)$ and lacked cultivar and tissue position variation on nodal roots. By contrast, wheat cultivars documented a significant variation along the root tissue position $(P<0.001)$ and its interaction with treatment $(P<0.05$; Supplementary Table S3). Wheat cultivars maintained higher SD:RD ( $\sim 35$ to $40 \%)$ than rice cultivars ( $\sim 20$ to $25 \%)$ across three root positions sectioned (Fig. 6). An increasing trend with SD:RD was observed in response to water-deficit stress in all three rice cultivars (Fig. 6), but this was least affected by water-deficit stress in wheat, except for a noticeable reduction at 10 to $15 \mathrm{~cm}$ from RA for SeriM82 (Fig. $6 \mathrm{~B})$.

\section{Late metaxylem diameter and number}

Late metaxylem diameter (LMXD) remained relatively constant in rice cultivars across different root tissue positions under both control and water-deficit stress, with a narrow variation $(P<0.05)$ recorded among cultivars (Supplementary Table S3). The effect of waterdeficit stress on LMXD was not significant, but a decreasing trend was observed across all three rice cultivars near the RSJ (Fig. 7A). Late metaxylem number (LMXN) varied significantly with root tissue position $(P<0.01)$, cultivar, and treatment $(P<0.05)$ in rice. Among rice cultivars, lowland-adapted IR64 had lower LMXN at $6 \mathrm{~cm}$ from RA in non-stress conditions, but, upon exposure to stress, LMXN increased significantly and was like that of other cultivars (Fig. 7F). Unlike in rice, LMXD varied with cultivar and tissue position and their interaction in wheat $(P<0.001$; Supplementary Table S3). In both control and water-deficit stress, wheat cultivars maintained higher LMXD at 10 to $15 \mathrm{~cm}$ from RA and at $6 \mathrm{~cm}$ from RA compared with RSJ, except for SeriM82 recording a 28\% lower LMXD at 10 to $15 \mathrm{~cm}$ from RA under water-deficit stress (Fig. 7B). LMXD increased greatly in both wheat cultivars at $6 \mathrm{~cm}$ from RA under water-deficit stress exposure, with the increase being higher in Weebill4 (51\%) than in SeriM82 (30\%; Fig. 7C). Additionally, LMXN displayed strong interaction between treatment and tissue position $(P<0.001)$. Exposure to water-deficit stress resulted in an increase in LMXN at RSJ in wheat cultivars (Fig. 7D), but this decreased at two other positions, with a highly significant reduction observed at $6 \mathrm{~cm}$ from RA (Fig. 7E-F). According to Hagen- 


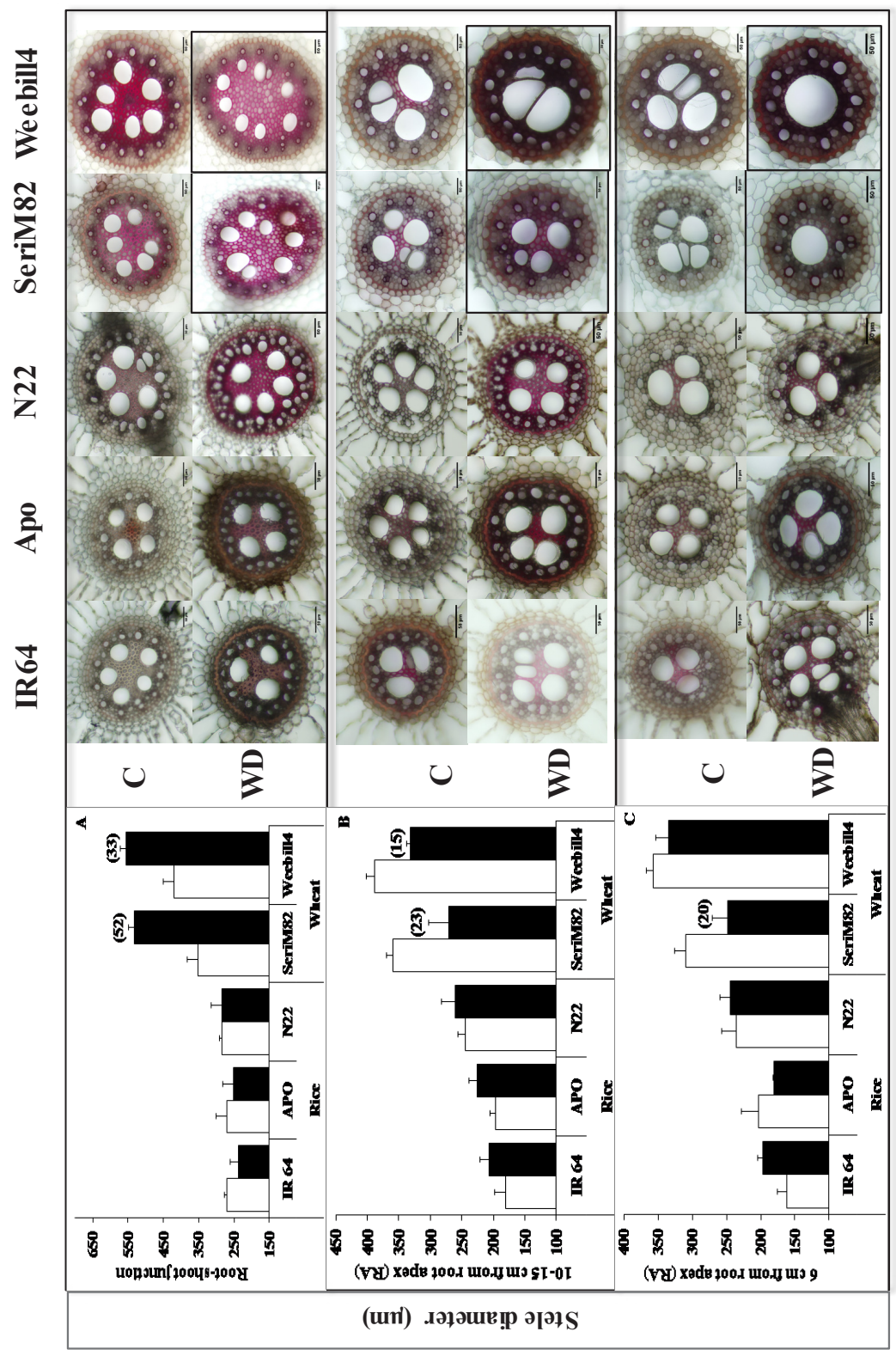

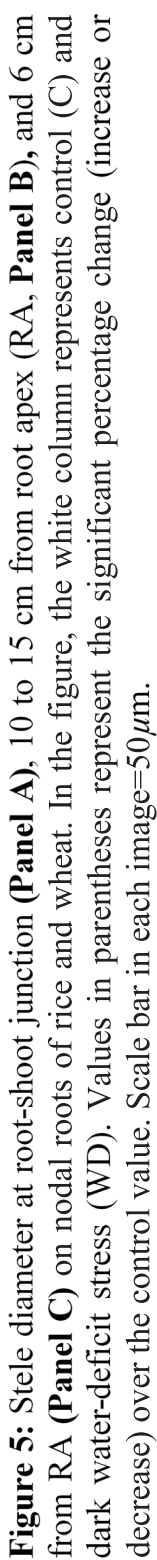



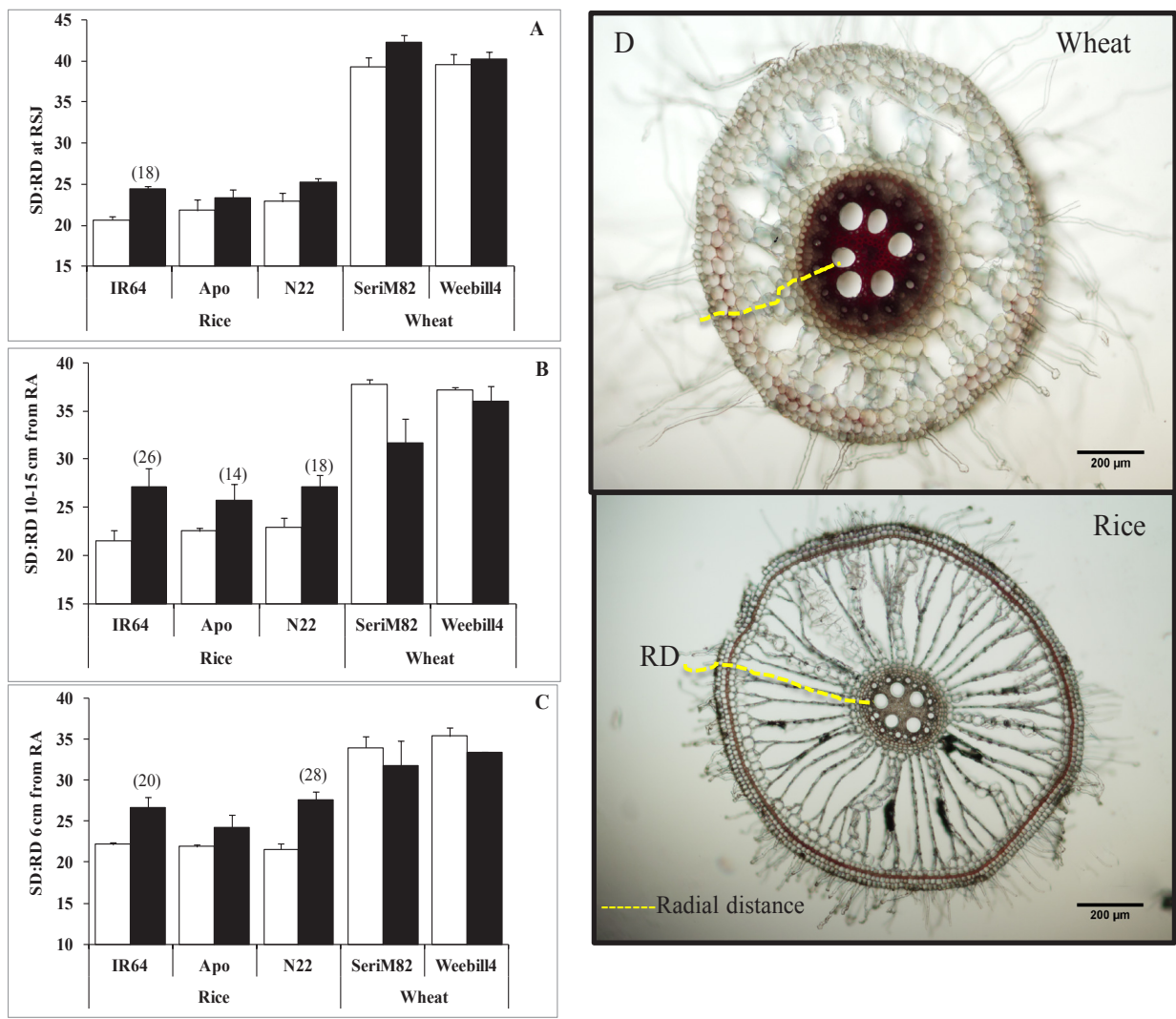

Figure 6: Stele diameter in proportion to root diameter (\%) (SD:RD) at root-shoot junction (RSJ, Panel A), 10 to $15 \mathrm{~cm}$ from root apex (RA, Panel B), and $6 \mathrm{~cm}$ from RA (Panel C) on nodal roots of rice and wheat. In the figure, the white column represents control and dark waterdeficit stress. A pictorial representation of radial distance in rice and wheat (Panel D). Values in parentheses represent the significant percentage change (increase or decrease) over the control value. Scale bar in image $=200 \mu \mathrm{m}$.

Poisseuille's law, the flow of water in any given conduit is the fourth power of the radius of the conduit. Theoretically calculated axial conductance by modified Hagen-Poisseuille's law also followed a pattern like that of LMXD across three different positions on nodal roots in both species (Fig. 8).

\section{Discussion}

We compared rice and wheat for their adaptive responses in root morphology and anatomy to water-deficit stress. Findings from our study are discussed below. 
Reduced specific leaf area is a determining factor for increased water use efficiency under stress in wheat

Reducing SLA in response to water-deficit stress to conserve water has been documented across crop species (Rao and Wight, 1994; Araus et al., 1997; Craufurd et al., 1999; Nautiyal et al., 2002), and the same was observed with wheat cultivars (Fig. 2A). Increased WUE under waterdeficit conditions is well known (Blum, 2009). Our results also documented increased WUE with cultivars of both species except for rice cultivar N22 under water-deficit stress; in wheat, this increased WUE (Fig. 2B) could be due to reduced SLA. Variation in $\Delta^{13} \mathrm{C}$ is determined by the balance between stomatal conductance $\left(\mathrm{g}_{\mathrm{s}}\right)$ and carboxylation efficiency (Farquhar et al., 1989). Both species had a comparable reduction in $\Delta^{13} \mathrm{C}$ in water-deficit stress (Fig. 2C), but possibly for different reasons. Lower SLA leads to higher carboxylation rate, but under water-deficit condition limitation of $g_{s}$ also reduces photosynthesis. Therefore, reduced $\Delta^{13} \mathrm{C}$ under water-deficit stress in wheat could be due to both lower $g_{s}$ and higher carboxylation rate (cf. Condon et al., 1990), but in rice due to lower $\mathrm{g}_{\mathrm{s}}$ only.

\section{Specific root length displays opposing responses among rice and wheat cultivars under water-deficit stress}

SRL captures the overall effect of both root thickness and root weight density (Fitter, 2002). In our study, SRL increased under water-deficit stress in rice cultivars (IR64 and Apo) because of reduced average root thickness, while lower SRL in wheat cultivars resulted from increased average root thickness and total root weight density (Fig. 3). Our results suggest that rice cultivars (except for N22) aimed for rapid water acquisition strategy, since thinner roots (higher SRL) increase overall root hydraulic conductance by exploring more soil volume for water and enabling rapid uptake of water (Reich et al., 1998; Eissenstat and Achor, 1999; Solari et al., 2006, Hernandez et al., 2010). This strategy could lead to higher susceptibility to water-deficit stress due to quicker water depletion (Ryser, 2006). On the other hand, the two wheat cultivars employed a conservative strategy by developing thicker roots and exploring less soil volume for water by reducing root length density. Thicker roots enhance soil penetrating ability to access deeper layers in drying soil conditions (Davis and Bacon, 2003). This can be substantiated by our results on maximum root length. Although full potential to express maximum root length of wheat could be influenced by limited pot size in our study, it however increased in response to water-deficit stress in wheat, while this was not the case with rice (Supplementary Table S2; Supplementary Figure S2). Among the rice cultivars, tolerant N22 

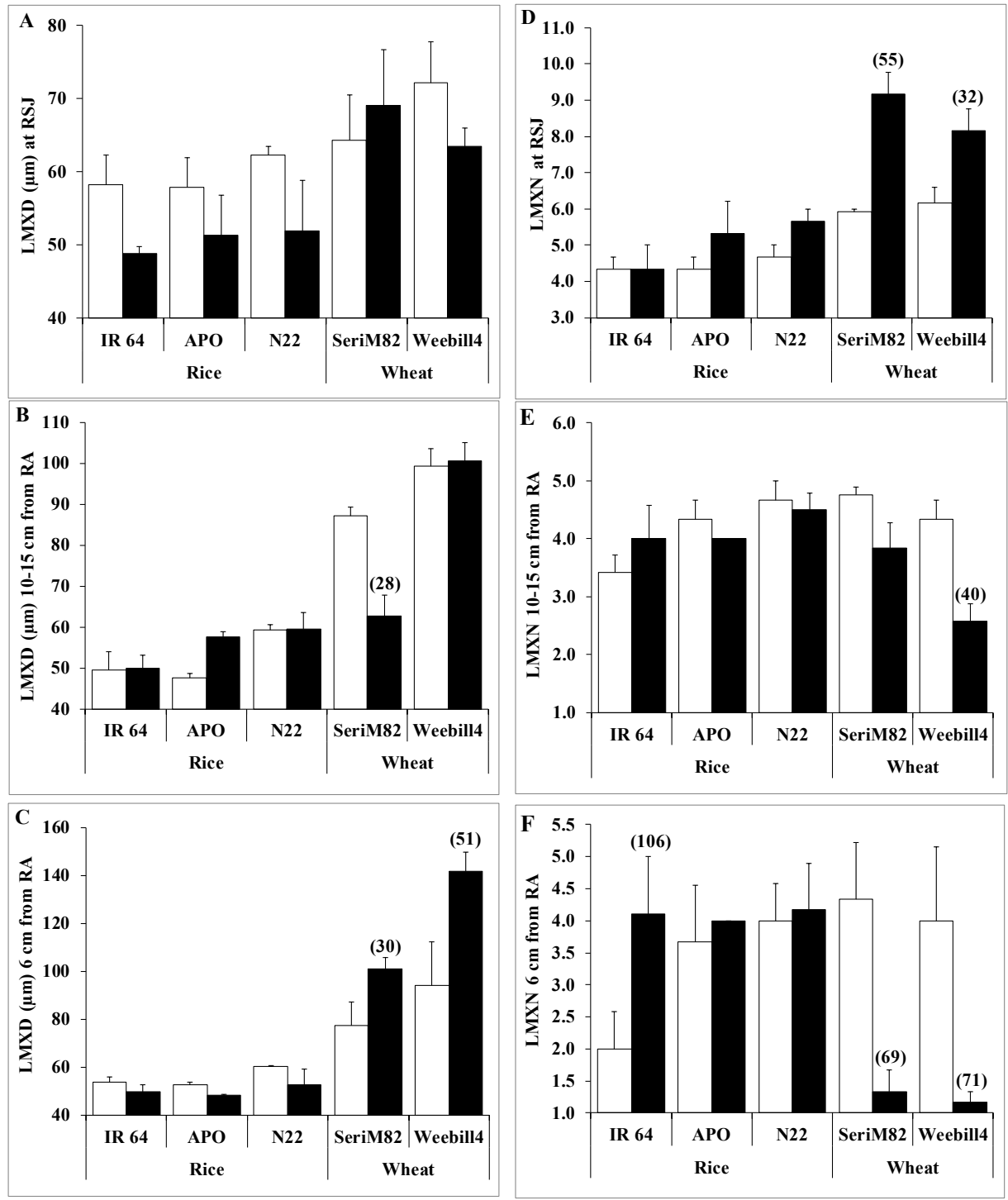

Figure 7: LMXD and LMXN at RSJ (Panels A \& D), 10 to $15 \mathrm{~cm}$ from root apex (RA, Panels B \& E), and $6 \mathrm{~cm}$ from RA (Panels $\mathbf{C} \& \mathbf{F}$ ) on nodal roots of rice and wheat (mean $\pm \mathrm{SE}$ ). White column represents control and dark water-deficit stress. Values in parenthesis represent the significant percentage change (increase or decrease) over the control value.

followed a conservative strategy by reducing root length density (Supplementary Table S2), but, unlike wheat, it lacked plasticity in SRL, average root thickness, and total root weight density (Fig. 3). 

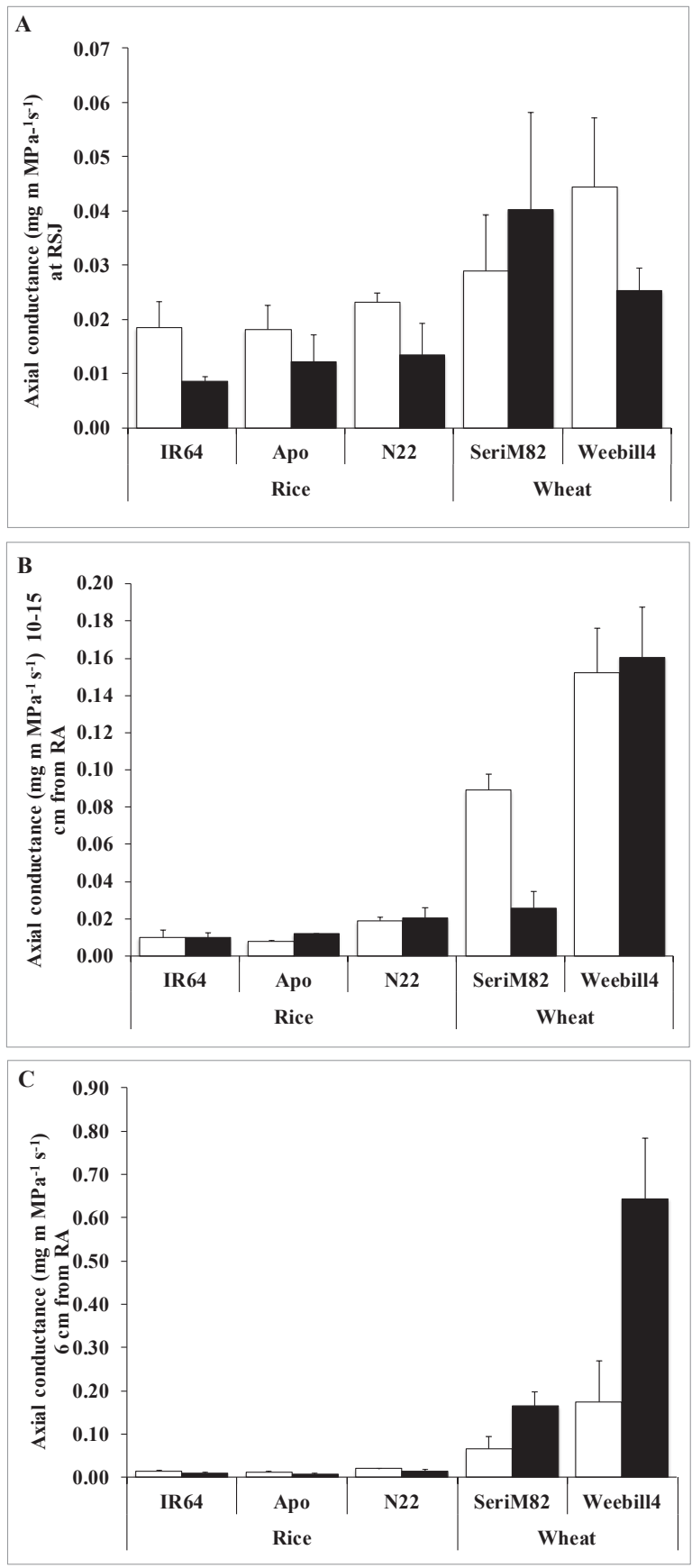

Figure 8: Theoretically calculated axial hydraulic conductance at root-shoot junction (RSJ) (Panel A), 10 to $15 \mathrm{~cm}$ from root apex (RA, Panel B), and $6 \mathrm{~cm}$ from RA (Panel C) on nodal roots of rice and wheat cultivars. In the figure, the white column represents control and dark water-deficit stress. 


\section{Stele diameter was more responsive to water-deficit stress in wheat than in rice}

The proportion of stele diameter to root diameter (SD:RD) provides an indirect measure of cortex tissue area/width. The stele size and SD:RD are lower in wetland than in dry-land plants (McDonald et al., 2002), and our result confirms this difference between rice and wheat (Figs. 5-6). This anatomical feature in wetland species aims to optimize the consumption of $\mathrm{O}_{2}$ under water-logging (Armstrong and Becket, 1987; Armstrong et al., 1991; Aguilar, 1998). A distinct sclerenchyma layer as an apoplastic barrier to impede radial oxygen loss was observed in rice, even in the absence of water-logging (Supplementary Figure S3). These root anatomical features in rice have an advantage under flooded conditions, but could affect root water uptake. An inverse relationship between overall radial hydraulic conductance and cortex width has been documented (Rieger and Litvin, 1999). Radial hydraulic conductance is lower in rice than in other cereals (Miyamoto et al., 2001), possibly because of larger aerenchyma in the cortex (Ranathunge et al., 2003). There was a significant position effect on the stele tissue, with higher stele diameter at the RSJ than at the other two positions (10 to $15 \mathrm{~cm}$ and $6 \mathrm{~cm}$ from RA) in both species but more conspicuously with wheat (Figs. 5-6). Although the exact ecophysiological significance of such gradient in stele tissue is unclear, it could play an important role in maintaining water uptake by improving internal aeration of roots, particularly near the root tip. The availability of $\mathrm{O}_{2}$ is known to decrease with increasing depth of the soil and smaller stele tissue tends to prevent $\mathrm{O}_{2}$ deficiency to support uninterrupted xylem transport (Gibbs et al., 1998). Further, water uptake by a region close to the root tip appears to be a predominant feature of all cereal roots (Greacen et al., 1976). An increased stele diameter near the RSJ in wheat may play a supportive role in water transport rather than in direct water uptake, but smaller stele diameter at the other two positions near the root apex (Fig. 5) could help in maintaining water uptake under stress. Contrary to this, stele diameter did not differ significantly under water-deficit in rice, but increasing trends of SD:RD were documented across all three positions along the root (Fig. 6). A similar increased SD:RD under water-deficit stress was previously identified in rice (Henry et al., 2012). The above response demonstrates the attempt of rice to reduce radial distance under water-deficit stress by decreasing cortex width (i.e. an increase in SD:RD without changing stele diameter) to improve radial hydraulic conductance (see Fig. 6D, pictorial representation of radial distance in rice $\&$ wheat). Together, the observed variation in root diameter under water-deficit in wheat was mostly due to a change in stele diameter, but in rice it was due to variation in cortex width (Fig. 4). Both wheat cultivars and the tolerant rice cultivar N22 maintained higher stele diameter, substantiating its role in 
water-deficit stress adaptation.

\section{Xylem developmental plasticity was more responsive to water-deficit stress in wheat than in rice}

The development of late metaxylem diameter (LMXD) and number (LMXN) varied strongly along the root length in wheat, with lower xylem diameter but a higher number near the RSJ and higher diameter but a lower number at 10 to $15 \mathrm{~cm}$ from RA and $6 \mathrm{~cm}$ from RA. Unlike in wheat, xylem diameter and number were least affected by either water-deficit stress or along the three different positions in rice roots (Fig. 7). Bulk flow of water or axial conductance is known to be closely related to the cross-sectional area or diameter of xylem vessels (Niklas, 1985). Hence, wheat cultivars would have higher water uptake than rice cultivars because of higher xylem diameter (Fig. 7), which was confirmed with calculated axial conductance (Fig. 8). Recently, it has been hypothesized that combining low axial conductance (narrow xylem diameter) at the base of the root system (i.e. closer to the RSJ) with higher axial conductivity (higher xylem diameter) near the root tips in deeper soil facilitates effective water use until flowering and grain development (Wasson et al., 2012). This is a pilot report, showing such a developmental gradient in xylem diameter along the root length in both wheat cultivars and it was confirmed with calculated axial hydraulic conductance. A large proportion of the lateral roots are generally developed towards the RSJ part compared to the root tip (Bramley et al., 2009). Therefore, under water-deficit stress increase in LMXN near the RSJ can help increase uptake of water by lateral roots from the top soil layers, but a strong decrease at the root tip (6 $\mathrm{cm}$ from RA) to conserve moisture in lower soil profiles. In summary, the response of xylem diameter and number to water-deficit stress in wheat was a novel finding and this provides additional mechanistic understanding of wheat root plasticity toward adapting to water-deficit stress.

\section{Conclusion}

A comprehensive analysis of two diverged species, one adapted to flooded conditions and the other to aerobic conditions, allowed us to demonstrate the functional role of organ/tissue plasticity for adapting to water-deficit stress. Both wheat cultivars had thicker leaves and roots and moderate tillering that help conserve soil moisture during vegetative-stage water-deficit stress (see summarized responses in Table 1). Plasticity in stele and xylem diameter, and xylem number along the root length in wheat cultivars facilitates efficient use of available moisture 
under water-deficit stress. Therefore, future studies should aim towards establishing the relationship between root morphology, anatomy with yield and yield components under waterdeficit conditions. 


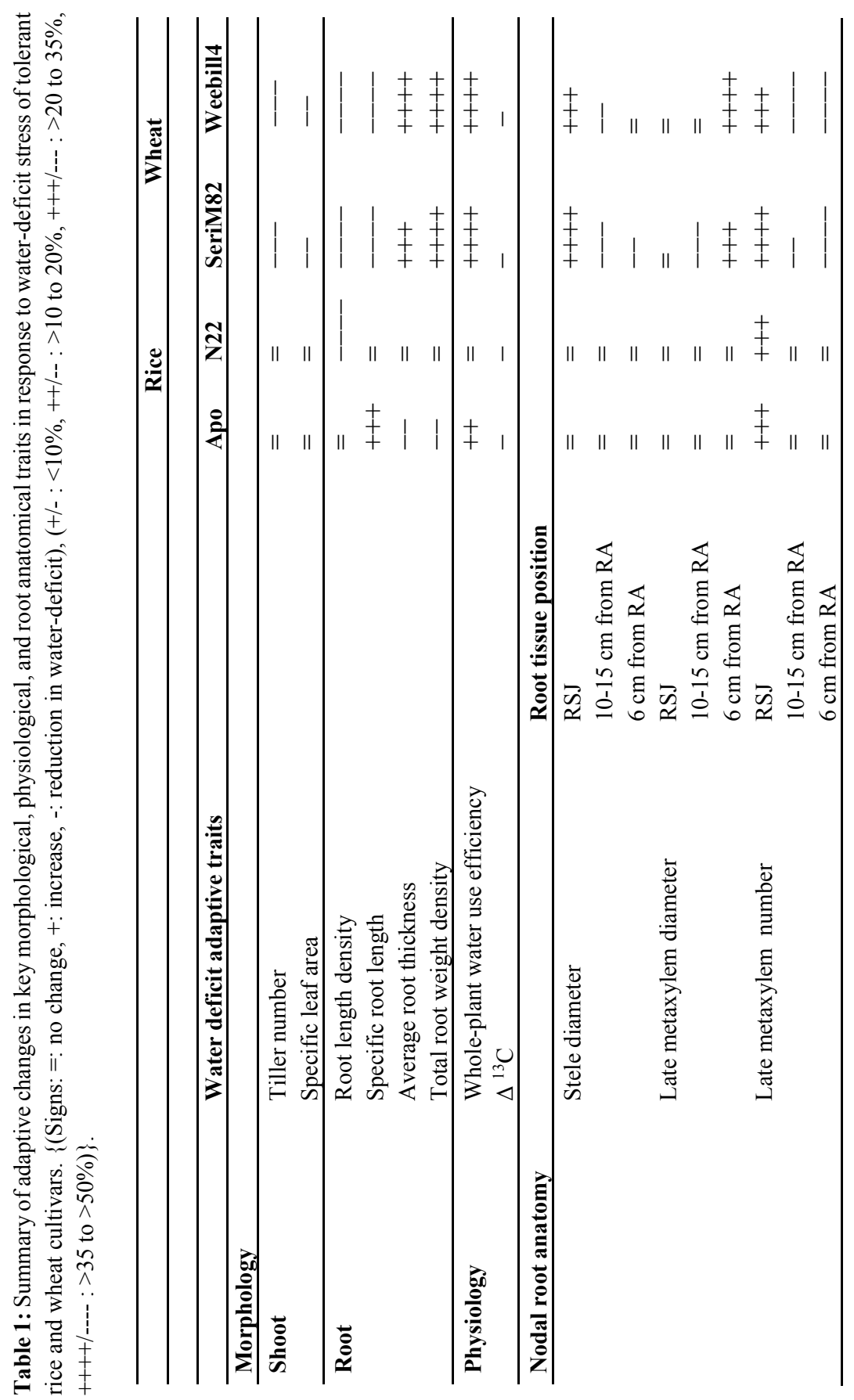


Supplementary information in Chapter 2 


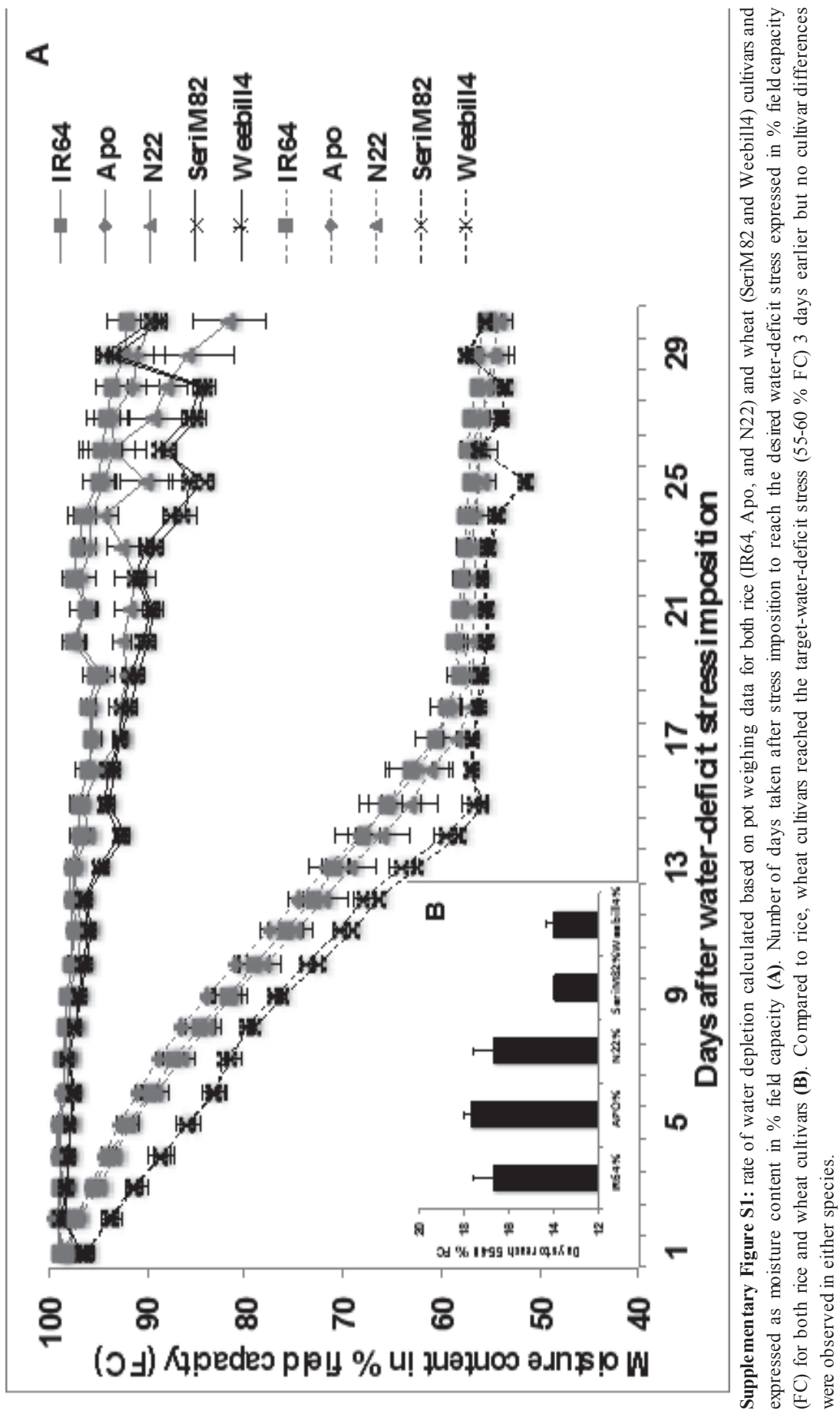




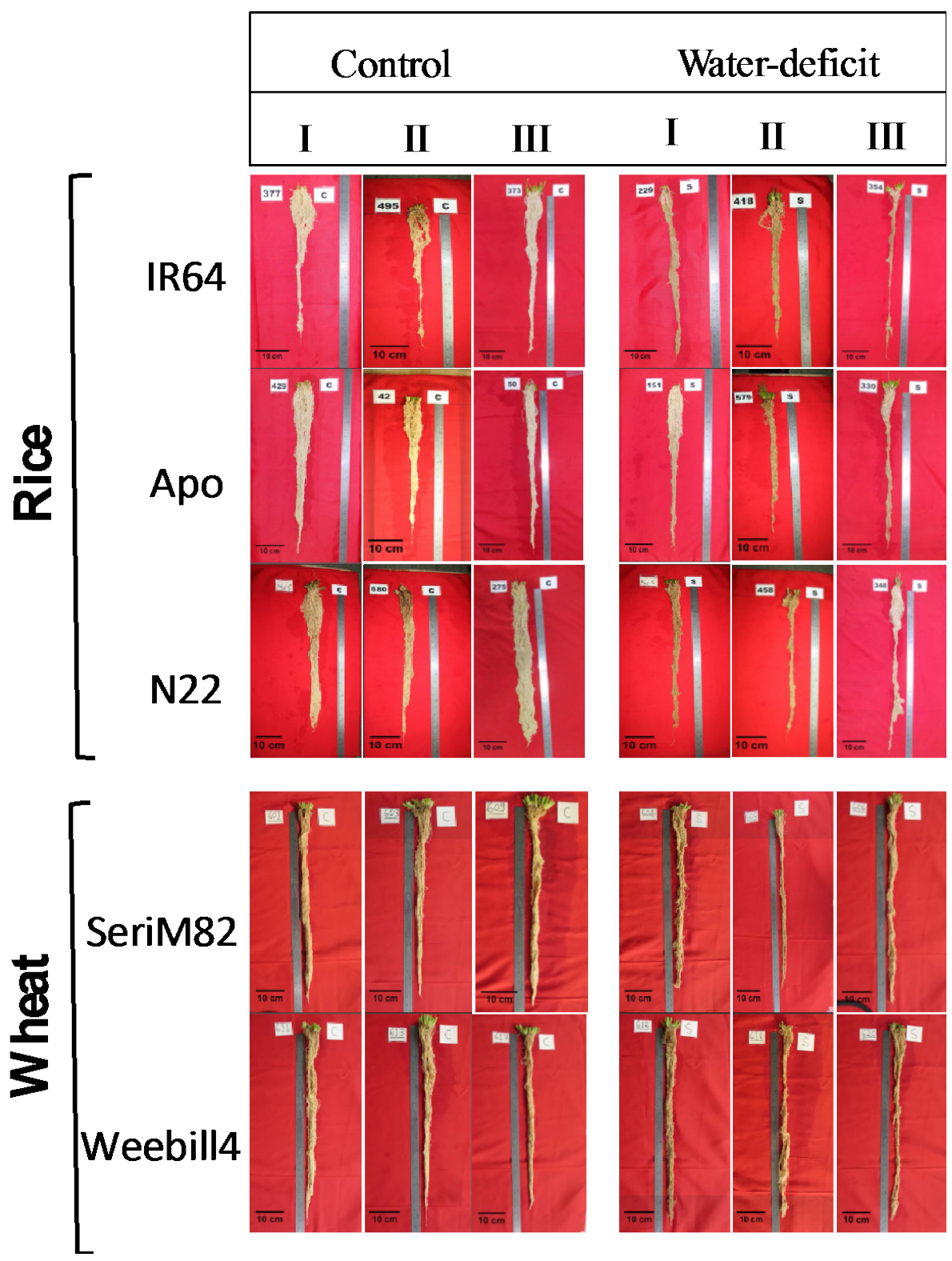

Supplementary Figure S2: Maximum root length (MRL) of rice (IR64, Apo, and N22) and wheat (SeriM82 and Weebill4) cultivars under control and water-deficit stress. Figure consists of replicated images $(n=3)$. Scale bar on each image represents $=10 \mathrm{~cm}$. 


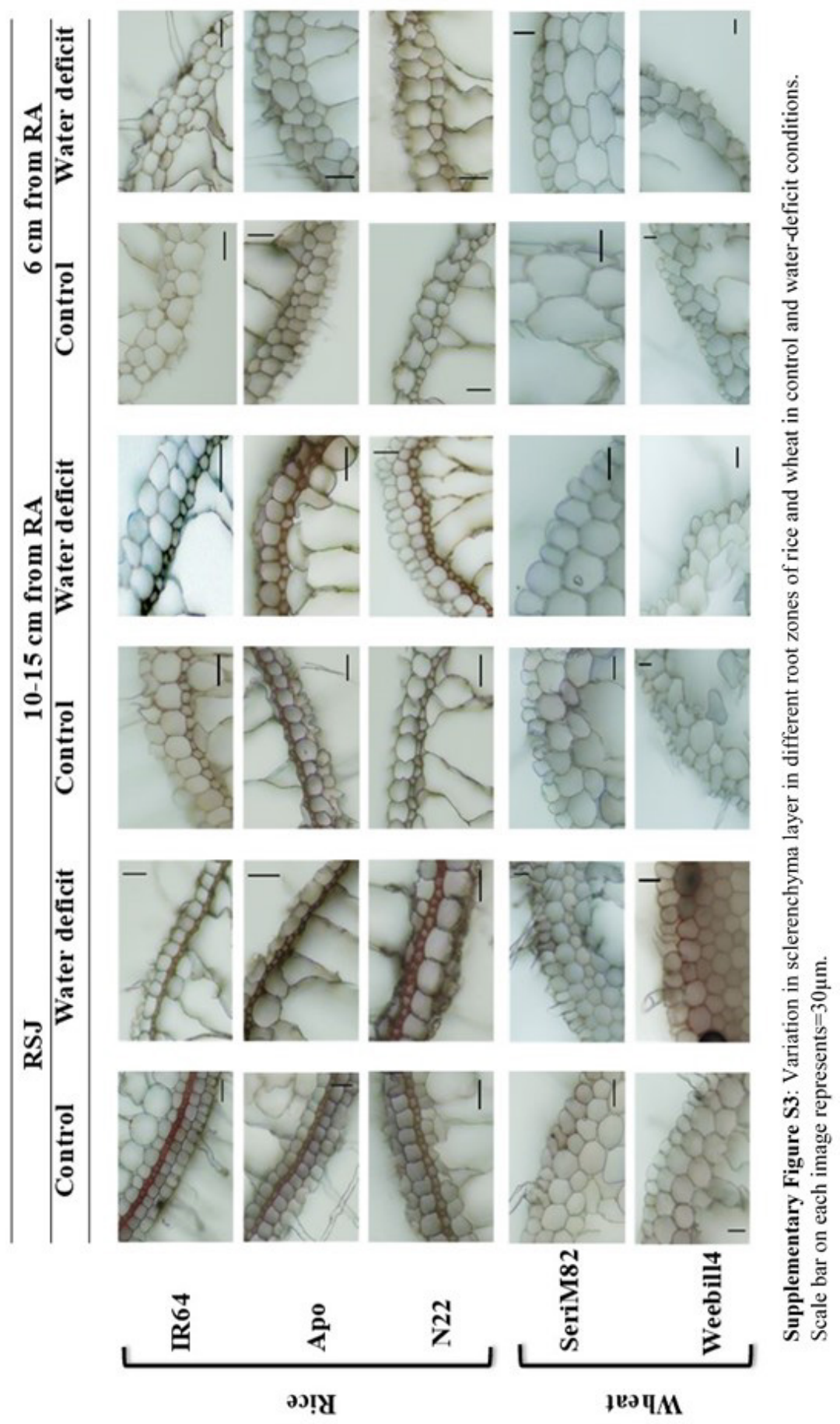




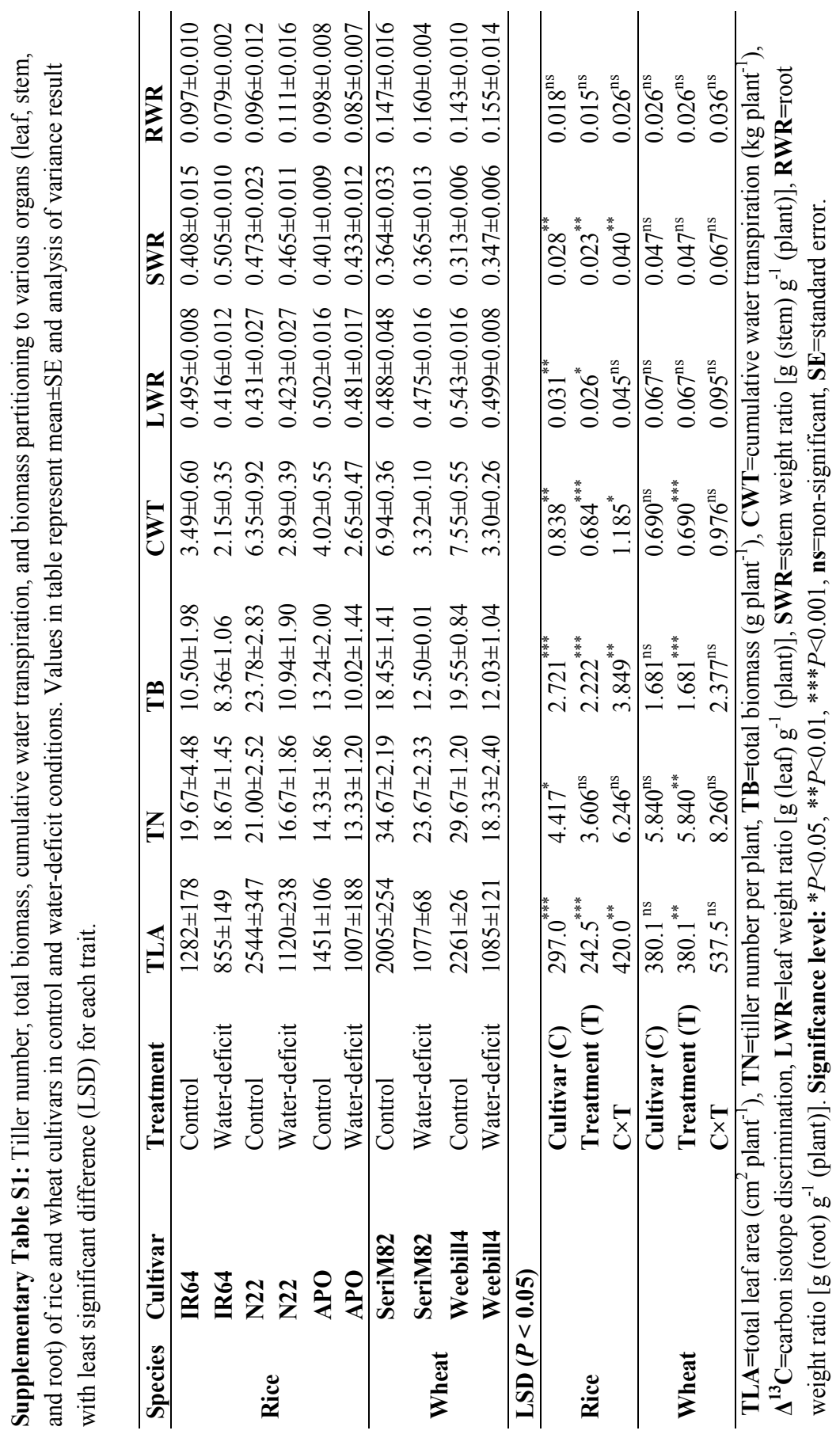




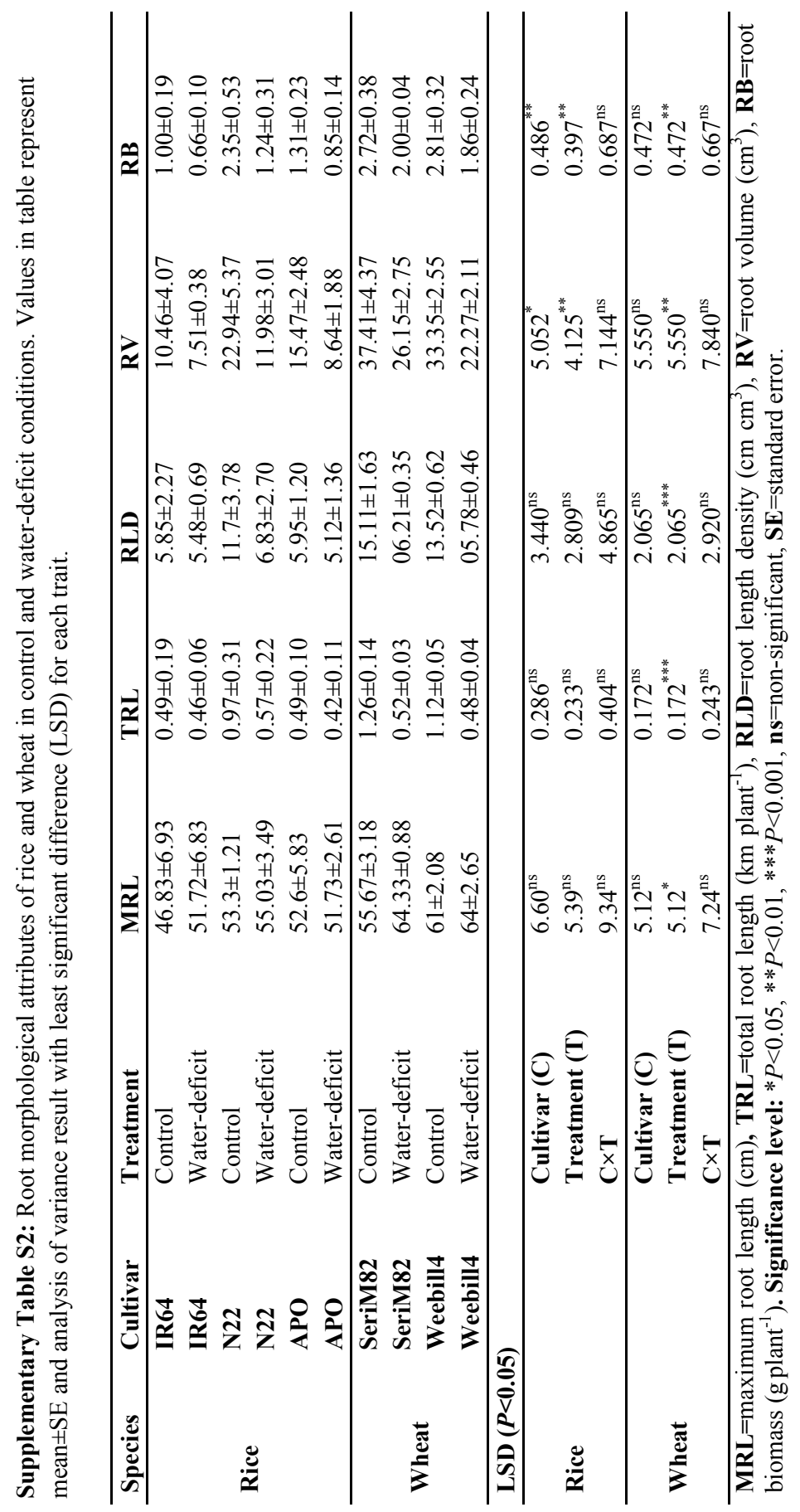




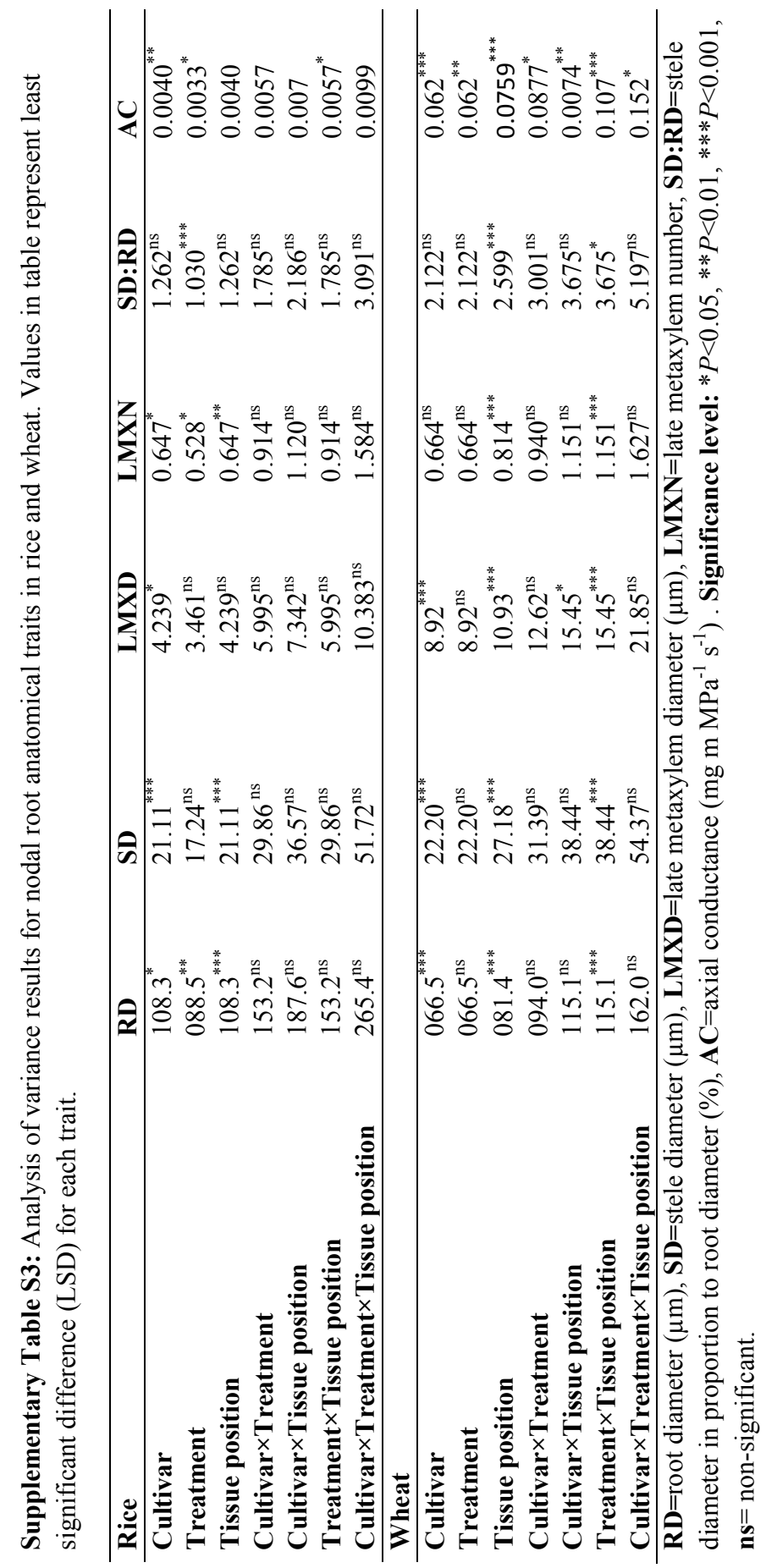





\title{
Genetic control of plasticity in root morphology and anatomy of rice in response to water-deficit
}

\begin{abstract}
Niteen N. Kadam ${ }^{1,2}$, Anandhan Tamilselvan ${ }^{1,3}$, Lovely M. F. Lawas ${ }^{1}$, Cherryl Quinones ${ }^{1}$, Rajeev N. Bahuguna ${ }^{1}$, Michael J. Thomson ${ }^{1,4}$, Michael Dingkuhn ${ }^{1}$, Raveendran Muthurajan ${ }^{3}$, Paul C. Struik ${ }^{2}$, Xinyou Yin ${ }^{2}$, Krishna S.V. Jagadish ${ }^{1,5}$
\end{abstract}

${ }^{1}$ International Rice Research Institute (IRRI), DAPO, Box 7777, Metro Manila, Philippines ${ }^{2}$ Centre for Crop Systems Analysis, Department of Plant Sciences, Wageningen University \& Research, PO Box 430, 6700 AK Wageningen, The Netherlands ${ }^{3}$ Tamil Nadu Agricultural University, Coimbatore, Tamil Nadu, India ${ }^{4}$ Department of Soil and Crop Sciences, Texas A \& M University, Texas, USA ${ }^{5}$ Department of Agronomy, Kansas State University, Manhattan, USA 


\begin{abstract}
Elucidating the genetic control of rooting behaviour under water-deficit stress is essential to breed climate-robust rice (Oryza sativa L.) cultivars. Using a diverse panel of 274 indica genotypes grown under control and water-deficit conditions during vegetative growth, we phenotyped 35 traits, mostly related to root morphology and anatomy, involving $\sim 45,000$ root scanning images and nearly $\sim 25,000$ cross-sections from the root-shoot junction. Phenotypic plasticity of these traits was quantified as the relative change in trait value under water-deficit compared to control conditions. We then carried out a genome-wide association analysis on these traits and their plasticity, using 45,608 high quality singlenucleotide polymorphisms. One hundred four significant loci were detected for these traits under control condition, 106 were detected under water-deficit stress, and 76 were detected for trait plasticity. We predicted 296 (control), 284 (water-deficit stress) and 233 (plasticity) a priori candidate genes within linkage disequilibrium (LD) blocks for these loci. We identified key a priori candidate genes regulating root growth and development and relevant alleles that upon validation can help improve rice adaptation to water-deficit stress.
\end{abstract}

Keywords: Oryza sativa L., root plasticity, linkage disequilibrium, loci, a priori candidate genes, multilocus analysis. 


\section{Introduction}

Increasing water scarcity, caused by global climate change and increasing competition for available water resources, is a major constraint for crop production and global food security (Rosegrant et al., 2009). Rice (Oryza sativa L.) is the most important staple cereal. It requires two-three times more water than dryland cereals, as it is grown predominately under flooded paddy cultivation. Improving rice adaptation to water-deficit conditions could support developing dryland rice production systems, thereby reducing the dependence of rice on large volumes of water. Therefore, current rice breeding programmes are striving to develop cultivars that are productive under water-deficit conditions (Bernier et al., 2009; Kumar et al., 2014; Sandhu et al., 2014). This will require a suite of morphological, anatomical and physiological adjustments of shoot and root traits (Kadam et al., 2015; Sandhu et al., 2016). Interactions among these traits in response to water-deficit are complex, rendering effective knowledgeintensive breeding strategies.

To adapt to water-deficit stress, rice needs to be plastic. Phenotypic plasticity is a characteristic of a given genotype to produce a distinct phenotype in response to changing environments (Nicotra et al., 2010). Mostly, the plasticity of traits is desirable for better stress adaptation. Both natural and human selection have created many rice types that are sensitive and tolerant to water scarcity and have different levels of (desired or undesirable) plasticity. Climate change and increased water scarcity demand a new compromise among stress resistance, stress escape or avoidance, and potential productivity through phenotypic plasticity. Previous studies have shown the role of root trait plasticity in improving water-deficit stress adaptation. For instance, the plasticity of root-length density in water-deficit stress contributes to rice grain yield stability (Sandhu et al., 2016). Similarly, the comparative analysis between water-deficit tolerant rice and wheat (Triticum aestivum L.) has demonstrated the functional relevance of plasticity in shoot and root traits to better adapt to water-deficit stress (Kadam et al., 2015). However, phenotypic traits that express constitutively with no plasticity could also provide stress adaptation. For example, changes in the root angle during early development resulted in constitutive expression of deep root architecture that helps in later stages to increase rice grain yield under water-deficit (Uga et al., 2013).

Although phenotypic plasticity is heritable (Nicotra and Davidson, 2010), plasticity per $s e$ is usually not targeted when breeding rice for water-deficit conditions. Breeding for plasticity in traits other than yield would offer alternative routes to enhance resilience to stress conditions (Sambatti and Caylor, 2007) and to tap into a larger rice genetic diversity pool for adapting to 
stressful environments (McCouch et al., 2013). The plasticity of traits is controlled by key environment-sensing genes (Juenger, 2013). Yet, no study has been undertaken to comprehensively demonstrate the quantitative variation in root and shoot plasticity and the underlying genetic control using diverse rice genotypes grown under water-deficit stress.

We report here a genome-wide association study (GWAS) in rice to unravel the genetic control of phenotypic traits in control and water-deficit stress and their plasticity. Given our diverse indica rice panel, which incorporates more evolutionary recombination events compared with biparental mapping populations (Ingvarsson and Street, 2011), we expect to detect phenotype associations with narrow genomic regions or even nearby/within causal genes. Specific objectives were (1) to assess natural genetic variability in root and shoot morphological and anatomical traits in control and water-deficit conditions and their plasticity as a relative change, (2) to associate genetic variation in root and shoot phenotypic plasticity with adaptive significance under water-deficit stress, and (3) to elucidate the genetic architecture of phenotypic traits and their plasticity by identifying the genomic loci with underlying a priori candidate genes.

\section{Materials and Methods}

\section{Plant materials}

For our GWAS study, we used a diverse collection of 274 genotypes covering traditional and improved indica rice sub-species, originating from major rice growing countries of tropical regions (Supplementary Figure S1 and Supplementary Data Set S1). This panel was carefully assembled at the International Rice Research Institute (IRRI) for the Phenomics of Rice Adaptation and Yield potential (PRAY) project for use in GWAS studies (Al-Tamimi et al., 2016; Rebolledo et al., 2016; Kikuchi et al., 2017) in the context of the GRiSP Global Rice Phenotyping Network (http://ricephenonetwork.irri.org/).

\section{Stress imposition and plant growth conditions}

A pot experiment was carried out in natural greenhouse conditions at the International Rice Research Institute (IRRI), for phenotyping root and shoot traits under two moisture regimes: (1) control, i.e., $100 \%$ field capacity (FC), which is defined as the maximum soil moisture content after draining excess water, and (2) water-deficit stress at 55 to $60 \% \mathrm{FC}$. The experiment was laid out in a randomized complete block design and replicated over three different time periods, due to space and labour constraints, during 2012 and 2013 
(Supplementary Figure S2A). Before sowing, rice seeds were exposed to $50{ }^{\circ} \mathrm{C}$ for 3 days to break dormancy and pregerminated seeds were sown in white-coloured painted pots $(55 \mathrm{~cm}$ long and $15 \mathrm{~cm}$ diameter) to minimize confounding effects of increasing temperature of pot surface and soil (Poorter et al., 2012). The pots were lined with polythene bags on the inside, filled with $11 \mathrm{~kg}$ of clay loam soil, and care was taken to avoid over compaction of the soil. Each pot had two holes at the bottom for imposing controlled stress. Water-deficit stress was imposed 15 days after seedling emergence (after ensuring healthy seedling establishment) and until then all pots were maintained at 100\% FC (Supplementary Figure S2B). A standardized gravimetric approach of daily pot weighing (Kadam et al., 2015) was followed on 1649 (5 pots were empty to measure evaporation) pots to gradually attain 55 to $60 \% \mathrm{FC}$ and thereafter maintained at the same level until the end of the experiment (Supplementary Figure S2C). Once the target stress level was reached, daily water loss due to evapotranspiration was replenished by adding back an exact amount of water to bring back the moisture content to the desired target in each pot. The soil surface was covered with a circular polythene sheet to protect direct evaporative loss of water and a slit across the radius of the polythene prevented heat build-up on the soil surface. Additionally, a set of soil-filled pots without a plant was also maintained to correct for evaporative loss of water from the opening created by slit in the circular shaped polythene sheet. Daily pot weights recorded for 30 consecutive days of stress period were used to calculate the daily evapotranspiration. After correcting for evaporative loss obtained from empty pots, actual transpiration was calculated. Finally, daily actual transpiration was summed for the 30-day period to calculate cumulative water transpired. Whole plant water use efficiency $\left(\mathrm{g} \mathrm{kg}^{-1}\right)$ was calculated as a ratio of total weight (root and shoot) to cumulative water transpired. Air temperature and humidity were constantly measured at 10-minute intervals by sensors installed in the greenhouse. The average daily temperature (day and night) and air humidity were recorded (Supplementary Figure S2D).

\section{Shoot and root harvesting}

After 30 days of water-deficit stress exposure, plants were harvested at 45 days after sowing and tiller numbers were counted and total leaf area was estimated by a leaf area meter (Li-3000, LI-COR, Lincoln, NE, USA). Leaves and stems were separately oven-dried at $70{ }^{\circ} \mathrm{C}$ for $72 \mathrm{~h}$ to compute the specific leaf area and shoot weight. The entire column of soil along with the roots was placed on a large $1 \mathrm{~mm}$ sieve and meticulously washed using a gentle stream of water to minimize the loss of small roots and root hairs. 
A strong plasticity in wheat root anatomy primarily near root-shoot junction (RSJ) and root tips under water-deficit stress has been confirmed following a similar approach (Kadam et al., 2015). Hence, three replicate root sections were collected near the RSJ $(\sim 7-10 \mathrm{~cm})$ from control $(274 \times 3=822)$ and water-deficit stressed $(274 \times 3=822)$ samples (1644 samples). Collected samples were stored in $40 \%(\mathrm{v} / \mathrm{v})$ alcohol to assess root anatomy. The remaining whole-plant root samples were placed in $20 \%$ (v/v) alcohol and stored at $4{ }^{\circ} \mathrm{C}$ for root scanning and image analysis.

\section{Root image acquisition and processing in WinRHIZO}

Root samples stored in $20 \%(\mathrm{v} / \mathrm{v})$ alcohol were cut to smaller segments to fit the scanner tray and aligned vertically on scanning plates to avoid overlapping (Supplementary Figure S3). An eight-bit greyscale image was acquired by scanning with an Epson Perfection 7000 scanner at a resolution of 600 dots per inch next to a ruler. After capturing the images, root samples were oven dried at $70{ }^{\circ} \mathrm{C}$ for $72 \mathrm{~h}$ to record the root weight. In total, we captured $\sim 45,000$ images from 274 genotypes across treatments and replications. The root morphological attributes such as total root length, average root thickness, root length classified based on root thickness, root volume, root surface area was computed by analysing images with WinRHIZO Reg 2012b (Supplementary Figure S3) software (http://regent.qc.ca/assets/winrhizo_about.html). To avoid underestimation of fine root lengths during image processing, the threshold that separates the roots and background was adjusted to automatic mode (Bouma et al., 2000).

\section{Root anatomical study}

To study the root anatomical parameters near root-shoot junction $(\sim 7-10 \mathrm{~cm}$; Supplementary Figure S4), samples stored in $40 \%$ alcohol were hand sectioned with a razor blade under the dissection microscope. Images of root sections were acquired with Zeiss Axioplan 2 compound microscope (Zeiss, Germany) with $50 \times$ and $100 \times$ magnification. At least three to five root images per replicate were considered for measuring anatomical parameters such as root crosssection diameter, stele diameter and late meta xylem diameter, with image $\mathrm{J}$ software (Schneider et al., 2012).

\section{Derived shoot, root and water uptake parameters}

Average specific leaf area was calculated as the ratio of total leaf area to leaf dry weight. Ratios of leaf weight, stem weight and root weight to total weight were also calculated. Root length 
density was calculated as the ratio of total root length to the soil volume in pot, and total root weight density was calculated as the ratio of root weight to root length density. Specific root length was calculated as the ratio of total root length to root weight. Root length per unit leaf area was calculated as the ratio of total root length to leaf area.

\section{Calculation of phenotypic plasticity}

The phenotypic plasticity of all traits was calculated as a relative change in water-deficit stress compared with control conditions, using the following formula (Sandhu et al., 2016).

$$
\text { Phenotypic plasticity }=\frac{\text { stress }- \text { control }}{\text { control }}
$$

To distinguish trait plasticity from the trait per se, all acronyms for plasticity starts with lowercase letter "r" (Table 1).

\section{Statistical data analysis}

The observed variation in a phenotypic trait can be partitioned to a source of variation in genotype $(\mathrm{G})$, treatment $(\mathrm{T})$ and their interaction $(\mathrm{G} \times \mathrm{T})$. The analysis of variance was performed using mixed linear model (MLM) for each phenotypic trait in Genstat release 17.1, as defined by

$$
\mathrm{y}_{\mathrm{ijk}}=\mu+\mathrm{G}_{\mathrm{i}}+\mathrm{T}_{\mathrm{j}}+(\mathrm{G} \times \mathrm{T})_{\mathrm{ij}}+\mathrm{r}_{\mathrm{k}(\mathrm{j})}+\mathrm{e}_{\mathrm{ijk}}
$$

where $y_{i j k}$ is the measured trait, $\mu$ is the overall mean, $G_{i}$ is the effect of $i^{\text {th }}$ genotype, $T_{j}$ is the effect of $\mathrm{j}^{\text {th }}$ treatment, $(\mathrm{G} \times \mathrm{T})_{\mathrm{ij}}$ is the interaction between $\mathrm{i}^{\text {th }}$ genotype and $\mathrm{j}^{\text {th }}$ treatment, $\mathrm{r}_{\mathrm{k}(\mathrm{j})}$ is the effect of replication $\mathrm{k}$ within the $\mathrm{j}^{\text {th }}$ treatment and $\mathrm{e}_{\mathrm{ijk}}$ is the random error. Genotypic and treatment effects were considered as fixed effect with their interaction $(\mathrm{G} \times \mathrm{T}$ term $)$ in the model, and replications were treated as random effect. The best linear unbiased estimator (BLUE) value of each phenotypic trait was computed separately across treatments by MLM. The BLUE value of traits was later used for histograms, box plots, principal component analysis (PCA) and Pearson's correlation analysis. The PCA analysis was performed in XLSTAT and correlation heat maps were compiled using the $\mathrm{R}$ package "corrplot" in $\mathrm{R}$ studio. The $P$ values of correlation coefficient were calculated by two-sided t-test using the cor.mtest function in $\mathrm{R}$ and only significant $(P<0.05)$ correlation was plotted on the heat maps. 


\section{SNPs genotyping data}

The studied panel is a large subset of 329 indica genotypes that were genotyped using the genotype-by-sequencing (GBS) protocol (Elshire et al., 2011) at Cornell University, USA. The reads were demultiplexed and aligned to the rice reference genome (Os-Nipponbare-ReferenceIRGSP-1.0; Kawahara et al., 2013), and variants were identified using the NGSEP pipeline (Duitama et al., 2014). Missing data were imputed with the implementation of the Fast Phase Hidden Markov Model (Scheet and Stephens, 2006).

Two different datasets with different missing SNPs imputation from GBS data were recently used in GWAS analysis for this panel, i.e., the 90K SNPs dataset with $22.8 \%$ missing imputation by (Rebolledo et al., 2016) and the 45K SNPs dataset with $8.75 \%$ missing imputation by (Kikuchi et al., 2017). In addition, this panel was also genotyped with a 700K SNPs dataset and recently used in a GWAS (Al-Tamimi et al., 2016). However, only 240 out of 274 genotypes used in our study were overlapped with quality SNPs. Thus, we used the $45 \mathrm{~K}$ SNPs data set with $8.75 \%$ missing imputation that was more precise than the 90K SNPs dataset with higher percentage of missing imputation. The original dataset contains 46,999 SNPs with minor allele frequency $(\mathrm{MAF}) \geq 0.05$ and $8.75 \%$ missing data for 329 genotypes. We selected the SNP data for 274 genotypes phenotyped in our study with another round of MAF $(\geq 0.05)$ filtering resulting in the final dataset containing 45,608 SNPs. MAF $\geq 0.05$ was used to reduce the spurious association caused by rare variants.

\section{Single-locus genome-wide association analysis}

The single-locus GWAS analysis was performed on 45,608 SNPs and phenotypic traits by compressed mixed linear model (CMLM; Zhang et al., 2010) in the Genomic Association and Prediction Integrated Tool (GAPIT; Lipka et al., 2012). We incorporated population structure (Q matrix as a PCA component) matrix (Supplementary Figure S5A-B) and family kinships (K) matrix (Supplementary Figure S6) calculated with 45,608 SNPs:

$$
\mathrm{Y}=\mathrm{X} \alpha+\mathrm{P} \beta+\mathrm{K} \mu+\mathrm{e}
$$

where $\mathrm{Y}$ and $\mathrm{X}$ represent the vector of phenotype (BLUE) and genotype (SNP) respectively, $\mathrm{P}$ is the PCA matrix and $\mathrm{K}$ is the relative kinship matrix. $\mathrm{X} a$ and $\mathrm{P} \beta$ are the fixed effects, and $\mathrm{K} \mu$ is the random effect and e represents the random error. The $\mathrm{P}$ and $\mathrm{K}$ terms were introduced to correct for false-positive association. Although correction for the population structure substantially reduces false positives, it sometimes eliminates the true-positive association due to overcorrection (Zhao et al., 2011). Therefore, the optimal number of PCs were determined 
for each trait before incorporating into CMLM, based on forward model selection using the Bayesian information criterion. Such statistical methods help to control both false positive and false negative associations effectively although they cannot eliminate both completely. Most of the root traits are complex polygenic in nature and we expected that the effect of the individual underlying loci would be small. Therefore, we chose a suggestive threshold of the probability $P$ value $\leq 1.00 \mathrm{E}-04$ to detect significant associations, as followed recently for the same population (Rebolledo et al., 2016) and in many other rice GWAS studies (Zhao et al., 2011; Norton et al., 2014; Dimkpa et al., 2016). The similar threshold was also used in another GWAS study for rice root traits (Courtois et al., 2013).

\section{Broad-sense and narrow-sense heritability}

Phenotypic variance can be decomposed into variance caused by genetic and environmental factors. The broad sense heritability $\left(\mathrm{H}^{2}\right)$ is the proportion of phenotypic variance that is due to genetic variance. Genetic variance can be a result of additive, dominance or epistatic effects. The broad-sense heritability $\left(\mathrm{H}^{2}\right)$ of traits was calculated across each treatment using following equation.

$$
H^{2}=\frac{\sigma_{G}^{2}}{\sigma_{G}^{2}+\frac{\sigma_{E}^{2}}{r}}
$$

where $\sigma_{\mathrm{G}}^{2}$ and $\sigma_{\mathrm{E}}^{2}$ are the genotypic and residual variance respectively and $\mathrm{r}$ is the number of replications. The restricted maximum likelihood estimate was used to calculate the variance components in Genstat 17.1. The narrow-sense heritability is the proportion of phenotypic variance that is due to additive genetic variance. The marker-based narrow sense heritability $\left(h^{2}\right)$ was obtained from above mentioned CMLM equation and was calculated using following equation in GAPIT.

$$
h^{2}=\frac{\sigma_{\mathrm{a}}^{2}}{\sigma_{\mathrm{a}}^{2}+\sigma_{\mathrm{e}}^{2}}
$$

where $\sigma^{2}{ }_{\mathrm{a}}$ is the additive genetic variance and $\sigma^{2}$ is the residual variance.

\section{Multi-locus genome wide association analysis}

In addition to correcting the confounding effect of population structure (first three PCA components) and family kinships (K) matrix, multi-locus linear mixed model (MLMM) corrects the confounding effect of background loci may be present due to LD in the genome (Segura et al., 2012). This was done by explicitly using loci as cofactors in the statistical model, 
similar to standard composite interval mapping of biparental analysis (Jansen and Stam, 1994). The multi-locus GWAS was implemented in the modified version of MLMM in R studio (R script for mlmm.cof.r available at https://cynin.gmi.oeaw.ac.at/home/resources $/ \mathrm{mlmm}$ ). First, we ran the complete model as recommended with stepwise forward inclusion of the strongest significant markers as a cofactor until the heritability reached close to zero, and after that backward elimination of the least significant markers from the model was carried out with estimating the variance components and $P$ values at each step (Segura et al., 2012). In the second step we checked the optimal model selection using the available criteria in MLMM: (1) extended Bayesian information and (2) the multiple Bonferroni. However, both these criteria were too conservative to identify loci for most of the traits in our study and identified significant loci for very few traits (LMXN, RS, SW and SWR) only in water-deficit stress condition. Therefore, we checked the $P$ value of markers at first step (similar to single locus GWAS analysis with no cofactor in the model) before including them as a cofactor and continued the model with inclusion of markers as a cofactor on an arbitrary cut-off significance threshold $P$ value $\leq 1.00 \mathrm{E}-04$ as used in the single-locus GWAS analysis. The model was stopped when no significant loci appeared above the cut-off threshold $P$ value and all significant cofactors with this approach were considered as significant genetic loci.

\section{Linkage disequilibrium (LD) analysis}

The pair wise LD was calculated for the whole panel using the correlation coefficient $\left(r^{2}\right)$ between pairs of SNPs on each chromosome by setting the sliding window at 100 in TASSEL 5.0 (Bradbury et al., 2007). A total of 45,608 SNPs with MAF $\geq 0.05$ were considered for LD analysis. To investigate the LD decay rate, the $r^{2}$ values of the chromosome and average across the chromosomes representing the whole genome LD pattern were plotted against the physical distance $(\mathrm{kb})$ among the markers. The LD decay rate was measured as the physical distance $(\mathrm{kb})$ at which $\mathrm{r}^{2}$ value drops to half of its initial value.

\section{A priori candidate gene selections}

The variation in recombination rates (an essential determinant of LD structure) could have broken the chromosome into a series of discrete haplotype LD blocks that determined the actual resolution of association mapping. The upper limit of LD decay rate is $\sim 500 \mathrm{~kb}$ in rice (Mather et al., 2007). Therefore, we selected $\sim 0.5$ to $0.6 \mathrm{Mb}$ (total $\sim 1.1 \mathrm{Mb}$ ) region on each side of the significant SNPs identified through GWAS analysis, to investigate the local LD pattern near to 
the significant SNPs (Huang et al., 2010). The Haploview 4.2 program was used to calculate LD structure near the significant SNPs (Barrett et al., 2005) and visualize the discrete haplotype block in $\sim 1.1 \mathrm{Mb}$ region. The LD haplotype block harbouring the significant SNP or more than one significant SNPs was identified and considered as a unique significant locus. The known genes (genes with known annotation) located within LD blocks were collected. The closest Arabidopsis (Arabidopsis thaliana L.) orthologue genes were obtained from the MSU7 Rice genome database (http://rice.plantbiology.msu.edu/cgi-bin/gbrowse/rice/). All the genes described as a transposon and retro transposon were not selected and genes described as an expressed protein (EP) were considered only when there was relevant information available from Arabidopsis orthologue.

\section{Results and Discussion}

\section{Genotypic variation in phenotypic traits and their interrelations}

Rice exhibits large functional diversity due to strong natural and human selection pressure, which underlies evolutionary variation in traits inducing stress adaptation (McCouch et al., 2013). A set of 274 rice indica genotypes assembled from major rice growing regions across the world was evaluated to assess the variation in phenotypic traits (Supplementary Figure S1 and Supplementary Data Set S1). In total, 35 phenotypic traits, broadly classified into five categories (shoot morphology, whole-plant physiology, root morphology, root anatomy, and dry matter production), were evaluated on plants grown in control and water-deficit stress conditions during the vegetative phase (Table 1).

Genotypic variation observed in all traits across treatments was strong $(P \leq 0.001)$, except in root length classes RL3035 and RL35 (Supplementary Table S1). The broad-sense heritability $\left(\mathrm{H}^{2}\right)$ ranged from 0.10 to 0.89 in the control and from 0.03 to 0.88 under waterdeficit stress (Supplementary Table S2). A principal component analysis (PCA) identified eight significant principal components (PCs) with eigenvalue $>1$, cumulatively explaining $>80 \%$ of the total variation for the 35 traits across the panel in each treatment (Supplementary Figure S7). The first PC, explaining more than $35 \%$ of the total variation, was associated with genotypic variation in most morphological (shoot and root), dry matter and cumulative water transpiration (CWT) traits in both treatments (Fig. 1A-B) and with substantial correlations among these traits (Supplementary Figure S8A-B). The second PC, explaining more than 12\% of the total variation, was mainly associated with root anatomical traits but a portion of the variation was also accounted for by root morphological traits such as specific root length (SRL) 
Table 1. The list of measured and derived phenotypic traits broadly classified into five categories (A-E) with trait acronyms and units.

\begin{tabular}{|c|c|c|c|}
\hline Traits & Trait acronym & Unit & Phenotypic plasticity acronym \\
\hline \multicolumn{4}{|l|}{ (A) Shoot morphological traits } \\
\hline Plant height & PHT & $\mathrm{cm}$ & rPHT \\
\hline Tiller number & $\mathrm{TN}$ & plant $^{-1}$ & $\mathrm{rTN}$ \\
\hline Total leaf area & TLA & $\mathrm{m}^{2}$ plant $^{-1}$ & rTLA \\
\hline Specific leaf area & SLA & $\mathrm{m}^{2} \mathrm{~g}^{-1}$ & rSLA \\
\hline \multicolumn{4}{|l|}{ (B) Physiological traits } \\
\hline Cumulative water transpiration & CWT & kg plant $^{-1}$ & rCWT \\
\hline Water use efficiency & WUE & $\mathrm{g} \mathrm{kg}^{-1}$ & rWUE \\
\hline \multicolumn{4}{|l|}{ (C) Root morphological traits } \\
\hline Total root length & TRL & $\mathrm{m}_{\text {plant }}{ }^{-1}$ & rTRL \\
\hline \multicolumn{4}{|c|}{ Root length $(\mathrm{RL})$ with diameter $(\mathrm{mm})$ class } \\
\hline RL_0-0.5 & RL005 & $\mathrm{m}$ plant ${ }^{-1}$ & rRL005 \\
\hline $\mathrm{RL}_{-} 0.5-1.0$ & RL0510 & $\mathrm{m}$ plant ${ }^{-1}$ & rRL0510 \\
\hline $\mathrm{RL}_{-} 1.0-1.5$ & RL1015 & $\mathrm{m} \mathrm{plant}^{-1}$ & rRL1015 \\
\hline $\mathrm{RL}_{-} 1.5-2.0$ & RL1520 & $\mathrm{m}$ plant ${ }^{-1}$ & rRL1520 \\
\hline $\mathrm{RL}$-2.0-2.5 & RL2025 & $\mathrm{m}$ plant ${ }^{-1}$ & rRL2025 \\
\hline $\mathrm{RL}_{-} 2.5-3.0$ & RL2530 & $\mathrm{m}$ plant ${ }^{-1}$ & rRL2530 \\
\hline $\mathrm{RL}_{-} 3.0-3.5$ & RL3035 & $\mathrm{m}$ plant ${ }^{-1}$ & rRL3035 \\
\hline RL_3 3.5 & RL35 & $\mathrm{m}$ plant ${ }^{-1}$ & rRL35 \\
\hline Maximum root length & MRL & $\mathrm{cm}$ & rMRL \\
\hline Surface area & SA & $\mathrm{cm}^{2}$ plant $^{-1}$ & rSA \\
\hline Root volume & $\mathrm{RV}$ & $\mathrm{cm}^{3}$ plant $^{-1}$ & $\mathrm{rRV}$ \\
\hline Average root thickness & ART & $\mathrm{mm}$ & rART \\
\hline Specific root length & SRL & $\mathrm{m} \mathrm{g}^{-1}$ & rSRL \\
\hline Total root weight density & TRWD & $\mathrm{g} \mathrm{cm}^{-3}$ & rTRWD \\
\hline Root length per unit leaf area & RLLA & $\mathrm{m} \mathrm{m}^{-2}$ & rRLLA \\
\hline \multicolumn{4}{|l|}{ (D) Root anatomical traits } \\
\hline Root diameter & $\mathrm{RD}$ & $\mu \mathrm{m}$ & rRD \\
\hline Cortex diameter & $\mathrm{CD}$ & $\mu \mathrm{m}$ & $\mathrm{rCD}$ \\
\hline Stele diameter & SD & $\mu \mathrm{m}$ & $\mathrm{rSD}$ \\
\hline Late metaxylem diameter & LMXD & $\mu \mathrm{m}$ & rLMXD \\
\hline Late metaxylem number & LMXN & $\mu \mathrm{m}$ & rLMXN \\
\hline $\begin{array}{l}\text { Stele diameter in proportion of root } \\
\text { diameter }\end{array}$ & SD:RD & $\%$ & rSDRD \\
\hline \multicolumn{4}{|l|}{ (E) Dry matter traits } \\
\hline Leaf weight & LW & g plant $^{-1}$ & rLW \\
\hline Stem weight & SW & g plant $^{-1}$ & rSW \\
\hline Root weight & RW & g plant ${ }^{-1}$ & rRW \\
\hline Total weight & TW & g plant ${ }^{-1}$ & rTW \\
\hline Root: shoot ratio & RS & - & rRS \\
\hline Leaf weight ratio & LWR & - & rLWR \\
\hline Stem weight ratio & SWR & - & rSWR \\
\hline
\end{tabular}

and two of its components: total root weight density (TRWD) and average root thickness (ART; Fig. 1A-B). Moreover, these root anatomical and morphological traits were correlated with each other. For instance, SRL showed a negative correlation with TRWD (on average $r=-0.87$ ), ART $(r=-0.73)$, and all root anatomical traits $(r=$ ca -0.30$)$ in both treatments, except with late metaxylem number $(\mathrm{LMXN})$ in control and stele diameter in proportion of root diameter 
(SD:RD) in both control and stress (Supplementary Figure S8A-B). These results clearly indicate, that an increase in SRL could result in reducing the root thickness, stele diameter (SD) and late metaxylem diameter (LMXD). The first two components in control and water-deficit stress explained many of these complex relationships for most of the traits in this study (Fig. 1). In general, such relationships among traits might be due to pleiotropic or tightly linked genetic loci or gene, although that cannot be inferred directly from their positive and negative relationships.

\section{High degree of trait variability in response to water-deficit stress underlies phenotypic plasticity}

Phenotypic plasticity can have adaptive significance, while in some cases it can be an inevitable response under resource limitations (Nicotra et al., 2010). Significant treatment effects $(P<$ $0.001)$ on all traits indicate expression of phenotypic plasticity under water-deficit stress. For most traits water-deficit stress resulted in lower values than observed for the control, with reductions ranging from 2 to $66 \%$. Most of the root traits showed significant reductions. However, SRL, SD:RD, stem weight ratio (SWR), root length per unit leaf area (RLLA) and water use efficiency (WUE) were increased for plants grown under water-deficit stress than for plants under control conditions (Supplementary Table S1). Roots were thinner under waterdeficit stress than under control conditions as indicated by SRL (22\% increase over control) and two of its components TRWD (20\% decrease) and ART (11\% decrease; Fig. 2A-C).

The rice root anatomy is adapted to semiaquatic conditions with characteristic outer sclerenchymatous layer, large cortex diameter, small stele and xylem (Coudert et al., 2010; Kadam et al., 2015). However, to what extent natural and human selection has shaped root anatomical plasticity in response to water-deficit stress remains to be elucidated. In this study, all root anatomical traits showed phenotypic plasticity to stress treatment $(\mathrm{T}: P<0.001)$ but lacked genotypic variability for plasticity $(\mathrm{G} \times \mathrm{T}: P \geq 0.05)$ (Supplementary Table $\mathrm{S} 1$ and Fig. 2D-I). Cortex diameter (CD) showed a strong response (18\% decrease; Fig. 2E) with low level of plasticity for stele diameter (SD; 4\% decrease, Fig. 2F), LMXD (7\% decrease; Fig. 2H) and LMXN (2 \% decrease; Fig. 2I). These results are in agreement with a recent study involving three rice genotypes (Kadam et al., 2015). The reduced CD increases the relative area Constituted by the stele (increased SD:RD; Fig. 2G) in roots, decreases radial distance, and improves radial hydraulic conductivity. The reduced CD could also significantly reduce the roots metabolic cost of soil exploration, thereby improving the water and nutrient acquisition 


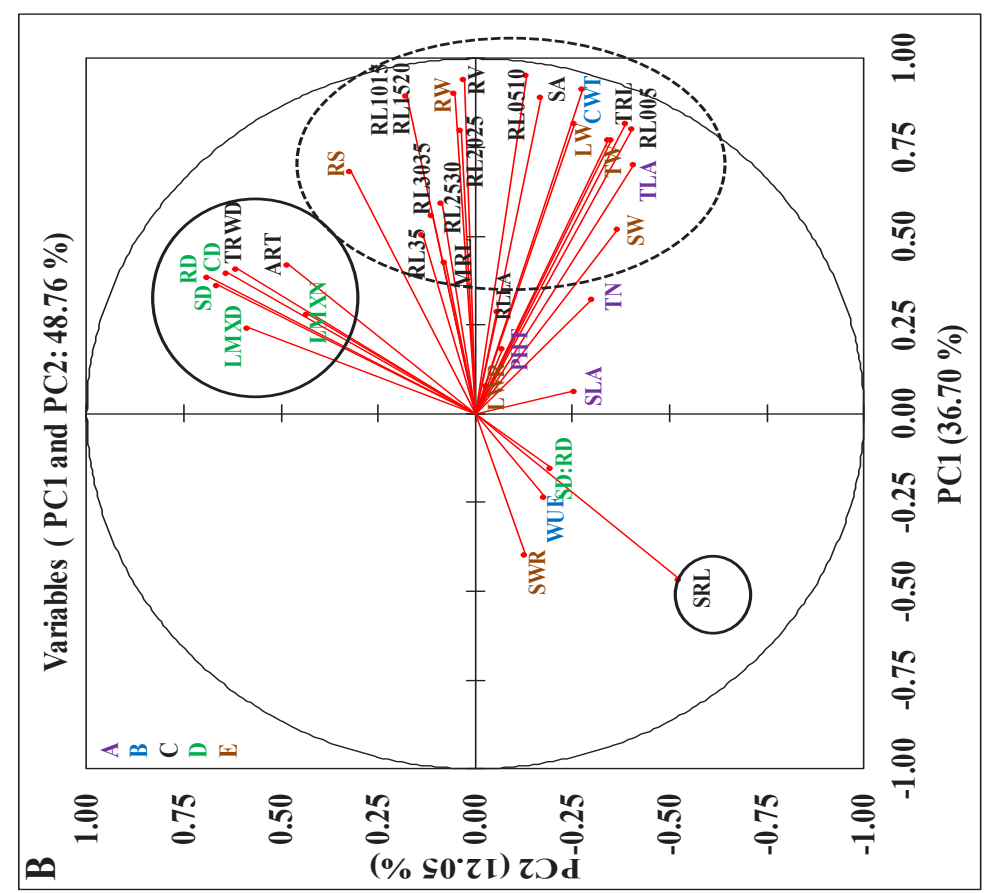

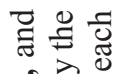
安总志 氙帚 응 范产 옹 t] एँ on 의 言骂寻 के 焉 들 융르 客咍

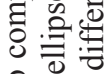
过 荡 현 중융

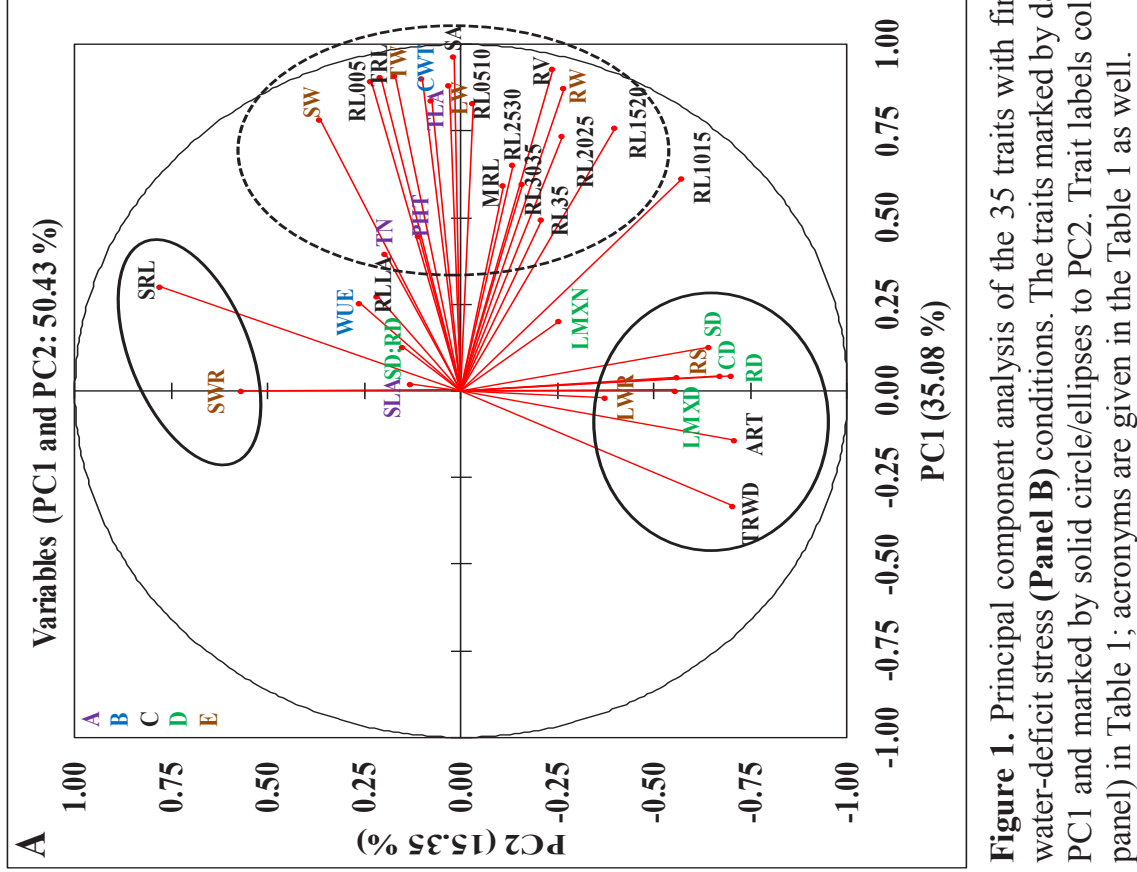


in water-deficit and nutrient stress (Chimungu et al., 2014; Vejchasarn et al., 2016). However, reduced $\mathrm{CD}$ reduces the root thickness (Fig. 2D) and thereby mechanical strength of the root, which is a key to penetrating soil hardening under water-deficit stress (Yoshida and Hasegawa, 1982).

\section{Population structure and whole-genome linkage disequilibrium}

A balanced population structure and an optimal amount of linkage disequilibrium (LD) are important prerequisites for a successful GWAS, because the former corrects any confounding effect to avoid spurious associations whereas the LD is critical to infer the results (Mackay and Powell, 2007). The PCA with 46K SNPs (MAF $\geq 0.05$ ) revealed continuous distribution with no deep substructure in the 274 rice indica genotypes as, indicated by the limited amount of genetic variation (only 19\%) explained by the first four PCs (Supplementary Figure S5A-B). Likewise, the LD on average across chromosomes dropped to half of its initial value at $\sim 55$ to $65 \mathrm{~kb}$ and to the background levels $\left(\mathrm{r}^{2} \leq 0.1\right)$ at around $\sim 600 \mathrm{~kb}$ to $1 \mathrm{Mb}$ (Supplementary Figure $\mathrm{S} 10$ ). The observed LD decay distance was significantly shorter than previously observed values in rice indica subgroups at $\sim 100-125 \mathrm{~kb}$ (Huang et al., 2010; Zhao et al., 2011), indicating more historical recombination events in our studied population likely due to the diverse sampling of a wide range of landraces and breeding lines with a low degree of genetic relatedness. Hence, a higher resolution can be expected from the mapping efforts, although it would also depend on the local LD pattern near the significant peaks.

\section{Single-locus and multi-locus mapping identifying core regions of rice genome associated with phenotypic traits}

To elucidate the genetic architecture, we conducted GWAS on 33 traits (excluding two traits [RL3035 \& RL35] that lacked genotypic variation) across treatments and of their plasticity with $46 \mathrm{~K}$, SNPs (MAF $\geq 0.05)$ using a single-locus compressed mixed linear model (CMLM) and a multi-locus mixed model (MLMM; more details in Materials and Methods). Table 2 provides a summary of GWAS for 33 traits from five categories. In total, we detected a nearly equal number of associations in control (104) and the water-deficit stress (106), although the significant loci varied across and within trait categories and treatments. Furthermore, 22 out of 104 associations in control and 10 out of 106 in water-deficit conditions were linked with more than one trait, possibly due to tight linkages or pleiotropic effects of loci or genes. For plasticity of traits, we identified 76 associations (Table 2 and Supplementary Tables S3-S5), of which 

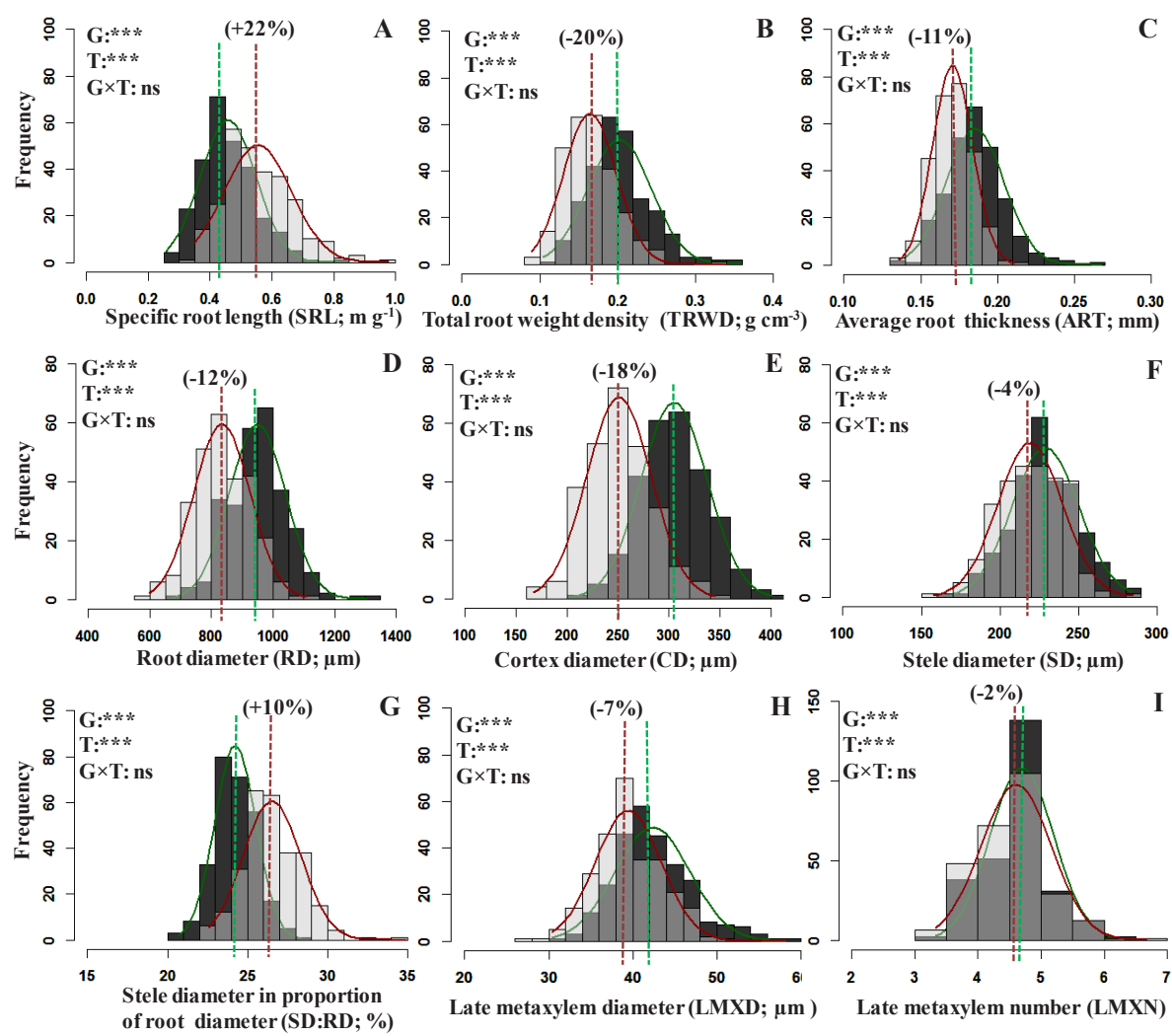

Figure 2. Overlying histograms with normal distribution curves (control: green line, dark grey bars; water-deficit stress: red line, light grey bars; intermediate grey: overlap for the treatment with the lower frequency value) showing the phenotypic distribution of root morphological (Panel A-C) and anatomical (Panel D-I) traits. The vertical lines in the histograms show population mean values in control (green) and water-deficit stress (red) conditions and values in parentheses represent the significant percentage change ( + : increase or - : decrease) in waterdeficit stress conditions over the control. Levels of significance for Genotype (G), Treatment $(\mathrm{T})$ and their interaction $(\mathrm{G} \times \mathrm{T})$ effects from ANOVA are given in the histograms $(* * *$, $P<0.001$; ns, not significant).

nine were linked with more than one trait (Supplementary Table S6). Of the total loci, 22\% in control, $33 \%$ in water-deficit stress and $27 \%$ for plasticity of the traits were detected commonly by both approaches, with statistically improved power (lower $P$ value) for most of the loci using the MLMM approach. In addition, MLMM identified additional novel loci in both treatments and for trait plasticity. In particular, MLMM identified significant loci for some traits where CMLM failed to identify any loci, and the identified loci was mostly novel, although in a few cases, they were already found to be associated with other traits in this study. For instance, we 
identified four and three loci for total root length (TRL) in control, and water-deficit stress conditions, respectively, only with MLMM, and one locus on chromosome 4 under stress was associated with root weight (RW) and root: shoot ratio (RS; Supplementary Figures S11-S12). Similarly, we identified three loci for CWT and four for WUE in water-deficit condition only through MLMM (Supplementary Figure S13). Thus, MLMM approach proved to be valuable in dissecting the genetic architecture of complex traits by identifying additional novel loci (Segura et al., 2012). The detailed GWAS results through CMLM and MLMM approach are given in Supplementary Tables S3-S5.

\section{Quantitative variation of root morphology in two moisture regimes and their plasticity provides insights into a complex genetic pattern}

The genetic architecture of root traits is complex; determined by multiple small effect loci and studied extensively on mapping populations of rice representing the narrow genotypic base (Courtois et al., 2009). The genetic variations of root traits are relatively less characterized in diverse rice genotypes (Courtois et al., 2013; Phung et al., 2016; Biscarini et al., 2016) and can be a potential source for evolutionary beneficial alleles. Further, most of these studies have characterized the genetic variations in single isolated environments and not considered the two moisture regimes simultaneously, typically due to difficulty in the root phenotyping (space, time and cost). In this study, we carefully phenotyped the root traits in two moisture regimes and extracted the root morphology in various hierarchies by automated digital image analysis tool WinRHIZO (Table 1; Materials and Methods for root phenotyping). Through GWAS analysis, we detected 34 loci for 11 morphological, one for RW and three for RS in control and 52 loci for 12 morphological, four for RW and four for RS ratio under water-deficit (Table 2 and Supplementary Tables S3-S4). The SRL is one of the important root morphological traits and often used as a proxy for root thickness. We observed three and eight loci for SRL in control and stress conditions through CMLM and MLMM (Fig. 3 and Supplementary Tables S3-S4). The mean narrow-sense heritability $\left(h^{2}\right)$ of root traits that showed significantly associated loci varied between 0.20 and 0.89 in control and between 0.32 and 0.78 in stress conditions (Supplementary Table S2). In addition, we identified 33 loci for 12 root morphological plasticity traits, one locus for rRW and four loci for rRS ratio, with mean $h^{2}=0.40$ for traits that showed significant associations (Table 2; Supplementary Tables S2 and S5). Above results clearly illustrate that variation in root plasticity is heritable and determined by the genetic factors. 
Table 2. Summary of significant loci identified by GWAS analysis using two approaches (comprised mixed linear model (CMLM) and multi-locus mixed model (MLMM) for 35 traits across five categories (A-E) in control (C) and water-deficit (WD) conditions and for phenotypic plasticity $(\mathrm{PP})$ of traits as a relative measure

\begin{tabular}{lccc}
\hline Trait classification & C & WD & PP \\
\hline (A) Shoot morphological traits & 6 & 11 & 8 \\
(B) Physiological traits & 16 & 6 & 6 \\
(C) Root morphological traits & 34 & 52 & 33 \\
(D) Root anatomical traits & 14 & 17 & 15 \\
(E) Dry matter traits & 34 & 20 & 14 \\
\hline Total loci & $\mathbf{1 0 4}(\mathbf{2 2})$ & $\mathbf{1 0 6}(\mathbf{1 0 )}$ & $\mathbf{7 6}(\mathbf{9})$ \\
\hline Loci detected by CMLM approach & $\mathbf{3 9}[\mathbf{3 2 \%}]$ & $\mathbf{2 6}[\mathbf{2 4 \%}]$ & $\mathbf{1 9}[\mathbf{2 5 \%}]$ \\
\hline Loci detected by MLMM approach & $\mathbf{4 2}[\mathbf{4 0 \%}]$ & $\mathbf{4 5}[\mathbf{4 2 \%}]$ & $\mathbf{3 6}[\mathbf{4 7 \%}]$ \\
\hline Loci detected by both approaches & $\mathbf{2 3}[\mathbf{2 2 \%}]$ & $\mathbf{3 5}[\mathbf{3 3 \%}]$ & $\mathbf{2 1}[\mathbf{2 7 \%}]$ \\
\hline Total predicted $\boldsymbol{a}$ priori genes & $\mathbf{2 9 6}$ & $\mathbf{2 8 4}$ & $\mathbf{2 3 3}$ \\
\hline Genes responsive to abiotic stress stimulus & $\mathbf{4 8}$ & $\mathbf{6 1}$ & $\mathbf{3 8}$ \\
\hline The values in parenthesis are loci associated with more than one trait (see Supplementary Table S6) and values \\
in square brackets are the percentages of loci out of total loci detected by CMLM, MLMM and both the \\
approaches. The total $a$ priori genes are predicted in expected LD block of peak SNP/SNPs.
\end{tabular}

Dividing a trait into multiple component traits unravels the underlying inherited complexity (Yin et al., 2002). We detected an increased number of genetic loci for root length classified on root thickness than for TRL across treatments (Supplementary Tables S3, S4 and S7). For instance, we identified four loci in control and three loci in water-deficit stress for TRL. Mapping with root length traits of different root thickness classes resulted in identifying the additional 10 loci in control and 18 loci under water-deficit stress that were not detected by TRL per se (Supplementary Table S7). Similar result was observed for total weight (TW) and for its three component traits namely leaf weight (LW), stem weight (SW) and RW (Supplementary Tables S3-S4). These results clearly suggested that separating the complex trait into component traits improves the power to detect significant associations, perhaps by minimizing the variance between raw value, thereby increases the chance to detect variation in its component traits in agreement with a previous study (Crowell et al., 2016). However, for plasticity, we identified only five loci for root length of different root thickness classes, of which one was common with rTRL and four were novel loci (Supplementary Table S7). This lower number of loci for plasticity could also be due to the fact that plasticity is the trait ratio estimated from measurements across two treatments. Nevertheless, our ability to identify these distinct genetic loci when mapping the component traits might be capturing the key causal genetic regulator controlling the various aspects of root morphology. Moreover, there were no common loci detected either for TRL or its component traits across treatments, and this suggests 
that genetic control of root morphology is different across moisture regimes and strongly influenced by water-deficit. This could be further substantiated by all the novel loci identified for plasticity in the above traits, which might be a specific stress responsive genetic loci determining the plastic response.

\section{Colocalization of root morphology loci explains underlying genetics and physiology}

Many of the root traits and other traits result from complex combination of biological mechanisms controlling the expression in coordination, as explained by their correlation. This correlation between traits could results from pleiotropic action of genetic loci on different traits or due to tight linkage between genetic loci. The root system supports the aboveground shoot growth through absorption of water and nutrients. In this study, one locus on chromosome 5 (7131196) was commonly associated with root morphology (root volume [RV], RL1015, RL1520), RW, CWT and TW in control condition (Supplementary Table S6). All these traits showed a positive $(\mathrm{r}=\mathrm{ca}$ 0.65) correlation with CWT in control condition (Supplementary Figure S8A). In water-deficit stress, one locus on chromosome 1 (a different SNP but falls within same LD block) was commonly associated with CWT (23207640) and SRL (23218344) and both these traits were negatively correlated ( $\mathrm{r}=-0.34$; Supplementary Figure S8B). Similarly, for plasticity, one locus on chromosome 7 (9463744) was commonly associated with rTRL, rSA (9463899; different SNP but falls within same LD block), rTLA and rCWT (Supplementary Table S6). To comprehend, these results clearly illustrate the common genetic control of root morphology and water transpiration possibly to maintain the balanced hydraulic continuum between water uptake and transpiring organ. One locus on chromosome 9 (14829621) was commonly associated with RV, leaf weight ratio (LWR) and stem weight ratio SWR), in water-deficit (Fig. 4). The minor allele at this locus had a positive effect on SWR and negative on RV and LWR (Supplementary Table S4). This further elucidates the negative correlation of SWR with RV and LWR (Supplementary Figure S8B). The same locus was associated with root length 0.5 to $1.0 \mathrm{~mm}$ diameter class (RL0510) and surface area (SA) in water-deficit stress (Supplementary Table S6). The ratio of root to shoot is more often used as an index of water-deficit stress tolerance and surrogate for root morphology. One locus on chromosome 4 (29111186) was commonly associated with TRL, RL005, RW and RS in waterdeficit. The minor allele of this locus had a positive effect on all these traits (Supplementary Table S4). Furthermore, one of the significant loci was commonly detected in both the moisture regimes; associated with maximum root length (MRL) in control and SRL in water-deficit stress 


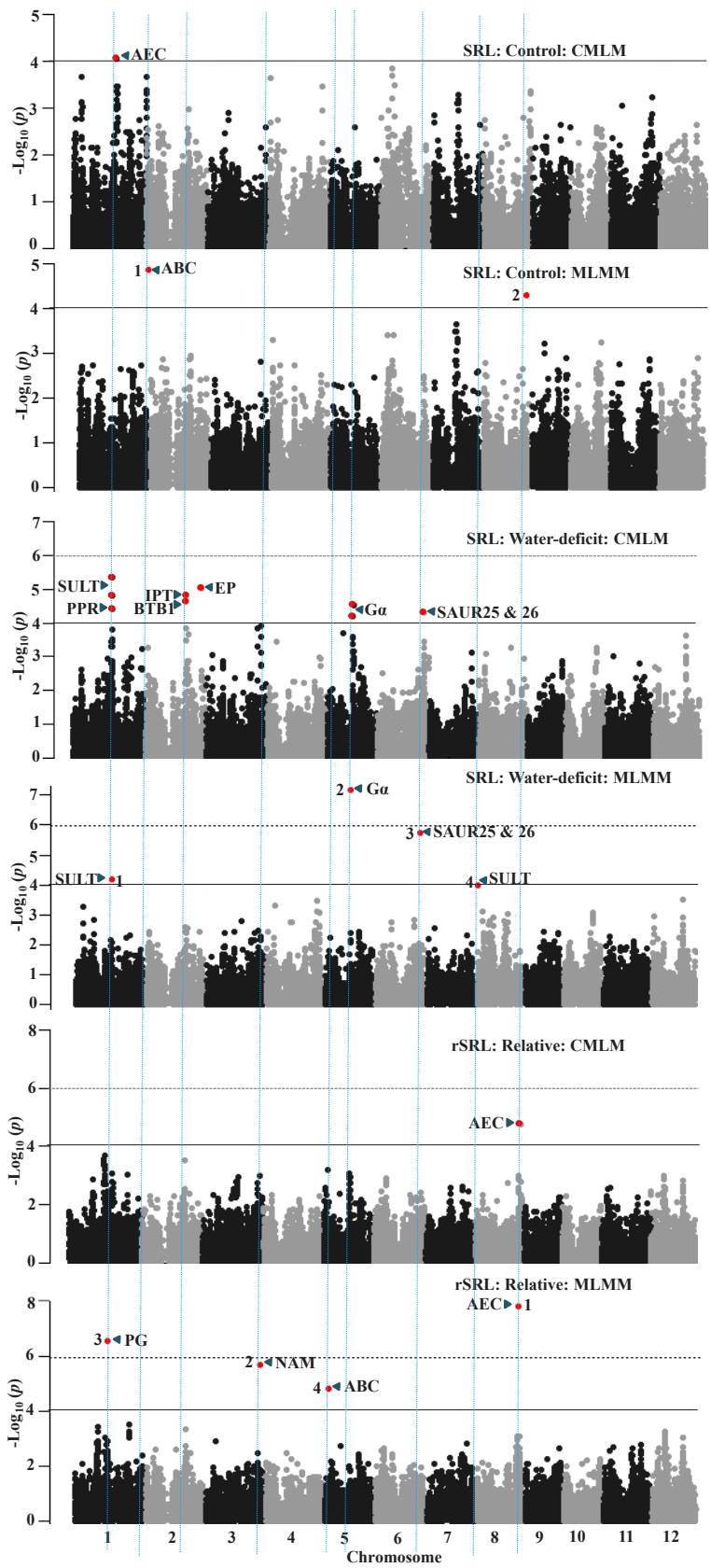

Figure 3. GWAS results through the compressed mixed linear model (CMLM) and the multi locus mixed model (MLMM) approaches for specific root length (SRL) in control (the two upper panels) and water-deficit conditions (the two middle panels) and the trait plasticity calculated as the relative value of the water-deficit stress conditions over the control (the two bottom panels). Significant SNPs (coloured red in the Manhattan plots) are distinguished by 
Figure 3. (Continued)

threshold $P$ value lines (solid black $=\left[-\log _{10} P>4\right]$ and dotted black= Bonferroni-corrected threshold). Significant SNPs in MLMM Manhattan plots are numbered in the order that they were included in the model as a cofactor. A priori candidate genes (Supplementary Tables S9S11) are indicated near to peak SNP/SNPs in the Manhattan plot. AEC: auxin efflux carrier; ABC: ATP-binding cassette transporters; SULT: Sulfate transporter; PPR: Pentatricopeptide; IPT: Inorganic phosphate transporter; BTB1: Brick-Brack, Tramtrack, Broad Complex BTB, EP: Expressed protein; Ga: G-protein alpha subunit; SAUR: Small auxin UP-RNA; PG: Polygalacturonase; NAM: No apical meristem.

(Supplementary Tables S3-S4). We also identified locus on chromosome 12 (25006932) commonly associated with plasticity of root morphology traits (rTRL, rRL005, rSA, rRV, rRTN and rRLD) and rTN (Supplementary Table S6). These identified loci influencing multiple traits could be a potential marker for the marker assisted selection after validating in the elite genetic background.

\section{Genetic basis of radial root anatomy}

The functioning of roots is strongly depending on radial organization of root anatomy, which is regulated by the asymmetric cell division. The genetic control of radial root organization is less studied in rice, with largely unknown underlying genetic mechanisms. Understanding the genetic control of radial root anatomy is more challenging in rice because the complexity and size of the fibrous root system presented several phenotyping challenges. To date, only one study in rice has identified the genomic regions for radial root anatomy (Uga et al., 2008). Through GWAS analyses, we identified 14 significant loci for five anatomical traits in control; 17 loci for four anatomical traits in water-deficit and 15 loci for the plasticity of four anatomical traits (Table 2 and Supplementary Tables S3-S5). Root diameter (RD; anatomical) of the adventitious root and ART (morphological) of the complete root system are positively correlated (control: $\mathrm{r}=0.22$ and water-deficit: $\mathrm{r}=0.25$ ) and a locus on chromosome 1 (1099857/1111294; different marker but fall within same LD block) was commonly associated in control condition (Supplementary Table S6). Both these traits are measures of root thickness, thus illustrate that measuring the $\mathrm{RD}$ at one position (near root-shoot junction) to some extent, was able to capture genetic variation of complete root system thickness. Three anatomical traits, namely RD, CD and SD:RD, were highly correlated with each other in control (Supplementary Figure S8A), and we found one common locus (21266079) associated with them on chromosome 7 (Supplementary Table S6). Stele tissue is the central part of the root enclosing the vascular cylinder (xylem and phloem), and one locus on chromosome 9 (13788883) and 5 
(3057869) was commonly associated with SD and LMXD in stress (Supplementary Table S6). However, no locus was commonly detected across moisture regimes clearly suggest that genetic control of radial root anatomy is strongly influenced by stress. For anatomical plasticity, we observed two loci (11038867 and 11596350) on chromosome 1 common to rRD, rCD and rSD (Supplementary Figure S14) and plasticity of these traits was positively correlated with each other (Supplementary Figure S9). Hence, relative change in these traits in response to the waterdeficit is partly under similar genetic control because they also have another independently associated genetic loci.

\section{A priori candidate genes underlying the genetic loci of phenotypic traits}

A lower LD decay rate results in larger LD block and lower mapping resolution, which makes the GWAS not straightforward in identifying the causal genes. On average across genome LD decay rate was 55 to $65 \mathrm{~kb}$ in the studied population but then again, the association resolution varied with loci due to local LD pattern. Hence, we have calculated the LD pattern near to all the significant loci identified in this study (See Materials and Method). In total, we have collected a list of 296, 284, and 233 a priori candidate gene within the expected LD block in control, water-deficit and for their plasticity, respectively. Of the total a priori candidate genes, 48 (control), 61 (water-deficit) and 38 (plasticity) genes were responsive to abiotic stress stimulus (Table 2 and Supplementary Data Sets S2-S4). Furthermore, we have identified the list of 70 a priori genes close to significant loci for shoot morphological, physiological, dry matter traits in control (32 genes), water-deficit (21 genes) and for their plasticity (17 genes; Supplementary Table S8). For instance, one locus on chromosome 6 (13412649) for CWT and one on chromosome 9 (15426362) for WUE under stress was near to AQUAPORIN (AQP; 4 $\mathrm{kb})$ and the WAX2 (66 kb) genes, respectively (Supplementary Figure S13 and Supplementary Table S8). The AQP gene is known to maintain root hydraulic conductivity, cell turgor, mesophyll conductance, water transpiration and thereby growth (Flexas et al., 2006; Henry et al., 2012), whereas WAX2 gene regulates epicuticular wax production, maintains cellular water status and improves the WUE (Premachandra et al., 1994; Chen et al., 2003). Similarly, one locus on chromosome 2 (31650233) for tiller number (TN) in control was within ethyleneresponsive transcription factor (ERFTF) gene and homologue of this gene was known to regulate rice tillering (Qi et al., 2011). Likewise, for all the root traits (root morphology and anatomy, RW and RS), we have identified a list of 40, 57 and 41 a priori candidate genes in control, water-deficit and for their plasticity, respectively, with a role in root growth and 

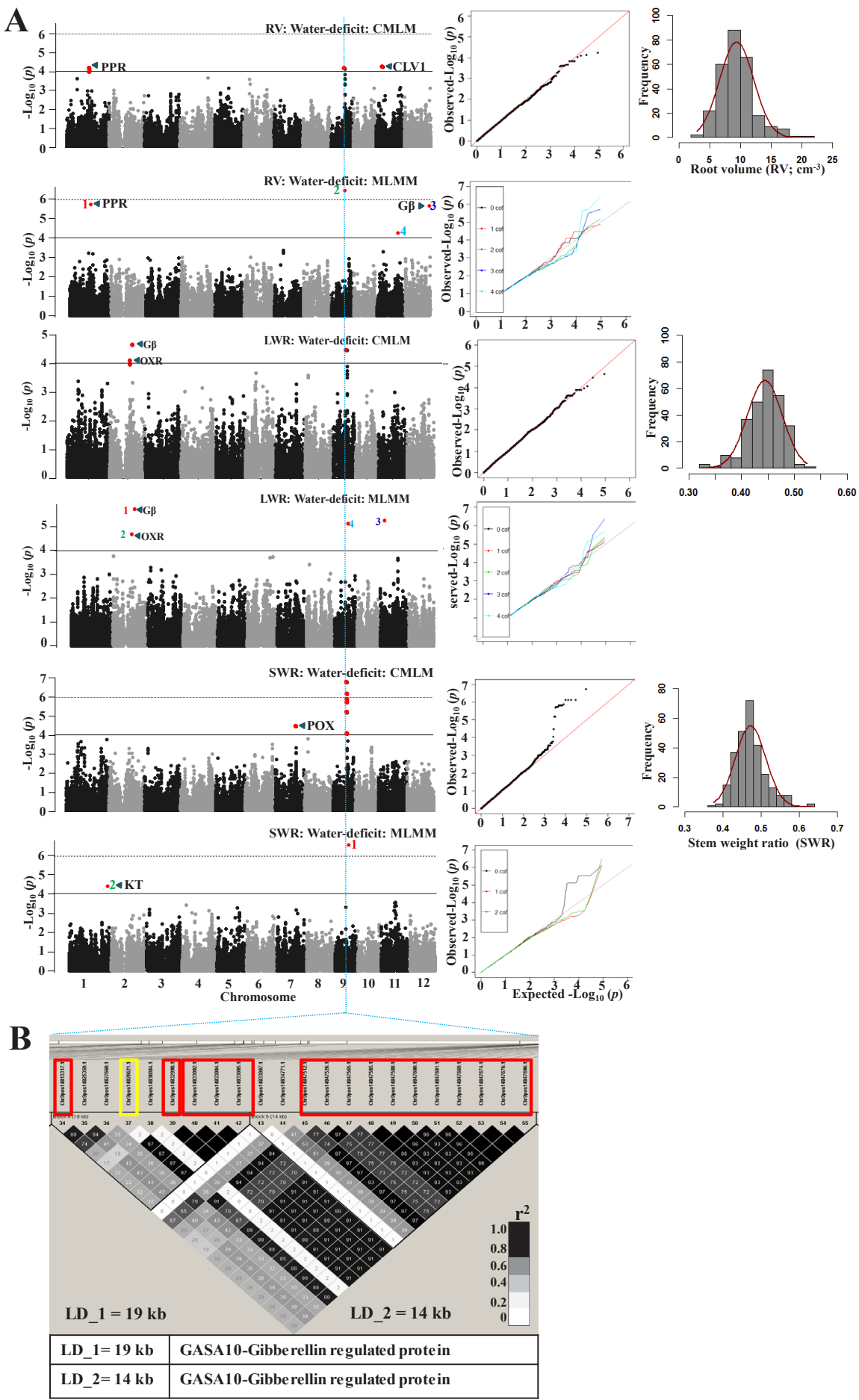
Figure 4. GWAS results through compressed mixed linear model (CMLM) and multi-locus mixed model (MLMM) approaches (Manhattan and Quantile-Quantile plots) for root volume (RV), leaf weight ratio (LWR) and stem weight ratio (SWR) in water-deficit stress. Significant SNPs (coloured red in the Manhattan plots) are distinguished by threshold $P$ value lines (solid black $=\left[\begin{array}{ll}-\log _{10} & P>4\end{array}\right]$ and dotted black $=$ Bonferroni-corrected significance threshold) and coloured red in the Manhattan plots (Panel A). Significant SNPs on MLMM Manhattan plots are numbered in the order that they were included in the model as a cofactor. Identified LD blocks based on pairwise $r^{2}$ values between SNPs on chromosome 9 (Panel B) with a priori candidate gene in the underneath table (for more details see Supplementary Tables S8 and S10). The colour intensity of the box corresponds with $\mathrm{r}^{2}$ value (multiplied by 100) according to the legend. Significant SNP ("14829621") marked in yellow rectangle was commonly associated with RV, LWR and SWR (Panel B). PPR: Pentatricopeptide, CLV1: CLAVATA1; Gß: Gprotein beta subunit; OXR: Oxidoreductase; POX: Peroxidase; KT: Potassium transporter.

development (Supplementary Tables S9-S11). Several genes were regulating root growth and development through phytohormone transport and signalling (Auxin, ABA, GA, ethylene and brassinosteroid); cell division and differentiation; cellular redox homeostasis; molecular chaperone; water and nutrient transporter; cellular component organization and cell wall remodelling. For instance, one locus on chromosome 6 (366330) for RL0510 in control (Supplementary Table S9) was within the SCARECROW (SCR) gene that regulates radial root and shoot anatomy and root hair tip growth through cell division and differentiation (Gao et al., 2004). One locus on chromosome 1 (40526762) for RV in control was within the OsSAUR3 gene, an early auxin responsive gene that regulates root elongation (Markakis et al., 2013). The two homologues of this gene were close (OsSAUR25=11 kb and OsSAUR26=42 kb) to the locus on chromosome 6 (27819933) for MRL in control (Supplementary Table S9). Likewise, in water-deficit conditions, a locus on chromosome 9 (14829621) was commonly associated with RV, RL0510, SA, LWR and SWR and was found within the GASA10 gene (Supplementary Table S10). The GASA10 gene is known to participate in phytohormone crosstalk leading to redox homeostasis, and regulates root, stem and other organs growth (Nahirñak et al., 2012). For plasticity, one locus on chromosome 8 (26362631) for rSRL was near $(30 \mathrm{~kb})$ to an auxin efflux carrier component protein (AEC; Supplementary Table S11) and this gene is known to regulate auxin transport with mutant showing defective root development (Grieneisen et al., 2007).

Three interesting a priori candidate genes were recognized for radial root anatomy loci in this study. A locus on chromosome 11 (2838776) for LMXN in control was near (7 kb) to bHLH (basic helix-loop helix protein). The Arabidopsis orthologue LONESOME HIGHWAY having sequence similarity to bHLH, regulates the stele and xylem development (Supplementary Table S9). Similarly, a locus on chromosome 11 (28871551) for LMXD in 
stress was within SCR (3 homologous copies in LD block), a gene that regulate radial anatomy of root and shoot (Supplementary Table S10); its homologue was associated with root morphology traits as discussed earlier. The LONESOME HIGHWAY gene regulates vascular tissue differentiation and number with involvement of auxin in Arabidopsis (Ohashi-Ito et al., 2013), while SCR is an auxin responsive gene regulating radial patterning in both root and shoot in Arabidopsis (Gao et al., 2004). Likewise, one of the locus on chromosome 9 (13788883) commonly associated with SD and LMXD in stress (Supplementary Table S10). This locus was near (24 kb) KANADI gene that regulates root development (Hawker and Bowman, 2004), and expressed during vascular tissue development (Zhao et al., 2005). In summary, many a priori candidate gene regulating the root morphology and radial root anatomy has been identified in this study.

\section{Conclusions}

In the past mainly root morphological differences have been extensively (phenotypically and genetically) characterized with very little attention to radial root anatomy in rice. To our knowledge, for the first time, we have characterized phenotypic variation for root morphological traits through powerful and intensive image-based systems and anatomical traits through microscopic dissection of root in a diverse set of rice indica genotypes across two moisture regimes. The single-locus and multi-locus GWAS analyses provided novel genetic insights that can help explain the observed genotypic variation of root morphological and anatomical traits across two moisture regimes. The phenotypic plasticity of the root morphology and anatomy was moderately heritable and had sufficient genetic control that resulted in identifying key core regions of rice genome. Thus, variation in root traits is valuable resources that can result in identifying the potential novel genetic loci. Favourable alleles of these identified loci could after validation be directly used for marker-assisted selection. Many of these loci were either close to known genes or within genes themselves that play a role in root growth and development. For example, several phytohormone genes influencing transport and signalling were found close to our identified loci, confirming well-known dominant role of these genes in root growth and development. The cloning and characterization of these genes can provide additional checkpoints in rice root growth and development. A further holistic approach of root system genetics is needed to be complemented with GWAS studies to understand the complexity of gene networks in controlling root growth and development. Future studies should also aim for more efficient high-throughput root phenotyping approaches 
both in field and control glasshouse conditions, to help advance root genetics.

\section{URLs.}

WinRHIZO root image analysis, http://regent.qc.ca/assets/winrhizo_about.html/; R version of MLMM, https://cynin.gmi.oeaw.ac.at/home/resources/mlmm/; Michigan State University (MSU) Genome Browser, http://rice.plantbiology.msu.edu/cgi-bin/gbrowse/rice/. 
Supplementary information in Chapter 3 


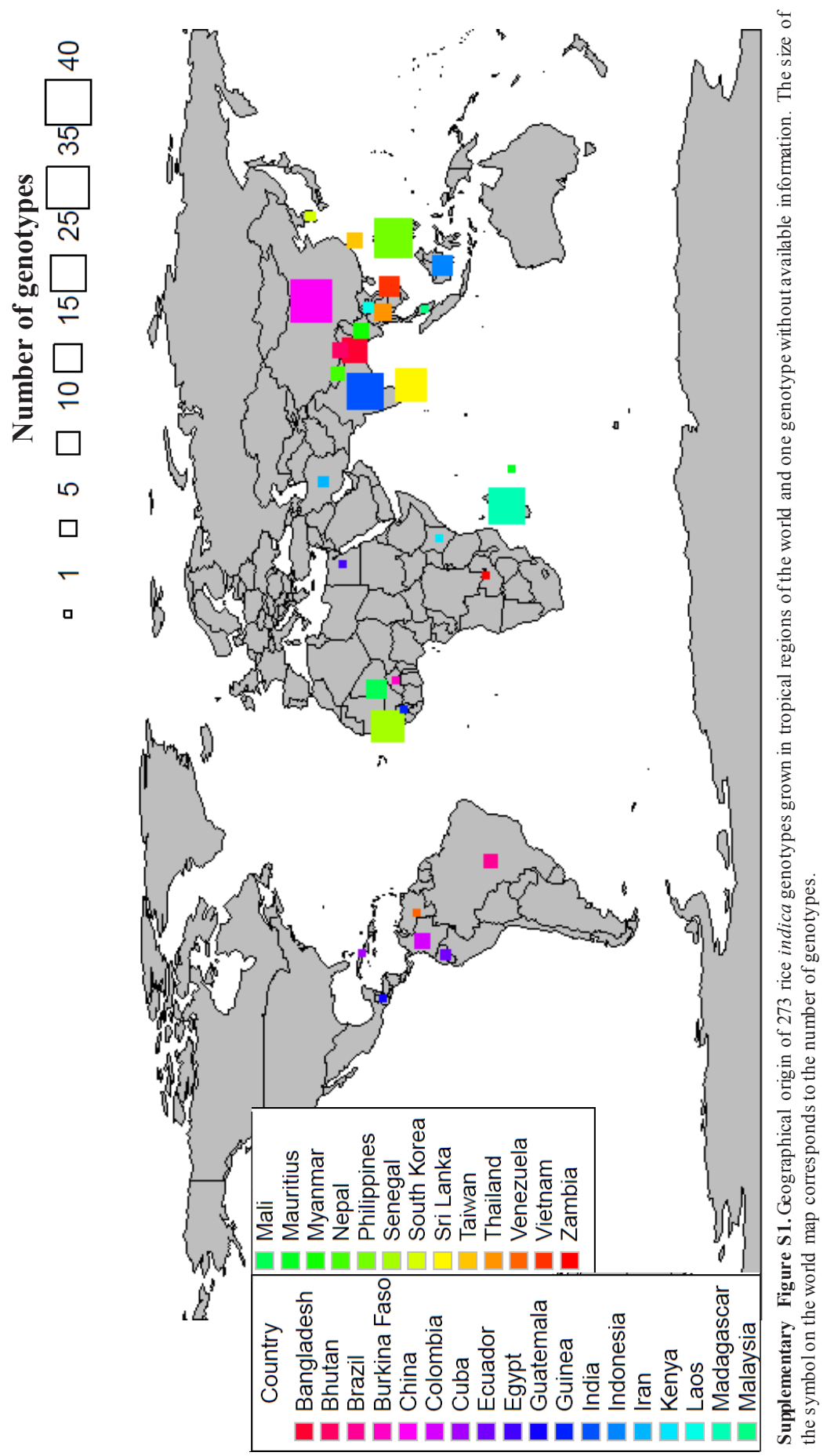



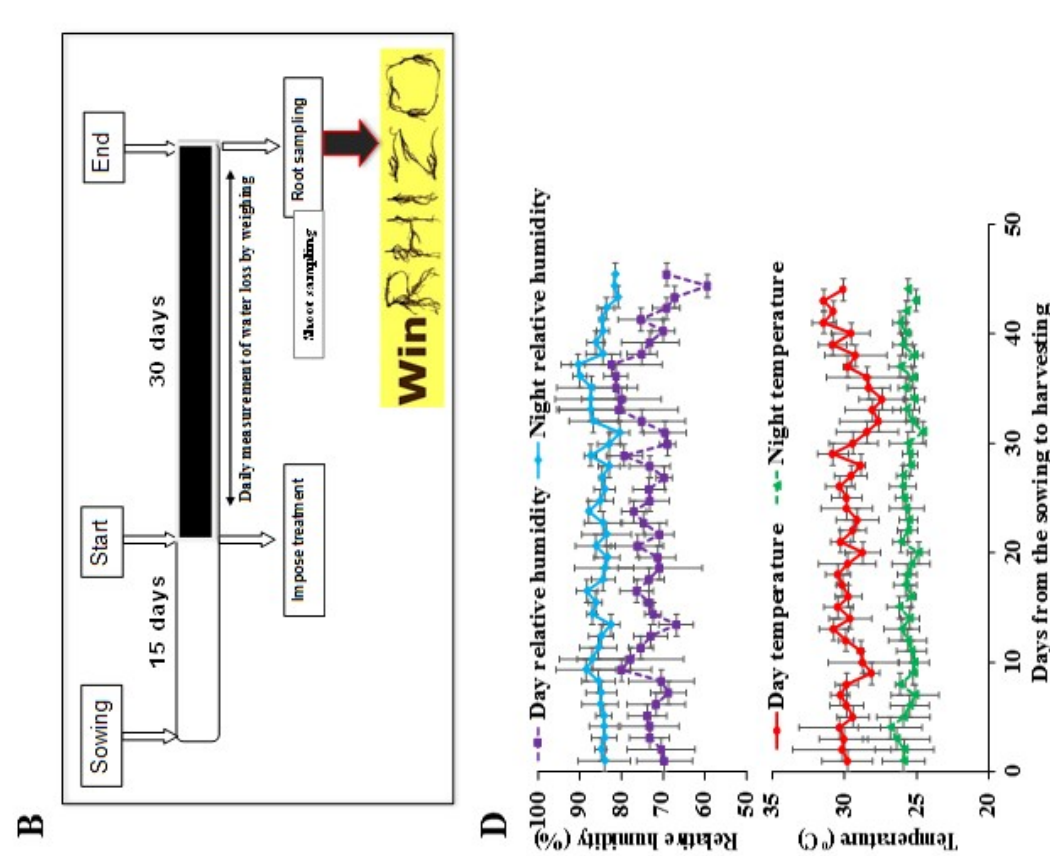

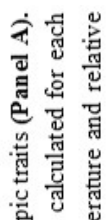

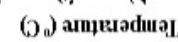
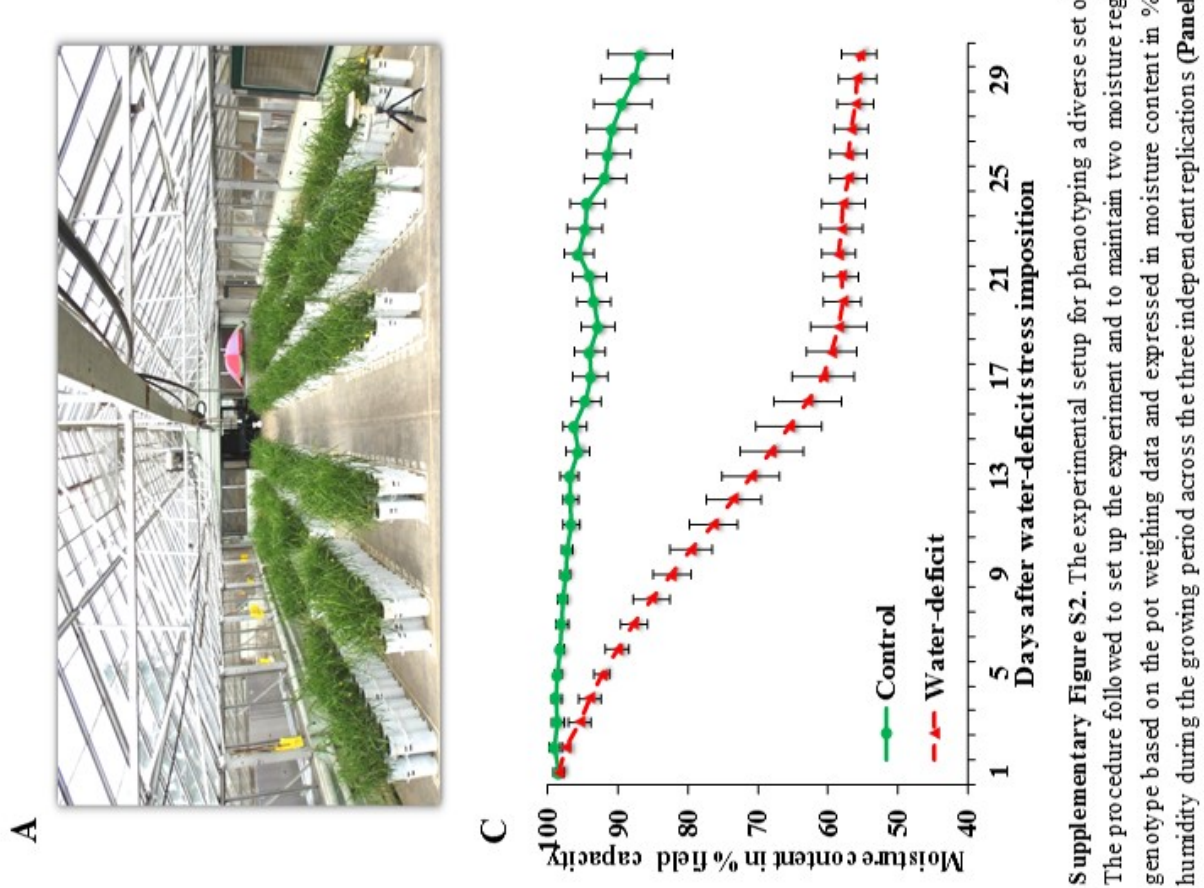

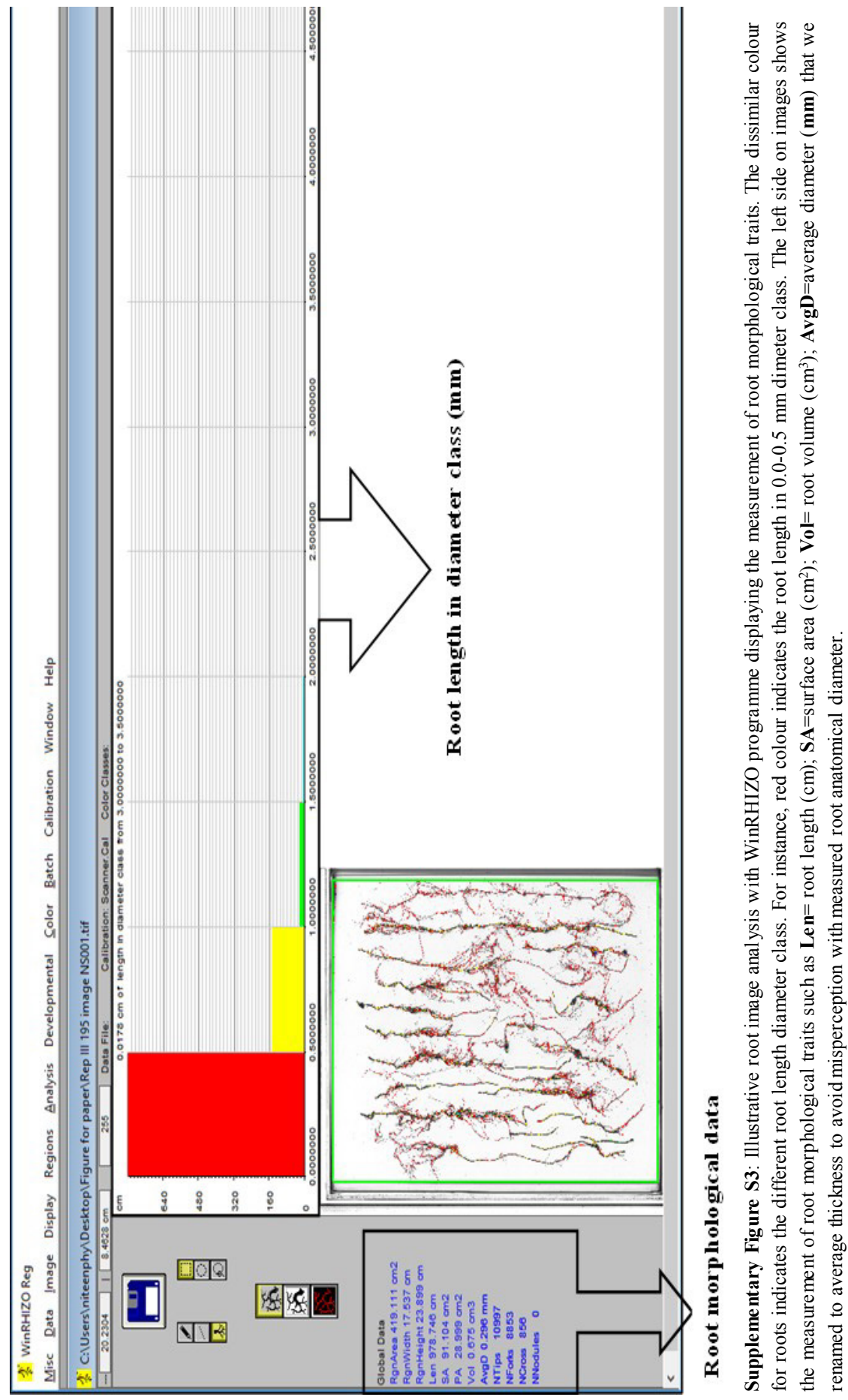


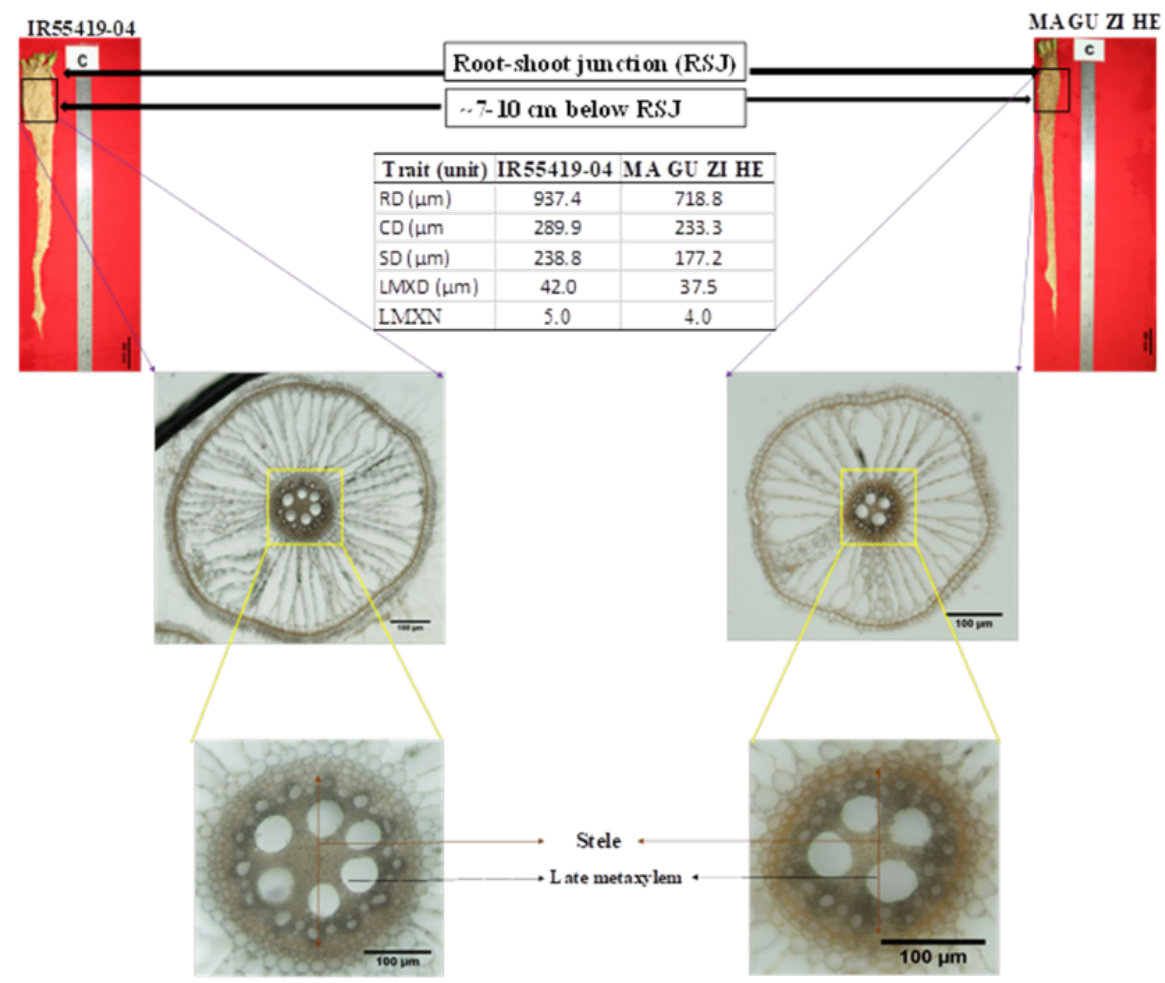

Supplementary Figure S4: The root anatomical trait variation of two rice genotypes near root-shoot junction in control conditions. RD: root diameter, CD: cortex diameter, SD: stele diameter, LMXD: late metaxylem diameter and LMXN: late metaxylem number. Scale bar on root morphology image is $50 \mathrm{~cm}$ and on root anatomy is 100 $\mu \mathrm{m}$. The table on image displays mean root anatomical variation measured across three replications. 


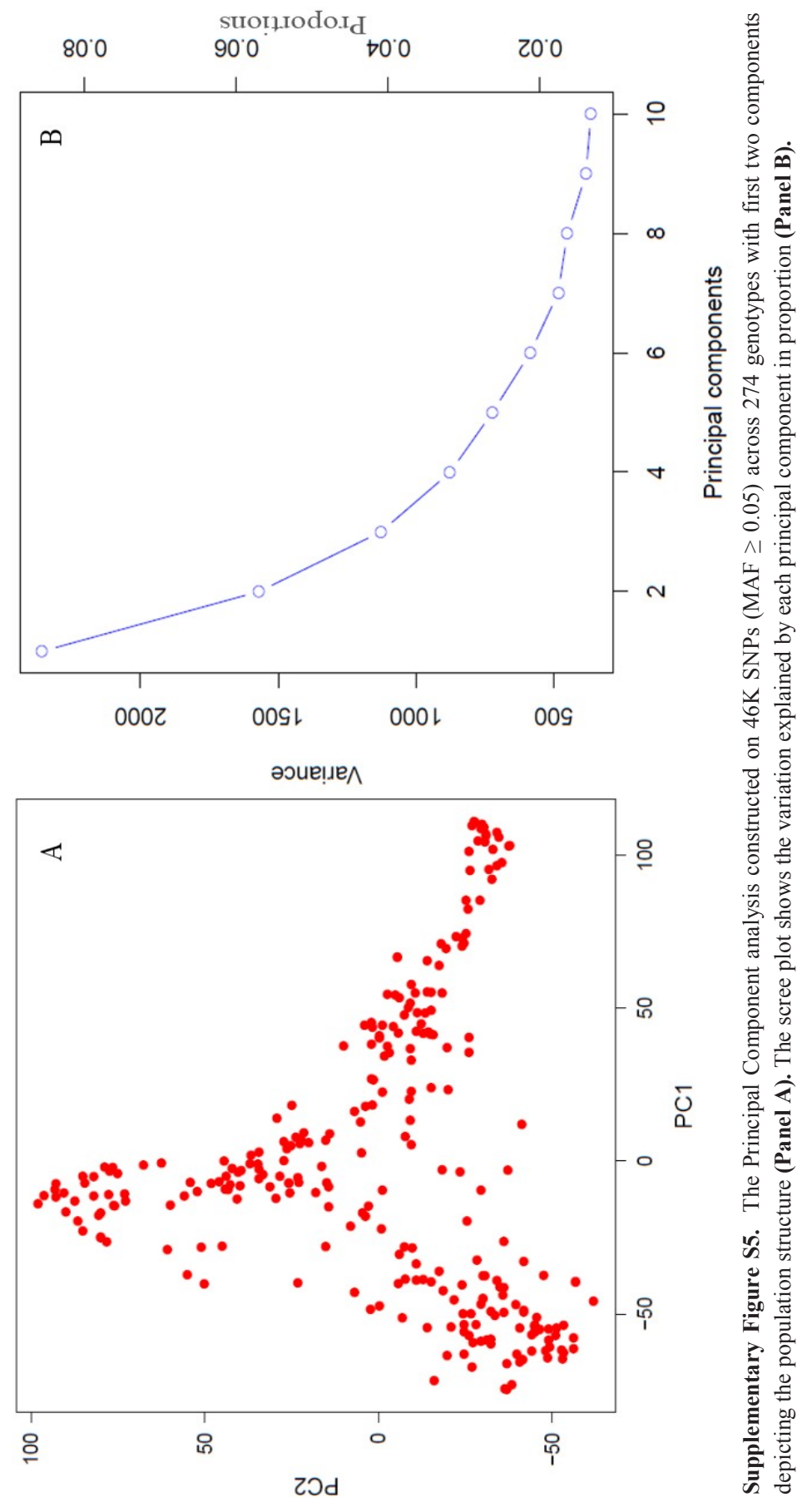



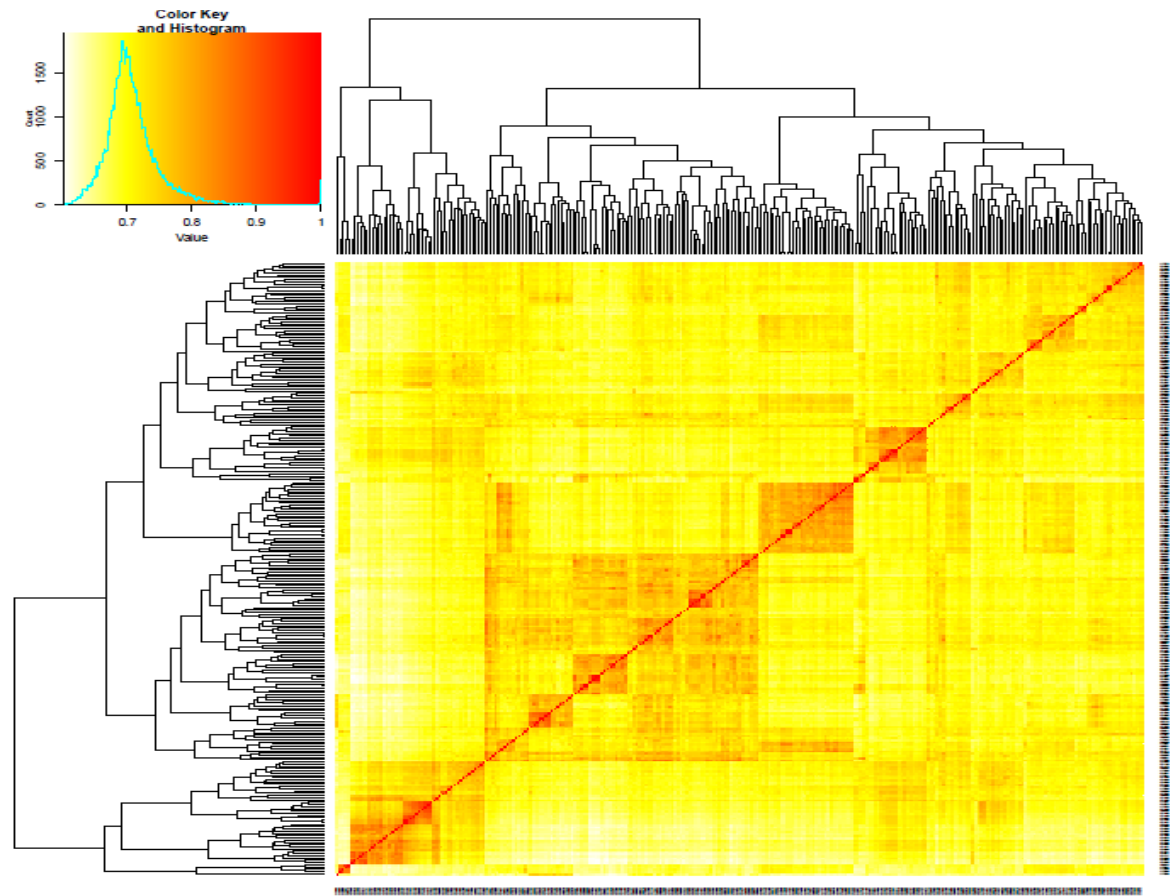

Supplementary Figure S6. The heat map of kinship matrix defining genetic relatedness across 274 genotypes with red and yellow colour indicates the highest and lowest correlation between pairs of the genotypes respectively. A hierarchical clustering between genotypes is based on kinship values. 

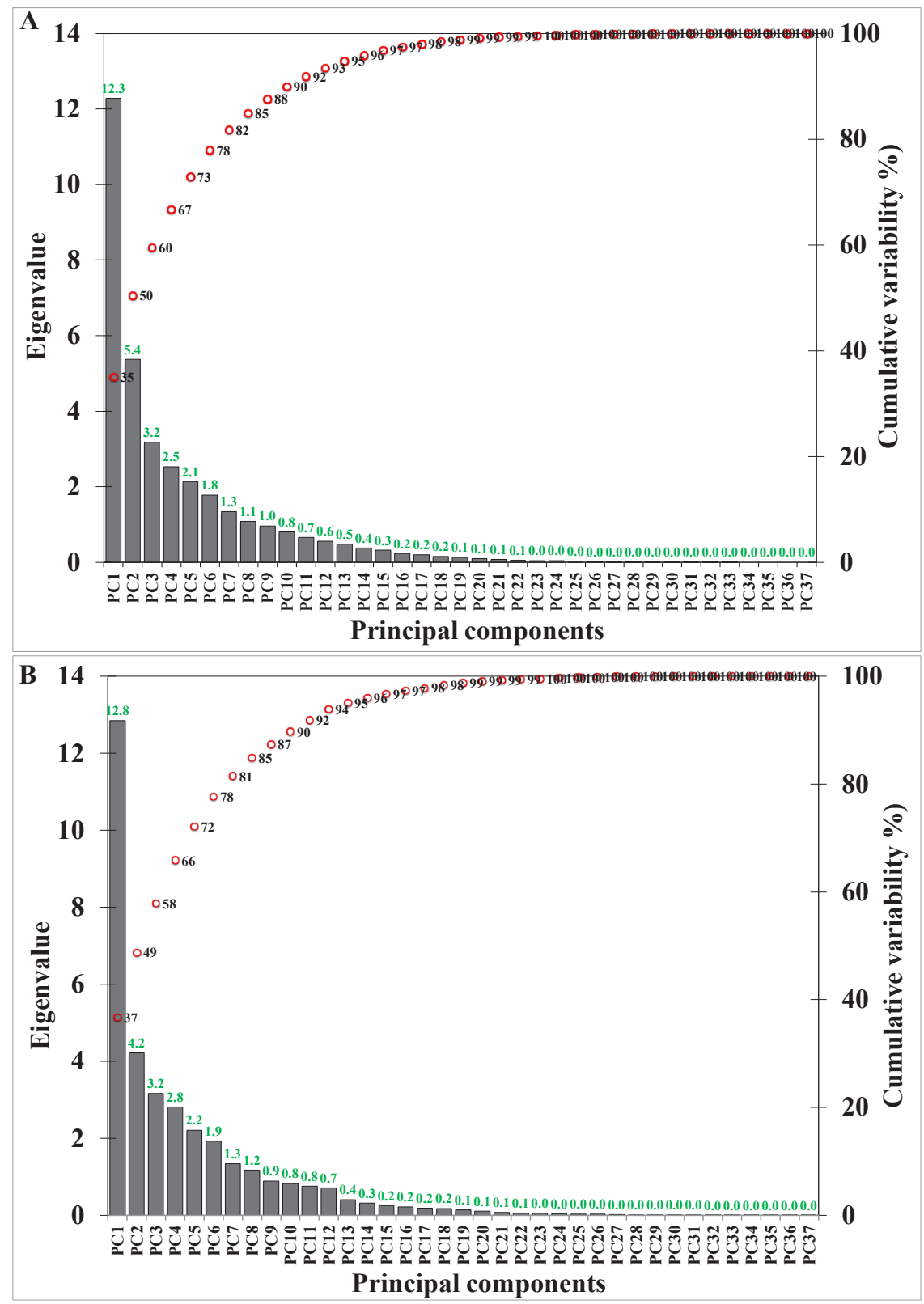

Supplementary Figure S7. The Principal Component Analysis scree plot of 35 phenotypic traits across 274 genotypes depicting the variation explained by each component (PC) in control (Panel A) or water-deficit stress (Panel B) conditions. The PC1 to PC8 with eigenvalues greater than 1.0 (green value above bars) were considered significant and cumulatively explained $>80 \%$ total variation. 


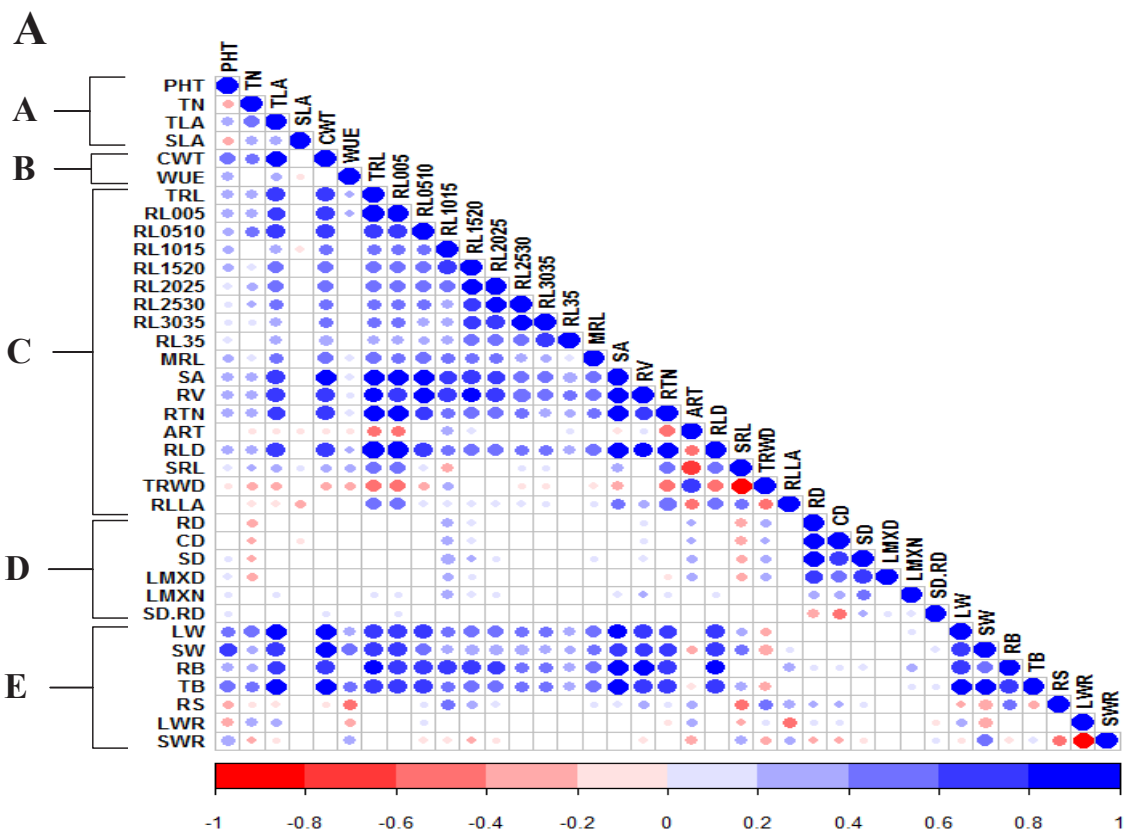

B

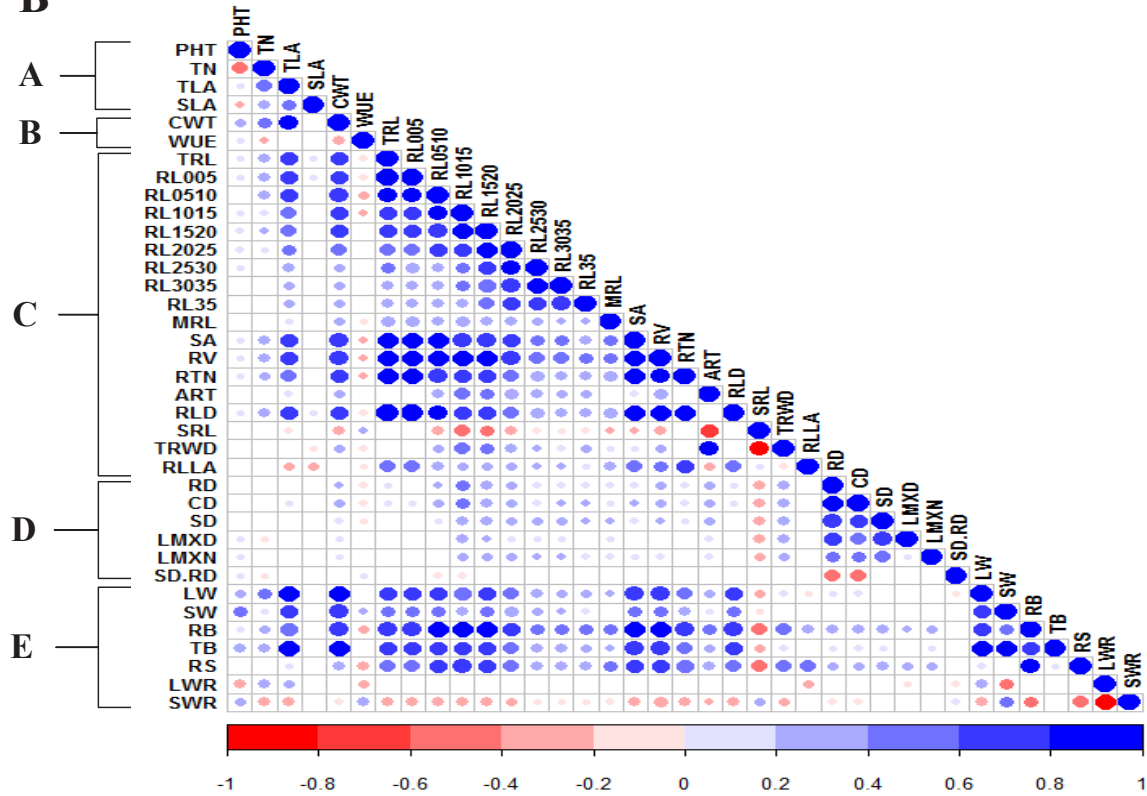

Supplementary Figure S8. Pearson correlation coefficients between 35 phenotypic traits in control (Panel A) and water-deficit stress (Panel B) conditions. The blue and red colours indicate positive and negative correlations, respectively. Colour intensity and size of the circle are proportional to the strength of correlation coefficients between the pair of traits. Uppercase letters on the left panels of the figure correspond with trait classifications as in Table 1; for trait acronyms and units see the Table 1. 


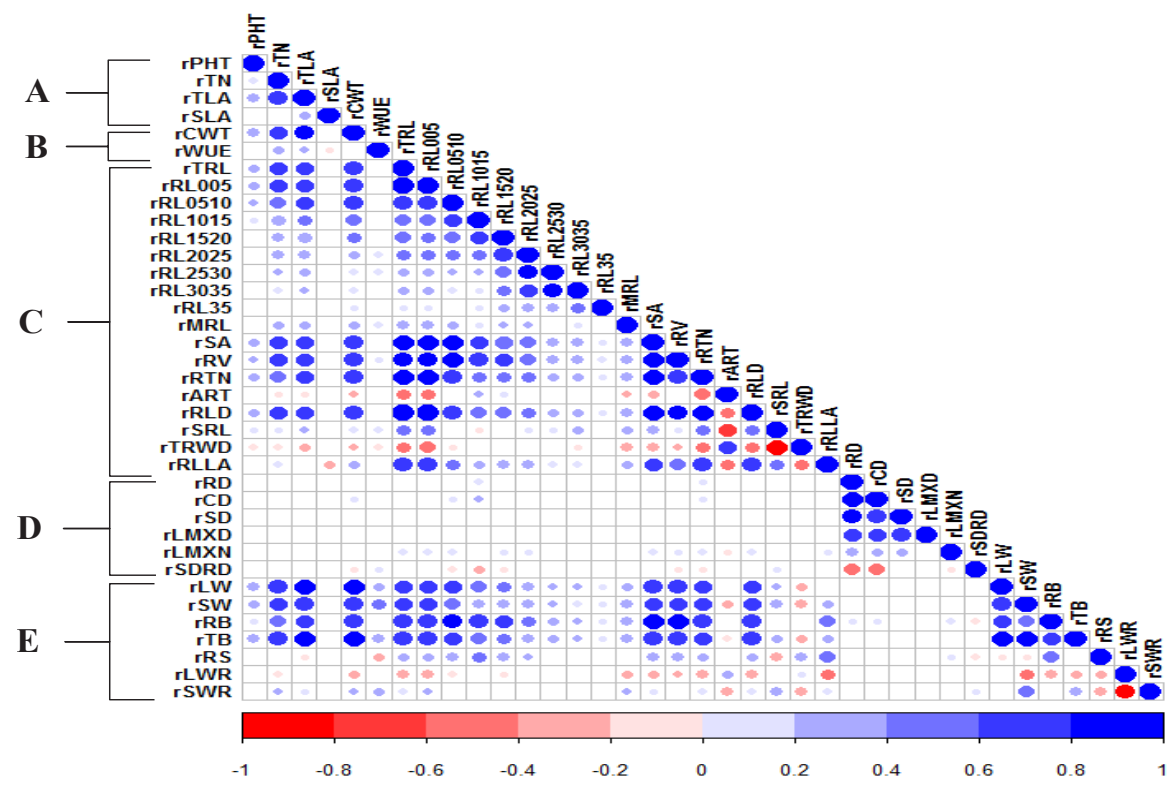

Supplementary Figure S9. Pearson correlation coefficients for the plasticity of 35 phenotypic traits (Panel C). The blue and red colours indicate positive and negative correlations, respectively. Colour intensity and size of the circle are proportional to the strength of correlation coefficients between the pair of traits. Uppercase letters on the left panels of the figure correspond with trait classifications as in Table 1; for trait acronyms and units see the Table 1. 


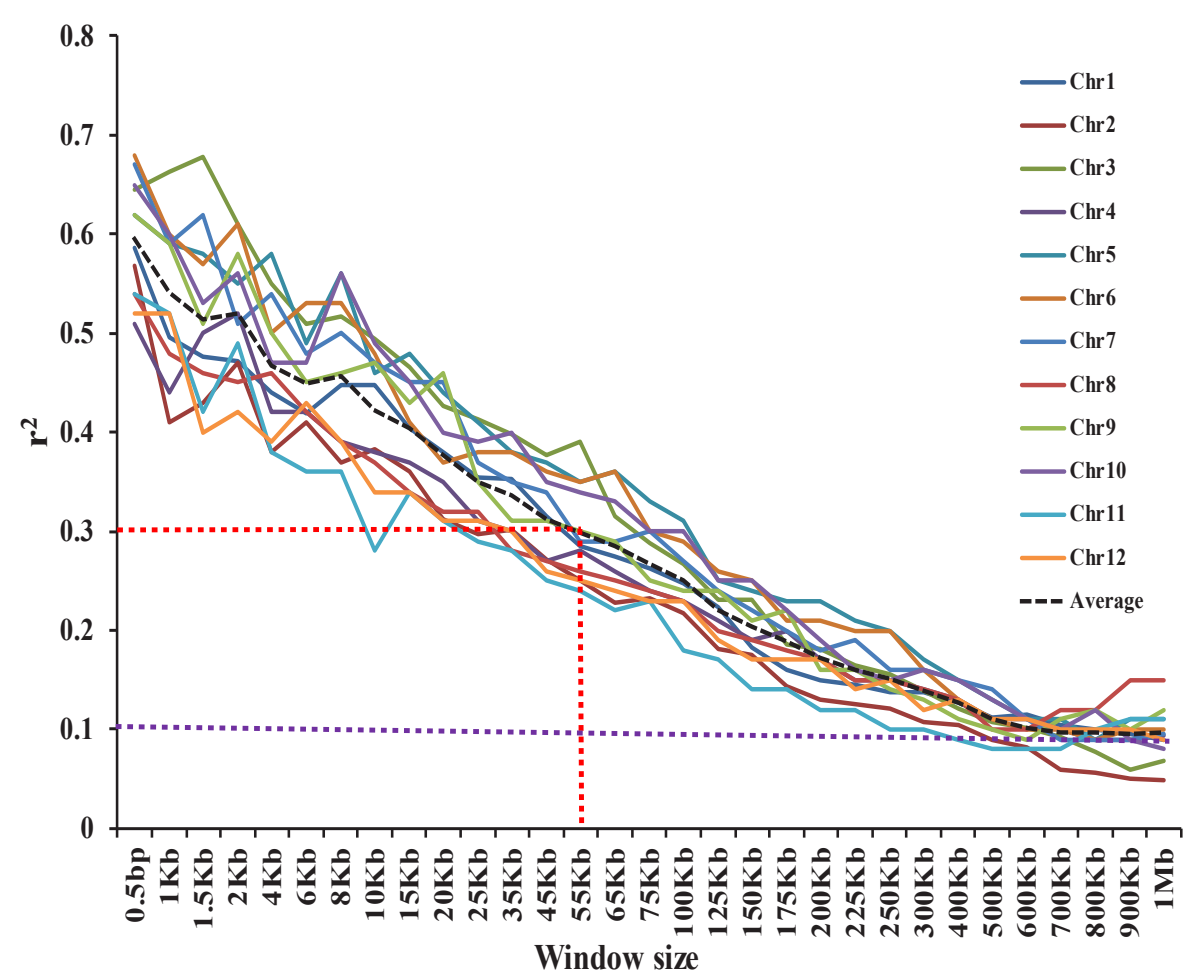

Supplementary Figure S10. Individual chromosome and average genome wide linkage disequilibrium decay as a measure of $r^{2}$ between the pairs of SNPs over the physical distance on the genome. The $r^{2}$ was calculated using the 100 bp sliding window in the TASSEL 5 programme. 

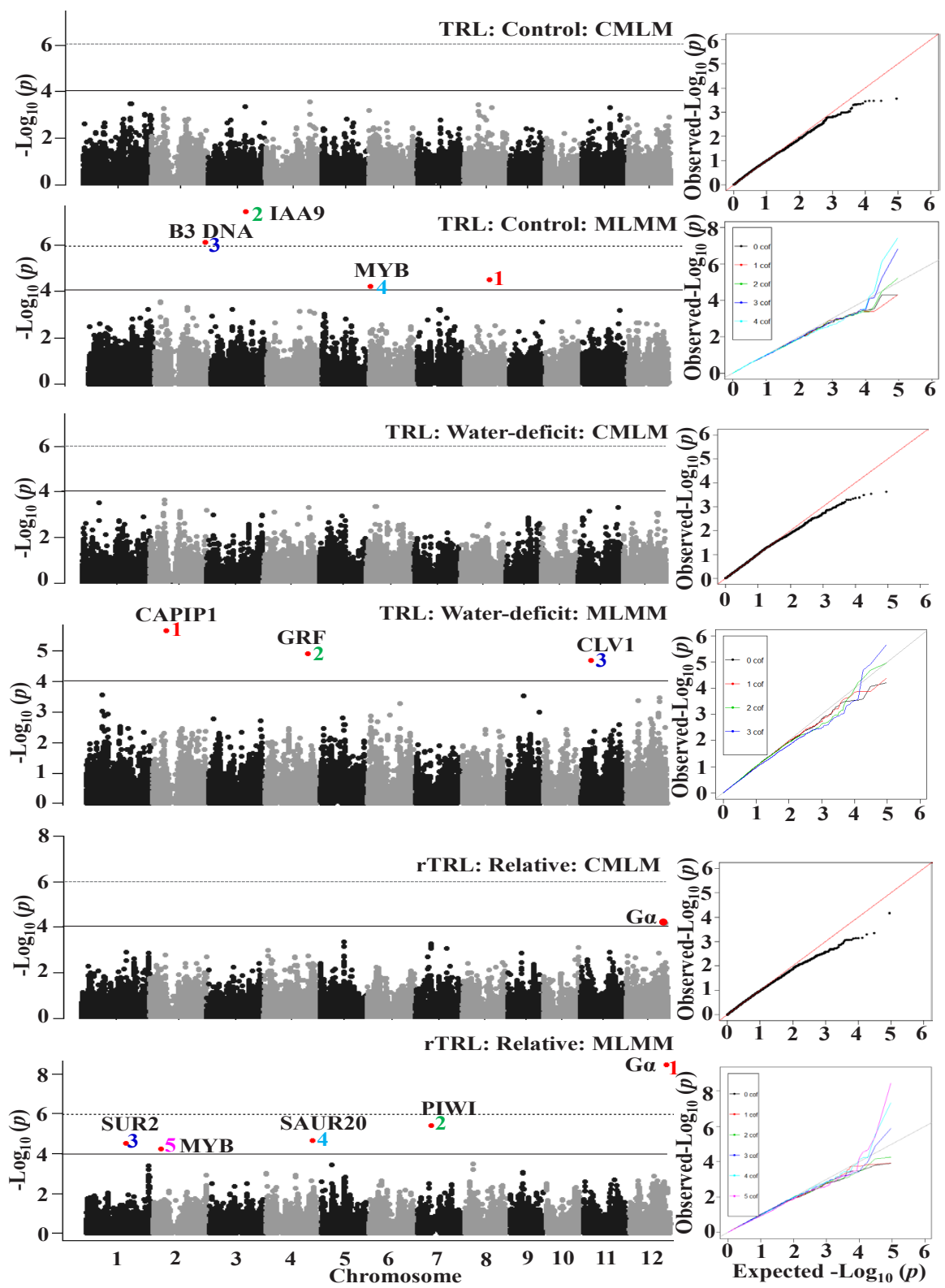

Supplementary Figure S11. The GWAS result through the compressed mixed linear model (CMLM) and the multi-locus mixed model (MLMM) approaches for total root length (TRL) in control and water-deficit stress conditions and for its plasticity as a relative measure. Significant SNPs (coloured red in the Manhattan plots) are distinguished by a threshold $P$ value lines (solid black $=\left[-\log _{10} P>4\right]$ and dotted black=Bonferroni-corrected significance threshold). Significant SNP on MLMM Manhattan plots are numbered in the order that they were included in the model as a cofactor (cof in Quantile-Quantile plot is for cofactor). A priori candidate genes (see the Supplementary Tables S9-S11) are indicated near to peak SNP in the Manhattan plot. 

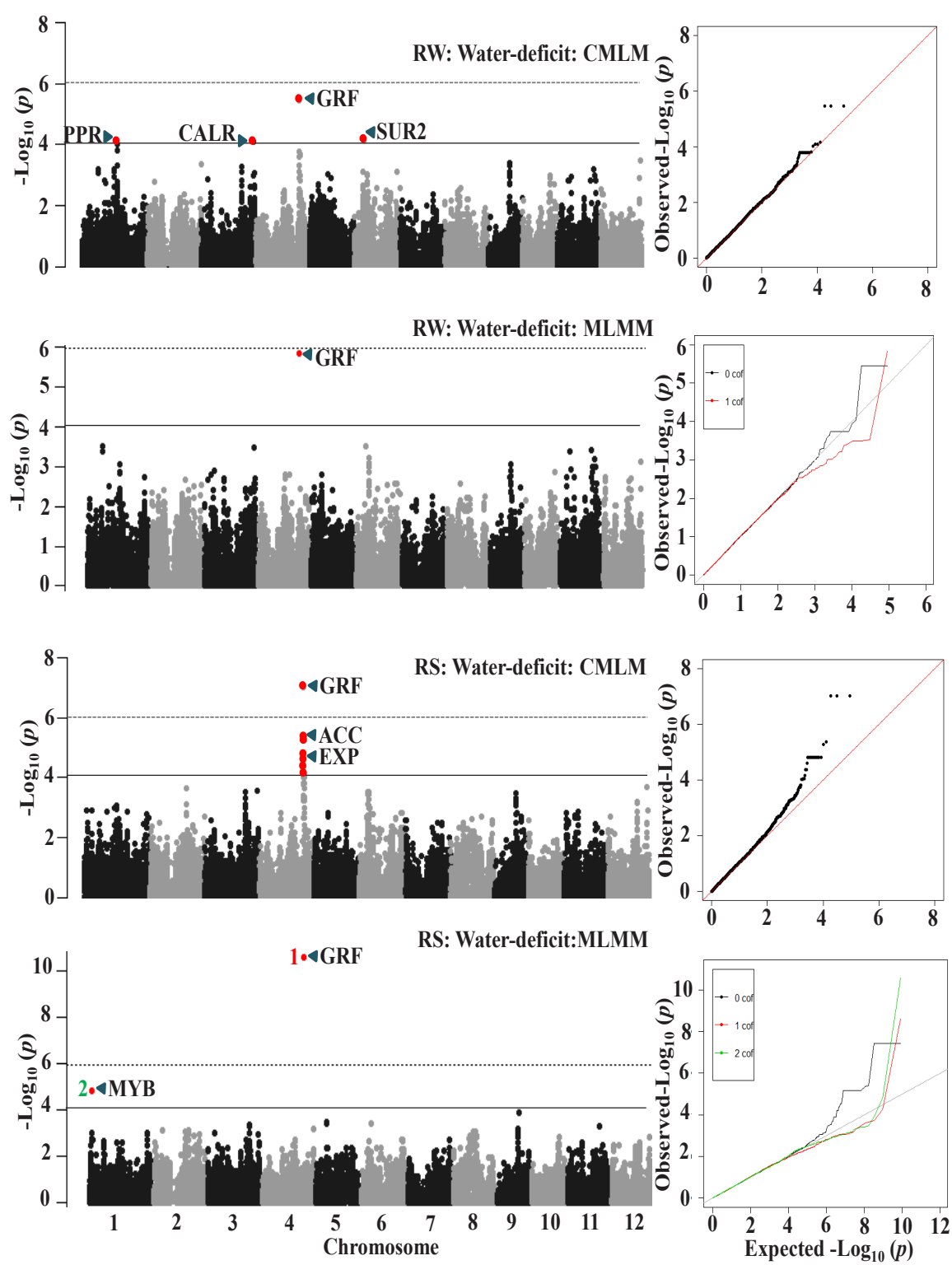

Supplementary Figure S12. The GWAS result through the compressed mixed linear model (CMLM) and multilocus mixed model (MLMM) approaches for root weight (RW) and root: shoot ratio (RS) in water-deficit stress condition. Significant SNPs (coloured red in the Manhattan plots) are distinguished by a threshold $P$ value lines (solid black= $\left[-\log _{10} P>4\right]$ and dotted black=Bonferroni-corrected significance threshold). Significant SNP on MLMM Manhattan plots are numbered in the order that they were included in the model as a cofactor (cof in Quantile-Quantile plot is for cofactor). A Priori candidate gene (see the Supplementary Table S10) are indicated near to peak SNP/SNPs in the Manhattan plot. 

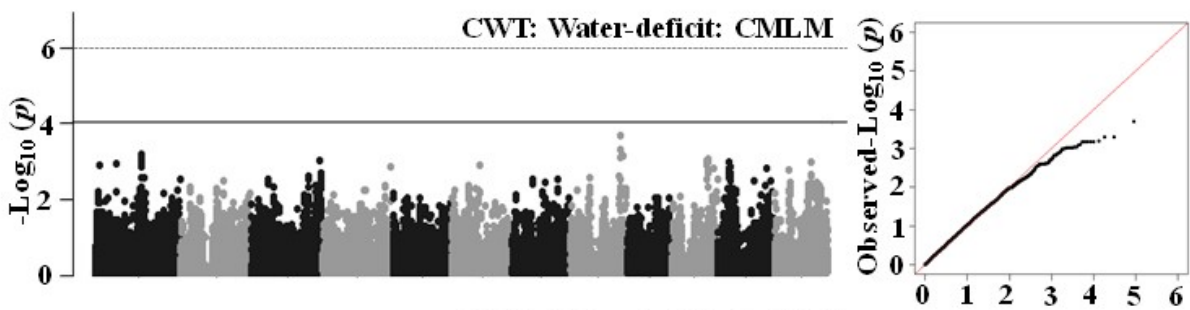

CWT: Water-deficit: MLMM
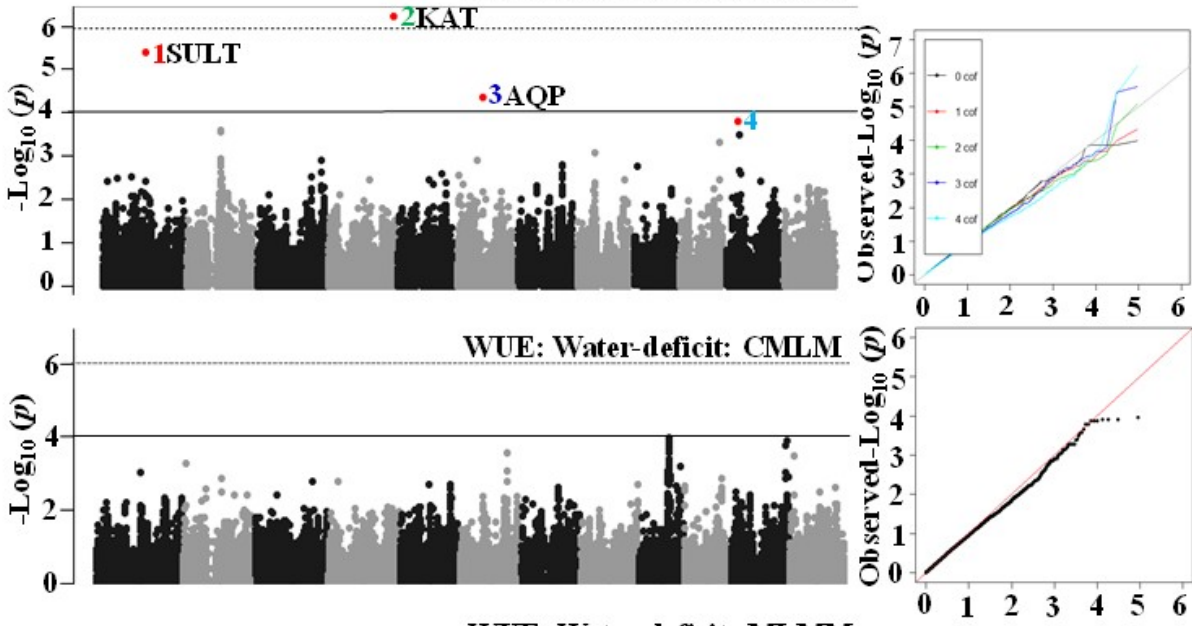

WUE: Water-deficit: MLMM

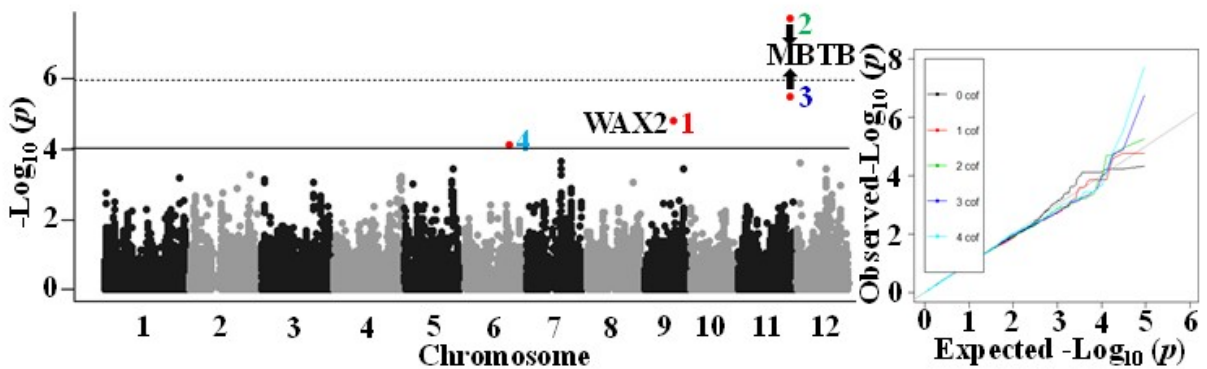

Supplementary Figure S13. The GWAS result through the compressed mixed linear model (CMLM) and multilocus mixed model (MLMM) approaches for cumulative water transpiration (CWT) and water use efficiency (WUE) in water-deficit stress condition. Significant SNPs (coloured red in the Manhattan plots) are distinguished by a threshold $P$ value lines (solid black $=\left[-\log _{10} P>4\right]$ and dotted black=Bonferroni-corrected significance). Significant SNP on MLMM Manhattan plots are numbered in the order that they were included in the model as a cofactor (cof in Quantile-Quantile plot is for cofactor). A priori candidate genes (see the Supplementary Table S8) are indicated near to peak SNP/SNPs in the Manhattan plot. 

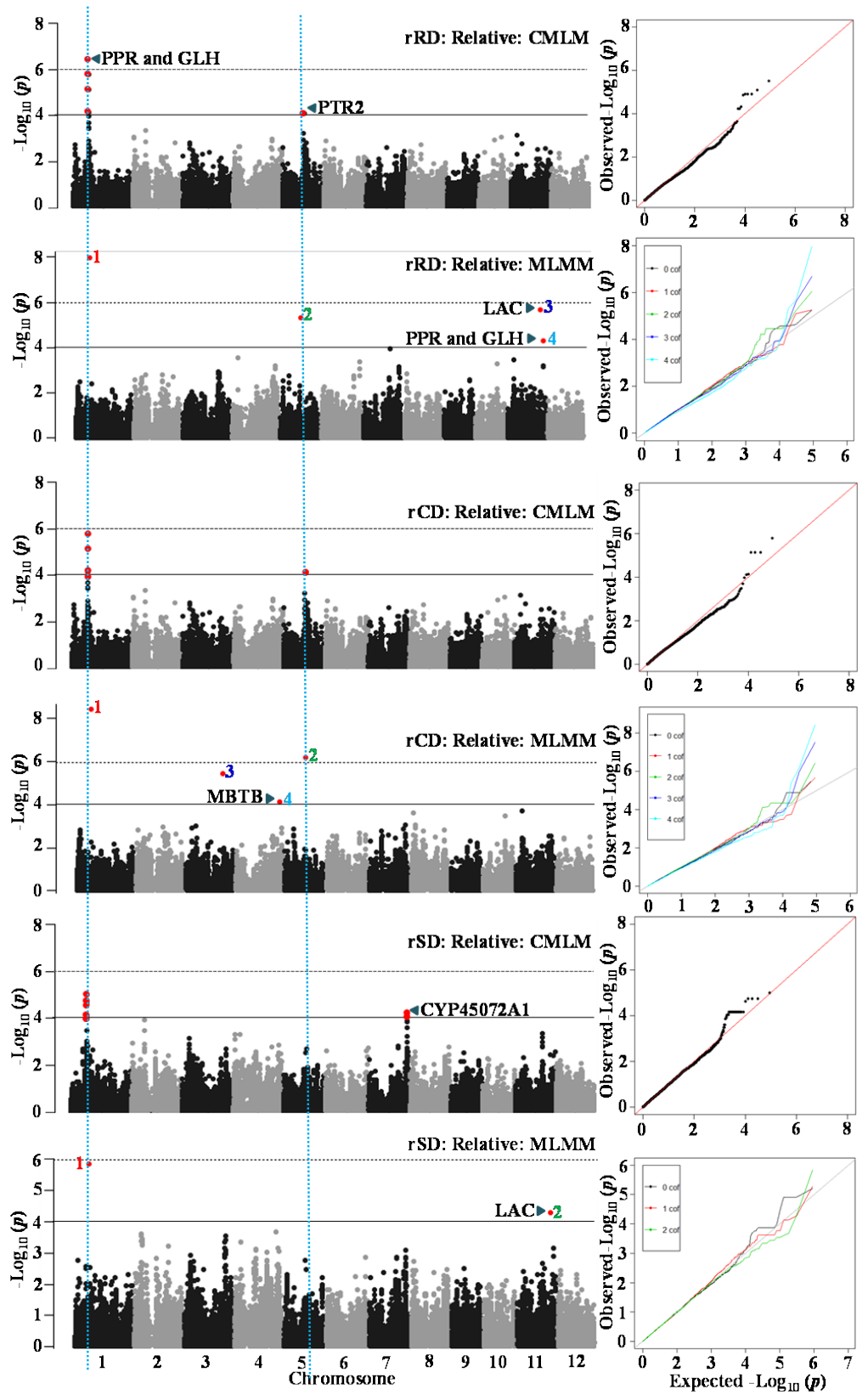

Supplementary Figure S14. The GWAS result through the compressed mixed linear model (CMLM) and multilocus mixed model (MLMM) approaches for plasticity as the relative value of the water-deficit stress over the control conditions for root diameter (rRD), cortex diameter (rCD) and stele diameter (rSD). Significant SNPs (coloured red in the Manhattan plots) are distinguished by a threshold $P$ value lines (solid black=[-Log $P>4]$ and dotted black=Bonferroni-corrected significance). Significant SNP on MLMM Manhattan plots are numbered in the order that they were included in the model as a cofactor (cof in Quantile-Quantile plot is for cofactor). $A$ priori candidate genes (see the Supplementary Table S11) are indicated near to peak SNP in the Manhattan plot. 
Supplementary Table S1. Descriptive statistics and the significance of $P$ (Wald test summary) value based on a linear mixed model for genotype $(\mathrm{G})$, treatment $(\mathrm{T})$ and their interactions $(\mathrm{G} \times \mathrm{T})$. For more details on trait acronyms and units see the Table 1 .

\begin{tabular}{|c|c|c|c|c|c|c|c|c|c|c|}
\hline \multirow[b]{2}{*}{ Traits } & \multicolumn{3}{|c|}{ Control (C) } & \multicolumn{3}{|c|}{ Water-deficit (WD) } & \multirow[b]{2}{*}{$\% \mathrm{C}$} & \multicolumn{3}{|c|}{$P$ value (Wald test) } \\
\hline & Mean \pm SD & Min & Max & Mean \pm SD & Min & Max & & G & $\mathbf{T}$ & $\mathbf{G} \times \mathbf{T}$ \\
\hline \multicolumn{11}{|c|}{ (A) Shoot morphological traits } \\
\hline PHT & $107.00 \pm 16.40$ & 63.47 & 150.10 & $84.40 \pm 13.60$ & 51.70 & 120.40 & -21 & $<0.001$ & $<0.001$ & 0.027 \\
\hline $\mathrm{TN}$ & $19.10 \pm 6.03$ & 7.67 & 38.70 & $14.70 \pm 4.14$ & 6.00 & 28.00 & -23 & $<0.001$ & $<0.001$ & 0.133 \\
\hline TLA & $0.19 \pm 0.05$ & 0.06 & 0.37 & $0.09 \pm 0.02$ & 0.04 & 0.18 & -53 & $<0.001$ & $<0.001$ & $<0.001$ \\
\hline SLA & $0.02 \pm 0.002$ & 0.02 & 0.03 & $0.02 \pm 0.002$ & 0.02 & 0.03 & -2 & $<0.001$ & $<0.001$ & 0.131 \\
\hline \multicolumn{11}{|c|}{ (B) Physiological traits } \\
\hline CWT & $5.57 \pm 1.30$ & 1.72 & 8.90 & $2.40 \pm 0.49$ & 1.30 & 4.09 & -56 & $<0.001$ & $<0.001$ & $<0.001$ \\
\hline WUE & $3.24 \pm 0.27$ & 2.40 & 4.06 & $4.00 \pm 0.44$ & 2.90 & 6.60 & +23 & $<0.001$ & $<0.001$ & 0.038 \\
\hline \multicolumn{11}{|c|}{ (C) Root morphological traits } \\
\hline TRL & $0.76 \pm 0.25$ & 0.19 & 1.67 & $0.40 \pm 0.11$ & 0.15 & 0.88 & -47 & $<0.001$ & $<0.001$ & 0.004 \\
\hline RL005 & $0.71 \pm 0.24$ & 0.17 & 1.60 & $0.39 \pm 0.10$ & 0.15 & 0.84 & -45 & $<0.001$ & $<0.001$ & 0.005 \\
\hline RL0510 & $41.70 \pm 11.15$ & 13.64 & 87.40 & $16.80 \pm 5.45$ & 5.19 & 39.00 & -60 & $<0.001$ & $<0.001$ & $<0.001$ \\
\hline RL1015 & $8.27 \pm 3.90$ & 1.64 & 29.30 & $2.80 \pm 1.23$ & 0.48 & 7.54 & -66 & $<0.001$ & $<0.001$ & $<0.001$ \\
\hline RL1520 & $1.20 \pm 0.46$ & 0.33 & 3.55 & $0.68 \pm 0.28$ & 0.11 & 1.90 & -43 & $<0.001$ & $<0.001$ & 0.043 \\
\hline RL2025 & $0.51 \pm 0.24$ & 0.11 & 2.02 & $0.31 \pm 0.14$ & 0.04 & 0.88 & -39 & $<0.001$ & $<0.001$ & 0.235 \\
\hline RL2530 & $0.27 \pm 0.15$ & 0.04 & 0.96 & $0.16 \pm 0.08$ & 0.01 & 0.50 & -41 & 0.004 & $<0.001$ & 0.411 \\
\hline RL3035 & $0.12 \pm 0.08$ & 0.01 & 0.48 & $0.07 \pm 0.04$ & 0.004 & 0.23 & -42 & 0.116 & $<0.001$ & 0.657 \\
\hline RL35 & $10.80 \pm 10.30$ & 0.41 & 94.70 & $7.04 \pm 4.70$ & 0.28 & 30.20 & -35 & 0.711 & $<0.001$ & 0.922 \\
\hline MRL & $58.10 \pm 5.60$ & 41.8 & 73.90 & $55.50 \pm 5.60$ & 37.80 & 69.40 & -4 & $<0.001$ & $<0.001$ & 0.262 \\
\hline SA & $0.40 \pm 0.12$ & 0.11 & 0.94 & $0.21 \pm 0.06$ & 0.07 & 0.47 & -48 & $<0.001$ & $<0.001$ & 0.006 \\
\hline RV & $19.00 \pm 5.70$ & 5.85 & 45.48 & $9.43 \pm 2.80$ & 2.91 & 21.70 & -50 & $<0.001$ & $<0.001$ & 0.002 \\
\hline ART & $0.19 \pm 0.02$ & 0.14 & 0.27 & $0.17 \pm 0.01$ & 0.14 & 0.21 & -11 & $<0.001$ & $<0.001$ & 0.026 \\
\hline SRL & $0.46 \pm 0.09$ & 0.26 & 0.85 & $0.56 \pm 0.13$ & 0.35 & 1.71 & +22 & $<0.001$ & $<0.001$ & 0.612 \\
\hline TRWD & $0.20 \pm 0.04$ & 0.10 & 0.36 & $0.16 \pm 0.03$ & 0.09 & 0.34 & -20 & $<0.001$ & $<0.001$ & 0.832 \\
\hline RLLA & $3.94 \pm 0.92$ & 2.04 & 7.40 & $4.40 \pm 0.98$ & 2.00 & 8.50 & +12 & $<0.001$ & $<0.001$ & 0.359 \\
\hline \multicolumn{11}{|c|}{ (D) Root anatomical traits } \\
\hline $\mathrm{RD}$ & $949.50 \pm 92$ & 668.10 & 1305.90 & $834.17 \pm 91.60$ & 597.60 & 1106.40 & -12 & $<0.001$ & $<0.001$ & 0.507 \\
\hline CD & $305.01 \pm 32.60$ & 202.00 & 401.00 & $250.80 \pm 31.70$ & 167.10 & 345.60 & -18 & $<0.001$ & $<0.001$ & 0.545 \\
\hline SD & $228.90 \pm 21.22$ & 166.90 & 283.40 & $219.18 \pm 20.60$ & 157.70 & 284.40 & -4 & $<0.001$ & $<0.001$ & 0.490 \\
\hline LMXD & $42.36 \pm 4.50$ & 30.40 & 58.20 & $39.46 \pm 3.90$ & 27.82 & 51.38 & -7 & $<0.001$ & $<0.001$ & 0.454 \\
\hline LMXN & $4.70 \pm 0.50$ & 3.33 & 6.33 & $4.60 \pm 0.56$ & 3.33 & 6.67 & -2 & $<0.001$ & $<0.001$ & 0.361 \\
\hline SD:RD & $24.20 \pm 1.30$ & 20.70 & 28.30 & $26.52 \pm 1.80$ & 22.57 & 34.87 & +10 & $<0.001$ & $<0.001$ & 0.648 \\
\hline \multicolumn{11}{|c|}{ (E) Dry matter and dry matter partitioning traits } \\
\hline LW & $8.40 \pm 2.21$ & 2.57 & 14.97 & $4.15 \pm 0.82$ & 1.87 & 6.93 & -51 & $<0.001$ & $<0.001$ & $<0.001$ \\
\hline SW & $8.01 \pm 2.30$ & 2.33 & 14.73 & $4.46 \pm 0.93$ & 2.00 & 7.53 & -44 & $<0.001$ & $<0.001$ & 0.002 \\
\hline RW & $1.60 \pm 0.46$ & 0.54 & 3.72 & $0.79 \pm 0.25$ & 0.23 & 1.93 & -51 & $<0.001$ & $<0.001$ & 0.007 \\
\hline TW & $18.09 \pm 4.55$ & 5.44 & 31.55 & $9.39 \pm 0.75$ & 4.84 & 14.81 & -48 & $<0.001$ & $<0.001$ & $<0.001$ \\
\hline RS & $0.10 \pm 0.02$ & 0.05 & 0.22 & $0.09 \pm 0.02$ & 0.03 & 0.18 & -10 & $<0.001$ & $<0.001$ & 0.019 \\
\hline LWR & $0.47 \pm 0.04$ & 0.30 & 0.55 & $0.44 \pm 0.03$ & 0.32 & 0.52 & -6 & $<0.001$ & $<0.001$ & 0.055 \\
\hline SWR & $0.44 \pm 0.05$ & 0.35 & 0.64 & $0.47 \pm 0.04$ & 0.38 & 0.63 & +7 & $<0.001$ & $<0.001$ & 0.002 \\
\hline
\end{tabular}

Bold $P$ values are not statistically significant $(P=>0.05) . \% \mathrm{C}: \%$ change $(+:$ increase or $-:$ decrease $)$ over control condition. 
Supplementary Table S2. Broad-sense $\left(\mathrm{H}^{2}\right)$ heritability for 35 phenotypic traits classified in 5 (A-E) categories in control (C) and water-deficit stress (WD) conditions. The narrow-sense $\left(h^{2}\right)$ heritability of 35 phenotypic traits in $\mathrm{C}$, and WD conditions and for their phenotypic plasticity (PP). The details on trait acronyms and units are given in the Table 1.

\begin{tabular}{|c|c|c|c|c|c|}
\hline & \multicolumn{2}{|c|}{$\mathbf{H}^{2}$} & \multicolumn{3}{|c|}{$h^{2}$} \\
\hline Trait acronym & $\mathbf{C}$ & WD & $\mathbf{C}$ & WD & $\mathbf{P P}$ \\
\hline \multicolumn{6}{|c|}{ (A) Shoot morphological traits } \\
\hline PHT (rPHT) & 0.89 & 0.88 & 0.84 & 0.86 & 0.46 \\
\hline $\mathrm{TN}(\mathrm{rTN})$ & 0.70 & 0.77 & 0.86 & 0.86 & 0.46 \\
\hline TLA (rTLA) & 0.61 & 0.58 & 0.84 & 0.65 & 0.57 \\
\hline SLA (rSLA) & 0.63 & 0.52 & 0.73 & 0.64 & 0.34 \\
\hline \multicolumn{6}{|c|}{\begin{tabular}{|l|} 
(B) Physiological traits \\
\end{tabular}} \\
\hline $\mathrm{CWT}(\mathrm{rCWT})$ & 0.53 & 0.42 & 0.70 & 0.39 & 0.56 \\
\hline WUE (rWUE) & 0.48 & 0.22 & 0.59 & 0.68 & 0.58 \\
\hline \multicolumn{6}{|c|}{ (C) Root morphological traits } \\
\hline TRL (rTRL) & 0.46 & 0.45 & 0.82 & 0.56 & 0.48 \\
\hline RL005 (rRL005) & 0.46 & 0.44 & 0.82 & 0.55 & 0.49 \\
\hline RL0510 (rRL0510) & 0.47 & 0.47 & 0.81 & 0.32 & 0.34 \\
\hline RL1015 (rRL1015) & 0.71 & 0.6 & 0.89 & 0.48 & 0.51 \\
\hline RL1520 (rRL1520) & 0.46 & 0.45 & 0.52 & 0.48 & 0.08 \\
\hline RL2025 (rRL2025) & 0.28 & 0.30 & 0.20 & 0.55 & 0.21 \\
\hline RL2530 (rRL2530) & 0.10 & 0.08 & 0.47 & 0.60 & 0.30 \\
\hline RL3035 (rRL3035) & - & 0.08 & - & - & - \\
\hline RL35 (rRL35) & - & 0.04 & - & - & - \\
\hline MRL (rMRL) & 0.34 & 0.13 & 0.52 & 0.52 & 0.28 \\
\hline $\mathrm{SA}(\mathrm{rSA})$ & 0.49 & 0.5 & 0.80 & 0.52 & 0.35 \\
\hline RV (rRV) & 0.54 & 0.52 & 0.79 & 0.47 & 0.56 \\
\hline ART (rART) & 0.58 & 0.03 & 0.57 & 0.57 & 0.64 \\
\hline SRL (rSRL) & 0.57 & 0.36 & 0.78 & 0.57 & 0.61 \\
\hline TRWD (rTRWD) & 0.44 & 0.42 & 0.60 & 0.58 & 0.49 \\
\hline RLLA (rRLLA) & 0.25 & 0.40 & 0.71 & 0.69 & 0.17 \\
\hline \multicolumn{6}{|c|}{ (D) Root anatomical traits } \\
\hline $\mathrm{RD}$ (rRD) & 0.38 & 0.36 & 0.70 & 0.59 & 0.29 \\
\hline $\mathrm{CD}(\mathrm{rCD})$ & 0.31 & 0.33 & 0.76 & 0.54 & 0.31 \\
\hline $\mathrm{SD}(\mathrm{rSD})$ & 0.47 & 0.44 & 0.80 & 0.72 & 0.26 \\
\hline LMXD (rLMXD) & 0.56 & 0.46 & 0.71 & 0.72 & 0.33 \\
\hline LMXN (rLMXN) & 0.27 & 0.46 & 0.56 & 0.78 & 0.50 \\
\hline SD:RD (rSDRD) & 0.31 & 0.43 & 0.75 & 0.41 & 0.24 \\
\hline \multicolumn{6}{|c|}{ (E) Dry matter traits } \\
\hline LW (rLW) & 0.57 & 0.57 & 0.72 & 0.69 & 0.63 \\
\hline SW (rSW) & 0.51 & 0.42 & 0.72 & 0.33 & 0.77 \\
\hline RW (rRW) & 0.57 & 0.58 & 0.78 & 0.53 & 0.35 \\
\hline TW (rTW) & 0.51 & 0.47 & 0.78 & 0.40 & 0.63 \\
\hline RS (rRS) & 0.44 & 0.64 & 0.71 & 0.71 & 0.43 \\
\hline LWR (rLWR) & 0.25 & 0.65 & 0.77 & 0.65 & 0.61 \\
\hline SWR (rSWR) & 0.82 & 0.71 & 0.76 & 0.45 & 0.65 \\
\hline
\end{tabular}


Supplementary Table S3. Summary of identified genome-wide significant association loci for phenotypic traits in control condition using compressed mixed linear model (CMLM) and multi-locus mixed model (MLMM) approaches. The loci commonly detected through both the approaches were marked by an asterisk sign $(*)$ and those detected through only MLMM were marked by a hashtag sign (\#). All the other unmarked loci were detected only through the CMLM approach. Trait acronyms are given in the Table 1.

\begin{tabular}{|c|c|c|c|c|c|c|c|c|c|c|}
\hline \multirow[t]{2}{*}{ Traits } & \multirow[t]{2}{*}{ Chr } & \multirow[t]{2}{*}{ Position } & \multirow[t]{2}{*}{ Allele } & \multirow[t]{2}{*}{ MAF } & \multirow[t]{2}{*}{$P$ valuecmlм } & \multirow[t]{2}{*}{$P$ valueмцмм } & \multirow[t]{2}{*}{ M RAE } & \multicolumn{2}{|c|}{ LD block } & \multirow[t]{2}{*}{ Size (kb) } \\
\hline & & & & & & & & From & To & \\
\hline \multicolumn{11}{|c|}{ (A) Shoot morphological traits } \\
\hline \multirow[t]{2}{*}{ PHT } & 5 & 2173057 & $\mathrm{~A}: \mathrm{G}$ & 0.201 & $1.22 \mathrm{E}-05$ & - & 0.036 & 2102183 & 2197641 & 95 \\
\hline & $1^{*}$ & 38286772 & $\mathrm{G}: \mathrm{A}$ & 0.288 & 0.0001 & $1.23 \mathrm{E}-05$ & -0.051 & 38178239 & 38437530 & 259 \\
\hline \multirow[t]{3}{*}{$\mathrm{TN}$} & $2^{*}$ & 31650233 & $\mathrm{~A}: \mathrm{G}$ & 0.307 & $1.75 \mathrm{E}-05$ & $4.83 \mathrm{E}-08$ & 0.095 & 31624655 & 31770057 & 145 \\
\hline & $5^{\#}$ & 16091302 & $\mathrm{C}: \mathrm{T}$ & 0.106 & - & 4.94E-07 & 0.241 & 15933499 & 16151637 & 218 \\
\hline & $12^{\#}$ & 22528624 & A:G & 0.088 & - & $5.48 \mathrm{E}-05$ & 0.246 & 22511727 & 22544593 & 32 \\
\hline SLA & $11^{*}$ & 19707755 & $\mathrm{~A}: \mathrm{G}$ & 0.493 & $3.08 \mathrm{E}-05$ & $1.56 \mathrm{E}-05$ & -0.030 & 19695820 & 19762199 & 66 \\
\hline \multicolumn{11}{|c|}{ (B) Physiological traits } \\
\hline \multirow[t]{11}{*}{ CWT } & 1 & 29132939 & G:A & 0.062 & $1.36 \mathrm{E}-05$ & - & 0.111 & 29124263 & 29250090 & 125 \\
\hline & $1^{*}$ & 29441428 & $\mathrm{~A}: \mathrm{T}$ & 0.066 & $5.17 \mathrm{E}-07$ & $1.75 \mathrm{E}-06$ & 0.127 & 29394012 & 29470779 & 76 \\
\hline & 1 & 29575437 & $\mathrm{~A}: \mathrm{G}$ & 0.058 & $1.40 \mathrm{E}-06$ & - & 0.127 & 29483935 & 29597597 & 113 \\
\hline & 1 & 29600620 & $\mathrm{C}: \mathrm{T}$ & 0.058 & $1.40 \mathrm{E}-06$ & - & 0.127 & 29597627 & 29606402 & 8 \\
\hline & 1 & 29620240 & $\mathrm{~T}: \mathrm{C}$ & 0.058 & $1.40 \mathrm{E}-06$ & - & 0.127 & 29606625 & 29918762 & 312 \\
\hline & 1 & 29981149 & $\mathrm{~T}: \mathrm{G}$ & 0.055 & $3.73 \mathrm{E}-06$ & - & 0.129 & 29935443 & 29999692 & 64 \\
\hline & 1 & 30060278 & G:A & 0.055 & $3.73 \mathrm{E}-06$ & - & 0.129 & 30027570 & 30172920 & 145 \\
\hline & 2 & 22518040 & $\mathrm{~T}: \mathrm{C}$ & 0.062 & $2.60 \mathrm{E}-05$ & - & 0.113 & 22518040 & 22554488 & 36 \\
\hline & 5 & 7131196 & $\mathrm{~A}: \mathrm{G}$ & 0.091 & $7.95 \mathrm{E}-05$ & - & 0.093 & 7021512 & 7216325 & 194 \\
\hline & 11 & 24219301 & $\mathrm{C}: \mathrm{A}$ & 0.124 & $9.73 \mathrm{E}-05$ & - & -0.072 & 24219246 & 24244335 & 25 \\
\hline & $11^{\#}$ & 7124411 & $\mathrm{~T}: \mathrm{C}$ & 0.091 & - & $1.28 \mathrm{E}-05$ & 0.252 & 7095402 & 7124411 & 29 \\
\hline \multirow[t]{5}{*}{ WUE } & 4 & 3707267 & G:A & 0.128 & $4.65 \mathrm{E}-05$ & - & 0.031 & 3707245 & 3798274 & 91 \\
\hline & 3 & 26299468 & $\mathrm{~A}: \mathrm{G}$ & 0.378 & 8.03E-05 & - & 0.023 & 26078859 & 26333221 & 254 \\
\hline & 8 & 20079154 & $\mathrm{~A}: \mathrm{G}$ & 0.066 & $9.82 \mathrm{E}-05$ & - & 0.040 & 20021659 & 20091332 & 69 \\
\hline & $7^{\#}$ & 20000202 & $\mathrm{C}: \mathrm{T}$ & 0.191 & - & $6.20 \mathrm{E}-06$ & -0.026 & 19968125 & 20085468 & 117 \\
\hline & $12^{\#}$ & 22169193 & $\mathrm{~T}: \mathrm{G}$ & 0.310 & - & $1.91 \mathrm{E}-05$ & 0.031 & 22161720 & 22194001 & 32 \\
\hline \multicolumn{11}{|c|}{ (C) Root morphological traits } \\
\hline \multirow[t]{4}{*}{ TRL } & $8^{\#}$ & 17150092 & C:A & 0.098 & - & $3.20 \mathrm{E}-05$ & -0.055 & 17032320 & 17158399 & 126 \\
\hline & $3^{\#}$ & 23497636 & $\mathrm{~T}: \mathrm{C}$ & 0.084 & - & 3.74E-08 & -0.186 & 23497636 & 23514021 & 16 \\
\hline & $2^{\#}$ & 34358656 & C:A & 0.153 & - & 7.87E-07 & 0.174 & 34337530 & 34358688 & 21 \\
\hline & $6^{\#}$ & 416782 & $\mathrm{~T}: \mathrm{C}$ & 0.271 & - & $6.20 \mathrm{E}-05$ & 0.217 & 397816 & 457474 & 59 \\
\hline \multirow[t]{3}{*}{ RL005 } & $8^{\#}$ & 17150092 & $\mathrm{C}: \mathrm{A}$ & 0.098 & - & $3.13 \mathrm{E}-05$ & -0.052 & 17032320 & 17158399 & 126 \\
\hline & $3^{\#}$ & 23497636 & $\mathrm{~T}: \mathrm{C}$ & 0.084 & - & $3.42 \mathrm{E}-08$ & 0.192 & 23497636 & 23514021 & 16 \\
\hline & $2^{\#}$ & 34358656 & $\mathrm{C}: \mathrm{A}$ & 0.153 & - & $6.36 \mathrm{E}-07$ & 0.180 & 34337530 & 34358688 & 21 \\
\hline \multirow[t]{2}{*}{ RL05100 } & $8^{\#}$ & 9082010 & $\mathrm{C}: \mathrm{T}$ & 0.084 & - & $1.44 \mathrm{E}-06$ & 0.200 & 9049321 & 9090378 & 41 \\
\hline & $6^{\#}$ & 366330 & $\mathrm{~A}: \mathrm{G}$ & 0.449 & - & 4.25E-05 & 0.168 & 132127 & 366436 & 234 \\
\hline RL1015 & $5^{*}$ & 7131196 & $\mathrm{~A}: \mathrm{G}$ & 0.091 & $5.97 \mathrm{E}-05$ & $5.05 \mathrm{E}-09$ & 0.213 & 7021512 & 7216325 & 194 \\
\hline & 5 & 16517488 & $\mathrm{~T}: \mathrm{C}$ & 0.234 & - & 7.19E-07 & 0.165 & 16218476 & 16580411 & 361 \\
\hline & 7 & 6865357 & $\mathrm{~A}: \mathrm{G}$ & 0.062 & - & $7.06 \mathrm{E}-09$ & 0.444 & 6705724 & 6865357 & 159 \\
\hline & 2 & 24961280 & $\mathrm{~T}: \mathrm{A}$ & 0.062 & - & $2.27 \mathrm{E}-06$ & -0.156 & 24937103 & 24981552 & 44 \\
\hline RL1520 & $5^{*}$ & 7131196 & $\mathrm{~A}: \mathrm{G}$ & 0.091 & $1.46 \mathrm{E}-05$ & $5.05 \mathrm{E}-09$ & 0.192 & 7021512 & 7216325 & 194 \\
\hline & 2 & 25265958 & G:T & 0.055 & $4.65 \mathrm{E}-05$ & - & 0.208 & 25125170 & 25265958 & 140 \\
\hline RL2025 & $2^{*}$ & 25265958 & G:T & 0.055 & $3.61 \mathrm{E}-05$ & $4.30 \mathrm{E}-06$ & 0.235 & 25125170 & 25265958 & 140 \\
\hline & $8^{\#}$ & 17321714 & $\mathrm{~A}: \mathrm{T}$ & 0.474 & - & $1.93 \mathrm{E}-06$ & -0.124 & 17254709 & 17338132 & 83 \\
\hline & $1^{\#}$ & 28515026 & $\mathrm{C}: \mathrm{G}$ & 0.109 & - & $2.69 \mathrm{E}-06$ & 0.394 & 28449249 & 28917868 & 468 \\
\hline & $2^{\#}$ & 24210664 & G:A & 0.161 & - & $7.83 \mathrm{E}-05$ & -0.194 & 24210664 & 24225612 & 14 \\
\hline MRL & $6^{*}$ & 27819933 & $\mathrm{~T}: \mathrm{C}$ & 0.062 & 4.14E-05 & $5.93 \mathrm{E}-07$ & -0.049 & 27819933 & 27872692 & 52 \\
\hline
\end{tabular}


Supplementary Table S3. (Continued)

\begin{tabular}{|c|c|c|c|c|c|c|c|c|c|c|}
\hline \multirow[t]{6}{*}{ RV } & $5^{*}$ & 7131196 & $\mathrm{~A}: \mathrm{G}$ & 0.091 & $3.66 \mathrm{E}-05$ & - & 0.140 & 7021512 & 7216325 & 194 \\
\hline & $7^{\#}$ & 6865357 & $A: G$ & 0.062 & - & $1.23 \mathrm{E}-11$ & 0.327 & 6705724 & 6865357 & 159 \\
\hline & $5^{\#}$ & 16517488 & $\mathrm{~T}: \mathrm{C}$ & 0.234 & - & $1.42 \mathrm{E}-06$ & 0.127 & 16218476 & 16580411 & 361 \\
\hline & $2^{\#}$ & 24961280 & $\mathrm{~T}: \mathrm{A}$ & 0.062 & - & $8.18 \mathrm{E}-06$ & -0.062 & 24937103 & 24981552 & 44 \\
\hline & $1^{\#}$ & 40526762 & $\mathrm{~T}: \mathrm{G}$ & 0.288 & - & $1.55 \mathrm{E}-05$ & -0.025 & 40526762 & 40614822 & 88 \\
\hline & $11^{\#}$ & 24219246 & $\mathrm{C}: \mathrm{T}$ & 0.124 & - & $4.01 \mathrm{E}-05$ & -0.098 & 24219246 & 24244335 & 25 \\
\hline \multirow[t]{3}{*}{ ART } & $11^{*}$ & 28808353 & $\mathrm{G}: \mathrm{C}$ & 0.489 & $1.34 \mathrm{E}-05$ & $1.76 \mathrm{E}-05$ & 0.026 & 28808219 & 28839108 & 30 \\
\hline & 1 & 10998576 & $\mathrm{G}: \mathrm{T}$ & 0.190 & 0.0001 & - & 0.037 & 10947296 & 11220396 & 273 \\
\hline & 1 & 34378789 & $\mathrm{C}: \mathrm{G}$ & 0.255 & - & $1.08 \mathrm{E}-04$ & 0.041 & 34378789 & 34396661 & 17 \\
\hline \multirow[t]{3}{*}{ SRL } & 1 & 25773127 & $\mathrm{C}: \mathrm{A}$ & 0.069 & $8.93 \mathrm{E}-05$ & - & 0.091 & 25666408 & 26101392 & 434 \\
\hline & $1^{\#}$ & 43108024 & $\mathrm{G}: \mathrm{C}$ & 0.277 & - & $1.37 \mathrm{E}-05$ & -0.057 & 43065133 & 43245089 & 179 \\
\hline & $8^{\#}$ & 27104372 & $\mathrm{~A}: \mathrm{G}$ & 0.388 & - & 4.97E-05 & 0.354 & 27104372 & 27142568 & 38 \\
\hline \multirow[t]{2}{*}{ RLLA } & $2^{*}$ & 10134429 & $\mathrm{G}: \mathrm{A}$ & 0.343 & $9.63 \mathrm{E}-05$ & $4.57 \mathrm{E}-05$ & 0.069 & 10134419 & 10188662 & 54 \\
\hline & $8^{\#}$ & 24892309 & G:A & 0.241 & - & $7.03 \mathrm{E}-05$ & -0.002 & 24831278 & 24907343 & 76 \\
\hline \multicolumn{11}{|c|}{ (D) Root anatomical traits } \\
\hline \multirow[t]{5}{*}{$\mathrm{RD}$} & $7^{*}$ & 21266079 & $\mathrm{C}: \mathrm{T}$ & 0.099 & $2.02 \mathrm{E}-05$ & $2.18 \mathrm{E}-12$ & -0.044 & 21245869 & 21290877 & 45 \\
\hline & $1^{*}$ & 11112944 & $\mathrm{C}: \mathrm{T}$ & 0.175 & 0.0001 & $2.68 \mathrm{E}-07$ & -0.032 & 10947296 & 11220396 & 273 \\
\hline & $3^{\#}$ & 4913579 & $\mathrm{~A}: \mathrm{G}$ & 0.372 & - & $1.57 \mathrm{E}-06$ & -0.033 & 4882848 & 5008438 & 125 \\
\hline & $3^{\#}$ & 29686521 & $\mathrm{G}: \mathrm{T}$ & 0.350 & - & $1.69 \mathrm{E}-06$ & 0.032 & 29614230 & 29742101 & 127 \\
\hline & $5^{\#}$ & 28880728 & $\mathrm{~A}: \mathrm{C}$ & 0.408 & - & $3.86 \mathrm{E}-05$ & 0.018 & 28862506 & 28957228 & 94 \\
\hline $\mathrm{CD}$ & $7^{*}$ & 21266079 & $\mathrm{C}: \mathrm{T}$ & 0.099 & $9.86 \mathrm{E}-06$ & $3.12 \mathrm{E}-06$ & -0.052 & 21245869 & 21290877 & 45 \\
\hline \multirow[t]{4}{*}{ LMXD } & $4^{*}$ & 29450620 & $\mathrm{~A}: \mathrm{G}$ & 0.095 & $4.58 \mathrm{E}-05$ & 4.91E-06 & 0.046 & 29398010 & 29526672 & 128 \\
\hline & 4 & 29606053 & $\mathrm{~A}: \mathrm{C}$ & 0.168 & $7.88 \mathrm{E}-05$ & - & 0.043 & 29531026 & 29621778 & 90 \\
\hline & $1^{\#}$ & 19177575 & $\mathrm{~A}: \mathrm{G}$ & 0.190 & - & $4.32 \mathrm{E}-06$ & 0.108 & 19174820 & 19262944 & 88 \\
\hline & $12^{\#}$ & 22371182 & $\mathrm{C}: \mathrm{T}$ & 0.377 & - & $5.49 \mathrm{E}-05$ & -0.013 & 22194036 & 22425011 & 230 \\
\hline \multirow[t]{2}{*}{ LMXN } & $11^{\#}$ & 2838776 & G:T & 0.297 & - & $6.59 \mathrm{E}-06$ & 0.046 & 2723682 & 2942360 & 218 \\
\hline & $1^{\#}$ & 39902281 & $\mathrm{~T}: \mathrm{C}$ & 0.190 & - & $2.66 \mathrm{E}-05$ & -0.035 & 39886933 & 40061573 & 174 \\
\hline \multirow[t]{2}{*}{ SD:RD } & 7 & 21266079 & C:T & 0.099 & $7.28 \mathrm{E}-05$ & - & 0.020 & 21245869 & 21290877 & 45 \\
\hline & $4^{\#}$ & 31749561 & $\mathrm{G}: \mathrm{T}$ & 0.095 & - & $5.29 \mathrm{E}-05$ & -0.052 & 31728015 & 31778051 & 50 \\
\hline \multicolumn{11}{|c|}{ (E) Dry matter traits } \\
\hline \multirow[t]{8}{*}{ SW } & 1 & 29132939 & $\mathrm{G}: \mathrm{A}$ & 0.062 & $6.42 \mathrm{E}-05$ & - & 0.140 & 29124263 & 29250090 & 125 \\
\hline & $1^{*}$ & 29441428 & A:T & 0.066 & $7.82 \mathrm{E}-06$ & $5.53 \mathrm{E}-06$ & 0.150 & 29394012 & 29470779 & 76 \\
\hline & 1 & 29575437 & $\mathrm{~A}: \mathrm{G}$ & 0.058 & $1.50 \mathrm{E}-05$ & - & 0.154 & 29483935 & 29597597 & 113 \\
\hline & 1 & 29600620 & $\mathrm{C}: \mathrm{T}$ & 0.058 & $1.50 \mathrm{E}-05$ & - & 0.154 & 29597627 & 29606402 & 8 \\
\hline & 1 & 29620240 & $\mathrm{~T}: \mathrm{C}$ & 0.058 & $1.50 \mathrm{E}-05$ & - & 0.154 & 29606625 & 29918762 & 312 \\
\hline & 1 & 29981149 & $\mathrm{~T}: \mathrm{G}$ & 0.055 & $5.15 \mathrm{E}-05$ & - & 0.149 & 29935443 & 29999692 & 64 \\
\hline & 1 & 30060278 & $\mathrm{G}: \mathrm{A}$ & 0.055 & $5.15 \mathrm{E}-05$ & - & 0.149 & 30027570 & 30172920 & 145 \\
\hline & 5 & 7131196 & $\mathrm{~A}: \mathrm{G}$ & 0.091 & $2.59 \mathrm{E}-05$ & - & 0.127 & 7021512 & 7216325 & 194 \\
\hline \multirow[t]{4}{*}{ LW } & 6 & 366330 & G:A & 0.449 & 7.71E-05 & - & 0.067 & 132127 & 366436 & 234 \\
\hline & 5 & 7131196 & $A: G$ & 0.091 & $8.85 \mathrm{E}-05$ & - & 0.114 & 7021512 & 7216325 & 194 \\
\hline & $11^{*}$ & 7124411 & $\mathrm{~T}: \mathrm{C}$ & 0.091 & $9.18 \mathrm{E}-05$ & $1.77 \mathrm{E}-05$ & 0.108 & 7095402 & 7149638 & 54 \\
\hline & $7^{\#}$ & 15930391 & G:A & 0.285 & - & 5.63E-05 & -0.020 & 15930391 & 16109354 & 178 \\
\hline RW & $5^{*}$ & 7131196 & $\mathrm{~A}: \mathrm{G}$ & 0.091 & $6.78 \mathrm{E}-05$ & $4.05 \mathrm{E}-05$ & 0.138 & 7021512 & 7216325 & 194 \\
\hline \multirow[t]{3}{*}{ TW } & $1^{*}$ & 29441428 & A:T & 0.066 & $7.57 \mathrm{E}-05$ & $3.39 \mathrm{E}-05$ & 0.127 & 29394012 & 29470779 & 76 \\
\hline & 1 & 39058787 & $\mathrm{~T}: \mathrm{G}$ & 0.248 & $9.20 \mathrm{E}-05$ & - & 0.072 & 39047133 & 39165647 & 118 \\
\hline & $5^{\#}$ & 7131196 & $\mathrm{~A}: \mathrm{G}$ & 0.091 & - & $5.03 \mathrm{E}-05$ & 0.213 & 7021512 & 7216325 & 194 \\
\hline \multirow[t]{3}{*}{ RS } & $1^{*}$ & 39255482 & C:T & 0.350 & $2.24 \mathrm{E}-05$ & $1.28 \mathrm{E}-06$ & 0.100 & 39225855 & 39394856 & 169 \\
\hline & $12^{*}$ & 25497648 & $\mathrm{C}: \mathrm{A}$ & 0.232 & $7.25 \mathrm{E}-05$ & $5.70 \mathrm{E}-06$ & 0.080 & 25482817 & 25632919 & 150 \\
\hline & $1^{\#}$ & 1562911 & $\mathrm{~A}: \mathrm{C}$ & 0.365 & - & $1.07 \mathrm{E}-07$ & -0.160 & 1532281 & 1562911 & 30 \\
\hline \multirow[t]{6}{*}{ LWR } & $8^{*}$ & 3265446 & C:T & 0.084 & $6.54 \mathrm{E}-06$ & $5.28 \mathrm{E}-07$ & -0.043 & 3248294 & 3318437 & 70 \\
\hline & 9 & 14127114 & $\mathrm{C}: \mathrm{T}$ & 0.193 & $3.56 \mathrm{E}-05$ & - & -0.026 & 14053172 & 14127114 & 73 \\
\hline & 1 & 14788199 & $\mathrm{C}: \mathrm{T}$ & 0.069 & $3.99 \mathrm{E}-05$ & - & -0.040 & 14749963 & 14788222 & 38 \\
\hline & 3 & 32750373 & $\mathrm{~A}: \mathrm{C}$ & 0.223 & $9.26 \mathrm{E}-05$ & - & -0.023 & 32637987 & 32847633 & 209 \\
\hline & $7^{\#}$ & 21266079 & $\mathrm{C}: \mathrm{T}$ & 0.096 & - & $6.12 \mathrm{E}-06$ & -0.087 & 21245869 & 21290877 & 45 \\
\hline & $5^{\#}$ & 23606267 & C:T & 0.124 & - & $1.03 \mathrm{E}-05$ & 0.047 & 23606267 & 23623230 & 16 \\
\hline SWR & $1^{*}$ & 14788199 & C:T & 0.069 & $1.54 \mathrm{E}-06$ & $2.07 \mathrm{E}-09$ & 0.059 & 14749963 & 14788222 & 38 \\
\hline
\end{tabular}


Supplementary Table S3. (Continued)

\begin{tabular}{cccccccccc}
\hline 8 & 3265446 & C:T & 0.084 & $4.29 \mathrm{E}-06$ & - & 0.052 & 3248294 & 3318437 & 70 \\
9 & 14127114 & C:T & 0.193 & $8.13 \mathrm{E}-05$ & - & 0.030 & 14053172 & 14127114 & 73 \\
12 & 5829054 & G:T & 0.197 & $8.34 \mathrm{E}-05$ & - & 0.036 & 5829054 & 5839553 & 10 \\
9 & 14829621 & G:A & 0.142 & $9.20 \mathrm{E}-05$ & - & 0.036 & 14813317 & 14833005 & 19 \\
$6^{\#}$ & 6744100 & G:A & 0.387 & - & $2.91 \mathrm{E}-05$ & 0.048 & 6684474 & 6769503 & 85 \\
$12^{\#}$ & 141599 & G:A & 0.124 & - & $6.14 \mathrm{E}-06$ & 0.080 & 141599 & 148272 & 6 \\
$11^{\#}$ & 18947620 & T:C & 0.179 & - & $1.14 \mathrm{E}-05$ & 0.052 & 18922273 & 18954436 & 32 \\
$11^{\#}$ & 22302172 & G:A & 0.051 & - & $7.45 \mathrm{E}-05$ & 0.120 & 22274274 & 22302172 & 27 \\
\hline
\end{tabular}

Chr: chromosome, MAF: minor allele frequency, RAE: relative allelic effect calculated as a ratio of minor allele effect trait value to population average trait value, LD: linkage disequilibrium 
Supplementary Table S4. Summary of identified genome-wide significant association loci for phenotypic traits in water-deficit condition using compressed mixed linear model (CMLM) and multi-locus mixed model (MLMM) approaches. The loci commonly detected through both the approaches were marked by an asterisk sign $(*)$ and those detected through only MLMM were marked by a hashtag sign (\#). All the other unmarked loci were detected only through the CMLM approach. Trait acronyms are given in the Table 1 .

\begin{tabular}{|c|c|c|c|c|c|c|c|c|c|c|}
\hline \multirow[t]{2}{*}{ Traits } & \multirow[t]{2}{*}{ Chr } & \multirow[t]{2}{*}{ Position } & \multirow[t]{2}{*}{ Allele } & \multirow[t]{2}{*}{ MAF } & \multirow[t]{2}{*}{$P$ valuecmlm } & \multirow[t]{2}{*}{$P$ valuemlмm } & \multirow[t]{2}{*}{ RAE } & \multicolumn{2}{|c|}{ LD block } & \multirow[t]{2}{*}{$\begin{array}{l}\text { Size } \\
\text { (kb) }\end{array}$} \\
\hline & & & & & & & & From & To & \\
\hline \multicolumn{11}{|c|}{ (A) Shoot morphological traits } \\
\hline \multirow[t]{4}{*}{ PHT } & $1^{*}$ & 38286772 & G:A & 0.288 & $8.88 \mathrm{E}-06$ & $3.14 \mathrm{E}-07$ & -0.065 & 38178239 & 38437530 & 259 \\
\hline & $3^{*}$ & 30407838 & $\mathrm{C}: \mathrm{G}$ & 0.297 & $2.75 \mathrm{E}-05$ & 5.32E-06 & -0.039 & 30407838 & 30499464 & 91 \\
\hline & $5^{*}$ & 2173057 & $A: G$ & 0.201 & $2.81 \mathrm{E}-05$ & $3.88 \mathrm{E}-06$ & 0.040 & 2102183 & 2197641 & 95 \\
\hline & $6^{\#}$ & 22909435 & A:G & 0.051 & - & $5.95 \mathrm{E}-06$ & 0.213 & 22850994 & 22969216 & 118 \\
\hline \multirow[t]{4}{*}{$\mathrm{TN}$} & $3^{*}$ & 9995472 & $\mathrm{C}: \mathrm{T}$ & 0.265 & $5.80 \mathrm{E}-06$ & $1.03 \mathrm{E}-06$ & 0.089 & 9967155 & 10106317 & 139 \\
\hline & 2 & 25053043 & $\mathrm{C}: \mathrm{T}$ & 0.398 & $2.58 \mathrm{E}-05$ & - & 0.059 & 25044454 & 25109632 & 65 \\
\hline & 8 & 4505925 & $\mathrm{C}: \mathrm{A}$ & 0.058 & $3.68 \mathrm{E}-05$ & - & 0.173 & 4505925 & 4629870 & 123 \\
\hline & $1^{*}$ & 24821998 & $\mathrm{~T}: \mathrm{A}$ & 0.401 & $7.61 \mathrm{E}-05$ & $1.69 \mathrm{E}-05$ & 0.071 & 24792895 & 24869656 & 76 \\
\hline \multirow[t]{3}{*}{ TLA } & $8^{*}$ & 25121944 & $\mathrm{C}: \mathrm{T}$ & 0.077 & $5.42 \mathrm{E}-05$ & $2.47 \mathrm{E}-06$ & 0.111 & 25091497 & 25238735 & 147 \\
\hline & $3^{\#}$ & 29050414 & $\mathrm{C}: \mathrm{T}$ & 0.088 & - & $1.22 \mathrm{E}-05$ & 0.178 & 29018521 & 29050414 & 33 \\
\hline & $3^{\#}$ & 11725360 & $\mathrm{C}: \mathrm{A}$ & 0.172 & - & $7.85 \mathrm{E}-05$ & 0.189 & 11693492 & 11725360 & 31 \\
\hline \multicolumn{11}{|c|}{ (B) Physiological traits } \\
\hline \multirow[t]{3}{*}{ CWT } & $1^{\#}$ & 23207640 & G:A & 0.066 & - & $8.30 \mathrm{E}-06$ & -0.147 & 23095746 & 23298172 & 202 \\
\hline & $4^{\#}$ & 34640918 & C:A & 0.175 & - & $3.59 \mathrm{E}-05$ & 0.047 & 34608671 & 34640918 & 32 \\
\hline & $6^{\#}$ & 13412649 & $\mathrm{G}: \mathrm{C}$ & 0.077 & - & 4.33E-05 & -0.078 & 13360689 & 13465974 & 105 \\
\hline \multirow[t]{3}{*}{ WUE } & $9^{\#}$ & 15426362 & $\mathrm{~T}: \mathrm{G}$ & 0.263 & - & $1.49 \mathrm{E}-05$ & 0.074 & 15394548 & 15559339 & 164 \\
\hline & $11^{\#}$ & 27574096 & $\mathrm{C}: \mathrm{T}$ & 0.08 & - & $1.92 \mathrm{E}-08$ & 0.112 & 27478419 & 27603835 & 125 \\
\hline & $6^{\#}$ & 23297154 & $A: G$ & 0.336 & - & 7.41E-05 & 0.047 & 23243062 & 23338253 & 95 \\
\hline \multicolumn{11}{|c|}{ (C) Root morphological traits } \\
\hline \multirow[t]{3}{*}{ TRL } & $2^{\#}$ & 8835096 & G:T & 0.149 & - & $2.20 \mathrm{E}-06$ & 0.145 & 8769237 & 8874697 & 105 \\
\hline & $4^{\#}$ & 29111186 & $\mathrm{~T}: \mathrm{C}$ & 0.084 & - & $1.20 \mathrm{E}-05$ & 0.235 & 28701604 & 29126558 & 424 \\
\hline & $11^{\#}$ & 7124411 & $\mathrm{~T}: \mathrm{C}$ & 0.091 & - & $2.04 \mathrm{E}-05$ & 0.228 & 7095402 & 7124411 & 29 \\
\hline \multirow[t]{3}{*}{ RL005 } & $2^{\#}$ & 8835096 & G:T & 0.149 & - & $1.56 \mathrm{E}-06$ & 0.144 & 8769237 & 8874697 & 105 \\
\hline & $4^{\#}$ & 29111186 & $\mathrm{~T}: \mathrm{C}$ & 0.084 & - & $1.34 \mathrm{E}-05$ & 0.228 & 28701604 & 29126558 & 424 \\
\hline & $11^{\#}$ & 7124411 & $\mathrm{~T}: \mathrm{C}$ & 0.091 & - & $2.50 \mathrm{E}-05$ & 0.215 & 7095402 & 7124411 & 29 \\
\hline RL0510 & 11 & 24625645 & $\mathrm{~T}: \mathrm{C}$ & 0.069 & $2.24 \mathrm{E}-05$ & - & 0.165 & 24423060 & 24639658 & 216 \\
\hline & 11 & 25317141 & $\mathrm{G}: \mathrm{C}$ & 0.235 & $9.42 \mathrm{E}-05$ & - & 0.092 & 25317141 & 25426798 & 109 \\
\hline & $1^{*}$ & 23079331 & $\mathrm{~A}: \mathrm{G}$ & 0.113 & $9.51 \mathrm{E}-05$ & $2.50 \mathrm{E}-06$ & -0.122 & 22992632 & 23095676 & 103 \\
\hline & $12^{\#}$ & 26195748 & A:G & 0.080 & - & $2.34 \mathrm{E}-05$ & 0.263 & 26124690 & 26202838 & 78 \\
\hline & $9^{\#}$ & 14829621 & G:A & 0.142 & - & $1.18 \mathrm{E}-04$ & -0.211 & 14813317 & 14833005 & 19 \\
\hline RL1015 & $1^{*}$ & 23044334 & $\mathrm{~T}: \mathrm{C}$ & 0.113 & $6.18 \mathrm{E}-05$ & $6.53 \mathrm{E}-05$ & -0.175 & 22992632 & 23095676 & 103 \\
\hline RL2025 & $1^{*}$ & 24421833 & G:A & 0.217 & 9.36E-06 & 6.77E-08 & 0.174 & $\overline{4421833}$ & 44438552 & 16 \\
\hline & 1 & 42799636 & $\mathrm{C}: \mathrm{A}$ & 0.192 & $9.23 \mathrm{E}-05$ & - & 0.145 & 42799636 & 42856304 & 56 \\
\hline & $2^{*}$ & 5180480 & $A: G$ & 0.434 & $2.05 \mathrm{E}-05$ & $3.90 \mathrm{E}-05$ & 0.129 & 5054863 & 5189396 & 134 \\
\hline & 5 & 10646015 & C:T & 0.153 & 7.69E-05 & - & 0.161 & 10584713 & 10646015 & 61 \\
\hline & 6 & 6996556 & $\mathrm{~T}: \mathrm{G}$ & 0.091 & $8.14 \mathrm{E}-05$ & - & -0.194 & 6951049 & 7001492 & 50 \\
\hline & 6 & 7006358 & $\mathrm{C}: \mathrm{G}$ & 0.091 & $8.14 \mathrm{E}-05$ & - & -0.210 & 7001506 & 7106699 & 105 \\
\hline & $3^{\#}$ & 6374082 & $\mathrm{~T}: \mathrm{C}$ & 0.219 & - & $4.25 \mathrm{E}-05$ & -0.045 & 6217543 & 6439952 & 222 \\
\hline & $10^{\#}$ & 11555942 & $\mathrm{~A}: \mathrm{G}$ & 0.117 & - & $9.72 \mathrm{E}-05$ & -0.181 & 11390755 & 11575800 & 185 \\
\hline RL2530 & $11^{*}$ & 24267277 & A:T & 0.066 & $3.69 \mathrm{E}-05$ & 7.30E-08 & 0.294 & 24267277 & 24279832 & 12 \\
\hline & 2 & 503016 & $\mathrm{~A}: \mathrm{G}$ & 0.124 & $5.23 \mathrm{E}-05$ & - & 0.213 & 503016 & 536341 & 11 \\
\hline & $10^{\#}$ & 18971155 & $\mathrm{C}: \mathrm{T}$ & 0.234 & $2.42 \mathrm{E}-05$ & - & -0.244 & 18898657 & 19018639 & 119 \\
\hline & $6^{\#}$ & 7045303 & $\mathrm{C}: \mathrm{T}$ & 0.088 & $3.24 \mathrm{E}-05$ & - & -0.281 & 7001506 & 7106699 & 105 \\
\hline MRL & $12^{*}$ & 17607622 & G:T & 0.131 & $1.15 \mathrm{E}-05$ & $3.28 \mathrm{E}-06$ & -0.042 & 17563026 & 17617181 & 54 \\
\hline & 12 & 17780100 & C:T & 0.135 & $4.82 \mathrm{E}-05$ & - & -0.039 & 17779917 & 17952275 & 172 \\
\hline
\end{tabular}


Supplementary Table S4. (Continued)

\begin{tabular}{|c|c|c|c|c|c|c|c|c|c|c|}
\hline & 12 & 17977281 & $\mathrm{G}: \mathrm{A}$ & 0.117 & $2.46 \mathrm{E}-05$ & - & -0.043 & 17973553 & 18060777 & 87 \\
\hline & $2^{*}$ & 2783451 & $\mathrm{C}: \mathrm{T}$ & 0.206 & $1.35 \mathrm{E}-05$ & $1.02 \mathrm{E}-05$ & 0.035 & 2749982 & 2802120 & 52 \\
\hline \multirow[t]{5}{*}{ RV } & 11 & 7124411 & $\mathrm{~T}: \mathrm{C}$ & 0.091 & $5.68 \mathrm{E}-05$ & & 0.135 & 7095402 & 7124411 & 29 \\
\hline & $9^{*}$ & 14829621 & $\mathrm{G}: \mathrm{A}$ & 0.142 & $7.31 \mathrm{E}-05$ & $3.84 \mathrm{E}-07$ & -0.109 & 14813317 & 14833005 & 19 \\
\hline & $1^{*}$ & 23044334 & $\mathrm{~T}: \mathrm{C}$ & 0.113 & $7.93 \mathrm{E}-05$ & $1.94 \mathrm{E}-06$ & -0.119 & 22992632 & 23095676 & 103 \\
\hline & $12^{\#}$ & 26195748 & A:G & 0.08 & - & $2.24 \mathrm{E}-06$ & 0.220 & 26124690 & 26202838 & 78 \\
\hline & $11^{\#}$ & 22973837 & A:G & 0.153 & - & $5.41 \mathrm{E}-05$ & -0.168 & 22703332 & 22976412 & 273 \\
\hline \multirow[t]{5}{*}{ SA } & $1^{\#}$ & 23044334 & $\mathrm{~T}: \mathrm{C}$ & 0.113 & - & $3.23 \mathrm{E}-06$ & -0.157 & 22992632 & 23095676 & 103 \\
\hline & $9^{\#}$ & 14829621 & $\mathrm{G}: \mathrm{A}$ & 0.142 & - & $1.26 \mathrm{E}-05$ & -0.190 & 14813317 & 14833005 & 19 \\
\hline & $2^{\#}$ & 8835096 & $\mathrm{G}: \mathrm{T}$ & 0.149 & - & $5.91 \mathrm{E}-05$ & 0.114 & 8769237 & 8874697 & 105 \\
\hline & $3^{\#}$ & 35654626 & $\mathrm{~A}: \mathrm{C}$ & 0.106 & - & $6.12 \mathrm{E}-06$ & -0.095 & 35605462 & 35654703 & 49 \\
\hline & $5^{\#}$ & 14825506 & G:A & 0.066 & - & $7.12 \mathrm{E}-05$ & 0.271 & 14818383 & 14881083 & 62 \\
\hline \multirow[t]{2}{*}{ ART } & $11^{*}$ & 6245556 & $\mathrm{C}: \mathrm{T}$ & 0.255 & $5.85 \mathrm{E}-05$ & $4.23 \mathrm{E}-06$ & -0.024 & 6232379 & 6350995 & 118 \\
\hline & 9 & 20970824 & $\mathrm{~T}: \mathrm{C}$ & 0.088 & 0.0001 & & -0.029 & 20953059 & 21030489 & 77 \\
\hline \multirow[t]{8}{*}{ SRL } & $1^{*}$ & 23218344 & $\mathrm{~T}: \mathrm{C}$ & 0.058 & $4.31 \mathrm{E}-06$ & $6.56 \mathrm{E}-05$ & 0.148 & 23095746 & 23298172 & 202 \\
\hline & 1 & 23095621 & $\mathrm{~A}: \mathrm{G}$ & 0.062 & $3.73 \mathrm{E}-05$ & - & 0.125 & 22992632 & 23095676 & 103 \\
\hline & 2 & 33496059 & G:A & 0.051 & $9.36 \mathrm{E}-06$ & - & 0.143 & 33487245 & 33529812 & 42 \\
\hline & 2 & 24006148 & $\mathrm{G}: \mathrm{C}$ & 0.073 & $1.53 \mathrm{E}-05$ & - & 0.138 & 23898412 & 24059505 & 161 \\
\hline & 2 & 24122043 & $\mathrm{C}: \mathrm{G}$ & 0.084 & $2.39 \mathrm{E}-05$ & - & 0.121 & 24108015 & 24136056 & 28 \\
\hline & $5^{*}$ & 15673557 & $\mathrm{G}: \mathrm{T}$ & 0.073 & $3.00 \mathrm{E}-05$ & 7.05E-08 & 0.121 & 15469660 & 15756954 & 287 \\
\hline & $6^{*}$ & 27819933 & $\mathrm{~T}: \mathrm{C}$ & 0.062 & $4.96 \mathrm{E}-05$ & $1.85 \mathrm{E}-06$ & 0.121 & 27819933 & 27872692 & 52 \\
\hline & $8^{\#}$ & 51541 & $\mathrm{~T}: \mathrm{C}$ & 0.102 & & $1.05 \mathrm{E}-04$ & -0.168 & 51541 & 148519 & 96 \\
\hline \multirow[t]{4}{*}{ RLLA } & $6^{*}$ & 5760641 & $\mathrm{~T}: \mathrm{C}$ & 0.087 & $1.31 \mathrm{E}-05$ & $9.80 \mathrm{E}-07$ & 0.116 & 5758331 & 5783531 & 25 \\
\hline & $2^{*}$ & 25154659 & $\mathrm{~T}: \mathrm{C}$ & 0.113 & $5.54 \mathrm{E}-05$ & $1.17 \mathrm{E}-07$ & 0.093 & 25125170 & 25212962 & 87 \\
\hline & $8^{\#}$ & 27563586 & $\mathrm{~T}: \mathrm{C}$ & 0.456 & - & $7.05 \mathrm{E}-06$ & 0.086 & 27563572 & 27583372 & 19 \\
\hline & $3^{\#}$ & 27177614 & $\mathrm{C}: \mathrm{A}$ & 0.223 & - & $3.47 \mathrm{E}-05$ & 0.145 & 27144252 & 27252032 & 107 \\
\hline \multicolumn{11}{|c|}{ (D) Root anatomical traits } \\
\hline \multirow[t]{4}{*}{ SD } & $9^{*}$ & 13788883 & $\mathrm{C}: \mathrm{A}$ & 0.068 & $7.75 \mathrm{E}-05$ & $1.63 \mathrm{E}-07$ & -0.045 & 13665706 & 13788883 & 123 \\
\hline & $5^{\#}$ & 3057869 & $\mathrm{~A}: \mathrm{G}$ & 0.450 & - & 5.92E-06 & 0.069 & 3048995 & 3074221 & 25 \\
\hline & $2^{\#}$ & 17151144 & $\mathrm{C}: \mathrm{T}$ & 0.165 & - & $3.08 \mathrm{E}-05$ & -0.086 & 17075796 & 17222194 & 146 \\
\hline & $3^{\#}$ & 31345248 & $\mathrm{~A}: \mathrm{G}$ & 0.322 & - & $4.92 \mathrm{E}-05$ & -0.040 & 31311835 & 31345248 & 33 \\
\hline \multirow[t]{3}{*}{ LMXN } & $11^{*}$ & 25552124 & $\mathrm{C}: \mathrm{A}$ & 0.053 & $2.47 \mathrm{E}-06$ & $1.84 \mathrm{E}-07$ & 0.091 & 25542935 & 25854764 & 311 \\
\hline & $7^{*}$ & 1316016 & A:T & 0.319 & $8.15 \mathrm{E}-05$ & $1.36 \mathrm{E}-06$ & -0.039 & 1316016 & 1523942 & 207 \\
\hline & $3^{\#}$ & 34487907 & $\mathrm{G}: \mathrm{T}$ & 0.161 & - & $5.30 \mathrm{E}-05$ & -0.073 & 34418455 & 34496574 & 78 \\
\hline \multirow[t]{7}{*}{ LMXD } & $9^{\#}$ & 13788883 & C:A & 0.066 & - & $2.25 \mathrm{E}-08$ & -0.080 & 13665706 & 13788883 & 123 \\
\hline & $2^{\#}$ & 6749649 & $\mathrm{~A}: \mathrm{G}$ & 0.405 & - & $1.77 \mathrm{E}-08$ & 0.064 & 6706866 & 6752341 & 45 \\
\hline & $3^{\#}$ & 31305902 & $\mathrm{~A}: \mathrm{G}$ & 0.478 & - & $2.47 \mathrm{E}-05$ & -0.017 & 31305888 & 31311496 & 5 \\
\hline & $5^{\#}$ & 10668631 & $\mathrm{C}: \mathrm{T}$ & 0.197 & - & $4.52 \mathrm{E}-06$ & -0.036 & 10641707 & 10668631 & 26 \\
\hline & $5^{\#}$ & 3057869 & $\mathrm{~A}: \mathrm{G}$ & 0.451 & - & $3.38 \mathrm{E}-09$ & 0.063 & 3048995 & 3074221 & 25 \\
\hline & $6^{\#}$ & 471179 & $\mathrm{G}: \mathrm{A}$ & 0.070 & - & $6.16 \mathrm{E}-07$ & 0.075 & 467003 & 531468 & 64 \\
\hline & $11^{\#}$ & 28871551 & $\mathrm{G}: \mathrm{A}$ & 0.095 & - & $2.48 \mathrm{E}-05$ & 0.101 & 28864998 & 28907095 & 42 \\
\hline \multirow[t]{3}{*}{ SD:RD } & $11^{*}$ & 25175343 & $\mathrm{~T}: \mathrm{C}$ & 0.058 & $2.82 \mathrm{E}-05$ & $2.99 \mathrm{E}-06$ & 0.038 & 25160136 & 25181615 & 21 \\
\hline & 11 & 25182643 & $\mathrm{C}: \mathrm{T}$ & 0.064 & $5.67 \mathrm{E}-05$ & - & 0.036 & 25182643 & 25288987 & 106 \\
\hline & $1^{*}$ & 1676898 & $\mathrm{C}: \mathrm{G}$ & 0.237 & $5.74 \mathrm{E}-05$ & $1.90 \mathrm{E}-06$ & 0.023 & 1676850 & 1833926 & 157 \\
\hline \multicolumn{11}{|c|}{ (E) Dry matter traits } \\
\hline \multirow[t]{2}{*}{ LW } & 1 & 15402532 & $\mathrm{~T}: \mathrm{C}$ & 0.164 & $6.23 \mathrm{E}-05$ & - & -0.075 & 15393938 & 15402532 & 8 \\
\hline & $1^{*}$ & 15402601 & $\mathrm{C}: \mathrm{T}$ & 0.164 & $6.23 \mathrm{E}-05$ & $6.69 \mathrm{E}-06$ & -0.075 & 15402553 & 15441315 & 38 \\
\hline \multirow[t]{2}{*}{ SW } & $12^{*}$ & 17786153 & G:T & 0.355 & $3.67 \mathrm{E}-05$ & $2.11 \mathrm{E}-05$ & 0.067 & 17779917 & 17952275 & 172 \\
\hline & $3^{*}$ & 11713631 & $\mathrm{~T}: \mathrm{C}$ & 0.197 & 4.12E-05 & $1.05 \mathrm{E}-05$ & 0.081 & 11693492 & 11725360 & 31 \\
\hline \multirow[t]{4}{*}{ RW } & $4^{*}$ & 29111186 & $\mathrm{~T}: \mathrm{C}$ & 0.083 & $3.44 \mathrm{E}-06$ & $1.47 \mathrm{E}-06$ & 0.177 & 28701604 & 29126558 & 424 \\
\hline & 6 & 6579613 & $\mathrm{~T}: \mathrm{A}$ & 0.120 & 7.07E-05 & - & 0.122 & 6579551 & 6598292 & 18 \\
\hline & 3 & 34943958 & $\mathrm{~T}: \mathrm{C}$ & 0.339 & $8.21 \mathrm{E}-05$ & - & 0.097 & 34927423 & 34977879 & 50 \\
\hline & 1 & 23079331 & $\mathrm{~A}: \mathrm{G}$ & 0.113 & $9.51 \mathrm{E}-05$ & - & -0.122 & 22992632 & 23095676 & 103 \\
\hline RS & $4^{*}$ & 29111186 & $\mathrm{~T}: \mathrm{C}$ & 0.083 & $9.31 \mathrm{E}-08$ & $2.75 \mathrm{E}-11$ & 0.222 & 28701604 & 29126558 & 424 \\
\hline & 4 & 29184866 & G:A & 0.113 & 4.37E-06 & - & 0.111 & 29126713 & 29377299 & 250 \\
\hline & 4 & 29450620 & $A: G$ & 0.094 & $7.06 \mathrm{E}-05$ & - & 0.111 & 29398010 & 29526672 & 128 \\
\hline & $1^{\#}$ & 1562911 & $\mathrm{~A}: \mathrm{C}$ & 0.365 & $1.54 \mathrm{E}-05$ & - & -0.133 & 1532281 & 1562911 & 30 \\
\hline LWR & $2^{*}$ & 23246559 & $\mathrm{C}: \mathrm{A}$ & 0.113 & $2.26 \mathrm{E}-05$ & $1.94 \mathrm{E}-06$ & 0.030 & 23184521 & 23514586 & 330 \\
\hline & $2^{*}$ & 20169674 & $A: G$ & 0.427 & $8.53 \mathrm{E}-05$ & $2.16 \mathrm{E}-05$ & -0.016 & 20169653 & 20202576 & 32 \\
\hline
\end{tabular}


Supplementary Table S4. (Continued)

\begin{tabular}{ccccccccccc} 
& $9^{*}$ & 14829621 & G:A & 0.142 & $3.49 \mathrm{E}-05$ & $7.63 \mathrm{E}-06$ & -0.027 & 14813317 & 14833005 & 19 \\
& $11^{\#}$ & 4823624 & T:C & 0.109 & $5.67 \mathrm{E}-06$ & - & 0.041 & 4583780 & 4823732 & 239 \\
\hline SWR & $9^{*}$ & 14829621 & G:A & 0.142 & $1.81 \mathrm{E}-07$ & $3.01 \mathrm{E}-07$ & 0.040 & 14813317 & 14833005 & 19 \\
& 9 & 14847601 & A:T & 0.131 & $1.34 \mathrm{E}-06$ & - & 0.038 & 14833007 & 14847696 & 14 \\
& 7 & 20874845 & C:G & 0.166 & $3.41 \mathrm{E}-05$ & - & 0.030 & 20774955 & 20901272 & 126 \\
& $1^{\#}$ & 40813452 & T:C & 0.168 & - & $4.06 \mathrm{E}-05$ & 0.051 & 40813452 & 40832763 & 19 \\
\hline
\end{tabular}

Chr: chromosome, MAF: minor allele frequency, RAE: relative allelic effect calculated as a ratio of minor allele effect trait value to population average trait value, LD: linkage disequilibrium. 
Supplementary Table S5. Summary of identified genome-wide significant association loci for plasticity of phenotypic traits using compressed mixed linear model (CMLM) and multi-locus mixed model (MLMM) approaches. The loci commonly detected through both the approaches were marked by asterisk sign (*) and those detected through only MLMM were marked by a hashtag sign (\#). All the other unmarked loci were detected only through the CMLM approach. Trait acronyms are given in the Table 1.

\begin{tabular}{|c|c|c|c|c|c|c|c|c|c|c|}
\hline \multirow[t]{2}{*}{ Traits } & \multirow[t]{2}{*}{ Chr } & \multirow[t]{2}{*}{ Pos } & \multirow[t]{2}{*}{ Allele } & \multirow[t]{2}{*}{ MAF } & \multirow[t]{2}{*}{$P$ value } & \multirow[t]{2}{*}{$P$ value } & \multirow[t]{2}{*}{$\mathbf{A E}$} & \multicolumn{2}{|c|}{ LD block } & \multirow[t]{2}{*}{ Size (kb) } \\
\hline & & & & & & & & From & To & \\
\hline \multicolumn{11}{|c|}{ (A) Shoot morphological traits } \\
\hline \multirow[t]{2}{*}{ rPHT } & $2^{*}$ & 24405418 & $\mathrm{~T}: \mathrm{C}$ & 0.058 & $4.68 \mathrm{E}-06$ & $8.53 \mathrm{E}-07$ & -0.030 & 24405418 & 24432576 & 27 \\
\hline & $6^{\#}$ & 19606050 & $\mathrm{C}: \mathrm{T}$ & 0.464 & - & 4.64E-05 & 0.021 & 19496375 & 19730704 & 234 \\
\hline rTN & $12^{*}$ & 25006932 & $\mathrm{C}: \mathrm{T}$ & 0.091 & $4.63 \mathrm{E}-05$ & $4.40 \mathrm{E}-05$ & -0.080 & 24762153 & 25006932 & 244 \\
\hline \multirow[t]{3}{*}{ rTLA } & $7^{\#}$ & 15930391 & $\mathrm{~A}: \mathrm{G}$ & 0.285 & - & $1.52 \mathrm{E}-05$ & 0.026 & 15930391 & 16109354 & 178 \\
\hline & $7^{\#}$ & 9463744 & $A: G$ & 0.080 & - & $1.27 \mathrm{E}-05$ & -0.088 & 9317314 & 10004086 & 186 \\
\hline & $3^{\#}$ & 1706123 & $\mathrm{~T}: \mathrm{A}$ & 0.066 & - & $5.22 \mathrm{E}-05$ & -0.068 & 1705565 & 1730221 & 24 \\
\hline \multirow[t]{2}{*}{ rSLA } & $11^{*}$ & 19245430 & $\mathrm{C}: \mathrm{T}$ & 0.124 & $6.18 \mathrm{E}-05$ & $2.56 \mathrm{E}-05$ & -0.030 & 19197921 & 19245506 & 47 \\
\hline & 3 & 34125791 & $\mathrm{~T}: \mathrm{G}$ & 0.270 & $9.80 \mathrm{E}-05$ & - & -0.023 & 34069089 & 34183243 & 114 \\
\hline \multicolumn{11}{|c|}{ (B) Physiological traits } \\
\hline \multirow[t]{6}{*}{ rCWT } & $7^{*}$ & 9463744 & G:A & 0.080 & $1.29 \mathrm{E}-05$ & $1.77 \mathrm{E}-05$ & -0.052 & 9317314 & 10004086 & 186 \\
\hline & 1 & 33277486 & $\mathrm{~T}: \mathrm{C}$ & 0.467 & $2.62 \mathrm{E}-05$ & - & -0.030 & 32994632 & 33284067 & 289 \\
\hline & 1 & 33293954 & $\mathrm{G}: \mathrm{A}$ & 0.471 & $5.65 \mathrm{E}-05$ & - & -0.030 & 33293954 & 33727514 & 433 \\
\hline & 1 & 33755921 & $\mathrm{~T}: \mathrm{G}$ & 0.493 & $4.48 \mathrm{E}-05$ & - & -0.028 & 33755921 & 34165612 & 409 \\
\hline & 1 & 34280616 & $\mathrm{G}: \mathrm{A}$ & 0.453 & 7.83E-05 & - & 0.028 & 34184887 & 34357192 & 172 \\
\hline & $1^{*}$ & 42575227 & $A: G$ & 0.069 & $3.81 \mathrm{E}-05$ & $8.09 \mathrm{E}-05$ & -0.050 & 42467072 & 42587728 & 120 \\
\hline \multicolumn{11}{|c|}{ (C) Root morphological traits } \\
\hline \multirow[t]{5}{*}{ rTRL } & $12^{*}$ & 25006932 & C:T & 0.093 & $6.73 \mathrm{E}-05$ & $3.66 \mathrm{E}-09$ & -0.094 & 24762153 & 25006932 & 244 \\
\hline & $7^{\#}$ & 9463744 & G:A & 0.080 & - & $3.81 \mathrm{E}-06$ & -0.150 & 9317314 & 10004086 & 186 \\
\hline & $1^{\#}$ & 26826635 & $\mathrm{~T}: \mathrm{C}$ & 0.120 & - & $2.91 \mathrm{E}-05$ & -0.123 & 26709644 & 26847391 & 137 \\
\hline & $4^{\#}$ & 30764890 & $A: G$ & 0.354 & - & $2.20 \mathrm{E}-05$ & -0.107 & 30690751 & 30790417 & 99 \\
\hline & $2^{\#}$ & 4943157 & $\mathrm{C}: \mathrm{T}$ & 0.343 & - & $5.81 \mathrm{E}-05$ & -0.050 & 4868972 & 4950264 & 95 \\
\hline rRL005 & 12 & 25006932 & $\mathrm{C}: \mathrm{T}$ & 0.093 & $8.33 \mathrm{E}-05$ & - & -0.098 & 24762153 & 25006932 & 244 \\
\hline rRL1015 & $3^{\#}$ & 35780154 & $\overline{A: G}$ & 0.343 & - & $3.23 \mathrm{E}-07$ & 0.054 & 35765758 & 35793182 & 27 \\
\hline & $12^{\#}$ & 6154896 & $A: G$ & 0.120 & - & $1.67 \mathrm{E}-05$ & 0.109 & 6141695 & 6218230 & 76 \\
\hline & $3^{\#}$ & 26828159 & $\mathrm{~A}: \mathrm{G}$ & 0.073 & - & $9.66 \mathrm{E}-05$ & -0.131 & 26756997 & 26978105 & 221 \\
\hline rRL2025 & $4^{\#}$ & 16463674 & A:G & 0.423 & & $4.35 \mathrm{E}-05$ & -0.170 & 16397482 & 16542176 & 144 \\
\hline rRL2530 & 3 & 2553785 & $\mathrm{~A}: \mathrm{G}$ & 0.055 & $1.70 \mathrm{E}-05$ & - & -0.370 & 2497861 & 2572474 & 74 \\
\hline & 3 & 2714299 & $\mathrm{C}: \mathrm{A}$ & 0.062 & $5.95 \mathrm{E}-05$ & - & -0.320 & 2630128 & 2714299 & 84 \\
\hline & $3^{*}$ & 34392848 & $\mathrm{C}: \mathrm{T}$ & 0.080 & $5.64 \mathrm{E}-05$ & $3.71 \mathrm{E}-05$ & -0.280 & 34389921 & 34415828 & 25 \\
\hline $\mathrm{rSA}$ & $12^{*}$ & 25006932 & C:T & 0.093 & $4.46 \mathrm{E}-05$ & $6.33 \mathrm{E}-05$ & -0.079 & 24762153 & 25006932 & 244 \\
\hline & $7^{\#}$ & 9463899 & $\mathrm{~T}: \mathrm{C}$ & 0.091 & - & $1.47 \mathrm{E}-05$ & -0.128 & 9317314 & 10004086 & 186 \\
\hline rRV & $12^{*}$ & 25006932 & $\mathrm{C}: \mathrm{T}$ & 0.093 & $5.43 \mathrm{E}-05$ & $2.07 \mathrm{E}-05$ & -0.068 & 24762153 & 25006932 & 244 \\
\hline & $1^{\#}$ & 42575227 & $A: G$ & 0.069 & - & $2.07 \mathrm{E}-05$ & -0.137 & 42467072 & 42587728 & 120 \\
\hline rMRL & 12 & 17977281 & G:A & 0.117 & $2.31 \mathrm{E}-05$ & - & 0.052 & 17973553 & 18060777 & 87 \\
\hline & 12 & 17607622 & G:T & 0.131 & 0.0001 & - & 0.050 & 17563026 & 17617181 & 54 \\
\hline & $2^{*}$ & 26794003 & A:T & 0.084 & $3.88 \mathrm{E}-05$ & $2.58 \mathrm{E}-06$ & -0.060 & 26585953 & 26796794 & 210 \\
\hline & $6^{\#}$ & 25183518 & $\mathrm{C}: \mathrm{T}$ & 0.142 & - & $3.33 \mathrm{E}-06$ & 0.077 & 25177943 & 25227042 & 49 \\
\hline rSRL & $8^{*}$ & 26362631 & $\mathrm{~T}: \mathrm{A}$ & 0.051 & $1.77 \mathrm{E}-05$ & $1.66 \mathrm{E}-08$ & -0.150 & 26333486 & 26384762 & 51 \\
\hline & $3^{\#}$ & 34024418 & $\mathrm{C}: \mathrm{T}$ & 0.080 & - & $2.08 \mathrm{E}-06$ & 0.180 & 34004938 & 34069043 & 64 \\
\hline & $1^{\#}$ & 20574500 & $\mathrm{C}: \mathrm{T}$ & 0.307 & - & $2.97 \mathrm{E}-07$ & -0.147 & 20478562 & 20579687 & 101 \\
\hline & $5^{\#}$ & 2184238 & $\mathrm{~A}: \mathrm{C}$ & 0.324 & - & $1.56 \mathrm{E}-05$ & -0.100 & 2102183 & 2197641 & 95 \\
\hline rART & 1 & 29981149 & $\mathrm{~T}: \mathrm{G}$ & 0.055 & $9.06 \mathrm{E}-05$ & - & -0.056 & 29935443 & 30019818 & 84 \\
\hline & 1 & 30060278 & G:A & 0.055 & $9.06 \mathrm{E}-05$ & - & -0.056 & 30027570 & 30084144 & 56 \\
\hline & $1^{*}$ & 34378789 & G:C & 0.254 & $6.43 \mathrm{E}-05$ & $1.43 \mathrm{E}-07$ & 0.029 & 34378789 & 34396661 & 17 \\
\hline & $5^{\#}$ & 28880707 & $A: G$ & 0.165 & $8.13 \mathrm{E}-06$ & - & -0.063 & 28862506 & 28957228 & 94 \\
\hline & $5^{\#}$ & 2184238 & $\mathrm{~A}: \mathrm{C}$ & 0.324 & $5.93 \mathrm{E}-05$ & - & 0.027 & 2102183 & 2197641 & 95 \\
\hline rTRWD & $1^{*}$ & 25703110 & G:T & 0.095 & $2.07 \mathrm{E}-05$ & $6.51 \mathrm{E}-06$ & -0.090 & 25666408 & 26101392 & 434 \\
\hline & $1^{\#}$ & 20571077 & $\mathrm{~A}: \mathrm{C}$ & 0.270 & - & $7.82 \mathrm{E}-05$ & 0.090 & 20478562 & 20579687 & 101 \\
\hline rRLLA & 5 & 213976 & $\mathrm{C}: \mathrm{A}$ & 0.151 & $6.44 \mathrm{E}-05$ & $2.63 \mathrm{E}-05$ & -0.100 & 192969 & 219803 & 26 \\
\hline
\end{tabular}


Supplementary Table S5. (Continued)

\begin{tabular}{|c|c|c|c|c|c|c|c|c|c|c|}
\hline \multicolumn{11}{|c|}{ (D) Root anatomical traits } \\
\hline \multirow[t]{4}{*}{ rRD } & $1^{*}$ & 11038867 & $\mathrm{~T}: \mathrm{G}$ & 0.307 & $3.17 \mathrm{E}-06$ & $2.09 \mathrm{E}-07$ & -0.036 & 10947296 & 11220396 & 273 \\
\hline & 1 & 11596350 & $\mathrm{~A}: \mathrm{G}$ & 0.276 & $8.12 \mathrm{E}-06$ & - & -0.034 & 11502214 & 11611147 & 108 \\
\hline & $5^{*}$ & 15841709 & $\mathrm{G}: \mathrm{A}$ & 0.279 & 4.77E-05 & $2.51 \mathrm{E}-06$ & -0.032 & 15709743 & 15874456 & 164 \\
\hline & $11^{\#}$ & 25434106 & $\mathrm{G}: \mathrm{C}$ & 0.338 & $1.86 \mathrm{E}-05$ & - & 0.040 & 25434106 & 25522849 & 88 \\
\hline \multirow[t]{5}{*}{$\mathrm{rCD}$} & $1^{*}$ & 11038867 & $\mathrm{~T}: \mathrm{G}$ & 0.306 & $1.65 \mathrm{E}-06$ & $3.79 \mathrm{E}-09$ & -0.073 & 10947296 & 11220396 & 273 \\
\hline & 1 & 11596350 & $A: G$ & 0.275 & $7.48 \mathrm{E}-05$ & - & -0.990 & 11502214 & 11611147 & 108 \\
\hline & $5^{*}$ & 15841709 & G:A & 0.279 & 7.87E-05 & $6.64 \mathrm{E}-07$ & 0.042 & 15709743 & 15874456 & 164 \\
\hline & $3^{\#}$ & 27843993 & C:T & 0.237 & - & $3.74 \mathrm{E}-06$ & 0.027 & 27703252 & 27847143 & 143 \\
\hline & $4^{\#}$ & 33647561 & G:A & 0.179 & - & $7.35 \mathrm{E}-05$ & 0.061 & 33647561 & 33661659 & 14 \\
\hline \multirow[t]{4}{*}{ rSD } & $1^{*}$ & 11038867 & $\mathrm{~T}: \mathrm{G}$ & 0.307 & $1.00 \mathrm{E}-05$ & $1.49 \mathrm{E}-06$ & -0.029 & 10947296 & 11220396 & 273 \\
\hline & 1 & 11596350 & $A: G$ & 0.276 & $2.39 \mathrm{E}-05$ & - & -0.028 & 11502214 & 11611147 & 108 \\
\hline & 7 & 27122192 & $\mathrm{G}: \mathrm{T}$ & 0.215 & 7.09E-05 & - & 0.028 & 27021782 & 27141446 & 119 \\
\hline & 11 & 25434106 & $\mathrm{G}: \mathrm{C}$ & 0.338 & - & $5.23 \mathrm{E}-05$ & 0.032 & 25434106 & 25522849 & 88 \\
\hline \multirow[t]{2}{*}{ rLMXD } & $7^{*}$ & 27141434 & C:T & 0.219 & $4.06 \mathrm{E}-05$ & $3.41 \mathrm{E}-05$ & 0.028 & 27021782 & 27141446 & 119 \\
\hline & $4^{\#}$ & 32411349 & $\mathrm{~T}: \mathrm{C}$ & 0.410 & - & $8.11 \mathrm{E}-05$ & 0.042 & 32404768 & 32451098 & 46 \\
\hline \multicolumn{11}{|c|}{ (E) Dry matter traits } \\
\hline rLW & $7^{\#}$ & 15930391 & $\mathrm{~A}: \mathrm{G}$ & 0.285 & - & $3.69 \mathrm{E}-05$ & 0.023 & 15930391 & 16109354 & 178 \\
\hline rSW & $6^{\#}$ & 7840678 & $\mathrm{~A}: \mathrm{G}$ & 0.296 & - & $4.90 \mathrm{E}-05$ & -0.114 & 7785975 & 7909286 & 123 \\
\hline rRW & $4^{\#}$ & 29184866 & $\mathrm{~A}: \mathrm{G}$ & 0.113 & - & $1.21 \mathrm{E}-05$ & -0.108 & 29126713 & 29377299 & 250 \\
\hline \multirow[t]{4}{*}{ rRS } & $2^{*}$ & 651557 & $\mathrm{~A}: \mathrm{C}$ & 0.292 & $1.78 \mathrm{E}-05$ & $5.11 \mathrm{E}-06$ & -0.061 & 636695 & 672438 & 35 \\
\hline & $3^{*}$ & 26825291 & $\mathrm{G}: \mathrm{C}$ & 0.102 & $2.00 \mathrm{E}-05$ & $1.70 \mathrm{E}-06$ & -0.096 & 26756997 & 26978105 & 221 \\
\hline & $8^{\#}$ & 17221046 & $\mathrm{C}: \mathrm{T}$ & 0.055 & - & 5.37E-05 & 0.084 & 17176998 & 17243358 & 66 \\
\hline & 12 & 19648498 & $\mathrm{G}: \mathrm{A}$ & 0.073 & $2.91 \mathrm{E}-05$ & - & -0.100 & 19628587 & 19662212 & 33 \\
\hline \multirow[t]{4}{*}{ rLWR } & $9^{\#}$ & 1450424 & C:T & 0.237 & - & $4.36 \mathrm{E}-07$ & -0.031 & 1317383 & 1826588 & 509 \\
\hline & $9^{\#}$ & 14127114 & $\mathrm{C}: \mathrm{T}$ & 0.193 & - & $1.88 \mathrm{E}-06$ & -0.038 & 14053172 & 14127114 & 73 \\
\hline & $2^{\#}$ & 25453820 & $\mathrm{~T}: \mathrm{G}$ & 0.252 & - & $1.36 \mathrm{E}-05$ & 0.021 & 25442913 & 25550826 & 107 \\
\hline & $5^{\#}$ & 925555 & C:T & 0.394 & - & $9.62 \mathrm{E}-06$ & 0.019 & 925555 & 966011 & 40 \\
\hline \multirow[t]{3}{*}{ rSWR } & $2^{\#}$ & 35635147 & G:T & 0.102 & - & $5.65 \mathrm{E}-06$ & -0.053 & 35378463 & 35635147 & 256 \\
\hline & $2^{\#}$ & 24133875 & $\mathrm{C}: \mathrm{G}$ & 0.143 & - & $2.72 \mathrm{E}-07$ & -0.065 & 24108015 & 24136056 & 28 \\
\hline & $2^{\#}$ & 25596944 & $\mathrm{~A}: \mathrm{G}$ & 0.172 & - & 7.99E-05 & -0.046 & 25568600 & 25609348 & 40 \\
\hline
\end{tabular}

Chr: chromosome, MAF: minor allele frequency, RAE: relative allelic effect calculated as a ratio of minor allele effect trait value to population average trait value, LD: linkage disequilibrium. 


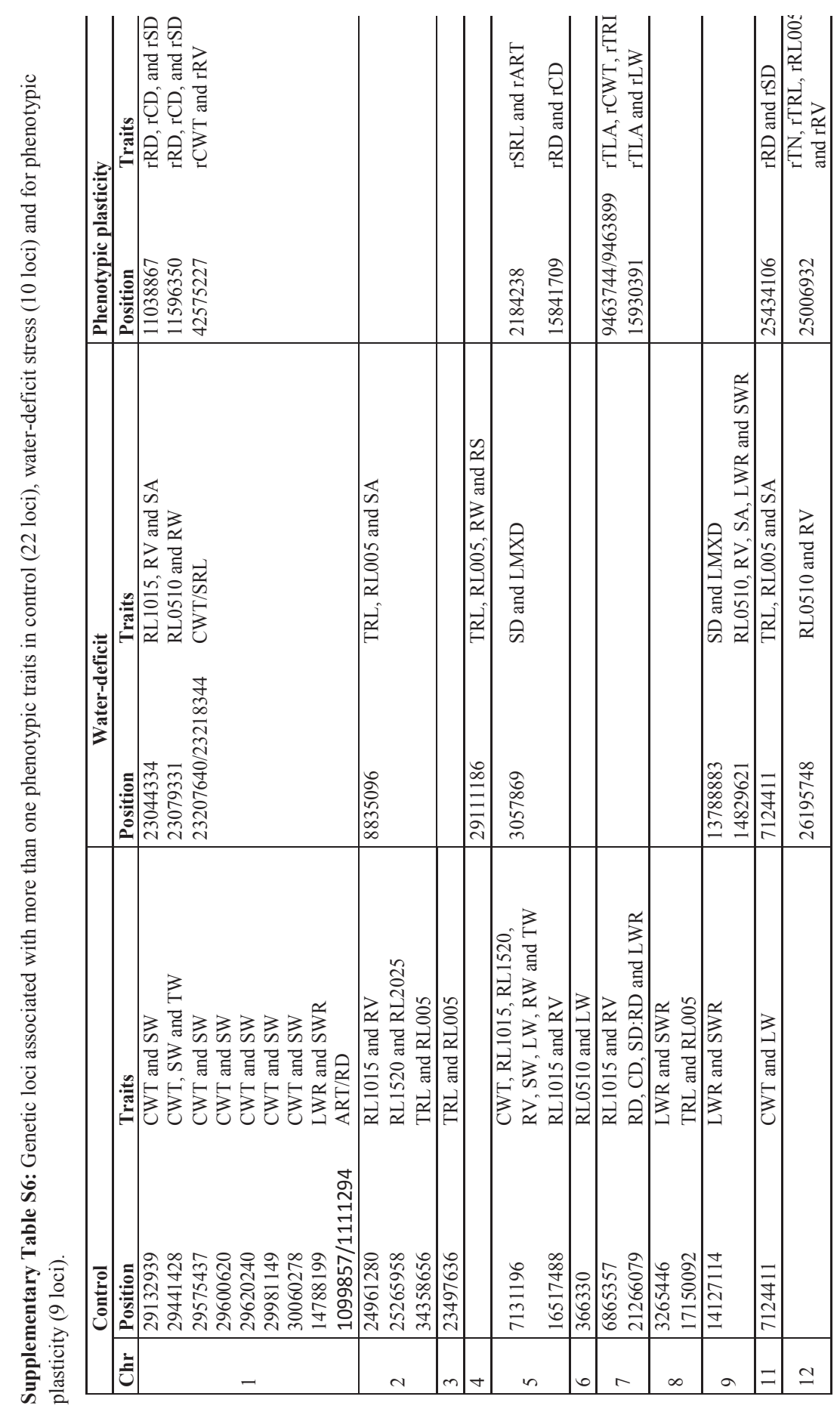




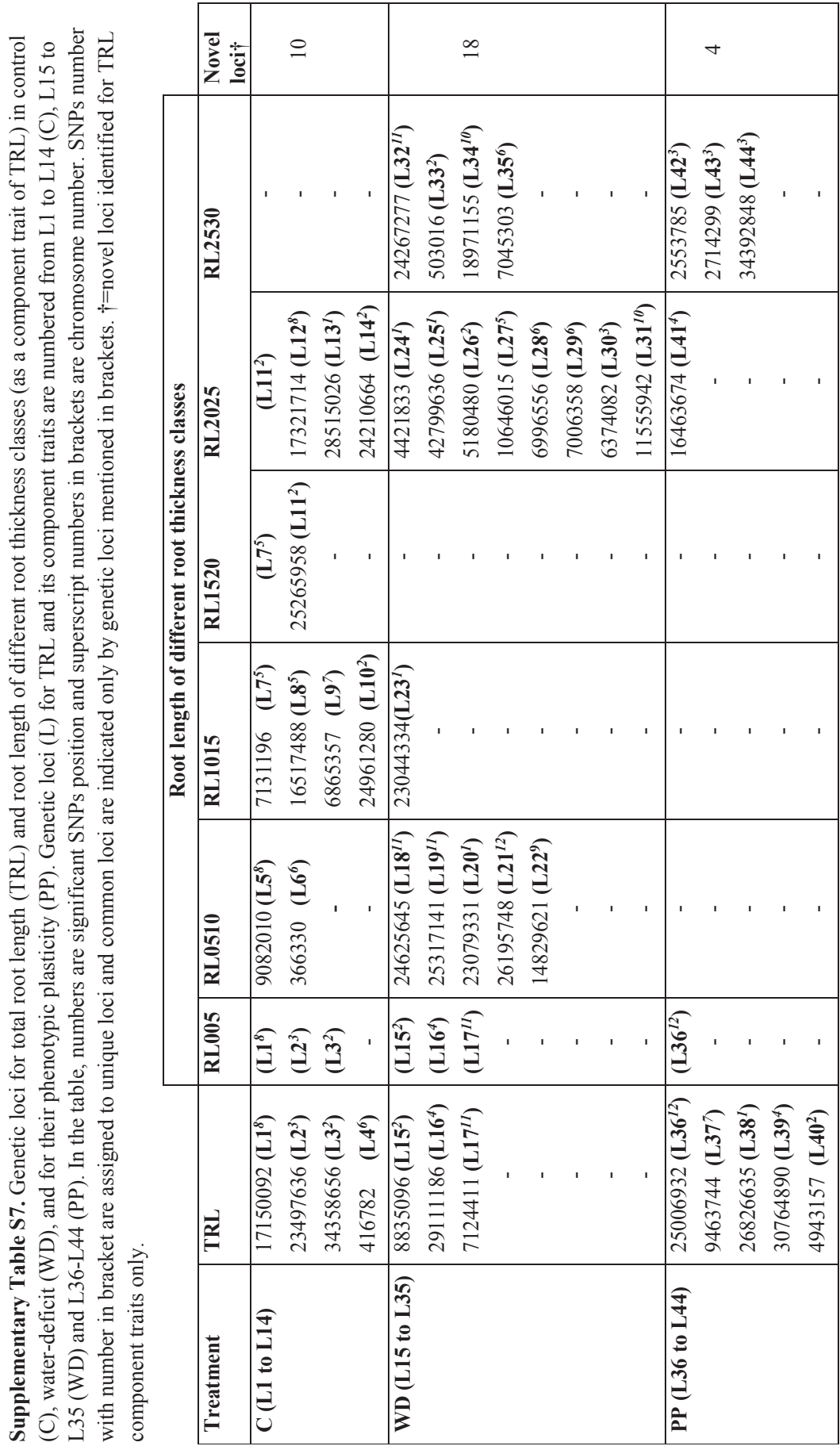


Supplementary Table S8: The a priori candidate genes underlying different loci/locus of shoot morphological, physiological, dry matter traits in control (C; 32 genes), water-deficit stress conditions (WD; 21 genes) and for its phenotypic plasticity (PP;17 genes) as a relative measure. A priori candidate gene annotations in bold were responsive to abiotic stress stimulus (Gene Ontology:0009628) according to Rice genome browser database. Trait acronyms are given in the Table $1 . \dagger=$ Distance of gene from peak SNP.

\begin{tabular}{|c|c|c|c|c|c|c|c|c|c|}
\hline Traits & Trt & Chr & SNP & Gene ID & $\begin{array}{l}\begin{array}{l}\text { Distance } \\
(\text { kbp }) \dagger\end{array} \\
\end{array}$ & Acr & Gene annotation & General description & Ref \\
\hline \multirow[t]{2}{*}{ PHT } & \multirow[t]{2}{*}{ C } & $1^{*}$ & 38286772 & LOC_Os01g66100.1 & 95 & GA200X & $\begin{array}{l}\text { Gibberellin } 20 \text { oxidase } \\
2\end{array}$ & $\begin{array}{l}\text { The mutant semi dwarf (sdl-"green revolution rice") } \\
\text { phenotype in rice is the result of a deficiency of active } \\
\text { GA in the elongating stem arising from a defective } 20 \text { - } \\
\text { oxidase GA biosynthetic enzyme. }\end{array}$ & $\begin{array}{l}\text { (Spielmeyer et } \\
\text { al., 2002) }\end{array}$ \\
\hline & & 5 & 2173057 & LOC_Os05g04610.1 & 4 & $\mathrm{ABC}$ & $\begin{array}{l}\text { ABC transporter, ATP- } \\
\text { binding protein }\end{array}$ & $\begin{array}{l}\text { Basipetal and acropetal auxin transport and previous } \\
\text { study predicted this candidate gene in one of the QTLs } \\
\text { on chromosome } 1 \text { for plant height in rice. }\end{array}$ & $\begin{array}{l}\text { (Ishimaru et al., } \\
\text { 2004) }\end{array}$ \\
\hline \multirow{3}{*}{$\mathrm{TN}$} & \multirow{3}{*}{$\mathrm{C}$} & $2^{*}$ & 31650233 & LOC_Os02g 51670.1 & Within & ERFTF & $\begin{array}{l}\text { Ethylene-responsive } \\
\text { transcription factor }\end{array}$ & $\begin{array}{l}\text { Involved in the cross talk between ethylene and GA; } \\
\text { down regulates ethylene-induced enhancement of GA } \\
\text { synthase. Rice ERF }(O S E A T B) \text { regulates tillering. }\end{array}$ & (Qi et al., 2011) \\
\hline & & $5^{\#}$ & 16091302 & $\begin{array}{l}\text { LOC_Os09g } 26590.1 \\
\text { LOC_Os09g } 26610.1\end{array}$ & $\begin{array}{l}34 \\
53\end{array}$ & SAUR & $\begin{array}{l}\text { OsSAUR37 - Auxin- } \\
\text { responsive SAUR } \\
\text { OsSAUR38 - Auxin- } \\
\text { responsive SAUR } \\
\end{array}$ & $\begin{array}{l}\text { Early auxin responsive gene, multicellular organism } \\
\text { development, rice mutant showed dwarfism, sterility } \\
\text { and lower tillering. }\end{array}$ & (Jain et al., 2006) \\
\hline & & $12^{\#}$ & 22528624 & LOC_Os12g 36770.2 & 2 & LipIII & $\begin{array}{l}\text { Lipase class } 3 \text { family } \\
\text { protein }\end{array}$ & $\begin{array}{l}\text { Regulates tillering, plant height and spikelet fertility in } \\
\text { rice. Possibly involved in phytohormone signalling of } \\
\text { strigolactone and auxin. }\end{array}$ & (Liu et al., 2013) \\
\hline \multirow{3}{*}{$\begin{array}{l}\text { CWT and } \\
\text { TW }\end{array}$} & \multirow{3}{*}{$\mathrm{C}$} & 1 & 29132939 & LOC_Os01g50720.1 & Within & MYB & $\begin{array}{l}\text { MYB family } \\
\text { transcription factor }\end{array}$ & $\begin{array}{l}\text { Phytohormone signalling, response to water-deficit and } \\
\text { salt stress, regulates root development and lateral root } \\
\text { by modulating auxin inducible genes. }\end{array}$ & $\begin{array}{l}\text { (Ambawat et al., } \\
\text { 2013) }\end{array}$ \\
\hline & & \multirow[b]{2}{*}{$1^{*}$} & \multirow[b]{2}{*}{29441428} & LOC_Os01g51154.1 & 35 & MYB & Single myb histone & $\begin{array}{l}\text { Phytohormone signalling, response to water-deficit and } \\
\text { salt stress, regulates root development and lateral root by } \\
\text { modulating auxin inducible genes. }\end{array}$ & $\begin{array}{l}\text { (Ambawat et al., } \\
\text { 2013) }\end{array}$ \\
\hline & & & & LOC_Os01g51260.1 & 28 & MYB & $\begin{array}{l}\text { MYB family } \\
\text { transcription factor }\end{array}$ & $\begin{array}{l}\text { Phytohormone signalling, response to water-deficit and } \\
\text { salt stress, regulates root development and lateral root } \\
\text { by modulating auxin inducible genes. }\end{array}$ & $\begin{array}{l}\text { (Ambawat et al., } \\
\text { 2013) }\end{array}$ \\
\hline \multirow{5}{*}{$\begin{array}{l}\text { CWT and } \\
\text { SW }\end{array}$} & \multirow{5}{*}{ C } & \multirow[t]{2}{*}{1} & \multirow[t]{2}{*}{29575437} & LOC_Os01g51300.1 & 74 & $\mathrm{G} \beta$ & $\begin{array}{l}\text { WD domain, G-beta } \\
\text { repeat domain } \\
\text { containing protein }\end{array}$ & $\begin{array}{l}\text { Subunit of large heterotrimeric G protein }(\alpha \beta \gamma) \\
\text { negatively regulates auxin induced signalling. Loss of } \\
\text { function mutant in G-protein have altered auxin } \\
\text { mediated cell division during formation of lateral and } \\
\text { adventitious root primordia. }\end{array}$ & $\begin{array}{l}\text { (Ullah et al., } \\
\text { 2003) }\end{array}$ \\
\hline & & & & LOC_Os01g51420.1 & 7 & CALB & Calcineurin B & $\begin{array}{l}\text { Plays critical role in diverse } \mathrm{Ca}^{2+} \text { dependent processes in } \\
\text { plants. Interacts with protein kinase } \mathrm{CIPK} 23 \text { and } \\
\text { regulates leaf transpiration and root potassium uptake in } \\
\text { Arabidopsis. }\end{array}$ & $\begin{array}{l}\text { (Cheong et al., } \\
\text { 2007) }\end{array}$ \\
\hline & & \multirow[t]{2}{*}{1} & \multirow[t]{2}{*}{29620240} & LOC_Os01g51780.1 & 158 & $\mathrm{AEC}$ & $\begin{array}{l}\text { Auxin efflux carrier } \\
\text { component }\end{array}$ & $\begin{array}{l}\text { Polar auxin transport and homoeostasis, auxin activated } \\
\text { signalling and mutant shows defects in root } \\
\text { development. Regulates leaf formation and phyllotaxis. }\end{array}$ & $\begin{array}{l}\text { (Grieneisen et } \\
\text { al., 2007), } \\
\text { (Reinhardt et al., } \\
\text { 2003) } \\
\end{array}$ \\
\hline & & & & LOC_Os01g51700.1 & 114 & RAS & Ras-related protein & $\begin{array}{l}\text { Small GTPase mediated signal transduction, root hair } \\
\text { initiation and root tip growth. }\end{array}$ & $\begin{array}{l}\text { (Molendijk et al., } \\
\text { 2001), (Jones et } \\
\text { al., 2002) }\end{array}$ \\
\hline & & 1 & 29981149 & LOC_Os01g52110.1 & 10 & CHYR1 & $\begin{array}{l}\text { RING finger and CHY } \\
\text { zinc finger domain- } \\
\text { containing protein } 1\end{array}$ & $\begin{array}{l}\text { An ubiquitin } \mathrm{E} 3 \text { ligase protein regulate } \mathrm{ABA} \text { induced } \\
\text { stomatal closer in water-deficit stress. }\end{array}$ & $\begin{array}{l}\text { (Ding et al., } \\
\text { 2015) }\end{array}$ \\
\hline \multirow[t]{2}{*}{$\begin{array}{l}\text { CWT, } \\
\mathrm{LW}, \mathrm{SW} \\
\text { and TW }\end{array}$} & \multirow[t]{2}{*}{ C } & \multirow[t]{2}{*}{5} & \multirow[t]{2}{*}{7131196} & LOC_Os05g12180.1 & 96 & PIP1 & CAPIP1 & $\begin{array}{l}\text { The Arabidopsis orthologue encodes regulatory elements } \\
\text { (PYL8 or RCAR3) of ABA receptor. Positive regulation } \\
\text { of abscisic acid-activated signalling pathway. Promotes } \\
\text { lateral root development by enhancing MYB } 77 \text { - } \\
\text { dependent transcription of auxin-responsive genes. }\end{array}$ & $\begin{array}{l}\text { (Zhao et al., } \\
\text { 2014) }\end{array}$ \\
\hline & & & & $\begin{array}{l}\text { LOC_Os05g12400.1 } \\
\text { LOC_Os05g12410.1 }\end{array}$ & $\begin{array}{ll}4 \\
6\end{array}$ & - BURP & $\begin{array}{l}\text { BURP domain } \\
\text { contaning protein }\end{array}$ & $\begin{array}{l}\text { ABA responsive and loss of function increased moisture } \\
\text { stress resistance }\end{array}$ & $\begin{array}{l}\text { (Harshavardhan } \\
\text { et al., 2014) }\end{array}$ \\
\hline CWT & C & $11^{\#}$ & 7124411 & LOC_Os11g12620.1 & 20 & CLV1 & $\begin{array}{l}\text { Receptor protein kinase } \\
\text { CLAVATA1 }\end{array}$ & $\begin{array}{l}\text { Regulates root meristems, regulation of root plasticity in } \\
\text { response to nitrogen. }\end{array}$ & $\begin{array}{l}\text { (Stahl et al., } \\
\text { 2013), (Araya et } \\
\text { al., 2014) }\end{array}$ \\
\hline \multirow{4}{*}{ WUE } & \multirow{4}{*}{$\mathrm{C}$} & 4 & 3707267 & LOC_Os04g07150.1 & 84 & AGAP & AGAP002737-PA & $\begin{array}{l}\text { The Arabidopsis orthologue of unknown protein } \\
\text { response to ABA. }\end{array}$ & $\mathrm{NA}$ \\
\hline & & & & LOC_Os07g33440.1 & 14 & & & The Arabidopsis orthologue catalyses essential oxidative & \\
\hline & & $7^{\#}$ & 20000202 & LOC_Os $07 \mathrm{~g} 33480.1$ & 12 & CYP450 & Cytochrome P450 & $\begin{array}{l}\text { step in the biosynthesis of brassinosteroid (BR). BR in } \\
\text { interaction with auxin promotes lateral root } \\
\text { developments and regulates stomatal development. }\end{array}$ & $\begin{array}{l}\text { 2004), (Kim et } \\
\text { al., 2012) }\end{array}$ \\
\hline & & $12^{\#}$ & 22169193 & LOC_Os12g36194.1 & 14 & NDPK & $\begin{array}{l}\text { Nucleoside diphosphate } \\
\text { kinase }\end{array}$ & $\begin{array}{l}\text { Regulates expression of antioxidant enzymes and multiple } \\
\text { environmental stress tolerance. }\end{array}$ & (Tang et al., 2008) \\
\hline \multirow[t]{2}{*}{ LW } & \multirow[t]{2}{*}{$\mathrm{C}$} & 6 & 366330 & LOC_Os06g01620.1 & Within & SCR & Scarecrow & $\begin{array}{l}\text { Transcription factor (TF) from GRAS family contributes } \\
\text { to the specification and determination of root quiescent } \\
\text { centre (QC). Together with SHORTROOT (another } \\
\text { GRAS TF) controls the division of endoderm/cortex } \\
\text { cells. Regulates radial patterning mechanism of root and } \\
\text { shoot. }\end{array}$ & $\begin{array}{l}\text { (Mai et al., } \\
\text { 2014), } \\
\text { (Wysocka-Diller } \\
\text { et al., 2000) }\end{array}$ \\
\hline & & $11^{*}$ & 7124411 & LOC_Os11g12620.1 & 20 & CLV1 & $\begin{array}{l}\text { Receptor protein kinase } \\
\text { CLAVATA1 }\end{array}$ & $\begin{array}{l}\text { Regulates root and shoot apical meristems, regulation of } \\
\text { root plasticity in response to nitrogen. }\end{array}$ & $\begin{array}{l}\text { (Stahl et al., } \\
\text { 2013), (Araya et } \\
\text { al., 2014), (Kalve } \\
\text { et al., 2014) } \\
\end{array}$ \\
\hline TW & $\mathrm{C}$ & 1 & 39058787 & LOC_Os01g67410.1 & 86 & $\mathrm{AP} 2 / \mathrm{ERF}$ & $\begin{array}{l}\text { AP2/EREBP } \\
\text { transcription factor } \\
\text { BABY BOOM }\end{array}$ & $\begin{array}{l}\text { Regulates adventitious root growth, improves water- } \\
\text { deficit and other abiotic stress tolerance, involved in } \\
\text { auxin and other phytohormone activated signalling } \\
\text { pathway. }\end{array}$ & $\begin{array}{l}\text { (Kitomi et al., } \\
\text { 2011),(Quan et } \\
\text { al., 2010) }\end{array}$ \\
\hline
\end{tabular}


Supplementary Table S8. (Continued)

\begin{tabular}{|c|c|c|c|c|c|c|c|c|c|}
\hline \multirow{3}{*}{$\begin{array}{l}\text { LWR and } \\
\text { SWR }\end{array}$} & \multirow{3}{*}{$\mathrm{C}$} & $\begin{array}{c}8^{*} \\
\text { and } \\
8\end{array}$ & 3265446 & LOC_Os08g06060.1 & 42 & CLKC & $\begin{array}{l}\text { CGMC-includes CDA, } \\
\text { MAPK, GSK3, and } \\
\text { CLKC kinases }\end{array}$ & $\begin{array}{l}\text { CGMC kinase group includes MAP kinase (MAPK), } \\
\text { regulates biotic and abiotic stress response. }\end{array}$ & \multirow{2}{*}{$\begin{array}{l}\text { (Rohila and } \\
\text { Yang, 2007), } \\
\text { (Wang et al., } \\
\text { 2004) }\end{array}$} \\
\hline & & 9 & 14127114 & LOC_Os09g23650.1 & 67 & FAM10 & FAM10 family protein & $\begin{array}{l}\text { Also, known as HSC70 interacting protein belonging } \\
\text { from HSP70 family protein. Regulates cellular redox } \\
\text { homoeostasis, heat acclimation and protein folding. }\end{array}$ & \\
\hline & & $\begin{array}{c}1 \\
\text { and } \\
1^{*}\end{array}$ & 14788199 & LOC_Os01g26039.1 & 24 & EP & Expressed protein & $\begin{array}{l}\text { The Arabidopsis orthologue encodes PHYTYL ESTER } \\
\text { SYNTHASE } 1 \text { involved in the deposition of free phytol } \\
\text { and free fatty acids in the form of phytol esters in } \\
\text { chloroplasts that maintain the integrity of the } \\
\text { photosynthetic membrane during abiotic stress and } \\
\text { senescence. }\end{array}$ & $\begin{array}{l}\text { (Lippold et al., } \\
\text { 2012) }\end{array}$ \\
\hline \multirow{3}{*}{ LWR } & \multirow{3}{*}{$\mathrm{C}$} & \multirow[t]{2}{*}{3} & \multirow[t]{2}{*}{32750373} & LOC_Os03g57290.1 & 78 & CUL & Cullin & $\begin{array}{l}\text { Cullin proteins are molecular scaffolds that have crucial } \\
\text { roles in the post-translational modification of } \\
\text { cellular proteins involving ubiquitin (protein } \\
\text { ubiquitination). Combines with RING proteins to form } \\
\text { Cullin-RING ubiquitin ligases (CRLs) and plays a role in } \\
\text { cellular processes under abiotic stress pathway. }\end{array}$ & $\begin{array}{l}\text { (Guo et al, } \\
\text { 2013) }\end{array}$ \\
\hline & & & & LOC_Os03g57340.1 & 42 & DnaJ & $\begin{array}{l}\text { Chaperone protein } \\
\text { dnaJ }\end{array}$ & $\begin{array}{l}\text { Components of macromolecular chaperone complexes } \\
\text { (DnaJ-HSP70). DnaJ-like protein (ARG1) involved in } \\
\text { gravity signal transduction in root. Maintains cellular } \\
\text { protein homoeostasis in normal and stress conditions. }\end{array}$ & $\begin{array}{l}\text { (Rosen et al., } \\
\text { 1999) }\end{array}$ \\
\hline & & 3 & 32750373 & LOC_Os03g57560.1 & 63 & PIWI & $\begin{array}{l}\text { Piwi domain } \\
\text { containing protein }\end{array}$ & $\begin{array}{l}\text { Conserved domain protein of ARGOUNATE protein } \\
\text { regulates leaf and multicellular organisms development. }\end{array}$ & $\begin{array}{l}\text { (Bohmert et al., } \\
\text { 1998) }\end{array}$ \\
\hline \multirow[t]{2}{*}{ SWR } & \multirow[t]{2}{*}{$\mathrm{C}$} & 9 & $14829621 \mathrm{~L}$ & LOC_Os09g24840.1 & 13 & GASA & $\begin{array}{l}\text { GASR10 - Gibberellin- } \\
\text { regulated } \\
\text { GASA/GAST/Snakin } \\
\text { family protein }\end{array}$ & $\begin{array}{l}\text { Phytohormone cross-talk and redox homoeostasis, } \\
\text { regulates root, stem and other organ growth and } \\
\text { development. }\end{array}$ & $\begin{array}{l}\text { (Nahirñak et al., } \\
\text { 2012) }\end{array}$ \\
\hline & & $11^{\#}$ & $18947620 \mathrm{I}$ & LOC_Os1lg32110.1 & 6 & ARF & Auxin response factor & $\begin{array}{l}\text { Auxin activated signalling, mutant showed defects in } \\
\text { plant growth and development. }\end{array}$ & $\begin{array}{l}\text { (Guilfoyle and } \\
\text { Hagen, 2007) } \\
\end{array}$ \\
\hline \multirow{4}{*}{ PHT } & \multirow{4}{*}{ WD } & 1 & 38286772 & LOC_Os01g66100.1 & 95 & GA200X & $\begin{array}{l}\text { Gibberellin } 20 \text { oxidase } \\
2\end{array}$ & $\begin{array}{l}\text { The mutant semi dwarf (sdl-"green revolution rice") } \\
\text { phenotype in rice is the result of a deficiency of active } \\
\text { GA in the elongating stem arising from a defective 20- } \\
\text { oxidase GA biosynthetic enzyme. }\end{array}$ & $\begin{array}{l}\text { (Spielmeyer et } \\
\text { al., 2002) }\end{array}$ \\
\hline & & \multirow{2}{*}{$3^{*}$} & \multirow{2}{*}{30407838} & LOC_Os03g53020.1 & Within & BHLH & $\begin{array}{l}\text { Helix-loop-helix DNA- } \\
\text { binding domain } \\
\text { contaning protein }\end{array}$ & $\begin{array}{l}\text { Arabidopsis orthologue regulating stress-related } \\
\text { transcriptional changes and drought tolerance. }\end{array}$ & NA \\
\hline & & & & LOC_Os03g53150.1 & 73 & AUX/IAA & $\begin{array}{l}\text { OsIAA13 - Auxin- } \\
\text { responsive Aux/IAA } \\
\text { gene family member }\end{array}$ & $\begin{array}{l}\text { Auxin activated signalling, agravitopic root and shoot, } \\
\text { defect in root hairs. }\end{array}$ & (Reed, 2001) \\
\hline & & $5^{*}$ & 2173057 & LOC_Os05g04610.1 & 4 & $\mathrm{ABC}$ & $\mathrm{ABC}$ transporter & $\begin{array}{l}\text { Basipetal and acropetal auxin transport and previous } \\
\text { study predicted this candidate gene in one of the QTLs } \\
\text { on chromosome } 1 \text { for plant height in rice. }\end{array}$ & $\begin{array}{l}\text { (Ishimaru et al., } \\
\text { 2004) }\end{array}$ \\
\hline TN & WD & $3^{*}$ & 9995472 & LOC_Os03g18050.1 & 62 & SAUR13 & $\begin{array}{l}\text { OsSAUR13 - Auxin- } \\
\text { responsive SAUR gene } \\
\text { family member }\end{array}$ & $\begin{array}{l}\text { Early auxin responsive gene, multicellular organism } \\
\text { development, rice mutant showed dwarfism, sterility } \\
\text { and lower tillering. }\end{array}$ & (Jain et al., 2006) \\
\hline TLA & WD & $8^{*}$ & $25121944 \mathrm{I}$ & LOC_Os08g39840.1 & 102 & LOXs & $\begin{array}{l}\text { Lipoxygenase, } \\
\text { chloroplast precursor }\end{array}$ & $\begin{array}{l}\text { Increased activity in leaves and root and associated with } \\
\text { lipid peroxidation mechanism under water-deficit stress. }\end{array}$ & $\begin{array}{l}\text { (Sofo et al., } \\
2004 \text { ) }\end{array}$ \\
\hline $\begin{array}{l}\text { TLA and } \\
\text { SW }\end{array}$ & & $\begin{array}{c}3^{\#} \\
\text { and } \\
3^{*}\end{array}$ & $11725360 \mathrm{I}$ & LOC_Os03g20700.2 & 13 & $\mathrm{MgC}$ & Magnesium-chelatase & $\begin{array}{l}\text { Arabidopsis orthologue regulate chlorophyll biosynthesis } \\
\text { and photosynthesis }\end{array}$ & NA \\
\hline \multirow{3}{*}{ CWT } & \multirow{3}{*}{ WD } & $1^{\#}$ & 23207640 & LOC_Os0lg 41050.1 & 43 & SULT & Sulfate transporter & $\begin{array}{l}\text { Sulfate uptake, strongly regulated in roots under water- } \\
\text { deficit and salinity stress, response to ABA. }\end{array}$ & $\begin{array}{l}\text { (Gallardo et al., } \\
\text { 2014) }\end{array}$ \\
\hline & & $4^{\#}$ & 34640918 & LOC_Os04g58130.1 & 18 & KAT & $\begin{array}{l}\text { Katanin p80 WD40 } \\
\text { repeat-containing } \\
\text { subunit B1 homolog } 1\end{array}$ & $\begin{array}{l}\text { A microtubule severing enzyme. Overexpression of } \\
\text { OsKTN80a caused the retarded root growth of rice } \\
\text { seedlings. }\end{array}$ & $\begin{array}{l}\text { (Wan et al., } \\
\text { 2014) }\end{array}$ \\
\hline & & $6^{\#}$ & 13412649 & LOC_Os06g22960.1 & 4 & $\mathrm{AQP}$ & Aquaporin protein & $\begin{array}{l}\text { Cellular water homoeostasis and transport, response to } \\
\text { ABA, lateral root emergence and elongation, maintains } \\
\text { root hydraulic conductivity. }\end{array}$ & $\begin{array}{l}\text { (Reinhardt et al., } \\
\text { 2016) }\end{array}$ \\
\hline \multirow{3}{*}{ WUE } & \multirow{3}{*}{ WD } & $9^{\#}$ & 15426362 & LOC_0s09g25850.1 & 66 & WAX & WAX2 & $\begin{array}{l}\text { Involved in cuticle membrane development and wax } \\
\text { production. Arabidopsis mutant (wax2) showed altered } \\
\text { cuticle membrane. Higher epicuticular wax improves } \\
\text { water use efficiency (WUE). }\end{array}$ & $\begin{array}{l}\text { (Chen et al., } \\
2003)\end{array}$ \\
\hline & & $11^{\#}$ & 27574096 & LOC_Os1lg45560.1 & 3 & МBTB & $\begin{array}{l}\text { MBTB68 - Bric-a- } \\
\text { Brac, Tramtrack, } \\
\text { Broad Complex BTB } \\
\text { domain }\end{array}$ & $\begin{array}{l}\text { Response to salt, water-deficit stress and osmotic stress, } \\
\text { protein ubiquitination, interact with CULLIN } 3 \text { to } \\
\text { regulate root growth. }\end{array}$ & $\begin{array}{l}\text { (Thomann et al., } \\
\text { 2009) }\end{array}$ \\
\hline & & $6^{\#}$ & 23297154 & + LOC_Os06g39240.1 & 4 & EDR & $\begin{array}{l}\text { Endothelial } \\
\text { differentiation-related } \\
\text { factor } 1\end{array}$ & $\begin{array}{l}\text { Also known as MULTIPROTEIN BRIDGING FACTOR } \\
1 \mathrm{C} \text {, enhances the tolerances to heat and osmotic stress by } \\
\text { partially activating, or perturbing, the ethylene-response } \\
\text { signal transduction pathway. }\end{array}$ & $\begin{array}{l}\text { (Suzuki et al., } \\
2005 \text { ) }\end{array}$ \\
\hline SW & WD & $12^{*}$ & 17786153 & LOC_Os12g29980.1 & 129 & GRF & $\begin{array}{l}\text { Growth regulating factor } \\
\text { protein }\end{array}$ & $\begin{array}{l}\text { Cell expansion and proliferation, root development, } \\
\text { coordination of growth in water-deficit stress condition. }\end{array}$ & $\begin{array}{l}\text { (Omidbakhshfard } \\
\text { et al., 2015) }\end{array}$ \\
\hline$L W$ & WD & $1^{*}$ & 15402601 & LOC_Os01g27630.1 & 8 & GST & $\begin{array}{l}\text { Glutathione S- } \\
\text { transferase }\end{array}$ & $\begin{array}{l}\text { Over-expression of GST in soybean showed longer root } \\
\text { length and less growth retardation in drought and salinity } \\
\text { stress. }\end{array}$ & (Ji et al., 2010) \\
\hline
\end{tabular}


Supplementary Table S8. (Continued)

\begin{tabular}{|c|c|c|c|c|c|c|c|c|c|}
\hline \multirow{3}{*}{ LWR } & \multirow{3}{*}{ WD } & $2^{*}$ & 20169674 & LOC_Os02g33860.1 & 13 & $\mathrm{G} \beta$ & $\begin{array}{l}\text { WD domain, G-beta } \\
\text { repeat domain } \\
\text { containing protein }\end{array}$ & $\begin{array}{l}\text { Subunit of large heterotrimeric G protein }(\alpha \beta \gamma) \\
\text { negatively regulates auxin induced signalling. Loss of } \\
\text { function mutants in G-protein have altered auxin } \\
\text { mediated cell division during formation of lateral and } \\
\text { adventitious root primordia. }\end{array}$ & $\begin{array}{l}\text { (Ullah et al., } \\
\text { 2003) }\end{array}$ \\
\hline & & \multirow[t]{2}{*}{$2^{*}$} & \multirow[t]{2}{*}{23246559} & LOC_Os02g38440.1 & 5 & OXR & $\begin{array}{l}\text { Oxidoreductase, short } \\
\text { chain } \\
\text { dehydrogenase/reductase } \\
\text { family }\end{array}$ & $\begin{array}{l}\text { The Arabidopsis orthologue encodes BETA- } \\
\text { KETOACYL REDUCTASE } 1 \text { protein regulating } \\
\text { cuticular waxes production. }\end{array}$ & $\begin{array}{l}\text { (Hooker et al., } \\
\text { 2002) }\end{array}$ \\
\hline & & & & LOC_Os02g38480.1 & 18 & EP & Expressed protein & Arabidopsis orthologue regulating salt stress response & NA \\
\hline $\begin{array}{l}\text { LWR and } \\
\text { SWR }\end{array}$ & WD & $\begin{array}{c}9 \\
\text { and } \\
9^{*}\end{array}$ & 14829621 & LOC_Os09g24840.1 & Within & GASA & $\begin{array}{l}\text { GASR10-Gibberellin- } \\
\text { regulated } \\
\text { GASA/GAST/Snakin } \\
\text { family protein }\end{array}$ & $\begin{array}{l}\text { Phytohormone cross-talk and redox homoeostasis, } \\
\text { regulates root, stem and other organ growth and } \\
\text { development. }\end{array}$ & $\begin{array}{l}\text { (Nahirñak et al., } \\
\text { 2012) }\end{array}$ \\
\hline \multirow[t]{2}{*}{ SWR } & \multirow[t]{2}{*}{ WD } & 7 & 20874845 & LOC_Os07g34670.1 & 92 & POX & Peroxidase precursor & $\begin{array}{l}\text { Involved in the scavenging of reactive oxygen species } \\
\text { (ROS) in water-deficit and other abiotic stresses. }\end{array}$ & $\begin{array}{l}\text { (Chakrabarty et } \\
\text { al., 2016) }\end{array}$ \\
\hline & & $1^{\#}$ & 40813452 & LOC_Os01g70490.1 & 12 & KT & Potassium transporter & $\begin{array}{l}\text { Overexpression improved rice osmotic and drought } \\
\text { stress tolerance by increasing tissue levels of } \mathrm{K}^{+} \text {in the } \\
\text { root. }\end{array}$ & $\begin{array}{l}\text { (Ahmad et al., } \\
\text { 2016) }\end{array}$ \\
\hline \multirow[b]{3}{*}{ rPHT } & \multirow[b]{3}{*}{ PP } & $2^{*}$ & 24405418 & LOC_Os02g40320.1 & Within & PNH & PINHEAD & $\begin{array}{l}\text { Required for reliable formation of primary and axillary } \\
\text { shoot apical meristems. }\end{array}$ & $\begin{array}{l}\text { (Lynn et al., } \\
1999)\end{array}$ \\
\hline & & \multirow{2}{*}{\multicolumn{2}{|c|}{$6^{\#}$}} & LOC_Os06g33480.1 & 102 & PNH & PINHEAD & $\begin{array}{l}\text { Required for reliable formation of primary and axillary } \\
\text { shoot apical meristems. }\end{array}$ & $\begin{array}{l}\text { (Lynn et al., } \\
\text { 1999) }\end{array}$ \\
\hline & & & & LOC_Os06g33690.1 & Within & PIP1 & CAPIP1 & $\begin{array}{l}\text { The Arabidopsis orthologue encodes regulatory elements } \\
\text { (PYL8 or RCAR3) of ABA receptor. Positive regulation } \\
\text { of abscisic acid-activated signalling pathway. Promotes } \\
\text { lateral root development by enhancing MYB77- } \\
\text { dependent transcription of auxin-responsive genes. }\end{array}$ & $\begin{array}{l}\text { (Zhao et al., } \\
\text { 2014) }\end{array}$ \\
\hline rTN & PP & $12^{*}$ & 25006932 & LOC_Os12g40190.1 & 134 & $\mathrm{G} \alpha$ & $\begin{array}{l}\text { G-protein alpha } \\
\text { subunit }\end{array}$ & $\begin{array}{l}\text { Regulates root morphogenesis, mutant shows reduced } \\
\text { root growth in rice }(d l) \text { maize }(c t 2) \text { and Arabidopsis } \\
\text { (gpal). Arabidopsis orthologue involved in ABA } \\
\text { signalling and root morphogenesis. }\end{array}$ & $\begin{array}{l}\text { (Ullah et al., } \\
\text { 2001), (Urano et } \\
\text { al., 2016), }\end{array}$ \\
\hline $\begin{array}{l}\text { rTLA } \\
\text { and } \\
\text { rCWT }\end{array}$ & PP & $7^{\sharp}$ & 9463744 & LOC_Os07g16224.1 & Within & PIWI & $\begin{array}{l}\text { Piwi domain containing } \\
\text { protein }\end{array}$ & $\begin{array}{l}\text { Conserved domain protein of ARGOUNATE protein } \\
\text { regulates leaf and multicellular organisms development. }\end{array}$ & $\begin{array}{l}\text { (Bohmert et al., } \\
\text { 1998) }\end{array}$ \\
\hline rSLA & PP & $11^{*}$ & 19245430 & LOC_Os1lg32610.1 & 9 & CHS & $\begin{array}{l}\text { Chalcone and stilbene } \\
\text { synthases }\end{array}$ & $\begin{array}{l}\text { Flavonoid biosynthesis, regulates polar auxin transport, } \\
\text { mutant } t t 4(2 Y Y 6) \text { shows delayed root gravity response. }\end{array}$ & $\begin{array}{l}\text { (Buer et al., } \\
2006)\end{array}$ \\
\hline \multirow{6}{*}{ rCWT } & \multirow{6}{*}{ PP } & 1 & 33277486 & LOC_Os01g57210.1 & 226 & KAT & $\begin{array}{l}\text { Katanin p80 WD40 } \\
\text { repeat-containing } \\
\text { subunit B1 homolog } 1\end{array}$ & $\begin{array}{l}\text { A microtubule severing enzyme. Overexpression of } \\
\text { OsKTN80a caused the retarded root growth of rice } \\
\text { seedlings. Involved in the }\end{array}$ & $\begin{array}{l}\text { (Wan et al., } \\
\text { 2014), }\end{array}$ \\
\hline & & 1 & 33293954 & LOC_Os01g57730.1 & 88 & POD & Peroxidase precursor & $\begin{array}{l}\text { scavenging of reactive oxygen species (ROS) in water- } \\
\text { deficit and other abiotic stresses. }\end{array}$ & $\begin{array}{l}\text { (Chakrabarty et } \\
\text { al., 2016) }\end{array}$ \\
\hline & & \multirow{2}{*}{1} & \multirow{2}{*}{33755921} & LOC_Os01g58420.1 & 110 & AP2/ERF & $\begin{array}{l}\mathrm{AP} 2 \text { (ERF/AP2) domain } \\
\text { containing protein }\end{array}$ & $\begin{array}{l}\text { Regulates adventitious root growth, improves water- } \\
\text { deficit and other abiotic stress tolerance, involved in } \\
\text { auxin and other phytohormone activated signalling } \\
\text { pathways. }\end{array}$ & $\begin{array}{l}\text { (Kitomi et al., } \\
\text { 2011), (Quan et } \\
\text { al., 2010) }\end{array}$ \\
\hline & & & & LOC_Os0lg58860.1 & 80 & $\mathrm{AEC}$ & $\begin{array}{l}\text { Auxin efflux carrier } \\
\text { component, putative, } \\
\text { expressed }\end{array}$ & $\begin{array}{l}\text { Polar auxin transport and homoeostasis, auxin activated } \\
\text { signalling and mutant shows defects in root } \\
\text { development. Regulates leaf formation and phyllotaxis. }\end{array}$ & $\begin{array}{l}\text { (Grieneisen et } \\
\text { al., 2007), } \\
\text { (Reinhardt et al., } \\
\text { 2003) }\end{array}$ \\
\hline & & 1 & 34280616 & LOC_Os0lg59360.1 & 45 & CAMK & $\begin{array}{l}\text { CAMK- } \\
\text { calcium/calmodulin } \\
\text { dependent protein } \\
\text { kinases }\end{array}$ & ABA activated signalling pathway and stomatal closure. & $\begin{array}{l}\text { (Grabov and } \\
\text { Blatt, 1998) }\end{array}$ \\
\hline & & $1^{*}$ & 42575227 & LOC_Os01g73310.1 & 91 & $\mathrm{ACT}$ & Actin & $\begin{array}{l}\text { Actins function is essential for cytoplasmic streaming, } \\
\text { organelle orientation, cell elongation and root tip growth. } \\
\text { Actin filament regulates stomatal movement. }\end{array}$ & $\begin{array}{l}\text { (Gilliland et al., } \\
\text { 2003), (Kim et } \\
\text { al., 1995) }\end{array}$ \\
\hline rLWR & PP & $9^{\sharp}$ & 14127114 & LOC_Os09g23650.1 & 76 & FAM & FAM10 family protein & $\begin{array}{l}\text { Also known as HSC70 interacting protein belonging } \\
\text { from HSP70 family protein. Regulates cellular redox } \\
\text { homoeostasis, heat acclimation and protein folding. }\end{array}$ & $\begin{array}{l}\text { (Wang et al., } \\
\text { 2004) }\end{array}$ \\
\hline \multirow{3}{*}{ rSWR } & \multirow{3}{*}{ PP } & $2^{\#}$ & 35635147 & LOC_Os02g58020.1 & 117 & $\mathrm{ABC}$ & $\begin{array}{l}\mathrm{ABC} \text { transporter, ATP- } \\
\text { binding protein }\end{array}$ & $\begin{array}{l}\text { Basipetal and acropetal auxin transport and previous } \\
\text { study predicted this candidate gene in one of the QTLs } \\
\text { on chromosome } 1 \text { for plant height in rice. }\end{array}$ & $\begin{array}{l}\text { (Ishimaru et al., } \\
\text { 2004) }\end{array}$ \\
\hline & & $2^{\#}$ & 24133875 & LOC_Os02g39910.1 & Within & BTB & $\begin{array}{l}\text { B4-BTB1 - Bric-a-Brac, } \\
\text { Tramtrack, Broad } \\
\text { Complex BTB domain }\end{array}$ & $\begin{array}{l}\text { Response to salt, water-deficit stress and osmotic stress, } \\
\text { protein ubiquitination, interacts with CULLIN3 to } \\
\text { regulate root growth. }\end{array}$ & $\begin{array}{l}\text { (Thomann et al., } \\
\text { 2009) }\end{array}$ \\
\hline & & $2^{\#}$ & & LOC_Os02g42585.1 & Within & $\mathrm{AP} 2 / \mathrm{ERF}$ & $\begin{array}{l}\text { AP2 domain containing } \\
\text { protein }\end{array}$ & $\begin{array}{l}\text { Regulates adventitious root growth, improves water- } \\
\text { deficit stress tolerance, involved in auxin and other } \\
\text { phytohormone activated signalling. }\end{array}$ & $\begin{array}{l}\text { (Kitomi et al., } \\
\text { 2011), (Quan et } \\
\text { al., 2010) }\end{array}$ \\
\hline
\end{tabular}

Trt: treatment, Chr: chromosome, Acr: gene acronym, Ref: reference. 
Supplementary Table S9: The predicted a priori candidate genes (total 40 unique a priori genes excluding loci associated with more than one trait) underlying different loci/locus of root traits in control (C) condition and demonstrating to play a role in root growth and development. A priori candidate gene annotations in bold are responsive to abiotic stress stimulus (Gene Ontology:0009628) according to Rice genome browser database. Trait acronyms are given in the Table $1 . \dagger=$ Distance of gene from peak SNP.

\begin{tabular}{|c|c|c|c|c|c|c|c|c|}
\hline Traits & Chr & SNP & Gene & Distance $(k$ & bp) $\dagger$ Acr & Gene annotation & General description & Ref \\
\hline \multicolumn{9}{|c|}{ (A) Root morphological traits } \\
\hline \multirow{2}{*}{ TRL } & \multirow{2}{*}{$6^{\#}$} & \multirow{2}{*}{416782} & LOC_Os06g01670.1 & 13 & MYB & $\begin{array}{l}\text { MYB family transcription } \\
\text { factor }\end{array}$ & $\begin{array}{l}\text { Phytohormone signalling, response to water- } \\
\text { deficit and salt stress, regulates root } \\
\text { development and lateral root by modulating } \\
\text { auxin inducible genes. }\end{array}$ & $\begin{array}{l}\text { (Ambawat et } \\
\text { al., 2013) }\end{array}$ \\
\hline & & & LOC_Os06g01780.1 & 7 & $\mathrm{AP} 2 / \mathrm{ERF}$ & $\begin{array}{l}\text { Ethylene-responsive } \\
\text { element-binding protein }\end{array}$ & $\begin{array}{l}\text { Regulates adventitious root growth, improves } \\
\text { water-deficit stress tolerance, auxin and other } \\
\text { phytohormone activated signalling pathways. }\end{array}$ & $\begin{array}{l}\text { (Kitomi et } \\
\text { al., } \\
\text { 2011),(Quan } \\
\text { et al., 2010) }\end{array}$ \\
\hline $\begin{array}{l}\text { TRL and } \\
\text { RL005 }\end{array}$ & $2^{\#}$ & 34358656 & LOC_Os02g56120.2 & 10 & Aux/IAA & $\begin{array}{l}\text { OsIAA9 - Auxin- } \\
\text { responsive Aux/IAA }\end{array}$ & $\begin{array}{l}\text { Auxin activated signalling, agravitopic root and } \\
\text { shoot, defect in root hairs. }\end{array}$ & (Reed, 2001) \\
\hline RL0510 & $6^{\#}$ & 366330 & LOC_Os06g01620.1 & Within & SCR & Scarecrow & $\begin{array}{l}\text { Transcription factor (TF) from GRAS family } \\
\text { contributes to the specification and } \\
\text { determination of root quiescent centre (QC). } \\
\text { Together with SHORTROOT (another GRAS } \\
\text { TF) controls the division of endoderm/cortex } \\
\text { cells. Regulates radial patterning mechanism of } \\
\text { root and shoot. }\end{array}$ & $\begin{array}{l}\text { (Mai et al., } \\
\text { 2014),(Wyso } \\
\text { cka-Diller et } \\
\text { al., 2000) }\end{array}$ \\
\hline \multirow[t]{3}{*}{$\begin{array}{l}\text { RL1015, } \\
\text { RL1520, } \\
\text { RV and } \\
\text { RW }\end{array}$} & \multirow[t]{3}{*}{$5^{*}$} & \multirow[t]{3}{*}{7131196} & LOC_Os05g12180.1 & 96 & PIP1 & CAPIP1 & $\begin{array}{l}\text { The Arabidopsis orthologue encodes regulatory } \\
\text { elements (PYL8 or RCAR3) of ABA receptor. } \\
\text { Positive regulation of abscisic acid-activated } \\
\text { signalling pathway. Promotes lateral root } \\
\text { development by enhancing MYB77-dependent } \\
\text { transcription of auxin-responsive genes. }\end{array}$ & $\begin{array}{l}\text { (Zhao et al., } \\
2014 \text { ) }\end{array}$ \\
\hline & & & LOC_Os05g12400.1 & 4 & \multirow{2}{*}{ BURP } & \multirow{2}{*}{$\begin{array}{l}\text { BURP domain } \\
\text { contaning protein }\end{array}$} & \multirow{2}{*}{$\begin{array}{l}\text { ABA responsive and loss of function increased } \\
\text { moisture stress resistance }\end{array}$} & \multirow{2}{*}{$\begin{array}{l}\text { (Harshavardh } \\
\text { an et al., } \\
\text { 2014) }\end{array}$} \\
\hline & & & LOC_Os05g12410.1 & 6 & & & & \\
\hline $\begin{array}{l}\text { RL1015 } \\
\text { and RV }\end{array}$ & $5^{\#}$ & 16517488 & LOC_Os05g27930.1 & 217 & $\mathrm{AP} 2 / \mathrm{ERF}$ & $\begin{array}{l}\text { AP2 domain containing } \\
\text { protein }\end{array}$ & $\begin{array}{l}\text { Regulates adventitious root growth, improves } \\
\text { water-deficit stress tolerance, auxin and other } \\
\text { phytohormone activated signalling pathways. }\end{array}$ & $\begin{array}{l}\text { (Kitomi et } \\
\text { al., } \\
\text { 2011),(Quan } \\
\text { et al., 2010) }\end{array}$ \\
\hline RL1015 & $7^{\#}$ & 6865357 & LOC_Os07g12130.1 & 84 & MYB & $\begin{array}{l}\text { MYB family transcription } \\
\text { factor }\end{array}$ & $\begin{array}{l}\text { Phytohormone signalling, response to water- } \\
\text { deficit and salt stress, regulates root } \\
\text { development and lateral root by modulating } \\
\text { auxin inducible genes. }\end{array}$ & $\begin{array}{l}\text { (Ambawat et } \\
\text { al., 2013) }\end{array}$ \\
\hline \multirow{2}{*}{$\begin{array}{l}\text { RL1520 } \\
\text { and } \\
\text { RL2025 }\end{array}$} & \multirow{2}{*}{$\begin{array}{c}2 \\
\text { and } \\
2^{*}\end{array}$} & \multirow[t]{2}{*}{25265958} & LOC_Os02g41860.1 & 103 & $\mathrm{AQP}$ & Aquaporin protein & $\begin{array}{l}\text { Cellular water homoeostasis and transport, } \\
\text { response to } \mathrm{ABA} \text {, lateral root emergence and } \\
\text { elongation, maintains root hydraulic } \\
\text { conductivity. }\end{array}$ & $\begin{array}{l}\text { (Reinhardt et } \\
\text { al., 2016) }\end{array}$ \\
\hline & & & LOC_Os02g41800.1 & 132 & ARF & Auxin response factor & $\begin{array}{l}\text { Regulates auxin responsive genes, root cap } \\
\text { development and mutant showed reduced } \\
\text { adventitious and lateral roots. }\end{array}$ & $\begin{array}{l}\text { (Guilfoyle } \\
\text { and Hagen, } \\
\text { 2007) }\end{array}$ \\
\hline RL2025 & $1^{\#}$ & 28515026 & LOC_Os01g49710.1 & 52 & GST & Glutathione S-transferase & $\begin{array}{l}\text { Over-expression of GST in soybean showed } \\
\text { longer root length and less growth retardation in } \\
\text { drought and salinity stress. }\end{array}$ & $\begin{array}{l}\text { (Ji et al., } \\
2010)\end{array}$ \\
\hline \multirow{3}{*}{ MRL } & \multirow{3}{*}{$6^{*}$} & \multirow{3}{*}{27819933} & LOC_Os06g 45940.2 & Within & KT & Potassium transporter & Regulate root tip growth in Arabidopsis & $\begin{array}{l}\text { (Rigas et al., } \\
2001)\end{array}$ \\
\hline & & & LOC_Os06g45950.1 & 11 & \multirow[b]{2}{*}{ SAUR } & \multirow{2}{*}{$\begin{array}{l}\text { OsSAUR } 25 \text { and } \\
\text { OsSAUR26 - Small auxin } \\
\text { UP-RNA }\end{array}$} & \multirow{2}{*}{$\begin{array}{l}\text { Early auxin responsive gene, multicellular } \\
\text { organism development, regulation of growth by } \\
\text { root cell elongation, regulates root meristematic } \\
\text { activity. }\end{array}$} & \multirow[b]{2}{*}{$\begin{array}{l}\text { (Markakis et } \\
\text { al., 2013) }\end{array}$} \\
\hline & & & LOC_Os06g 45970.1 & 42 & & & & \\
\hline RV & $1^{\#}$ & 40526762 & LOC_Os01g70050.1 & Within & SAUR & $\begin{array}{l}\text { OsSAUR3 - Auxin- } \\
\text { responsive SAUR }\end{array}$ & $\begin{array}{l}\text { Early auxin responsive gene, multicellular } \\
\text { organism development, regulation of growth by } \\
\text { root cell elongation, regulates root meristematic } \\
\text { activity. }\end{array}$ & $\begin{array}{l}\text { (Markakis et } \\
\text { al., 2013) }\end{array}$ \\
\hline \multirow{4}{*}{ ART } & $11^{*}$ & 28808353 & LOC_Os11g47760.1 & Within & DnaK & $\begin{array}{l}\text { DnaK family protein, } \\
\text { putative }\end{array}$ & $\begin{array}{l}\text { Molecular chaperone protein response to heat } \\
\text { and drought, in interaction with brassinosteroid } \\
\text { signalling, regulates root development, regulates } \\
\text { root gravity response. }\end{array}$ & $\begin{array}{l}\text { (Bekh-Ochir } \\
\text { et al., } \\
\text { 2013),(Sedbr } \\
\text { ook et al., } \\
\text { 1999) }\end{array}$ \\
\hline & \multirow{2}{*}{1} & \multirow{2}{*}{10998576} & LOC_Os01g19380.1 & 33 & PPR & Pentatricopeptide & $\begin{array}{l}\text { Response to salt stress, regulates size of root } \\
\text { apical meristem and mutant ( } \operatorname{sg} l \text { ) shows } \\
\text { reduced root growth. }\end{array}$ & $\begin{array}{l}\text { (Hsieh et al., } \\
\text { 2015) }\end{array}$ \\
\hline & & & LOC_Os01g19750.1 & 209 & GLH & Glycosyl hydrolase & $\begin{array}{l}\text { Cell wall remodelling (loosening, degradation } \\
\text { and reorganization) and mutant }(g l h) \text { showed } \\
\text { reduced root growth. }\end{array}$ & $\begin{array}{l}\text { (Swarup et } \\
\text { al., 2008) }\end{array}$ \\
\hline & $1^{\#}$ & 34378789 & LOC_Os01g59440.1 & $307 b p$ & BRI1 & $\begin{array}{l}\text { BRASSINOSTEROID } \\
\text { INSENSITIVE } 1\end{array}$ & $\begin{array}{l}\text { Overexpression line of this gene shows impaired } \\
\text { root growth compared to wild type but under } \\
\text { heat and cold stress roots are more elongated in } \\
\text { overexpressed line than in wild type. }\end{array}$ & $\begin{array}{l}\text { (Singh et al., } \\
2016)\end{array}$ \\
\hline
\end{tabular}




\section{Supplementary Table S9. (Continued)}

\begin{tabular}{|c|c|c|c|c|c|c|c|c|}
\hline \multirow{5}{*}{ SRL } & 1 & 25773127 & LOC_Os01g45550.1 & 90 & $\mathrm{AEC}$ & $\begin{array}{l}\text { Auxin efflux carrier } \\
\text { component }\end{array}$ & $\begin{array}{l}\text { Polar auxin transport and homoeostasis, auxin } \\
\text { activated signalling and mutant showed defects } \\
\text { in root growth and development. }\end{array}$ & $\begin{array}{l}\text { (Grieneisen } \\
\text { et al., 2007) }\end{array}$ \\
\hline & \multirow{4}{*}{$1^{\#}$} & \multirow{4}{*}{43108024} & LOC_Os01g74450.1 & 9 & $\mathrm{AQP}$ & Aquaporin protein & $\begin{array}{l}\text { Cellular water homoeostasis and transport, } \\
\text { response to } \mathrm{ABA} \text {, lateral root emergence and } \\
\text { elongation, maintains root hydraulic } \\
\text { conductivity. }\end{array}$ & $\begin{array}{l}\text { (Reinhardt et } \\
\text { al., 2016) }\end{array}$ \\
\hline & & & LOC_Os01g74470.1 & 26 & $\mathrm{ABC}$ & $\begin{array}{l}\text { ABC transporter, ATP- } \\
\text { binding protein }\end{array}$ & $\begin{array}{l}\text { Basipetal and acropetal auxin transport and } \\
\text { involved in auxin mediated lateral and root hair } \\
\text { development. Mutant }(m d r l) \text { showed defect in } \\
\text { lateral root growth. }\end{array}$ & $\begin{array}{l}\text { (Santelia et } \\
\text { al., } \\
\text { 2005),(Wu et } \\
\text { al., 2007) }\end{array}$ \\
\hline & & & $\begin{array}{l}\text { LOC_Os01g74410.1 } \\
\end{array}$ & 12 & \multirow[b]{2}{*}{ MYB } & \multirow[b]{2}{*}{$\begin{array}{l}\text { MYB family transcription } \\
\text { factor }\end{array}$} & \multirow{2}{*}{$\begin{array}{l}\text { Phytohormone signalling, response to water- } \\
\text { deficit and salt stress, regulates root } \\
\text { development and lateral root by modulating } \\
\text { auxin inducible genes. }\end{array}$} & \multirow[b]{2}{*}{$\begin{array}{l}\text { (Ambawat et } \\
\text { al., 2013) }\end{array}$} \\
\hline & & & LOC_Os01g74590.1 & 87 & & & & \\
\hline \multicolumn{9}{|c|}{ (C) Root anatomical traits } \\
\hline \multirow{2}{*}{$\mathrm{RD}$} & \multirow{2}{*}{$1^{*}$} & \multirow{2}{*}{11112944} & LOC_Os01g19380.1 & 33 & PPR & Pentatricopeptide & $\begin{array}{l}\text { Response to salt stress, regulates size of root } \\
\text { apical meristem and mutant }(\operatorname{sg} 1) \text { shows } \\
\text { reduced root growth. }\end{array}$ & $\begin{array}{l}\text { (Hsieh et al., } \\
2015 \text { ) }\end{array}$ \\
\hline & & & LOC_Os01g19750.1 & $\begin{array}{c}20 \\
9\end{array}$ & GLH & Glycosyl hydrolase & $\begin{array}{l}\text { Cell wall remodelling (loosening, degradation } \\
\text { and reorganization) and mutant }(\mathrm{glh}) \text { showed } \\
\text { reduced root growth. }\end{array}$ & $\begin{array}{l}\text { (Swarup et } \\
\text { al., 2008) }\end{array}$ \\
\hline $\begin{array}{l}\mathrm{RD}, \mathrm{CD} \\
\text { and } \\
\mathrm{SD}: \mathrm{RD}\end{array}$ & $7^{*}$ & 21266079 & LOC_Os07g 35560.1 & 17 & GLUB3 & $\begin{array}{l}\text { Glucan endo-1,3-beta- } \\
\text { glucosidase precursor }\end{array}$ & $\begin{array}{l}\text { Belongs to cell wall remodelling (loosening, } \\
\text { degradation and reorganisation) enzyme } \\
\text { glycosyl hydrolase family protein and degrade } \\
\text { b-glucan-component of hemicelluloses which } \\
\text { builds cell walls. Some studies showed that the } \\
\text { increase in the synthesis of beta-glucosidase is } \\
\text { associated with a higher plant tolerance to } \\
\text { osmotic stress while another study showed } \\
\text { decreased expression in response to osmotic } \\
\text { stress in roots. }\end{array}$ & $\begin{array}{l}\text { (Budak et al., } \\
\text { 2013),(Grębo } \\
\text { sz et al., } \\
\text { 2014) }\end{array}$ \\
\hline \multirow{4}{*}{ RD } & $3^{\#}$ & 4913579 & LOC_Os03g09930.1 & 37 & SULT & Sulfate transporter & $\begin{array}{l}\text { Sulfate uptake, strongly regulated in roots under } \\
\text { water-deficit and salinity stress, response to } \\
\text { ABA. }\end{array}$ & $\begin{array}{l}\text { (Gallardo et } \\
\text { al., 2014) }\end{array}$ \\
\hline & \multirow[b]{2}{*}{$3^{\#}$} & \multirow[b]{2}{*}{29686521} & LOC_Os03g51710.1 & 54 & $\mathrm{HP}$ & $\begin{array}{l}\text { Homeobox protein } \\
\text { knotted-1 }\end{array}$ & $\begin{array}{l}\text { Multicellular organismal development and cell } \\
\text { differentiation, Arabidopsis orthologue regulate } \\
\text { xylem development }\end{array}$ & NA \\
\hline & & & LOC_Os03g51740.1 & 24 & $\mathrm{ACC}$ & $\begin{array}{l}\text { Aminotransferase similar } \\
\text { ACC Synthase }\end{array}$ & $\begin{array}{l}\text { Ethylene biosynthesis and regulates rice root } \\
\text { growth in deep water, induced by water-deficit } \\
\text { stress in rice. }\end{array}$ & $\begin{array}{l}\text { (Lorbiecke } \\
\text { and Sauter, } \\
\text { 1999) (Wang } \\
\text { et al., 2011) }\end{array}$ \\
\hline & $5^{\#}$ & 28880728 & LOC_Os05g50460.1 & 43 & PPR & Pentatricopeptide & $\begin{array}{l}\text { Response to salt stress, regulates size of root } \\
\text { apical meristem and mutant }(\operatorname{slg} 1) \text { shows } \\
\text { reduced root growth }\end{array}$ & $\begin{array}{l}\text { (Hsieh et al., } \\
2015 \text { ) }\end{array}$ \\
\hline \multirow{6}{*}{ LMXD } & \multirow{2}{*}{$4^{*}$} & \multirow{2}{*}{29450620} & LOC_Os04g49450.1 & 53 & MYB & $\begin{array}{l}\text { MYB family } \\
\text { transcription factor }\end{array}$ & $\begin{array}{l}\text { Phytohormone signalling, response to water- } \\
\text { deficit and salt stress, regulates root } \\
\text { development and lateral root by modulating } \\
\text { auxin inducible genes. }\end{array}$ & $\begin{array}{l}\text { (Ambawat et } \\
\text { al., 2013) }\end{array}$ \\
\hline & & & LOC_Os04g49410.1 & 35 & EXP & Expansin precursor & $\begin{array}{l}\text { Cell wall loosening and maintains root cell } \\
\text { elongation, supports acid growth theory. }\end{array}$ & $\begin{array}{l}\text { (Wu et al., } \\
\text { 1996; } \\
\text { Cosgrove, } \\
\text { 1998) }\end{array}$ \\
\hline & 4 & 29606053 & LOC_Os04g49570.2 & 36 & GLR & Glutamate receptor & $\begin{array}{l}\text { Calcium ion transport and homoeostasis and } \\
\text { transduction of gravitropism signal in root. }\end{array}$ & $\begin{array}{l}\text { (Miller et al., } \\
2010)\end{array}$ \\
\hline & $12^{\#}$ & 22371182 & LOC_Os12g36620.1 & 56 & PPR & Pentatricopeptide & $\begin{array}{l}\text { Response to salt stress, regulates size of root } \\
\text { apical meristem and mutant }(\operatorname{sg} 1) \text { shows } \\
\text { reduced root growth. }\end{array}$ & $\begin{array}{l}\text { (Hsieh et al., } \\
\text { 2015) }\end{array}$ \\
\hline & \multirow{2}{*}{$1^{\#}$} & \multirow{2}{*}{19177575} & LOC_Os01g34850.1 & 64 & HKT & $\begin{array}{l}\text { OsHKT2;3 }-\mathrm{Na}^{+} \\
\text {transporter }\end{array}$ & $\begin{array}{l}\text { HKT-type transporters play key roles in } \\
\mathrm{Na}^{+} \text {accumulation and salt sensitivity in plants. } \\
\text { Arabidopsis } \mathrm{HKT} 1 ; 1 \text { has been proposed to } \\
\text { influx Na+ into roots, recirculates } \mathrm{Na}^{+} \text {in the } \\
\text { phloem and controls root: shoot allocation of } \\
\mathrm{Na}^{+} \text {. }\end{array}$ & $\begin{array}{l}\text { (Davenport et } \\
\text { al., 2007) }\end{array}$ \\
\hline & & & LOC_Os01g34880.1 & 83 & CAS & Callose synthase & $\begin{array}{l}\text { Necessary for normal phloem development and } \\
\text { cell signalling. The roots of cals3- } d \text { gain-of- } \\
\text { function mutants were shown to accumulate } \\
\text { more callose at the plasmodesmata and defects } \\
\text { in root development, callose regulates } \\
\text { symplastic trafficking during root development. }\end{array}$ & $\begin{array}{l}\text { (Vatén et al., } \\
\text { 2011) }\end{array}$ \\
\hline LMXN & 11 & 2838776 & LOC_Os11g06010.1 & 7 & bHLH & $\begin{array}{l}\text { Helix-loop-helix DNA- } \\
\text { binding protein }\end{array}$ & $\begin{array}{l}\text { Arabidopsis orthologue LONESOME } \\
\text { HIGHWAY with sequence similarity to bHLH- } \\
\text { domain proteins. It promotes the production of } \\
\text { stele cells in root meristems and maintain the } \\
\text { normal vascular cell number in coordination } \\
\text { with auxin in primary and lateral roots. }\end{array}$ & $\begin{array}{l}\text { (Ohashi-Ito et } \\
\text { al., 2014), } \\
\text { (Ohashi-Ito et } \\
\text { al., 2013) }\end{array}$ \\
\hline \multicolumn{9}{|c|}{ (E) Dry matter trait } \\
\hline \multirow{2}{*}{ RS } & \multirow{2}{*}{$1^{*}$} & \multirow{2}{*}{39255482} & LOC_Os01g67650.1 & 63 & $\begin{array}{c}\text { DEL } \\
\text { LA }\end{array}$ & $\begin{array}{l}\text { Gibberellin response } \\
\text { modulator protein }\end{array}$ & \multirow{2}{*}{$\begin{array}{l}\text { Cell proliferation and expansion, root growth in } \\
\text { interaction with auxin. }\end{array}$} & \multirow{2}{*}{$\begin{array}{l}\text { (Fu and } \\
\text { Harberd, } \\
2003 \text { ) }\end{array}$} \\
\hline & & & LOC_Os01g67670.1 & 70 & $\begin{array}{c}\text { DEL } \\
\text { LA }\end{array}$ & $\begin{array}{l}\text { Gibberellin response } \\
\text { modulator protein }\end{array}$ & & \\
\hline
\end{tabular}

Trt: treatment, Chr: chromosome, Acr: gene acronym, Ref: reference. 
Supplementary Table S10: The predicted a priori candidate genes (total 57 unique a priori genes excluding loci associated with more than one trait) underlying different loci/locus of root traits in water-deficit stress (WD) conditions and demonstrating to have a role in root growth and development. Candidate a priori gene annotations in bold are responsive to abiotic stress stimulus (Gene Ontology:0009628) according to Rice genome browser database. Trait acronyms are given in the Table $1 . \dagger=$ Distance of gene from peak SNP.

\begin{tabular}{|c|c|c|c|c|c|c|c|c|}
\hline Traits & Chr & SNP & Gene & ce $(\mathrm{kbp}) \dagger$ & Acr & Gene annotation & Description & Ref \\
\hline \multicolumn{9}{|c|}{ (A) Root morphological traits } \\
\hline $\begin{array}{l}\text { TRL, } \\
\text { RL005 and } \\
\text { SA }\end{array}$ & $2^{\#}$ & 8835096 & LOC_Os02g15620.1 & 53 & PIP1 & CAPIP1 & $\begin{array}{l}\text { The Arabidopsis orthologue encodes a } \\
\text { regulatory element (PYL8 or RCAR3) of ABA } \\
\text { receptor. Positive regulation of abscisic acid- } \\
\text { activated signalling pathway. Promotes lateral } \\
\text { root development by enhancing MYB77- } \\
\text { dependent transcription of auxin-responsive } \\
\text { genes. }\end{array}$ & $\begin{array}{l}\text { (Zhao et al., } \\
\text { 2014) }\end{array}$ \\
\hline $\begin{array}{l}\text { TRL and } \\
\text { RL005 }\end{array}$ & $4^{\#}$ & 29111186 & LOC_Os04g 48510.1 & 189 & GRF & $\begin{array}{l}\text { Growth regulating } \\
\text { factor protein }\end{array}$ & $\begin{array}{l}\text { Cell expansion and proliferation, root } \\
\text { development, coordination of growth in water- } \\
\text { deficit stress condition. }\end{array}$ & $\begin{array}{l}\text { (Omidbakhsh } \\
\text { fard et al., } \\
\text { 2015) }\end{array}$ \\
\hline $\begin{array}{l}\text { TRL and } \\
\text { RL005 }\end{array}$ & $11^{\#}$ & 7124411 & LOC_Os11g12620.1 & 20 & CLV1 & $\begin{array}{l}\text { Receptor protein } \\
\text { kinase CLAVATA1 } \\
\text { precursor }\end{array}$ & $\begin{array}{l}\text { Regulates root and shoot meristems, regulation } \\
\text { of root plasticity in response to nitrogen. }\end{array}$ & $\begin{array}{l}\text { (Stahl et al., } \\
\text { 2013),(Araya } \\
\text { et al., 2014), } \\
\text { (Kalve et al., } \\
\text { 2014) }\end{array}$ \\
\hline $\begin{array}{l}\text { RL0510 and } \\
\text { RV }\end{array}$ & $12^{\#}$ & 26195748 & LOC_Os12g42150.1 & 65 & $\mathrm{G} \beta$ & $\begin{array}{l}\text { WD domain, G-beta } \\
\text { repeat domain } \\
\text { containing protein }\end{array}$ & $\begin{array}{l}\text { Subunit of large heterotrimeric } \mathrm{G} \text { protein }(\alpha \beta \gamma) \\
\text { negatively regulates auxin induced signalling. } \\
\text { Loss of function mutants in G-protein have } \\
\text { altered auxin mediated cell division - } \\
\text { formation of lateral and adventitious root } \\
\text { primordia. }\end{array}$ & $\begin{array}{l}\text { (Ullah et al., } \\
\text { 2003) }\end{array}$ \\
\hline $\begin{array}{l}\text { RL0510 and } \\
\text { SA }\end{array}$ & $9^{\#}$ & 14829621 & LOC_Os09g24840.1 & Within & GASA & $\begin{array}{l}\text { GASR10 - } \\
\text { Gibberellin- } \\
\text { regulated } \\
\text { GASA/GAST/Snaki } \\
\text { n family protein }\end{array}$ & $\begin{array}{l}\text { Phytohormone cross-talk and redox } \\
\text { homoeostasis, regulates root, stem and other } \\
\text { organ growth and development. }\end{array}$ & $\begin{array}{l}\text { (Nahirñak et } \\
\text { al., 2012) }\end{array}$ \\
\hline RL0510 & $11^{\#}$ & 25317141 & LOC_Os11g42200.1 & 106 & LAC & $\begin{array}{l}\text { Laccase precursor } \\
\text { protein }\end{array}$ & $\begin{array}{l}\text { Lignin synthesis, role in roots development } \\
\text { during acclimation to salinity stress. }\end{array}$ & $\begin{array}{l}\text { (Liang et al., } \\
2006 \text { ) }\end{array}$ \\
\hline $\begin{array}{l}\text { RL0510, } \\
\text { RL1015 and } \\
\text { RV } \\
\end{array}$ & $1^{*}$ & 23079331 & LOC_Os01g40680.1 & 87 & PPR & Pentatricopeptide & $\begin{array}{l}\text { Response to salt stress, regulates size of root } \\
\text { apical meristem and mutant ( } \operatorname{sg} l \text { ) showed } \\
\text { reduced root growth. }\end{array}$ & $\begin{array}{l}\text { (Hsieh et al., } \\
\text { 2015) }\end{array}$ \\
\hline \multirow{6}{*}{ RL2025 } & \multirow{2}{*}{$2^{*}$} & \multirow{2}{*}{5180480} & LOC_Os02g09910.1 & 49 & \multirow[b]{2}{*}{ - PHD } & \multirow{2}{*}{$\begin{array}{l}\text { PHD-finger domain } \\
\text { containing protein }\end{array}$} & \multirow{2}{*}{$\begin{array}{l}\text { Multicellular organismal development, } \\
\text { anatomical development and response to } \\
\text { abiotic stimulus in rice. }\end{array}$} & \multirow[b]{2}{*}{ NA } \\
\hline & & & LOC_Os02g09920.1 & 35 & & & & \\
\hline & 6 & 6996556 & LOC_Os06g12790.1 & 6 & RAS & Ras-related protein & $\begin{array}{l}\text { Small GTPase mediated signal transduction, } \\
\text { root hair initiation and root tip growth. }\end{array}$ & $\begin{array}{l}\text { (Molendijk et } \\
\text { al., 2001), } \\
\text { (Jones et al., } \\
\text { 2002) }\end{array}$ \\
\hline & \multirow{2}{*}{$3^{\#}$} & \multirow{2}{*}{6374082} & LOC_Os03g12236.1 & 48 & DnaJ & $\begin{array}{l}\text { Heat shock protein } \\
\text { DnaJ }\end{array}$ & $\begin{array}{l}\text { Components of macromolecular chaperone } \\
\text { complexes (DnaJ-HSP70). DnaJ-like protein } \\
\text { (ARG1) involved in gravity signal transduction } \\
\text { in root. Maintains cellular protein homoeostasis } \\
\text { in normal and stress condition. }\end{array}$ & $\begin{array}{l}\text { (Rosen et al., } \\
\text { 1999) }\end{array}$ \\
\hline & & & LOC_Os03g11910.1 & 127 & DnaK & $\begin{array}{l}\text { DnaK family } \\
\text { protein }\end{array}$ & $\begin{array}{l}\text { Molecular chaperone protein response to heat } \\
\text { and drought, in interaction with brassinosteroid } \\
\text { signalling, regulates root development, } \\
\text { regulates root gravity response. }\end{array}$ & $\begin{array}{l}\text { (Bekh-Ochir } \\
\text { et al., } \\
\text { 2013),(Sedbr } \\
\text { ook et al., } \\
\text { 1999) }\end{array}$ \\
\hline & $10^{\#}$ & 11555942 & LOC_Os10g22310.1 & 18 & GST & $\begin{array}{l}\text { Glutathione S- } \\
\text { transferase GST } 26\end{array}$ & $\begin{array}{l}\text { Over-expression of GST in soybean showed } \\
\text { longer root length and less growth retardation } \\
\text { in drought and salinity stress. }\end{array}$ & $\begin{array}{l}\text { (Ji et al., } \\
2010)\end{array}$ \\
\hline \multirow{3}{*}{ RL2530 } & $11^{*}$ & 24267277 & LOC_Os1lg40680.1 & 11 & MBTB & $\begin{array}{l}\text { MBTB64 - Bric-a- } \\
\text { Brac, Tramtrack, } \\
\text { Broad Complex } \\
\text { BTB domain }\end{array}$ & $\begin{array}{l}\text { Response to salt, water-deficit stress and } \\
\text { osmotic stress, protein ubiquitination, interacts } \\
\text { with CULLIN3 to regulate root growth. }\end{array}$ & $\begin{array}{l}\text { (Thomann et } \\
\text { al., 2009) }\end{array}$ \\
\hline & \multirow[t]{2}{*}{$10^{\#}$} & \multirow[t]{2}{*}{18971155} & LOC_Os10g35560.1 & 54 & EP & Expressed protein & $\begin{array}{l}\text { The Arabidopsis orthologue encodes a nuclear } \\
\text { targeted protein (ATSFR6) that plays a role in } \\
\text { the CBF pathway -downstream of CBF } \\
\text { translation. NAC transcription factor promotes } \\
\text { lateral root growth through activation of } \\
\text { DREB-CBF-COR pathway. }\end{array}$ & $\begin{array}{l}\text { (Hao et al., } \\
\text { 2011) }\end{array}$ \\
\hline & & & LOC_Os10g35460.1 & 5 & COBRA & COBRA & $\begin{array}{l}\text { Cellular component organization and response } \\
\text { to abiotic stress stimulus. Arabidopsis } \\
\text { orthologue regulates multidimensional cell } \\
\text { growth and response to salt stress. }\end{array}$ & NA \\
\hline
\end{tabular}


Supplementary Table S10. (Continued)

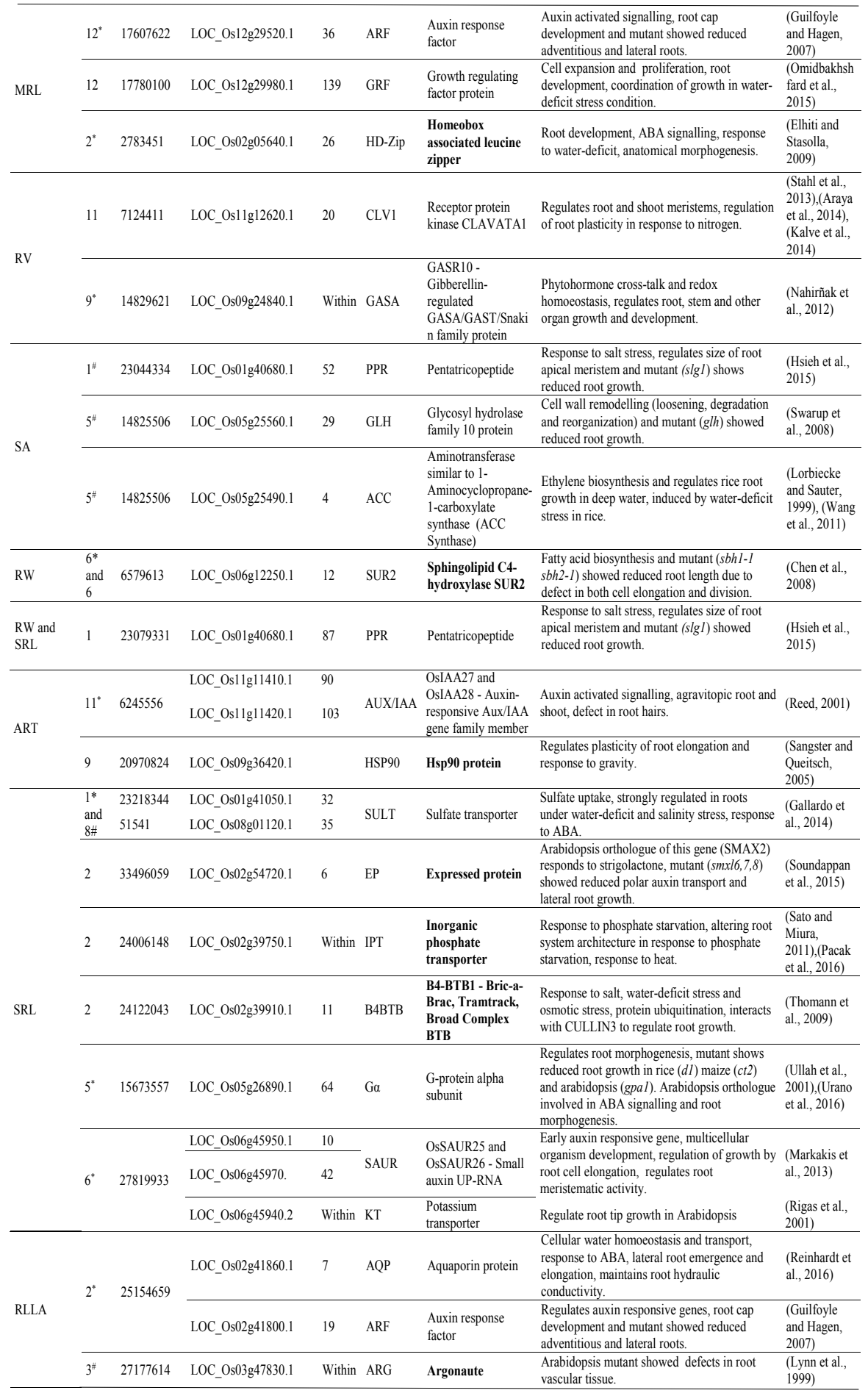


Supplementary Table S10. (Continued)

\begin{tabular}{|c|c|c|c|c|c|c|c|c|}
\hline \multicolumn{9}{|c|}{ (D) Root anatomical traits } \\
\hline \multirow{2}{*}{$\begin{array}{l}\text { SD and } \\
\text { LMXD }\end{array}$} & \multirow{2}{*}{$\begin{array}{l}9^{*} \text { and } \\
9^{\#}\end{array}$} & \multirow[t]{2}{*}{13788883} & LOC_Os09g 23200.1 & 24 & KAN1 & KANADI1 & $\begin{array}{l}\text { Lateral root development, vascular tissue } \\
\text { development in root. }\end{array}$ & $\begin{array}{l}\text { (Hawker and } \\
\text { Bowman, } \\
\text { 2004),(Zhao et } \\
\text { al., 2005) }\end{array}$ \\
\hline & & & LOC_Os09g 23220.1 & 8 & GLH16 & $\begin{array}{l}\text { Glycosyl hydrolases } \\
\text { family } 16\end{array}$ & $\begin{array}{l}\text { Cell wall remodelling (loosening, } \\
\text { degradation and reorganization) and mutant } \\
\text { (glh) showed reduced root growth. }\end{array}$ & $\begin{array}{l}\text { (Swarup et al., } \\
2008 \text { ) }\end{array}$ \\
\hline $\begin{array}{l}\text { SD and } \\
\text { LMXD }\end{array}$ & $5^{\#}$ & 3057869 & LOC_Os05g06110.1 & $843 b p$ & VIL & Villin & $\begin{array}{l}\text { Actin binding protein involved in root hair } \\
\text { growth through actin organisation. }\end{array}$ & $\begin{array}{l}\text { (Zhang et al., } \\
\text { 2011) }\end{array}$ \\
\hline \multirow[b]{2}{*}{$\mathrm{SD}: \mathrm{RD}$} & \multirow[b]{2}{*}{$1^{*}$} & \multirow[b]{2}{*}{1676898} & LOC_Os01g03950.1 & 21 & GLH31 & $\begin{array}{l}\text { Glycosyl hydrolase, } \\
\text { family } 31\end{array}$ & $\begin{array}{l}\text { Cell wall remodelling (loosening, } \\
\text { degradation and reorganization) and mutant } \\
\text { (glh) showed reduced root growth. }\end{array}$ & $\begin{array}{l}\text { (Swarup et al., } \\
2008 \text { ) }\end{array}$ \\
\hline & & & LOC_Os01g04020.2 & 69 & $\mathrm{AP} 2 / \mathrm{ERF}$ & $\begin{array}{l}\text { AP2 domain } \\
\text { containing protein }\end{array}$ & $\begin{array}{l}\text { Regulates adventitious root growth, } \\
\text { improves water-deficit stress tolerance, } \\
\text { auxin and other phytohormone activated } \\
\text { signalling pathways. }\end{array}$ & $\begin{array}{l}\text { (Kitomi et al., } \\
\text { 2011),(Quan et } \\
\text { al., 2010) }\end{array}$ \\
\hline \multirow{4}{*}{ LMXN } & \multirow{4}{*}{$3^{\#}$} & \multirow{4}{*}{34487907} & LOC_Os07g03270.1 & Within & DnaJ & $\begin{array}{l}\text { Heat shock protein } \\
\text { DnaJ }\end{array}$ & $\begin{array}{l}\text { Components of macromolecular chaperone } \\
\text { complexes (DnaJ-HSP70). DnaJ-like } \\
\text { protein (ARG1) involved in gravity signal } \\
\text { transduction in root. Maintains cellular } \\
\text { protein homoeostasis in normal and stress } \\
\text { conditions. }\end{array}$ & $\begin{array}{l}\text { (Rosen et al., } \\
\text { 1999) }\end{array}$ \\
\hline & & & LOC_Os03g60550.1 & 75 & THIF & $\begin{array}{l}\text { ThiF family } \\
\text { domain containing } \\
\text { protein }\end{array}$ & $\begin{array}{l}\text { Associated with production of ubiquitin- } \\
\text { activating enzyme E1. Controls root and } \\
\text { shoot architecture, Arabidopsis orthologue } \\
\text { plays a role in auxin activated signalling. }\end{array}$ & $\begin{array}{l}\text { (Prince et al., } \\
2015 \text { ) }\end{array}$ \\
\hline & & & LOC_Os03g60580.1 & 57 & $\mathrm{ADF}$ & $\begin{array}{l}\text { Actin- } \\
\text { depolymerizing } \\
\text { factor }\end{array}$ & $\begin{array}{l}\text { Capable of rapid and reversible disruption } \\
\text { of actin cytoskeleton. Disturbs root } \\
\text { elongation and root hair formation. }\end{array}$ & $\begin{array}{l}\text { (Baluška et al., } \\
\text { 2000),(Baluška } \\
\text { et al., 2001) }\end{array}$ \\
\hline & & & LOC_Os03g60620.1 & 37 & DnaK & $\begin{array}{l}\text { DnaK family } \\
\text { protein }\end{array}$ & $\begin{array}{l}\text { Molecular chaperone protein responds to } \\
\text { heat and drought, in interaction with } \\
\text { brassinosteroid signalling, regulates root } \\
\text { development, regulates root gravity } \\
\text { response. }\end{array}$ & $\begin{array}{l}\text { (Bekh-Ochir et } \\
\text { al., 2013), } \\
\text { (Sedbrook et } \\
\text { al., 1999) }\end{array}$ \\
\hline \multirow{4}{*}{ LMXD } & $6^{\#}$ & 471179 & LOC_Os06g01890.1 & 23 & MADS & $\begin{array}{l}\text { MADS-box } \\
\text { transcription factor }\end{array}$ & $\begin{array}{l}\text { Plays diverse roles in plant development } \\
\text { and one of the Arabidopsis MADS (ANR1) } \\
\text { is a key regulator of root developmental } \\
\text { plasticity in response to nitrate. }\end{array}$ & $\begin{array}{l}\text { (Zhang and } \\
\text { Forde, 1998) }\end{array}$ \\
\hline & $5^{\#}$ & 3057869 & LOC_Os05g06110.1 & $843 b p$ & VIL & Villin & $\begin{array}{l}\text { Actin binding protein involved in root hair } \\
\text { growth through actin organisation. }\end{array}$ & $\begin{array}{l}\text { (Zhang and } \\
\text { Forde, 1998) }\end{array}$ \\
\hline & $6^{\#}$ & 471179 & LOC_Os06g01920.1 & 41 & EXP & Expansin precursor & $\begin{array}{l}\text { Cell wall loosening and maintain root cell } \\
\text { elongation, supports acid growth theory. }\end{array}$ & $\begin{array}{l}\text { (Wu et al., } \\
\text { 1996),(Cosgrov } \\
\text { e, 1998) }\end{array}$ \\
\hline & $11^{\#}$ & 28871551 & $\begin{array}{l}\text { LOC_Os11g47870.1 } \\
\text { LOC_Os11g47900.1 }\end{array}$ & $\begin{array}{l}\text { Within } \\
19\end{array}$ & SCR & SCARECROW & $\begin{array}{l}\text { Auxin responsive transcription factor (TF) } \\
\text { from GRAS family contributes to the } \\
\text { specification and determination of root } \\
\text { quiescent centre (QC). Together with } \\
\text { SHORTROOT (another GRAS TF) controls } \\
\text { the division of endoderm/cortex cells. } \\
\text { Regulates radial patterning mechanism of } \\
\text { root and shoot. }\end{array}$ & $\begin{array}{l}\text { (Gao et al., } \\
\text { 2004; Mai et } \\
\text { al., } \\
\text { 2014),(Wysock } \\
\text { a-Diller et al., } \\
\text { 2000) }\end{array}$ \\
\hline \multicolumn{9}{|c|}{ (E) Dry matter traits } \\
\hline \multirow[t]{5}{*}{ RS } & \multirow[t]{2}{*}{4} & \multirow[t]{2}{*}{29184866} & LOC_Os04g48850.1 & 53 & $\mathrm{ACC}$ & $\begin{array}{l}\text { Aminotransferase } \\
\text { similar to 1- } \\
\text { Aminocyclopropane- } \\
\text { 1-carboxylate } \\
\text { synthase (ACC } \\
\text { Synthase) } \\
\end{array}$ & $\begin{array}{l}\text { Ethylene biosynthesis and regulates rice } \\
\text { root growth in deep water, induced by } \\
\text { water-deficit stress in rice. }\end{array}$ & $\begin{array}{l}\text { (Lorbiecke and } \\
\text { Sauter, 1999), } \\
\text { (Wang et al., } \\
\text { 2011) }\end{array}$ \\
\hline & & & LOC_Os04g49130.1 & 104 & SUMO & $\begin{array}{l}\text { Ubiquitin-conjugating } \\
\text { enzyme }\end{array}$ & $\begin{array}{l}\text { The Arabidopsis orthologue encodes } \\
\text { SUMO E3 ligase and one of the SUMO E } \\
\text { ligase AtMMS21 controls root cell } \\
\text { proliferation via cell cycle regulation and } \\
\text { Cytokinin signalling. }\end{array}$ & $\begin{array}{l}\text { (Huang et al., } \\
\text { 2009) }\end{array}$ \\
\hline & \multirow[t]{2}{*}{4} & \multirow[t]{2}{*}{29450620} & LOC_Os04g49450.1 & 50 & MYB & $\begin{array}{l}\text { MYB family } \\
\text { transcription factor }\end{array}$ & $\begin{array}{l}\text { Phytohormone signalling, response to } \\
\text { water-deficit and salt stress, regulates root } \\
\text { development and lateral root by } \\
\text { modulating auxin inducible genes. }\end{array}$ & $\begin{array}{l}\text { (Ambawat et } \\
\text { al., 2013) }\end{array}$ \\
\hline & & & LOC_Os04g49410.1 & 34 & EXP & Expansin precursor & $\begin{array}{l}\text { Cell wall loosening and maintains root cell } \\
\text { elongation, supports an acid growth theory. }\end{array}$ & $\begin{array}{l}\text { (Wu et al., } \\
1996),(\text { Cosgrov } \\
\text { e, 1998) }\end{array}$ \\
\hline & $1^{\#}$ & 1562911 & LOC_Os01g03720.1 & 12 & MYB & $\begin{array}{l}\text { MYB family } \\
\text { transcription factor }\end{array}$ & $\begin{array}{l}\text { Phytohormone signalling, response to } \\
\text { water-deficit and salt stress, regulates root } \\
\text { development and lateral root by modulating } \\
\text { auxin inducible genes. }\end{array}$ & $\begin{array}{l}\text { (Ambawat et } \\
\text { al., 2013) }\end{array}$ \\
\hline $\begin{array}{l}\text { RS and } \\
\text { RW }\end{array}$ & $4^{*}$ & 29111186 & LOC_Os04g48510.1 & 189 & GRF & $\begin{array}{l}\text { Growth regulating } \\
\text { factor protein }\end{array}$ & $\begin{array}{l}\text { Cell expansion and proliferation, root } \\
\text { development, coordination of growth in } \\
\text { water-deficit stress condition. }\end{array}$ & $\begin{array}{l}\text { (Omidbakhshfa } \\
\text { rd et al., 2015) }\end{array}$ \\
\hline RW & 3 & 34943958 & LOC_Os03g61670.1 & 8 & CRT & $\begin{array}{l}\text { Calreticulin } \\
\text { precursor }\end{array}$ & $\begin{array}{l}\text { Enhances root regeneration and response to } \\
\text { oxidative and salt stress. }\end{array}$ & $\begin{array}{l}\text { (Jin et al., } \\
2005 \text { ) }\end{array}$ \\
\hline
\end{tabular}

Chr: chromosome, Acr: gene acronym, Ref: reference. 
Supplementary Table S11: The a priori candidate genes (41 a priori genes) underlying different loci/locus for plasticity of root traits as the relative value of the water-deficit stress treatment over the control treatment and demonstrating to have a role in root growth and development. Candidate a priori gene annotations in bold are responsive to abiotic stress stimulus (Gene Ontology:0009628) according to the rice genome browser database. Trait acronyms are given in the Table $1 . \dagger=$ Distance of gene from peak SNP.

\begin{tabular}{|c|c|c|c|c|c|c|c|c|}
\hline Traits & $\mathrm{Chr}$ & SNP & Gene & $\begin{array}{l}\text { Distance } \\
\text { (kbp) } \dagger\end{array}$ & Acr & Gene annotation & General description & Ref \\
\hline \multicolumn{9}{|c|}{ (A) Root morphological } \\
\hline $\begin{array}{l}\text { rTRL and } \\
\text { rSA }\end{array}$ & $7^{\#}$ & 9463744 & LOC_Os07g16224.1 & Within & PIWI & $\begin{array}{l}\text { Piwi domain contaning } \\
\text { protein }\end{array}$ & $\begin{array}{l}\text { Conserved domain protein of } \\
\text { ARGOUNATE protein regulates leaf and } \\
\text { multicellular organisms development. }\end{array}$ & $\begin{array}{l}\text { (Bohmert et al., } \\
\text { 1998) }\end{array}$ \\
\hline $\begin{array}{l}\text { rTRL, } \\
\text { rRV, rSA } \\
\text { and }\end{array}$ & $12^{*}$ & 25006932 & LOC_Os12g40190.1 & 134 & $\mathrm{G} \alpha$ & G-protein alpha subunit & $\begin{array}{l}\text { Regulates root morphogenesis, mutant } \\
\text { shows reduced root growth in rice }(d l) \\
\text { maize }(\text { ct } 2 \text { ) and Arabidopsis ( }(\text { pal }) \text {. } \\
\text { Arabidopsis orthologue involved in } \mathrm{ABA} \\
\text { signalling and root morphogenesis. }\end{array}$ & $\begin{array}{l}\text { (Ullah et al., } \\
\text { 2001),(Urano et } \\
\text { al., 2016) }\end{array}$ \\
\hline \multirow{7}{*}{ rTRL } & \multirow{3}{*}{$1^{\#}$} & \multirow{3}{*}{26826635} & LOC_Os01g46860.1 & 101 & IPT & $\begin{array}{l}\text { Inorganic phosphate } \\
\text { transporter }\end{array}$ & $\begin{array}{l}\text { Response to phosphate starvation, altering } \\
\text { root system architecture in response to } \\
\text { phosphate starvation, response to heat. }\end{array}$ & $\begin{array}{l}\text { (Sato and Miura, } \\
\text { 2011),(Pacak et al., } \\
\text { 2016) }\end{array}$ \\
\hline & & & LOC_Os01g46870.1 & 93 & $\begin{array}{l}\mathrm{AP} 2 / \mathrm{E} \\
\mathrm{RF}\end{array}$ & $\begin{array}{l}\text { AP2 domain containing } \\
\text { protein }\end{array}$ & $\begin{array}{l}\text { Regulates adventitious root growth, } \\
\text { improves water-deficit stress tolerance, } \\
\text { auxin and other phytohormone activated } \\
\text { signalling pathways. }\end{array}$ & $\begin{array}{l}\text { (Kitomi et al., } \\
\text { 2011),(Quan et al., } \\
\text { 2010) }\end{array}$ \\
\hline & & & LOC_Os01g46940.1 & 36 & SUR2 & $\begin{array}{l}\text { Sphingolipid C4- } \\
\text { hydroxylase SUR2 }\end{array}$ & $\begin{array}{l}\text { Fatty acid biosynthesis and mutant (sbhl-1 } \\
s b h 2-1 \text { ) showed reduced root length due to } \\
\text { defect in both cell elongation and division. }\end{array}$ & (Chen et al., 2008) \\
\hline & \multirow{3}{*}{$4^{\#}$} & \multirow{3}{*}{30764890} & LOC_Os04g51890.1 & 19 & SAUR & $\begin{array}{l}\text { OsSAUR20 - Auxin- } \\
\text { responsive SAUR }\end{array}$ & $\begin{array}{l}\text { Early auxin responsive gene, multicellular } \\
\text { organism development, regulation of } \\
\text { growth by root cell elongation, regulates } \\
\text { root meristematic activity. }\end{array}$ & $\begin{array}{l}\text { (Markakis et al., } \\
\text { 2013) }\end{array}$ \\
\hline & & & LOC_Os04g51800.1 & 54 & MYB & MYB protein, putative & $\begin{array}{l}\text { Phytohormone signalling, response to } \\
\text { water-deficit and salt stress, regulates root } \\
\text { development and lateral root by } \\
\text { modulating auxin inducible genes. }\end{array}$ & $\begin{array}{l}\text { (Ambawat et al., } \\
\text { 2013) }\end{array}$ \\
\hline & & & LOC_Os04g51820.2 & 41 & HKT & $\begin{array}{l}\text { OsHKT1;1 }-\mathrm{Na}^{+} \\
\text {transporter }\end{array}$ & $\begin{array}{l}\text { HKT-type transporters play key roles in } \\
\mathrm{Na}^{+} \text {accumulation and salt sensitivity in } \\
\text { plants. Arabidopsis } \mathrm{HKT} 1 ; 1 \text { has been } \\
\text { proposed to influx } \mathrm{Na}+\text { into roots, } \\
\text { recirculate } \mathrm{Na}^{+} \text {in the phloem and control } \\
\text { root: shoot allocation of } \mathrm{Na}^{+} \text {. }\end{array}$ & $\begin{array}{l}\text { (Davenport et al., } \\
\text { 2007) }\end{array}$ \\
\hline & $2^{\#}$ & 4943157 & LOC_Os02g09480.1 & 75 & MYB & $\begin{array}{l}\text { MYB-like DNA-binding } \\
\text { domain containing } \\
\text { protein }\end{array}$ & $\begin{array}{l}\text { Phytohormone signalling, response to } \\
\text { water-deficit and salt stress, regulates root } \\
\text { development and lateral root by modulating } \\
\text { auxin inducible genes. }\end{array}$ & $\begin{array}{l}\text { (Ambawat et al., } \\
\text { 2013) }\end{array}$ \\
\hline rRL2025 & $4^{\#}$ & 16463674 & LOC_Os04g27980.1 & 56 & GLH & Glycosyl hydrolase & $\begin{array}{l}\text { Cell wall remodelling (loosening, } \\
\text { degradation and reorganization) and mutant } \\
\text { (glh) showed reduced root growth. }\end{array}$ & $\begin{array}{l}\text { (Swarup et al., } \\
2008 \text { ) }\end{array}$ \\
\hline \multirow{3}{*}{ rRL2530 } & 3 & 2553785 & LOC_Os03g05290.1 & 10 & $\mathrm{AQP}$ & Aquaporin protein & $\begin{array}{l}\text { Cellular water homoeostasis and transport, } \\
\text { response to ABA, lateral root emergence } \\
\text { and elongation, maintains root hydraulic } \\
\text { conductivity. }\end{array}$ & $\begin{array}{l}\text { (Reinhardt et al., } \\
\text { 2016) }\end{array}$ \\
\hline & $\begin{array}{l}3 \text { and } \\
3^{\prime \prime}\end{array}$ & $\begin{array}{l}2553785 \\
34392848 \\
\end{array}$ & $\begin{array}{l}\text { LOC_Os03g05280.1 } \\
\text { LOC_Os03g60530.1 }\end{array}$ & $\begin{array}{l}10 \\
14 \\
\end{array}$ & RAS & Ras-related protein & $\begin{array}{l}\text { Small GTPase mediated signal } \\
\text { transduction, root hair initiation and root tip } \\
\text { growth. }\end{array}$ & $\begin{array}{l}\text { (Molendijk et al., } \\
\text { 2001), (Jones et } \\
\text { al., 2002) }\end{array}$ \\
\hline & $3^{\circ}$ & 34392848 & LOC_Os03g60550.1 & 20 & THIF & $\begin{array}{l}\text { ThiF family domain } \\
\text { containing protein }\end{array}$ & $\begin{array}{l}\text { Associated with the production of } \\
\text { ubiquitin-activating enzyme E1 and } \\
\text { controls root and shoot architecture, } \\
\text { Arabidopsis orthologue plays role in auxin } \\
\text { activated signalling. }\end{array}$ & $\begin{array}{l}\text { (Prince et al., } \\
2015 \text { ) }\end{array}$ \\
\hline $\mathrm{rRV}$ & $1^{\#}$ & 42575227 & LOC_Os01g73310.1 & 91 & ACT & Actin & $\begin{array}{l}\text { Actin function is essential for cytoplasmic } \\
\text { streaming, organelle orientation, cell } \\
\text { elongation and root tip growth. }\end{array}$ & $\begin{array}{l}\text { (Gilliland et al., } \\
\text { 2003) }\end{array}$ \\
\hline \multirow{3}{*}{ rMRL } & 12 & 17607622 & LOC_Os12g29520.1 & 36 & $\mathrm{ARF}$ & Auxin response factor & $\begin{array}{l}\text { Auxin activated signalling, root cap } \\
\text { development and mutant showed reduced } \\
\text { adventitious and lateral roots. }\end{array}$ & $\begin{array}{l}\text { (Guilfoyle and } \\
\text { Hagen, 2007) }\end{array}$ \\
\hline & \multirow[b]{2}{*}{$2^{*}$} & \multirow[b]{2}{*}{26794003} & LOC_Os02g44108.1 & 185 & EXP & Expansin precursor & $\begin{array}{l}\text { Cell wall loosening and maintaining root } \\
\text { cell elongation, supports acid growth } \\
\text { theory. }\end{array}$ & $\begin{array}{l}\text { (Wu et al., } \\
1996),(\text { Cosgrove, } \\
1998)\end{array}$ \\
\hline & & & LOC_Os02g44080.1 & 143 & $\mathrm{AQP}$ & Aquaporin protein & $\begin{array}{l}\text { Cellular water homoeostasis and transport, } \\
\text { response to ABA, lateral root emergence } \\
\text { and elongation, maintains root hydraulic } \\
\text { conductivity. }\end{array}$ & $\begin{array}{l}\text { (Chaumont et al., } \\
\text { 2001) }\end{array}$ \\
\hline
\end{tabular}


Supplementary Table S11. (Continued)

\begin{tabular}{|c|c|c|c|c|c|c|c|c|}
\hline \multirow{3}{*}{ rART } & \multirow[b]{2}{*}{1} & \multirow[b]{2}{*}{29981149} & LOC_Os01g52130.1 & 13 & SULT & Sulfate transporter & $\begin{array}{l}\text { Sulfate uptake, strongly regulated in roots } \\
\text { under water-deficit stress, response to } \\
\text { ABA. }\end{array}$ & $\begin{array}{l}\text { (Gallardo et al., } \\
\text { 2014) }\end{array}$ \\
\hline & & & LOC_Os01g52070.1 & 37 & AKT1 & $\begin{array}{l}\text { Potassium channel } \\
\text { AKT1 }\end{array}$ & $\begin{array}{l}\text { Root potassium uptake and potassium } \\
\text { homoestasis and facilitates growth in } \\
\text { potassium limitation condition. Growth } \\
\text { retardation in Arabidopsis akt1-1 and atkcl- } \\
\text { f mutants under K starvation. }\end{array}$ & $\begin{array}{l}\text { (Geiger et al., } \\
\text { 2009) }\end{array}$ \\
\hline & $1^{*}$ & 34378789 & LOC_Os01g59440.1 & $307 b p$ & BRIl & $\begin{array}{l}\text { BRASSINOSTEROID } \\
\text { INSENSITIVE } 1- \\
\text { associated receptor } \\
\text { kinase } 1 \text { precursor }\end{array}$ & $\begin{array}{l}\text { Overexpression line of this gene shows } \\
\text { impaired root growth compared to wild type } \\
\text { but under heat and cold stress roots are } \\
\text { more elongated in overexpressed line than } \\
\text { in wild type. }\end{array}$ & (Singh et al., 2016) \\
\hline \multirow[b]{2}{*}{$\begin{array}{l}\text { rSRL and } \\
\text { rART }\end{array}$} & \multirow[b]{2}{*}{$5^{\#}$} & \multirow[b]{2}{*}{2184238} & LOC_Os05g04610.1 & 15 & \multirow[b]{2}{*}{$\mathrm{ABC}$} & \multirow[b]{2}{*}{$\begin{array}{l}\text { ABC transporter, ATP- } \\
\text { binding protein }\end{array}$} & \multirow{2}{*}{$\begin{array}{l}\text { Basipetal and acropetal auxin transport and } \\
\text { involved in auxin mediated lateral and root } \\
\text { hair development. Mutant }(m d r l) \text { showed } \\
\text { defect in lateral root growth. }\end{array}$} & \multirow[b]{2}{*}{$\begin{array}{l}\text { (Santelia et al., } \\
\text { 2005),(Wu et al., } \\
\text { 2007) }\end{array}$} \\
\hline & & & LOC_Os05g04600.1 & 19 & & & & \\
\hline \multirow{2}{*}{$\begin{array}{l}\text { rSRL } \\
\text { rTRWD }\end{array}$} & $8^{*}$ & 26362631 & LOC_Os08g41720.1 & 30 & \multirow{2}{*}{$\mathrm{AEC}$} & \multirow{2}{*}{$\begin{array}{l}\text { Auxin efflux carrier } \\
\text { component }\end{array}$} & \multirow{2}{*}{$\begin{array}{l}\text { Polar auxin transport and homoeostasis, } \\
\text { auxin activated } \\
\text { signalling and mutant shows defects in root } \\
\text { development. }\end{array}$} & \multirow{2}{*}{$\begin{array}{l}\text { (Grieneisen et al., } \\
\text { 2007) }\end{array}$} \\
\hline & $1^{*}$ & 25703110 & LOC_Os01g45550.1 & 160 & & & & \\
\hline \multirow[t]{2}{*}{ rSRL } & $3^{\#}$ & 34024418 & LOC_Os03g59730.1 & 21 & NAM & No apical meristem & $\begin{array}{l}\text { NAC domain containing protein regulates } \\
\text { multicellular }\end{array}$ & (He et al., 2005) \\
\hline & $1^{\#}$ & 20574500 & LOC_Os01g36830.1 & 86 & PG & Polygalacturonase & $\begin{array}{l}\text { Involved in cell elongation and expansion } \\
\text { and expressed during root and other organ } \\
\text { development. }\end{array}$ & (Xiao et al., 2014) \\
\hline \multicolumn{9}{|c|}{ (D) Root anatomical } \\
\hline \multirow{2}{*}{$\begin{array}{l}\mathrm{rRD}, \mathrm{rCD} \\
\text { and rSD }\end{array}$} & \multirow{2}{*}{$1^{*}$} & \multirow{2}{*}{11038867} & LOC_Os01g19380.1 & 74 & PPR & Pentatricopeptide & $\begin{array}{l}\text { Response to salt stress, regulates size of } \\
\text { root apical meristem and mutant }(\operatorname{slg} 1) \\
\text { shows reduced root growth. }\end{array}$ & (Hsieh et al., 2015) \\
\hline & & & LOC_Os01g19750.1 & 169 & GLH & Glycosyl hydrolase & $\begin{array}{l}\text { Cell wall remodelling (loosening, } \\
\text { degradation and reorganization) and mutant } \\
\text { (glh) showed reduced root growth. }\end{array}$ & $\begin{array}{l}\text { (Swarup et al., } \\
2008 \text { ) }\end{array}$ \\
\hline \multirow[b]{2}{*}{ rRD } & \multirow{2}{*}{$11^{\#}$} & \multirow{2}{*}{27205864} & LOC_Os1 lg44930.1 & 5 & PPR & Pentatricopeptide & $\begin{array}{l}\text { Response to salt stress, regulates size of } \\
\text { root apical meristem and mutant }(\operatorname{slg} 1) \\
\text { shows reduced root growth. }\end{array}$ & (Hsieh et al., 2015) \\
\hline & & & LOC_Os1 1g44950.2 & 2 & GLH & Glycosyl hydrolase 3 & $\begin{array}{l}\text { Cell wall remodelling (loosening, } \\
\text { degradation and reorganization) and mutant } \\
\text { ( } g l h) \text { showed reduced root growth. }\end{array}$ & $\begin{array}{l}\text { (Swarup et al., } \\
2008 \text { ) }\end{array}$ \\
\hline \multirow[t]{2}{*}{$\begin{array}{l}\text { rSD and } \\
\text { rLMXD }\end{array}$} & \multirow[t]{2}{*}{$\begin{array}{l}7 \text { and } \\
7^{*}\end{array}$} & \multirow[t]{2}{*}{27141434} & LOC_Os07g45290.1 & 122 & $\begin{array}{l}\text { CYP4 } \\
50\end{array}$ & Cytochrome P450 72A1 & $\begin{array}{l}\text { The Arabidopsis orthologue catalyses } \\
\text { essential oxidative step in the biosynthesis } \\
\text { of Brassinosteroids (BR). BR in interaction } \\
\text { with auxin promote lateral root } \\
\text { development. }\end{array}$ & (Bao et al., 2004) \\
\hline & & & LOC_Os07g45400.1 & 71 & EP & Expressed protein & $\begin{array}{l}\text { Arabidopsis orthologue regulate radial } \\
\text { pattern formation. }\end{array}$ & NA \\
\hline $\begin{array}{l}\text { rRD and } \\
\mathrm{rSD}\end{array}$ & $11^{\#}$ & 25434106 & LOC_Os1lg42220.1 & Within & LAC & Laccase precursor protein & $\begin{array}{l}\text { Lignin synthesis, role in roots development } \\
\text { during acclimation to salinity stress. }\end{array}$ & (Liang et al., 2006) \\
\hline $\mathrm{rCD}$ & $4^{\#}$ & 33647561 & LOC_Os04g56460.1 & 10 & MBTB & $\begin{array}{l}\text { MBTB9 - Bric-a-Brac, } \\
\text { Tramtrack, Broad } \\
\text { Complex BTB } \\
\end{array}$ & $\begin{array}{l}\text { Response to salt, water-deficit stress and } \\
\text { osmotic stress, protein ubiquitination, } \\
\text { interacts with CULLIN3 to regulate root } \\
\text { growth. }\end{array}$ & $\begin{array}{l}\text { (Thomann et al., } \\
\text { 2009) }\end{array}$ \\
\hline \multicolumn{9}{|c|}{ (E) Dry matter traits } \\
\hline \multirow[b]{2}{*}{ rRW } & \multirow[b]{2}{*}{$4^{\#}$} & \multirow[b]{2}{*}{29184866} & LOC_Os04g49130.1 & 104 & SUMO & $\begin{array}{l}\text { Ubiquitin-conjugating } \\
\text { enzyme }\end{array}$ & $\begin{array}{l}\text { The Arabidopsis orthologue encodes SUMO } \\
\text { E3 ligase and one of the SUMO E ligase } \\
\text { AtMMS } 21 \text { controls root cell proliferation via } \\
\text { cell cycle regulation and cytokinin signalling. }\end{array}$ & $\begin{array}{l}\text { (Huang et al., } \\
2009 \text { ) }\end{array}$ \\
\hline & & & LOC_Os04g 48850.1 & 53 & ACS & $\begin{array}{l}\text { Aminotransferase } \\
\text { similar to 1- } \\
\text { Aminocyclopropane-1- } \\
\text { carboxylate synthase } \\
\text { (ACC Synthase) } \\
\end{array}$ & $\begin{array}{l}\text { Ethylene biosynthesis and regulates rice root } \\
\text { growth in deep water, induced by water- } \\
\text { deficit stress in rice. }\end{array}$ & $\begin{array}{l}\text { (Lorbiecke and } \\
\text { Sauter, 1999), } \\
\text { (Wang et al., 2011) }\end{array}$ \\
\hline \multirow{4}{*}{$\mathrm{rRS}$} & \multirow{2}{*}{$2^{*}$} & 651557 & LOC_Os02g02190.1 & 15 & TR & $\begin{array}{l}\text { Transporter, major } \\
\text { facilitator family }\end{array}$ & $\begin{array}{l}\text { The Arabidopsis orthologue encodes high } \\
\text { affinity nitrate transporter, plays a role in } \\
\text { nitrate assimilation and regulates lateral root } \\
\text { development. }\end{array}$ & (Little et al., 2005) \\
\hline & & & LOC_Os02g02140.1 & 14 & CLV1 & $\begin{array}{l}\text { Receptor protein kinase } \\
\text { CLAVATAl }\end{array}$ & $\begin{array}{l}\text { Regulates root and shoot meristems, regulates } \\
\text { root plasticity in response to nitrogen. }\end{array}$ & $\begin{array}{l}\text { (Stahl et al., 2013), } \\
\text { (Araya et al., } \\
\text { 2014), (Kalve et } \\
\text { al., 2014) } \\
\end{array}$ \\
\hline & $3^{*}$ & 26825291 & LOC_Os03g47530.1 & 50 & GLT & $\begin{array}{l}\text { Glycosyl transferase } 8 \\
\text { domain protein }\end{array}$ & $\begin{array}{l}\text { Cell wall thickening, xylem pattern formation } \\
\text { and differentiation, mutant showed swollen } \\
\text { root and reduced growth. }\end{array}$ & $\begin{array}{l}\text { (Schuetz et al., } \\
\text { 2012),(Scheible } \\
\text { and Pauly, 2004) }\end{array}$ \\
\hline & $8^{\#}$ & 17221046 & LOC_Os08g28190.1 & 16 & ACT & Actin & $\begin{array}{l}\text { Actin function is essential for cytoplasmic } \\
\text { streaming, organelle orientation, cell } \\
\text { elongation and root tip growth. }\end{array}$ & $\begin{array}{l}\text { (Gilliland et al., } \\
\text { 2003) }\end{array}$ \\
\hline
\end{tabular}

Chr: chromosome, Acr: gene acronym, Ref: reference. 


\section{Chapter 3}

Supplementary Data Sets 1 to 4 are available online in Supplementary data of Plant Physiology: http://www.plantphysiol.org/content/174/4/2302 


\title{
Genome wide association reveals genetic basis of rice grain yield and its component traits under water-deficit stress during the reproductive stage
}

\begin{abstract}
Niteen N. Kadam ${ }^{1,2}$, Paul C. Struik ${ }^{2}$, Xinyou Yin ${ }^{2}$, Krishna S.V. Jagadish ${ }^{1,3}$
${ }^{1}$ International Rice Research Institute (IRRI), DAPO, Box 7777, Metro Manila, Philippines ${ }^{2}$ Centre for Crop Systems Analysis, Department of Plant Sciences, Wageningen University \& Research, PO Box 430, 6700 AK Wageningen, The Netherlands ${ }^{3}$ Department of Agronomy, Kansas State University, Manhattan, USA
\end{abstract}




\begin{abstract}
A diversity panel comprising of 296 indica rice genotypes was phenotyped under non-stress and reproductive stage water-deficit stress conditions during 2013 and 2014 dry seasons at IRRI, Philippines. We investigated the genotypic variability for grain yield as well as yield components and related traits, and conducted the single-locus and multi-locus genome-wide association studies (GWAS) using highdensity $45 \mathrm{~K}$ single nucleotide polymorphisms. One hundred two loci were detected in non-stress conditions (38 loci in 2013 and 64 loci in 2014) and 124 loci (69 loci in 2013 and 55 in 2014) in waterdeficit stress. Desynchronised flowering time strongly confounded the grain yield and its components in 2013 water-deficit. However, statistically corrected grain yield and yield component values using days to flowering allowed to minimise the confounding effect, and helped to detect 31 additional genetic loci for grain yield, its components and harvest index in 2013. These genetic analyses also provided important insights into genetic architecture of grain yield and its potential link with seed set and assimilate partitioning. Interestingly key a priori candidate genes were identified within the linkage disequilibrium block of grain yield loci regulating physiological, reproductive and abiotic stress tolerant biological processes.
\end{abstract}

Keywords: Oryza sativa, synchronized phenology, linkage disequilibrium, a priori candidate genes, multi-locus GWAS analysis, reproductive stage drought stress. 


\section{Introduction}

Rice (Oryza sativa L.) is a staple food crop for more than half of the world population, and its high yield potential with good yield stability is imperative for future food security. However, global climate change, with frequent episodes of abiotic stresses (water-deficit and heat stress), reduces the productivity of rice (Kadam et al., 2014; Reynolds et al., 2016), as rice is more sensitive to water-deficit than other cereals (Kadam et al., 2015). Nearly 20\% rice production is affected by water-deficit around the world (Bouman et al., 2005). Water-deficit can occur at any time of the growing season, but stress occurring during reproductive phase (i.e. from meiosis to flowering) causes the greatest grain yield losses (Liu et al., 2006). The physiological effects of water-deficit during the reproductive phase have been discussed (Saini and Lalonde, 1997; Saini et al., 1999; Barnabás et al., 2008).

Increasing tolerance to water-deficit has been considered as a breeding target, although knowledge on phenotypic traits linked with stress tolerance is limited. Increasing grain yield has been considered as the primary goal in breeding programmes. Recent evidence in rice has demonstrated that progress can be made through direct selection of grain yield under reproductive stage water-deficit (Venuprasad et al., 2007; Kumar et al., 2014). Physiologically, grain yield is a very complex trait determined by different component traits (Slafer, 2003). Hence, exploring ideotype breeding based on selection for component traits is proposed as a complementary route for further yield improvement (Donald, 1968).

Revealing the genetic basis of grain yield and its component traits is essential for providing the breeders the tools for efficient development of stress resilient crop cultivars. The genetic control of grain yield under reproductive stage water-deficit has been investigated extensively using linkage analysis of biparental crosses in rice. This approach is proven to be powerful in detecting quantitative trait loci (QTLs) for grain yield and its components under stress (Lanceras et al., 2004; Bernier et al., 2007; Vikram et al., 2011; Dixit et al., 2014; Kumar et al., 2014). Few of these QTLs regulating grain yield, for instance $q D T Y_{1.1}$, were introgressed into elite cultivars to improve stress tolerance (Vikram et al., 2011). However, most of these QTLs identified with the above approach are only based on a small fraction of the genetic variation present in the rice germplasm. Identifying the allelic variation assembled in a genetic pool that is large due to divergent selection pressure, has great potential in grain yield improvement under water-deficit stress. This approach using natural allelic variation is studied in rice under non-stress conditions for grain yield and its component traits through genomewide association studies (GWAS; Agrama et al., 2007; Borba et al., 2010; Huang et al., 2010; 
Zhao et al., 2011; Huang et al., 2012; Begum et al., 2015; Spindel et al., 2015; Rebolledo et al., 2016; Yano et al., 2016). Yet, very few studies are published for reproductive stage waterdeficit conditions (Ma et al., 2016; Pantalião et al., 2016; Swamy et al., 2017). This was partly due to difficulty in implementing the stress at this stage under field conditions for a large GWAS panel that usually consists of genotypes having diverse phenological characteristics. Only the study of Ma et al. (2016) followed a staggered sowing to account for variation in flowering phenology under stress.

This study is aimed at (1) exploring the natural variation in grain yield as well as components and related traits in non-stress and reproductive stage water-deficit conditions; (2) linking the variation of these phenotypic traits with single nucleotide polymorphisms (SNPs) through GWAS; and (3) identify the most likely underlying candidate genes near to the significant SNP markers.

\section{Materials and Methods}

\section{Association mapping population}

We used a diverse set of 296 indica rice genotypes consisting of improved and traditional genotypes with (sub)tropical adaptation. This panel was assembled at International Rice Research Institute (IRRI) for the Phenomics of Rice Adaptation and Yield potential (PRAY) project in the context of Global Rice Phenotyping Network (http://ricephenonetwork.irri.org). Recent studies have reported a GWAS analysis using this population for grain quality traits (Qiu et al., 2016), salinity tolerance (Al-Tamimi et al., 2016), panicle architecture (Rebolledo et al., 2016), planting density (Kikuchi et al., 2017), and root morphological and anatomical plasticity in Chapter 3 (Kadam et al., 2017).

\section{Strategy to cope with variation in flowering phenology}

The PRAY panel was screened in non-stress and reproductive stage water-deficit conditions under field experiments conducted at the upland farm of IRRI, Philippines ( $14^{\circ} 11^{\prime} \mathrm{N}, 121^{\circ} 15^{\prime} \mathrm{E}$; elevation $21 \mathrm{~m}$ above sea level) in 2013 and 2014 dry seasons (DS). Seeds were sown from December of the preceding year to late January or early February of each year (Fig. 1). As expected, a strong genotypic variation in flowering phenology was observed that confounds the true water-deficit response (Fukai et al., 1999) and inevitably induces bias with interpretation of genetic mapping outcomes (Pinto et al., 2010; Kumar et al., 2014). We followed staggered sowing in seedbeds and transplanting in main plots to synchronise flowering and thus minimize 
phenological differences under stress imposition (Fig. 1). Briefly, in the 2013 DS experiment, we divided 296 genotypes into six groups with a 10-day interval based on days to flowering data collected in a pre-experiment in the 2012 wet season (WS), our only source of flowering dates for this population grown at IRRI. While the expected date of flowering was 29 March to 08 April 2013 (Fig. 1A), we observed deviation in days to flowering in the 2013 DS experiment, where the staggered sowing was based on the 2012 WS data. Therefore, in the 2014 DS experiment, we regrouped the 296 genotypes into eight groups with a 7-day interval using 2013 DS flowering data to improve synchrony within the whole population. The expected date of flowering was 28 March to 05 April 2014 for these genotypes (Fig. 1B). In each year, the sowing date chosen for the stress treatment was the same for the non-stress treatment of the same genotype.

\section{Crop management}

The soil type of the upland farm is Maahas clay loam, isohyperthermic mixed Typic Tropudalf. The experiments were laid out in a group block design with three replications for each genotype in both treatments (Supplementary Figure S1). Seeds were first exposed to $50{ }^{\circ} \mathrm{C}$ for 3 days to break the dormancy and then hand sown in a seedbed nursery. Twenty-one-day-old seedlings were transplanted (two seedlings per hill) for each genotype in four rows per replication. In both years, row distance was $0.2 \mathrm{~m}$ and row length was $2.4 \mathrm{~m}$. The seeds of one genotype in 2013 and 8 genotypes in 2014 germinated poorly and hence were excluded. In addition, four genotypes completed flowering and maturity before stress imposition in 2013 and were excluded. This resulted in the final sets of 291 genotypes in 2013 and 288 genotypes in 2014; and with 3 replications and 2 treatments, these gave 1746 and 1728 plots for 2013 and 2014, respectively. One day before transplanting, $30 \mathrm{~kg} \mathrm{P} \mathrm{ha}^{-1}$ (as single superphosphate), $40 \mathrm{~kg} \mathrm{~K}$ $\mathrm{ha}^{-1}$ (as $\mathrm{KCl}$ ), and $5 \mathrm{~kg} \mathrm{Zn} \mathrm{ha}^{-1}$ (as zinc sulfate heptahydrate) fertilizers were manually applied. Nitrogen fertilizer as urea was applied in three splits: $45 \mathrm{~kg} \mathrm{ha}^{-1}$ before transplanting, $30 \mathrm{~kg} \mathrm{ha}^{-1}$ at mid-tillering, and $45 \mathrm{~kg} \mathrm{ha}^{-1}$ at panicle initiation. The IRRI standard management practices were followed to control weeds, insects and diseases. In both years, all plots were maintained like irrigated lowlands with $\sim 5 \mathrm{~cm}$ standing water until maturity except for the water-deficit plots during the stress period (see below).

\section{Reproductive stage water-deficit stress imposition}

There was variation in synchronizing days to flowering among rice genotypes in 2013, resulting 
in deviation from our expected flowering window (29 March to 08 April). In rice, the reproductive stage initiation ranges between from 19 and 25 days, starting at panicle initiation and ending with flowering (Moldenhauer and Slaton, 2001). Therefore, before imposing stress, we manually dissected the main tillers of the middle two plants of border rows from waterdeficit plot for all the genotypes, primarily to check the reproductive stage development. When majority of genotypes reached the panicle initiation stage, we imposed stress on 23 March 2013 by draining water out from the field. The stress continued for 14 days until 5 April 2013. In the 2014 experiment, the synchronisation was more precise with expected dates of flowering occurring between $28 \mathrm{March}$ and April 5, as predicted. The same dissection approach as in 2013 was followed and stress was imposed on 26 March 2014 and continued for 14 days until 8 April.

To quantify the stress intensity, 26 tensiometers were installed randomly across the entire stress field at $30 \mathrm{~cm}$ depth in each season. A polythene sheet was inserted at 2-meter depth by digging a deep and narrow trench in between stress and non-stress fields to prevent water seepage during the stress period from the adjacent non-stress field. The intensity of stress was higher in 2014 than in 2013 (Supplementary Figure S2A). There was no rainfall during the peak stress period in both seasons, except rainfall during the first day of stress period in 2013 (Supplementary Figure S2B). Higher stress intensity in 2014 compared to 2013 could be due to higher maximum temperature and higher vapour-pressure deficit (Supplementary Figure S3B and D), leading to quicker loss of soil moisture in 2014. A weather station was placed between the non-stress and water-deficit plots (see Supplementary Figure S1). Detailed weather data are given in Supplementary Figure S3.

\section{Observations}

At maturity, plants of 16 hills from the middle two rows i.e. $0.64 \mathrm{~m}^{2}$ plot area (excluding the border rows) were harvested to assess grain yield (14\% moisture), its components and related traits in both experiments, following Shi et al. (2016). Days to flowering was assessed as the interval between the date of sowing and the date when panicles of $50 \%$ plants per plot were fully exerted. Days to maturity was assessed as the interval between the flowering date and date when panicles on most plants in a plot turned yellow and ready for harvest. Plant height was measured from the base of the root-shoot junction to the tip of the flag leaf. Non-grain dry weight was assessed as the sum of leaf, stem and rachis dry weight. The total aboveground dry weight was the sum of non-grain dry weight and grain dry weight. Harvest index was the ratio of grain dry weight to total aboveground dry weight. 


\section{Statistical analysis of phenotypic data}

\section{Analysis of variance (ANOVA)}

A combined linear mixed model based ANOVA was performed to test the effect of genotype $(\mathrm{G})$, treatment $(\mathrm{T})$ and year $(\mathrm{Y})$ with their interactions using the following model in Genstat V17.1.

$$
\mathrm{Y}_{\mathrm{ijkl}}=\mu+\mathrm{G}_{\mathrm{i}}+\mathrm{T}_{\mathrm{j}}+\mathrm{Y}_{\mathrm{k}}+\mathrm{R}_{\mathrm{l}}\left[\left(\mathrm{T}_{\mathrm{j}}\left(\mathrm{Y}_{\mathrm{k}}\right)\right]+(\mathrm{G} \times \mathrm{T} \times \mathrm{Y})_{\mathrm{ijk}}+\mathrm{E}_{\mathrm{ijk} 1}\right.
$$

where $\mathrm{Y}_{\mathrm{ijkl}}$ is the phenotypic trait value recorded in a plot, $\mu$ is the overall mean, $\mathrm{G}_{\mathrm{i}}$ is the effect of the $\mathrm{i}^{\text {th }}$ genotype, $T_{j}$ is the effect of the $\mathrm{j}^{\text {th }}$ treatment, $\mathrm{Y}_{\mathrm{k}}$ is the effect of the $\mathrm{k}^{\text {th }}$ year, $\mathrm{R}_{1}\left[\left(\mathrm{~T}_{\mathrm{j}}\left(\mathrm{Y}_{\mathrm{k}}\right)\right]\right.$ is the effect of the $1^{\text {th }}$ replication within the $\mathrm{j}^{\text {th }}$ treatment of the $\mathrm{k}^{\text {th }}$ year, $(\mathrm{G} \times \mathrm{T} \times \mathrm{Y})_{\mathrm{ijk}}$ is the effect of three-way interaction between the $\mathrm{i}^{\text {th }}$ genotype, the $\mathrm{j}^{\text {th }}$ treatment and the $\mathrm{k}^{\text {th }}$ year, and $\mathrm{E}_{\mathrm{ijk}}$ is the error. Apart from three-way interaction, we also consider two-way interactions of main factors in all possible combinations.

\section{Linear mixed model to estimate best linear unbiased estimators}

We estimated the best linear unbiased estimators (BLUEs) of phenotypic traits for individual genotype across years and treatments, separately. The following linear mixed model was used in Genstat release V17.1 to estimate the BLUEs separately in non-stress and stress conditions across years, using genotypes as a fixed effect and replications as a random effect.

$$
\mathrm{Y}_{\mathrm{ij}}=\mu+\mathrm{G}_{\mathrm{i}}+\mathrm{R}_{\mathrm{j}}+\mathrm{E}_{\mathrm{ij}}
$$

where $\mathrm{Y}_{\mathrm{ij}}$ is the phenotypic trait value recorded in a plot, $\mu$ is the overall mean, $\mathrm{G}_{\mathrm{i}}$ is the effect of the $\mathrm{i}^{\text {th }}$ genotype, $\mathrm{R}_{\mathrm{j}}$ is the effect of the $\mathrm{j}^{\text {th }}$ replication, and $\mathrm{E}_{\mathrm{ij}}$ is the error.

Days to flowering had a strong confounding effect on grain yield and its components under stress, particularly in 2013 (Fig. 1C). Therefore, we performed the linear mixed model based ANOVA analysis using the above equation with days to flowering as covariate. If the effect of days to flowering was significant, corrected BLUEs of grain yield and its components and related traits were estimated in stress treatments.

\section{Principal component analysis, trait correlation and multiple regression analysis}

A multivariate principal component analysis (PCA) was performed in XLSTAT across years and treatments. The chart. Correlation() function within the $\mathrm{R}$ package "Performance 
Analytics" was used to generate the correlation scatter plot. The $\operatorname{lm}()$ function within the R statistical framework was used for multiple linear regression analysis of grain yield with its component and related traits.

\section{Heritability estimates}

Broad-sense heritability $\left(\mathrm{H}^{2}\right)$, capturing the proportion of phenotypic variance explained by genetic factors that is due to dominance, epistatic and additive effects, was calculated using the below equation across years and treatments separately:

$$
H^{2}=\frac{\sigma_{\mathrm{G}}^{2}}{\sigma_{\mathrm{G}}^{2}+\frac{\sigma_{\mathrm{E}}^{2}}{\mathrm{r}}}
$$

where $\sigma^{2}{ }_{G}$ and $\sigma^{2}$ are the genotypic and residual variances respectively and $r$ is the number of replications. The restricted maximum likelihood estimate was used to calculate the variance components in Genstat release 17.1. The narrow-sense heritability $\left(h^{2}\right)$, capturing the proportion of total phenotypic variance explained by the additive genetic variance, was estimated using the equation in Genomic Association and Prediction Integrated Tool (GAPIT) function:

$$
h^{2}=\frac{\sigma_{\mathrm{a}}^{2}}{\sigma_{\mathrm{a}}^{2}+\sigma_{\mathrm{e}}^{2}}
$$

where $\sigma^{2}{ }_{\mathrm{a}}$ is the additive genetic variance and $\sigma^{2}$ is the residual variance.

\section{Genetic analysis of marker-trait associations}

The 291 genotypes in 2013, and 288 genotypes in 2014 experiments had complete phenotypic data. However, 20 genotypes were missing in the data of 45,699 (46K) SNPs, meaning only 271 genotypes in 2013 and 268 in 2014 were used for our GWAS analysis. The detailed genotype-by- sequencing protocol of SNPs genotyping, population structure and linkage disequilibrium (LD) for this population are explained in Chapter 3 (Kadam et al., 2017). The GWAS was performed on a set of 271 (2013) and 268 (2014) genotypes separately, with the 267 genotypes being common across both years. Two GWAS methods were used to test the marker-trait associations: the single-locus and the multi-locus analysis.

\section{Single-locus GWAS analysis}

Single-locus analysis is a one-dimensional scan, typically identifying associations between single marker and traits in the population. We performed this analysis using a compressed mixed-linear model (CMLM; Zhang et al., 2010) in the GAPIT (Lipka et al., 2012). In the 
mixed model, we included population structure and family kinship (family relatedness), that were calculated by the GAPIT function using SNPs with $\geq 0.05$ minor allele frequency (MAF).

$$
\mathrm{Y}=\mathrm{X} \alpha+\mathrm{P} \beta+\mathrm{K} \mu+\mathrm{e}
$$

where Y represents the vector of phenotype, $\mathrm{X}$ represents the vector of SNPs, P is the PCA matrix and $\mathrm{K}$ is the relative kinship matrix. $\mathrm{X} \alpha$ and $\mathrm{P} \beta$ are the fixed effect, and $\mathrm{K} \mu$ is the random effects and e represents the random error. The $\mathrm{P}$ and $\mathrm{K}$ matrices help to reduce the spurious false positive associations. Correction for population structure $(\mathrm{P})$ substantially reduces the false positives but it sometimes eliminates true positive associations due to overcorrection. Therefore, the optimal number of principal components was estimated for each trait before incorporating them for CMLM tests, based on the forward model selection method using the Bayesian information criterion (BIC). This method helps to control both false-positive and false-negative associations more effectively although it cannot eliminate both completely. We used a lower suggestive threshold probability $P$ value 1.0E-04 and upper Bonferroni corrected threshold (at $\alpha=0.05$ ) to detect significant associations.

\section{Multi-locus GWAS analysis}

The single-locus analysis corrects the confounding effects of population structure and family kinship but does not consider the confounding effect of causal genetic loci. The multi-locus GWAS is a method that corrects not only the confounding effects of population structure and family kinship but also the confounding and/or interaction effects of causal loci present in the genome due to LD (Segura et al., 2012). We performed the multi-locus GWAS using a modified version of the multi-locus mixed linear model (MLMM) in R studio ( $\mathrm{R}$ script for mlmm.cof.r available at https://cynin.gmi.oeaw.ac.at/home/resources/mlmm). We ran the complete model as recommended with stepwise forward inclusion of the strongest significant markers (lower $P$ value) and stepwise backward elimination of the last forward model (that is least significant markers). Significant markers were selected based on the criteria explained in Chapter 3 (Kadam et al., 2017). Briefly, in the first step (like single-locus GWAS without any marker as a cofactor), we manually checked the $P$ value of SNPs before including them as a cofactor in model. Then we continued adding markers to the model as cofactor based on cut-off threshold $p$-value $\leq 1.00 \mathrm{E}-04$. Once there are no significant loci appeared above the threshold $P$ value, the model was stopped. All the significant cofactor identified were considered as significant genetic loci. 


\section{Selecting a priori candidate genes underlying the genetic loci}

The detailed protocol to select a priori candidate genes near to significant SNPs was followed as explained in Chapter 3 (Kadam et al., 2017).

\section{Results}

\section{The flowering time was sensitive to seasonal climate variations}

The flowering time synchronisation approach was followed to reduce the confounding effect of flowering time differences of rice genotypes on grain yield and its components and related traits under stress (Fig. 1A-B). However, we witnessed deviation of our observed days to flowering from expected days $\left(R^{2}=0.53\right.$ in non-stress and $R^{2}=0.46$ in stress; Fig. 1C) in 2013. As rice flowering time is regulated by internal genetic cues and external stimuli such as photoperiod and temperature (Yin et al., 1997), such deviations were anticipated, since the synchronisation in 2013 was based on 2012 WS pre-experiment data due to lack of DS data. Many genotypes exhibited photothermal sensitivity across wet and dry seasons. Therefore, some genotypes experienced stress during the flowering period (31\%), whereas others experienced stress either before $(60 \%)$ or immediately after flowering ( $8 \%$ ). In 2014, we restructured the synchronisation based on 2013 DS data. This resulted in better synchronisation with only small deviation observed from expected days to flowering $\left(\mathrm{R}^{2}=0.91\right.$ in non-stress and $\mathrm{R}^{2}=0.85$ in stress; Fig. 1D). Further, to test the effect of days to flowering, we performed the analysis with days to flowering as a covariate in the mixed model ANOVA. The moderate to strong significant effect of days to flowering on grain yield, its components and harvest index were detected in 2013 stress, most likely due to desynchronised flowering time. Conversely, the improved flowering synchronization caused no significant effect in 2014 stress. The marginal $(P<0.05)$ to moderate $(P<0.01)$ effect of days to flowering on grain yield, seed set and harvest index was detected in both years under non-stress conditions (Fig. 1C-D). This could be due to the pleiotropic effect of flowering genes on panicle development (Crowell et al., 2016), a key determinant of rice grain yield.

\section{Genotype effects and genotype-by-environment interactions accounted for variations in grain yield and other traits}

A combined mixed model ANOVA across years was carried out to divide the variation in genotype, treatment and year components and their interactions (Table 1). The variation in grain yield, its components and related traits differed significantly between genotype $(\mathrm{G} ; P<0.001)$, 


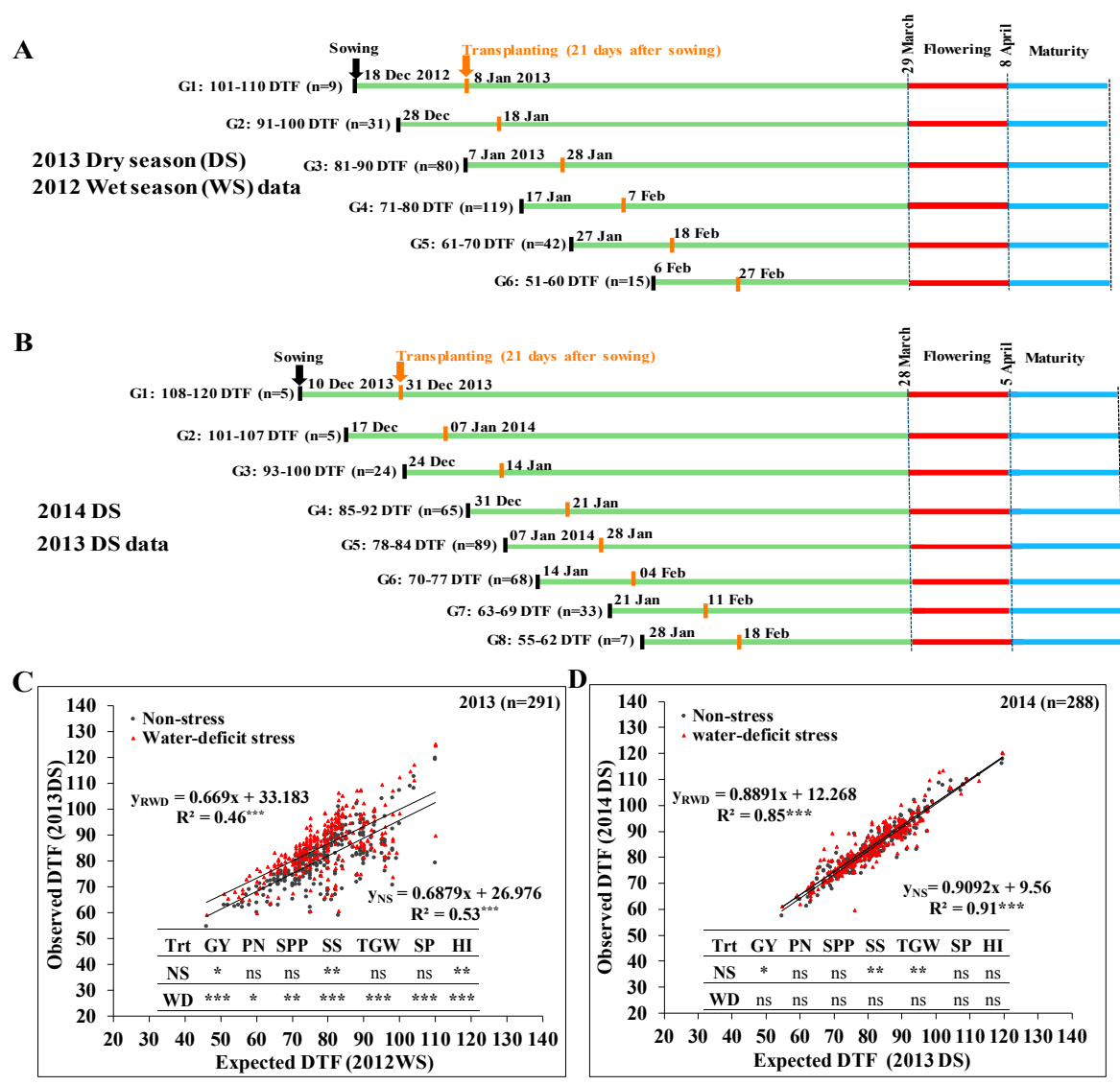

Figure 1: Schematic representation of staggered sowing and transplanting approach followed for screening of indica rice diversity panel under reproductive stage water-deficit stress in dry season (DS) of 2013 (Panel A) and 2014 (Panel B). Days to flowering interval was 10 days between groups $(\mathrm{G})$ in 2013 and 7 days in 2014 DS experiments. The expected and observed days to flowering (DTF) in non-stress (NS) and water-deficit stress (WD) in 2013 (Panel C) and 2014 dry season (Panel D) experiments. The ANOVA results with the effect of DTF (as a covariate in mixed linear model) on grain yield and its key component traits are given in panel. $\mathrm{n}=$ number of genotypes; Trt = treatments; $\mathrm{GY}=$ grain yield; $\mathrm{PN}=$ panicles per $\mathrm{m}^{2}$; $\mathrm{SPP}=$ spikelets per panicle; $\mathrm{SS}=$ seed set; $\mathrm{TGW}=$ thousand grain weight; $\mathrm{SP}=$ spikelets per $\mathrm{m}^{2}$; $\mathrm{HI}=$ harvest index. Significance levels: $* P<0.05, * * P<0.01, * * * P<0.001$.

treatment $(\mathrm{T} ; P<0.001)$ and year $(\mathrm{Y} ; P<0.01$ to $P<0.001)$. Further, the grain yield, its components and related traits of each genotype responded differently to treatment $(\mathrm{G} \times \mathrm{T}$; $P<0.001)$ and year $(\mathrm{G} \times \mathrm{Y} ; P<0.001)$. The detailed descriptive statistics of these traits are given in Supplementary Table S1. The traits showed different distributions in non-stress and stress conditions for both years (Fig. 2). Grain yield ranged from 106.3 to $727.0 \mathrm{~g} \mathrm{~m}^{-2}$ in non-stress, 
and from 16.7 to $622.6 \mathrm{~g} \mathrm{~m}^{-2}$ under stress in 2013, and from 102.8 to $839.7 \mathrm{~g} \mathrm{~m}^{-2}$ in non-stress, and from 78.1 to $761.1 \mathrm{~g} \mathrm{~m}^{-2}$ under stress conditions in 2014. Across all observations, $\mathrm{H}^{2}$ and $h^{2}$ estimates ranged from 0.73 to 0.99 and from 0.27 to 0.94 , respectively, in 2013; and from 0.62 to 0.99 and from 0.69 to 0.93 , respectively, in 2014 (Supplementary Table S1). The higher reduction of grain yield, seed set and harvest index under stress in 2014 was due to higher stress intensity during 2014 (-64 kPa) compared to 2013 (-46 kPa), because of higher vapour-pressure deficit (Supplementary Figures S2A and S3D). However, a higher reduction of spikelets per panicle and spikelets per $\mathrm{m}^{2}$ despite lower stress intensity was observed during 2013 than during 2014 (Fig. 2C-E). This could be due to variation in flowering time synchronisation with more genotypes experiencing stress before flowering in 2013 than in 2014. These results clearly illustrate that stress affects the number of spikelets per $\mathrm{m}^{2}$ when imposed before flowering, but spikelet fertility when imposed during flowering (Lanceras et al., 2004) as seen in Fig. 2C-E. The days to flowering differed significantly $(P=0.002)$ between non-stress and stress in 2013 , but not $(P>0.05)$ in 2014 (Fig. 2I). The first two principal components cumulatively explained $>55 \%$ (non-stress: 55.61\%; stress: 59.59\%) in 2013 and $>61 \%$ (non-stress: $61.26 \%$; stress: $63.66 \%$ ) in 2014 of the total phenotypic variation (Fig. 3). The genotypic variation in the first PC was mostly explained by grain yield, harvest index and spikelets per $\mathrm{m}^{2}$ in non-stress (2013: $\mathrm{PC} 1=29.09 \%$; 2014: $\mathrm{PC} 1=34.69 \%$ ) and grain yield, harvest index, spikelets per $\mathrm{m}^{2}$ and total dry weight in stress (2013: $\mathrm{PC} 1=35.51 \% ; 2014$ : $\mathrm{PC} 1=37.54 \%)$. The genotypic variation in second $\mathrm{PC}$ was explained by non-grain dry weight, days to flowering and total dry weight in non-stress (2013: $\mathrm{PC} 2=26.52 \% ; 2013: \mathrm{PC} 2=26.56 \%$ ) and plant height, non-grain dry weight and days to flowering in stress (2013: $\mathrm{PC} 2=24.08 \%$; 2014: $\mathrm{PC} 2=26.12 \%$ ). In addition, the phenotypic traits with their magnitude (the length of the vector) and orientation were elucidating that the principal component variations differed in response to treatment and year (Fig. 3). This confirms our ANOVA results. For instance, variation in traits were higher in 2014 than in 2013, indicating a $\mathrm{G} \times \mathrm{Y}$ interaction. The trait variation was higher in stress $(2013=59.59 \%$; $2014=63.66 \%)$ compared to non-stress $(2013=55.61 \% ; 2014=61.26 \%)$ conditions, indicating that stress increased genotypic variability $(\mathrm{G} \times \mathrm{T}$ interaction).

\section{Phenotypic trait correlations and contribution of component traits to grain yield}

Grain yield was significantly $(P<0.05)$ correlated with most of its components and related traits across treatments and years (Supplementary Figures S4-S7). However, non-significant $(P>0.05)$ correlations of grain yield were found with thousand grain weight and non-grain dry 
Table 1: Analysis of variance (ANOVA) of grain yield and its components and related traits in 2013 and 2014 dry season experiments.

\begin{tabular}{lllllllllc}
\hline Class & Trait & Unit & $\mathbf{G}$ & $\mathbf{T}$ & $\mathbf{Y}$ & $\mathbf{G} \times \mathbf{T}$ & $\mathbf{G} \times \mathbf{Y}$ & $\mathbf{T} \times \mathbf{Y}$ & $\mathbf{G} \times \mathbf{T} \times \mathbf{Y}$ \\
\hline $\mathbf{A}$ & Grain yield & $\mathrm{g} \mathrm{m}^{-2}$ & $* * *$ & $* * *$ & $* * *$ & $* * *$ & $* * *$ & $* * *$ & $* *$ \\
\hline $\mathbf{B}$ & Grain yield component traits & & & & & & & \\
\hline & Panicles per $\mathrm{m}^{2}$ & $\mathrm{~m}^{-2}$ & $* * *$ & $* * *$ & $* * *$ & $* * *$ & $* * *$ & $\mathrm{~ns}$ & $* * *$ \\
& Spikelets per panicle & - & $* * *$ & $* * *$ & $* * *$ & $* * *$ & $* * *$ & $\mathrm{~ns}$ & \\
& Seed set & $\%$ & $* * *$ & $* * *$ & $* * *$ & $* * *$ & $* * *$ & $* * *$ & $* * *$ \\
& Thousand grain weight & $\mathrm{g}$ & $* * *$ & $* * *$ & $* *$ & $* * *$ & $* * *$ & $\mathrm{~ns}$ & $* * *$ \\
& Spikelets per $\mathrm{m}^{2}\left(\times 10^{3}\right)$ & $\mathrm{m}^{-2}$ & $* * *$ & $* * *$ & $* * *$ & $* * *$ & $* * *$ & $* * *$ & \\
\hline C & Grain yield related traits & & & & & & & & \\
\hline & Harvest index & - & $* * *$ & $* * *$ & $* * *$ & $* * *$ & $* * *$ & $* * *$ & $* * *$ \\
& Total dry weight & $\mathrm{kg} \mathrm{m}^{-2}$ & $* * *$ & $* * *$ & $* * *$ & $* * *$ & $* * *$ & $\mathrm{~ns}$ & $\mathrm{~ns}$ \\
& Non-grain dry weight & $\mathrm{kg} \mathrm{m}^{-2}$ & $* * *$ & $* * *$ & $* * *$ & $* * *$ & $* * *$ & $* * *$ & $* * *$ \\
& Plant height & $\mathrm{cm}$ & $* * *$ & $* * *$ & $* * *$ & $* * *$ & $* * *$ & $*$ & $\mathrm{~ns}$ \\
& Days to flowering & - & $* * *$ & $* * *$ & $* * *$ & $* * *$ & $* * *$ & $* * *$ & $* * *$ \\
Days to maturity & - & - & - & - & - & - & - & - \\
\hline
\end{tabular}

$\mathrm{G}=$ genotype, $\mathrm{T}=$ treatment, $\mathrm{Y}=$ year, $\mathrm{ns}=$ non-significant. Spikelets per $\mathrm{m}^{2}$ is not an independent yield component and it is the product of panicles per $\mathrm{m}^{2}$ and spikelets per panicle. Significance level: ${ }^{*} P<0.05$, $* * P<0.01, * * * P<0.001$.

weight in non-stress, and with panicle number in 2013 stress. Grain yield was not significantly $(P>0.05)$ correlated with non-grain dry weight across treatments in 2014. The correlation of grain yield with spikelets per panicle was higher in stress $(2013: r=0.73 ; 2014: r=0.46)$ than in non-stress conditions $(2013: \mathrm{r}=0.40 ; 2014: \mathrm{r}=0.36)$ in both years, and increase was more in 2013. Similarly, the correlation between grain yield and seed set increased from 0.62 in nonstress to 0.75 in stress conditions in 2014. The increased correlation of grain yield with spikelets per panicle in 2013 and with seed set in 2014 in stress reflects the effect of variation in days to flowering synchronisation. Further, correlations of grain with days to flowering was increased weakly under stress in 2013 (non-stress: $r=0.16$; stress $r=0.29$ ), but remained unchanged across treatments in 2014 (non-stress and stress: $r=0.30$ ). The correlation between grain yield and harvest index was marginally increased in water-deficit $(2013: \mathrm{r}=0.85,2014: \mathrm{r}=0.86)$ compared with non-stress (2013: $\mathrm{r}=0.83,2014: \mathrm{r}=0.81$ ). We also tested the relative contribution of each component and related trait to grain yield through multiple linear regression. All components and related traits significantly contributed to grain yield except for plant height and days to flowering in non-stress and only days to flowering in stress during 2013 and 2014, respectively (Supplementary Table S2). The cumulative variation of grain yield explained by its components and related traits was marginally higher in $2014\left(R^{2}: 0.94\right.$ in nonstress and $\mathrm{R}^{2}=0.93$ in stress) than in $2013\left(\mathrm{R}^{2}: 0.89\right.$ in non-stress and $\mathrm{R}^{2}=0.88$ in stress). 


\section{Treatment and year specific genetic loci for grain yield and other traits}

Grain yield and its components and related traits followed a normal distribution (Supplementary Figures S4-S7), indicating the quantitative pattern suitable for genetic analysis. To identify marker-trait association, we used a lower suggestive threshold $P$ value $1.0 \mathrm{E}-04\left(-\log _{10} P=4\right)$ and superior Bonferroni corrected threshold as an upper limit $\left(2013:-\log _{10}(0.05 / 45,437)=6\right.$; 2014: $\left.-\log _{10}(0.05 / 45,414)=6\right)$. A summary of GWAS results is given in Table 2, while the detailed results are in Supplementary Tables S3-S6. In total, we identified 38 significant loci in non-stress, and 69 loci in stress during 2013, and 64 significant loci in non-stress, and 55 in stress during 2014. Most loci were specific across treatments within years and within treatments across the years. Nevertheless, we also detected 14 common loci (9 in 2013 and 5 in 2014) across treatments and 8 common loci within treatments $(6$ in non-stress and 2 in stress conditions) across years only for the same components and related traits (Supplementary Table S7).

\section{Genetic analysis after correcting for days to flowering under stress in 2013}

Flowering time synchronisation was strongly confounding the grain yield and its component traits in 2013 stress (Fig. 1C-D). We corrected grain yield and its components and related (only harvest index in this class) traits using days to flowering as a covariate in the mixed model. The single-locus and multi-locus analysis of corrected trait values evidenced 31 additional loci using similar threshold $P$-values as mentioned earlier (Table 2; Supplementary Table S8). Most genetic loci detected for non-corrected traits disappeared when corrected trait values were subject to GWAS analysis. This suggested that the trait variation associated with these loci were mostly explained by variation in days to flowering. Only five genetic loci (one on chromosome 4 for grain yield [Q9]; one on chromosome 12 for spikelets per $\mathrm{m}^{2}$ [1, 41,599] and 3 loci on chromosome 11 for harvest index $[10,627,944 ; 10,131,062 ; 10,329,677]$ were common to corrected and non-corrected trait values. The common loci detected for grain yield (Q9; Table 3 and Figure 4) and harvest index (Supplementary Figure S8; Supplementary Tables S4 and S8) recorded lower $P$-value (improved statistical power) for corrected value through singlelocus analysis. Despite correction, the novel locus Q10 on chromosome 3 for corrected grain yield, seed set and harvest index overlapped with days to flowering (Table 3 ). In conclusion, statistical correction helped to explain the confounding effect of days to flowering and could eliminate its effect on grain yield under water-deficit. Unless otherwise mentioned, all results discussed in the following sections were for the corrected trait loci in 2013 stress. 


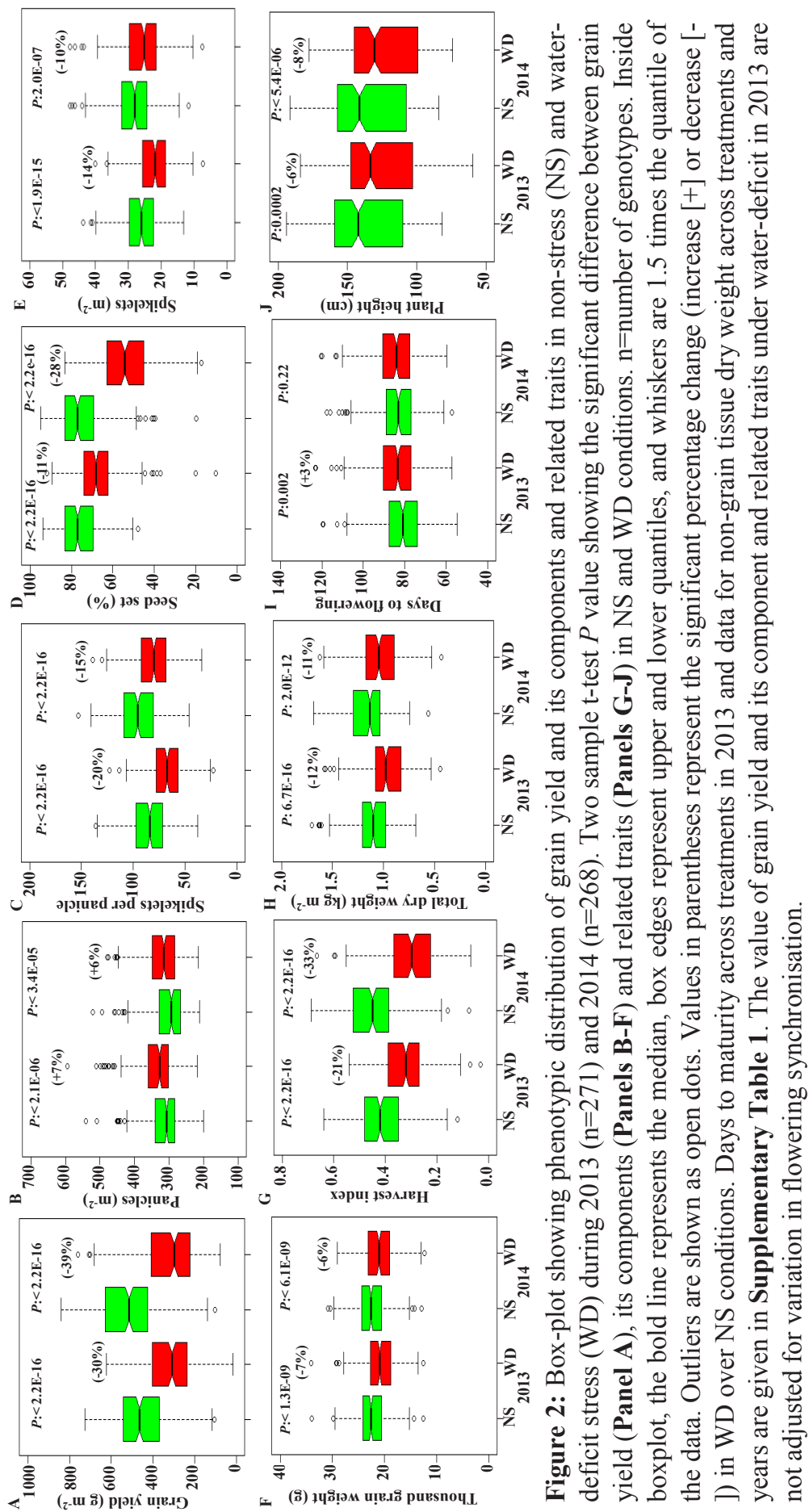



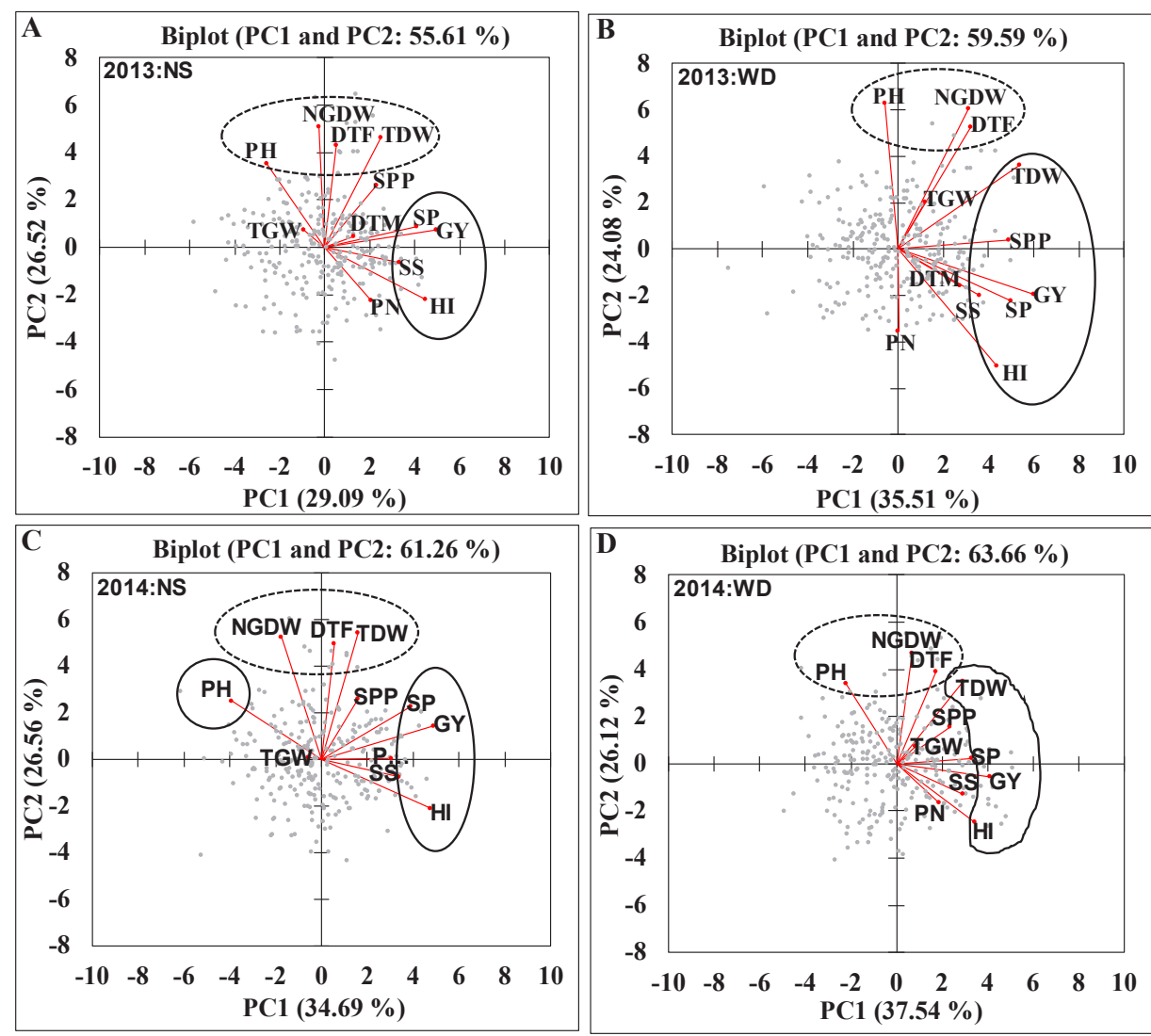

Figure 3: The principal component analysis of grain yield, its component and related traits with first two principal components (PC1 and PC2) in non-stress (NS) and water-deficit stress (WD) during 2013 (NS=Panel A; WD=Panel B) and 2014 DS (NS=Panel C; WD=Panel D). The traits marked inside the solid circle/ellipses contributed more to the variation explained by $\mathrm{PC} 1$ and marked inside the dashed ellipses to $\mathrm{PC} 2$. GY=grain yield; $\mathrm{PN}=$ panicles per $\mathrm{m}^{2}$; $\mathrm{SPP}=$ spikelets per panicle; $\mathrm{SS}=$ seed set; $\mathrm{TGW}=$ thousand grain weight; $\mathrm{SP}=$ spikelets per $\mathrm{m}^{2}$; $\mathrm{HI}=$ harvest index; TDW= total dry weight; $\mathrm{NGDW}=$ non-grain dry weight; $\mathrm{PH}=$ plant height; $\mathrm{DTF}=$ days to flowering; DTM=days to maturity.

\section{Eight grain-yield loci revealed small to medium allelic effect in non-stress conditions}

We identified two (Q1 and Q2) and six (Q3-Q8) loci for grain yield in 2013 and 2014, respectively (Table 3). There were no common loci across years, most likely due to significant variations in minimum and maximum temperature and vapour-pressure deficit (VPD; Supplementary Figure S3). These loci had a positive or negative effect (small to medium) on grain yield regarding its minor allele (allele refers to the 0.05 frequency in studied population). In 2013, the minor allele of Q1 $\left(30.13 \mathrm{~g} \mathrm{~m}^{-2}\right)$ had a positive effect on grain yield. Conversely, the minor allele of Q2 (-175.9 $\left.\mathrm{g} \mathrm{m}^{-2}\right)$ locus had a negative effect on grain yield. In 2014, Q3 
Table 2: Summary of genetic loci detected for grain yield; its components and related traits in 2013 and 2014 under non-stress (NS) and water-deficit stress (WD) conditions.

\begin{tabular}{llccccc}
\hline & & $\mathbf{2 0 1 3}$ & \multicolumn{2}{c}{$\mathbf{2 0 1 4}$} \\
\hline Class & Traits & NS & WD & WD $^{\dagger}$ & NS & WD \\
\hline A & Grain yield & 2 & 4 & 2 & 6 & 5 \\
\hline B & Grain yield component traits & & & & & \\
\hline & Panicles per m $^{2}$ & 6 & 12 & 7 & 9 & 3 \\
& Spikelets per panicle & 5 & 9 & 6 & 2 & na \\
& Seed set & 3 & 7 & 7 & 8 & 11 \\
& Thousand grain weight & 3 & 4 & na & 6 & 8 \\
& Spikelets per m ${ }^{2}$ & 1 & 4 & 1 & 4 & 3 \\
\hline Subtotal B & $\mathbf{1 8}$ & $\mathbf{3 6}$ & $\mathbf{2 1}$ & $\mathbf{2 9}$ & $\mathbf{2 5}$ \\
\hline Crain yield related traits & & & & & \\
\hline & Harvest index & 6 & 7 & 8 & 4 & 2 \\
& Total dry weight & 3 & 2 & - & 4 & 11 \\
& Non-grain dry weight & 3 & 3 & - & 5 & 2 \\
& Plant height & 2 & 6 & - & 6 & 4 \\
& Days to flowering & 3 & 11 & - & 10 & 6 \\
& Days to maturity & 1 & na & - & - & - \\
\hline Subtotal $\boldsymbol{C}$ & $\mathbf{1 8}$ & $\mathbf{2 9}$ & $\mathbf{8}$ & $\mathbf{2 9}$ & $\mathbf{2 5}$ \\
\hline Total (A+B+C) & $\mathbf{3 8}$ & $\mathbf{6 9}$ & $\mathbf{3 1}$ & $\mathbf{6 4}$ & $\mathbf{5 5}$ \\
\hline
\end{tabular}

na $=$ no marker-trait association; $\uparrow=$ marker-trait associations detected for corrected trait values in water-deficit stress.

(30.40 $\left.\mathrm{g} \mathrm{m}^{-2}\right)$, Q5 (13.98 $\left.\mathrm{g} \mathrm{m}^{-2}\right)$ and Q6 (74.08 $\left.\mathrm{g} \mathrm{m}^{-2}\right)$ all had a positive effect, while Q4 (-46.18 $\left.\mathrm{g} \mathrm{m}^{-2}\right)$, Q7 (-186.24 $\left.\mathrm{g} \mathrm{m}^{-2}\right)$ and Q8 (-97.80 $\left.\mathrm{g} \mathrm{m}^{-2}\right)$ all had a negative effect on grain yield regarding the minor allele. Eighteen and sixty-eight a priori (known or characterized) candidate genes were harboured within the expected LD block by Q1 and Q2 in 2013, and Q3-Q8 in 2014, respectively. Interestingly eight a priori candidate genes were identified. Some are presented below while others are given in Supplementary Table S9. Q1 was close to OsPTR2 (6 and 31 $\mathrm{kb}$; two copies in LD block). The rice homologue of this gene short panicle 1 (OsPTR2) regulates panicle and grain size and nitrate transport in rice ( $\mathrm{Li}$ et al., 2009). The homologue of OsPTR2 was recently detected at $q-28$ locus (OsPTR9) for spikelet number per panicle (a key determinant of grain yield) in the same rice association panel as we used in this study (Rebolledo et al., 2016). Likewise, Q4 was close (34 kb from peak SNP) to serine-threonine kinase (OsSTE). The Arabidopsis orthologue of OsSTE (AtSTE) is the major regulator of stomatal opening (Supplementary Table S9). 
Seven grain-yield loci revealed small to medium allelic effect in response to reproductive stage water-deficit

We identified two loci (Q9 and Q10) for grain yield under stress in 2013. The minor allele of both loci had a negative effect (Q9: $-81.29 \mathrm{~g} \mathrm{~m}^{-2}$ and Q10: $-40.61 \mathrm{~g} \mathrm{~m}^{-2}$ ) on grain yield. Five significant loci Q11-Q15 were detected for grain yield under stress in 2014, (Figure 5). The minor allele of Q11 (31.47 $\left.\mathrm{g} \mathrm{m}^{-2}\right)$, Q12 $\left(6.10 \mathrm{~g} \mathrm{~m}^{-2}\right)$ and Q15 (33.65 $\left.\mathrm{g} \mathrm{m}^{-2}\right)$ had a positive effect on grain yield. While the minor allele of two loci, Q13 (-49.86 $\left.\mathrm{g} \mathrm{m}^{-2}\right)$ and Q14 (-23.54 $\left.\mathrm{g} \mathrm{m}^{-2}\right)$, had a negative effect on grain yield. The Q9, Q10, and Q11-Q15 harboured 18 and 16 a priori candidate genes within the expected LD block region, respectively (Table 3). Seven a priori candidate genes, mostly near significant SNPs, are given in Supplementary Table S9. The Q9 locus was close $(13 \mathrm{~kb})$ to the Phosphomannomutase gene regulating L-Ascorbic acid (Asc) biosynthesis and response to abiotic stress stimulus (Gene Ontology [GO]: 0009628). Asc acts as a redox buffer to detoxify reactive oxygen species (ROS; Arrigoni and De Tullio, 2002). Q11 was close to Squalene monooxygenase or epoxidase (16 and $23 \mathrm{~kb}$; two copies in LD block) and response to abiotic stress stimulus (GO: 0009628). This gene is known to regulate ROS, stomatal responses and water-deficit stress tolerance in Arabidopsis (Posé et al., 2009).

\section{Only three loci for grain yield acted via change in seed set percentage}

Although rice grain yield is co-determined by panicle number, spikelets per panicle, seed set percentage and grain weight, very few loci of these component traits were coinciding with loci for grain yield per se. The seed set percentage is one of the most important yield components as indicated by its strong correlation with grain yield (Supplementary Figures S4-S7). Three loci were regulating grain yield through changes in seed set percentage, i.e., two loci designated as Q2 (2013) and Q7 (2014) in non-stress, and Q10 (2013) in stress. The major (allele refers to the 0.95 frequency in studied population) allele of these loci had a positive effect on grain yield, seed set and harvest index, respectively (Fig. 6). In addition, the Q10 was also detected for days to flowering. No loci were common for grain yield and seed set in 2014 stress, but one of the loci on chromosome $1(29,223,354)$ was commonly detected for seed set and harvest index (Supplementary Figure S9). Similarly, the major alleles had a positive effect on seed set, harvest index, and grain yield (irrespective of genetic significance), respectively (Fig. 7). Hence, these above loci were regulating grain yield through the effect of seed set on harvest index. Four $a$ priori candidate genes were predicted within the expected LD block of these loci. The Q2 was close (55 kb from peak SNP) to Plastocyanin gene that regulates flower development (GO: 
0009908) and pollination (GO: 0009856) in rice (Supplementary Table S9). The Arabidopsis orthologue of this gene regulates the seed set and pollen tube growth (Dong et al., 2005). Q7 was within the novel expressed protein, which provides an entry point for future study. Sugar transport or uptake is essential for normal pollen development (Reinders, 2016), while the lack of starch synthesis arrests the pollen development in water-deficit thereby regulating seed set (Sheoran and Saini, 1996).Our Q10 locus was within the sugar transporter gene that plays an important role in sugar distribution. The rice grain yield $M Q T L_{2.1}$ (meta-analysis QTL) detected in water-deficit was also containing sugar transporter gene (Swamy et al., 2011). Similarly, the locus on chromosome 1 for seed set and harvest index in 2014 stress was near (34 kb from peak $\mathrm{SNP}$ ) to the nitrate transporter gene that plays a role in rice grain yield increment (Fan et al., 2016).

\section{Discussion}

The main aim of this study was linking the phenotypic variation with genetic markers, thereby gaining insights about promising candidate genes and the genetic architecture controlling yield traits. To the best of our knowledge, this is the first study conducted on rice PRAY association mapping panel under reproductive stage water-deficit stress. The key findings from our study are discussed below.

\section{Statistical trait adjustment can reduce confounding effect of desynchronised flowering on genetic analysis under water-deficit stress}

The desynchronised flowering time may result in the identification of QTLs, often coinciding with QTLs for phenology and grain yield in reproductive stage stress (Pinto et al., 2010). Our genetic analysis of statistically corrected trait values was effective in minimizing the effect of desynchronised flowering time, as it led to detection of several novel loci that were not detected for non-corrected trait values. Despite of statistical adjustment for flowering time, our novel Q10 for grain yield was co-localised with flowering time (different SNP but falls within the same gene and LD block). In addition, it was also co-localised with seed set and harvest index. Previous studies in rice have identified several grain yield QTLs using linkage mapping under reproductive water-deficit stress conditions (Bernier et al., 2007; Venuprasad et al., 2009; Vikram et al., 2011; Swamy et al., 2013; Mishra et al., 2013), of which some were co-localised with plant height ( $\left.\mathrm{qDTY}_{6.2}\right)$, days to flowering $\left(\mathrm{qDTY}_{3.2}\right)$ or both $\left(\mathrm{qDTY}_{1.1}\right)$. Interestingly, the major effect of $\mathrm{qDTY}_{1.1}$ was consistent even after statistical correction of grain yield using 
flowering time and plant height as covariates (Vikram et al., 2011), and the recent detailed characterisation confirmed the tight linkage and not the pleiotropy of this QTL with plant phenology (Vikram et al., 2015). Our novel Q10 provided higher confidence of causative SNP placed directly within the sugar transporter gene. However, this SNP was just $5 \mathrm{~kb}$ away from the COP9 signalosome complex subunit 4 gene within the same LD block (Supplementary Table S9). The COP9 signalosome complex gene is known to regulate flower development in Arabidopsis (Wang et al., 2003), although no study so far reported the role of this gene in rice flowering. Therefore, a future characterisation of Q10 would be interesting to decipher the relationship with flowering time and stress tolerance to test linkage versus pleiotropy. Nevertheless, the effect of our consistent Q9 for grain yield (detected using either corrected or non-corrected values) was independent of flowering time stress conditions. More precise flowering time synchronisation in 2014, which allowed identifying the genetic loci without having any co-localisation with flowering time in stress conditions added value to the findings. To the best of our knowledge, this is the first report demonstrating the better synchronisation of flowering time phenology on a large GWAS panel under stress conditions at field level.

\section{Genetic control of grain yield, its components and related traits was mostly independent and environment specific}

Grain yield is a complex trait determined by many interactive physiological processes changing temporally during the growing period. These processes often match the development of the key yield components in cereals that are genetically less complex than yield per se (Yin et al., 2002). In rice, grain yield is the product of the panicle number/productive tiller (determined during the vegetative phase), spikelets per panicle (determined during panicle initiation), seed set percentage (determined during panicle initiation and anthesis) and individual grain weight (determined during grain filling). The genetic selection for each of these traits during rice domestication has given rise to rich genetic diversity (Doebley et al., 2006; Sweeney and McCouch, 2007). To date, molecular genetic studies have detected QTLs underlying these genetic changes in rice yield components (http://www.gramene.org/). From these QTLs some of the candidate genes were successfully identified, notably displaying the improvement in grain yield (Ashikari et al., 2005; Fan et al., 2006 ; Song et al., 2007; Shomura et al., 2008; Huang et al., 2009; Miura et al., 2010). For instance, the SPIKE gene/allele regulating the spikelet numbers indicated 13-36 \% yield increment in rice (Fujita et al., 2013). In the present study, genetic dissection of these yield components enabled us to detect more loci than yield 

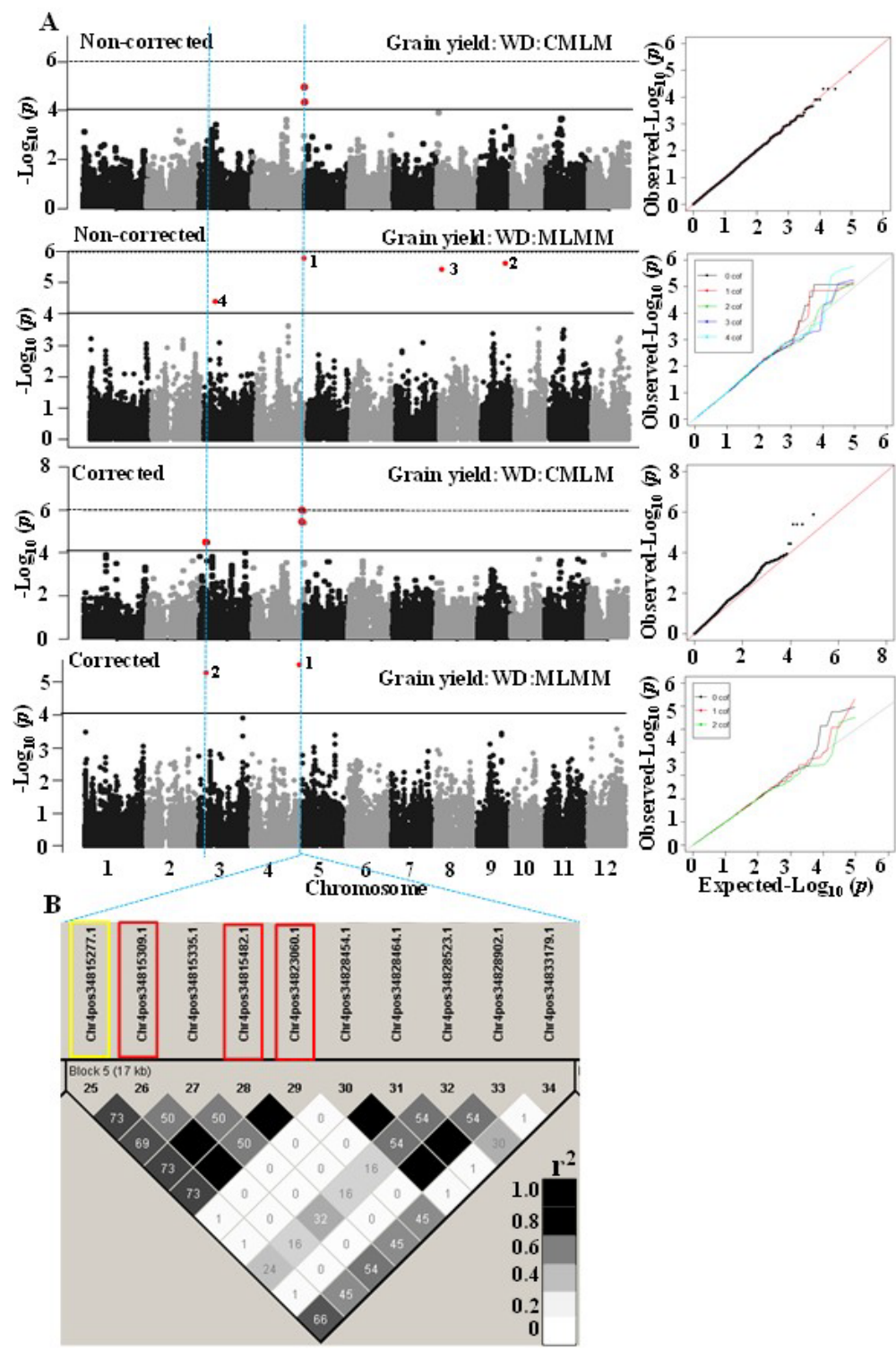

Figure 4: GWAS results (Manhattan and Quantile-Quantile plot) detected through single-locus compressed mixed linear model (CMLM) and multi-locus mixed model (MLMM) for noncorrected and corrected (using days to flowering as covariate) grain yield in 2013 water-deficit stress (WD) conditions (Panel A). Significant SNPs in the Manhattan plot of MLMM are numbered according to the order in which they were included as a cofactor in regression model. Identified LD block $(17 \mathrm{~kb})$ based on $\mathrm{r}^{2}$ value between SNPs on chromosome 4 and the colour intensity of the box on the LD plot corresponds with $\mathrm{r}^{2}$ (multiplied by 100) according to legend (Panel B). Significant SNP/SNPs marked in yellow rectangle was detected by CMLM and MLMM and in red rectangle only by CMLM approach. 


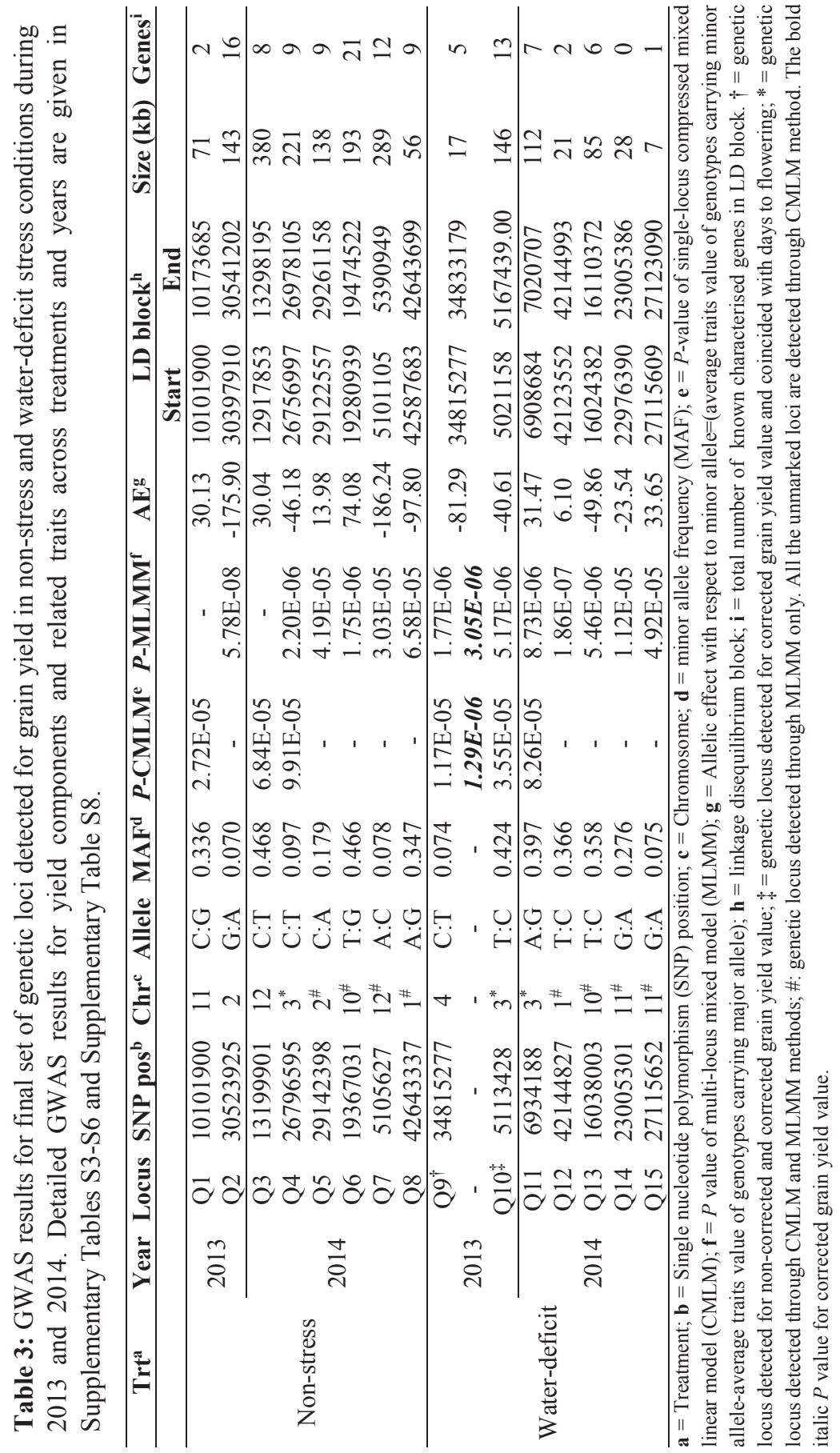



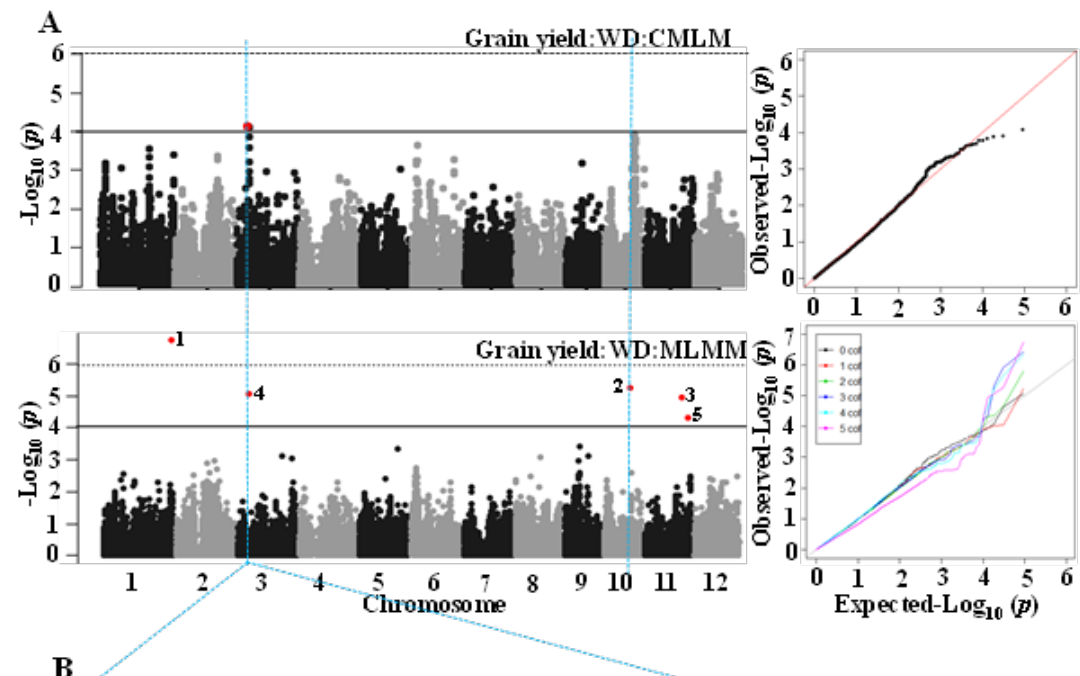

B

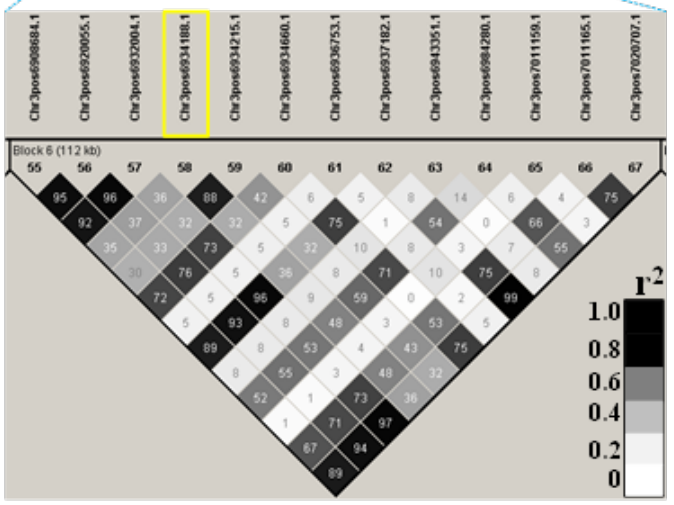

Figure 5: GWAS results (Manhattan and Quantile-Quantile plot) detected through single-locus compressed mixed linear model (CMLM) and multi-locus mixed model (MLMM) for grain yield in 2014 water-deficit stress (WD) conditions (Panel A). Significant SNPs on the Manhattan plot of MLMM are numbered according to the order in which they included as a cofactor in regression model. Identified LD block $(112 \mathrm{~kb})$ based on $\mathrm{r}^{2}$ value between SNPs on chromosome 3 and the colour intensity of the box on the LD plot corresponds with $\mathrm{r}^{2}$ (multiplied by 100) according to the legend (Panel B). Significant SNP marked in yellow rectangle was detected by CMLM and MLMM.

per se, which were directly or indirectly contributing to rice grain yield. The colocalization of grain yield loci with yield components was limited in this study compared to other studies in rice (Lanceras et al., 2004). This could be due to compensation among the yield components. In addition, these results emphasize the need for genetic analysis of yield components to identify additional genetic determinants having indirect effect on grain yield, providing alternative routes to enhance yield under water-deficit. 


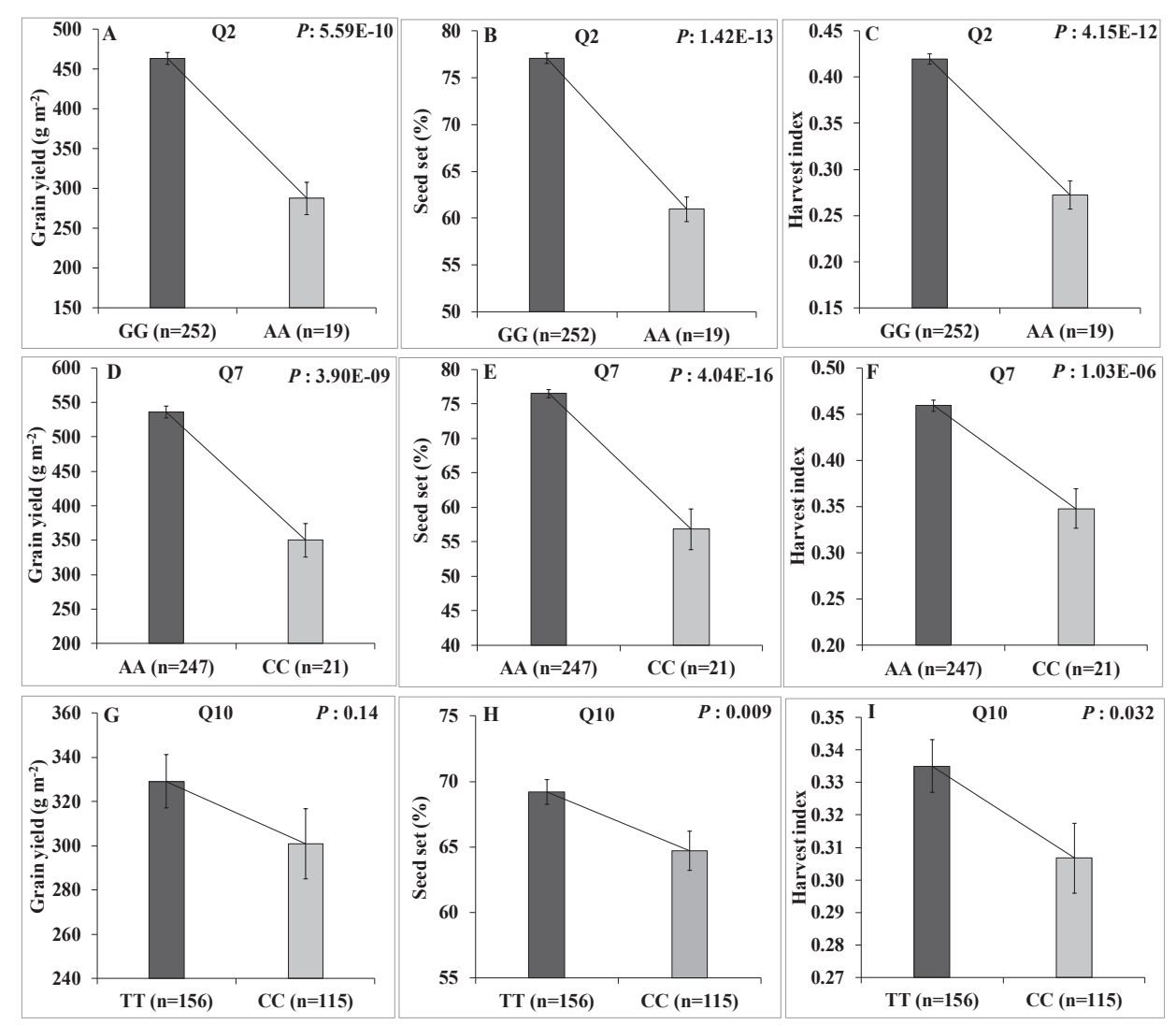

Figure 6: Allelic effect of Q2 (Panels A-C; 2013), Q7 (Panels D-F; 2014) in non-stress and Q10 (Panels G-I; 2013) in water-deficit stress conditions on grain yield, seed set and harvest index. Allelic effect of Q7 on harvest index was significant regardless of GWAS significance. Two sample t-test $P$ value showing significant allelic effect difference regarding major and minor allele.

Except for one locus on chromosome 12 for spikelets per $\mathrm{m}^{2}$ in 2014 , majority of the loci for grain yield and its component traits were specific to non-stress or stress conditions in both years. These results are in agreement with previous studies in rice (Lanceras et al., 2004; Vikram et al., 2011; Kumar et al., 2014) and other crop species (Yin et al., 2002; Millet et al., 2016). Hence, the greater dependence on environments appeared to be a common characteristic of QTLs, although this does not negate their importance in marker-assisted selection (MAS). Despite the strong variation in weather, we also detected four consistent loci: one each for panicles per $\mathrm{m}^{2}$ and spikelets per panicle on chromosome $10(19,903,199)$ and $4(23,423,399)$ respectively, and two loci on chromosome $2(30,699,332)$ and $5(53,664,89)$ for thousand grain weight across years in non-stress conditions (Supplementary Table S7). These consistent 

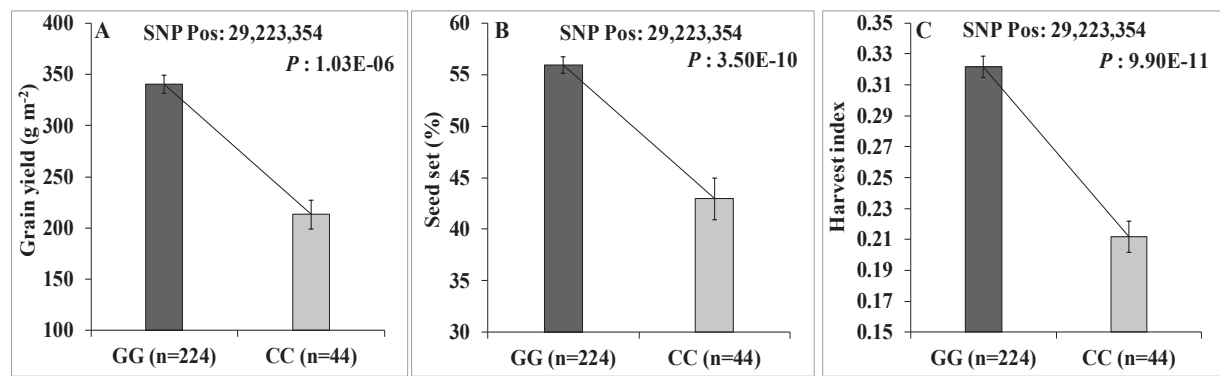

Figure 7: Allelic effect of chromosome 1 locus $(29,223,354)$ on grain yield (Panel A), seed set (Panel B), and harvest index (Panel C) in 2014 water-deficit stress conditions. Allelic effect on grain yield was significant regardless of GWAS significance. Two sample t-test $P$ value showing significant allelic effect difference regarding major and minor allele.

regions with favourable alleles could be used for improving yield.

The PRAY population have been previously used in GWAS for a range of phenotypic traits (Qiu et al., 2016; Al-Tamimi et al., 2016; Rebolledo et al., 2016; Kikuchi et al., 2017; Kadam et al., 2017). When comparing our results with those of these previous studies, we could not find any overlap between significant markers, except for plant height for which we detected a SNP marker (position: 38286772) that was also detected in our previous study (Kadam et al. 2017). The most likely reasons for such variation in results are the difference in target phenotypic traits, type and timing of stress treatments, population size, and genotypic marker data used by previous studies. Further, there was also no overlap of significant marker for grain yield and its components when comparing to other studies conducted under reproductive stage water-deficit for similar traits using different mapping panels (Ma et al., 2016; Pantalião et al., 2016; Swamy et al., 2017). The major reasons for this may be different rice accessions, population size and inherent environmental and field variation for stress treatment. Another possible reason could be use of indica subspecies genotypes in this study while previous studies either used japonica subspecies (Pantalião et al., 2016) or small population size ( 75 genotypes) with SSR markers for mapping (Swamy et al., 2017). In addition, it can be expected that the genomic regions/genes determining the trait difference across subspecies/genotypes could be difficult to identify.

\section{Seed set regulates the assimilate partitioning and grain yield}

Better optimisation of assimilate partitioning to reproductive organs with minimal competition among reproductive organs is essential to achieve stable and higher grain yield. So far, the physiological and genetic basis of above processes has been poorly understood in rice and other 
cereal crops. Our study showed that the co-localisation of grain yield loci with its components was rare. However, four genetic loci namely Q2, Q7 in non-stress, Q10 and 29,223,354 (SNP position) in stress conditions were regulating the grain yield and harvest index through changes in the seed set (Figs. 6-7). This indicates that the seed set is a critical determinant of assimilate partitioning (harvest index), thereby regulating the final expression of grain yield. A recent GWAS analysis confirmed these interactions in wheat (Guo et al., 2017). Hence, these identified loci could be pyramided into an "ideotype" at genomic level through MAS to enhance rice grain yield in non-stress and stress conditions. In addition, such loci could also be interesting in identifying the physiological and molecular basis of assimilates partitioning to reproductive organs.

\section{Promising a priori candidate genes for grain yield and water-deficit stress resilience}

We detected a priori candidate genes near of peak SNP/SNPs within the LD block for grain yield loci (Supplementary Table S9). A priori candidate genes of grain yield loci can indicate possible roles of underlying physiological (SET kinase, sugar and nitrate transporter genes) and reproductive developmental (Plastocyanin gene) processes in regulating the grain yield. Likewise, the abiotic stress tolerance candidate genes were detected near to grain yield loci in water-deficit, of which genes regulating the detoxification of ROS (Phosphomannomutase and Squalene epoxidase genes) seem to be critical in rice stress tolerance (Pyngrope et al., 2013; Selote and Chopra, 2004). In addition, these candidate genes need to be considered to detect the most likely causal genes. However, detailed large-scale molecular validations need to be conducted using the available approaches of RNAi, knockout mutants and transgenic overexpression. Similarly, the loci for components and related traits that were not co-localised with grain yield per se, could also be an interesting candidate for further identification of novel genes.

\section{Concluding remarks}

This study provides the genetic basis of grain yield of rice, its components and related traits in non-stress and stress conditions in field phenotyping experiments. We detected several favourable alleles regulating these traits that, upon validation, can be effectively used in improving grain yield. Additional genetic loci with limited overlap of yield component traits to grain yield per se clearly indicate the independent genetic architectures of these traits. Thus, many loci for component traits had an indirect effect on yield, which cannot be detected while 
mapping yield directly. This indicates the complexity of yield as a trait despite moderate to high $h^{2}$ which is most often used as a selection criterion to improve yield potential and stress tolerance. Hence, future studies should also explore the genetic basis of individual component traits that are genetically less complex-an approach expected to give additional useful information to further enhance yield. Present study suggest that maintenance of higher seed set is a vital component for enhancing yield potential and water-deficit tolerance. 
Supplementary information in Chapter 4 


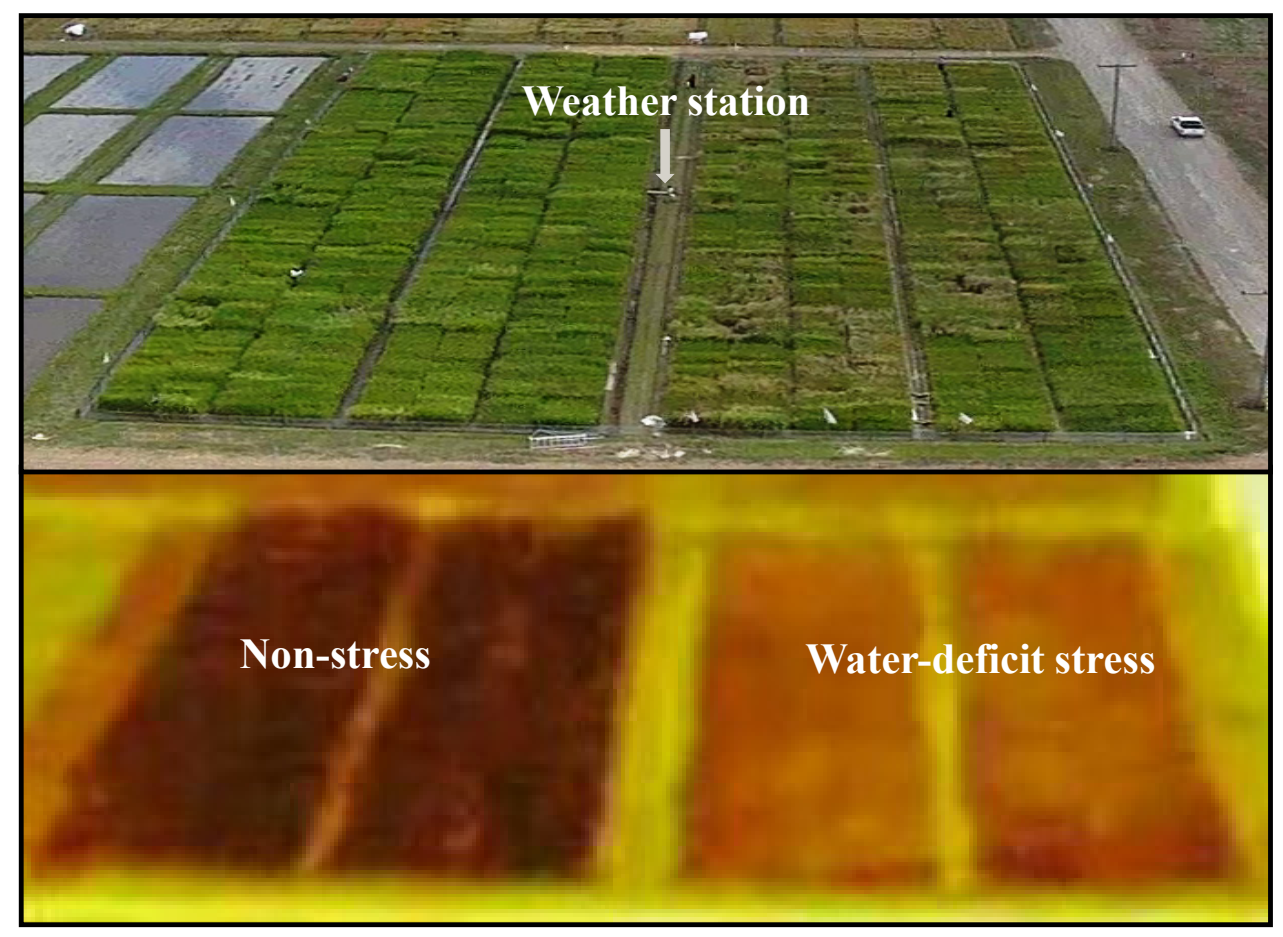

Supplementary Figure S1: Field set-up of 296 genotypes screened under non-stress and reproductive stage waterdeficit stress in 2013 and 2014 experiments. Aerial picture of experiment plot taken in 2014 and lower panel was the thermal image taken during stress period showing canopy temperature difference in non-stress and waterdeficit stress conditions. 
A

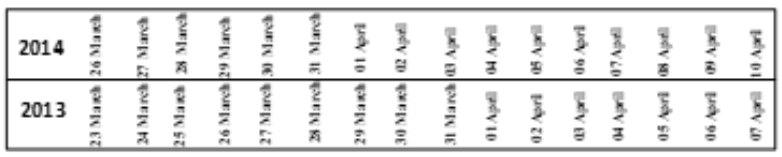

Days of stress period

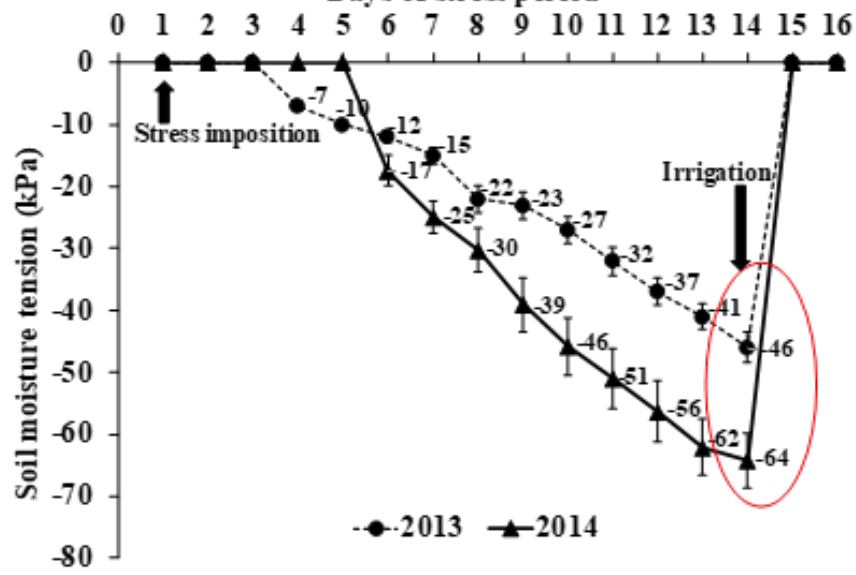

B

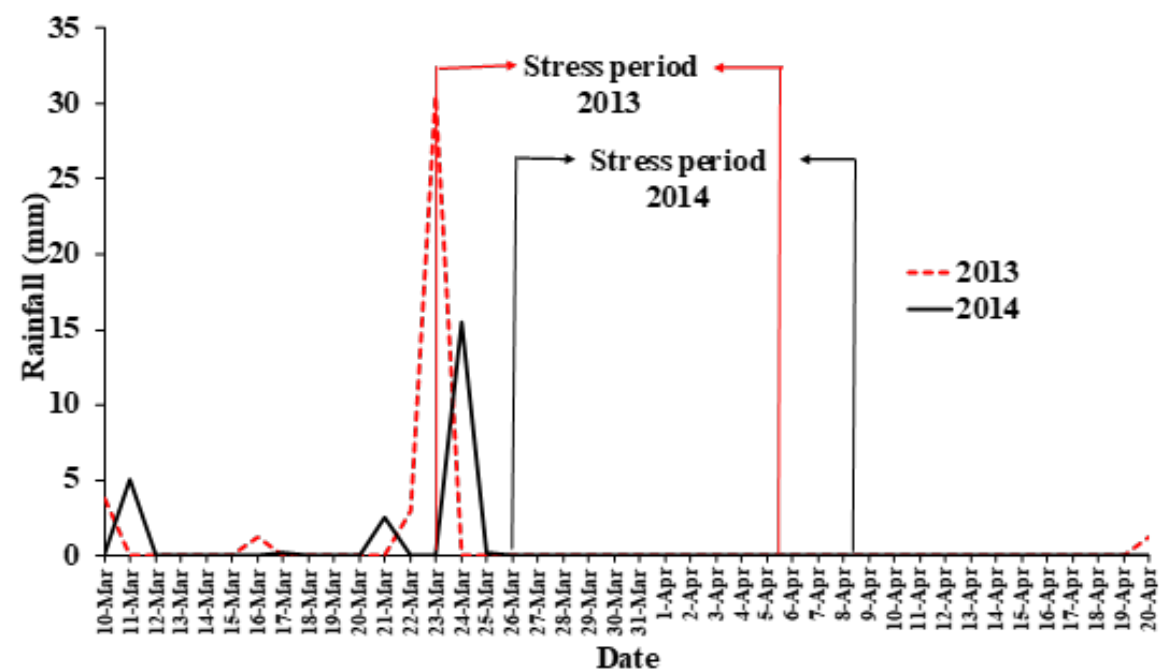

Supplementary Figure S2: Soil moisture tension measured using tensiometers in water-deficit stress field during 2013 and 2014 (Panel A), and rainfall pattern measured during stress period in 2013 and 2014 (Panel B). Soil moisture was measured using the 26 tensiometers randomly placed in the stress field at $30 \mathrm{~cm}$ depth and numbers above the symbols in Fig. 2A are the average soil moisture tension from 26 tensiometers. 

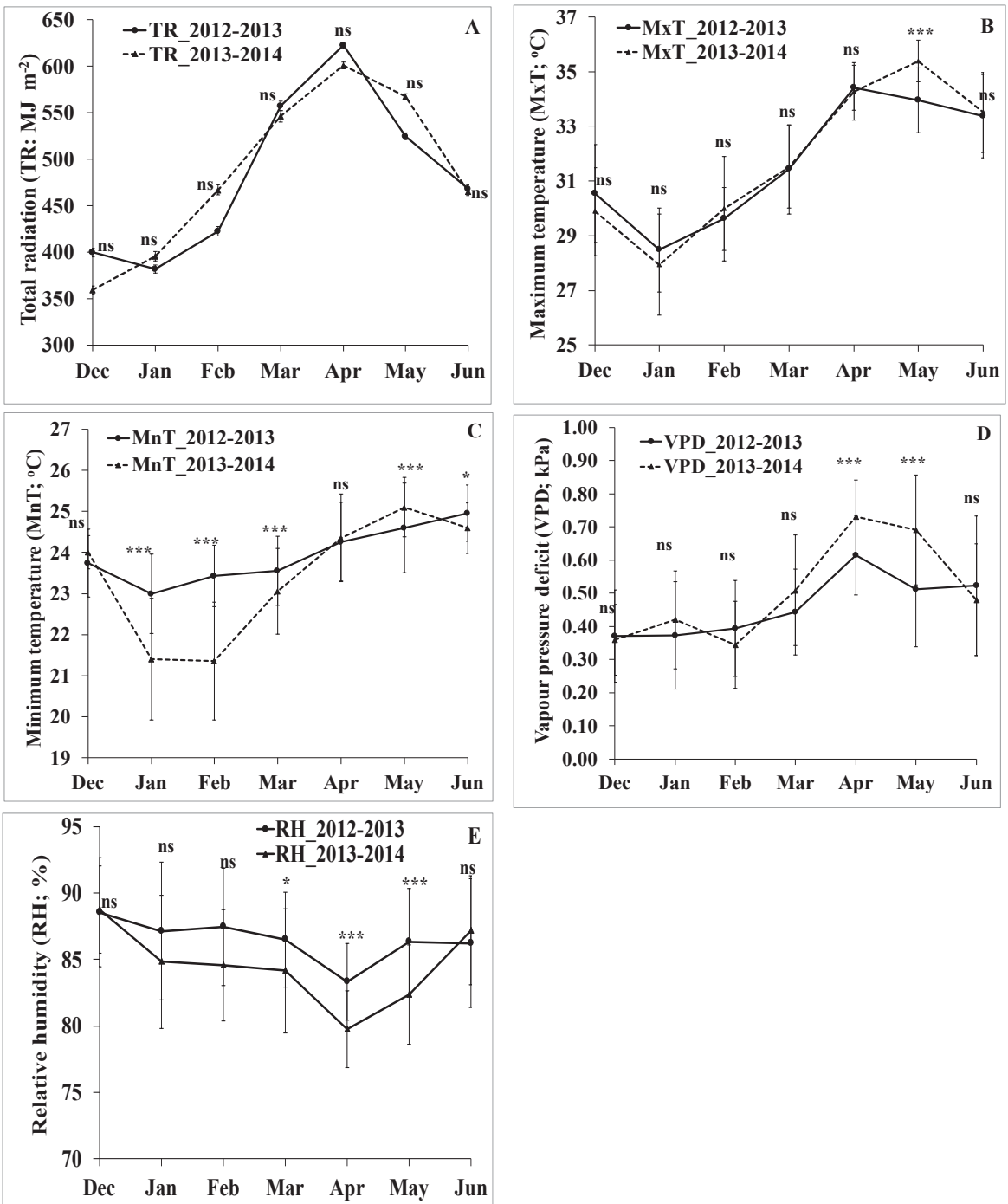

Supplementary Figure S3: Climate parameters observed during the growing period: Total radiation (Panel A), maximum temperature (Panel B), minimum temperature (Panel C), vapour pressure deficit (Panel D) and relative humidity (Panel E). Bar represent standard deviation. Paired t-test $P$ value is used to compare the monthly climate difference across years with significance level of $* P<0.05, * * P<0.01, * * * P<0.001$, and ns = non-significant. 


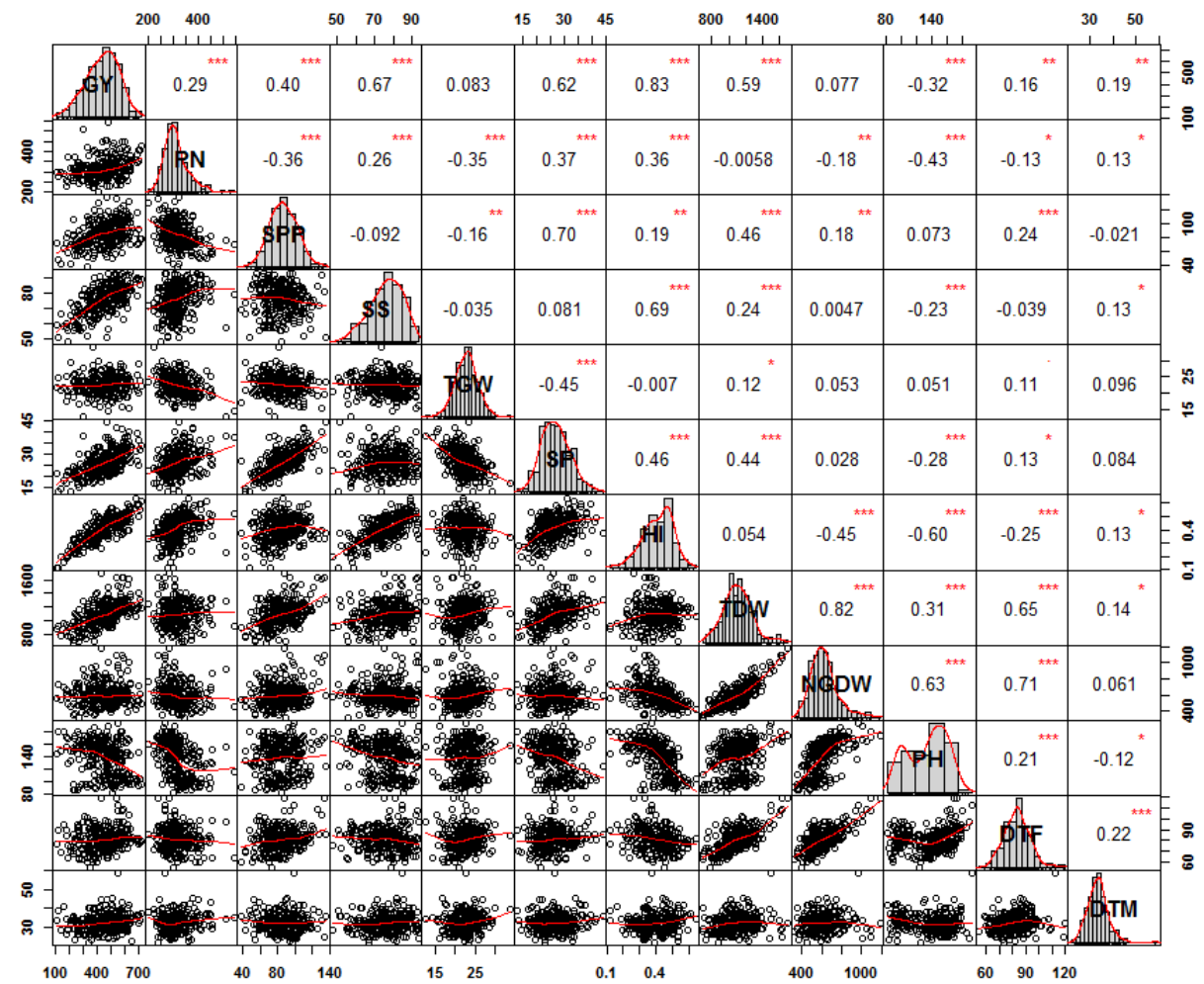

Supplementary Figure S4: Pearson correlation coefficient between grain yield and its components and related traits in 2013 non-stress conditions. Phenotypic traits with their histograms are given in the diagonal panel. Lower diagonal panel represents the scatter plot with red line depicting the best fit. The upper panel represents the Pearson correlation coefficient value and size of the correlation coefficient is proportional to the strength of the correlation. The correlation coefficient significance level: ${ }^{*} P<0.05,{ }^{*} P<0.01,{ }^{*} * * P<0.001$. GY=grain yield; $\mathrm{PN}=$ panicles per $\mathrm{m}^{2} ; \mathrm{SPP}=$ spikelets per panicle; $\mathrm{SS}=$ seed set; TGW=thousand grain weight; $\mathrm{SP}=$ spikelets per $\mathrm{m}^{2} ; \mathrm{HI}=$ harvest index; $\mathrm{TDW}=$ total dry weight; $\mathrm{NGDW}=$ non-grain dry weight; $\mathrm{PH}=$ plant height; $\mathrm{DTF}=$ days to flowering; $\mathrm{DTM}=$ days to maturity. 


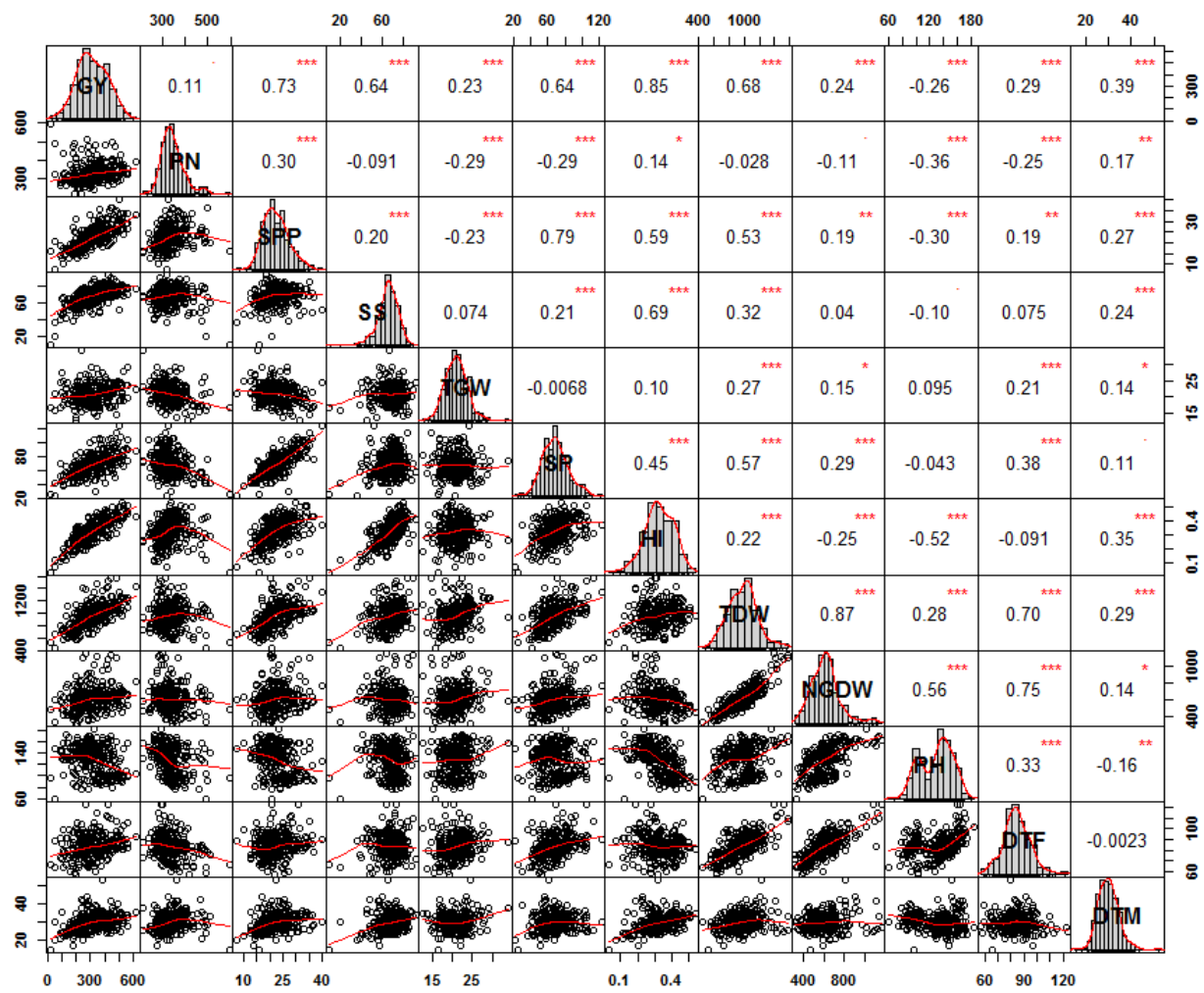

Supplementary Figure S5: Pearson correlation coefficient between grain yield and its components and related traits in 2013 water-deficit stress conditions. Phenotypic traits with their histograms are given in the diagonal panel. Lower diagonal panel represents the scatter plot with red line depicting the best fit. The upper panel represents the Pearson correlation coefficient value and size of the correlation coefficient is proportional to the strength of the correlation. The correlation coefficient significance level: ${ }^{*} P<0.05, * * P<0.01,{ }^{* * *} P<0.001$. $\mathrm{GY}=$ grain yield; $\mathrm{PN}=$ panicles per $\mathrm{m}^{2}$; $\mathrm{SPP}=$ spikelets per panicle; $\mathrm{SS}=$ seed set; $\mathrm{TGW}=$ thousand grain weight; $\mathrm{SP}=$ spikelets per $\mathrm{m}^{2} ; \mathrm{HI}=$ harvest index; TDW=total dry weight; $\mathrm{NGDW}=$ non-grain dry weight; $\mathrm{PH}=$ plant height; $\mathrm{DTF}=$ days to flowering; $\mathrm{DTM}=$ days to maturity. 


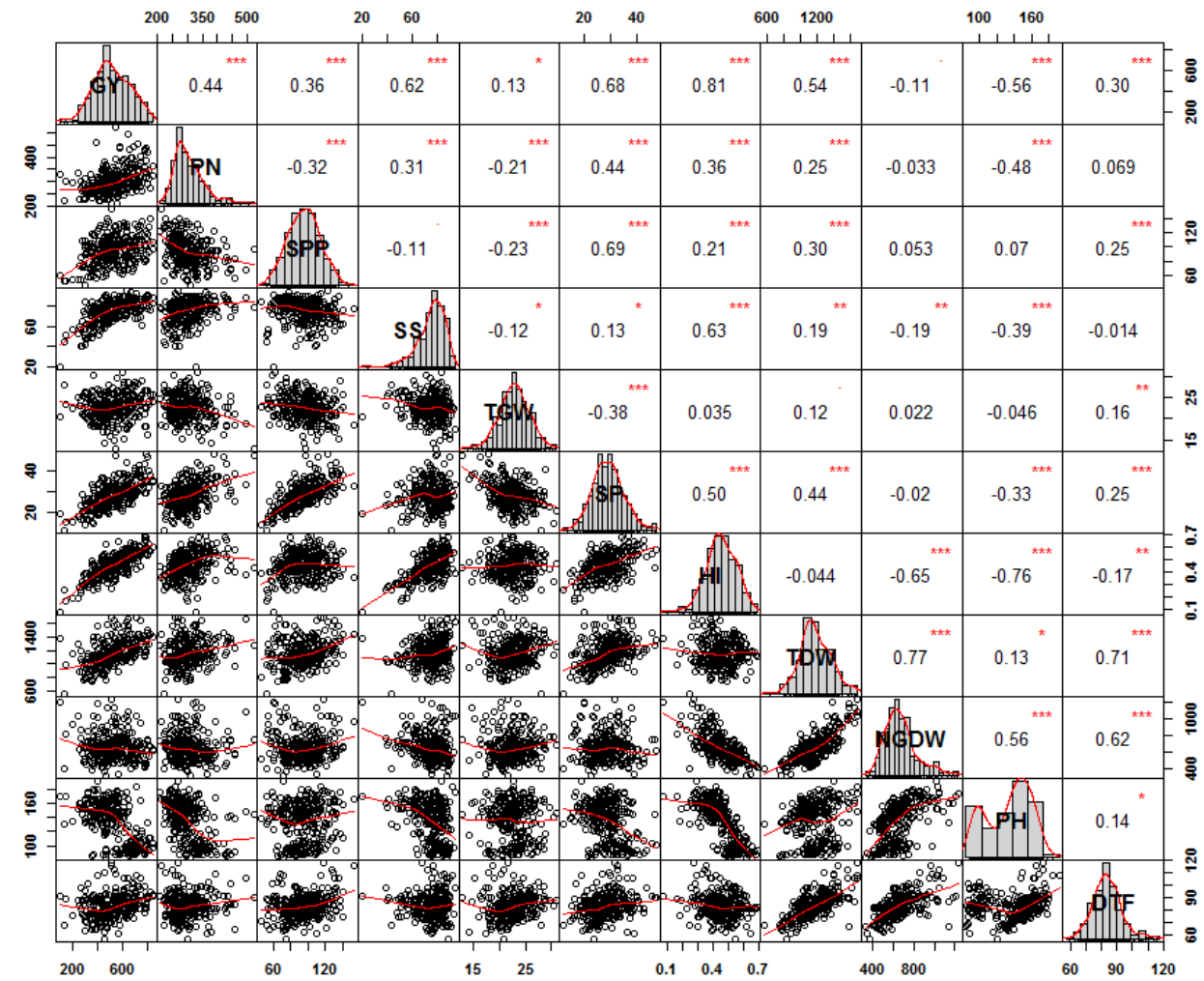

Supplementary Figure S6: Pearson correlation coefficient between grain yield and its components and related traits in 2014 non-stress conditions. Phenotypic traits with their histograms are given in the diagonal panel. Lower diagonal panel represents the scatter plot with the red line depicting the best fit. The upper panel represents the Pearson correlation coefficient value and size of the correlation coefficient is proportional to the strength of the correlation. The correlation coefficient significance level: ${ }^{*} P<0.05, * * P<0.01, * * * P<0.001$. GY=grain yield; $\mathrm{PN}=$ panicles per $\mathrm{m}^{2} ; \mathrm{SPP}=$ spikelets per panicle; $\mathrm{SS}=$ seed set; $\mathrm{TGW}=$ thousand grain weight; $\mathrm{SP}=$ spikelets per $\mathrm{m}^{2}$; $\mathrm{HI}=$ harvest index; TDW=total dry weight; $\mathrm{NGDW}=$ non-grain dry weight; $\mathrm{PH}=$ plant height; $\mathrm{DTF}=$ days to flowering. 


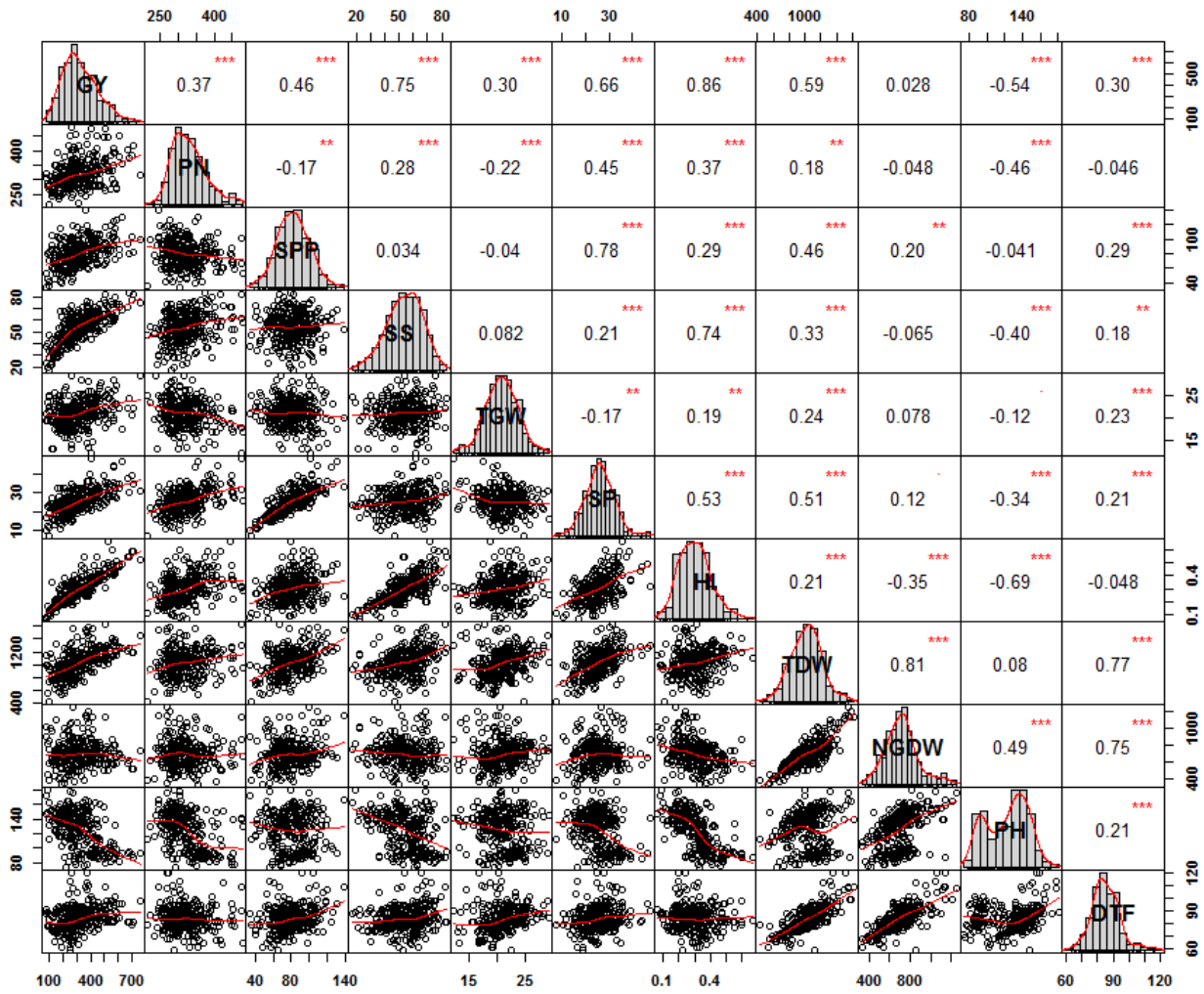

Supplementary Figure S7: Pearson correlation coefficient between grain yield and its components and related traits in 2014 water-deficit stress conditions. Phenotypic traits with their histograms are given in the diagonal panel. Lower diagonal panel represents the scatter plot with the red line depicting the best fit. The upper panel represents the Pearson correlation coefficient value and size of the correlation coefficient is proportional to the strength of the correlation. The correlation coefficient significance level: ${ }^{*} P<0.05, * * P<0.01,{ }^{*} * * P<0.001$. $\mathrm{GY}=$ grain yield; $\mathrm{PN}=$ panicles per $\mathrm{m}^{2}$; $\mathrm{SPP}=$ spikelets per panicle; $\mathrm{SS}=$ seed set; $\mathrm{TGW}=$ thousand grain weight; $\mathrm{SP}=$ spikelets per $\mathrm{m}^{2} ; \mathrm{HI}=$ harvest index; TDW=total dry weight; $\mathrm{NGDW=non-grain} \mathrm{dry} \mathrm{weight;} \mathrm{PH}=$ plant height; $\mathrm{DTF}=$ days to flowering. 

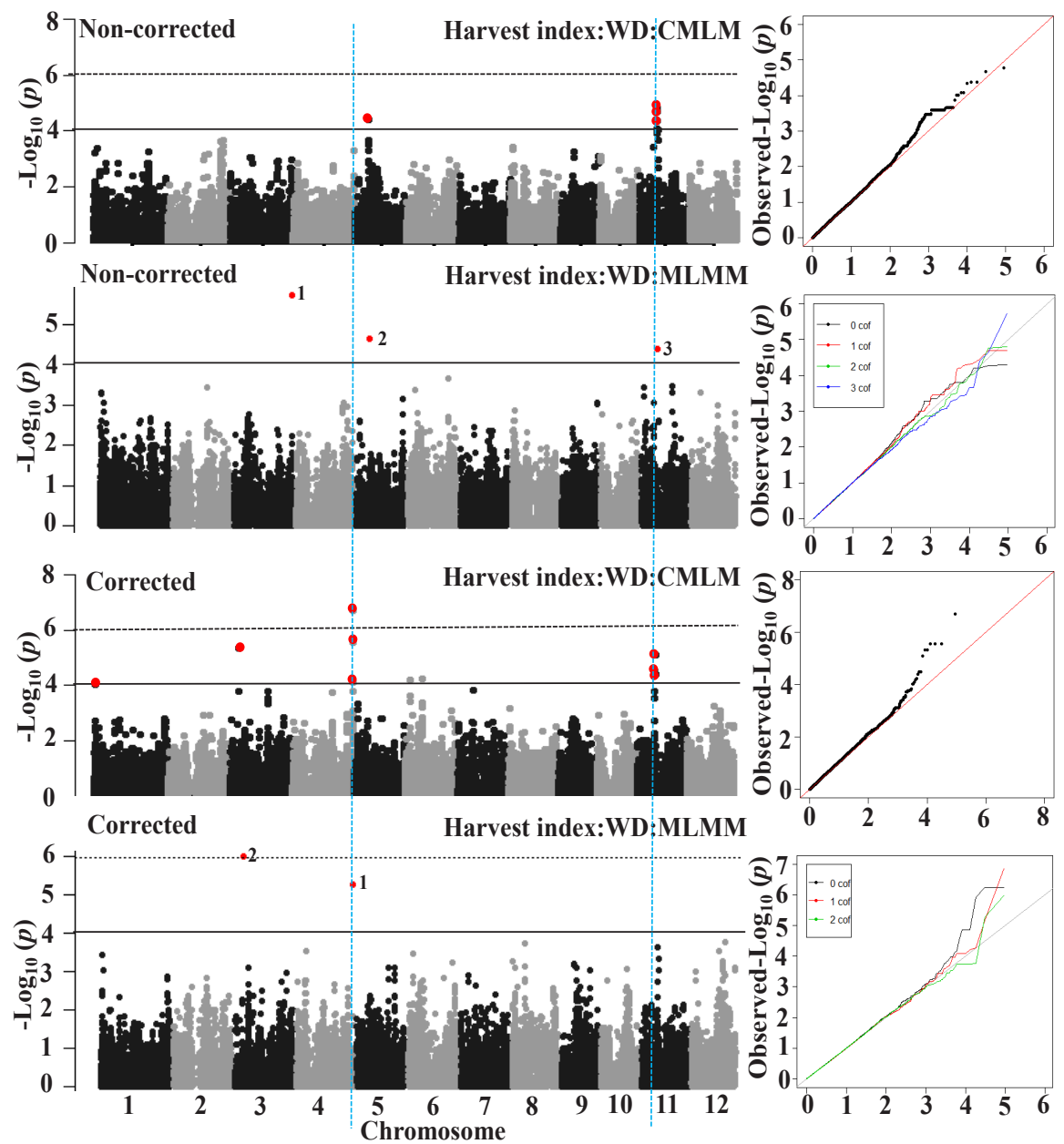

Supplementary Figure S8: GWAS results (Manhattan and Quantile-Quantile plot) detected through single-locus compressed mixed linear model (CMLM) and multi-locus mixed model (MLMM) for non-corrected and corrected harvest index (using days to flowering as a covariate) in 2013 water-deficit stress (WD) conditions. Significant SNPs in Manhattan plot of MLMM were numbered according to order in which they were included as a cofactor in regression model. 

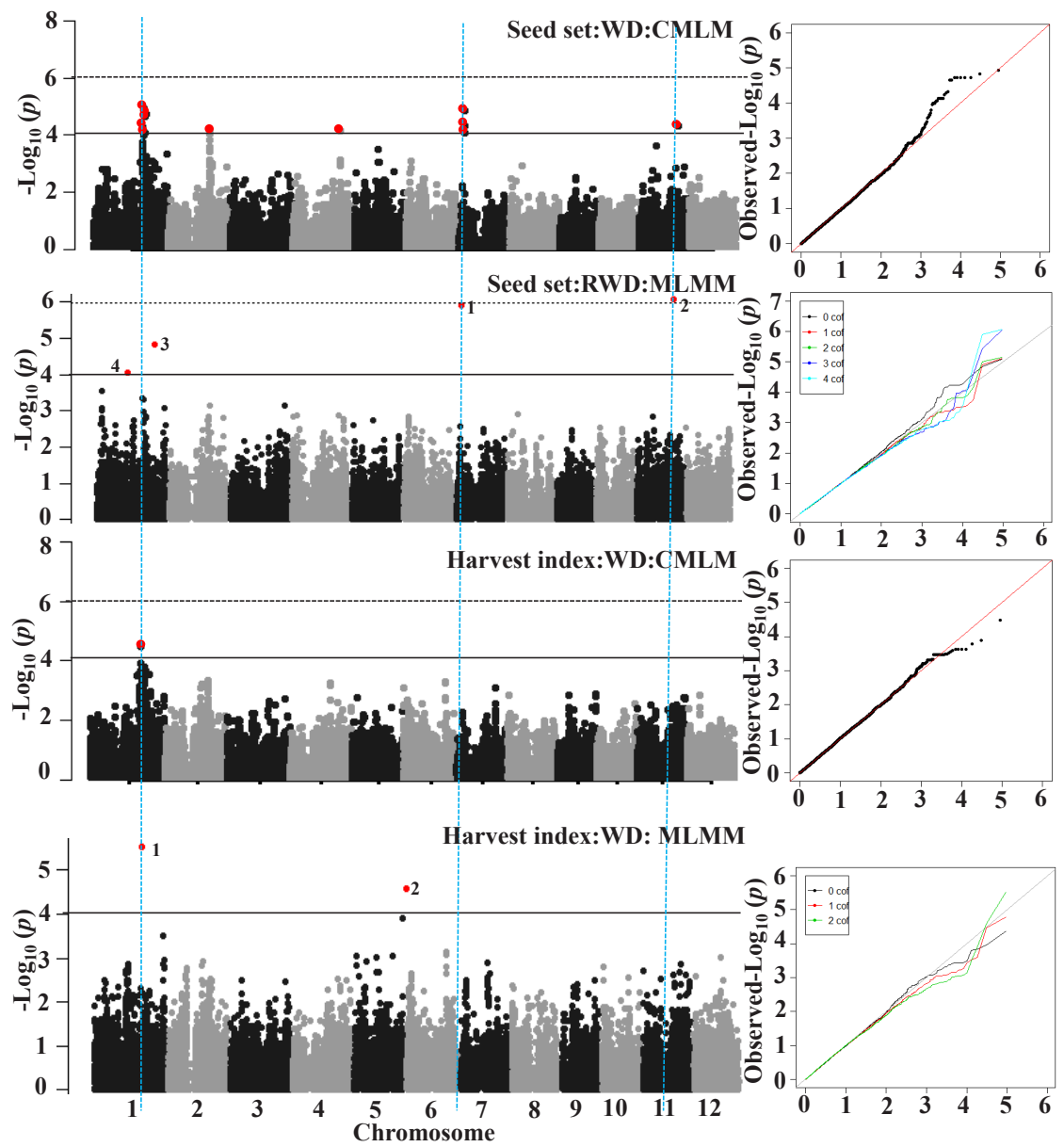

Supplementary Figure S9: GWAS results (Manhattan and Quantile-Quantile plot) detected through single-locus compressed mixed linear model (CMLM) and multi-locus mixed model (MLMM) for seed-set and harvest index in 2014 water-deficit stress (WD) conditions. Significant SNPs in Manhattan plot of MLMM were numbered according to order in which they were included as a cofactor in regression model. 
Supplementary Table S1: Summary statistics of grain yield and its components and related traits in 2013 and 2014 non-stress (NS) and water-deficit stress (WD) conditions.

\begin{tabular}{|c|c|c|c|c|c|c|c|c|c|c|c|}
\hline & & \multicolumn{5}{|c|}{2013} & \multicolumn{5}{|c|}{2014} \\
\hline Trait & Trt & $\mathbf{M} \pm$ SD & Min & Max & $\mathbf{H}^{2}$ & $h^{2}$ & $\mathbf{M} \pm \mathbf{S D}$ & Min & Max & $\mathbf{H}^{2}$ & $h^{2}$ \\
\hline \multirow{2}{*}{ GY } & NS & $451.1 \pm 123.2$ & 106.3 & 727.0 & 0.92 & 0.27 & $521.9 \pm 143.3$ & 102.8 & 839.7 & 0.93 & 0.75 \\
\hline & WD & $317.3 \pm 112.9$ & 16.7 & 622.6 & 0.84 & 0.64 & $319.5 \pm 134.3$ & 78.1 & 761.1 & 0.73 & 0.73 \\
\hline \multicolumn{12}{|c|}{ Grain yield component traits } \\
\hline \multirow{2}{*}{ PN } & NS & $316.8 \pm 56.7$ & 200.8 & 540.8 & 0.82 & 0.88 & $302.7 \pm 51.7$ & 212.0 & 520.3 & 0.89 & 0.92 \\
\hline & WD & $340.4 \pm 69.5$ & 219.2 & 593.6 & 0.87 & 0.87 & $321.0 \pm 50.0$ & 215.1 & 479.2 & 0.80 & 0.90 \\
\hline \multirow{2}{*}{ SPP } & NS & $84.9 \pm 17.6$ & 37.8 & 136.3 & 0.79 & 0.92 & $95.1 \pm 20.0$ & 46.4 & 153.2 & 0.89 & 0.90 \\
\hline & WD & $68.3 \pm 16.0$ & 22.8 & 122.9 & 0.77 & 0.77 & $80.4 \pm 18.3$ & 34.0 & 139.3 & 0.70 & 0.92 \\
\hline \multirow{2}{*}{ SS } & NS & $76.0 \pm 9.6$ & 47.7 & 93.7 & 0.85 & 0.65 & $75.0 \pm 11.3$ & 19.7 & 94.9 & 0.89 & 0.75 \\
\hline & WD & $67.3 \pm 10.5$ & 10.4 & 91.8 & 0.73 & 0.83 & $53.8 \pm 13.0$ & 17.2 & 83.3 & 0.62 & 0.72 \\
\hline \multirow{2}{*}{ TGW } & NS & $22.5 \pm 2.9$ & 12.5 & 34.0 & 0.99 & 0.86 & $22.6 \pm 3.0$ & 12.9 & 30.9 & 0.99 & 0.88 \\
\hline & WD & $20.9 \pm 3.0$ & 12.6 & 34.1 & 0.97 & 0.85 & $21.1 \pm 3.4$ & 12.3 & 29.1 & 0.77 & 0.90 \\
\hline \multirow{2}{*}{ SP } & NS & $26.3 \pm 5.4$ & 13.1 & 43.8 & 0.88 & 0.88 & $28.4 \pm 6.2$ & 11.7 & 47.8 & 0.91 & 0.92 \\
\hline & WD & $22.5 \pm 5.3$ & 7.3 & 40.1 & 0.85 & 0.84 & $25.5 \pm 6.3$ & 7.6 & 47.7 & 0.83 & 0.93 \\
\hline \multicolumn{12}{|c|}{ Grain yield related traits } \\
\hline \multirow{2}{*}{ HI } & NS & $0.4 \pm 0.09$ & 0.1 & 0.6 & 0.93 & 0.44 & $0.4 \pm 0.1$ & 0.08 & 0.7 & 0.95 & 0.90 \\
\hline & WD & $0.3 \pm 0.09$ & 0.03 & 0.5 & 0.83 & 0.63 & $0.3 \pm 0.1$ & 0.07 & 0.7 & 0.66 & 0.69 \\
\hline \multirow{2}{*}{ TDW } & NS & $1.1 \pm 0.1$ & 0.7 & 1.7 & 0.87 & 0.80 & $1.1 \pm 0.2$ & 0.5 & 1.7 & 0.89 & 0.80 \\
\hline & WD & $0.9 \pm 0.2$ & 0.4 & 1.5 & 0.90 & 0.87 & $1.0 \pm 0.2$ & 0.4 & 1.6 & 0.86 & 0.83 \\
\hline \multirow{2}{*}{ NGDW } & NS & $0.6 \pm 0.1$ & 0.3 & 1.1 & 0.93 & 0.94 & $0.6 \pm 0.1$ & 0.3 & 1.2 & 0.92 & 0.90 \\
\hline & WD & $0.6 \pm 0.1$ & 0.3 & 1.1 & 0.92 & 0.90 & $0.7 \pm 0.1$ & 0.3 & 1.2 & 0.89 & 0.89 \\
\hline \multirow{2}{*}{$\mathrm{PH}$} & NS & $136.6 \pm 27.6$ & 81.8 & 193.9 & 0.98 & 0.88 & $135.2 \pm 27.8$ & 84.3 & 191.4 & 0.98 & 0.86 \\
\hline & WD & $128.1 \pm 25.6$ & 59.7 & 184.1 & 0.97 & 0.90 & $125.0 \pm 27.0$ & 74.0 & 280.1 & 0.90 & 0.86 \\
\hline \multirow{2}{*}{ DTF } & NS & $81.0 \pm 10.4$ & 54.7 & 119.7 & 0.98 & 0.88 & $83.3 \pm 9.8$ & 57.3 & 117.7 & 0.99 & 0.91 \\
\hline & WD & $83.8 \pm 10.8$ & 57.3 & 123.3 & 0.99 & 0.88 & $84.4 \pm 9.9$ & 59.7 & 120.3 & 0.96 & 0.90 \\
\hline \multirow{2}{*}{ DTM } & NS & $32.3 \pm 4.7$ & 21.7 & 59.0 & 0.84 & 0.31 & - & - & - & - & - \\
\hline & WD & $29.7 \pm 4.7$ & 14.8 & 53.0 & 0.86 & 0.47 & - & - & - & - & - \\
\hline
\end{tabular}

$\mathrm{Trt}=$ treatment $\mathrm{M} \pm \mathrm{SD}=$ mean and standard deviation; $\mathrm{Min}=$ minimum; $\mathrm{Max}=$ maximum; $\mathrm{H}^{2}=$ broad-sense heritability; $h^{2}=$ narrow-sense heritability. $\mathrm{GY}=$ grain yield; $\mathrm{PN}=$ panicles per $\mathrm{m}^{2}$; $\mathrm{SPP}=$ spikelets per panicle; $\mathrm{SS}=$ seed set; $\mathrm{TGW}=$ thousand grain weight; $\mathrm{SP}=$ spikelets per $\mathrm{m}^{2} ; \mathrm{HI}=$ harvest index; $\mathrm{TDW}=$ total dry weight; $\mathrm{NGDW}=$ non-grain dry weight; $\mathrm{PH}=$ plant height; $\mathrm{DTF}=$ days to flowering; $\mathrm{DTM}=$ days to maturity. Data for days to maturity was not available for 2014 experiment. 
Supplementary Table S2: Multiple linear regression of grain yield with its components and related traits in nonstress and water-deficit stress conditions during 2013 and 2014. Note that spikelets per $\mathrm{m}^{2}$, harvest index and total dry weight were not included in the regression because spikelets per $\mathrm{m}^{2}$ is the product of panicles and spikelets per panicles, and not an independent component. Similarly harvest index is the ratio of grain yield to total dry weight. Total dry weight is the sum of non-grain dry weight and grain dry weight.

\begin{tabular}{|c|c|c|c|c|}
\hline Year & Treatment & Traits & $P$-value & $\mathbf{R}^{2}$ \\
\hline \multirow{12}{*}{2013} & \multirow{6}{*}{ Non-stress } & Panicles & $<0.001$ & \multirow{6}{*}{0.89} \\
\hline & & Spikelets per panicle & $<0.001$ & \\
\hline & & Seed set & $<0.001$ & \\
\hline & & Thousand grain weight & $<0.001$ & \\
\hline & & Plant height & 0.069 & \\
\hline & & Days to flowering & $\mathbf{0 . 0 5 5}$ & \\
\hline & \multirow{6}{*}{ Water-deficit } & Panicles & $<0.001$ & \multirow{6}{*}{0.88} \\
\hline & & Spikelets per panicle & $<0.001$ & \\
\hline & & Seed set & $<0.001$ & \\
\hline & & Thousand grain weight & $<0.001$ & \\
\hline & & Plant height & $<0.001$ & \\
\hline & & Days to flowering & $<0.001$ & \\
\hline \multirow{12}{*}{2014} & \multirow{6}{*}{ Non-stress } & Panicles & $<0.001$ & \multirow{6}{*}{0.94} \\
\hline & & Spikelets & $<0.001$ & \\
\hline & & Seed set & $<0.001$ & \\
\hline & & Thousand grain weight & $<0.001$ & \\
\hline & & Plant height & $<0.001$ & \\
\hline & & Days to flowering & $<0.01$ & \\
\hline & \multirow{6}{*}{ Water-deficit } & Panicles & $<0.001$ & \multirow{6}{*}{0.93} \\
\hline & & Spikelets per panicle & $<0.001$ & \\
\hline & & Seed set & $<0.001$ & \\
\hline & & Thousand grain weight & $<0.001$ & \\
\hline & & Plant height & $<0.001$ & \\
\hline & & Days to flowering & 0.404 & \\
\hline
\end{tabular}


Supplementary Table S3. The details of genetic loci detected for grain yield components and related traits in 2013 non-stress conditions using compressed mixed linear-model (CMLM) and multi-locus mixed model (MLMM) methods. The chromosome loci detected through both the methods were marked by asterisk sign (*); those detected through only by MLMM were marked by hashtag (\#) sign. The remaining all unmarked chromosome loci were detected only through CMLM method. Trait acronyms are given in Table 1 .

\begin{tabular}{|c|c|c|c|c|c|c|c|c|c|c|c|}
\hline \multirow[t]{2}{*}{ Traits } & \multirow[t]{2}{*}{ Chr } & \multirow[t]{2}{*}{ Pos } & \multirow[t]{2}{*}{ Allele } & \multirow[t]{2}{*}{ MAF } & \multirow[t]{2}{*}{$P$ valuecmlм } & \multirow[t]{2}{*}{$P$ valueмгмм } & \multirow[t]{2}{*}{$\mathbf{A E}$} & \multicolumn{2}{|c|}{ LD block } & \multirow[t]{2}{*}{ Size(kb) } & \multirow[t]{2}{*}{$\begin{array}{c}\text { Known } \\
\text { genes }\end{array}$} \\
\hline & & & & & & & & Start & End & & \\
\hline \multicolumn{12}{|c|}{ Grain yield component } \\
\hline \multirow[t]{6}{*}{$\mathrm{PN}$} & 4 & 2481502 & $\mathrm{~T}: \mathrm{A}$ & 0.100 & $4.11 \mathrm{E}-05$ & - & 28.53 & 2463707 & 2502427 & 38 & 2 \\
\hline & 12 & 1691509 & $\mathrm{C}: \mathrm{G}$ & 0.066 & 4.69E-05 & - & 27.81 & 1594015 & 1691770 & 97 & 12 \\
\hline & 6 & 9369614 & C:A & 0.059 & 7.89E-05 & - & 32.90 & 9329842 & 9371867 & 42 & 4 \\
\hline & 12 & 1691771 & $\mathrm{C}: \mathrm{T}$ & 0.068 & 8.33E-05 & - & 26.60 & 1691771 & 1734838 & 43 & 7 \\
\hline & 4 & 23514625 & $\mathrm{~A}: \mathrm{C}$ & 0.118 & $9.81 \mathrm{E}-05$ & - & 24.72 & 23514625 & 23597658 & 83 & 3 \\
\hline & $10^{\#}$ & 19903199 & $\mathrm{~T}: \mathrm{A}$ & 0.148 & - & $8.47 \mathrm{E}-19$ & 51.58 & 19882559 & 19916740 & 34 & 3 \\
\hline \multirow[t]{5}{*}{ SPP } & $4^{*}$ & 23423399 & $\mathrm{~A}: \mathrm{G}$ & 0.288 & $4.70 \mathrm{E}-06$ & $1.23 \mathrm{E}-07$ & -7.15 & 23423240 & 23512064 & 88 & 11 \\
\hline & $11^{*}$ & 19641458 & $\mathrm{C}: \mathrm{T}$ & 0.391 & $5.47 \mathrm{E}-05$ & $1.20 \mathrm{E}-05$ & -5.13 & 19609894 & 19645174 & 35 & 4 \\
\hline & 4 & 23417928 & $\mathrm{C}: \mathrm{G}$ & 0.256 & $6.95 \mathrm{E}-05$ & - & -6.31 & 23357356 & 23417928 & 60 & 5 \\
\hline & $3^{\#}$ & 15094434 & G:A & 0.236 & $5.31 \mathrm{E}-06$ & - & 9.21 & 14873722 & 15132484 & 258 & 17 \\
\hline & $2^{\#}$ & 24278919 & $\mathrm{G}: \mathrm{C}$ & 0.332 & $2.13 \mathrm{E}-05$ & - & 9.09 & 24265692 & 24283607 & 17 & 4 \\
\hline \multirow[t]{3}{*}{ SS } & $2^{*}$ & 30523925 & G:A & 0.072 & $3.85 \mathrm{E}-05$ & $5.56 \mathrm{E}-07$ & -7.55 & 30397910 & 30541202 & 143 & 16 \\
\hline & $10^{\#}$ & 18906753 & G:C & 0.303 & - & $1.76 \mathrm{E}-05$ & 6.50 & 18898657 & 19018639 & 119 & 14 \\
\hline & $2^{\#}$ & 17591863 & $\mathrm{~T}: \mathrm{C}$ & 0.491 & - & $7.03 \mathrm{E}-05$ & -5.18 & 17591863 & 17806312 & 214 & 3 \\
\hline \multirow[t]{3}{*}{ TGW } & 2 & 308723 & A:T & 0.458 & $5.35 \mathrm{E}-05$ & - & -0.73 & 221193 & 338309 & 117 & 8 \\
\hline & $5^{*}$ & 5366489 & G:A & 0.387 & $5.45 \mathrm{E}-05$ & $1.79 \mathrm{E}-05$ & 0.82 & 5365520 & 5448285 & 82 & 6 \\
\hline & $2^{*}$ & 30699332 & $\mathrm{~T}: \mathrm{C}$ & 0.295 & $7.75 \mathrm{E}-05$ & $1.94 \mathrm{E}-05$ & 0.81 & 30684655 & 30784063 & 99 & 9 \\
\hline SP & $7^{*}$ & 22699138 & $\mathrm{~T}: \mathrm{C}$ & 0.185 & $3.18 \mathrm{E}-05$ & $2.06 \mathrm{E}-05$ & 2.04 & 22653977 & 22805994 & 152 & 12 \\
\hline \multicolumn{12}{|c|}{ Grain yield related traits } \\
\hline \multirow[t]{6}{*}{$\mathrm{HI}$} & 8 & 20255596 & G:T & 0.063 & $5.45 \mathrm{E}-06$ & - & -0.06 & 20221039 & 20450490 & 229 & 12 \\
\hline & $2^{*}$ & 30523925 & $\mathrm{G}: \mathrm{A}$ & 0.072 & $7.68 \mathrm{E}-06$ & $3.29 \mathrm{E}-10$ & -0.07 & 30397910 & 30541202 & 143 & 16 \\
\hline & 8 & 20221030 & $\mathrm{G}: \mathrm{A}$ & 0.068 & $1.12 \mathrm{E}-05$ & - & -0.05 & 20165675 & 20221035 & 55 & 5 \\
\hline & 8 & 20160760 & A:T & 0.066 & $1.23 \mathrm{E}-05$ & - & -0.06 & 20144631 & 20165644 & 21 & 1 \\
\hline & 10 & 2163454 & C:T & 0.493 & $7.00 \mathrm{E}-05$ & - & -0.02 & 2151405 & 2181552 & 30 & 1 \\
\hline & $7^{\#}$ & 17712506 & $\mathrm{~T}: \mathrm{C}$ & 0.240 & $2.79 \mathrm{E}-05$ & - & -0.05 & 17539335 & 17785193 & 245 & 7 \\
\hline \multirow[t]{3}{*}{ TDW } & 4 & 21345052 & G:C & 0.063 & $1.84 \mathrm{E}-05$ & - & 90.75 & 21337500 & 21360699 & 23 & 2 \\
\hline & 5 & 26477176 & A:G & 0.063 & $9.24 \mathrm{E}-05$ & - & 83.90 & 26473392 & 26847502 & 374 & 41 \\
\hline & $4^{\#}$ & 34815309 & $\mathrm{G}: \mathrm{A}$ & 0.055 & $3.11 \mathrm{E}-05$ & - & -209.0 & 34815277 & 34833179 & 17 & 5 \\
\hline \multirow[t]{3}{*}{ NGDW } & $2^{*}$ & 945729 & $\mathrm{~A}: \mathrm{T}$ & 0.225 & $1.14 \mathrm{E}-05$ & $5.10 \mathrm{E}-07$ & 43.23 & 944109 & 972602 & 28 & 4 \\
\hline & 10 & 19874918 & C:T & 0.232 & $9.57 \mathrm{E}-05$ & - & 36.54 & 19874875 & 19874918 & $44 b p$ & 0 \\
\hline & $12^{\#}$ & 24162384 & G:C & 0.306 & $2.18 \mathrm{E}-08$ & - & 83.60 & 24070904 & 24389670 & 318 & 17 \\
\hline \multirow[t]{2}{*}{$\mathrm{PH}$} & $1^{*}$ & 38286772 & $\mathrm{G}: \mathrm{A}$ & 0.292 & $2.75 \mathrm{E}-07$ & $9.46 \mathrm{E}-08$ & -12.37 & 38178239 & 38437530 & 259 & 29 \\
\hline & 1 & 34203951 & $\mathrm{~T}: \mathrm{A}$ & 0.454 & $9.80 \mathrm{E}-05$ & - & 5.99 & 34184887 & 34357192 & 172 & 6 \\
\hline \multirow[t]{3}{*}{ DTF } & 3 & 21686259 & $\mathrm{~T}: \mathrm{C}$ & 0.185 & $5.26 \mathrm{E}-05$ & - & 2.92 & 21660582 & 21686259 & 25 & 4 \\
\hline & 3 & 21686358 & $\mathrm{~T}: \mathrm{C}$ & 0.185 & $5.26 \mathrm{E}-05$ & - & 2.92 & 21686358 & 21944343 & 257 & 11 \\
\hline & $3^{\#}$ & 5113428 & $\mathrm{~T}: \mathrm{C}$ & 0.424 & $2.15 \mathrm{E}-05$ & - & -2.61 & 5021158 & 5167439 & 146 & 13 \\
\hline DTM & $2^{*}$ & 19163866 & $\mathrm{~T}: \mathrm{G}$ & 0.093 & $5.91 \mathrm{E}-05$ & $2.92 \mathrm{E}-05$ & 2.08 & 19151240 & 19163870 & 12 & 1 \\
\hline
\end{tabular}

Chr=chromosome; Pos= physical position of SNP; MAF=minor allele frequency; $\mathrm{AE}=$ allelic effect regarding the minor allele (average traits value of genotypes carrying minor allele - average traits value of genotypes carrying major allele). $\mathrm{LD}=$ linkage disequilibrium. Known genes $=$ total known genes observed within the LD block. 
Supplementary Table S4. The details of genetic loci detected for uncorrected grain yield, its components and related traits in 2013 water-deficit stress conditions using compressed mixed linear-model (CMLM) and multilocus mixed model (MLMM) methods. The chromosome loci detected through both the methods were marked by asterisk sign (*); those detected through only by MLMM were marked by hashtag (\#) sign. The remaining all unmarked chromosome loci were detected only through CMLM method. Trait acronyms are given in Table 1. For legends see the Supplementary Table S3.

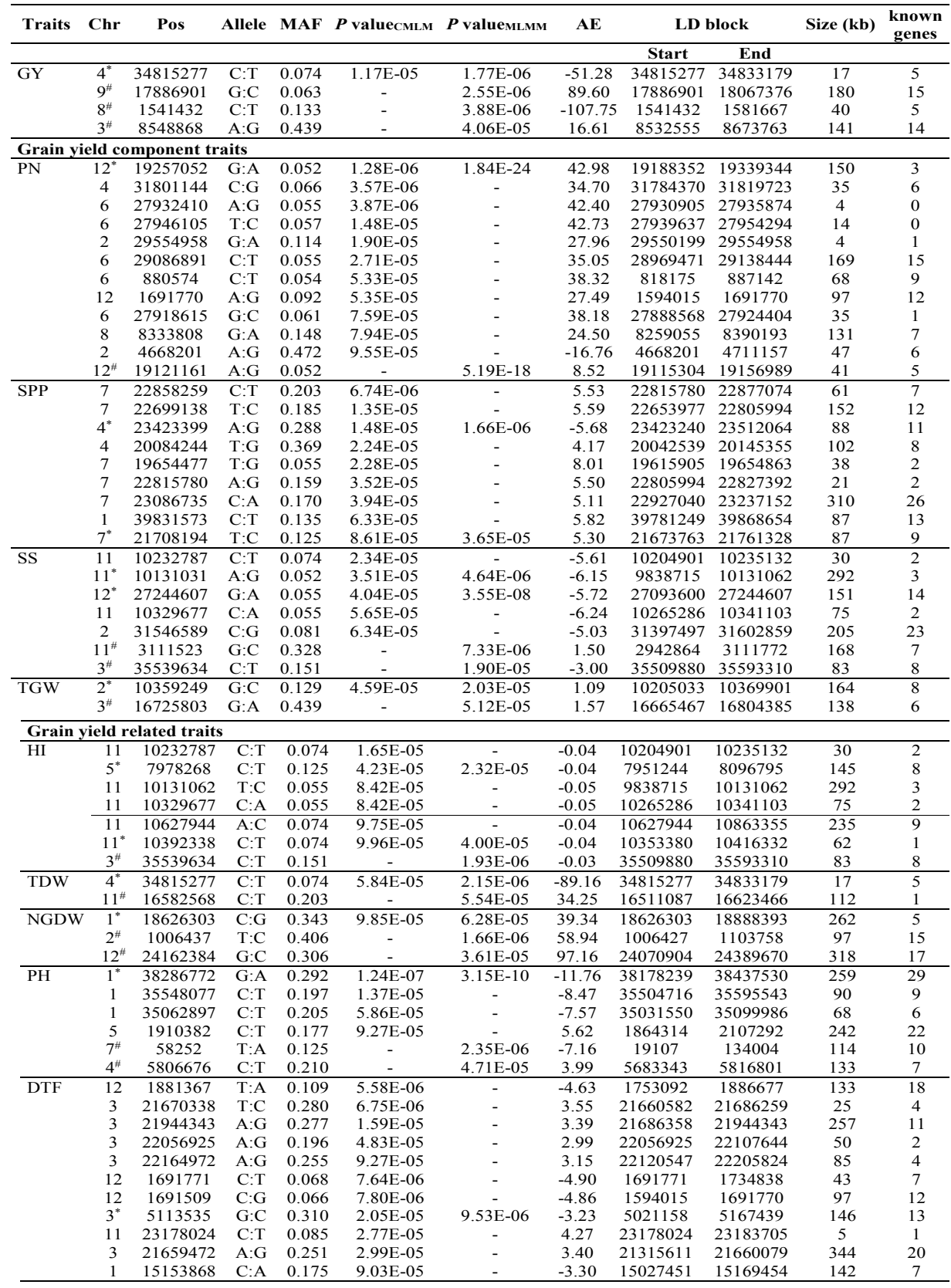


Supplementary Table S5. The details of genetic loci detected for grain yield components and related traits in 2014 non-stress conditions using compressed mixed linear-model (CMLM) and multi-locus mixed model (MLMM) methods. The chromosome loci detected through both the methods were marked by asterisk sign (*); those detected through only by MLMM were marked by hashtag (\#) sign. The remaining all unmarked chromosome loci were detected only through CMLM method. Trait acronyms are given in Table 1. For legends see the Supplementary Table S3.

\begin{tabular}{|c|c|c|c|c|c|c|c|c|c|c|c|}
\hline \multirow[t]{2}{*}{ Traits } & \multirow[t]{2}{*}{ Chr } & \multirow[t]{2}{*}{ Pos } & \multirow[t]{2}{*}{ Allele } & \multirow[t]{2}{*}{ MAF $I$} & \multirow[t]{2}{*}{$P$ valuecmLм } & \multirow[t]{2}{*}{$P$ valueмLмм } & \multirow[t]{2}{*}{$\mathbf{A E}$} & \multicolumn{2}{|c|}{ LD block } & \multirow[t]{2}{*}{ Size (kb) } & \multirow[t]{2}{*}{$\begin{array}{c}\text { Known } \\
\text { genes }\end{array}$} \\
\hline & & & & & & & & Start & End & & \\
\hline \multicolumn{12}{|c|}{ Grain yield component traits } \\
\hline $\mathrm{PN}$ & 10 & 19650831 & $\mathrm{~A}: \mathrm{C}$ & 0.149 & $2.05 \mathrm{E}-06$ & - & 19.35 & 19474964 & 19665687 & 190 & 14 \\
\hline & $10^{*}$ & 19903199 & $\mathrm{~T}: \mathrm{A}$ & 0.146 & $2.41 \mathrm{E}-06$ & $1.16 \mathrm{E}-06$ & 19.55 & 19882559 & 19916740 & 34 & 3 \\
\hline & 10 & 19713719 & $\mathrm{~T}: \mathrm{G}$ & 0.157 & $6.46 \mathrm{E}-06$ & - & 17.98 & 19665880 & 19778630 & 112 & 11 \\
\hline & 10 & 19463253 & $\mathrm{~T}: \mathrm{C}$ & 0.153 & $2.08 \mathrm{E}-05$ & - & 16.79 & 19280939 & 19474522 & 193 & 21 \\
\hline & 10 & 19107872 & $\mathrm{~A}: \mathrm{T}$ & 0.175 & $2.32 \mathrm{E}-05$ & - & 17.78 & 19049329 & 19219240 & 169 & 21 \\
\hline & 10 & 19788019 & $A: G$ & 0.157 & $4.75 \mathrm{E}-05$ & - & 16.93 & 19787326 & 19827002 & 39 & 1 \\
\hline & $4^{*}$ & 28829512 & $\mathrm{G}: \mathrm{A}$ & 0.101 & $6.10 \mathrm{E}-05$ & $8.74 \mathrm{E}-05$ & 21.71 & 28701604 & 29126558 & 424 & 31 \\
\hline & 8 & 27367304 & $\mathrm{C}: \mathrm{T}$ & 0.175 & $7.25 \mathrm{E}-05$ & - & 15.97 & 27364583 & 27381695 & 17 & 5 \\
\hline & $3^{\#}$ & 30407838 & $\mathrm{C}: \mathrm{G}$ & 0.295 & - & $1.18 \mathrm{E}-06$ & 14.84 & 30407838 & 30499464 & 91 & 10 \\
\hline SPP & $4^{*}$ & 23423399 & $\mathrm{~A}: \mathrm{G}$ & 0.287 & $2.44 \mathrm{E}-05$ & $2.41 \mathrm{E}-05$ & -7.40 & 23423240 & 23512064 & 88 & 11 \\
\hline & $11^{*}$ & 22233662 & $\mathrm{G}: \mathrm{T}$ & 0.104 & $8.24 \mathrm{E}-05$ & $5.36 \mathrm{E}-05$ & -8.48 & 22203908 & 22242728 & 38 & 2 \\
\hline SS & $6^{*}$ & 1174802 & $\mathrm{~T}: \mathrm{C}$ & 0.084 & $8.61 \mathrm{E}-05$ & $2.64 \mathrm{E}-08$ & -4.54 & 1117344 & 1208291 & 90 & 2 \\
\hline & 10 & 22428992 & $\mathrm{~A}: \mathrm{T}$ & 0.052 & 8.77E-05 & - & -5.57 & 22419446 & 22436125 & 16 & 3 \\
\hline & $1^{\#}$ & 19327482 & $\mathrm{~T}: \mathrm{C}$ & 0.183 & - & $1.09 \mathrm{E}-06$ & -14.32 & 19247447 & 19360189 & 112 & 6 \\
\hline & $8^{\#}$ & 10059172 & $\mathrm{G}: \mathrm{A}$ & 0.056 & - & $1.72 \mathrm{E}-06$ & -4.12 & 9997926 & 10079318 & 81 & 4 \\
\hline & $11^{\#}$ & 19108095 & $A: G$ & 0.224 & - & $1.77 \mathrm{E}-06$ & 6.09 & 19075632 & 19130323 & 54 & 4 \\
\hline & $12^{\#}$ & 5105627 & $\mathrm{~A}: \mathrm{C}$ & 0.078 & - & $1.42 \mathrm{E}-05$ & -19.68 & 5101105 & 5390949 & 289 & 12 \\
\hline & $2^{\#}$ & 4862726 & $\mathrm{G}: \mathrm{A}$ & 0.239 & - & $1.60 \mathrm{E}-05$ & -7.84 & 4774730 & 4869814 & 95 & 15 \\
\hline & $4^{\#}$ & 21381516 & $\mathrm{C}: \mathrm{A}$ & 0.063 & - & 7.34E-05 & 2.37 & 21360906 & 21381516 & 20 & 5 \\
\hline TGW & 2 & 30699332 & $\mathrm{~T}: \mathrm{C}$ & 0.291 & $4.21 \mathrm{E}-05$ & - & 0.90 & 30684655 & 30784063 & 99 & 9 \\
\hline & $2^{\#}$ & 10359249 & $\mathrm{G}: \mathrm{C}$ & 0.131 & - & $8.66 \mathrm{E}-08$ & 2.42 & 10205033 & 10369901 & 164 & 8 \\
\hline & $7^{\#}$ & 22413176 & $\mathrm{C}: \mathrm{G}$ & 0.396 & - & $3.01 \mathrm{E}-14$ & -1.04 & 22404388 & 22606330 & 201 & 14 \\
\hline & $5^{\#}$ & 5366489 & $\mathrm{G}: \mathrm{A}$ & 0.388 & - & $1.32 \mathrm{E}-06$ & 1.19 & 5365520 & 5448285 & 82 & 6 \\
\hline & $3^{\#}$ & 16736753 & $\mathrm{C}: \mathrm{T}$ & 0.455 & - & $4.58 \mathrm{E}-06$ & -1.47 & 16665467 & 16804385 & 138 & 6 \\
\hline & $3^{\#}$ & 12760491 & $\mathrm{~A}: \mathrm{C}$ & 0.075 & - & $1.39 \mathrm{E}-05$ & 2.38 & 12717890 & 12764642 & 46 & 5 \\
\hline SP & $11^{*}$ & 18102156 & $\mathrm{~A}: \mathrm{C}$ & 0.295 & $6.13 \mathrm{E}-05$ & $1.81 \mathrm{E}-06$ & -1.75 & 18095189 & 18129687 & 34 & 2 \\
\hline & $12^{\#}$ & 10443628 & $\mathrm{C}: \mathrm{A}$ & 0.478 & - & $1.38 \mathrm{E}-06$ & 2.26 & 10320934 & 10622432 & 301 & 10 \\
\hline & $4^{\#}$ & 4550145 & $\mathrm{C}: \mathrm{T}$ & 0.313 & - & $1.41 \mathrm{E}-05$ & -0.47 & 4521317 & 4558051 & 36 & 1 \\
\hline & $11^{\#}$ & 22065446 & $\mathrm{~T}: \mathrm{C}$ & 0.134 & - & $2.08 \mathrm{E}-05$ & -5.65 & 22065446 & 22150769 & 85 & 3 \\
\hline Graln & eld rel & lated traits & & & & & & & & & \\
\hline $\mathrm{HI}$ & 10 & 22419446 & $\mathrm{~A}: \mathrm{G}$ & 0.056 & $1.71 \mathrm{E}-05$ & - & -0.05 & 22419446 & 22436125 & 16 & 3 \\
\hline & 8 & 16617975 & $\mathrm{~T}: \mathrm{C}$ & 0.259 & $7.73 \mathrm{E}-05$ & - & -0.02 & 16611066 & 16619402 & 8 & 3 \\
\hline & $1^{*}$ & 42643328 & $\mathrm{G}: \mathrm{A}$ & 0.272 & $9.78 \mathrm{E}-05$ & $3.36 \mathrm{E}-06$ & -0.02 & 42627969 & 42643337 & 15 & 3 \\
\hline & $8^{\#}$ & 16324317 & A:T & 0.097 & - & $2.04 \mathrm{E}-06$ & -0.10 & 16308107 & 16398979 & 90 & 5 \\
\hline TDW & $2^{*}$ & 945729 & $\mathrm{~A}: \mathrm{T}$ & 0.224 & $4.92 \mathrm{E}-05$ & $1.29 \mathrm{E}-06$ & 63.09 & 944109 & 972602 & 28 & 4 \\
\hline & $4^{\#}$ & 30764890 & $A: G$ & 0.354 & - & $2.59 \mathrm{E}-08$ & -3.62 & 30690751 & 30790417 & 99 & 7 \\
\hline & $1^{\#}$ & 143282 & $\mathrm{G}: \mathrm{A}$ & 0.116 & - & $2.40 \mathrm{E}-05$ & 20.85 & 19837 & 197790 & 177 & 16 \\
\hline & $3^{\#}$ & 33546549 & $\mathrm{C}: \mathrm{A}$ & 0.291 & - & $4.08 \mathrm{E}-05$ & -40.90 & 33518876 & 33569432 & 50 & 5 \\
\hline NGDW & $5^{*}$ & 21385305 & $\mathrm{C}: \mathrm{T}$ & 0.090 & $1.37 \mathrm{E}-05$ & $9.65 \mathrm{E}-05$ & 76.41 & 21312370 & 21385305 & 72 & 4 \\
\hline & $1^{*}$ & 42363099 & $\mathrm{C}: \mathrm{T}$ & 0.101 & $1.37 \mathrm{E}-05$ & $2.92 \mathrm{E}-05$ & 73.35 & 42326848 & 42367533 & 40 & 3 \\
\hline & 11 & 10867613 & $\mathrm{C}: \mathrm{T}$ & 0.153 & $6.24 \mathrm{E}-05$ & - & 58.86 & 10834263 & 10928827 & 94 & 3 \\
\hline & 3 & 34032565 & A:G & 0.246 & $6.71 \mathrm{E}-05$ & - & -48.68 & 34004938 & 34069043 & 64 & 5 \\
\hline & $7^{\#}$ & 21850303 & $\mathrm{C}: \mathrm{T}$ & 0.437 & - & $2.59 \mathrm{E}-05$ & 100.45 & 21814261 & 21908474 & 94 & 13 \\
\hline $\mathrm{PH}$ & $1^{*}$ & 38286772 & $\mathrm{G}: \mathrm{A}$ & 0.291 & $6.03 \mathrm{E}-09$ & $2.57 \mathrm{E}-14$ & -13.34 & 38178239 & 38437530 & 259 & 29 \\
\hline & 1 & 34280616 & G:A & 0.455 & $9.54 \mathrm{E}-06$ & - & 6.22 & 34184887 & 34357192 & 172 & 6 \\
\hline & 1 & 35548077 & $\mathrm{C}: \mathrm{T}$ & 0.196 & $2.51 \mathrm{E}-05$ & - & -8.04 & 35504716 & 35595543 & 90 & 9 \\
\hline & 1 & 35062897 & $\mathrm{C}: \mathrm{T}$ & 0.203 & $2.93 \mathrm{E}-05$ & - & -7.80 & 35031550 & 35099986 & 68 & 6 \\
\hline & 1 & 33059505 & $\mathrm{~T}: \mathrm{A}$ & 0.332 & $4.31 \mathrm{E}-05$ & - & -5.36 & 32994632 & 33284067 & 289 & 30 \\
\hline & $9^{\#}$ & 20537268 & $\mathrm{C}: \mathrm{T}$ & 0.078 & - & $3.52 \mathrm{E}-06$ & 4.87 & 20428722 & 20537316 & 108 & 6 \\
\hline DTF & 12 & 19403471 & $\mathrm{~T}: \mathrm{C}$ & 0.356 & $8.37 \mathrm{E}-05$ & - & -2.24 & 19400490 & 19412883 & 12 & 0 \\
\hline & $3^{\#}$ & 5113428 & $\mathrm{~T}: \mathrm{C}$ & 0.425 & - & $5.53 \mathrm{E}-09$ & -2.03 & 5021158 & 5167439 & 146 & 13 \\
\hline & $3^{\#}$ & 72105 & $\mathrm{C}: \mathrm{T}$ & 0.086 & - & $1.36 \mathrm{E}-10$ & 7.98 & 6480 & 197654 & 191 & 22 \\
\hline & $3^{\#}$ & 28533036 & $\mathrm{C}: \mathrm{A}$ & 0.056 & - & $2.57 \mathrm{E}-09$ & 13.33 & 28529762 & 28761862 & 232 & 28 \\
\hline & $7^{\#}$ & 21266079 & $\mathrm{C}: \mathrm{T}$ & 0.09 & - & $2.12 \mathrm{E}-05$ & -4.68 & 21245869 & 21290877 & 45 & 3 \\
\hline & $4^{\#}$ & 30764890 & $A: G$ & 0.354 & - & $1.01 \mathrm{E}-07$ & -0.05 & 30690751 & 30790417 & 99 & 7 \\
\hline & $9^{\#}$ & 11299373 & $\mathrm{C}: \mathrm{T}$ & 0.164 & - & $8.11 \mathrm{E}-07$ & 4.08 & 11299269 & 11315063 & 15 & 1 \\
\hline & $12^{\#}$ & 24162384 & $\mathrm{G}: \mathrm{C}$ & 0.310 & - & $1.44 \mathrm{E}-07$ & 6.04 & 24070904 & 24389670 & 318 & 17 \\
\hline & $11^{\#}$ & 18168801 & G:T & 0.052 & - & $8.16 \mathrm{E}-06$ & -7.33 & 18134653 & 18242389 & 107 & 1 \\
\hline & $6^{\#}$ & 10389819 & G:T & 0.071 & - & $2.17 \mathrm{E}-05$ & -4.58 & 10279684 & 10410579 & 130 & 8 \\
\hline
\end{tabular}


Supplementary Table S6. The details of genetic loci detected for grain yield components and related traits in 2014 water-deficit stress conditions using compressed mixed linear-model (CMLM) and multi-locus mixed model (MLMM) methods. The chromosome loci detected through both the methods were marked by asterisk sign (*); those detected through only by MLMM were marked by hashtag (\#) sign. The remaining all unmarked chromosome loci were detected only through CMLM method. Trait acronyms are given in Table 1. For legends see the Supplementary Table S3.

\begin{tabular}{|c|c|c|c|c|c|c|c|c|c|c|c|}
\hline \multirow[t]{2}{*}{ Traits } & \multirow[t]{2}{*}{ Chr } & \multirow[t]{2}{*}{ Pos } & \multirow[t]{2}{*}{ Allele } & \multirow[t]{2}{*}{ MAF } & \multirow[t]{2}{*}{$P$ value } & \multirow[t]{2}{*}{$P$ value } & \multirow[t]{2}{*}{$\mathbf{A E}$} & \multicolumn{2}{|c|}{ LD block } & \multirow{2}{*}{$\begin{array}{l}\text { Size } \\
(\mathbf{k b})\end{array}$} & \multirow{2}{*}{$\begin{array}{c}\text { Known } \\
\text { genes }\end{array}$} \\
\hline & & & & & & & & Start & End & & \\
\hline \multicolumn{12}{|c|}{ Grain yield component traits } \\
\hline \multirow[t]{3}{*}{$\mathrm{PN}$} & $11^{*}$ & 2170439 & C:A & 0.073 & $2.67 \mathrm{E}-05$ & $3.21 \mathrm{E}-06$ & 29.60 & 1940541 & 2291962 & 351 & 39 \\
\hline & $1^{\#}$ & 39886933 & $\mathrm{G}: \mathrm{C}$ & 0.254 & - & $3.60 \mathrm{E}-05$ & -37.99 & 39886933 & 40061573 & 174 & 15 \\
\hline & $3^{\#}$ & 32507536 & $\mathrm{~T}: \mathrm{G}$ & 0.489 & - & $4.56 \mathrm{E}-05$ & -14.37 & 32507536 & 32594052 & 86 & 9 \\
\hline \multirow[t]{11}{*}{ SS } & 1 & 29223354 & G:C & 0.164 & $1.17 \mathrm{E}-05$ & - & -5.16 & 29135405 & 29300574 & 165 & 13 \\
\hline & $7^{*}$ & 3293128 & $\mathrm{G}: \mathrm{T}$ & 0.172 & $1.46 \mathrm{E}-05$ & $1.27 \mathrm{E}-06$ & 5.18 & 3293128 & 3297350 & 4 & 2 \\
\hline & 1 & 30657333 & G:A & 0.198 & $1.86 \mathrm{E}-05$ & - & -4.63 & 30583128 & 30819501 & 236 & 25 \\
\hline & 1 & 29483935 & $\mathrm{~T}: \mathrm{A}$ & 0.104 & $2.22 \mathrm{E}-05$ & - & -5.94 & 29394012 & 29600705 & 206 & 19 \\
\hline & $11^{*}$ & 23110189 & $\mathrm{G}: \mathrm{A}$ & 0.063 & $4.73 \mathrm{E}-05$ & $8.69 \mathrm{E}-07$ & -6.95 & 23066834 & 23133274 & 66 & 6 \\
\hline & 1 & 28427790 & $\mathrm{C}: \mathrm{T}$ & 0.097 & $5.69 \mathrm{E}-05$ & - & -5.47 & 28406849 & 28444503 & 37 & 1 \\
\hline & 4 & 27444465 & $\mathrm{G}: \mathrm{T}$ & 0.175 & $7.38 \mathrm{E}-05$ & - & -4.90 & 27316695 & 27568586 & 251 & 27 \\
\hline & 2 & 23767444 & $\mathrm{G}: \mathrm{T}$ & 0.067 & $7.47 \mathrm{E}-05$ & - & -6.78 & 23754037 & 23767444 & 13 & 3 \\
\hline & 2 & 24234634 & $\mathrm{~T}: \mathrm{G}$ & 0.093 & $9.39 \mathrm{E}-05$ & - & -6.55 & 24228897 & 24236361 & 7 & 1 \\
\hline & $1^{\#}$ & 35548077 & $\mathrm{C}: \mathrm{T}$ & 0.194 & $\begin{array}{c}-0000 \\
-\end{array}$ & $1.47 \mathrm{E}-05$ & 11.27 & 35504716 & 35595543 & 90 & 9 \\
\hline & $1^{\#}$ & 19262986 & G:A & 0.138 & - & $8.75 \mathrm{E}-05$ & -2.72 & 19248831 & 19360189 & 111 & 6 \\
\hline \multirow[t]{8}{*}{ TGW } & $1^{*}$ & 3398710 & A:G & 0.338 & $1.76 \mathrm{E}-05$ & $9.80 \mathrm{E}-08$ & -1.14 & 3398710 & 3538828 & 140 & 16 \\
\hline & 3 & 16725803 & G:A & 0.435 & $2.23 \mathrm{E}-05$ & - & 1.23 & 16665467 & 16804385 & 138 & 6 \\
\hline & 4 & 16574303 & $\mathrm{C}: \mathrm{T}$ & 0.104 & $7.98 \mathrm{E}-05$ & - & 1.58 & 16574289 & 16642369 & 68 & 5 \\
\hline & $5^{\#}$ & 7021512 & $\mathrm{C}: \mathrm{T}$ & 0.063 & - & $2.94 \mathrm{E}-07$ & 3.00 & 7021378 & 7039434 & 18 & 1 \\
\hline & $1^{\#}$ & 21362367 & G:A & 0.086 & - & $1.77 \mathrm{E}-08$ & -1.24 & 21348660 & 21627407 & 278 & 8 \\
\hline & $3^{\#}$ & 22959891 & $\mathrm{C}: \mathrm{T}$ & 0.075 & - & 2.62E-06 & 2.16 & 22817514 & 22959891 & 142 & 7 \\
\hline & $9^{\#}$ & 17852034 & $A: G$ & 0.06 & - & $1.10 \mathrm{E}-05$ & 2.85 & 17844173 & 17979314 & 135 & 13 \\
\hline & $3^{\#}$ & 7043553 & $\mathrm{~T}: \mathrm{C}$ & 0.44 & - & $1.98 \mathrm{E}-05$ & -0.93 & 7030587 & 7070877 & 40 & 6 \\
\hline \multirow[t]{3}{*}{ SP } & $12^{*}$ & 10611754 & $\mathrm{C}: \mathrm{A}$ & 0.496 & $4.26 \mathrm{E}-05$ & $1.01 \mathrm{E}-08$ & 1.83 & 10320934 & 10622432 & 301 & 10 \\
\hline & $12^{\#}$ & 16565406 & $\mathrm{G}: \mathrm{A}$ & 0.407 & $\frac{-}{2-00}$ & 7.30E-05 & 2.77 & 16565406 & 16584455 & 19 & 1 \\
\hline & $11^{\#}$ & 3518037 & G:A & 0.101 & - & $6.62 \mathrm{E}-05$ & 2.61 & 3368572 & 3562283 & 198 & 18 \\
\hline Grain y & yield $r$ & elated trait & & & & & & & & & \\
\hline $\mathrm{HI}$ & $1^{*}$ & 29223354 & G:C & 0.164 & $3.31 \mathrm{E}-05$ & $3.03 \mathrm{E}-06$ & -0.03 & 29135405 & 29300574 & 165 & 13 \\
\hline & $6^{\#}$ & 217858 & $\mathrm{C}: \mathrm{T}$ & 0.119 & - & $2.59 \mathrm{E}-05$ & -0.001 & 132127 & 366436 & 234 & 25 \\
\hline TDW & $3^{*}$ & 15532341 & $\mathrm{~T}: \mathrm{C}$ & 0.481 & $5.81 \mathrm{E}-05$ & $3.76 \mathrm{E}-05$ & 58.79 & 15532341 & 15564883 & 32 & 2 \\
\hline & 12 & 23011365 & $\mathrm{~A}: \mathrm{C}$ & 0.067 & $7.82 \mathrm{E}-05$ & - & -102.57 & 23004415 & 23141150 & 136 & 13 \\
\hline & 12 & 2589690 & $\mathrm{C}: \mathrm{T}$ & 0.146 & $9.78 \mathrm{E}-05$ & - & -67.86 & 2567973 & 2594603 & 26 & 4 \\
\hline & $6^{*}$ & 9774102 & C:T & 0.144 & $9.93 \mathrm{E}-05$ & $1.01 \mathrm{E}-09$ & -75.66 & 9655595 & 9774102 & 118 & 2 \\
\hline & $7^{\#}$ & 27620959 & $\mathrm{C}: \mathrm{T}$ & 0.09 & - & $4.12 \mathrm{E}-10$ & 71.75 & 27479689 & 27620959 & 141 & 8 \\
\hline & $1^{\#}$ & 42643699 & $\mathrm{C}: \mathrm{T}$ & 0.104 & - & $4.22 \mathrm{E}-06$ & -88.94 & 42627969 & 42691537 & 63 & 5 \\
\hline & $7^{\#}$ & 26457561 & $\mathrm{G}: \mathrm{A}$ & 0.06 & - & $5.90 \mathrm{E}-06$ & 248.12 & 26450722 & 26548855 & 98 & 12 \\
\hline & $9^{\#}$ & 6323526 & $\mathrm{G}: \mathrm{A}$ & 0.299 & - & $6.60 \mathrm{E}-07$ & 136.50 & 6195580 & 6323526 & 127 & 7 \\
\hline & $10^{\#}$ & 17454693 & A:G & 0.078 & - & $2.00 \mathrm{E}-06$ & 134.78 & 17378773 & 17548721 & 169 & 9 \\
\hline & $6^{\#}$ & 2721526 & $\mathrm{~A}: \mathrm{T}$ & 0.231 & - & $1.94 \mathrm{E}-06$ & -39.32 & 2662180 & 2726347 & 64 & 10 \\
\hline & $6^{\#}$ & 7135140 & $\mathrm{~A}: \mathrm{G}$ & 0.306 & - & $1.90 \mathrm{E}-05$ & 12.67 & 7110053 & 7136325 & 26 & 4 \\
\hline NGDW & $11^{*}$ & 10867613 & $\mathrm{C}: \mathrm{T}$ & 0.153 & $4.42 \mathrm{E}-05$ & $2.44 \mathrm{E}-05$ & 61.13 & 10834263 & 10928827 & 94 & 3 \\
\hline & 7 & 3488686 & C:G & 0.312 & $5.84 \mathrm{E}-05$ & - & -55.51 & 3454851 & 3619076 & 164 & 15 \\
\hline$\overline{\mathrm{PH}}$ & $1^{*}$ & 38286772 & $\mathrm{G}: \mathrm{A}$ & 0.291 & $1.39 \mathrm{E}-07$ & $3.49 \mathrm{E}-08$ & -13.23 & 38178239 & 38437530 & 259 & 29 \\
\hline & 2 & 23720396 & C:G & 0.295 & $4.62 \mathrm{E}-05$ & - & 5.87 & 23720396 & 23720592 & $197 b p$ & 1 \\
\hline & 11 & 586603 & C:T & 0.093 & $9.70 \mathrm{E}-05$ & - & -10.55 & 566590 & 642837 & 76 & 16 \\
\hline & 5 & 16676691 & C:T & 0.070 & $2.86 \mathrm{E}-05$ & - & 8.89 & 16588982 & 16785352 & 196 & 9 \\
\hline DTF & $3^{*}$ & 72105 & C:T & 0.086 & $6.61 \mathrm{E}-08$ & $3.74 \mathrm{E}-08$ & $\begin{array}{l}0.09 \\
1.82\end{array}$ & 6480 & 197654 & 191 & 22 \\
\hline & $4^{\#}$ & 23430194 & $\mathrm{~T}: \mathrm{C}$ & 0.257 & - & $6.09 \mathrm{E}-07$ & -3.18 & 23424327 & 23483270 & 58 & 6 \\
\hline & $1^{\#}$ & 855970 & $\mathrm{G}: \mathrm{C}$ & 0.172 & - & $8.52 \mathrm{E}-07$ & 1.94 & 769982 & 931087 & 161 & 19 \\
\hline & $4^{\#}$ & 34314696 & G:T & 0.052 & - & $2.23 \mathrm{E}-05$ & -2.33 & 34284403 & 34314696 & 30 & 3 \\
\hline & $12^{\#}$ & 24162384 & G:C & $\begin{array}{c}0.032 \\
0.31\end{array}$ & - & $8.53 \mathrm{E}-06$ & 5.83 & 24070904 & 24389670 & 318 & 17 \\
\hline & $11^{\#}$ & 10867613 & $\mathrm{C}: \mathrm{T}$ & 0.153 & - & $2.26 \mathrm{E}-05$ & 3.48 & 10834263 & 10928827 & 94 & 3 \\
\hline
\end{tabular}




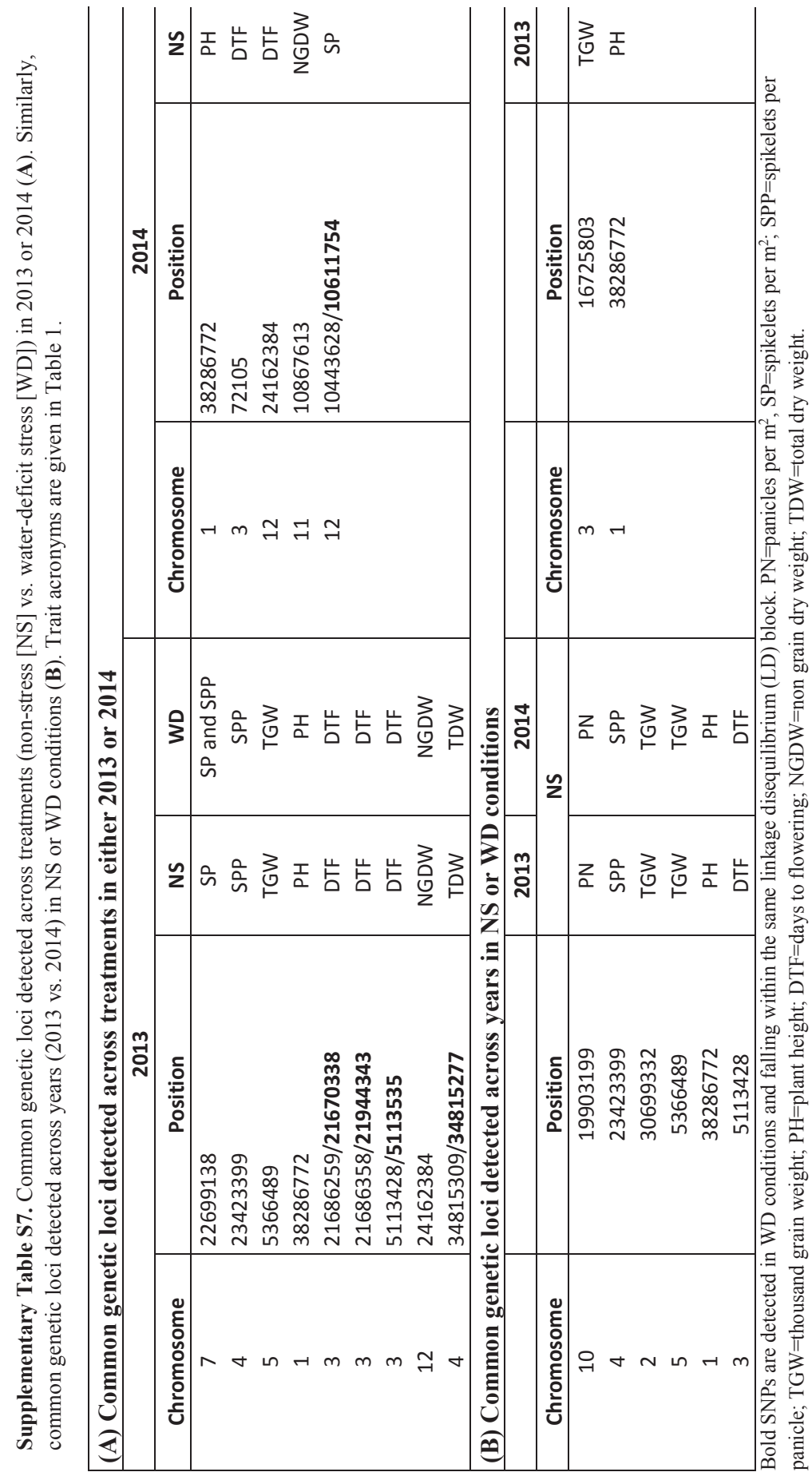


Supplementary Table S8: The details of genetic loci detected for corrected grain yield components and related traits (only on harvest index excluding the other traits in this class) in 2013 water-deficit stress conditions using compressed mixed linear-model (CMLM) and multi-locus mixed model (MLMM) methods. The chromosome loci detected through both the methods were marked by asterisk sign (*); those detected through only by MLMM were marked by hashtag (\#) sign. The remaining all unmarked chromosome loci were detected only through CMLM method. Trait acronyms are given in Table 1.

\begin{tabular}{|c|c|c|c|c|c|c|c|c|c|c|c|}
\hline \multirow[t]{2}{*}{ Traits } & \multirow[t]{2}{*}{ Chr } & \multirow[t]{2}{*}{ Pos } & \multirow[t]{2}{*}{ Allele } & \multirow[t]{2}{*}{ MAF } & \multirow[t]{2}{*}{$P$ valuecmlм } & \multirow[t]{2}{*}{$P$ valueмсмм } & \multirow[t]{2}{*}{$\mathbf{A E}$} & \multicolumn{2}{|c|}{ LD block } & \multirow[t]{2}{*}{ Size (kb) } & \multirow{2}{*}{$\begin{array}{c}\text { Known } \\
\text { genes }\end{array}$} \\
\hline & & & & & & & & Start & End & & \\
\hline \multicolumn{12}{|c|}{ Grain yield component traits } \\
\hline \multirow[t]{7}{*}{$\mathrm{PN}$} & $8^{*}$ & 20408464 & G:T & 0.052 & $1.39 \mathrm{E}-06$ & $1.77 \mathrm{E}-06$ & 44.65 & 20199466 & 20450490 & 251 & 14 \\
\hline & $6^{*}$ & 9774102 & $\mathrm{C}: \mathrm{T}$ & 0.151 & 7.57E-06 & $5.05 \mathrm{E}-11$ & 28.01 & 9774102 & 9992897 & 218 & 10 \\
\hline & 2 & 4668201 & A:G & 0.476 & $9.44 \mathrm{E}-05$ & - & -15.91 & 4668201 & 4711157 & 42 & 6 \\
\hline & $7^{\#}$ & 15365358 & $\mathrm{~T}: \mathrm{C}$ & 0.351 & - & $1.57 \mathrm{E}-06$ & -16.42 & 15293375 & 15388056 & 94 & 5 \\
\hline & $4^{\#}$ & 22006507 & C:T & 0.264 & - & $1.85 \mathrm{E}-05$ & 15.47 & 21989957 & 22015135 & 25 & 3 \\
\hline & $8^{\#}$ & 27943348 & G:A & 0.052 & - & $1.25 \mathrm{E}-05$ & 24.89 & 27905391 & 27943348 & 37 & 8 \\
\hline & $4^{\#}$ & 15853443 & $\mathrm{C}: \mathrm{G}$ & 0.374 & - & $4.76 \mathrm{E}-05$ & 14.6 & 15637881 & 15853443 & 215 & 2 \\
\hline \multirow[t]{6}{*}{ SPP } & $4^{*}$ & 23471311 & $\mathrm{C}: \mathrm{G}$ & 0.365 & $7.00 \mathrm{E}-06$ & $3.09 \mathrm{E}-08$ & -8.96 & 23428565 & 23483270 & 54 & 5 \\
\hline & 4 & 20027177 & A:G & 0.052 & 4.32E-05 & - & 14.81 & 20014494 & 20066427 & 51 & 4 \\
\hline & $6^{*}$ & 9871701 & $\mathrm{~A}: \mathrm{G}$ & 0.144 & $6.33 \mathrm{E}-05$ & $8.99 \mathrm{E}-06$ & -9.89 & 9774102 & 9992897 & 218 & 10 \\
\hline & $3^{\#}$ & 29663197 & C:T & 0.257 & - & 4.69E-06 & -6.79 & 29614230 & 29742101 & 127 & 7 \\
\hline & $1^{\#}$ & 855970 & $\mathrm{G}: \mathrm{C}$ & 0.167 & - & $1.85 \mathrm{E}-05$ & 5.76 & 769982 & 931087 & 161 & 19 \\
\hline & $11^{\#}$ & 23178024 & $\mathrm{C}: \mathrm{T}$ & 0.086 & - & $3.18 \mathrm{E}-05$ & 8.37 & 23178024 & 23183705 & 5 & 1 \\
\hline \multirow[t]{7}{*}{ SS } & $6^{*}$ & 9871701 & $\mathrm{~A}: \mathrm{G}$ & 0.144 & $1.37 \mathrm{E}-05$ & $5.46 \mathrm{E}-09$ & -5.93 & 9774102 & 9992897 & 218 & 10 \\
\hline & 11 & 17886595 & $\mathrm{C}: \mathrm{T}$ & 0.297 & $7.11 \mathrm{E}-05$ & - & -3.64 & 17874496 & 18026910 & 152 & 2 \\
\hline & $3^{*}$ & 5113428 & $\mathrm{~T}: \mathrm{C}$ & 0.424 & 8.63E-05 & 4.12E-08 & -3.76 & 5021158 & 5167439 & 146 & 13 \\
\hline & 6 & 10086748 & $\mathrm{C}: \mathrm{G}$ & 0.188 & $9.81 \mathrm{E}-05$ & - & -4.26 & 10086745 & 10132707 & 45 & 4 \\
\hline & $7^{\#}$ & 21266079 & $\mathrm{C}: \mathrm{T}$ & 0.096 & - & $6.95 \mathrm{E}-06$ & -3.49 & 21245869 & 21290877 & 45 & 3 \\
\hline & $5^{\#}$ & 29213653 & C:A & 0.140 & - & $2.96 \mathrm{E}-05$ & 3.38 & 29213653 & 29238030 & 24 & 4 \\
\hline & $12^{\#}$ & 27244607 & $\mathrm{G}: \mathrm{A}$ & 0.055 & - & $7.01 \mathrm{E}-05$ & -5.56 & 27093600 & 27244607 & 151 & 14 \\
\hline SP & $12^{*}$ & 141599 & $\mathrm{G}: \mathrm{A}$ & 0.122 & $2.66 \mathrm{E}-05$ & $2.52 \mathrm{E}-05$ & -2.29 & 141599 & 148272 & 6 & 1 \\
\hline \multicolumn{12}{|c|}{ Grain yield related traits } \\
\hline \multirow[t]{8}{*}{$\mathrm{HI}$} & $4^{*}$ & 34815277 & $\mathrm{C}: \mathrm{T}$ & 0.074 & $1.98 \mathrm{E}-07$ & $5.40 \mathrm{E}-06$ & -0.06 & 34815277 & 34833179 & 17 & 5 \\
\hline & $3^{*}$ & 5113428 & $\mathrm{~T}: \mathrm{C}$ & 0.424 & 4.62E-06 & $1.03 \mathrm{E}-06$ & -0.03 & 5021158 & 5167439 & 146 & 13 \\
\hline & 11 & 10627944 & $\mathrm{~A}: \mathrm{C}$ & 0.074 & 8.20E-06 & - & -0.05 & 10627944 & 10863355 & 235 & 9 \\
\hline & 11 & 10131062 & $\mathrm{~T}: \mathrm{C}$ & 0.055 & $3.20 \mathrm{E}-05$ & - & -0.06 & 9838715 & 10131062 & 292 & 3 \\
\hline & 11 & 10329677 & $\mathrm{C}: \mathrm{A}$ & 0.055 & $3.20 \mathrm{E}-05$ & - & -0.06 & 10265286 & 10341103 & 75 & 2 \\
\hline & 6 & 10086748 & $\mathrm{C}: \mathrm{G}$ & 0.188 & $5.88 \mathrm{E}-05$ & - & -0.03 & 10036641 & 10086748 & 50 & 1 \\
\hline & 6 & 2950054 & G:T & 0.092 & $6.57 \mathrm{E}-05$ & - & -0.04 & 2888879 & 2981224 & 92 & 12 \\
\hline & 1 & 604746 & $\mathrm{~A}: \mathrm{G}$ & 0.452 & $9.43 \mathrm{E}-05$ & - & -0.03 & 557715 & 717021 & 159 & 12 \\
\hline
\end{tabular}

$\mathrm{Chr}=$ chromosome; Pos= physical position of SNP; MAF=minor allele frequency; $\mathrm{AE}=$ allelic effect regarding the minor allele (average traits value of genotypes carrying minor allele - average traits value of genotypes carrying major allele). Known genes=total known genes observed within the LD block. 


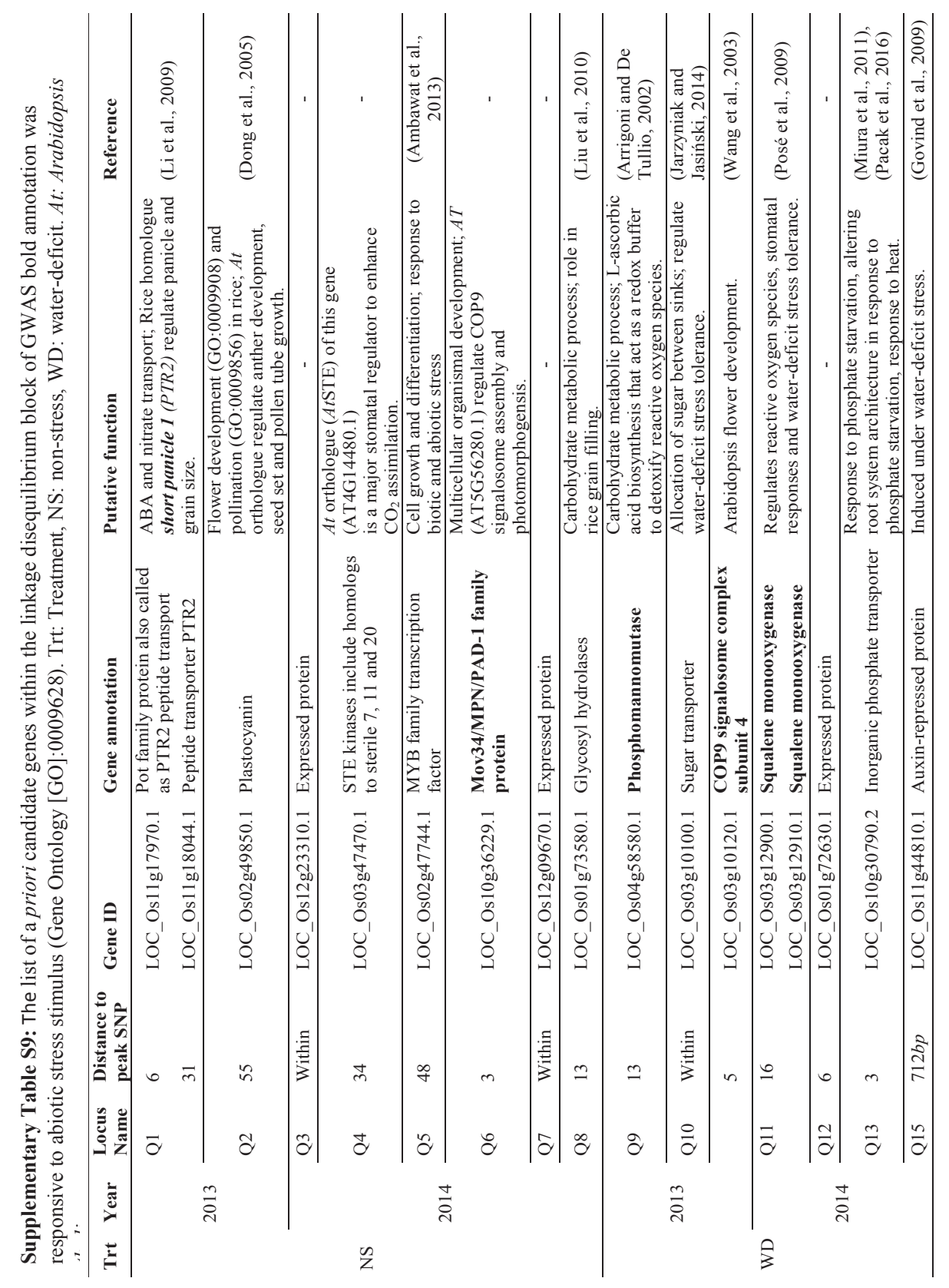




\section{Linking eco-physiological modelling with genome wide association} mapping to design crop ideotypes: A case study on rice under water-deficit conditions

Niteen N. Kadam ${ }^{1,2}$, Krishna S.V. Jagadish ${ }^{1,3}$, Paul C. Struik ${ }^{2}$, C.G. van der Linden ${ }^{4}$, and
Xinyou Yin ${ }^{2}$

${ }^{1}$ International Rice Research Institute, DAPO, Box 7777, Metro Manila, Philippines ${ }^{2}$ Centre for Crop Systems Analysis, Department of Plant Sciences, Wageningen University \& Research, PO Box 430, 6700 AK Wageningen, The Netherlands ${ }^{3}$ Department of Agronomy, Kansas State University, Manhattan, Kansas 66506, USA ${ }^{4}$ Plant Breeding, Department of Plant Sciences, Wageningen University \& Research, P.O. Box 386, 6700 AJ Wageningen, The Netherlands 


\begin{abstract}
Genetic markers can be used in combination with eco-physiological models to predict the performance of genotypes in various environments. We explore the use of crop models to identify markers and design ideotypes of rice (Oryza sativa L.) yield under control and water-deficit conditions. Using GECROS, crop yield was dissected into eight parameters, which were estimated from the control treatment in one season for an indica rice panel consisting of 267 genotypes. Some parameters had more significant effect on yield than other parameters. The model accounted for $58 \%$ of yield variation of 267 genotypes in control and $40 \%$ under water-deficit conditions. For each parameter, associated single nucleotide polymorphism (SNP) loci were identified via GWAS in randomly selected 213 genotypes as the traning dataset and remaning 54 genotypes were used as testing dataset. The SNP-based parameter values were calculated from estimated effects of the loci, and were fed into the model. The SNP-based model accounted for $37 \%$ and $29 \%$ of yield variation under control and water-deficit, respectively in training set. However, SNP-based model accounted for $10 \%$ of yield variation in control and $15 \%$ under waterdeficit stress in testing set. In addition, performance was also lower, using either original or SNP-based parameter values, when the model was used to simulate yields in an independent season. Overall, the correlation between simulated yields using original and SNP-based parameter values was above 0.70 . The rank of the SNP loci for their relative importance in explaining yield variation in the genotypes, as determined by model-based sensitivity analysis, differed greatly between control and water-deficit environments. The GECROS-based dissection approach detected more SNP loci than the analysis using yield per se. Virtual ideotypes based on SNPs identified by modelling had higher yield than those based on SNPs for yield per se. Eco-physiological modelling can potentially guide the design of crops for improving grain yields under contrasting conditions, but the resolution of the model in distinguishing the genotypic variation has to be improved.
\end{abstract}

Keywords: genotype-phenotype relationships, GWAS panel, model-based ideotyping, SNP. Oryza sativa L., rice. 


\section{Introduction}

In the past, genomic information has proven to provide opportunities for detecting genes and quantitative trait loci (QTLs) associated with various morphological and physiological traits. Rice breeding currently exploits these genes and QTLs to improve grain yield potential and yield stability of rice cultivars when exposed to major abiotic stresses (water-deficit, high temperature, salinity and submergence; Zhang et al., 2009; Singh et al., 2009; Vikram et al., 2011; Ali et al., 2013). The recent advent of high-throughput and cost-effective genome sequencing technologies has made it possible to conduct in-depth genome analyses of thousands of individual genotypes and breeding material in many crops. For example, complete genome sequencing was carried out on 3000 diverse genotypes of rice (Oryza sativa L.), and this allowed to detect many mutations ( $\mathrm{Li}$ et al., 2014) and to explain the diversity at genome level in the form of single nucleotide polymorphisms (SNPs). Despite recent advances in knowledge and technological tools in crop genetics, several scientific and technical challenges need to be overcome to exploit this information to further improve grain yield. Grain yield is a complex trait showing a low heritability and strong response to environment (genotype $x$ environment interaction). To further improve grain yield, a deeper understanding of the complex morphological and physiological traits contributing to grain yield, and how genes or QTLs regulating these traits interact with the environment (gene / QTL $\times$ environment interaction) is required.

Genotype $\times$ environment interaction $(\mathrm{G} \times \mathrm{E})$ is a complex phenomenon relevant to both genetics and crop physiology. Its quantification involves the build-up of a model based on the information generated by phenotyping many genotypes in several characterised environments. Then the model application can be illustrated in a step-wise approach using observed information to predict the phenotypic performance of: (1) genotypes phenotyped in new environments, (2) new genotypes in characterised environments, and (3) new genotypes in new environments (Bustos-Korts et al., 2016). While this step-wise approach was proposed largely from the viewpoint of statistical modelling of $\mathrm{G} \times \mathrm{E}$, it can also be applied to the eco physiological modelling of $\mathrm{G} \times \mathrm{E}$ using crop models.

Process-based eco-physiological modelling of crop growth has been widely used to resolve the complexity of grain yield formation under different environments (Soltani et al., 1999; Mo et al., 2005; Tang et al., 2009; Yin and Struik, 2010; Martre et al., 2011). A model can dissect the complex traits such as grain yield into its component traits at lower hierarchical levels. Most traits in the model are believed to be controlled genetically; yet, these traits are 


\section{Chapter 5}

commonly estimated from phenotyping experiments and their genetic basis is largely unknown (Kromdijk et al., 2014). To overcome this limitation several studies have tried to link crop modelling with genetics (Yin and Struik, 2015). Using such an approach grain yield was first predicted in barley (Yin et al., 2000), later followed by grain yield in rice under control and water-deficit conditions (Gu et al., 2014). In addition, such QTL-based crop modelling helps to design virtual ideotypes (hypothetical crop plants combining ideal characteristics known to enhance grain yield), and support marker-assisted selection (MAS) to accelerate traditional crop breeding (Gu et al., 2014; Yin et al., 2016; Xu and Buck-Sorlin, 2016; Hammer et al., 2016).

However, most studies linking crop growth modelling with genetics were conducted on biparental mapping populations representing only a small part of the available genetic diversity. Recently, genome wide association studies (GWAS) have become increasingly popular to dissect the genetic architecture of complex traits, using wider genetic diversity in crops. Only recently, it was recommended to extend the biparental QTL-based eco-physiological modelling to a wider genetic diversity using the GWAS approach (Yin et al., 2016).

To the best of our knowledge only few (and very recent) studies were conducted on linking GWAS with crop growth modelling (Mangin et al., 2017; Dingkuhn et al., 2017a; Dingkuhn et al., 2017b). Mangin et al. (2017) showed that crop models can be used to develop "stress indicators" that explain yield variation across multiple environments, facilitating GWAS application to identify relevant QTLs for yield in response to environmental stresses. Similarly, Dingkuhn et al. (2017a) and Dingkuhn et al. (2017b) have shown that the crop model RIDEV can dissect phenology and spikelet sterility, respectively, into their components, thereby heuristically strengthening the phenotyping and GWAS analysis of these two traits. These studies demonstrated benefit of crop modelling in GWAS analysis. However, whether the genetic approach for GWAS can facilitate the application of crop modelling in plant breeding, e.g. in designing crop ideotypes, has yet to be demonstrated.

Our current study is the first attempt to explore the QTL-based eco-physiological modelling approach on a genome wide association panel of rice following the principles explained for traditional linkage analysis (Yin et al., 2000; Gu et al., 2014). This approach allows dissecting the $\mathrm{G} \times \mathrm{E}$ and integrating the effects of multiple component traits regulating the complex grain yield trait. To that end, we applied the GECROS (Genotype-by-Environment interaction on CROp growth Simulator; Yin and Van Laar, 2005) model to a rice association mapping panel as a case in point. The model was first parameterised from the control condition in the experiment of one growing season, and evaluated by simulating and estimating the grain 
yield across control and water-deficit treatments in the same and other growing seasons. Then GWAS analysis was performed on model input parameters to identify significant SNP markers, and a model-based sensitivity analysis was used to rank the identified SNP markers based on their relative importance in determining the grain yield variation. Based on these analyses, grain yield ideotypes were designed for control and water-deficit conditions.

\section{Material and Methods}

We modified the methodology that was explained by (Gu et al., 2014) for model application to a biparental population (Supplementary Figure 1). Each step in this modification sequence is briefly explained in the following sections.

\section{Association mapping panel and field phenotyping}

An association mapping panel of indica genotypes of rice was developed and assembled at the International Rice Research Institute (IRRI), Philippines, in the context of the Global Rice Phenotyping Network project (http://ricephenonetwork.irri.org). Recently, this population was extensively used to study the genetic architecture of a wide range of phenotypic traits (AlTamimi et al., 2016; Rebolledo et al., 2016; Kikuchi et al., 2017; Kadam et al., 2017). We phenotyped this population to quantify the variation in grain yield and its component traits under well-watered (control) conditions throughout the crop cycle and under water-deficit conditions during the reproductive stage (focussing on flowering stage). Two field experiments were executed at the upland farm of IRRI, Philippines (14 $11^{\prime} \mathrm{N}, 121^{\circ} 15^{\prime} \mathrm{E}$; elevation $21 \mathrm{~m}$ above sea level) during the dry seasons (DS) of 2013 and 2014. A systematic and well designed staggered sowing and transplanting scheme was followed to synchronise flowering, and thereby the timing of the water-deficit stress with respect to plant developmental stage, for the entire panel. Data on the environmental conditions such as daily radiation, maximum and minimum temperature, vapour pressure, rainfall and wind speed were collected from an on-site weather station. The detailed experimental setup, stress imposition and other relevant details on agronomic management practices are described in Chapter 4.

\section{The GECROS model and its modification}

The GECROS model was first described by Yin and Van Laar (2005) and recently updated by Yin and Struik (2017). GECROS simulates crop growth on a daily basis, but with subroutines for photosynthesis, transpiration, and phenology implemented on a shorter time step. The model 
simulates yield by considering the effects of interactions and feedback mechanism of physiological processes during crop growth and development. These physiological processes include photosynthesis-transpiration coupled via stomatal conductance, carbon-nitrogen interaction, functional balance between shoot and root activities, and interplay between source supply and sink demand.

$\mathrm{Gu}$ et al. (2014) showed poorer performance of GECROS in simulating yield of rice genotypes under drought than under control, due to the model's inability to correctly simulate spikelet number under drought. The number of spikelets per $\mathrm{m}^{2}$ in the model is assumed to be co-determined by the amounts of carbon and nitrogen accumulated in the plant during the reproductive phase around flowering. However, the percentage of filled spikelets, or grain set, depends on panicle temperature during flowering hours, especially when stress occurs during this phase (Jagadish et al., 2007; Julia and Dingkuhn, 2013). Therefore, we modified the GECROS model to account for the direct effect of panicle temperature on sink size. The simulation of panicle temperature was done using the same algorithms in GECROS (Yin and Struik, 2017) for simulating leaf-surface energy balance, based on a coupled conductance photosynthesis-transpiration routine, whereby panicles were treated as a photosynthesizing organ and its conductance was calculated using a semi-empirical leaf stomatal conductance model. Because the panicle temperature is most crucial in determining the spikelet sterility only during flowering hours of a day (Julia and Dingkuhn, 2013), upscaling instantaneous photosynthesis and transpiration to daily total was changed from the five-point Gaussian integration in GECROS to hourly computation. A factor for reduction induced by any high panicle temperature at flowering hours under stress, relative to the control, was introduced to simulate the actual spikelet fertility under water-deficit stress, based on the linear relationship between sterility and panicle temperature reported by Julia \& Dingkuhn (2013). The grain set in control was herein called "the baseline grain set".

\section{Measurement of model input parameters, model calibration and testing}

The model requires a certain set of genotype-specific input parameters to simulate grain yield. These input parameters were classified into (1) phenological; (2) morphological; and (3) physiological categories (Table 1). Phenological parameters included pre-flowering duration $\left(m_{\mathrm{V}}\right)$, post-flowering duration $\left(m_{\mathrm{R}}\right)$, and photoperiod sensitivity $(\delta)$. Morphological parameters included maximum plant height $\left(H_{\max }\right)$ and single-seed weight $\left(S_{\mathrm{w}}\right)$. Similarly, physiological parameters included grain set $\left(g_{\text {set }}\right)$, grain nitrogen concentration $\left(n_{\mathrm{so}}\right)$, and total crop nitrogen 
uptake capacity $\left(N_{\max }\right)$. A complete set of model input parameters for the association mapping panel of rice was determined from control condition data of the 2013 DS experiment (Chapter 4). The exception was photoperiod sensitivity parameter $\delta$ that was estimated using preflowering phenology data collected from the 2013 DS as well as an additional 2012 wet-season phenology experiment, because $\delta$ requires at least two photoperiods to estimate. A bell-shaped nonlinear function of the phenological response to temperature in the GECROS model was used to calculate the parameters $m_{\mathrm{V}}$ and $\delta$ using the measured flowering time, and $m_{\mathrm{R}}$ calculated using the harvest time, of the association mapping panel. These parameters were estimated, based on daily photoperiod and hourly temperature generated from weather data, using daily maximum and minimum temperatures (Yin et al., 2005). Values of $H_{\max }, S_{\mathrm{w}}$, and $g_{\text {set }}$ were determined directly from the experimental measurements (described in Chapter 4). The value of $n_{\text {so }}$ was measured using the micro-Kjeldahl method. $N_{\max }$ is not an input parameter in the default GECROS model, and is used here as a genotype-specific parameter to avoid the confounding effect of the inherent model inaccuracy in simulating crop nitrogen uptake from soil. The value of $N_{\max }$ was assessed based on dry weight and nitrogen concentration in the various plant organs. While calculating $N_{\max }$, nitrogen concentration was assumed to be $0.463 \%$ in the straw (Singh et al., 1998) and 5.0\% in the roots (Yin and Van Laar, 2005). Other parameters for which genotype-specific values were lacking were kept at default synthesized from previous studies for the whole panel in the crop model (Yin and Van Laar, 2005).

The GECROS model, calibrated as described above using the model input parameters from the control conditions in the 2013 experiment, was then used to simulate values of grain yield of the genotypes in the water-deficit condition of 2013, as well as in 2014 environments under both control and water-deficit conditions. Relative root mean square error (rRMSE) was used to inspect the quality of model simulation (Brun et al., 2006), and the $\mathrm{R}^{2}$ coefficient of the linear regression of simulated versus observed values of grain yield was used to show the percentage of phenotypic variation accounted for by the model.

\section{Multiple linear regression to identify the contribution of model input parameters to grain yield}

We also performed a multiple linear regression (MLR) analysis to test the contribution of each individual model input parameter (Table 1) to grain yield. The $\operatorname{lm}($ ) function in $\mathrm{R}$ was used to perform this analysis. 
Identifying SNP markers for model input parameters and grain yield, and estimating SNP-based values of these traits

In this study, we have followed a strategy that was explained by (Gu et al., 2014) for a biparental population, but with some modifications (see Supplementary Figure 1). Firstly, the rice association mapping population of 267 genotypes was randomly divided into a training (213 genotypes; $80 \%$ of the population) and a testing (54 genotypes; $20 \%$ of the population) set. Then we followed the two-step approach to identify the SNP markers and to calculate the marker-based estimates for the model input parameters and grain yield using the training set. In the first step, we used both the single-locus and the multi-locus GWAS analysis to identify the significant SNP markers for the model input parameters and grain yield. In the second step, these significant SNP markers were fed into a multiple-linear regression framework to estimate the additive effects of the markers, which were subsequently used to estimate the "GECROS" model input parameters and grain yield. A description of each step is explained in more detail below.

\section{Step 1: Single-locus and multi-locus GWAS analysis to identify the significant markers}

The single-locus GWAS analysis was performed on model input parameters and grain yield using a 45K SNP dataset by a compressed mixed linear model (CMLM) in the Genomic Association and Prediction Integrated Tool (GAPIT). The detailed protocol was explained in Chapter 4. Using this protocol, we selected the top ten significant markers with lowest $P$ value after excluding the redundant markers within the linkage disequilibrium (LD) of $\sim 55$ to $65 \mathrm{~kb}$ reported for this population (Kadam et al., 2017). Similarly, we conducted a multi-locus GWAS analysis that in addition to correcting the confounding effect of population structure (PC) and family relatedness $(\mathrm{K})$, corrected for the confounding effect of background loci present due to LD in the genome. We ran the complete model with stepwise forward inclusion of the lowest $P$ value marker as a cofactor until the heritability reached a value close to zero, followed by backward elimination of the least significant markers from the model (Segura et al., 2012). With this protocol, we selected all significant SNP markers associated to traits were incorporated as a cofactor in the model. In fact, multi-locus analysis also corrects the confounding effect of genome LD (Segura et al., 2012). Thus, significant SNP markers associated with traits identified through multi-locus analysis were not within LD region of $\sim 55$ to $65 \mathrm{~kb}$ reported for this population (Kadam et al., 2017). 
Step 2: Multiple-linear regression to estimate the model input parameters and grain yield All significant SNPs identified in Step 1 were fed into a multiple linear regression (MLR) using the $\operatorname{lm}()$ function in $\mathrm{R}$ with equation 1 .

$$
\mathrm{Y}_{\mathrm{k}}=\mu+\sum_{n=1}^{N} a_{\mathrm{n}} \mathrm{M}_{\mathrm{k}, \mathrm{n}}
$$

where $\mu=$ intercept, $a_{n}=$ additive effect of the $n^{\text {th }}$ marker, $M_{k, n}=$ genetic score of the $n^{\text {th }}$ of the individual genotypes $\mathrm{k}$ that takes either the value -1 (major allele with 0.95 frequency in studied population) or 1 (minor allele with 0.05 frequency in studied population). This analysis with all the SNP markers identified the non-significant markers due to collinearity of markers, which were removed in the next round of the MLR analysis. In addition, we also performed one more round of MLR analysis to remove the markers with cut-off threshold $P$ value $<0.01$. Finally, we estimated the SNP markers-based model input parameters using equation 1 in the GECROS model with estimated additive effects of the individual markers, and marker allelic data for each genotype in the whole panel.

\section{Sensitivity analysis to rank the relative importance of individual SNP markers}

Sensitivity analysis was performed using the GECROS model to test the effect of individual SNP markers on grain yield simulation following the principle explained by (Yin et al., 2000). First, we conducted the baseline simulation with genotype-specific allelic values of markers as model input to test the percentage of variation in grain yield explained by all markers. In the second step, to identify the important markers, we fixed one marker at a time to zero (i.e. excluding the effect of this marker in the analysis) to examine the variation in grain yield accounted for by the model. We performed such an analysis on all significant SNP markers, and assessed by what percentage the explained variation in grain yield dropped in comparison with the explained percentage of the baseline simulation. Using this protocol, we ranked the relative importance of the markers in determining grain yield variation.

\section{Virtual designing of an ideotype}

We followed two approaches to virtually design the ideotype for grain yield using GECROS by pyramiding the positive alleles of significant SNPs detected for model input parameters or of SNPs detected for grain yield. In the first approach, we regressed model input parameters against all the significant SNPs from the GWAS, detected for each model parameter, using equation 1. Similarly, we also regressed model input parameters against the SNPs detected for 
grain yield. The top four SNPs for model input parameters were selected based on relative importance in $\mathrm{R}$ package relaimpo (). Then, we regressed model input parameters against these top four SNP markers (eq. 1) to estimate the additive effect, and to calculate the marker-based value of the model input parameter. We used these marker-based model input parameters to design Ideotype I (using SNPs for model input parameters) and Ideotype II (using SNPs for grain yield). In the second approach, instead of using the top four SNPs, we selected all significant SNPs with $P$ values of $<0.01$ using equation 1 to estimate the additive effect, and to calculate the marker-based value of model input parameters. This was done for SNPs detected for each model input parameter, and for SNPs detected for grain yield. We used these markerbased model input parameters to design Ideotype III (using significant SNPs for model input parameters) and Ideotype IV (using significant SNPs for grain yield).

\section{Results}

Genotypic variation in model input parameters and their relative contribution to yield We used the control conditions of the 2013 experiment to parameterise or calibrate GECROS. Measured or estimated model input parameters (Table 1) showed a strong genotypic variation (Fig. 1). We used regression analysis to test the relative contribution of each of these model input parameters to grain yield variation. The model input parameter of total crop nitrogen uptake $\left(N_{\max }\right)$ accounted for the highest percentage of the grain yield variation in the association mapping panel (72.43\%; Table 2). Therefore, multiple linear regression analysis was performed with $N_{\max }$ as a cofactor in the model. Grain yield was significantly correlated with four other input parameters (post-flowering period $\left[m_{\mathrm{R}}\right]$, maximum plant height $\left[H_{\max }\right]$, grain set $\left[g_{\text {set }}\right]$, and grain nitrogen concentration $\left.\left[n_{\mathrm{so}}\right]\right)$, but not with pre-flowering period $\left(m_{\mathrm{V}}\right)$, photoperiod sensitivity $[\delta]$ or single-grain weight $\left(S_{\mathrm{w}}\right)$.

\section{Model performance in control and water-deficit conditions in the 2013 experiment}

We ran the model using the model input parameters of control conditions in the 2013 experiment. Simulating the grain yield under control conditions accounted for $58 \%$ of the total variation in grain yield with an rRMSE value of 0.19 in the association mapping panel (Fig. 2A). Using the same input parameter values calibrated with the data from the control conditions to simulate the situation under water-deficit stress of the same year 2013, the model accounted for $40 \%$ of the variation in grain yield with an rRMSE value of 0.28 (Fig. 2B). 
Table 1: Details of genotype-specific GECROS model input parameters classified in three categories.

\begin{tabular}{|c|c|c|}
\hline Trait & Description & Unit \\
\hline \multicolumn{3}{|c|}{ (A) Phenological } \\
\hline$m_{\mathrm{V}}$ & Pre-flowering period & thermal day \\
\hline$m_{\mathrm{R}}$ & Post-flowering period & thermal day \\
\hline$\delta$ & Photoperiod sensitivity & $\mathrm{hr}^{-1}$ \\
\hline \multicolumn{3}{|c|}{ (B) Morphological } \\
\hline$H_{\max }$ & Maximum plant height & $\mathrm{m}$ \\
\hline$\underline{S_{\mathrm{w}}}$ & Single-grain weight & $\mathrm{g}$ \\
\hline \multicolumn{3}{|c|}{ (C) Physiological } \\
\hline$g_{\text {set }}$ & Grain set & $\%$ \\
\hline$n_{\mathrm{so}}$ & Grain nitrogen concentration & $\mathrm{g} \mathrm{N} \mathrm{g}^{-1} \mathrm{DM}$ \\
\hline$N_{\max }$ & Total crop nitrogen uptake at maturity & $\mathrm{g} \mathrm{N} \mathrm{m}^{-2}$ \\
\hline $\begin{array}{l}\text { Thermal } \\
\text { on hourl } \\
\text { thermal c } \\
\text { temperat }\end{array}$ & $\begin{array}{l}\text { e bell-shaped temperature response equation } \\
\text { from weather data on daily maximum and } \\
\text { ual day only if temperature at each hour of the } \\
\text { elopment. So, } m_{\mathrm{V}} \text { or } m_{\mathrm{R}} \text { in thermal days are }\end{array}$ & $\begin{array}{l}\text { SECROS, base } \\
\text { temperatures; } \\
\text { to the optimun } \\
\text { their values i } \\
\text { en. }\end{array}$ \\
\hline
\end{tabular}

\section{Model performance in the 2014 experiment}

To simulate grain yield in the control and water-deficit conditions of the 2014 experiment, GECROS was used again with input parameters values from 2013 control conditions. In the 2014 experiment, the simulation was less accurate in both treatments, only accounting for $20 \%$ and $13 \%$ of the variation in grain yield under control and water-deficit conditions with rRMSE values of 0.31 and 0.40 , respectively (Fig. $2 \mathrm{C}$-D). The model tended to underestimate the grain yield in control conditions for most genotypes in the association panel (Fig. 2C). The model overestimated the grain yield at the lower tail of observed grain yield values, and underestimated grain yield at the upper end of the observed grain yield in water-deficit conditions (Fig. 2D).

\section{Identifying SNP markers for model input parameters and for grain yield}

To identify the SNP markers for model input parameters and grain yield, a single-locus and a multi-locus GWAS analysis (for more details see Materials and Methods) were performed on the 213 genotypes of the training set from 2013 control conditions. The remaining 54 genotypes were treated as the testing set. In total, we identified 104 SNP markers associated with model input parameters, and 12 SNP markers with grain yield in control conditions (Table 3). In the next step, we selected the final set of 90 out of 104 SNP markers for model input parameters with cut-off threshold $P$ values $<0.01$ using the MLR equation 1 (Supplementary Table 1 ). The 
Table 2: Linear regression of grain yield $\left(\mathrm{Y}\right.$ in $\left.\mathrm{g} \mathrm{m}^{-2}\right)$ with total crop nitrogen uptake $\left(N_{\max }\right.$ in $\mathrm{g} \mathrm{N} \mathrm{m}^{-2}$ ) and other individual model input parameters (Table 1) in 2013 control conditions.

\begin{tabular}{lcccc}
\hline \multicolumn{1}{c}{ Equation } & $\boldsymbol{\mu}$ & $\mathbf{a}_{\mathbf{1}}$ & $\mathbf{a}_{\mathbf{2}}$ & $\mathbf{R}^{\mathbf{2}}(\mathbf{\%})$ \\
\hline $\mathrm{Y}=\mu+\mathrm{a}_{1} N_{\max }$ & -207.29 & $81.28^{* * *}$ & & 72.43 \\
$\mathrm{Y}=\mu+\mathrm{a}_{1} N_{\max }+\mathrm{a}_{2} m_{\mathrm{V}}$ & -202.74 & $81.38^{* * *}$ & $-0.08^{\mathrm{ns}}$ & 72.43 \\
$\mathrm{Y}=\mu+\mathrm{a}_{1} N_{\max }+\mathrm{a}_{2} m_{\mathrm{R}}$ & -301.61 & $79.93^{* * *}$ & $3.11^{* *}$ & 73.39 \\
$\mathrm{Y}=\mu+\mathrm{a}_{1} N_{\max }+\mathrm{a}_{2} \delta$ & -211.24 & $82.28^{* * *}$ & $-102.65^{\text {ns }}$ & 72.79 \\
$\mathrm{Y}=\mu+\mathrm{a}_{1} N_{\max }+\mathrm{a}_{2} H_{\max }$ & -9.60 & $83.23^{* * *}$ & $-156.79^{* * *}$ & 85.05 \\
$\mathrm{Y}=\mu+\mathrm{a}_{1} N_{\max }+\mathrm{a}_{2} S_{\mathrm{w}}$ & -266.67 & $81.14^{* * *}$ & $2688.87^{\mathrm{ns}}$ & 72.85 \\
$\mathrm{Y}=\mu+\mathrm{a}_{1} N_{\max }+\mathrm{a}_{2} g_{\text {set }}$ & -355.65 & $66.87^{* * *}$ & $349.30^{* * *}$ & 77.92 \\
$\mathrm{Y}=\mu+\mathrm{a}_{1} N_{\max }+\mathrm{a}_{2} n_{\mathrm{so}}$ & 134.63 & $76.88^{* * *}$ & $-22861.21^{* * *}$ & 82.00 \\
\hline
\end{tabular}

phenotypic variation explained by the final set of SNPs detected for individual model input parameters ranged from $42.2 \%\left(g_{\text {set }}\right)$ to $77.0 \%$ ( $H_{\max }$; Supplementary Table 1$)$. In comparison, 12 SNP markers detected when grain yield of the same experiment was subjected to the GWAS analysis, together explaining $44.4 \%$ of the total variation in grain yield. No common SNP markers were found among model input parameters. Two markers on chromosome 8 (2341829) and 5 (658940) for $N_{\max }$ however were also associated with grain yield.

\section{Performance of SNP-based GECROS simulations on 2013 and 2014 experiments}

In the next step, a SNP-based GECROS model was created by linking the additive effect of each SNP for model input parameters estimated from the MLR analysis (eq. 1), and allelic data of each SNP (-1 for major allele and 1 for minor allele) for the whole association mapping panel (including training and testing sets). SNP-based model input parameter values calculated using equation 1 were fed to GECROS to simulate grain yield. The performance of such a model was assessed individually for training and testing sets. In training set, the SNP-based model accounted for $37 \%$ and $29 \%$ of variation in grain yield under control and water-deficit conditions with rRMSE values of 0.23 and 0.30 , respectively, during the 2013 experiment (Fig. $3 \mathrm{~A})$. However, model simulation was less robust on testing set, accounting only for $10 \%$ of yield variation under control conditions (rRMSE $=0.26$ ), and accounting for $15 \%$ of yield variation under water-deficit conditions (rRMSE=0.33; Fig. 3B) in 2013.

We also tested the marker-based GECROS model on data from the 2014 experiment. In training set, the model accounted for only $23 \%$ and $17 \%$ of variation in grain yield under control and water-deficit conditions, respectively (Fig. 3C). For the testing set, the model accounted for only $1 \%$ of the variation in grain yield in control and $9 \%$ of the variation in grain yield in water-deficit conditions (Fig. 3D). Across both years and both treatments, the model 


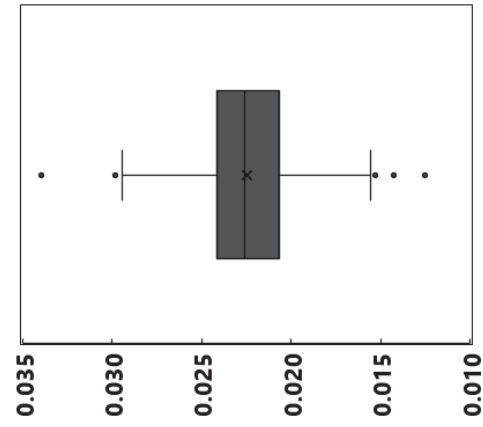

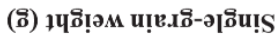

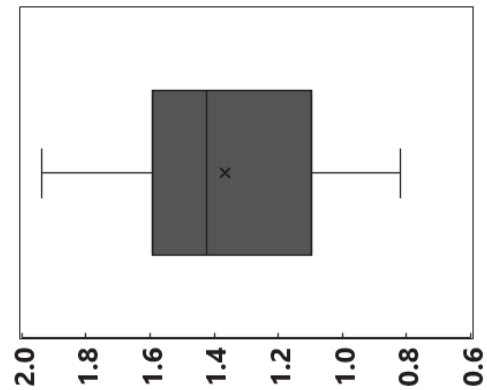

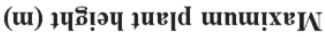
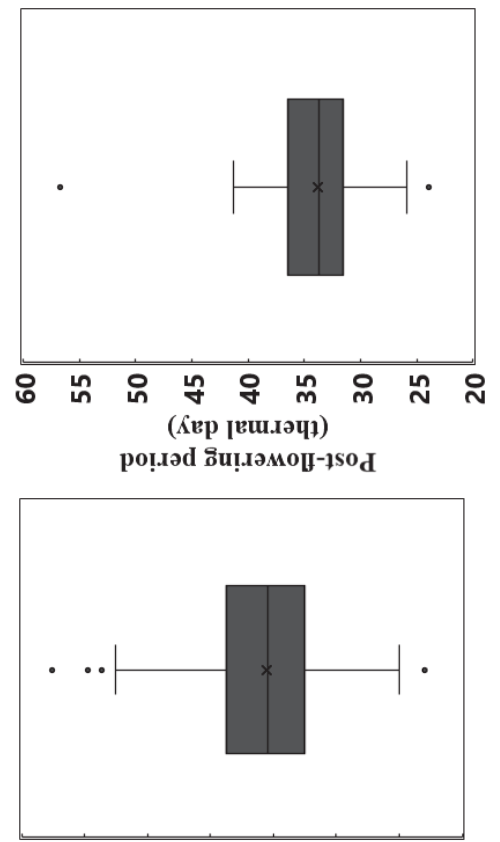

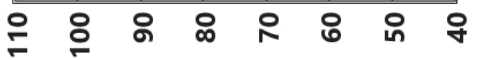

(Кер [ви.ләч1)

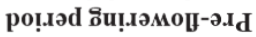

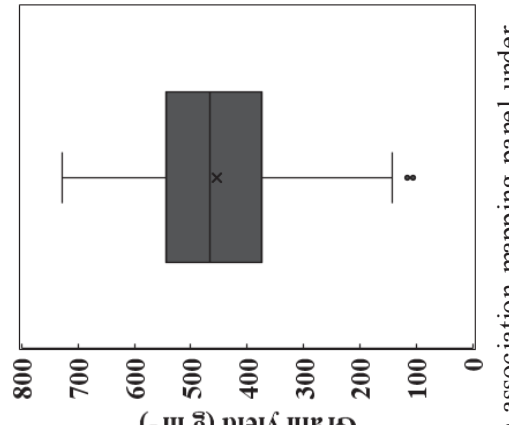

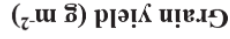

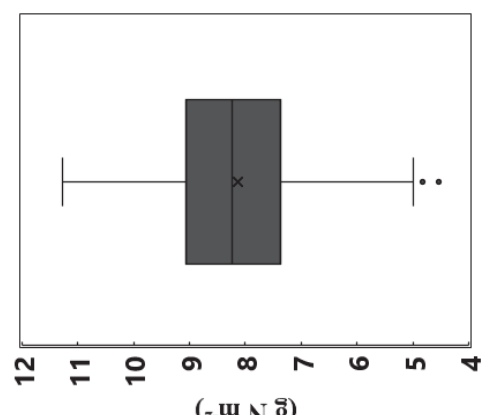

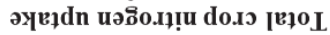

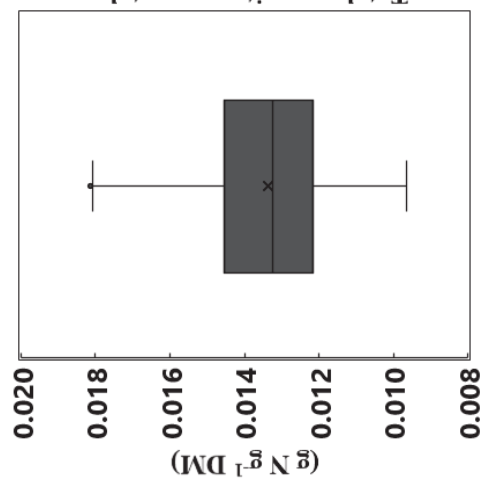

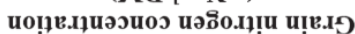

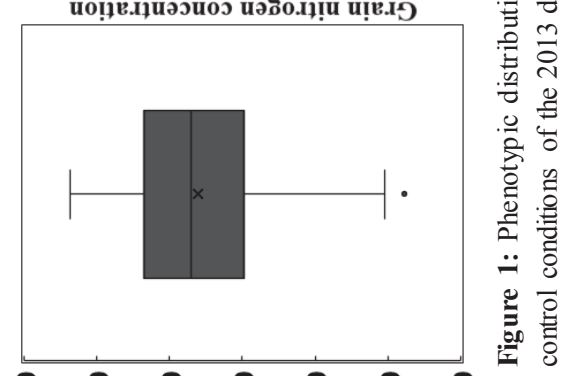

요 \& \&

(\%) ^əs u!̣..19 
overestimated the grain yield for genotypes having lower observed grain yield (lower end), and underestimated the grain yield for genotypes having higher observed grain yield (upper end) value (Fig. 3). We also correlated the original parameter-based simulations with SNP-based simulations, for the whole association mapping panel. The SNP-based simulations were well correlated with original parameter-based simulations under control conditions (2013: $r=0.72$ and 2014: $r=0.70$ ) and water-deficit conditions (2013: $r=0.77$ and 2014: $r=0.74$ ) in both years (Fig. 4).

\section{Sensitivity analysis to rank the relative importance of SNP markers in determining yield}

As stated in an earlier section, we detected 90 significant SNP markers for model input parameters. So, the sensitivity analysis by fixing these markers one at a time involved a total of 180 (90 in control and 90 in water-deficit) simulations to determine their relative importance in determining grain yield under control as well as water-stress conditions of the 2013 experiment. The top four SNP markers on chromosome 6 (1360962; rank 1), 7 (23760855; rank 2), 12 (6720935; rank 3), and 1 (1360962; rank 4) for $N_{\max }$ contributed to variation in grain yield under control conditions. For the top ranked SNP on chromosome 6 (1360962; rank 1), the phenotypic variation accounted for by GECROS decreased from $31.6 \%$ to $25.9 \%$ in control conditions for $N_{\max }$ (Supplementary Table 2). These results are supported by the linear regression of $N_{\max }$ that explained most of the variation in grain yield (Table 2). Similarly, the top 3 SNP markers on chromosome 4 (19591930; rank 1), 1 (9243669; rank 2), and 2 (4390533; rank 3) for $m_{\mathrm{V}}$ contributed most to grain yield under water-deficit conditions (Supplementary Table 2). The phenotypic variation accounted for by the model for the top ranked SNP on chromosome 4 (19591930; rank 1) decreased from $26.1 \%$ to $14.9 \%$ in water-deficit. Likewise, the fourth ranked SNP marker on chromosome 7 (58252) contributing to variation in grain yield was detected for $H_{\max }$ under water-deficit. These results clearly indicate that phenology plays a major role in influencing grain yield under stress comparable to that of $N_{\max }$ in control conditions. Nevertheless, the SNP marker on chromosome 6 (1360962; rank 6) influencing $N_{\max }$ and the marker on chromosome 3 (16529108; rank 7) influencing $n_{\text {So }}$ had significant effects on grain yield even under water-deficit (Supplementary Table 2). In addition, we also noticed that excluding the effect of some markers did not affect or change the variation in grain yield explained by the model, while in another situation it increased the explained grain yield variation. For instance, excluding one of the SNPs on chromosome 9 linked with $m_{\mathrm{R}}$ in control increased the explained variation in grain yield from $31.6 \%$ (baseline simulations) to $33.5 \%$ 

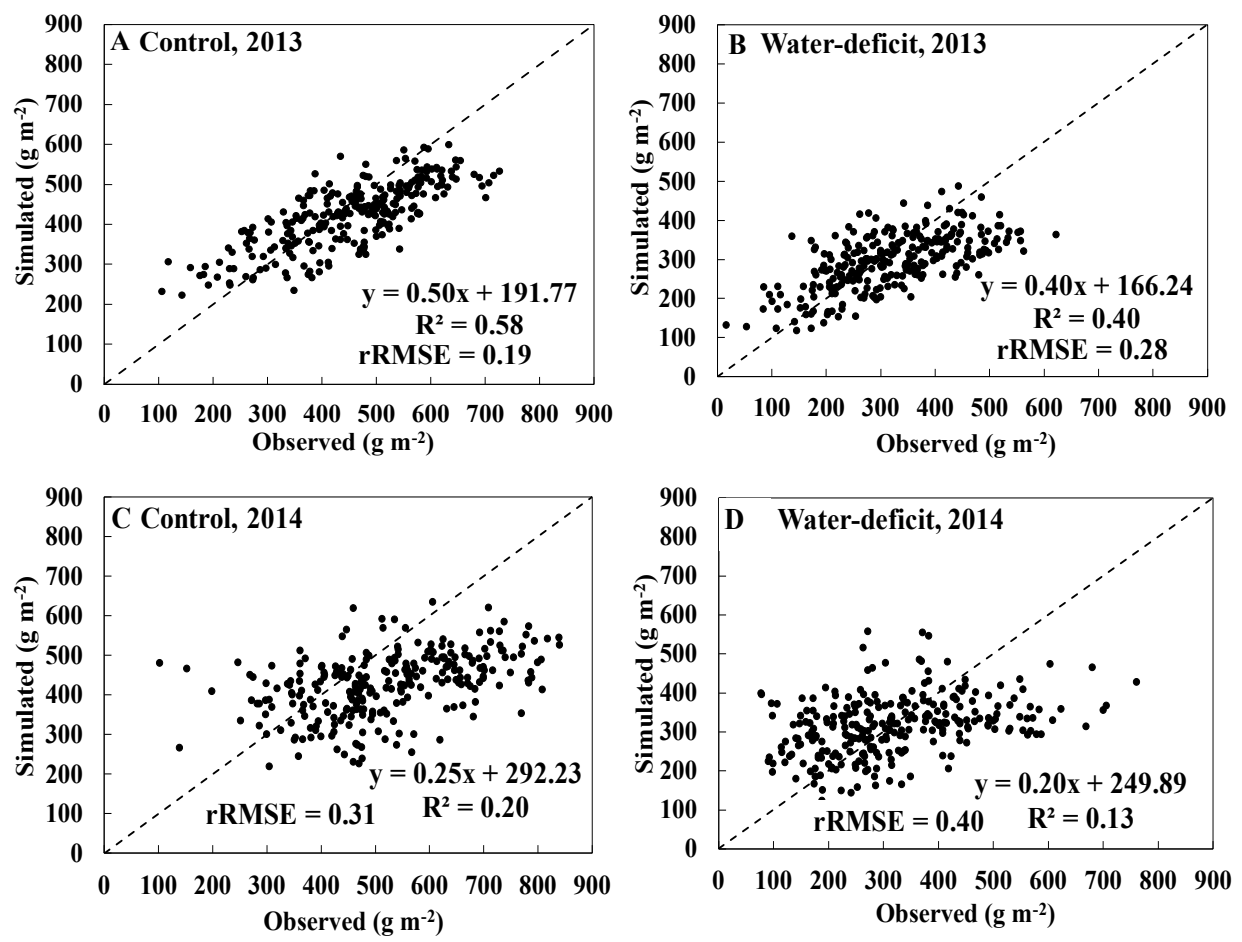

Figure 2: Relationship between simulated and observed values of grain yield in 267 genotypes of rice genome-wide association mapping population under control and water-deficit conditions in the 2013 (Panels A-B), and 2014 (Panels C-D) dry season experiments.

(Supplementary Table 2).

Designing virtual ideotypes using SNP alleles detected for model input parameters and for grain yield per se

Ideotype I showed only 3\% simulated grain yield advantage compared with Ideotype II in both treatments (Fig. 5). However, Ideotype III showed $89 \%$ and $75 \%$ simulated grain yield advantage compared to that of Ideotype IV under control and water-deficit stress, respectively (Fig. 5).

\section{Discussion}

In this study, we tried to link the process-based crop growth model GECROS with SNP markers identified through GWAS to simulate variation in grain yield among different rice genotypes in an association mapping panel. Key findings from our analysis are discussed below in detail. 
Table 3: Total number of significant SNPs detected through multiple linear regression (MLR) for eight GECROS model input parameters and grain yield of the rice training population $(n=213)$ under control conditions in the 2013 experiment. Percentage of phenotypic variations $\left(\mathrm{R}^{2}\right)$ explained by significant SNPs of model parameters and yield are derived from MLR (equation 1). The number mentioned in brackets refers to the number of significant SNP markers originally detected through the genome-wide association mapping study before putting them into the MLR analysis (for more details see Materials and Methods). Coefficients of equation 1 and additive effect of each significant SNP for model input parameters and grain yield are given in the Supplementary Table 1 .

\begin{tabular}{ccc}
\hline Trait & Significant SNPs & $\mathbf{R}^{\mathbf{2}}(\mathbf{\%})$ \\
\hline (A) Phenological & & \\
\hline$m_{\mathrm{V}}$ & $16(20)$ & 74.2 \\
$m_{\mathrm{R}}$ & $9(9)$ & 51.6 \\
$\delta$ & $9(9)$ & 65.1 \\
\hline (B) Morphological & & \\
\hline$H_{\max }$ & $13(17)$ & 77.0 \\
$S_{\mathrm{w}}$ & $8(9)$ & 47.3 \\
\hline (C) Physiological & & 42.2 \\
$g_{\text {set }}$ & $6(6)$ & 70.0 \\
$n_{\mathrm{SO}}$ & $16(19)$ & 66.8 \\
$N_{\max }$ & $13(15)$ & \\
\hline Total SNPs & $\mathbf{9 0 ( 1 0 4 )}$ & 44.3 \\
\hline Grain yield & 12 & \\
\hline
\end{tabular}

Model-based grain yield simulation in a new environment was less accurate than in the tested environment

It is often difficult to simulate the performance of given genotypes under contrasting environments or simulate the phenotypes of a set of genotypes under a given environment. GECROS works based on the principle of carbon-nitrogen interaction to simulate crop growth and development (Yin and Van Laar, 2005; Yin and Struik, 2010; Yin, 2013). This model was used to simulate grain yield and biomass differences in a biparental segregating population of rice ( $\mathrm{Gu}$ et al., 2014). For our study, the model was calibrated using eight model input parameters (Table 1) under control conditions from the 2013 experiment. This calibrated model satisfactorily simulated the observed differences in grain yield among the rice association mapping population in tested environments (2013 experiment, Fig. 2A). However, the variation accounted for was lower than in a previous study with a biparental population of introgression lines (Gu et al., 2014). This was mostly because the association mapping panel used in our study contained more much diversed or unrelated genotypes while population derived from biparental crosses are related with each other. The calibrated model showed poor simulation accuracy of 

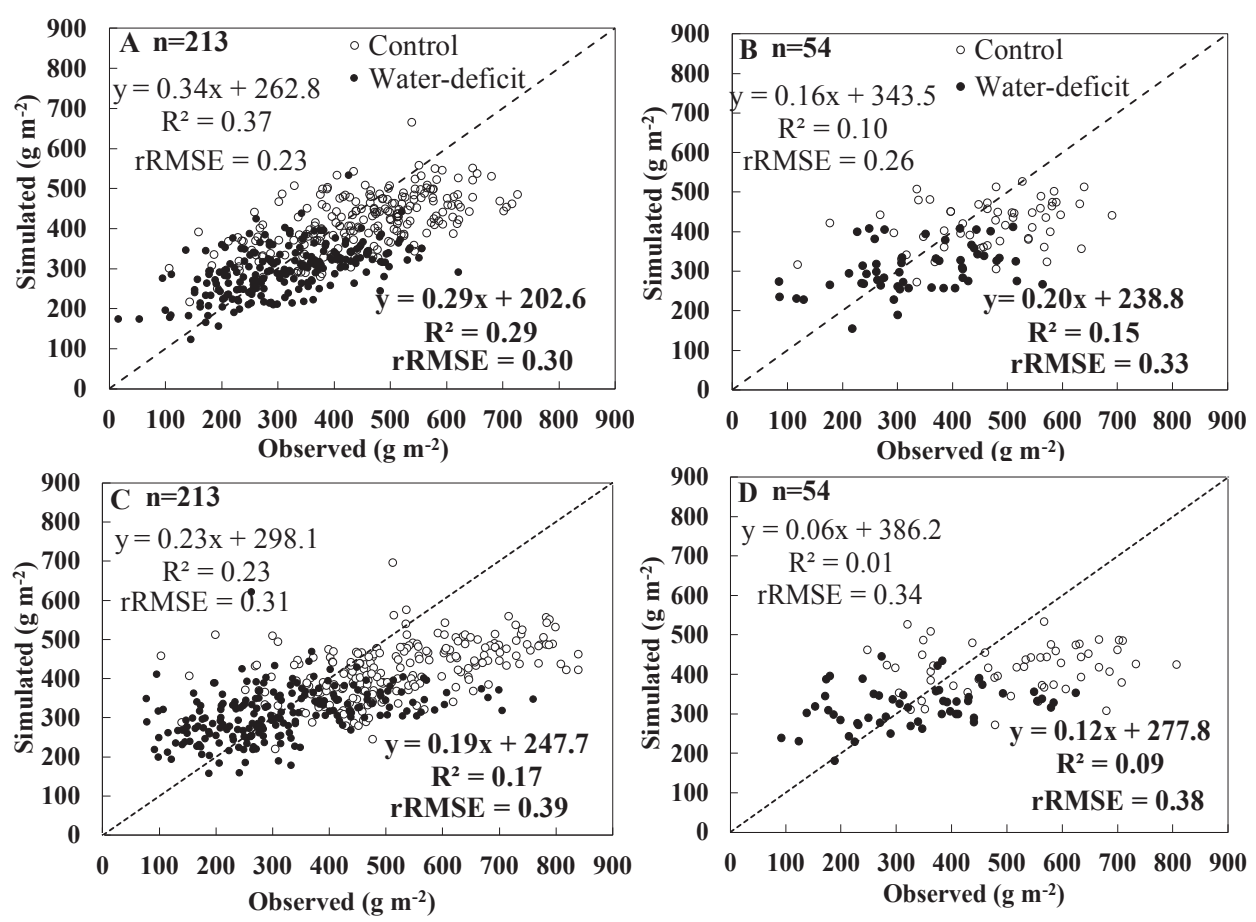

Figure 3: Relationship between SNP-based simulated and observed values of grain yield for training $(n=213)$ and testing $(n=54)$ populations of rice under control (open circle, statistical indicators in non-bold) and water-deficit (closed circle, statistical indicators in bold) conditions during 2013 (Panels A and B) and 2014 (Panels C and D) dry season experiments.

variation in grain yield in new environments, the 2013 water-deficit condition (Fig. 2B) and both control and water-deficit conditions in the 2014 experiment (Fig. 2C-D), due to strong genotype-by-environment $(\mathrm{G} \times \mathrm{E})$ interaction. These results also suggest that variations for morpho-physiological traits in our GWAS panel (see Kadam et al., 2017) important for yield determination are not completely accounted for by using the eight GECROS model input parameters chosen in the present study.

Water-deficit reduces transpiration cooling and increases tissue and organ temperature leading to higher spikelets sterility in rice (Jagadish et al., 2007). Potential seed number was determined by carbon and nitrogen accumulation during the vegetative phase in an earlier version of GECROS (Yin and Van Laar, 2005). Hence, the model originally did not have the capacity to account for the effect of organ temperature on spikelet sterility under stress conditions. In the present study, an upgraded version of the model was used to account for organ temperature effects on spikelet fertility while determining variation in grain yield under stress conditions. This indeed allowed to simulate $40 \%$ and $13 \%$ of the grain yield variation in the 

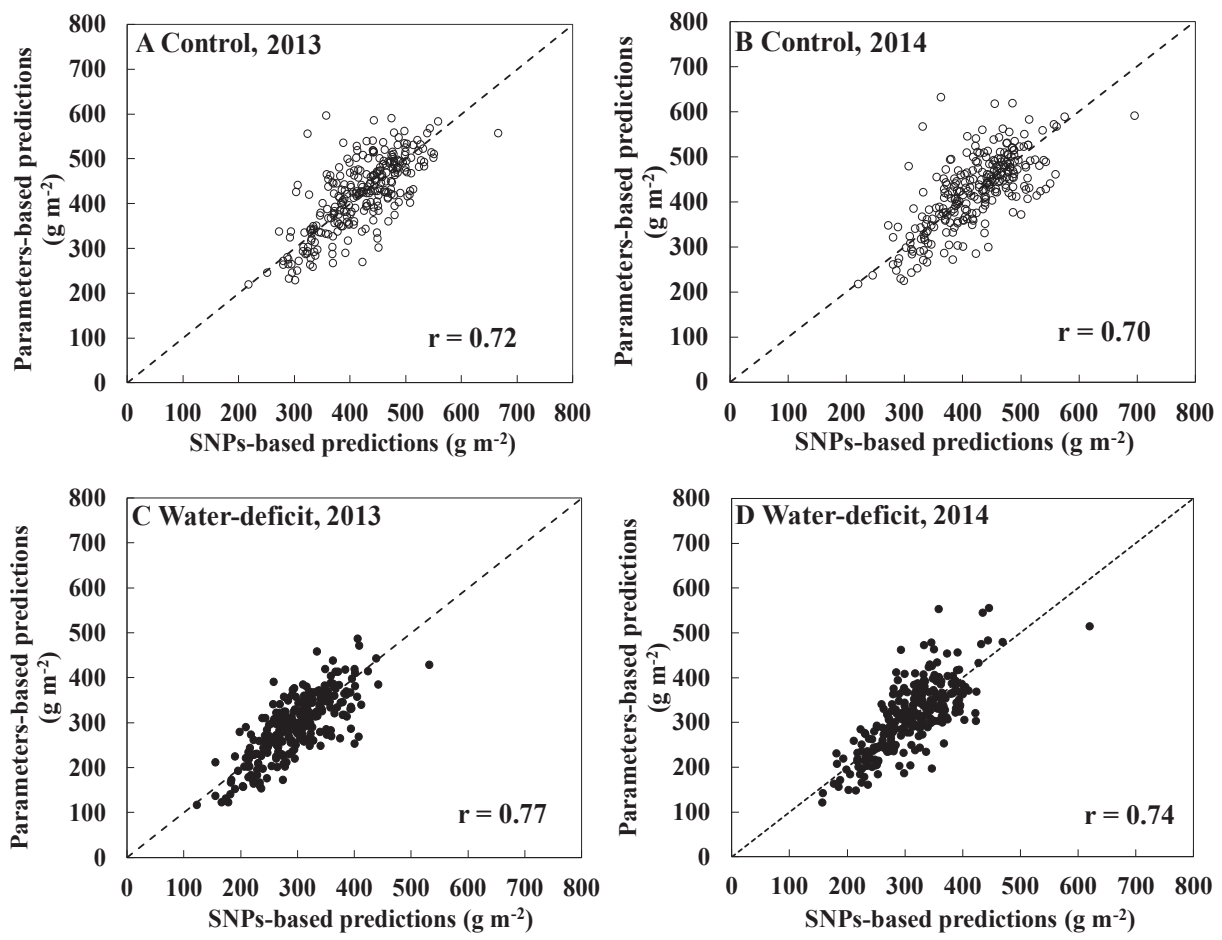

Figure 4: Correlations between GECROS input parameters based prediction of grain yield values and those predicted based on SNP-based model parameters for 267 rice genotypes under control (open circles; Panels A-B) and water-deficit (filled circles; Panels C-D) conditions during 2013 and 2014 dry season experiments.

association mapping panel under stress conditions during the 2013 (Fig. 2B) and 2014 experiments, respectively (Fig. 2D). The decreased simulated yield for the stress condition was due to an increased spikelet sterility because of simulated warmer panicle temperature by ca $2{ }^{\circ} \mathrm{C}$. Such an extent of panicle warming was in line with measurements of canopy temperature in the same experiment (Melandri, personal communication). Individual genotypes may respond differently both in their panicle temperature to water deficit and in their sensitivity of spikelet fertility to warmer panicle temperature. However, we did not have sufficient data on these possible differences; so, a uniform sensitivity parameter was applied to all genotypes, based on the recent report of Julia and Dingkuhn (2013). This may cause the poorer performance of the model in explaining yield differences among genotypes under stress environments, compared to the control conditions (Fig. 2).

The SNP-based model was created by estimating the genetic effect of model input parameters. To evaluate the predictive quality of the SNP-based model, special cross-validation 


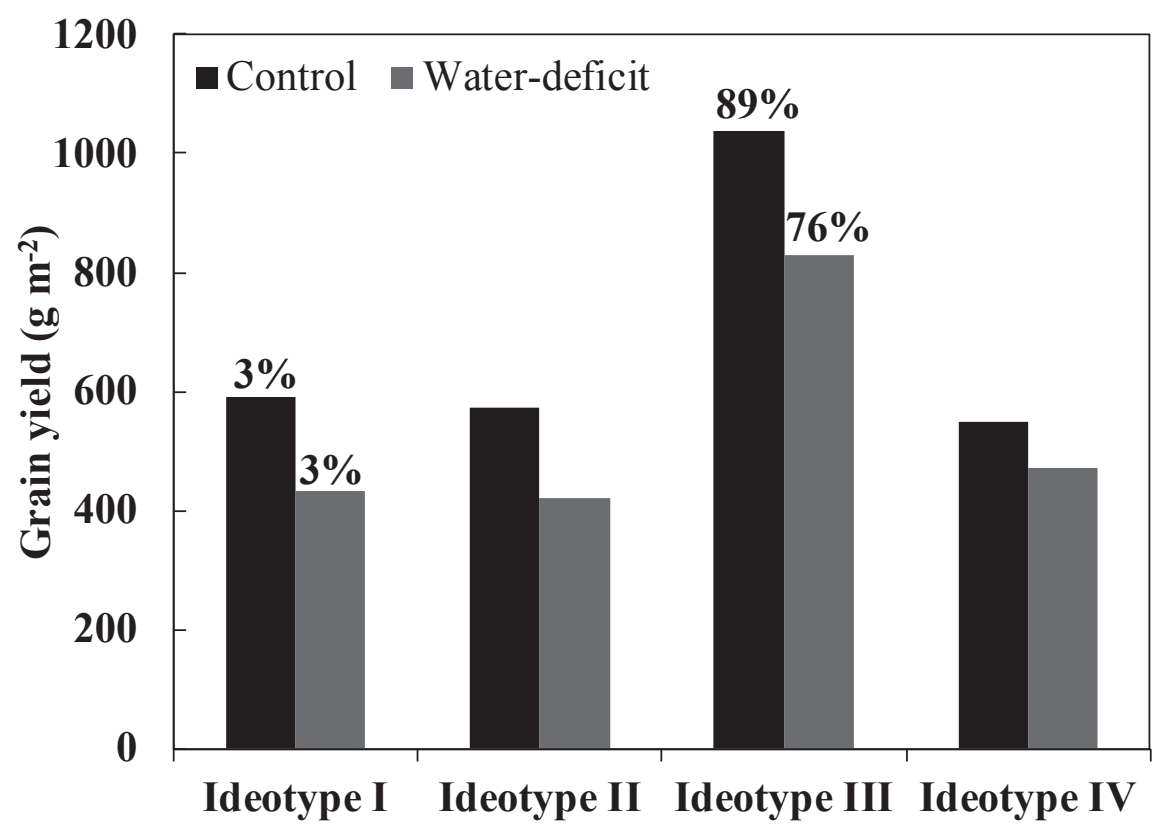

Figure 5: Simulated grain yields using the marker-based GECROS model for four ideotypes in control and water-deficit conditions. Ideotype I is the hypothetical genotype designed by pyramiding the positive alleles of the top 4 SNP markers selected based on relative importance in a multiple linear regression analysis for each model input parameter. Ideotype II is the hypothetical genotype designed by pyramiding the positive alleles of the top 4 SNP markers selected based on relative importance in a multiple linear regression analysis (regardless of significance of the $P$ value) for grain yield per se. Ideotype III is the hypothetical genotype designed by pyramiding the positive alleles of only significant SNP markers $(P<0.01)$ identified by multiple linear regression analysis for each model input parameter. Ideotype IV is the hypothetical genotype designed by pyramiding the positive alleles of only significant SNP markers $(P<0.01)$ identified by multiple linear regression analysis for grain yield per se. Percentage value indicates the relative advantage of Ideotype I over Ideotype II, and of Ideotype III over Ideotype IV.

schemes were used. In these schemes, the genotypes were randomly subdivided into a training and a testing set. The SNP-based model showed good potential to quantify the grain yield variation in the training set under 2013 control and new environments (Fig. 3A-C). However, the model showed poor simulation in a testing set (Fig. 3B-D). The population size is important for reliable GWAS analysis. Further, the phenotypic variance is strongly determined by how the two allelic variants differ in their phenotypic effect and their allelic frequency in the population sample. Hence, the lower simulation accuracy for the testing set suggests that excluding the testing set of genotypes in the GWAS analysis changed the allelic frequency of a given SNP in the population that had a strong influence on the phenotypic variance and on 
detecting the significant marker. Therefore, testing set was not representing the similar genetic diversity or population structure as training set and SNPs alleles may not be similarly represented in both the set. Such distant genetic relationship between training and testing sets might have lower down the prediction accuracy (Isidro et al., 2015). Therefore, it is very important to optimize the population structure using marker data while designing the training and testing sets to maximize the prediction accuracy.

Crop modelling helps to elucidate the genetic control of grain yield by identifying SNPs for yield-determining model input parameters

Complex traits such as grain yield are determined by many interactive physiological processes at the organ, plant and crop canopy level changing over time during the growing period. A deeper understanding of the way these processes contribute to grain yield is a prerequisite for designing the future new plant type for improved grain yield under changing climatic conditions (Peng et al., 2008). Crop growth models have been widely used as a tool to dissect complex traits (e.g., grain yield) as a function of its meaningful physiological components (Yin et al., 2004; Chenu et al., 2008; Hammer et al., 2010). This is the basis of using crop modelling to enhance phenotyping, i.e. what Dingkuhn et al. $(2017 \mathrm{a}, \mathrm{b})$ called "the heuristic phenotyping" of complex crop traits. We used the model to dissect grain yield into eight simple model input parameters to quantify genetic variation in a rice association mapping panel (Table 1). The multiple linear regression analysis confirmed that $N_{\max }$ had the strongest effect on grain yield among the model input parameters in agreement with a previous study (Gu et al., 2014). The genetic analysis also confirmed this by demonstrating that the two SNP markers for $N_{\max }$ colocalised with grain yield. This indicates that dissecting complex traits into their physiological components helps to pinpoint the exact genetic control and fundamental insights of complex traits such as grain yield (Yin et al., 2002). In addition, the number of QTLs identified for a single trait is always inadequate; however, model-based dissection allows detecting more markers than grain yield per se (Table 3). This clearly indicates that model based dissecting of the complex trait into individual components helps to detect more markers than for the complex trait such as grain yield alone (Gu et al., 2014; Amelong et al., 2015). Similar results have been recently reported for flowering time as a complex trait (Dingkuhn et al. 2017a). Despite this advantage of model-based dissection analysis over complex traits like grain yield per se, the latter approach cannot be replaced completely. Grain yield analysis identified SNP markers that were not detected by the model-based dissection, except two SNP 
markers for $N_{\max }$ colocalised with grain yield. This could be due to the fact that markers detected for the aggregated trait (such as grain yield) might have less impact on component traits (Yin et al., 2002). Another possibility could be that some of the yield-determining physiological mechanisms are not incorporated in the current GECROS model.

Further, we could not find any common SNP markers between model input parameters. This result is in line with that of Dingkuhn et al. $(2017 \mathrm{a}, \mathrm{b})$ for a rice association panel, but in contrast to a previous report on a biparental population of introgression lines ( $\mathrm{Gu}$ et al., 2014). Such contrasting results could also be due to the fact that in a biparental population of introgression lines one or two major segregating genes/QTLs might have a strong influence on multiple phenotypic traits (Yin et al., 2016). However, QTLs detected through GWAS analysis were having smaller effects on the main traits. In addition, their effect on other traits might also be too small, which cannot be detectable by current GWAS threshold $P$ value.

SNP-based GECROS modelling helps to evaluate the benefits of single markers at a time to improve the efficiency of marker assisted selection

In this study, we have demonstrated that GECROS is a useful tool to enhance the efficiency of selection for grain yield. The SNP-based modelling approach was used to rank the relative importance of markers identified for various grain yield determining model input parameters. This enabled to identify the most important yield determining markers that breeders can prioritize to improve the efficiency of MAS for specific environments. In addition, the relative performance of detected markers was different for control and water-deficit conditions of 2013 experiment (Supplementary Table 2). This indicates that the contribution of different physiological and morphological traits to grain yield varies under different environments.

Plant phenology such as flowering time is not only an essential part of reproductive processes but also a critical stage sensitive to various abiotic stresses (e.g. drought and heat) causing highest grain yield losses (Barnabás et al., 2008). In addition, it is evident that altering the flowering time is an avoidance strategy adopted by crops to maximise the fitness under reproductive stage stresses (Kazan and Lyons, 2016). Our SNP-based modelling analysis identified SNP markers linked with flowering time that strongly influenced variation in grain yield under water-deficit conditions in the rice association mapping panel (Supplementary Table 2). However, these SNP markers did not have a strong effect under control conditions, in which markers for $N_{\max }$ were more important (Supplementary Table 2). Hence, the marker based modelling analysis can help to understand how environmental variables affect the relative 
importance of phenotypic components and genotypic markers for complex traits. Further, this can greatly improve the selection efficiency for future genetic manipulation of crops to improve the productivity under changing climatic conditions.

\section{Virtual designing new plant types by pyramiding yield-determining positive alleles}

Grain yield results from many actions and interacting biochemical, morphological, and physiological processes taking place at different temporal and spatial scales in a crop. There are several lines of evidence for a shift in phenotypic characteristics while breeding the rice for improved grain yield. For instance, $15-20 \%$ grain yield increment was obtained by heterosis combined with phenotypic characteristics of new plant type (new plant type concept developed at IRRI was inspired by ideotype breeding) to develop a super hybrid rice (Peng et al., 2008; Yuan et al., 2003). Further, conventional crop models have become effective tools in identifying the best suitable combination of parameters, which helps in designing ideotypes for different environmental conditions (Aggarwal et al., 1997; Dingkuhn et al., 2007), thereby assisting crop breeding. However, such an approach lacks the connection of model input parameters to genetic information while designing the model based ideotype (Hammer et al., 2006; Martre et al., 2015). Recently, attempts were made to connect model input parameters to quantitative genetics and design the ideotype with yield advantage by pyramiding the marker alleles detected for model input parameters rather than grain yield per se (Letort et al., 2008; Gu et al., 2014).

Our simulation using a SNP-based model showed that the ideotype designed by pyramiding the positive alleles of only significant markers for model input parameters had higher grain yield potential than the ideotype designed based on markers of grain yield per se across control and water-deficit conditions (Fig. 5). The results clearly indicate the great potential of model-based dissection of a complex trait such as grain yield into its meaningful physiological component traits at different levels of biological organisation, and virtual pyramiding of their marker alleles to improve grain yield under different environments. Nevertheless, often ideotypes designed by crop growth modelling are contradictory and we are still far away from developing these virtual genotypes through molecular breeding and testing them under real field conditions. This could be due to the gap existing between model input parameters and genes or physiological function in response to changing environments $(\mathrm{G} \times \mathrm{E} / \mathrm{QTL} \times \mathrm{E}$ interactions). Therefore, to progress in this work, there is a need to narrow down the gap between genetic control relating to model input parameters and physiological processes in the model (Génard et al., 2016). 


\section{Challenges in linking the eco-physiological model with genome-wide association mapping}

Despite promising results, there are several intrinsic problems when combining ecophysiological modelling with GWAS. First, the calibrated model was only moderately accounting for the genotypic variation in grain yield across treatments in tested environments, and poorly performed under a new environment. Eight simple genotype-specific model input parameters (component traits), which could be estimated from the phenotyping data available in the present study, were not enough to realize a reasonably good yield prediction in diverse rice genotypes under different environmental conditions. Some of the eight parameters even did not contribute much to yield (Table 2). Hence, the current GECROS model needs to be further upgraded in terms of both model structure and model-input parameters, to capture more genotype-specific physiological and morphological processes measured using modern highthroughput phenotyping platforms for better simulation accuracy. Second, in contrast to a biparental QTL analysis, identification and estimation of QTL effects in a GWAS analysis indeed need to account for the population structure and genetic relatedness. We have accounted for both population structure and genetic relatedness while identifying the QTL for model input parameters using GWAS. Yet, later while using equation 1 to deriving model input parameter values from the identified QTL, population structure and genetic relatedness were ignored. To what extent the estimates of additive effect of QTLs on model input parameters using equation 1 without population structure and genetic relatedness could affect the simulation accuracy of grain yield by the crop model would need a further analysis. For that, there is a need for statistical algorithms that can better account for population structure and genetic relatedness inside the model while linking the modelling with the GWAS approach.

\section{Conclusion}

In this study, we reported on a genotype-to-phenotype modelling exercise, and how whole crop eco-physiological modelling provides an effective link with quantitative genetics to enhance the efficiency of molecular breeding for crop improvement. Unlike statistical genotype-tophenotype approaches that require many experiments (although on a single trait) to create a prediction model, eco-physiological genotype-to-phenotype modelling can, in principle, rely on few experiments for model parameterisation because the prediction is made largely based on eco-physiological principles as captured in the models. Our preliminary results when applying marker based modelling to identify key input parameters, were promising accounting for a large portion of the variation in grain yield under different environments, although largely 
based on only one treatment in the 2013 experiment for model parameterisation. This approach not only provided more SNP markers for model input parameters than grain yield per se, but also ranked the relative importance of these markers for molecular breeding for improved grain yield. This complemented the analysis of grain yield per se, and went beyond the existing reports of Mangin et al. (2017) and Dingkuhn et al. (2017a, b), who only illustrated the use of crop modelling to enhance the phenotyping for GWAS. Nevertheless, we also identified several pitfalls of such a modelling approach, which need to be addressed in future studies. 
Supplementary information in Chapter 5 


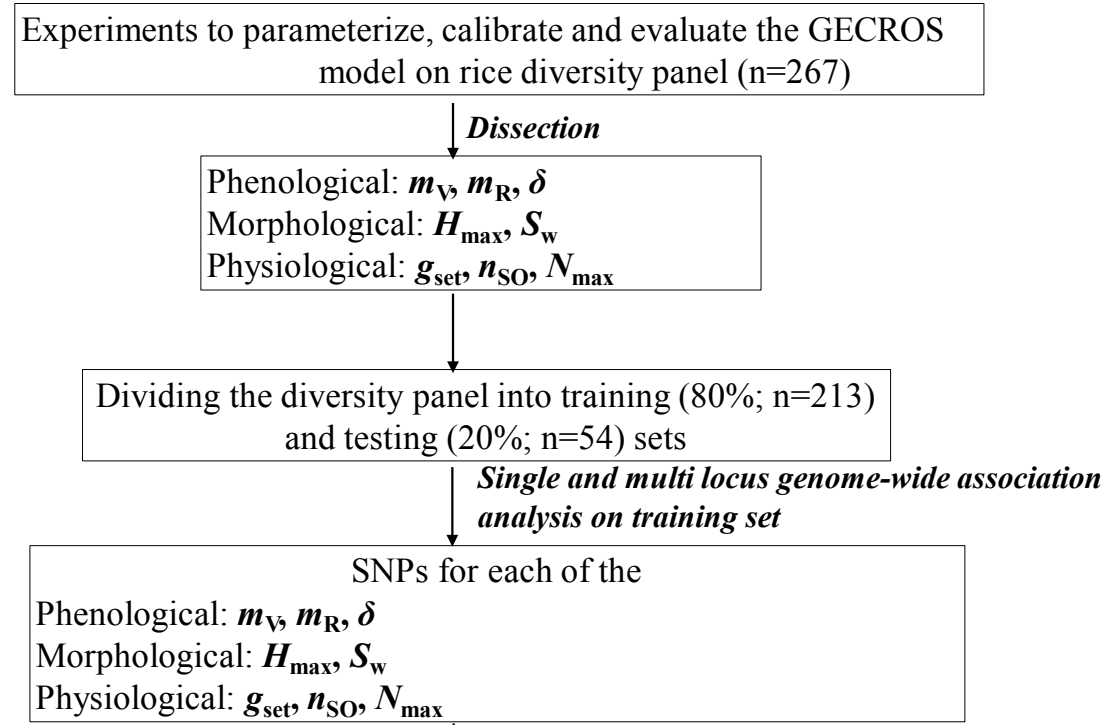

Calculation using equation (1) and allelic data of training set

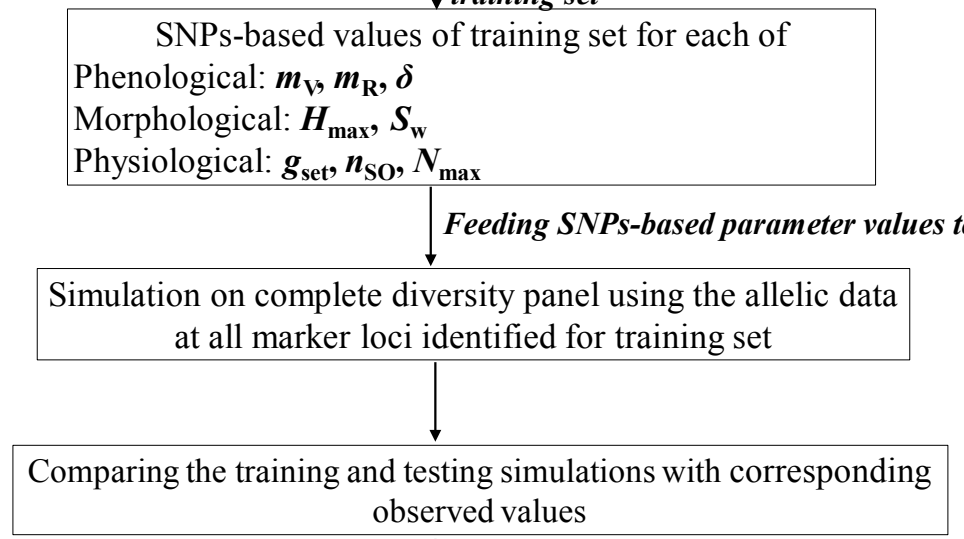

Fixing one SNP at a time, by assuming that its allelic genotype is 0 for all the diversity panel

\begin{tabular}{c} 
Sensitivity analysis to rank the importance of the \\
SNPs \\
$\qquad \begin{array}{l}\text { Pyramiding yield increasing alleles of } \\
\text { relevant SNPS }\end{array}$ \\
\hline Design of
\end{tabular}

Design of virtual ideotypes with increased grain yield

Supplementary Figure 1: Flow chart explaining the stepwise methodology adapted to combine the genome-wide association study (GWAS) with an eco-physiological crop modelling (GECROS). This flow chart was modified from Gu et al. (2014). 
Supplementary Table 1: Multiple linear regression (MLR) analysis of significant SNPs detected through the genome-wide association analysis against the model input parameters and grain yield. $\mathrm{R}^{2}$ value represents the percentage of phenotypic variation explained by all the SNP markers for a given trait.

\begin{tabular}{|c|c|c|c|c|c|c|}
\hline Parameter & Chromosome & Position & $u$ & Additive effects $\left(a_{n}\right)$ & $P$ value & $\mathbf{R}^{2}(\%)$ \\
\hline \multicolumn{7}{|c|}{ (A) Phenological } \\
\hline \multirow{16}{*}{$m_{\mathrm{V}}$} & 1 & 28262688 & \multirow{16}{*}{64.19} & 2.5371 & 0.001111 & \multirow{16}{*}{74.2} \\
\hline & 1 & 9243669 & & 3.7197 & 5.24E-08 & \\
\hline & 1 & 7344741 & & -1.5835 & 0.000138 & \\
\hline & 2 & 4390533 & & 3.6114 & $3.17 \mathrm{E}-12$ & \\
\hline & 2 & 23538671 & & -2.58 & $1.76 \mathrm{E}-11$ & \\
\hline & 3 & 10106310 & & -2.3681 & 0.000467 & \\
\hline & 4 & 19591930 & & 4.8726 & $5.21 \mathrm{E}-11$ & \\
\hline & 4 & 1258958 & & -2.6171 & 0.000878 & \\
\hline & 6 & 26238067 & & -2.467 & $1.54 \mathrm{E}-05$ & \\
\hline & 7 & 99783 & & -4.3277 & $1.48 \mathrm{E}-13$ & \\
\hline & 9 & 18375567 & & -1.2878 & 0.000274 & \\
\hline & 10 & 4760551 & & -1.3883 & 0.00039 & \\
\hline & 12 & 1691770 & & -4.0529 & 4.73E-09 & \\
\hline & 12 & 18314444 & & 2.5202 & $9.73 \mathrm{E}-12$ & \\
\hline & 12 & 3640868 & & -2.1222 & $6.73 \mathrm{E}-05$ & \\
\hline & 12 & 7323204 & & -2.7144 & $1.81 \mathrm{E}-07$ & \\
\hline \multirow{9}{*}{$m_{\mathrm{R}}$} & 4 & 33336957 & \multirow{9}{*}{32.95} & 0.6348 & 0.003947 & \multirow{9}{*}{51.6} \\
\hline & 4 & 3661992 & & -2.5713 & $1.60 \mathrm{E}-09$ & \\
\hline & 4 & 20195438 & & 3.362 & $2.53 \mathrm{E}-11$ & \\
\hline & 4 & 20014494 & & -1.7035 & 7.79E-06 & \\
\hline & 5 & 16151637 & & 0.8617 & $3.29 \mathrm{E}-05$ & \\
\hline & 9 & 22561421 & & -13.2386 & $4.75 \mathrm{E}-16$ & \\
\hline & 9 & 22561709 & & 11.0251 & $1.79 \mathrm{E}-12$ & \\
\hline & 9 & 16415255 & & 0.6762 & 0.002308 & \\
\hline & 11 & 19245430 & & 1.1817 & 0.000162 & \\
\hline \multirow{9}{*}{$\delta$} & 1 & 27113695 & \multirow{9}{*}{0.13} & -0.022173 & 0.001154 & \multirow{9}{*}{65.1} \\
\hline & 2 & 5508005 & & 0.024534 & 0.000143 & \\
\hline & 2 & 30150945 & & 0.039265 & $3.27 \mathrm{E}-08$ & \\
\hline & 4 & 4499266 & & 0.03125 & $4.75 \mathrm{E}-08$ & \\
\hline & 5 & 5619386 & & 0.017076 & 0.000126 & \\
\hline & 10 & 2718469 & & -0.028512 & $5.49 \mathrm{E}-07$ & \\
\hline & 11 & 22826451 & & 0.019679 & 0.000269 & \\
\hline & 11 & 6520591 & & 0.026071 & $2.78 \mathrm{E}-05$ & \\
\hline & 12 & 15123621 & & 0.043865 & $<2 \mathrm{e}-16$ & \\
\hline \multicolumn{7}{|c|}{ (B) Morphological } \\
\hline \multirow{13}{*}{$H_{\max }$} & 1 & 39255482 & \multirow{13}{*}{1.29} & -0.06984 & 0.000147 & \multirow{13}{*}{77.0} \\
\hline & 1 & 38286772 & & -0.1849 & $<2 \mathrm{e}-16$ & \\
\hline & 1 & 21100541 & & 0.10041 & $1.64 \mathrm{E}-06$ & \\
\hline & 1 & 852462 & & 0.04606 & 0.000382 & \\
\hline & 1 & 37707297 & & 0.06128 & $1.44 \mathrm{E}-05$ & \\
\hline & 1 & 6077035 & & -0.03864 & 0.000458 & \\
\hline & 2 & 24267632 & & -0.047 & 0.000128 & \\
\hline & 2 & 774705 & & 0.05459 & $1.56 \mathrm{E}-05$ & \\
\hline & 4 & 16556278 & & 0.03247 & 0.001486 & \\
\hline & 7 & 58252 & & -0.11813 & $1.34 \mathrm{E}-13$ & \\
\hline & 8 & 7880762 & & -0.10699 & $7.32 \mathrm{E}-12$ & \\
\hline & 8 & 19677137 & & 0.08531 & 0.000217 & \\
\hline & 10 & 2506985 & & -0.06651 & 0.002954 & \\
\hline
\end{tabular}


Supplementary Table 1. (Continued)

\begin{tabular}{|c|c|c|c|c|c|c|}
\hline \multirow{8}{*}{$S_{\mathrm{w}}$} & 2 & 554478 & \multirow{8}{*}{0.022} & 0.0008908 & $3.21 \mathrm{E}-08$ & \multirow{8}{*}{47.3} \\
\hline & 2 & 30699332 & & 0.0008471 & $3.22 \mathrm{E}-06$ & \\
\hline & 2 & 17222218 & & -0.0015312 & $2.41 \mathrm{E}-06$ & \\
\hline & 2 & 11071729 & & 0.0005774 & 0.000404 & \\
\hline & 3 & 16725807 & & 0.0009015 & $1.12 \mathrm{E}-07$ & \\
\hline & 3 & 12717890 & & -0.0007547 & 7.25E-06 & \\
\hline & 7 & 23227646 & & -0.0008358 & $3.21 \mathrm{E}-07$ & \\
\hline & 12 & 7731908 & & 0.0011273 & $1.92 \mathrm{E}-10$ & \\
\hline \multicolumn{7}{|l|}{ (C) Physiological } \\
\hline \multirow{6}{*}{$g_{\text {set }}$} & 2 & 29373768 & \multirow{6}{*}{0.732} & -0.05334 & $2.54 \mathrm{E}-07$ & \multirow{6}{*}{42.2} \\
\hline & 6 & 6585943 & & 0.031565 & $2.00 \mathrm{E}-07$ & \\
\hline & 10 & 14926494 & & 0.02464 & $1.97 \mathrm{E}-05$ & \\
\hline & 10 & 18906753 & & 0.023826 & $9.76 \mathrm{E}-05$ & \\
\hline & 12 & 20014218 & & -0.032433 & $3.48 \mathrm{E}-06$ & \\
\hline & 12 & 21173768 & & 0.016498 & 0.00755 & \\
\hline \multirow{16}{*}{$n_{\mathrm{SO}}$} & 1 & 42643627 & \multirow{16}{*}{$1.13 \mathrm{E}-02$} & $4.52 \mathrm{E}-04$ & $9.10 \mathrm{E}-05$ & \multirow{16}{*}{70.0} \\
\hline & 1 & 6765299 & & $2.66 \mathrm{E}-04$ & 0.001454 & \\
\hline & 1 & 22900197 & & $-2.82 \mathrm{E}-04$ & 0.000543 & \\
\hline & 2 & 34358656 & & $6.35 \mathrm{E}-04$ & $7.67 \mathrm{E}-10$ & \\
\hline & 3 & 25074645 & & $-5.29 \mathrm{E}-04$ & $5.34 \mathrm{E}-06$ & \\
\hline & 3 & 16529108 & & 5.79E-04 & $1.92 \mathrm{E}-12$ & \\
\hline & 4 & 1982000 & & $-3.23 \mathrm{E}-04$ & 0.000173 & \\
\hline & 4 & 31449324 & & $-3.09 \mathrm{E}-04$ & 0.000703 & \\
\hline & 5 & 14030811 & & $-3.43 \mathrm{E}-04$ & $1.59 \mathrm{E}-05$ & \\
\hline & 7 & 467419 & & $-7.43 \mathrm{E}-04$ & $1.95 \mathrm{E}-15$ & \\
\hline & 7 & 4568023 & & $-4.87 \mathrm{E}-04$ & $5.93 \mathrm{E}-10$ & \\
\hline & 7 & 9524268 & & $-6.50 \mathrm{E}-04$ & $1.34 \mathrm{E}-05$ & \\
\hline & 9 & 7207743 & & $-4.18 \mathrm{E}-04$ & 0.000483 & \\
\hline & 11 & 25041651 & & $-7.32 \mathrm{E}-04$ & 4.89E-07 & \\
\hline & 12 & 20907521 & & $8.55 \mathrm{E}-04$ & $<2 \mathrm{e}-16$ & \\
\hline & 12 & 17569836 & & $-5.84 \mathrm{E}-04$ & $1.54 \mathrm{E}-07$ & \\
\hline \multirow{13}{*}{$N_{\max }$} & 1 & 22221764 & \multirow{13}{*}{5.84} & -0.43691 & $3.05 \mathrm{E}-06$ & \multirow{13}{*}{66.8} \\
\hline & 1 & 18893159 & & 0.37043 & $7.18 \mathrm{E}-05$ & \\
\hline & 1 & 41741982 & & -0.40857 & 0.000239 & \\
\hline & 4 & 34815309 & & -0.77981 & $1.62 \mathrm{E}-10$ & \\
\hline & 5 & 658940 & & -0.2115 & 0.000765 & \\
\hline & 6 & 1360962 & & 0.34729 & $3.56 \mathrm{E}-05$ & \\
\hline & 7 & 23760855 & & -0.75225 & $3.80 \mathrm{E}-08$ & \\
\hline & 8 & 2341829 & & 0.28059 & 0.000122 & \\
\hline & 8 & 20492803 & & -0.32529 & $8.02 \mathrm{E}-08$ & \\
\hline & 11 & 10143495 & & 0.27417 & 7.34E-06 & \\
\hline & 11 & 965990 & & -0.3564 & 0.001213 & \\
\hline & 12 & 6720935 & & -0.45876 & $5.80 \mathrm{E}-09$ & \\
\hline & 12 & 19666909 & & -0.46896 & $1.32 \mathrm{E}-06$ & \\
\hline \multirow{12}{*}{ Grain yield } & 1 & 37302008 & \multirow{12}{*}{406.63} & -10.331 & 0.27545 & \multirow{12}{*}{44.3} \\
\hline & 1 & 537855 & & 1.933 & 0.78126 & \\
\hline & 2 & 26263170 & & 15.201 & 0.16315 & \\
\hline & 2 & 26654759 & & 19.416 & 0.05706 & \\
\hline & 4 & 10502119 & & 31.805 & 0.02564 & \\
\hline & 5 & 658940 & & -43.576 & $3.53 \mathrm{E}-09$ & \\
\hline & 6 & 10086748 & & -20.301 & 0.01844 & \\
\hline & 8 & 2756338 & & 1.809 & 0.83464 & \\
\hline & 8 & 2341829 & & 24.607 & 0.02028 & \\
\hline & 11 & 7789963 & & -48.323 & 0.0011 & \\
\hline & 11 & 10101900 & & -20.479 & 0.00836 & \\
\hline & 12 & 22741407 & & -46.749 & $8.56 \mathrm{E}-07$ & \\
\hline
\end{tabular}


Supplementary Table 2: The SNPs-based GECROS model accounting for the phenotypic variance of grain yield in a rice association mapping panel $(n=267)$ by different sets of simulations by stepwise fixing one marker at a time.

\begin{tabular}{|c|c|c|c|c|c|c|}
\hline \multirow{2}{*}{ Parameter } & \multirow[b]{2}{*}{ Chr } & \multirow[b]{2}{*}{ Position } & \multicolumn{2}{|c|}{ Control } & \multicolumn{2}{|c|}{ Water-deficit } \\
\hline & & & $R^{2}(\%)$ & Rank & $R^{2}(\%)$ & Rank \\
\hline \multicolumn{7}{|l|}{ (A) Phenological } \\
\hline \multirow{16}{*}{$m_{\mathrm{v}}$} & 1 & 28262688 & 30.5 & 29 & 20.5 & 5 \\
\hline & 1 & 9243669 & 29.4 & 12 & 16.0 & 2 \\
\hline & 1 & 7344741 & 31.5 & 49 & 29.4 & 86 \\
\hline & 2 & 4390533 & 29.1 & 10 & 18.0 & 3 \\
\hline & 2 & 23538671 & 29.6 & 14 & 24.0 & 14 \\
\hline & 3 & 10106310 & 33.1 & 87 & 29.4 & 85 \\
\hline & 4 & 19591930 & 28.6 & 8 & 14.9 & 1 \\
\hline & 4 & 1258958 & 31.8 & 65 & 29.1 & 84 \\
\hline & 6 & 26238067 & 31.5 & 47 & 28.7 & 80 \\
\hline & 7 & 99783 & 29.9 & 19 & 30.0 & 88 \\
\hline & 9 & 18375567 & 31.6 & 60 & 26.6 & 62 \\
\hline & 10 & 4760551 & 30.9 & 34 & 26.9 & 66 \\
\hline & 12 & 1691770 & 31.9 & 69 & 31.0 & 89 \\
\hline & 12 & 18314444 & 31.7 & 64 & 23.6 & 9 \\
\hline & 12 & 3640868 & 32.1 & 75 & 29.0 & 83 \\
\hline & 12 & 7323204 & 32.7 & 82 & 29.5 & 87 \\
\hline \multirow{9}{*}{$m_{\mathrm{R}}$} & 4 & 33336957 & 32.0 & 70 & 25.8 & 40 \\
\hline & 4 & 3661992 & 29.9 & 20 & 25.9 & 44 \\
\hline & 4 & 20195438 & 32.6 & 81 & 25.9 & 45 \\
\hline & 4 & 20014494 & 30.7 & 31 & 25.6 & 37 \\
\hline & 5 & 16151637 & 32.0 & 71 & 26.2 & 57 \\
\hline & 9 & 22561421 & 29.8 & 18 & 26.0 & 49 \\
\hline & 9 & 22561709 & 33.5 & 90 & 25.0 & 33 \\
\hline & 9 & 16415255 & 31.6 & 50 & 25.4 & 35 \\
\hline & 11 & 19245430 & 31.7 & 61 & 25.8 & 41 \\
\hline \multirow{9}{*}{$\delta$} & 1 & 27113695 & 31.6 & 53 & 26.1 & 50 \\
\hline & 2 & 5508005 & 30.6 & 30 & 24.5 & 24 \\
\hline & 2 & 30150945 & 30.7 & 32 & 23.9 & 12 \\
\hline & 4 & 4499266 & 30.2 & 24 & 24.2 & 19 \\
\hline & 5 & 5619386 & 30.5 & 27 & 24.9 & 30 \\
\hline & 10 & 2718469 & 31.9 & 66 & 26.8 & 63 \\
\hline & 11 & 22826451 & 30.3 & 25 & 24.9 & 31 \\
\hline & 11 & 6520591 & 30.1 & 21 & 24.5 & 25 \\
\hline & 12 & 15123621 & 30.1 & 22 & 24.1 & 18 \\
\hline
\end{tabular}


Supplementary Table 2. (Continued)

\begin{tabular}{|c|c|c|c|c|c|c|}
\hline \multicolumn{7}{|c|}{ (B) Morphological } \\
\hline \multirow{13}{*}{$H_{\text {max }}$} & 1 & 39255482 & 32.3 & 78 & 28.9 & 82 \\
\hline & 1 & 38286772 & 32.0 & 73 & 31.7 & 90 \\
\hline & 1 & 21100541 & 32.3 & 79 & 28.4 & 79 \\
\hline & 1 & 852462 & 31.5 & 46 & 27.4 & 72 \\
\hline & 1 & 37707297 & 30.5 & 28 & 27.3 & 71 \\
\hline & 1 & 6077035 & 31.2 & 39 & 25.7 & 39 \\
\hline & 2 & 24267632 & 31.4 & 42 & 24.8 & 28 \\
\hline & 2 & 774705 & 31.6 & 57 & 28.2 & 76 \\
\hline & 4 & 16556278 & 31.1 & 35 & 26.3 & 59 \\
\hline & 7 & 58252 & 28.8 & 9 & 20.5 & 4 \\
\hline & 8 & 7880762 & 31.4 & 41 & 25.8 & 42 \\
\hline & 8 & 19677137 & 32.0 & 74 & 28.2 & 77 \\
\hline & 10 & 2506985 & 30.4 & 26 & 23.6 & 8 \\
\hline \multirow{8}{*}{$S_{w}$} & 2 & 554478 & 31.5 & 43 & 26.2 & 58 \\
\hline & 2 & 30699332 & 31.7 & 62 & 26.0 & 47 \\
\hline & 2 & 17222218 & 31.4 & 40 & 26.1 & 55 \\
\hline & 2 & 11071729 & 31.5 & 45 & 26.1 & 54 \\
\hline & 3 & 16725807 & 31.6 & 54 & 26.1 & 51 \\
\hline & 3 & 12717890 & 31.6 & 55 & 26.1 & 53 \\
\hline & 7 & 23227646 & 32.0 & 72 & 26.1 & 56 \\
\hline & 12 & 7731908 & 31.5 & 44 & 26.1 & 52 \\
\hline \multicolumn{7}{|l|}{ (C) Physiological } \\
\hline \multirow{6}{*}{$g_{\text {set }}$} & 2 & 29373768 & 31.5 & 48 & 24.6 & 26 \\
\hline & 6 & 6585943 & 31.6 & 58 & 26.5 & 60 \\
\hline & 10 & 14926494 & 31.6 & 51 & 24.4 & 21 \\
\hline & 10 & 18906753 & 29.6 & 17 & 24.0 & 15 \\
\hline & 12 & 20014218 & 31.6 & 59 & 24.0 & 17 \\
\hline & 12 & 21173768 & 31.6 & 52 & 25.3 & 34 \\
\hline \multirow{16}{*}{$n_{\mathrm{so}}$} & 1 & 42643627 & 31.2 & 37 & 26.0 & 48 \\
\hline & 1 & 6765299 & 31.7 & 63 & 27.0 & 68 \\
\hline & 1 & 22900197 & 31.2 & 38 & 25.0 & 32 \\
\hline & 2 & 34358656 & 29.6 & 15 & 24.0 & 16 \\
\hline & 3 & 25074645 & 32.9 & 85 & 26.9 & 65 \\
\hline & 3 & 16529108 & 27.7 & 5 & 22.4 & 7 \\
\hline & 4 & 1982000 & 33.1 & 86 & 27.8 & 74 \\
\hline & 4 & 31449324 & 31.9 & 68 & 27.1 & 70 \\
\hline & 5 & 14030811 & 32.6 & 80 & 27.0 & 69 \\
\hline & 7 & 467419 & 31.9 & 67 & 23.7 & 10 \\
\hline & 7 & 4568023 & 33.4 & 89 & 27.7 & 73 \\
\hline & 7 & 9524268 & 33.2 & 88 & 26.8 & 64 \\
\hline & 9 & 7207743 & 32.9 & 84 & 27.9 & 75 \\
\hline & 11 & 25041651 & 31.6 & 56 & 26.0 & 46 \\
\hline & 12 & 20907521 & 32.9 & 83 & 24.7 & 27 \\
\hline & 12 & 17569836 & 31.2 & 36 & 26.9 & 67 \\
\hline
\end{tabular}


Supplementary Table 2. (Continued)

\begin{tabular}{cclcccc}
\hline & 1 & 22221764 & 27.5 & 4 & 24.4 & 22 \\
& 1 & 18893159 & 29.2 & 11 & 24.5 & 23 \\
& 1 & 41741982 & 30.2 & 23 & 25.8 & 43 \\
& 4 & 34815309 & 32.2 & 77 & 25.7 & 38 \\
& 5 & 658940 & 29.4 & 13 & 25.5 & 36 \\
$N_{\max }$ & 6 & 1360962 & $\mathbf{2 5 . 9}$ & $\mathbf{1}$ & 21.5 & 6 \\
& 7 & 23760855 & 26.0 & 2 & 24.8 & 29 \\
& 8 & 2341829 & 28.1 & 7 & 24.0 & 13 \\
& 8 & 20492803 & 32.2 & 76 & 28.7 & 81 \\
& 11 & 10143495 & 30.8 & 33 & 28.3 & 78 \\
& 11 & 965990 & 29.6 & 16 & 26.5 & 61 \\
\hline Baseline simulation & 12 & 6720935 & 27.2 & 3 & 24.3 & 20 \\
& 12 & 19666909 & 27.9 & 6 & 23.8 & 11 \\
\hline
\end{tabular}



CHAPTER 6

General discussion 
The current global climate change is well characterized with more frequent occurrence of the major abiotic stresses such as water-deficit and high temperature severely affecting crop productivity. Among the cereals, rice (Oryza sativa L.) is likely to become more affected by water-deficit stress than other cereals due to its adaptation to semi-aquatic conditions. Although water-deficit stress during the reproductive stage is most common and devastating, stress during the vegetative stage is also evidenced and can have significant carry-over effects on integrative traits like yield in rice (see Chapter 1). Therefore, it is imperative to develop rice genotypes with improved tolerance to water-deficit stress at any time during the growing period to ensure current and future food security.

During evolution, plants have developed several morphological, physiological and biochemical defence strategies to deal with water-deficit stress, and in most cases these strategies operate in synergy. Because of the multifaceted network of interactions among these strategies, tolerance to water-deficit stress is a complex, quantitative trait. Traditional breeding efforts to improve tolerance to water-deficit stress are indeed hampered by this complexity and by the quantitative nature of the trait (Xiong et al., 2006). Therefore, a better understanding of the above-mentioned defence strategies with their underlying physiological, genetic and molecular determinants is required to improve this stress tolerance.

Progress in developing high-throughput genome sequencing has been rapid over the last decade (Metzker, 2010). This rapid development has made it possible to sequence the whole genome of thousands of crop genotypes. Consequently science is now able to dissect the natural genetic variation resulting in a better scientific understanding of the genotype-to-phenotype relationship through mapping quantitative trait loci (QTLs), and thus provides a new strategic tool to breeders for rapid improvement of stress tolerance (Tuberosa and Salvi, 2006).

Most of the previous ecophysiological crop growth models were developed to predict crop yield, most likely of a few genotypes, in response to environmental variations. However, present generation ecophysiological crop growth models have been developed in a way that they can better handle the genetic difference of quantitative traits (under conditions with and without stress) among many genotypes, notably using genetically mapped QTL information as model input (see review of Yin \& Struik, 2016). This further helped to narrow down the gap between genotype and phenotype based on the impact of changing environmental conditions on complex traits (Gu et al., 2014). Such QTL-based crop growth modelling can dissect complex traits (e.g. grain yield) into biologically meaningful, physiological component traits that are genetically less complex. Once such models are properly validated they can assist 
marker-assisted selection and can support the design of virtual crop ideotypes (Gu et al., 2014), thereby accelerating traditional crop breeding. The simultaneous development of a new generation of crop models, state-of-the-art genotyping tools and enhanced insight in the crop physiology of stress tolerance opened new opportunities to advance breeding for stress tolerance in rice. Hence, in this dissertation, I have followed a multidisciplinary approach including the physiology, genetics and crop modelling, to better understand the adaptation of rice to water-deficit stress.

In this general discussion, I will provide a comprehensive analysis of my research findings and will cover the following major aspects.

- Physiological, morphological and root anatomical plasticity of rice to water stress during the vegetative stage, in comparison with the plasticity of wheat;

- Genetic control of root morphological and anatomical plasticity to water-deficit stress during the vegetative stage of rice;

- Challenges in phenotyping root traits for a genetic mapping study;

- Genetic control of grain yield and its components under water-deficit stress during the reproductive stage of rice;

- Linking ecophysiological modelling with genome wide association mapping to design a rice ideotype for grain yield.

I strongly believe that the knowledge generated in this study is an invaluable source for future scientific studies, and provides useful fundamental biological insights that help to understand the tolerance to water-deficit stress. Further, these findings can also help to improve the selection efficiency of rice breeding to improve stress adaptation. Finally, further research needs will be discussed.

\section{Rice displays weaker phenotypic plasticity in response to water-deficit stress during the vegetative stage than wheat}

Plants display many responses to water-deficit stress including changes in gene expression, metabolite production, gas exchange physiology, and morphology (Chaves and Oliveira, 2004). These responses can be measured as phenotypic plasticity, i.e. the ability of a given genotype to produce multiple phenotypes in response to changing environments (Sultan, 2000). Because 
of the primary role in water and nutrient uptake, plasticity in traits related to root morphology and anatomy are of great importance to improve rice stress adaptation and productivity. During its evolution, rice has developed a phenotypic plasticity to adapt to a wide range of moisture regimes, i.e. from traditional lowland (paddy rice) to upland/aerobic (moderately stress conditions) or severe water-deficit stress conditions (Khush, 1997). Indeed, several previous studies on rice have reported the role of root morphological plasticity in water-deficit adaptation. Plasticity in root length density (Kano-Nakata et al., 2011; Tran et al., 2015) and in lateral root length and/or branching (Suralta et al., 2010; Kano et al., 2011; Kano-Nakata et al., 2013) has been known to improve the water uptake and photosynthesis and thereby shoot biomass in rice under water-deficit conditions. Despite these research findings, rice is still considered to be relatively weakly adapted to water-deficit, certainly in comparison to other dryland cereal crops, such as wheat, maize and sorghum. In addition, there is inadequate knowledge on how rice differs from dryland cereals, and on which phenotypic traits related to water-deficit stress adaptation matter most (Praba et al., 2009). To fill this knowledge gap, I have evaluated the physiological, morphological and anatomical response of rice genotypes well adapted to different moisture regimes (lowland, aerobic and water-deficit conditions) with wheat genotypes that are drought tolerant under water-deficit stress during the vegetative stage. In this experiment, a comprehensive analysis between these two divergent species allowed to demonstrate the functional role of organ/tissue plasticity to independently validate known strategies and to identify novel complementary mechanistic means for adapting to water-deficit stress. In general, rice genotypes demonstrated a weaker morphological and anatomical plasticity in the shoot and in the root than wheat under water-deficit stress (Chapter 2).

Passioura (1997) suggested that wheat subjected to water-deficit during the vegetative stage tends to save soil water for sensitive stages in yield formation; the same tendency was suggested for pearl millet (Kholová et al., 2010). Both wheat genotypes studied in my experiment developed thicker leaves, thicker roots and showed moderate tillering under waterdeficit stress during the vegetative stage. These responses help to conserve soil moisture for use during later stages of development. The plant ideotype of rice proposed during the 1980s for water-deficit adaption includes most of the traits identified and mentioned above (for details see Henry, 2013). In contrast, two rice genotypes used in the experiment developed thinner roots in response to water-deficit stress, suggesting they were primed towards rapid water consumption, since having thinner roots is associated with an increase in overall root hydraulics enabling rapid uptake of water (Reich et al., 1998; Eissenstat and Achor, 1999; Solari et al., 
2006; Hernández et al., 2010). Such a rapid depletion of soil water reserves by thinner roots can result in higher susceptibility to water-deficit stress (Ryser, 2006).

The effects of water-deficit stress on radial root anatomy is often less studied than the effects on root morphology, but differences in root anatomical characteristics strongly influence water transport. Water transport in the root is proportional to the fourth power of the radius of the xylem conduit (Tyree and Ewers, 1991). The xylem conduit appears to be four times larger in deep roots than in a shallow root system (Jackson et al., 2000). In my study, the xylem diameter was smaller in rice with its shallow root system than in wheat, which has a deep root system. Increasing the water uptake by increasing the xylem size has been suggested in rice (Nguyen et al., 1997). Moreover, the plasticity of xylem diameter and number of xylem vessels in response to water-deficit in wheat was a novel finding, which provided additional mechanistic understanding of the plasticity of wheat roots towards water-deficit stress (Chapter 2). Further, (Parent et al., 2010) suggested that rice could be a species with acceptable waterdeficit tolerance after potential genetic improvements of root characteristics. Recently, the relationship between root architectural plasticity and yield stability was established under water-deficit in the field in a rice population derived from crossing traditional and improved genotypes (Sandhu et al., 2016). Therefore, growing rice like wheat is possible, provided additional efforts are made to identify traits that are related to a stress tolerant phenotype or to plasticity towards water-deficit stress, and to integrate such traits in breeding programs with a major emphasis on root morphological and anatomical plasticity.

\section{Genetic control of plasticity in root morphology and anatomy in response to water-deficit stress during the vegetative stage of rice}

The comparison of rice with wheat water-deficit tolerant genotypes made it possible to identify the functional relevance of root morphological and anatomical plasticity in water-deficit tolerance (Chapter 2). In addition, previous studies have proven that phenotypic plasticity is heritable (Nicotra and Davidson, 2010), and is controlled by key regulatory genes that regulate the growth and development in response to changing environments (Juenger, 2013). For instance, quantitative genotypic variation has been assessed and relevant QTL regions have been identified for plasticity of root hair length (Zhu et al., 2005a) and lateral root number (Zhu et al., 2005b) in maize under low phosphorus conditions. Similarly, it was demonstrated that genomic regions regulating the plasticity of increased root biomass in response to water-deficit stress were located on chromosome 1BS in wheat (Ehdaie et al., 2012). In addition to root 
morphological plasticity, plasticity in root anatomy, water-use efficiency (WUE) and phenology were associated with improved plant performance across changing environments in several species (Sadras et al., 2009; Niones et al., 2012; Niones et al., 2013; Kenney et al., 2014). In the past, hundreds of root traits/water-deficit tolerant QTLs were identified in rice, but genomic regions regulating the phenotypic plasticity of roots traits were rarely addressed. To the best of my knowledge, only QTLs regulating the plasticity of aerenchyma development (Niones et al., 2013), root length density (Kano-Nakata et al., 2011; Tran et al., 2015), lateral root growth (Suralta et al., 2010; Kano-Nakata et al., 2011) and root architectural plasticity (Sandhu et al., 2016) in response to water-deficit were identified in rice. Understanding the genetic and molecular mechanisms controlling the phenotypic plasticity of root morphology and anatomy will be essential for effective selection and breeding to improve rice water-deficit tolerance.

Diverse rice genotypes are a pool of naturally occurring mutations that can give fundamental insights into plant function as well as a vital resource of novel beneficial alleles for crop plant improvement (McCouch et al., 2013). In the experiment described in Chapter 3 , diverse indica rice genotypes were used to investigate the genetic architecture of root traits (root morphology and anatomy) across two moisture regimes (control and water-deficit) and of their calculated plasticity through a genome-wide association study (GWAS). Compared to those of many dicot species, the root traits of rice and other cereals are complex due to the fibrous root system consisting of many hierarchical orders of seminal, nodal, and lateral roots and root hairs. These different types of roots are highly plastic in response to water-deficit and nutrient stress and are strongly regulated by a complex network of many small-effect QTLs/genes. Our GWAS analysis identified that the genetic basis of root morphology and anatomy was different for the control and water-deficit conditions. We detected a strong QTL $\times$ environment interaction, in agreement with another recent study in rice ( $\mathrm{Li}$ et al., 2017). This was further strengthened by the novel loci for plasticity of root traits detected in my study (Chapter 3), which indicated that plasticity of root traits is heritable and under genetic control.

The genetic loci associated with root traits and their plasticity were in proximity to phytohormone genes regulating biosynthesis, transport or signalling. Among the phytohormones, predominately auxin plays a major role in root growth and development (Jung and McCouch, 2013; Wu and Cheng, 2014; Mai et al., 2014). Several of the loci that were significantly associated with root traits across both water-regimes or with the plasticity of these traits was placed near to the auxin transport (e.g. AUXIN EFFLUX CARRIER [PIN]) and 
signalling (e.g. auxin response factor [ARF], SMALL AUXIN UP-RNAs [SAUR]) genes. The PIN protein regulates the polar auxin transport and creates a differential auxin level within root system to regulate overall root architecture and growth with mutant alleles (pin1 \& 2) altering root growth (Grieneisen et al., 2007). Genes regulating the cell division and differentiation (e.g. SCARECROW, EXPANSIN), redox homeostasis, hormone cross-talk (e.g. GASA10) and water transport (e.g. AQUAPORIN) were also detected near the root traits loci. Hence, genetic loci identified in my study (Chapter 3) provide an important basis for revealing the molecular mechanism of plastic root development, to improve the water-deficit tolerance of rice in future.

\section{Phenotyping root systems under greenhouse and field conditions:}

\section{Challenges in genetic research on root characteristics}

There has been a rapid development in high-throughput genomic technologies enabling to accurately sequence thousands of crop species genotypes. However, exploiting this genomic information in genetic mapping analysis is still challenging because of the lack of a reliable high-throughput phenotyping platform. The GWAS analysis as described in Chapter 3 also highlights the importance of phenotyping in genetic research on root traits of rice, which merits additional discussion here as presented in this section.

Root phenotyping under real field conditions is extremely challenging, and is rapidly becoming the major bottleneck for genetic studies on water-deficit stress tolerance. In the past, several QTLs for root traits in rice were identified by experimenting in polyvinyl chloride (PVC) tubes containing a soil from the field and /or by growing plants under hydroponic conditions in a glasshouse (http://tropgenedb.cirad.fr/tropgene/JSP/interface.jsp? Module $=$ RICE). Nevertheless, very few of these QTLs have been validated under field conditions, in terms of their effects on root traits as well as on grain yield or yield components. This is mainly because phenotyping root traits on a large set of genotypes under field conditions is labour intensive, time consuming and costly. It is well known that conditions in pot experiments in the greenhouse strongly differ from field conditions, in terms of soil temperature (especially when the colour of the pot is black), compaction, water content and soil aeration (Poorter et al., 2012). For these reasons, QTLs identified under greenhouse conditions, in most cases, were not reproducible under field conditions.

Although phenotyping the root traits under field conditions is a more reliable and exciting approach, yet it is extremely complicated to retain complete root systems from the field soil. For this reason, PVC pots or hydroponic methods under greenhouse conditions were the 
preferred choice in most of the previous studies and in the present study as well. Presently many image analysis tools are available for capturing the root system and extracting quantitative data on root architecture from captured images. For more details please see the online resource (www.plant-image-analysis.org) and few of them have been systematically discussed by Lobet and Draye (2013). In our study, we used the WinRHIZO root image analysis tool (Chapters 23 ), which provided quantitative data on different components of the root system such as root thickness, total root length, root surface area, root volume, root thickness, root length classified for various classes of root thickness and root topology. Such analysis allowed investigating the entire root system and expanding our analysis beyond what could be traditionally measured by hand such as maximum root length. In addition, our genetic analysis identified the core regions of the rice genomes (SNPs or sometime termed QTLs) that had most significant impact on root development and their plasticity in response to water-deficit stress. Further, our gene content analysis also identified the most likely underlying a priori candidate genes, although the detailed future molecular characterization of these genes is essential to validate their roles in root development. Most of the root image analysis tools, including the one I have used in this study, are requiring destructive root sampling, which do not allow understanding the dynamics of root growth over time and space. Therefore, in the future, there is a need of non-invasive root phenotyping tools to measure the dynamic nature of root growth during different phases of plant development under target field conditions. Availability of such root phenotyping tools will be expected to provide tremendous information on the network of QTLs or genes regulating the root growth and development during the entire growing period of the plant or crop.

\section{Harnessing the QTL alleles for tolerance to water-deficit stress during the reproductive stage to improve rice yield}

High yielding semi-dwarf rice varieties that have been developed during the Green Revolution by the introduction of the dwarfing $s d l$ allele are better suited to optimal conditions, but typically sensitive to water-deficit stress (Vikram et al., 2015). Rice is affected by water-deficit stress throughout its life cycle, with the greatest grain yield losses reported for water-deficit stress during the reproductive stage (Venuprasad et al., 2007). To date, breeders have improved the tolerance to water-deficit stress during the reproductive stage by QTL alleles for grain yield and its components, detected in bi-parental mapping populations of rice (Venuprasad et al., 2009; Bernier et al., 2009; Vikram et al., 2011). This strategy has made promising progress in tolerance to water-deficit stress in rice, but still many of the QTLs/genes/alleles remain hidden 
in the genetic diversity that rice possesses and cannot be explored through traditional linkage analysis. In the experiments described in Chapter 4, I have explored the indica rice diversity panel to identify those hidden QTLs/genes for grain yield and its components through a GWAS under well-watered conditions and under water-deficit stress during the reproductive stage for two years. To date very few studies have considered the water-deficit stress during the reproductive stage using a GWAS approach (Ma et al., 2016; Pantalião et al., 2016; Swamy et al., 2017). The reason for this is that it is difficult to manage the plant phenology, especially the flowering time, in such a way that all genotypes are synchronized in terms of the timing of the stress treatment. In my study, I have followed staggered sowing of rice genotypes to synchronize their phenology and thus the phenological timing of exposure to water-deficit stress. Significant numbers of QTLs (SNPs) were detected for grain yield and its component traits, but most of these QTLs were specific to treatment and years, which means that there was a high QTL $\times$ environment interaction $(\mathrm{QEI})$.

Crop plants have developed a remarkable ability to respond to changing environments, and understanding the proximate and final mechanism behind these responses remains challenging. It is important to note that genotype $\times$ environment interactions are common for both QTL and gene expression, which are primarily determined by differential sensitivity of alleles (Marais et al., 2013). In our study, most of the QTLs for yield and its components were showing QEI. Sometimes these QEI can also be termed a QTL-effect plasticity. Grain yield is a complex trait with low heritability and strong environment interaction, mostly controlled by small-effect loci, and seldom by large-effect loci. To date, breeders have often used large- and consistent-effect grain yield QTLs under varying environments, to improve tolerance to waterdeficit stress during the reproductive stage in rice (Bernier et al., 2009; Vikram et al., 2011). QTLs identified through the GWAS of this study for grain yield and its component traits were small-effect loci with a strong QEI.

The QEI plays an important role in adaptation to changing environments, and is regulated by key environmental sensing genes (Marais et al., 2013). To understand the molecular basis of QEI for yield and its components, it is necessary to identify the underlying genes. The grain yield QTL loci Q9 (2013) and Q11 (2014) detected under water-deficit stress (Chapter 4) were placed near Phosphomannose mutase and Squalene epoxidase genes. Both genes were strongly responsive to abiotic stress and have a role in reactive oxygen species detoxification. Therefore, cloning and characterizing the genes from QTLs displaying QEI in 
water-deficit will provide much needed molecular causes of genotype $\times$ environment interactions, to improve stress tolerance of rice.

Under water-deficit stress, the selection for secondary traits such as canopy temperature could be used effectively to screen many genotypes. Low canopy temperature contributing to stress resistance through efficient water uptake by the roots helps to maintain cooler canopy (Richards et al., 2010). In my experiment, canopy temperature showed a negative correlation with grain yield under water-deficit $(r=-0.48$; data from measurements in the same experiment conducted by another $\mathrm{PhD}$ candidate), and therefore identifying the QTLs for desirable secondary traits, in addition to grain yield, could be an effective approach to improve stress tolerance of rice. Moreover, I could not find any root trait QTLs (Chapter 3) that were colocalized with QTLs identified for grain yield and its components under water-deficit stress (Chapter 4), despite using the same rice accessions in both chapters. This was mainly because QTLs for root traits were identified in PVC pots with water deficit stress during the vegetative stage under natural greenhouse conditions, while QTLs identified for grain yield and its components were identified under field water-deficit stress during the reproductive stage. Such results are obvious because the plant responses to water-deficit stress during the vegetative stage differ from those to water-deficit stress during the reproductive stage. In addition, growth conditions and stress level in pot experiments in the greenhouse (Chapter 3) strongly differ from those in real field experiments (Chapter 4), which might have also reduced the reproducibility of our root QTLs under field conditions. Thus, a tolerance mechanism observed for one type of water-deficit stress may not be effective in another type of stress under different growth and management conditions, which will eventually cause additional complexity in the breeding programme for tolerance to water-deficit stress.

\section{Merging eco-physiological modelling with genome-wide association mapping genetics}

The use of crop growth models is rapidly increasing to understand complex traits such as grain yield under water-deficit stress. Crop models dissect the complex grain yield and its response to environment into simple, biologically meaningful, physiological components. In addition, Yin et al. (2002) proposed the integration of QTL information into a process-based mechanistic crop growth model to design the grain yield ideotype and to support marker assisted selection under target environments. Further, linking the QTL information into an ecophysiological model can create a model that predicts the final phenotype (e.g. grain yield) from the 
combination of alleles by analysis of QTLs for model input traits. For example, grain yield was predicted under water-deficit, and was broken down into seven component physiological traits in rice (Gu et al., 2014). Similarly, the response of leaf growth to high temperature and waterdeficit stress was broken down to three components in maize (Reymond et al., 2003). In Chapter 5, the same principal was also followed, and I tried to link the QTL information detected through a GWAS analysis to the processed-based ecophysiological GECROS model. The QTLbased model moderately simulated the grain yield variation of rice diversity panel in tested environments (2013 experiment) but poorly in completely new environments (2014 experiment). The grain yield variation in the well-watered treatment was mainly explained by the input trait total crop nitrogen uptake $\left(N_{\max }\right)$ and in the treatment with water-deficit stress during the reproductive stage by pre-flowering time $\left(m_{\mathrm{V}}\right)$. Hence, crop phenology is a critical component of yield physiology strongly influenced by prevalent environmental conditions (Boonjung and Fukai, 1996). The QTLs identified for most of the model input trait did not coincide with the regions of the genome for grain yield per se except for $N_{\max }$ in control conditions, suggesting that mapping the input traits identified additional QTLs affecting grain yield. In addition, my analysis not only identified more markers for model input traits but also assessed their relative importance in explaining the grain yield variation, which can enhance the efficacy of marker-assisted selection. Moreover, my ideotype designing analysis clearly indicated that pyramiding the markers of model input traits had higher grain yield advantage than pyramiding the markers for grain yield per se. Therefore, the approach of linking crop modelling with GWAS has added values to studies on mapping grain yield per se. Most existing modelling studies have shown that crop models can assist to improve the phenotyping of GWAS (Mangin et al., 2017; Dingkuhn et al., 2017b; Dingkuhn et al., 2017a). Chapter 5 demonstrates that crop modelling not only assists the phenotyping, but also can integrate with GWAS into a promising strategy of crop ideotype design. In conclusion, the use of models provides an efficient platform to integrate the genetics and crop physiology to narrow down the genotypephenotype gap.

\section{Final remarks and future research}

The data generated in my study is very rich and powerful. However, the information collected requires further analysis and suggests the need to carry out additional trials, especially to validate candidate genes and to supplement our understanding of the diverse mechanisms of tolerance to water-deficit stress. The combination of genetics and crop growth modelling offers 
great potential to rapidly assess the importance of model input traits and their impact on final grain yield in response to chaining environments. Nevertheless, combining the crop modelling with GWAS to predict grain yield identified several challenges (see Chapter 5).

Current advanced crop models may miss important traits that determine yield differences among genotypes. For example, many morphological traits were identified relevant for genotypic differences in response to water-deficit stress (Chapters 3 and 4), but they are not yet captured in generic crop models like GECROS that was used in my study, for expressing differences among rice genotypes.

- Population structure and genetic relatedness strongly influencing the QTL genetic effect on trait was accounted for while identifying the markers for model input traits in GWAS analysis. However, while feeding the effect of identified QTLs for model input traits to the ecophysiological crop growth model, the population structure and genetic relatedness was ignored due to lack of statistical algorithms.

- Recently, genomic selection has been revolutionizing the marker-assisted breeding mainly because it includes all marker information in the prediction model, avoiding the biased estimation of QTL effect -a major disadvantage retained by GWAS (Meuwissen et al., 2001), thereby capturing the large part of phenotypic variation explained by small effect QTLs. Therefore, future studies should consider integrating crop growth models and genomic selection, with better optimisation of population structure in training and testing sets.

There is no doubt that generic crop models like GECROS need refining and further calibration, when applied to specific crops for analysing genotype-to-phenotype relationships. Another route is to seek advanced statistical approaches to enhance the resolution of the genetic prediction. One way of improvement is to integrate crop growth modelling with genomic selection or prediction. I expect that a new class of refined ecophysiological crop growth models, when integrated with advanced genomic and genetic prediction tools, will further narrow down genotype-phenotype gaps, thereby improving the efficiency of applied genetics and traditional breeding for tolerance to water-deficit stress.

In addition, studies for unravelling the mechanism related to abiotic stress in cereals, including this study for water-deficit stress, have been mostly independent, not considering multiple stress (drought, high temperature and flooding) imposition. However, under actual field conditions often these abiotic stresses occur simultaneously and interact with each other (Jagadish et al., 2012). Among the abiotic stresses, combined occurrence of water-deficit stress 
and high-temperature stress is identified to be the most commonly occurring companion stress (Mittler, 2006). In this context, I have systematically reviewed some progress on individual and combined effects of water-deficit stress and high-temperature stress in interaction with elevated $\mathrm{CO}_{2}$ on agronomic and physiological responses (Kadam et al., 2014). However, it is essential to dissect the tolerance of complex abiotic stresses in interaction through an integrative multidisciplinary approach of crop physiology, genetics and crop modelling, to create the next generation rice that can cope with climate change. 



\section{References}

Abdurakhmonov, I.Y., and Abdukarimov, A. (2008) Application of association mapping to understanding the genetic diversity of plant germplasm resources. International Journal of Plant Genomics 2008: 18.

Abramoff, M.D., Magalhaes, P.J., Ram, S.J. (2004) Image processing with ImageJ. Biophotonics International 11: 36-42.

Acevedo, E.H., Silva, P.C., Silva, H.R., Solar, B.R. (1999) Wheat production in Mediterranean environments. In: Satorre, E.H., Slafer, G.A. (Eds.), Wheat: ecology and physiology of yield determination. Food Products Press, Binghamton, NY, pp. 295331 .

Adachi, S., Tsuru, Y., Kondo, M., Yamamoto, T., Arai-Sanoh, Y., Ando, T., Ookawa, T., Yano, M., and Hirasawa, T. (2010) Characterization of a rice variety with high hydraulic conductance and identification of the chromosome region responsible using chromosome segment substitution lines. Annals of Botany 106: 803-811.

Aggarwal, P.K., Kropff, M.J., Cassman, K.G., and ten Berge, H.F.M. (1997) Simulating genotypic strategies for increasing rice yield potential in irrigated, tropical environments. Field Crops Research 51: 5-17.

Agrama, H.A., Eizenga, G.C., Yan, W. (2007) Association mapping of yield and its components in rice cultivars. Molecular Breeding 19: 341-356.

Aguilar, E.A. (1998) Responses of banana roots to oxygen deficiency and its implications for Fusarium wilt. PhD Thesis. The University of Western Australia, Australia.

Ahmad, I., Mian, A., Maathuis, F.J.M. (2016) Overexpression of the rice AKT1 potassium channel affects potassium nutrition and rice drought tolerance. Journal of Experimental Botany 67: 2689-2698.

Ali, S., Gautam, R.K., Mahajan, R., Krishnamurthy, S.L., Sharma, S.K., Singh, R.K., and Ismail, A.M. (2013) Stress indices and selectable traits in SALTOL QTL introgressed rice genotypes for reproductive stage tolerance to sodicity and salinity stresses. Field Crops Research 154: 65-73.

Al-Tamimi, N., Brien, C., Oakey, H., Berger, B., Saade, S., Ho, Y.S., Schmöckel, S.M., Tester, M., Negrão, S. (2016) Salinity tolerance loci revealed in rice using highthroughput non-invasive phenotyping. Nature Communications 7: 13342.

Ambawat, S., Sharma, P., Yadav, N.R., Yadav, R.C. (2013) MYB transcription factor genes as regulators for plant responses: an overview. Physiology and Molecular Biology of Plants 19: 307-321.

Amelong, A., Gambín, B.L., Severini, A.D., and Borrás, L. (2015) Predicting maize kernel number using QTL information. Field Crops Research 172: 119-131.

Araus, J.L., Amaro, T., Zuhair, Y., Nachit, M.M. (1997) Effect of leaf structure and water status on carbon isotope discrimination in field-grown durum wheat. Plant, Cell \& Environment 20: 1484-1494.

Araus, J.L., Slafer, G.A., Reynolds, M.P., and Royo, C. (2002) Plant breeding and drought in $\mathrm{C}_{3}$ cereals: What should we breed for? Annals of Botany 89: 925-940.

Araya, T., Miyamoto, M., Wibowo, J., Suzuki, A., Kojima, S., Tsuchiya, Y.N., Sawa, S., Fukuda, H., von Wirén N., Takahashi, H. (2014) CLE-CLAVATA1 peptide-receptor signaling module regulates the expansion of plant root systems in a nitrogen-dependent manner. Proceedings of the National Academy of Sciences 111: 2029-2034.

Armstrong, W., Beckett, P.M. (1987) Internal aeration and the development of stellar anoxia in submerged roots. A multishelled mathematical model combining axial diffusion of oxygen in the cortex with radial losses to the stele, the wall layers and the rhizosphere. New Phytologist 105: 221-245. 
Armstrong, W., Beckett, P.M., Justin, S.H.F.W., Lythe, S. (1991) Modelling, and other aspects of root aeration by diffusion. In MB Jackson, DD Davies and H Lambers, eds, Plant Life Under Oxygen Deprivation, SPB Academic Publishing, The Hague, pp 267282.

Arrigoni, O., De Tullio, M.C. (2002) Ascorbic acid: much more than just an antioxidant. Biochimica et Biophysica Acta (BBA)-General Subjects 1569: 1-9.

Ashikari, M., Sakakibara, H., Lin, S., Ymamoto, T., Takashi, T., Nishimura, A., Angeles, E.R., Qian, Q., Kitano, H., Matsuoka, M. (2005) Cytokinin oxidase regulates rice grain production. Science 309: 741-745.

Aslam, M., Zamir, M.S.I., Afzal, I., Yaseen, M., Mubeen, M., Shoai, B.A. (2013) Drought stress, its effect on maize production and development of drought tolerance through potassium application. Cercetări Agronomice in Moldova 46: 99-114.

Baluška, F., Jasik, J., Edelmann, H.G., Salajová, T., Volkmann, D. (2001) Latrunculin Binduced plant dwarfism: Plant cell elongation is F-actin-dependent. Developmental Biology 231: 113-124.

Baluška, F., Salaj, J., Mathur, J., Braun, M., Jasper, F., Šamaj, J., Chua, N.H., Barlow, P.W., Volkmann, D. (2000) Root hair formation: F-actin-dependent tip growth is initiated by local assembly of profilin-supported F-actin meshworks accumulated within expansin-enriched bulges. Developmental Biology 227: 618-632.

Bao, F., Shen, J., Brady, S.R., Muday, G.K., Asami, T., Yang, Z. (2004) Brassinosteroids interact with auxin to promote lateral root development in Arabidopsis. Plant Physiology 134: 1624-1631.

Barnabás, B., Jäger, K., and Fehér, A. (2008) The effect of drought and heat stress on reproductive processes in cereals. Plant, Cell \& Environment 31: 11-38.

Barrett, J.C., Fry, B., Maller, J., Daly, M.J. (2005) Haploview: analysis and visualization of LD and haplotype maps. Bioinformatics 21: 263-265.

Begum, H., Spindel, J.E., Lalusin, A., Borromeo, T., Gregorio, G., Hernandez, J., Virk, P., Collard, B., McCouch, S.R. (2015) Genome-wide association mapping for yield and other agronomic traits in an elite breeding population of tropical rice (Oryza sativa). PLOS ONE 10: e0119873.

Bekh-Ochir, D., Shimada, S., Yamagami, A., Kanda, S., Ogawa, K., Nakazawa, M., Matsui, M., Sakuta, M., Osada, H., Asami, T., Nakano, T. (2013) A novel mitochondrial DnaJ/Hsp40 family protein BIL2 promotes plant growth and resistance against environmental stress in brassinosteroid signaling. Planta 237: 1509-1525.

Bernier, J., Serraj, R., Kumar, A., Venuprasad, R., Impa, S., Gowda, R.P.V., Oane, R., Spaner, D., Atlin, G. (2009) The large-effect drought-resistance QTL qtl12.1 increases water uptake in upland rice. Field Crops Research 110: 139-146.

Bernier, J., Kumar, A., Venuprasad, R., Spaner, D., and Atlin, G. N. (2007) A large-effect QTL for grain yield under reproductive-stage drought stress in upland rice. Crop Science 47: 507-516.

Bernier, J., Kumar, A., Venuprasad, R., Spaner, D., Verulkar, S., Mandal, N. P., Sinha, P. K., Peeraju, P., Dongre, P. R., Mahto, R. N., and Atlin, G. (2009) Characterization of the effect of a QTL for drought resistance in rice, qtl12.1, over a range of environments in the Philippines and eastern India. Euphytica 166: 207-217.

Bindi, M., and Olesen, J.E. (2011) The responses of agriculture in Europe to climate change. Regional Environmental Change 11: 151-158.

Bindraban, P.S., Hengsdijk, H., Cao, W., Shi, Q., Thiyagarajan, T.M., Van der Krogt, W., Wardana, I.P. (2006) Transforming inundated rice cultivation. International Journal of Water Resources Development 22: 87-100. 
Biscarini, F., Cozzi, P., Casella, L., Riccardi, P., Vattari, A., Orasen, G., Perrini, R., Tacconi, G., Tondelli, A., Biselli, C., Cattivelli, L., Spindel, J., McCouch, S., Abbruscato, P., Valé, G., Piffanelli, P., Greco, R. (2016) Genome-wide association study for traits related to plant and grain morphology, and root architecture in temperate rice accessions. PLoS ONE 11: e0155425.

Blum, A. (2009) Effective use of water (EUW) and not water-use efficiency (WUE) is the target of crop yield improvement under drought stress. Field Crops Research 112: 119123

Bohmert, K., Camus, I., Bellini, C., Bouchez, D., Caboche, M., Benning, C. (1998) AGO1 defines a novel locus of Arabidopsis controlling leaf development. The EMBO Journal 17: $170-180$.

Boonjung, H., and Fukai, S. (1996) Effects of soil water deficit at different growth stages on rice growth and yield under upland conditions. 2. Phenology, biomass production and yield. Field Crops Research 48: 47-55.

Borba, T.C.D.O., Brondani, R.P.V., Breseghello, F., Coelho, A.S.G., Mendonça, J.A., Rangel P.H.N., Brondani, C. (2010) Association mapping for yield and grain quality traits in rice (Oryza sativa L.). Genetics and Molecular Biology 33: 515-524.

Bouma, T.J., Nielsen, K.L., Koutstaal, B. (2000) Sample preparation and scanning protocol for computerised analysis of root length and diameter. Plant and Soil 218: 185-196.

Bouman, B.A.M., Peng, S., Castañeda, A.R., and Visperas, R.M. (2005) Yield and water use of irrigated tropical aerobic rice systems. Agricultural Water Management 74: 87105.

Bouman, B.A.M., Yang, X., Wang, H., Wang, Z., Zhao, J., and Chen, B. (2006) Performance of aerobic rice varieties under irrigated conditions in North China. Field Crops Research 97: 53-65.

Bradbury, P.J., Zhang, Z., Kroon, D.E., Casstevens, T.M., Ramdoss, Y., Buckler, E.S. (2007) TASSEL: software for association mapping of complex traits in diverse samples. Bioinformatics 23: 2633-2635.

Bramley, H., Turner, N.C., Turner, D.W., Tyerman, S.D. (2009) Roles of morphology, anatomy, and aquaporins in determining contrasting hydraulic behavior of roots. Plant Physiology 150: 348-364.

Brun, F., Wallach, D., Makowski, D., and Jones, J.W. (2006) "Working with dynamic crop models: evaluation, analysis, parameterization, and applications," Elsevier.

Budak, H., Akpinar, B.A., Unver, T., Turktas, M. (2013) Proteome changes in wild and modern wheat leaves upon drought stress by two-dimensional electrophoresis and nanoLC-ESI-MS/MS. Plant Molecular Biology 83: 89-103.

Buer, C.S., Sukumar, P., Muday, G.K. (2006) Ethylene modulates flavonoid accumulation and gravitropic responses in roots of Arabidopsis. Plant Physiology 140: 1384-1396.

Bustos-Korts, D., Malosetti, M., Chapman, S., and van Eeuwijk, F. (2016) Modelling of genotype by environment interaction and prediction of complex traits across multiple environments as a synthesis of crop growth modelling, genetics and statistics. In "Crop Systems Biology: Narrowing the gaps between crop modelling and genetics" (X. Yin and P. C. Struik, eds.), pp. 55-82. Springer International Publishing, Cham.

Chakrabarty, A., Aditya, M., Dey, N., Banik, N., Bhattacharjee, S. (2016) Antioxidant signaling and redox regulation in drought- and salinity-stressed plants. In MA Hossain, SH Wani, S Bhattacharjee, DJ Burritt, L-SP Tran, eds, Drought Stress Tolerance in Plants, Vol 1: Physiology and Biochemistry. Springer International Publishing, Cham, pp 465-498. 
Chaumont, F., Barrieu, F., Wojcik, E., Chrispeels, M.J., Jung, R. (2001) Aquaporins constitute a large and highly divergent protein family in maize. Plant Physiology 125: 1206-1215.

Chaves, M.M., and Oliveira, M.M. (2004) Mechanisms underlying plant resilience to water deficits: prospects for water-saving agriculture. Journal of Experimental Botany 55: 2365-2384.

Chaves, M.M., Maroco, J.P., and Pereira, J.S. (2003) Understanding plant responses to drought-from genes to the whole plant. Functional Plant Biology 30: 239-264.

Chen, M., Markham, J.E., Dietrich, C.R., Jaworski, J.G., Cahoon, E.B. (2008) Sphingolipid long-chain base hydroxylation is important for growth and regulation of sphingolipid content and composition in Arabidopsis. The Plant Cell 20: 1862-1878.

Chen, X., Goodwin, S.M., Boroff, V.L., Liu, X., Jenks, M.A. (2003) Cloning and characterization of the WAX2 gene of Arabidopsis involved in cuticle membrane and wax production. The Plant Cell 15: 1170-1185.

Chenu, K., Chapman, S.C., Hammer, G.L., McLean, G., Salah, H.B.H., and Tardieu, F. (2008) Short-term responses of leaf growth rate to water deficit scale up to whole-plant and crop levels: an integrated modelling approach in maize. Plant, Cell \& Environment 31: 378-391.

Cheong, Y.H., Pandey, G.K., Grant, J.J., Batistic, O., Li, L., Kim, B.G., Lee, S.C., Kudla, J., Luan, S. (2007) Two calcineurin B-like calcium sensors, interacting with protein kinase CIPK23, regulate leaf transpiration and root potassium uptake in Arabidopsis. The Plant Journal 52: 223-239.

Chimungu, J.G., Brown, K.M., Lynch, J.P. (2014) Reduced root cortical cell file number improves drought tolerance in maize. Plant Physiology 166: 1943-1955.

Ciais, P., Reichstein, M., Viovy, N., Granier, A., Ogee, J., Allard, V., Aubinet, M., Buchmann, N., Bernhofer, C., Carrara, A., Chevallier, F., De Noblet, N., Friend, A. D., Friedlingstein, P., Grunwald, T., Heinesch, B., Keronen, P., Knohl, A., Krinner, G., Loustau, D., Manca, G., Matteucci, G., Miglietta, F., Ourcival, J. M., Papale, D., Pilegaard, K., Rambal, S., Seufert, G., Soussana, J. F., Sanz, M. J., Schulze, E. D., Vesala, T., and Valentini, R. (2005) Europe-wide reduction in primary productivity caused by the heat and drought in 2003. Nature 437: 529-533.

Condon, A.G., Farquhar, G.D., Richards, R.A. (1990) Genotypic variation in carbon isotope discrimination and transpiration efficiency in wheat. Leaf gas exchange and whole plant studies. Functional Plant Biology 17: 9-22.

Cooper, M., Rajatasereekul, S., Immark, S., Fukai, S., and Basnayake, J. (1999a) Rainfed lowland rice breeding strategies for Northeast Thailand.: I. Genotypic variation and genotype $\times$ environment interactions for grain yield. Field Crops Research 64: 131-151.

Cooper, M., Rajatasereekul, S., Somrith, B., Sriwisut, S., Immark, S., Boonwite, C., Suwanwongse, A., Ruangsook, S., Hanviriyapant, P., Romyen, P., Porn-uraisanit, P., Skulkhu, E., Fukai, S., Basnayake, J., and Podlich, D. W. (1999b) Rainfed lowland rice breeding strategies for Northeast Thailand II. Comparison of intrastation and interstation selection. Field Crops Research 64: 153-176.

Cosgrove, D.J. (1998) Cell wall loosening by expansins. Plant Physiology 118: 333-339.

Coudert, Y., Périn, C., Courtois, B., Khong, N.G., Gantet, P. (2010) Genetic control of root development in rice, the model cereal. Trends in Plant Science 15: 219-226.

Courtois, B., Ahmadi, N., Khowaja, F., Price, A.H., Rami, J.F., Frouin, J., Hamelin, C., Ruiz, M. (2009) Rice root genetic architecture: Meta-analysis from a drought QTL database. Rice 2: 115-128. 
Courtois, B., Audebert, A., Dardou, A., Roques, S., Ghneim-Herrera, T., Droc, G., Frouin, J., Rouan, L., Gozé, E., Kilian, A., Ahmadi, N., Dingkuhn, M. (2013) Genome-wide association mapping of root traits in a japonica rice panel. PLOS ONE 8: e78037.

Craufurd, P.Q., Wheeler, T.R., Ellis, R.H., Summerfield, R.J., Williams, J.H. (1999) Effect of temperature and water deficit on water-use efficiency, carbon isotope discrimination, and specific leaf area in peanut. Crop Science 39: 136-142.

Crowell, S., Korniliev, P., Falcao, A., Ismail, A., Gregorio, G., Mezey, J., McCouch, S. (2016) Genome-wide association and high-resolution phenotyping link Oryza sativa panicle traits to numerous trait-specific QTL clusters. Nature Communications 7: 10527.

Davenport, R.J., MuÑOz-Mayor, A., Jha, D., Essah, P.A., Rus, A.N.A., Tester, M. (2007) The $\mathrm{Na}^{+}$transporter AtHKT1; 1 controls retrieval of $\mathrm{Na}^{+}$from the xylem in Arabidopsis. Plant, Cell \& Environment 30: 497-507.

Davies, W.J., Bacon, M.A. (2003) Adaptation of roots to drought. In H Kroon, EJW Visser, eds, Root ecology, Vol 168. Springer, Berlin, pp 173-192.

De Datta, S.K. (1975) Major research in upland rice. International Rice Research Institute. Los Baños, Philippines.

Dimkpa, S.O.N., Lahari, Z., Shrestha, R., Douglas, A., Gheysen, G., Price, A.H. (2016) A genome-wide association study of a global rice panel reveals resistance in Oryza sativa to root-knot nematodes. Journal of Experimental Botany 67: 1191-1200.

Ding, S., Zhang, B., Qin, F. (2015) Arabidopsis RZFP34/CHYR1, a ubiquitin E3 ligase, regulates stomatal movement and drought tolerance via SnRK2.6-mediated phosphorylation. The Plant Cell 27: 3228-3244.

Dingkuhn, M., Luquet, D., Clément-Vidal, A., Tambour, L., Kim, H.K., and Song, Y. (2007) Is plant growth driven by sink regulation? Implications for crop models, phenotyping approaches and ideotypes. Frontis 21: 155-168.

Dingkuhn, M., Pasco, R., Pasuquin, J.M., Damo, J., Soulié, J.C., Raboin, L.M., Dusserre, J., Sow, A., Manneh, B., Shrestha, S., Balde, A., and Kretzschmar, T. (2017a) Cropmodel assisted phenomics and genome-wide association study for climate adaptation of indica rice. 1. Phenology. Journal of Experimental Botany 68: 4369-4388.

Dingkuhn, M., Pasco, R., Pasuquin, J.M., Damo, J., Soulié, J.C., Raboin, L.M., Dusserre, J., Sow, A., Manneh, B., Shrestha, S., and Kretzschmar, T. (2017b) Crop-model assisted phenomics and genome-wide association study for climate adaptation of indica rice. 2. Thermal stress and spikelet sterility. Journal of Experimental Botany 68: 43894406.

Dixit, S., Singh, A., Sta Cruz, M.T., Maturan, P.T., Amante, M., Kumar, A. (2014) Multiple major QTL lead to stable yield performance of rice cultivars across varying drought intensities. BMC Genetics 15: 16.

Doebley, J.F., Gaut, B.S., Smith, B.D. (2006) The molecular genetics of crop domestication. Cell 127: 1309-1321.

Donald, C.M. (1968) The breeding of crop ideotypes. Euphytica 17: 385-403.

Dong, J., Kim, S.T., Lord, E.M. (2005) Plantacyanin plays a role in reproduction in Arabidopsis. Plant Physiology 138: 778-789.

Duitama, J., Quintero, J.C., Cruz, D.F., Quintero, C., Hubmann, G., Foulquié-Moreno, M.R., Verstrepen, K.J., Thevelein, J.M., Tohme, J. (2014) An integrated framework for discovery and genotyping of genomic variants from high-throughput sequencing experiments. Nucleic Acids Research 42: e44-e44.

Ehdaie, B., Layne, A.P., and Waines, J.G. (2012) Root system plasticity to drought influences grain yield in bread wheat. Euphytica 186: 219-232. 
Eissenstat, D.M., Achor, D.S. (1999) Anatomical characteristics of roots of citrus rootstocks that vary in specific root length. New Phytologist 141: 309-321.

Elhiti, M., Stasolla, C. (2009) Structure and function of homodomain-leucine zipper (HD-Zip) proteins. Plant Signaling \& Behavior 4: 86-88.

Elshire, R.J., Glaubitz, J.C., Sun, Q., Poland, J.A., Kawamoto, K., Buckler, E.S., Mitchell, S.E. (2011) A robust, simple genotyping-by-sequencing (GBS) approach for high diversity species. PLoS ONE 6: e19379.

Fan, C., Xing, Y., Mao, H., Lu, T., Han, B., Xu, C., Li, X., Zhang, Q. (2006) GS3, a major QTL for grain length and weight and minor QTL for grain width and thickness in rice, encodes a putative transmembrane protein. Theoretical and Applied Genetics 112: 1164-1171.

Fan, X., Tang, Z., Tan, Y., Zhang, Y., Luo, B., Yang, M., Lian, X., Shen, Q., Miller, A.J., Xu, G. (2016) Overexpression of a $\mathrm{pH}$-sensitive nitrate transporter in rice increases crop yields. Proceedings of the National Academy of Sciences 113: 7118-7123.

Farquhar, G.D., Ehleringer, J.R., Hubick, K.T. (1989) Carbon isotope discrimination and photosynthesis. Annual Review of Plant Biology 40: 503-537.

Fischer, R.A., and Edmeades, G.O. (2010) Breeding and cereal yield progress. Crop Science 50: S-85-S-98.

Fitter, A. (2002) Characteristics and functions of root systems. In Y Waisel, A Eshel, T Beeckman, U Kafkaf, eds, Plant Roots: The Hidden Half, ED 3, NY: Marcel Dekker, Inc., New York, pp 15-32.

Fleury, D., Jefferies, S., Kuchel, H., and Langridge, P. (2010) Genetic and genomic tools to improve drought tolerance in wheat. Journal of Experimental Botany 61: 3211-3222.

Flexas, J., Ribas-Carbó, M., Hanson, D.T., Bota, J., Otto, B., Cifre, J., McDowell, N., Medrano, H., Kaldenhoff, R. (2006) Tobacco aquaporin NtAQP1 is involved in mesophyll conductance to CO2 in vivo. The Plant Journal 48: 427-439.

Foulkes, M.J., Sylvester-Bradley, R., Weightman, R., and Snape, J.W. (2007) Identifying physiological traits associated with improved drought resistance in winter wheat. Field Crops Research 103: 11-24.

Fu, X., Harberd, N.P. (2003) Auxin promotes Arabidopsis root growth by modulating gibberellin response. Nature 421: 740-743.

Fujita, D., Trijatmiko, K.R., Tagle, A.G., Sapasap, M.V., Koide, Y., Sasaki, K., Tsakirpaloglou, N., Gannaban, R.B., Nishimura, T., Yanagihara, S., Fukuta, Y., Koshiba, T., Slamet-Loedin, I.H., Ishimaru, T., Kobayashi, N. (2013) NAL1 allele from a rice landrace greatly increases yield in modern indica cultivars. Proceedings of the National Academy of Sciences 110: 20431-20436.

Fukai, S., Pantuwan, G., Jongdee, B., Cooper, M. (1999) Screening for drought resistance in rainfed lowland rice. Field Crops Research 64: 61-74.

Gallardo, K., Courty, P.E., Le Signor, C., Wipf, D., Vernoud, V. (2014) Sulfate transporters in the plant's response to drought and salinity: regulation and possible functions. Frontiers in Plant Science 5: 580.

Gao, M.J., Parkin, I., Lydiate, D., Hannoufa, A. (2004) An auxin-responsive SCARECROWlike transcriptional activator interacts with histone deacetylase. Plant Molecular Biology 55: 417-431.

Geiger, D., Becker, D., Vosloh, D., Gambale, F., Palme, K., Rehers, M., Anschuetz, U., Dreyer, I., Kudla, J., Hedrich, R. (2009) Heteromeric AtKC1·AKT1 channels in Arabidopsis roots facilitate growth under $\mathrm{K}^{+}$limiting conditions. Journal of Biological Chemistry 284: 21288-21295.

Génard, M., Memmah, M.M., Quilot-Turion, B., Vercambre, G., Baldazzi, V., Le Bot, J., Bertin, N., Gautier, H., Lescourret, F., and Pagès, L. (2016) Process-based 
simulation models are essential tools for virtual profiling and design of ideotypes: example of fruit and root. In "Crop Systems Biology: Narrowing the gaps between crop modelling and genetics" (X. Yin and P. C. Struik, eds.), pp. 83-104. Springer International Publishing, Cham.

Gilliland, L.U., Pawloski, L.C., Kandasamy, M.K., Meagher, R.B. (2003) Arabidopsis actin gene ACT7 plays an essential role in germination and root growth. The Plant Journal 33: 319-328.

Govind. G., Vokkaliga ThammeGowda, H., Jayaker Kalaiarasi, P., Iyer, D.R., Muthappa, S.K., Nese, S., Makarla, U.K. (2009) Identification and functional validation of a unique set of drought induced genes preferentially expressed in response to gradual water stress in peanut. Molecular Genetics and Genomics 281: 591-605.

Gowda, V.R.P., Henry, A., Yamauchi, A., Shashidhar, H.E., and Serraj, R. (2011) Root biology and genetic improvement for drought avoidance in rice. Field Crops Research 122: 1-13.

Grabov, A., Blatt, M.R. (1998) Membrane voltage initiates $\mathrm{Ca}^{2+}$ waves and potentiates $\mathrm{Ca}^{2+}$ increases with abscisic acid in stomatal guard cells. Proceedings of the National Academy of Sciences 95: 4778-4783.

Greacen, E.L., Ponsana, P., Barley, K.P. (1976) Resistance to water flow in the roots of cereals. In OL Lango, L Kappen and ED Schulze, eds, Water and Plant Life, Ed 1 Vol 19. Springer, Berlin, pp 86-100.

Grębosz, J., Badowiec, A., Weidner, S. (2014) Changes in the root proteome of Triticosecale grains germinating under osmotic stress. Acta Physiologiae Plantarum 36: 825-835.

Grieneisen, V.A., Xu, J., Maree, A.F.M., Hogeweg, P., Scheres, B. (2007) Auxin transport is sufficient to generate a maximum and gradient guiding root growth. Nature 449: 1008-1013.

Gu, J., Yin, X., Struik, P.C., Stomph, T.J., and Wang, H. (2012) Using chromosome introgression lines to map quantitative trait loci for photosynthesis parameters in rice (Oryza sativa L.) leaves under drought and well-watered field conditions. Journal of Experimental Botany 63: 455-469.

Gu, J., Yin, X., Zhang, C., Wang, H., and Struik, P.C. (2014) Linking ecophysiological modelling with quantitative genetics to support marker-assisted crop design for improved yields of rice (Oryza sativa) under drought stress. Annals of Botany 114: 499511.

Guilfoyle, T.J., Hagen, G. (2007) Auxin response factors. Current Opinion in Plant Biology 10: 453-460.

Guo, L., Nezames, C.D., Sheng, L., Deng, X., Wei, N. (2013) Cullin-RING ubiquitin ligase family in plant abiotic stress pathways. Journal of Integrative Plant Biology 55: 21-30.

Guo, Z., Chen, D., Alqudah, A.M., Röder, M.S., Ganal, M.W., Schnurbusch, T.C. (2017) Genome-wide association analyses of 54 traits identified multiple loci for the determination of floret fertility in wheat. New Phytologist 214: 257-270.

Hammer, G.L., van Oosterom, E., McLean, G., Chapman, S.C., Broad, I., Harland, P., and Muchow, R. C. (2010) Adapting APSIM to model the physiology and genetics of complex adaptive traits in field crops. Journal of Experimental Botany 61: 2185-2202.

Hammer, G., Cooper, M., Tardieu, F., Welch, S., Walsh, B., van Eeuwijk, F., Chapman, S., and Podlich, D. (2006) Models for navigating biological complexity in breeding improved crop plants. Trends in Plant Science 11: 587-593.

Hammer, G., Messina, C., van Oosterom, E., Chapman, S., Singh, V., Borrell, A., Jordan, D., and Cooper, M. (2016) Molecular breeding for complex adaptive traits: How integrating crop ecophysiology and modelling can enhance efficiency. In "Crop 
Systems Biology: Narrowing the gaps between crop modelling and genetics" (X. Yin and P. C. Struik, eds.), pp. 147-162. Springer International Publishing, Cham.

Hao, Y.J., Wei, W., Song, Q.X., Chen, H.W., Zhang, Y.Q., Wang, F., Zou, H.F., Lei, G., Tian, A.G., Zhang, W.K., Ma, B., Zhang, J.S., Chen, S.Y. (2011) Soybean NAC transcription factors promote abiotic stress tolerance and lateral root formation in transgenic plants. The Plant Journal 68: 302-313.

Harshavardhan, V.T., Van Son, L., Seiler C., Junker A., Weigelt-Fischer K., Klukas C., Altmann T., Sreenivasulu N., Bäumlein H., Kuhlmann, M. (2014) AtRD22 and AtUSPL1, members of the plant-specific BURP domain family involved in Arabidopsis thaliana drought tolerance. PLoS ONE 9: e110065.

Hawker, N.P., Bowman, J.L. (2004) Roles for class III HD-Zip and KANADI genes in Arabidopsis root development. Plant Physiology 135: 2261-2270.

He, X.J., Mu, R.L., Cao, W.H., Zhang, Z.G., Zhang, J.S., Chen, S.Y. (2005) AtNAC2, a transcription factor downstream of ethylene and auxin signaling pathways, is involved in salt stress response and lateral root development. The Plant Journal 44: 903-916.

Henry, A., Gowda, V.R., Torres, R.O., McNally, K.L., Serraj, R. (2011) Variation in root system architecture and drought response in rice (Oryza sativa): phenotyping of the OryzaSNP panel in rainfed lowland fields. Field Crops Research 120: 205-214.

Henry, A. (2013) IRRI's drought stress research in rice with emphasis on roots: accomplishments over the last 50 years. Plant Root 7: 92-106.

Henry, A., Cal, A.J., Batoto, T.C., Torres, R.O., and Serraj, R. (2012) Root attributes affecting water uptake of rice (Oryza sativa) under drought. Journal of Experimental Botany 63: 4751-4763.

Hernández, E.I., Vilagrosa, A., Pausas, J.G., Bellot, J. (2010) Morphological traits and water use strategies in seedlings of Mediterranean coexisting species. Plant Ecology 207: 233244.

Hooker, T.S., Millar, A.A., Kunst, L. (2002) Significance of the expression of the CER6 condensing enzyme for cuticular wax production in Arabidopsis. Plant Physiology 129: 1568-1580.

Hsieh, W.Y., Liao, J.C., Hsieh, M.H. (2015) Dysfunctional mitochondria regulate the size of root apical meristem and leaf development in Arabidopsis. Plant Signaling \& Behavior 10: e1071002.

Huang, L., Yang, S., Zhang, S., Liu, M., Lai, J., Qi, Y., Shi, S., Wang, J., Wang, Y., Xie, Q., Yang, C. (2009) The Arabidopsis SUMO E3 ligase AtMMS21, a homologue of NSE2/MMS21, regulates cell proliferation in the root. The Plant Journal 60: 666-678.

Huang, X., Qian, Q., Liu, Z., Sun, H., He, S., Luo, D., Xia, G., Chu, C., Li, J., Fu, X. (2009) Natural variation at the DEPl locus enhances grain yield in rice. Nature Genetics 41: 494-497.

Huang, X., Wei, X., Sang, T., Zhao, Q., Feng, Q., Zhao, Y., Li, C., Zhu, C., Lu, T., Zhang, Z., Li, M., Fan, D., Guo, Y., Wang, A., Wang, L., Deng, L., Li, W., Lu, Y., Weng, Q., Liu, K., Huang, T., Zhou, T., Jing, Y., Li, W., Lin, Z., Buckler, E.S., Qian, Q., Zhang, Q.F., Li, J., Han, B. (2010) Genome-wide association studies of 14 agronomic traits in rice landraces. Nature Genetics 42: 961-967.

Huang, X., Zhao, Y., Wei, X., Li, C., Wang, A., Zhao, Q., Li, W., Guo, Y., Deng, L., Zhu, C., Fan, D., Lu, Y., Weng, Q., Liu, K., Zhou, T., Jing, Y., Si, L., Dong, G., Huang, T., Lu, T., Feng, Q., Qian, Q., Li, J., Han, B. (2012) Genome-wide association study of flowering time and grain yield traits in a worldwide collection of rice germplasm. Nature Genetics 44: 32-39. 
Impa, S.M., Nadaradjan, S., Boominathan, P., Shashidhar, G., Bindumadhava, H.Y., Sheshshayee, M.S. (2005) Carbon isotope discrimination accurately reflects variability in WUE measured at a whole plant level in rice. Crop Science 45: 2517-2522

Ingvarsson, P.K., Street, N.R. (2011) Association genetics of complex traits in plants. New Phytologist 189: 909-922.

IPCC (2013). Working group, I contribution to the IPCC fifth assessment report climate change: The physical science basis, summary for policymakers. www. climatechange2013.org/images/uploads/WGIAR5-SPM_Approved27Sep2013.pdf.

Ishimaru, K., Ono, K., Kashiwagi, T. (2004) Identification of a new gene controlling plant height in rice using the candidate-gene strategy. Planta 218: 388-395.

Isidro, J., Jannink, J.L., Akdemir, D., Poland, J., Heslot, N., and Sorrells, M.E. (2015) Training set optimization under population structure in genomic selection. Theoretical and Applied Genetics 128: 145-158.

Jackson, R.B., Sperry, J.S., and Dawson, T.E. (2000) Root water uptake and transport: using physiological processes in global predictions. Trends in Plant Science 5: 482-488.

Jagadish, S.K., Muthurajan, R., Rang, Z.W., Malo, R., Heuer, S., Bennett, J., Craufurd, P.Q. (2011) Spikelet proteomic response to combined water deficit and heat stress in rice (Oryza sativa cv. N22). Rice 4: 1-11.

Jagadish, S.V.K., Craufurd, P.Q., and Wheeler, T.R. (2007) High temperature stress and spikelet fertility in rice (Oryza sativa L.). Journal of Experimental Botany 58: 16271635.

Jagadish, S.V.K., Septiningsih, E.M., Kohli, A., Thomson, M.J., Ye, C., Redoña, E., Kumar, A., Gregorio, G.B., Wassmann, R., Ismail, A.M., and Singh, R.K. (2012) Genetic advances in adapting rice to a rapidly changing climate. Journal of Agronomy and Crop Science 198: 360-373.

Jain, M., Tyagi, A.K., Khurana, J.P. (2006) Genome-wide analysis, evolutionary expansion, and expression of early auxin-responsive SAUR gene family in rice (Oryza sativa). Genomics 88: 360-371.

Jansen, R.C., Stam, P. (1994) High resolution of quantitative traits into multiple loci via interval mapping. Genetics 136: 1447-1455.

Jarzyniak, K.M., Jasiński, M. (2014) Membrane transporters and drought resistance-a complex issue. Frontiers in Plant Science 5: 687.

Jensen, W.A. (1962) Botanical histochemistry: Principles and practice. W.H. Freeman, San Francisco.

Ji, W., Zhu, Y., Li, Y., Yang, L., Zhao, X., Cai, H., Bai, X. (2010) Over-expression of a glutathione S-transferase gene, GsGST, from wild soybean (Glycine soja) enhances drought and salt tolerance in transgenic tobacco. Biotechnology Letters 32: 1173-1179.

Jin, Z.L., Hong, J.K., Yang, K.A., Koo, J.C., Choi, Y.J., Chung, W.S., Yun, D.J., Lee, S.Y., Cho, M.J., Lim, C.O. (2005) Over-expression of chinese cabbage calreticulin 1, $\operatorname{BrCRT1}$, enhances shoot and root regeneration, but retards plant growth in transgenic tobacco. Transgenic Research 14: 619-626.

Jones, M.A., Shen, J.J., Fu, Y., Li, H., Yang, Z., Grierson, C.S. (2002) The Arabidopsis Rop2 GTPase is a positive regulator of both root hair initiation and tip growth. The Plant Cell 14: 763-776.

Juenger, T.E. (2013) Natural variation and genetic constraints on drought tolerance. Current Opinion in Plant Biology 16: 274-281.

Julia, C., and Dingkuhn, M. (2013) Predicting temperature induced sterility of rice spikelets requires simulation of crop-generated microclimate. European Journal of Agronomy 49: 50-60. 
Jung, J., and McCouch, S. (2013) Getting to the roots of it: Genetic and hormonal control of root architecture. Frontiers in Plant Science 4: 186.

Kadam, N., Tamilselvan, A., Lawas, L.M.F., Quinones, C., Bahuguna, R., Thomson, M.J., Dingkuhn, M., Muthurajan, R., Struik, P., Yin, X., Jagadish, S.V.K. (2017) Genetic control of plasticity in root morphology and anatomy of rice in response to water-deficit. Plant Physiology 174: 2302-2315.

Kadam, N.N., Yin, X., Bindraban, P.S., Struik, P.C., Jagadish, K.S.V. (2015) Does morphological and anatomical plasticity during the vegetative stage make wheat more tolerant of water deficit stress than rice? Plant Physiology 167: 1389-1401.

Kadam, N.N., Xiao, G., Melgar, R.J., Bahuguna, R.N., Quinones, C., Tamilselvan, A., Prasad, P. V.V., and Jagadish, K.S.V. (2014) Agronomic and physiological responses to high temperature, drought, and elevated $\mathrm{CO}_{2}$ interactions in cereals. Advances in Agronomy 127: 111-156.

Kalve, S., De Vos, D., Beemster, G.T.S. (2014) Leaf development: a cellular perspective. Frontiers in Plant Science 5: 362.

Kamoshita, A., Babu, R.C., Boopathi, N.M., and Fukai, S. (2008) Phenotypic and genotypic analysis of drought-resistance traits for development of rice cultivars adapted to rainfed environments. Field Crops Research 109: 1-23.

Kang, Y., Khan, S., and Ma, X. (2009) Climate change impacts on crop yield, crop water productivity and food security-A review. Progress in Natural Science 19: 1665-1674.

Kano M, Inukai Y, Kitano H, Yamauchi, A. (2011) Root plasticity as the key root trait for adaptation to various intensities of drought stress in rice. Plant and Soil 342: 117-128.

Kano-Nakata, M., Gowda, V.R.P., Henry, A., Serraj, R., Inukai, Y., Fujita, D., Kobayashi, N., Suralta, R.R., and Yamauchi, A. (2013) Functional roles of the plasticity of root system development in biomass production and water uptake under rainfed lowland conditions. Field Crops Research 144: 288-296.

Kano-Nakata, M., Inukai, Y., Wade, L.J., Siopongco, J. D. L.C., and Yamauchi, A. (2011) Root development, water uptake, and shoot dry matter production under water deficit conditions in two CSSLs of rice: Functional roles of root plasticity. Plant Production Science 14: 307-317.

Kato, Y., Abe, J., Kamoshita, A., Yamagishi, J. (2006) Genotypic variation in root growth angle in rice (Oryza sativa L.) and its association with deep root development in upland fields with different water regimes. Plant and Soil 287: 117-129.

Kato, Y., Kamoshita, A., Yamagishi, J., Imoto, H., Abe, J. (2007) Growth of rice (Oryza sativa L.) cultivars under upland conditions with different levels of water supply. 3. Root system development, soil moisture change and plant water status. Plant Production Science 10: 3-13.

Kato, Y., Okami, M. (2011) Root morphology, hydraulic conductivity and plant water relations of high-yielding rice grown under aerobic conditions. Annals of Botany 108: 575-583.

Kato, Y., Okami, M., Tajima, R., Fujita, D., Kobayashi, N. (2010) Root response to aerobic conditions in rice, estimated by Comair root length scanner and scanner-based image analysis. Field Crops Research 118: 194-198.

Kato, Y., Kamoshita, A., and Yamagishi, J. (2008) Preflowering abortion reduces spikelet number in upland rice (Oryza sativa L.) under water stress. Crop Science 48: 23892395.

Kawahara, Y., De la Bastide, M., Hamilton, J.P., Kanamori, H., McCombie, W.R., Ouyang, S., Schwartz, D.C., Tanaka, T., Wu, J., Zhou, S., Childs, K.L., Davidson, R.M., Lin, H., Quesada-Ocampo, L., Vaillancourt, B., Sakai, H., Lee, S.S., Kim J., Numa, H., Itoh, T., Buell, C.R., Matsumoto, T. (2013) Improvement of the Oryza 
sativa nipponbare reference genome using next generation sequence and optical map data. Rice 6: 4.

Kazan, K., and Lyons, R. (2016) The link between flowering time and stress tolerance. Journal of Experimental Botany 67: 47-60.

Kenney, A.M., McKay, J.K., Richards, J.H., and Juenger, T.E. (2014) Direct and indirect selection on flowering time, water-use efficiency (WUE, $\delta^{13} \mathrm{C}$ ), and WUE plasticity to drought in Arabidopsis thaliana. Ecology and Evolution 4: 4505-4521.

Kholová, J., Hash, C.T., Kumar, P.L., Yadav, R.S., Kočová, M., and Vadez, V. (2010) Terminal drought-tolerant pearl millet [Pennisetum glaucum (L.) R. Br.] have high leaf ABA and limit transpiration at high vapour pressure deficit. Journal of Experimental Botany 61: 1431-1440.

Khush, G.S. (1997) Origin, dispersal, cultivation and variation of rice. In Oryza: Plant Molecular Biology 35: 25-34.

Kikuchi, S., Bheemanahalli, R., Jagadish, K.S.V., Kumagai, E., Masuya, Y., Kuroda, E., Raghavan, C., Dingkuhn, M., Abe, A., Shimono, H.C.P.C.E.R. (2017) Genome-wide association mapping for phenotypic plasticity in rice. Plant, Cell \& Environment 40: 1565-1575.

Kim, M., Hepler, P.K., Eun, S.O., Ha, K.S., Lee, Y. (1995) Actin filaments in mature guard cells are radially distributed and involved in stomatal movement. Plant Physiology 109: 1077-1084.

Kim, T.W., Michniewicz, M., Bergmann, D.C., Wang, Z.Y. (2012) Brassinosteroid regulates stomatal development by GSK3-mediated inhibition of a MAPK pathway. Nature 482: 419-422.

Kitomi, Y., Ito, H., Hobo, T., Aya, K., Kitano, H., Inukai, Y. (2011) The auxin responsive AP2/ERF transcription factor CROWN ROOTLESS5 is involved in crown root initiation in rice through the induction of $O S R R 1$, a type-A response regulator of cytokinin signaling. The Plant Journal 67: 472-484.

Kobata, T., Tanaka, S., Utumi, M., Hara, S., and Imaki, T. (1994) Sterility in rice (Oryza Sativa L.) subject to drought during the booting stage occurs not because of lack of assimilate or of water deficit in the shoot but because of dehydration of the root zone. Japanese journal of crop science 63: 510-517.

Kromdijk, J., Bertin, N., Heuvelink, E., Molenaar, J., de Visser, P.H.B., Marcelis, L.F. M., and Struik, P.C. (2014) Crop management impacts the efficiency of quantitative trait loci (QTL) detection and use: case study of fruit load $\times$ QTL interactions. Journal of Experimental Botany 65: 11-22.

Kumar, A., Dixit, S., Ram, T., Yadaw, R.B., Mishra, K.K., Mandal, N.P. (2014) Breeding high-yielding drought-tolerant rice: genetic variations and conventional and molecular approaches. Journal of Experimental Botany 65: 6265-6278.

Kumar, R., Venuprasad, R., and Atlin, G.N. (2007) Genetic analysis of rainfed lowland rice drought tolerance under naturally-occurring stress in eastern India: heritability and QTL effects. Field Crops Research 103: 42-52.

Lanceras, J.C., Pantuwan, G., Jongdee, B., and Toojinda, T. (2004) Quantitative trait loci associated with drought tolerance at reproductive stage in rice. Plant Physiology 135: 384-399.

Leakey, A.D.B., Ainsworth, E.A., Bernacchi, C.J., Rogers, A., Long, S.P., and Ort, D.R. (2009) Elevated $\mathrm{CO}_{2}$ effects on plant carbon, nitrogen, and water relations: six important lessons from FACE. Journal of Experimental Botany 60: 2859-2876.

Letort, V., Mahe, P., Cournède, P.H., de Reffye, P., and Courtois, B. (2008) Quantitative genetics and functional-structural plant growth models: Simulation of quantitative trait 
loci detection for model parameters and application to potential yield optimization. Annals of Botany 101: 1243-1254.

Li, S., Qian, Q., Fu, Z., Zeng, D., Meng, X., Kyozuka, J., Maekawa, M., Zhu, X., Zhang, J., Li, J., Wang, Y. (2009) Short paniclel encodes a putative PTR family transporter and determines rice panicle size. The Plant Journal 58: 592-605.

Li, J.Y., Wang, J., and Zeigler, R.S. (2014) The 3,000 rice genomes project: new opportunities and challenges for future rice research. Giga Science 3: 8.

Li, X., Guo, Z., Lv, Y., Cen, X., Ding, X., Wu, H., Li, X., Huang, J., and Xiong, L. (2017) Genetic control of the root system in rice under normal and drought stress conditions by genome-wide association study. PLoS Genetics 13: e1006889.

Liang, M., Haroldsen, V., Cai, X., Wu, Y. (2006) Expression of a putative laccase gene, ZmLAC1, in maize primary roots under stress. Plant, Cell \& Environment 29: 746-753.

Lipiec, J., Doussan, C., Nosalewicz, A., and Kondracka, K. (2013) Effect of drought and heat stresses on plant growth and yield: a review. International Agrophysics 27: 463477.

Lipka, A.E., Tian, F., Wang, Q., Peiffer, J., Li, M., Bradbury, P.J., Gore, M.A., Buckler, E.S., Zhang, Z. (2012) GAPIT: genome association and prediction integrated tool. Bioinformatics 28: 2397-2399.

Lippold, F., vom Dorp, K., Abraham, M., Hölzl, G., Wewer, V., Yilmaz, J.L., Lager, I., Montandon, C., Besagni, C., Kessler, F., Stymne, S., Dörmann, P. (2012) Fatty acid phytyl ester synthesis in chloroplasts of Arabidopsis. The Plant Cell 24: 2001-2014.

Little, D.Y., Rao, H., Oliva, S., Daniel-Vedele, F., Krapp, A., Malamy, J.E. (2005) The putative high-affinity nitrate transporter NRT2.1 represses lateral root initiation in response to nutritional cues. Proceedings of the National Academy of Sciences 102: 13693-13698.

Liu, J.X., Liao, D.Q., Oane, R., Estenor, L., Yang, X.E., Li, Z.C., Bennett, J. (2006) Genetic variation in the sensitivity of anther dehiscence to drought stress in rice. Field Crops Research 97: 87-100.

Liu, W., Zhang, D., Tang, M., Li, D., Zhu, Y., Zhu, L., Chen, C. (2013) THIS1 is a putative lipase that regulates tillering, plant height, and spikelet fertility in rice. Journal of Experimental Botany 64: 4389-4402.

Liu, X., Guo, T., Wan, X., Wang, H., Zhu, M., Li, A., Su, N., Shen, Y., Mao, B., Zhai, H., Mao, L., Wan, J. (2010) Transcriptome analysis of grain-filling caryopses reveals involvement of multiple regulatory pathways in chalky grain formation in rice. $B M C$ Genomics 11: 730 .

Lobell, D.B., Schlenker, W., and Costa-Roberts, J. (2011) Climate trends and global crop production since 1980. Science 333: 616-620.

Lobet, G., and Draye, X. (2013) Novel scanning procedure enabling the vectorization of entire rhizotron-grown root systems. Plant Methods 9: 1-1.

Lopes, M.S., Reynolds, M.P. (2010) Partitioning of assimilates to deeper roots is associated with cooler canopies and increased yield under drought in wheat. Functional Plant Biology 37: 147-156.

Lorbiecke, R., Sauter, M. (1999) Adventitious root growth and cell-cycle induction in deepwater rice. Plant Physiology 119: 21-30.

Ludlow, M.M., and Muchow, R.C. (1990) A critical evaluation of traits for improving crop yields in water-limited environments. Advances in Agronomy 43:107-153.

Lynn, K., Fernandez, A., Aida, M., Sedbrook, J., Tasaka, M., Masson, P., Barton, M.K. (1999) The PINHEAD/ZWILLE gene acts pleiotropically in Arabidopsis development and has overlapping functions with the ARGONAUTE1 gene. Development 126: 469481. 
Ma, X., Feng, F., Wei, H., Mei, H., Xu, K., Chen, S., Li, T., Liang, X., Liu, H., Luo, L. (2016) Genome-wide association study for plant height and grain yield in rice under contrasting moisture regimes. Frontiers in Plant Science 7: 1801.

Mackay, I., Powell, W. (2007) Methods for linkage disequilibrium mapping in crops. Trends in Plant Science 12: 57-63.

Mai, C.D., Phung, N.T., To, H.T., Gonin, M., Hoang, G.T., Nguyen, K.L., Do, V.N., Courtois, B., and Gantet, P. (2014) Genes controlling root development in rice. Rice 7: 30 .

Mangin, B., Casadebaig, P., Cadic, E., Blanchet, N., Boniface, M.-C., Carrère, S., Gouzy, J., Legrand, L., Mayjonade, B., Pouilly, N., André, T., Coque, M., Piquemal, J., Laporte, M., Vincourt, P., Muños, S., and Langlade, N. B. (2017) Genetic control of plasticity of oil yield for combined abiotic stresses using a joint approach of crop modelling and genome-wide association. Plant, Cell \& Environment 40: 2276-2291.

Marais, D.L.D., Hernandez, K.M., and Juenger, T.E. (2013) Genotype-by-Environment interaction and plasticity: Exploring genomic responses of plants to the abiotic environment. Annual Review of Ecology, Evolution, and Systematics 44: 5-29.

Markakis, M.N., Boron, A.K., Van Loock, B., Saini, K., Cirera, S., Verbelen, J.P., Vissenberg, K. (2013) Characterization of a small auxinup RNA (SAUR)-like gene involved in Arabidopsis thaliana development. PLoS ONE 8: e82596.

Martre, P., Bertin, N., Salon, C., and Génard, M. (2011) Modelling the size and composition of fruit, grain and seed by process-based simulation models. New Phytologist 191: 601618.

Martre, P., Quilot-Turion, B., Luquet, D., Memmah, M.-M. O.-S., Chenu, K., and Debaeke, P. (2015) Model-assisted phenotyping and ideotype design. Crop physiology: applications for genetic improvement and agronomy, 349-373.

Mather, K.A., Caicedo, A.L., Polato, N.R., Olsen, K.M., McCouch, S., Purugganan, M.D. (2007) The extent of linkage disequilibrium in rice (Oryza sativa L.). Genetics 177: 2223

Matsuo, N., Ozawa, K., Mochizuki, T. (2009) Genotypic differences in root hydraulic conductance of rice (Oryza sativa L.) in response to water regimes. Plant and Soil 316: 25-34.

McCouch, S., Baute, G.J., Bradeen, J., Bramel, P., Bretting, P.K., Buckler, E., Burke, J.M., Charest, D., Cloutier, S., Cole, G., Dempewolf, H., Dingkuhn, M., Feuillet, C., Gepts, P., Grattapaglia, D., Guarino, L., Jackson, S., Knapp, S., Langridge, P., Lawton-Rauh, A., Lijua, Q., Lusty, C., Michael, T., Myles, S., Naito, K., Nelson, R.L., Pontarollo, R., Richards, C.M., Rieseberg, L., Ross-Ibarra, J., Rounsley, S., Hamilton, R.S., Schurr, U., Stein, N., Tomooka, N., van der Knaap, E., van Tassel, D., Toll, J., Valls, J., Varshney, R.K., Ward, J., Waugh, R., Wenzl, P., Zamir, D. (2013) Agriculture: Feeding the future. Nature 499: 23-24.

McDonald, M.P., Galwey, N.W., Colmer, T.D. (2002) Similarity and diversity in adventitious root anatomy as related to root aeration among a range of wetland and dryland grass species. Plant, Cell \& Environment 25: 441-451.

Metzker, M. L. (2010) Sequencing technologies-the next generation. Nature Reviews Genetics 11: 31-46.

Meuwissen, T.H.E., Hayes, B.J., and Goddard, M.E. (2001) Prediction of total genetic value using genome-wide dense marker maps. Genetics 157: 1819-1829.

Miller, N.D., Durham Brooks, T.L., Assadi, A.H., Spalding, E.P. (2010) Detection of a gravitropism phenotype in glutamate receptor-like 3.3 mutants of Arabidopsis thaliana using machine vision and computation. Genetics 186: 585-593. 
Millet, E., Welcker, C., Kruijer, W., Negro, S., Nicolas, S., Praud, S., Ranc, N., Presterl, T., Tuberosa, R., Bedo, Z., Draye, X., Usadel, B., Charcosset, A., van Eeuwijk, F., Tardieu, F., Coupel-Ledru, A., Bauland, C. (2016) Genome-wide analysis of yield in Europe: allelic effects as functions of drought and heat scenarios. Plant Physiology 172: 749-764.

Mir, R.R., Zaman-Allah, M., Sreenivasulu, N., Trethowan, R., and Varshney, R.K. (2012) Integrated genomics, physiology and breeding approaches for improving drought tolerance in crops. Theoretical and Applied Genetics 125: 625-645.

Mishra, K.K., Vikram, P., Yadaw, R.B., Swamy, B.M., Dixit, S., Cruz, M.T.S., Maturan, P., Marker, S., Kumar, A. (2013) qDTY12.1: a locus with a consistent effect on grain yield under drought in rice. BMC Genetics 14: 12.

Mittler, R. (2006) Abiotic stress, the field environment and stress combination. Trends in Plant Science 11: 15-19.

Miura, K., Ikeda, M., Matsubara, A., Song, X.J., Ito, M., Asano, K., Matsuoka, M., Kitano, H., Ashikari, M. (2010) OSSPL14 promotes panicle branching and higher grain productivity in rice. Nature Genetics 42: 545-549.

Miura, K., Lee, J., Gong, Q., Ma, S., Jin, J.B., Yoo, C.Y., Miura, T., Sato, A., Bohnert, H.J., Hasegawa, P.M. (2011) SIZ1 regulation of phosphate starvation-induced root architecture remodeling involves the control of auxin accumulation. Plant Physiology 155: 1000-1012.

Miyamoto, N., Steudle, E., Hirasawa, T., Lafitte, R. (2001) Hydraulic conductivity of rice roots. Journal of Experimental Botany 52: 1835-1846.

Mo, X., Liu, S., Lin, Z., Xu, Y., Xiang, Y., and McVicar, T.R. (2005) Prediction of crop yield, water consumption and water use efficiency with a SVAT-crop growth model using remotely sensed data on the North China Plain. Ecological Modelling 183: 301322.

Molden, D., Oweis, T., Steduto, P., Bindraban, P., Hanjra, M.A., and Kijne, J. (2010) Improving agricultural water productivity: Between optimism and caution. Agricultural Water Management 97: 528-535.

Moldenhauer, K., Slaton, N. (2001) Rice growth and development. Rice production handbook, 7-14.

Molendijk, A.J., Bischoff, F., Rajendrakumar, C.S.V., Friml, J., Braun, M., Gilroy, S., Palme, K. (2001) Arabidopsis thaliana Rop GTPases are localized to tips of root hairs and control polar growth. The EMBO Journal 20: 2779-2788.

Nahirñak, V., Almasia, N.I., Hopp, H.E., Vazquez-Rovere, C. (2012) Snakin/GASA proteins: Involvement in hormone crosstalk and redox homeostasis. Plant Signaling \& Behavior 7: 1004-1008.

Nakagawa, H., Yamagishi, J., Miyamoto, N., Motoyama, M., Yano, M., and Nemoto, K. (2005) Flowering response of rice to photoperiod and temperature: a QTL analysis using a phenological model. Theoretical and Applied Genetics 110: 778-786.

Nautiyal, P.C., Rachaputi, N.R., Joshi, Y.C. (2002) Moisture-deficit-induced changes in leafwater content, leaf carbon exchange rate and biomass production in groundnut cultivars differing in specific leaf area. Field Crops Research 74: 67-79.

Nguyen, H.T., Babu, R.C., and Blum, A. (1997) Breeding for drought resistance in rice: Physiology and molecular genetics considerations. Crop Science 37: 1426-1434.

Nicotra, A.B., Atkin, O.K., Bonser, S.P., Davidson, A.M., Finnegan, E.J., Mathesius, U., Poot, P., Purugganan, M.D., Richards, C.L., Valladares, F., van Kleunen, M. (2010) Plant phenotypic plasticity in a changing climate. Trends in Plant Science 15: 684-692. 
Nicotra, A.B., Davidson, A. (2010) Adaptive phenotypic plasticity and plant water use. Functional Plant Biology 37: 117-127.

Niklas, K.J. (1985) The evolution of tracheid diameter in early vascular plants and its implications on the hydraulic conductance of the primary xylem strand. Evolution 39: 1110-1122.

Niones, J.M., Suralta, R.R., Inukai, Y., and Yamauchi, A. (2012) Field evaluation on functional roles of root plastic responses on dry matter production and grain yield of rice under cycles of transient soil moisture stresses using chromosome segment substitution lines. Plant and Soil 359: 107-120.

Niones, J. M., Suralta, R. R., Inukai, Y., and Yamauchi, A. (2013) Roles of root aerenchyma development and its associated QTL in dry matter production under transient moisture stress in rice. Plant Production Science 16: 205-216.

Norton, G.J., Douglas, A., Lahner, B., Yakubova, E., Guerinot, M.L., Pinson, S.R.M., Tarpley, L., Eizenga, G.C., McGrath, S.P., Zhao, F.J., Islam, M.R., Islam, S., Duan, G., Zhu, Y., Salt, D.E., Meharg, A.A., Price, A.H. (2014) Genome wide association mapping of grain arsenic, copper, molybdenum and zinc in rice (Oryza sativa L.) grown at four international field sites. PLoS ONE 9: e89685.

Ohashi-Ito, K., Oguchi, M., Kojima, M., Sakakibara, H., Fukuda, H. (2013) Auxinassociated initiation of vascular cell differentiation by LONESOME HIGHWAY. Development 140: 765-769.

Ohashi-Ito, K., Saegusa, M., Iwamoto, K., Oda, Y., Katayama, H., Kojima, M., Sakakibara, H., Fukuda, H. (2014) A $b H L H$ complex activates vascular cell division via cytokinin action in root apical meristem. Current Biology 24: 2053-2058.

Olesen, J. E., Trnka, M., Kersebaum, K. C., Skjelvåg, A.O., Seguin, B., Peltonen-Sainio, P., Rossi, F., Kozyra, J., and Micale, F. (2011) Impacts and adaptation of European crop production systems to climate change. European Journal of Agronomy 34: 96-112.

Olivares-Villegas J.J., Reynolds, M.P., McDonald, G.K. (2007) Drought-adaptive attributes in the Seri/Babax hexaploid wheat population. Functional Plant Biology 34: 189-203.

Omidbakhshfard Mohammad, A., Proost, S., Fujikura, U., Mueller-Roeber, B. (2015) Growth-regulating factors (GRFs): A small transcription factor family with important functions in plant biology. Molecular Plant 8: 998-1010.

Ostonen, I., Puttsepp, U., Biel, C., Alberton, O., Bakker, M.R., Lohmus, K., Brunner, I. (2007) Specific root length as an indicator of environmental change. Plant Biosystems 141: 426-442.

Pacak, A., Barciszewska-Pacak, M., Swida-Barteczka, A., Kruszka, K., Sega, P., Milanowska, K., Jakobsen, I., Jarmolowski, A., Szweykowska-Kulinska, Z. (2016) Heat stress affects Pi-related genes expression and inorganic phosphate deposition/accumulation in barley. Frontiers in Plant Science 7: 926.

Pandey, S., Bhandari, H., Hardy, B., eds (2007) Economic costs of drought and rice farmers coping mechanisms: A cross-country comparative analysis, International Rice Research Institute, Manila, pp 203.

Pantalião, G.F., Narciso, M., Guimarães, C., Castro, A., Colombari, J.M., Breseghello, F., Rodrigues, L., Vianello, R.P., Borba, T.O., Brondani, C. (2016) Genome wide association study (GWAS) for grain yield in rice cultivated under water deficit. Genetica 144: 651-664.

Parent, B., Suard, B., Serraj, R., and Tardieu, F. (2010) Rice leaf growth and water potential are resilient to evaporative demand and soil water deficit once the effects of root system are neutralized. Plant, Cell \& Environment 33: 1256-1267.

Passioura, J.B. (1977) Grain yield harvest index and water use of wheat. Journal of the Australian Institute of Agricultural Science 43: 117-120. 
Pearson, P.N., and Palmer, M.R. (2000) Atmospheric carbon dioxide concentrations over the past 60 million years. Nature 406: 695-699.

Peng, S., Bouman, B., Visperas, R.M., Castañeda, A., Nie, L., and Park, H.K. (2006) Comparison between aerobic and flooded rice in the tropics: Agronomic performance in an eight-season experiment. Field Crops Research 96: 252-259.

Peng, S., Khush, G.S., Virk, P., Tang, Q., and Zou, Y. (2008) Progress in ideotype breeding to increase rice yield potential. Field Crops Research 108: 32-38.

Pfeiffer, W.H. (1988) Drought tolerance in bread wheat - analysis of yield improvement over the years in CIMMYT germplasm. In AR Klatt eds, Wheat production constraints in tropical environments, Proceedings of the international conference, CIMMYT, Mexico City, pp 274-284.

Phung, N.T.P., Mai, C.D., Hoang, G.T., Truong, H.T.M., Lavarenne, J., Gonin, M., Nguyen, K.L., Ha, T.T., Do, V.N., Gantet, P., Courtois, B. (2016) Genome-wide association mapping for root traits in a panel of rice accessions from Vietnam. BMC Plant Biology 16: 64.

Poorter, H., Bühler, J., van Dusschoten, D., Climent, J., Postma, J.A. (2012) Pot size matters: a meta-analysis of the effects of rooting volume on plant growth. Functional Plant Biology 39: 839-850.

Poorter, H., Fiorani, F., Stitt, M., Schurr, U., Finck, A., Gibon, Y., Usadel, B., Munns, R., Atkin, O.K., Tardieu, F., Pons, T.L. (2012) The art of growing plants for experimental purposes: a practical guide for the plant biologist. Functional Plant Biology 39: 821838.

Posé, D., Castanedo, I., Borsani, O., Nieto, B., Rosado, A., Taconnat, L., Ferrer, A., Dolan, L., Valpuesta, V., Botella, M.A. (2009) Identification of the Arabidopsis dry2/sqe1-5 mutant reveals a central role for sterols in drought tolerance and regulation of reactive oxygen species. The Plant Journal 59: 63-76.

Powell, N., Ji, X., Ravash, R., Edlington, J., and Dolferus, R. (2012) Yield stability for cereals in a changing climate. Functional Plant Biology 39: 539-552.

Praba, M.L., Cairns, J.E., Babu, R.C., and Lafitte, H.R. (2009) Identification of physiological traits underlying cultivar differences in drought tolerance in rice and wheat. Journal of Agronomy and Crop Science 195: 30-46.

Premachandra, G.S., Hahn, D.T., Axtell, J.D., Joly, R.J. (1994) Epicuticular wax load and water-use efficiency in bloomless and sparse-bloom mutants of Sorghum bicolor L. Environmental and Experimental Botany 34: 293-301.

Prentice, I.C. (2001) The carbon cycle and atmospheric carbon dioxide. In: Houghton, J.T., Ding, Y., Griggs, D.J., Noguer, M., van der Linden, P.J., Dai, X., Maskell, K., Johnson, C.A. (Eds.), Climate Change 2001: The Scientific Basis. Cambridge University Press, Cambridge, pp. 183-237.

Prince, S.J., Song, L., Qiu, D., Maldonado dos Santos, J.V., Chai, C., Joshi, T., Patil, G., Valliyodan, B., Vuong, T.D., Murphy, M., Krampis, K., Tucker, D.M., Biyashev, R., Dorrance, A.E., Maroof, M.S., Xu, D., Shannon, J.G., Nguyen, H.T. (2015) Genetic variants in root architecture-related genes in a Glycine soja accession, a potential resource to improve cultivated soybean. BMC Genomics 16: 1-20.

Pyngrope, S., Bhoomika, K., Dubey, R.S. (2013) Reactive oxygen species, ascorbateglutathione pool, and enzymes of their metabolism in drought-sensitive and tolerant indica rice (Oryza sativa L.) seedlings subjected to progressing levels of water deficit. Protoplasma 250: 585-600.

Qi, W., Sun, F., Wang, Q., Chen, M., Huang, Y., Feng, Y.Q., Luo, X., Yang, J. (2011) Rice ethylene-response AP2/ERF factor OSEATB restricts internode elongation by downregulating a gibberellin biosynthetic gene. Plant Physiology 157: 216-228. 
Qin, L.X., Li, Y., Li, D.D., Xu, W.L., Zheng, Y., Li, X.B. (2014) Arabidopsis drought-induced protein Di19-3 participates in plant response to drought and high salinity stresses. Plant Molecular Biology 86: 609-625.

Qiu, X., Pang, Y., Yuan, Z., Xing, D., Xu, J., Dingkuhn, M., Li, Z., and Ye, G. (2016) Genome-wide association study of grain appearance and milling quality in a worldwide collection of indica rice germplasm. PLOS ONE 10: e0145577.

Quan, R., Hu, S., Zhang, Z., Zhang, H., Zhang, Z., Huang, R. (2010) Overexpression of an ERF transcription factor TSRF1 improves rice drought tolerance. Plant Biotechnology Journal 8: 476-488.

Rafalski, J.A. (2010) Association genetics in crop improvement. Current Opinion in Plant Biology 13: 174-180.

Raju, B.R., Narayanaswamy, B.R., Mohankumar, M.V., Sumanth, K.K., Rajanna, M.P., Mohanraju, B., Udaykumar, M., Sheshshayee, M.S. (2014) Root traits and cellular level tolerance hold the key in maintaining higher spikelet fertility of rice under water limited conditions. Functional Plant Biology 41: 930-939.

Ranathunge, K., Steudle, E., Lafitte, R. (2003) Control of water uptake by rice (Oryza sativa L.): role of the outer part of the root. Planta 217: 193-205.

Rang, Z.W., Jagadish, S.V.K., Zhou, Q.M., Craufurd, P.Q., Heuer, S. (2011) Effect of high temperature and water stress on pollen germination and spikelet fertility in rice. Environmetal and Exprimental Botany 70: 58-65.

Rao, R.C., Wright, G.C. (1994) Stability of the relationship between specific leaf area and carbon isotope discrimination across environments in peanut. Crop Science 34: 98-103.

Ravi, K., Vadez, V., Isobe, S., Mir, R.R., Guo, Y., Nigam, S. N., Gowda, M.V.C., Radhakrishnan, T., Bertioli, D.J., Knapp, S.J., and Varshney, R.K. (2011) Identification of several small main-effect QTLs and a large number of epistatic QTLs for drought tolerance related traits in groundnut (Arachis hypogaea L.). Theoretical and Applied Genetics 122: 1119-1132.

Rebolledo, M.C., Peña, A.L., Duitama, J., Cruz, D.F., Dingkuhn, M., Grenier, C., Tohme, J. (2016) Combining image analysis, genome wide association studies and different field trials to reveal stable genetic regions related to panicle architecture and the number of spikelets per panicle in rice. Frontiers in Plant Science 7: 1384.

Reed, J.W. (2001) Roles and activities of Aux/IAA proteins in Arabidopsis. Trends in Plant Science 6: 420-425.

Reich, P.B., Walters, M.B., Tjoelker, M.G., Vanderklein, D., and Buschena, C. (1998) Photosynthesis and respiration rates depend on leaf and root morphology and nitrogen concentration in nine boreal tree species differing in relative growth rate. Functional Ecology 12: 395-405.

Reinders, A. (2016) Fuel for the road-sugar transport and pollen tube growth. Journal of Experimental Botany 67: 2121-2123.

Reinhardt, D., Pesce, E.R., Stieger, P., Mandel, T., Baltensperger, K., Bennett, M., Traas, J., Friml, J., Kuhlemeier, C. (2003) Regulation of phyllotaxis by polar auxin transport. Nature 426: 255-260.

Reinhardt, H., Hachez, C., Bienert, M.D., Beebo, A., Swarup, K., Voß, U., Bouhidel, K., Frigerio, L., Schjoerring, J.K., Bennett, M.J., Chaumont, F. (2016) Tonoplast aquaporins facilitate lateral root emergence. Plant Physiology 170: 1640-1654.

Reymond, M., Muller, B., Leonardi, A., Charcosset, A., and Tardieu, F. (2003) Combining quantitative trait loci analysis and an ecophysiological model to analyze the genetic variability of the responses of maize leaf growth to temperature and water deficit. Plant Physiology 131: 664-675. 
Reynolds, M., Dreccer, F., Trethowan, R. (2007) Drought-adaptive traits derived from wheat wild relatives and landraces. Journal of Experimental Botany 58: 177-186.

Reynolds, M.P., Quilligan, E., Aggarwal, P.K., Bansal, K.C., Cavalieri, A.J., Chapman, S.C., Chapotin, S.M., Datta, S.K., Duveiller, E., Gill, K.S., Jagadish, K.S.V., Joshi, A.K., Koehler, A.K., Kosina, P., Krishnan, S., Lafitte, R., Mahala, R.S., Muthurajan, R., Paterson, A.H., Prasanna, B.M., Rakshit, S., Rosegrant, M.W., Sharma, I., Singh, R.P., Sivasankar, S., Vadez, V., Valluru, R., Vara Prasad, P.V., Yadav, O.P. (2016) An integrated approach to maintaining cereal productivity under climate change. Global Food Security 8: 9-18.

Richards, R.A., Rebetzke, G.J., Watt, M., Condon, A.G., Spielmeyer, W., and Dolferus, R. (2010) Breeding for improved water productivity in temperate cereals: phenotyping, quantitative trait loci, markers and the selection environment. Functional Plant Biology 37: 85-97.

Rieger, M., Litvin, P. (1999) Root system hydraulic conductivity in species with contrasting root anatomy. Journal of Experimental Botany 50: 201-209.

Rigas, S., Debrosses, G., Haralampidis, K., Vicente-Agullo, F., Feldmann, K.A., Grabov, A., Dolan, L., Hatzopoulos, P. (2001) TRH1 encodes a potassium transporter required for tip growth in Arabidopsis root hairs. The Plant Cell 13: 139-151.

Ristic, Z., and Cass, D.D. (1992) Chloroplast structure after water and high-temperature stress in two lines of maize that differ in endogenous levels of abscisic acid. International Journal of Plant Sciences 153: 186-196.

Rohila, J.S., Yang, Y. (2007) Rice mitogen-activated protein kinase gene family and its role in biotic and abiotic stress response. Journal of Integrative Plant Biology 49: 751-759.

Rosegrant, M.W., Ringler, C., Sulser, T.B., Ewing, M., Palazzo, A., Zhu, T., Nelson, G.C., Koo, J., Robertson, R., Msangi, S., Batka, M. (2009) Agriculture and food security under global change: Prospects for 2025/2050. Background note for supporting the development of CGIAR Strategy and Results Framework. International Food Policy Research Institute: Washington, DC.

Rosen, E., Chen, R., Masson, P.H. (1999) Root gravitropism: a complex response to a simple stimulus? Trends in Plant Science 4: 407-412.

Ryser, P. (2006) The mysterious root length. Plant Soil 286: 1-6.

Sadras, V.O., Reynolds, M.P., de la Vega, A.J., Petrie, P.R., and Robinson, R. (2009) Phenotypic plasticity of yield and phenology in wheat, sunflower and grapevine. Field Crops Research 110: 242-250.

Saini, H.S., Lalonde, S. (1997) Injuries to reproductive development under water stress, and their consequences for crop productivity. Journal of Crop Production 1: 223-248.

Saini, H.S., Westgate, M.E., Donald, L.S. (1999) Reproductive development in grain crops during drought. Advances in Agronomy 68: 59-96.

Samarah, N.H. (2005) Effects of drought stress on growth and yield of barley. Agronomy for Sustainable Development 25: 145-149.

Sambatti, J.B.M., Caylor, K.K. (2007) When is breeding for drought tolerance optimal if drought is random? New Phytologist 175: 70-80.

Sandhu, N., Raman, K.A., Torres, R.O., Audebert A, Dardou A, Kumar A, Henry, A. (2016) Rice root architectural plasticity traits and genetic regions for adaptability to variable cultivation and stress conditions. Plant Physiology 171: 2562-2576.

Sandhu, N., Singh, A., Dixit, S., Sta Cruz, M.T., Maturan, P.C., Jain, R.K., Kumar, A. (2014) Identification and mapping of stable QTL with main and epistasis effect on rice grain yield under upland drought stress. BMC Genetics 15: 1-15. 
Sangster, T.A., Queitsch, C. (2005) The HSP90 chaperone complex, an emerging force in plant development and phenotypic plasticity. Current Opinion in Plant Biology 8: 8692.

Santelia, D., Vincenzetti, V., Azzarello, E., Bovet, L., Fukao, Y., Düchtig, P., Mancuso, S., Martinoia, E., Geisler, M. (2005) MDR-like ABC transporter AtPGP4 is involved in auxin-mediated lateral root and root hair development. FEBS Letters 579: 5399-5406.

Sato, A., Miura, K. (2011) Root architecture remodeling induced by phosphate starvation. Plant Signaling \& Behavior 6: 1122-1126.

Scheet, P., Stephens, M. (2006) A fast and flexible statistical model for large-scale population genotype data: applications to inferring missing genotypes and haplotypic phase. American Journal of Human Genetics 78: 629-644.

Scheible, W.R., Pauly, M. (2004) Glycosyltransferases and cell wall biosynthesis: novel players and insights. Current Opinion in Plant Biology 7: 285-295.

Schneider, C.A., Rasband, W.S., Eliceiri, K.W. (2012) NIH Image to ImageJ: 25 years of image analysis. Nature Methods 9: 671-675.

Schuetz, M., Smith, R., Ellis, B. (2012) Xylem tissue specification, patterning, and differentiation mechanisms. Journal of Experimental Botany 64: 11-31.

Sedbrook, J.C., Chen, R., Masson, P.H. (1999) ARG1 (Altered Response to Gravity) encodes a DnaJ-like protein that potentially interacts with the cytoskeleton. Proceedings of the National Academy of Sciences 96: 1140-1145.

Segura, V., Vilhjalmsson, B.J., Platt, A., Korte, A., Seren, U., Long, Q., Nordborg, M. (2012) An efficient multi-locus mixed-model approach for genome-wide association studies in structured populations. Nature Genetics 44: 825-830.

Selote, D.S., Chopra, R.K. (2004) Drought induced spikelet sterility is associated with an inefficient antioxidant defense in rice panicles. Physiologia Plantarum 121: 462-471.

Sheoran, I.S., Saini, H.S. (1996) Drought-induced male sterility in rice: Changes in carbohydrate levels and enzyme activities associated with the inhibition of starch accumulation in pollen. Sexual Plant Reproduction 9: 161-169.

Shi, W., Yin, X., Struik, P.C., Xie, F., Schmidt, R.C., Jagadish, K.S.V. (2016) Grain yield and quality responses of tropical hybrid rice to high night-time temperature. Field Crops Research 190: 18-25.

Shomura, A., Izawa, T., Ebana, K., Ebitani, T., Kanegae, H., Konishi, S., Yano, M. (2008) Deletion in a gene associated with grain size increased yields during rice domestication. Nature Genetics 40: 1023-1028.

Singh, A., Breja, P., Khurana, J.P., Khurana, P. (2016) Wheat Brassinosteroid-Insensitive1 (TaBRII) interacts with members of TaSERK gene family and cause early flowering and seed yield enhancement in Arabidopsis. PLOS ONE 11: e0153273.

Singh, S., Mackill, D.J., and Ismail, A.M. (2009) Responses of SUB1 rice introgression lines to submergence in the field: Yield and grain quality. Field Crops Research 113: 12-23.

Singh, U., Ladha, J. K., Castillo, E. G., Punzalan, G., Tirol-Padre, A., and Duqueza, M. (1998) Genotypic variation in nitrogen use efficiency in medium and long-duration rice. Field Crops Research 58: 35-53.

Slafer, G.A. (2003) Genetic basis of yield as viewed from a crop physiologist's perspective. Annals of Applied Biology 142: 117-128.

Sofo, A., Dichio, B., Xiloyannis, C., Masia, A. (2004) Lipoxygenase activity and proline accumulation in leaves and roots of olive trees in response to drought stress. Physiologia Plantarum 121: 58-65.

Solari, L.I., Pernice, F., DeJong, T.M. (2006) The relationship of hydraulic conductance to root system characteristics of peach (Prunus persica) rootstocks. Physiologia Plantarum 128: 324-333. 
Soltani, A., Ghassemi-Golezani, K., Khooie, F.R., and Moghaddam, M. (1999) A simple model for chickpea growth and yield. Field Crops Research 62: 213-224.

Song, X.J., Huang, W., Shi, M., Zhu, M.Z., Lin, H.X. (2007) A QTL for rice grain width and weight encodes a previously unknown RING-type E3 ubiquitin ligase. Nature Genetics 39: 623-630.

Sorrells, M.E., La Rota, M., Bermudez-Kandianis, C.E., Greene, R.A., Kantety, R., Munkvold, J.D., Miftahudin Mahmoud, A., Ma, X., Gustafson, P.J., Qi, L.L., Echalier, B., Gill, B.S., Matthews, D.E., Lazo, G.R., Chao, S., Anderson, O.D., Edwards, H., Linkiewicz, A.M., Dubcovsky, J., Akhunov, E.D., Dvorak, J., Zhang, D., Nguyen, H.T., Peng, J., Lapitan, N.L., Gonzalez-Hernandez, J.L., Anderson, J.A., Hossain, K., Kalavacharla, V., Kianian, S.F., Choi, D.W., Close, T.J., Dilbirligi, M., Gill, K.S., Steber, C., Walker-Simmons, M.K., McGuire, P.E., Qualset, C.O. (2003) Comparative DNA sequence analysis of wheat and rice genomes. Genome Research 13: 1818-1827.

Soundappan, I., Bennett, T., Morffy, N., Liang, Y., Stanga, J.P., Abbas, A., Leyser, O., Nelson, D.C. (2015) SMAX1-LIKE/D53 family members enable distinct MAX2 dependent responses to strigolactones and karrikins in Arabidopsis. The Plant Cell 27: 3143-3159.

Spielmeyer, W., Ellis, M.H., Chandler, P.M. (2002) Semidwarf ( $s d-1)$, "green revolution" rice, contains a defective gibberellin 20-oxidase gene. Proceedings of the National Academy of Sciences 99: 9043-9048.

Spindel, J., Begum, H., Akdemir, D., Virk, P., Collard, B., Redoña, E., Atlin, G., Jannink, J.L., McCouch, S.R. (2015) Genomic selection and association mapping in rice (Oryza sativa): Effect of trait genetic architecture, training population composition, marker number and statistical model on accuracy of rice genomic selection in elite, tropical rice breeding lines. PLoS Genetics 11: e1004982.

Stahl, Y., Grabowski, S., Bleckmann, A., Kühnemuth, R., Weidtkamp-Peters, S., Pinto Karine, G., Kirschner Gwendolyn, K., Schmid Julia, B., Wink René, H., Hülsewede, A., Felekyan, S., Seidel Claus, A.M., Simon, R. (2013) Moderation of Arabidopsis root stemness by CLAVATA1 and ARABIDOPSIS CRINKLY4 receptor kinase complexes. Current Biology 23: 362-371.

Stoop, W.A., Uphoff, N., and Kassam, A. (2002) A review of agricultural research issues raised by the system of rice intensification (SRI) from Madagascar: opportunities for improving farming systems for resource-poor farmers. Agricultural Systems 71: 249274.

Sultan, S.E. (2000) Phenotypic plasticity for plant development, function and life history. Trends in Plant Science 5, 537-542.

Suralta, R.R., Inukai, Y., and Yamauchi, A. (2010) Dry matter production in relation to root plastic development, oxygen transport, and water uptake of rice under transient soil moisture stresses. Plant and Soil 332: 87-104.

Suzuki, N., Rizhsky, L., Liang, H., Shuman, J., Shulaev, V., Mittler, R. (2005) Enhanced tolerance to environmental stress in transgenic plants expressing the transcriptional coactivator multiprotein bridging factor 1c. Plant Physiology 139: 1313-1322.

Swamy, B.PM., Ahmed, H.U., Henry, A., Mauleon, R., Dixit, S., Vikram, P., Tilatto, R., Verulkar, S.B., Perraju, P., Mandal, N.P., Variar, M.,S.R., Chandrababu, R., Singh, O.N., Dwivedi, J.L., Das, S.P., Mishra, K.K., Yadaw, R.B., Aditya, T.L., Karmakar, B., Satoh, K., Moumeni, A., Kikuchi, S., Leung, H., Kumar, A. (2013) Genetic, physiological, and gene expression analyses reveal that multiple QTL enhance yield of rice mega-variety IR64 under drought. PLoS ONE 8: e62795. 
Swamy, B.P.M., Shamsudin, N.A.A., Rahman, S.N.A., Mauleon, R., Ratnam, W., Sta Cruz, M.T., Kumar, A. (2017) Association mapping of yield and yield-related traits under reproductive stage drought stress in rice (Oryza sativa L.). Rice 10: 21.

Swamy, B.P.M., Vikram, P., Dixit, S., Ahmed, H.U., and Kumar, A. (2011) Meta-analysis of grain yield QTL identified during agricultural drought in grasses showed consensus. BMC Genomics 12: 319.

Swarup, K., Benkova, E., Swarup, R., Casimiro, I., Peret, B., Yang, Y., Parry, G., Nielsen, E., De Smet, I., Vanneste, S., Levesque, M.P., Carrier, D., James, N., Calvo, V., Ljung, K., Kramer, E., Roberts, R., Graham, N., Marillonnet, S., Patel, K., Jones, J.D.G., Taylor, C.G., Schachtman, D.P., May, S., Sandberg, G., Benfey, P., Friml, J., Kerr, I., Beeckman, T., Laplaze, L., Bennett, M.J. (2008) The auxin influx carrier LAX3 promotes lateral root emergence. Nature Cell Biology 10: 946-954.

Sweeney, M., McCouch, S. (2007) The complex history of the domestication of rice. Annals of Botany 100: 951-957.

Tang, L., Kim, M.D., Yang, K.S., Kwon, S.Y., Kim, S.H., Kim, J.S., Yun, D.J., Kwak, S.S., Lee, H.S. (2008) Enhanced tolerance of transgenic potato plants overexpressing nucleoside diphosphate kinase 2 against multiple environmental stresses. Transgenic Research 17: 705-715.

Tang, L., Zhu, Y., Hannaway, D., Meng, Y., Liu, L., Chen, L., and Cao, W. (2009) RiceGrow: A rice growth and productivity model. NJAS-Wageningen Journal of Life Sciences 57: 83-92.

Tester, M., and Langridge, P. (2010) Breeding technologies to increase crop production in a changing world. Science 327: 818-822.

Thomann, A., Lechner, E., Hansen, M., Dumbliauskas, E., Parmentier, Y., Kieber, J., Scheres, B., Genschik, P. (2009) Arabidopsis CULLIN3 genes regulate primary root growth and patterning by ethylene-dependent and-independent mechanisms. PLoS Genetics 5: e1000328.

Tilman, D., Balzer, C., Hill, J., and Befort, B.L. (2011) Global food demand and the sustainable intensification of agriculture. Proceedings of the National Academy of Sciences 108: 20260-20264.

Tombesi, S., Johnson, R.S., Day, K.R., DeJong, T.M. (2010) Relationships between xylem vessel characteristics, calculated axial hydraulic conductance and size-controlling capacity of peach rootstocks. Annals of Botany 105: 327-331.

Tran, T.T., Kano-Nakata, M., Suralta, R.R., Menge, D., Mitsuya, S., Inukai, Y., and Yamauchi, A. (2015) Root plasticity and its functional roles were triggered by water deficit but not by the resulting changes in the forms of soil $\mathrm{N}$ in rice. Plant and Soil 386: 65-76.

Tuberosa, R., and Salvi, S. (2006) Genomics-based approaches to improve drought tolerance of crops. Trends in Plant Science 11: 405-412.

Tyree, M.T., Ewers, F.W. (1991) The hydraulic architecture of trees and other woody plants. New Phytologist 119: 345-360.

Uga, Y., Okuno, K., Yano, M. (2008) QTLs underlying natural variation in stele and xylem structures of rice root. Breeding Science 58: 7-14.

Uga, Y., Sugimoto, K., Ogawa, S., Rane, J., Ishitani, M., Hara, N., Kitomi, Y., Inukai, Y., Ono, K., Kanno, N., Inoue, H., Takehisa, H., Motoyama, R., Nagamura, Y., Wu, J., Matsumoto, T., Takai, T., Okuno, K., and Yano, M. (2013) Control of root system architecture by DEEPER ROOTING 1 increases rice yield under drought conditions. Nature Genetics 45: 1097-1102.

Ullah, H., Chen, J.G., Temple, B., Boyes, D.C., Alonso, J.M., Davis, K.R., Ecker, J.R., Jones, A.M. (2003) The $\beta$-subunit of the Arabidopsis G protein negatively regulates 
auxin-induced cell division and affects multiple developmental processes. The Plant Cell 15: 393-409.

Ullah, H., Chen, J.G., Young, J.C., Im, K.H., Sussman, M.R., Jones, A.M. (2001) Modulation of cell proliferation by heterotrimeric G protein in Arabidopsis. Science 292: 2066-2069.

Urano, D., Miura, K., Wu, Q., Iwasaki, Y., Jackson, D., Jones, A.M. (2016) Plant morphology of heterotrimeric G protein mutants. Plant and Cell Physiology 57:437445.

Vatén, A., Dettmer, J., Wu, S., Stierhof, Y.D., Miyashima, S., Yadav Shri, R., Roberts Christina, J., Campilho, A., Bulone, V., Lichtenberger, R., Lehesranta, S., Mähönen Ari, P., Kim, J.Y., Jokitalo, E., Sauer, N., Scheres, B., Nakajima, K., Carlsbecker, A., Gallagher Kimberly, L., Helariutta, Y. (2011) Callose biosynthesis regulates symplastic trafficking during root development. Developmental Cell 21: 11441155.

Vejchasarn, P., Lynch, J.P., Brown, K.M. (2016) Genetic variability in phosphorus responses of rice root phenotypes. Rice 9: 29.

Venuprasad R, Bool ME, Quiatchon L, Cruz MS, Amante M, Atlin GN (2012) A largeeffect QTL for rice grain yield under upland drought stress on chromosome 1. Molecular Breeding 30: 535-547.

Venuprasad, R., Lafitte, H.R., Atlin, G.N. (2007) Response to direct selection for grain yield under drought stress in rice. Crop Science 47: 285-293.

Venuprasad, R., Sta Cruz, M.T., Amante, M., Magbanua, R., Kumar, A., Atlin, G.N. (2008) Response to two cycles of divergent selection for grain yield under drought stress in four rice breeding populations. Field Crops Research 107: 232-244.

Venuprasad, R., Bool, M. E., Dalid, C.O., Bernier, J., Kumar, A., and Atlin, G.N. (2009a) Genetic loci responding to two cycles of divergent selection for grain yield under drought stress in a rice breeding population. Euphytica 167: 261-269.

Venuprasad, R., Dalid, C.O., Del Valle, M., Zhao, D., Espiritu, M., Sta Cruz, M.T., Amante, M., Kumar, A., and Atlin, G.N. (2009b) Identification and characterization of large-effect quantitative trait loci for grain yield under lowland drought stress in rice using bulk-segregant analysis. Theoretical and Applied Genetics 120: 177-190.

Verulkar, S.B., Mandal, N.P., Dwivedi, J.L., Singh, B.N., Sinha, P.K., Mahato, R.N., Dongre, P., Singh, O.N., Bose, L.K., Swain, P., Robin, S., Chandrababu, R., Senthil, S., Jain, A., Shashidhar, H.E., Hittalmani, S., Vera Cruz, C., Paris, T., Raman, A., Haefele, S., Serraj, R., Kumar, A. (2010) Breeding resilient and productive genotypes adapted to drought-prone rainfed ecosystem of India. Field Crops Research 117: 197208.

Vikram, P., Swamy, B.P.M., Dixit, S., Singh, R., Singh, B.P., Miro, B., Kohli, A., Henry, A., Singh, N.K., Kumar, A. (2015) Drought susceptibility of modern rice varieties: an effect of linkage of drought tolerance with undesirable traits. Scientific Reports 5: 14799.

Vikram, P., Swamy, B.M., Dixit, S., Ahmed, H.U., Teresa Sta Cruz, M., Singh, A.K., and Kumar, A. (2011) qDTY 1.1, a major QTL for rice grain yield under reproductive-stage drought stress with a consistent effect in multiple elite genetic backgrounds. $B M C$ Genetics 12, 89.

Villareal, R.L., Del Toro, E., Mujeeb-Kazi, A., Rajaram, S. (1995) The $1 B L / 1 R S$ chromosome translocation effect on yield characteristics in a Triticum aestivum L. cross. Plant Breeding 114: 497-500.

Wan, L., Wang, X., Li, S., Hu, J., Huang, W., Zhu, Y. (2014) Overexpression of OsKTN80a, a katanin P80 ortholog, caused the repressed cell elongation and stalled cell division 
mediated by microtubule apparatus defects in primary root in Oryza sativa. Journal of Integrative Plant Biology 56: 622-634.

Wang, D., Pan, Y., Zhao, X., Zhu, L., Fu, B., Li, Z. (2011) Genome-wide temporal-spatial gene expression profiling of drought responsiveness in rice. BMC Genomics 12: 1-15.

Wang, W., Vinocur, B., Shoseyov, O., Altman, A. (2004) Role of plant heat-shock proteins and molecular chaperones in the abiotic stress response. Trends in Plant Science 9: 244252.

Wang, X., Feng, S., Nakayama, N., Crosby, W.L., Irish, V., Deng, X.W., Wei, N. (2003) The COP9 signalosome interacts with $S C F(U F O)$ and participates in Arabidopsis flower development. The Plant Cell 15: 1071-1082.

Wassmann, R., Jagadish, S. V. K., Sumfleth, K., Pathak, H., Howell, G., Ismail, A., Serraj, R., Redoña, E., Singh, R. K., and Heuer, S. (2009) Regional vulnerability of climate change impacts on Asian rice production and scope for adaptation. Advances in Agronomy 102: 91-133.

Wasson, A.P., Richards, R.A., Chatrath, R., Misra, S.C., Prasad, S.S., Rebetzke, G.J., Kirkegaard, J.A., Christopher, J., Watt, M. (2012) Traits and selection strategies to improve root systems and water uptake in water-limited wheat crops. Journal of Experimental Botany 63: 3485-3498.

Wu, G., Lewis, D.R., Spalding, E.P. (2007) Mutations in Arabidopsis multidrug resistancelike $\mathrm{ABC}$ transporters separate the roles of acropetal and basipetal auxin transport in lateral root development. The Plant Cell 19: 1826-1837.

Wu, Y., Sharp, R.E., Durachko, D.M., Cosgrove, D.J. (1996) Growth maintenance of the maize primary root at low water potentials involves increases in cell-wall extension properties, expansin activity, and wall susceptibility to expansins. Plant Physiology 111: 765-772

Wu, W., and Cheng, S. (2014) Root genetic research, an opportunity and challenge to rice improvement. Field Crops Research 165: 111-124.

Wysocka-Diller, J.W., Helariutta, Y., Fukaki, H., Malamy, J.E., Benfey, P.N. (2000) Molecular analysis of SCARECROW function reveals a radial patterning mechanism common to root and shoot. Development 127: 595-603.

Xiao, C., Somerville, C., Anderson, C.T. (2014) POLYGALACTURONASE INVOLVED IN EXPANSION1 functions in cell elongation and flower development in Arabidopsis. The Plant Cell 26: 1018-1035.

Xiong, L., Wang, R.G., Mao, G., and Koczan, J.M. (2006) Identification of drought tolerance determinants by genetic analysis of root response to drought stress and abscisic acid. Plant Physiology 142: 1065-1074.

Xu, J.L., Lafitte, H.R., Gao, Y.M., Fu, B.Y., Torres, R., and Li, Z.K. (2005) QTLs for drought escape and tolerance identified in a set of random introgression lines of rice. Theoretical and Applied Genetics 111: 1642-1650.

Xu, L., and Buck-Sorlin, G. (2016) Simulating genotype-phenotype interaction using extended functional-structural plant models: Approaches, applications and potential pitfalls. In "Crop Systems Biology: Narrowing the gaps between crop modelling and genetics" (X. Yin and P. C. Struik, eds.), pp. 33-53. Springer International Publishing, Cham.

Yano, K., Yamamoto, E., Aya, K., Takeuchi, H., Lo, P.C., Hu, L., Yamasaki, M., Yoshida, S., Kitano, H., Hirano, K., Matsuoka, M. (2016) Genome-wide association study using whole-genome sequencing rapidly identifies new genes influencing agronomic traits in rice. Nature Genetics 48: 927-934. 
Yao, F., Huang, J., Cui, K., Nie, L., Xiang, J., Liu, X., Wu, W., Chen, M., and Peng, S. (2012) Agronomic performance of high-yielding rice variety grown under alternate wetting and drying irrigation. Field Crops Research 126: 16-22.

Yin, X., Chasalow, S.D., Stam, P., Kropff, M.J., Dourleijn, C.J., Bos, I., Bindraban, P.S. (2002) Use of component analysis in QTL mapping of complex crop traits: a case study on yield in barley. Plant Breeding 121: 314-319.

Yin, X., Kropff, M.J., Horie, T., Nakagawa, H., Centeno, H.G.S., Zhu, D., Goudriaan, J. (1997) A model for photothermal responses of flowering in rice I. Model description and parameterization. Field Crops Research 51: 189-200.

Yin, X. (2013) Improving ecophysiological simulation models to predict the impact of elevated atmospheric $\mathrm{CO}_{2}$ concentration on crop productivity. Annals of Botany 112: 465-475.

Yin, X., and Struik, P.C. (2010) Modelling the crop: from system dynamics to systems biology. Journal of Experimental Botany 61: 2171-2183.

Yin, X., and Struik, P.C. (2015) Crop systems biology: Narrowing the gaps between crop modelling and genetics, Springer International Publishing, Chams.

Yin, X., and Struik, P.C. (2016) Crop Systems Biology: Where are we and where to go? In "Crop Systems Biology: "Narrowing the gaps between crop modelling and genetics" (X. Yin and P. C. Struik, eds.), pp. 219-227. Springer International Publishing, Cham.

Yin, X., and Struik, P.C. (2017) Can increased leaf photosynthesis be converted into higher crop mass production? A simulation study for rice using the crop model GECROS. Journal of Experimental Botany 68: 2345-2360.

Yin, X., Chasalow, S.D., Dourleijn, C.J., Stam, P., and Kropff, M.J. (2000) Coupling estimated effects of QTLs for physiological traits to a crop growth model: predicting yield variation among recombinant inbred lines in barley. Heredity 85: 539-549.

Yin, X., Kropff, M.J., and Stam, P. (1999) The role of ecophysiological models in QTL analysis: the example of specific leaf area in barley. Heredity 82: 415-421.

Yin, X., Struik, P.C., and Kropff, M.J. (2004) Role of crop physiology in predicting gene-tophenotype relationships. Trends in Plant Science 9: 426-432.

Yin, X., Struik, P.C., Gu, J., and Wang, H. (2016) Modelling QTL-trait-crop relationships: Past experiences and future prospects. In "Crop Systems Biology: Narrowing the gaps between crop modelling and genetics" (X. Yin and P. C. Struik, eds.), pp. 193-218. Springer International Publishing, Cham.

Yin, X., and Van Laar, H. (2005) Crop systems dynamics: an ecophysiological simulation model for genotype-by-environment interactions, Wageningen Academic Pub.

Yin, X., Struik, P.C., Tang, J., Qi, C., and Liu, T. (2005) Model analysis of flowering phenology in recombinant inbred lines of barley. Journal of Experimental Botany 56: 959-965.

Yin, X., Struik, P. C., van Eeuwijk, F. A., Stam, P., and Tang, J. (2005) QTL analysis and QTL-based prediction of flowering phenology in recombinant inbred lines of barley. Journal of Experimental Botany 56: 967-976.

Yoshida, S., Hasegawa, S. (1982) The rice root system: its development and function. In: Drought resistance in crops with emphasis on rice. International Rice Research Institute, Manila, Philippines, pp 97-134.

Yue, B., Xue, W., Xiong, L., Yu, X., Luo, L., Cui, K., Jin, D., Xing, Y., and Zhang, Q. (2006) Genetic basis of drought resistance at reproductive stage in rice: separation of drought tolerance from drought avoidance. Genetics 172: 1213-1228.

Zhang, H., Forde, B.G. (1998) An Arabidopsis MADS box gene that controls nutrient-induced changes in root architecture. Science 279: 407-409. 
Zhang, Y., Xiao, Y., Du, F., Cao, L., Dong, H., Ren, H. (2011) Arabidopsis VILLIN4 is involved in root hair growth through regulating actin organization in a $\mathrm{Ca}^{2+}$-dependent manner. New Phytologist 190: 667-682.

Zhang, Z., Ersoz, E., Lai, C.Q., Todhunter, R.J., Tiwari, H.K., Gore, M.A., Bradbury, P.J., Yu, J., Arnett, D.K., Ordovas, J.M., Buckler, E.S. (2010) Mixed linear model approach adapted for genome-wide association studies. Nature Genetics 42: 355-360.

Zhang, G.L., Chen, L.Y., Xiao, G.Y., Xiao, Y.H., Chen, X.B., and Zhang, S.T. (2009) Bulked segregant analysis to detect QTL related to heat tolerance in rice (Oryza sativa L.) using SSR markers. Agricultural Sciences in China 8: 482-487.

Zhang, T., and Huang, Y. (2012) Impacts of climate change and inter-annual variability on cereal crops in China from 1980 to 2008. Journal of the Science of Food and Agriculture 92: 1643-1652.

Zhao, C., Craig, J.C., Petzold, H.E., Dickerman, A.W., Beers, E.P. (2005) The xylem and phloem transcriptomes from secondary tissues of the Arabidopsis root-hypocotyl. Plant Physiology 138: 803-818.

Zhao, Y., Xing, L., Wang, X., Hou, Y.J., Gao, J., Wang, P., Duan, C.G., Zhu, X., Zhu, J.K. (2014) The ABA receptor PYL8 promotes lateral root growth by enhancing MYB77dependent transcription of auxin-responsive genes. Science signaling 7: ra53-ra53.

Zhao, K., Tung, C.W., Eizenga, G.C., Wright, M.H., Ali, M.L., Price, A.H., Norton, G.J., Islam, M.R., Reynolds, A., Mezey, J., McClung, A.M., Bustamante, C.D., and McCouch, S.R. (2011) Genome-wide association mapping reveals a rich genetic architecture of complex traits in Oryza sativa. Nature Communications 2: 467.

Zhu, J., Kaeppler, S.M., and Lynch, J.P. (2005a) Mapping of QTL controlling root hair length in maize (Zea mays L.) under phosphorus deficiency. Plant and Soil 270: 299310 .

Zhu, J., Kaeppler, S.M., and Lynch, J.P. (2005b) Mapping of QTLs for lateral root branching and length in maize (Zea mays L.) under differential phosphorus supply. Theoretical and Applied Genetics 111: 688-695.

Zorrilla, G., Martinez, C., Berrío, L., Corredor, E., Carmona, L., Pulver, E. (2012) Improving rice production systems in Latin America and the Caribbean. In: EcoEfficiency: From Vision to Reality. CIAT, Cali, Colombia, pp. 161-170. 



\section{Summary}

Rice (Oryza sativa L.) is an important component of food security; it provides food for more than half of the world population. However, a rapidly changing climate with more frequent occurrence of water-deficit and high-temperature stress severely reduces the productivity of rice and other cereals. Among the cereals, rice is most sensitive to water-deficit stress due to its semi-aquatic adaptation: it requires 2 to 3 times more water for cultivation than other cereals. Especially, water-deficit stress occurring during the sensitive reproductive stage seriously impedes the productivity of rice. Nevertheless, stress occurring during the vegetative stage is also observed in Asia, and can also have significantly reduce final grain yield. Therefore, one of the major challenges is to improve the tolerance of rice to stress at any time during the growing period to ensure food security.

Plants have evolved specific abilities to adjust their morphology, physiology and biochemistry in response to stress a phenomenon commonly known as a phenotypic plasticity. Phenotypic plasticity is the ability of a given genotype to produce an adapted phenotype in response to changing environments. Plasticity in root morphology and anatomy is of great importance to improve the adaptation of rice to stress due to the primary role of roots in the uptake of water and nutrients. During domestication, rice has evolved a phenotypic plasticity to adapt to a wide range of moisture regimes: from traditional lowland (paddy rice) to upland/aerobic (moderately stress conditions) or even severe water-deficit stress. Nevertheless, rice is still considered to be relatively poorly adapted to water-deficit, in comparison to other dryland cereal crops, such as wheat, maize and sorghum. In addition, there is no adequate knowledge on how rice differs from dryland cereals in water-deficit stress adaptation. Therefore, in this thesis, I have studied the physiological, morphological and anatomical response or plasticity of rice genotypes well adapted to different moisture regimes (lowland, aerobic and water-deficit conditions), in comparison with that of wheat genotypes that are drought tolerant, under water-deficit stress during the vegetative stage (Chapter 2). This study allowed to demonstrate that compared with wheat, rice genotypes have a weaker morphological and anatomical plasticity in the shoot and in the root traits in response to water-deficit stress. Specifically, rice cultivars adopted a rapid water acquisition strategy through developing thinner roots under water deficit stress, whereas wheat cultivars followed a water-conserving strategy by developing thicker roots and moderate tillering. Further, a comprehensive analysis between these two divergent species made it possible to identify the functional relevance of root morphological and anatomical plasticity in water-deficit tolerance (Chapter 2). In addition, 
previous studies have proven that phenotypic plasticity is under genetic control and is regulated by key environmental sensing genes. To date, very few quantitative trait loci (QTLs) regulating the phenotypic plasticity in response to water-deficit were identified in rice. Diverse rice genotypes are a pool of naturally occurring mutations, which can give fundamental insights into plant function. They are also a vital resource of novel beneficial alleles for crop plant improvement. Therefore, I have scaled the key findings from Chapter 2 up to an indica rice diversity panel and quantified the genotypic variation of phenotypic plasticity for physiological, morphological and root anatomical responses under water-deficit stress during the vegetative stage (Chapter 3). We then carried out a genome-wide association study (GWAS) on these traits and their plasticity, using 45,608 high-quality single nucleotide polymorphisms (SNPs). One hundred four significant loci were detected for these traits under control condition, 106 were detected under water-deficit stress, and 76 were detected for trait plasticity. The genetic basis of root morphology and anatomy was different across both water-regimes (strong QTLX Environment interactions), in line with that so many loci were detected for the plasticity of these traits. In addition, these genetic loci associated with root traits and their plasticity was associated with genes regulating biosynthesis, transport or signalling of phytohormones. Hence, genetic loci identified in Chapter 3 provide an important basis for understanding the molecular mechanism of plastic root development in response to water-deficit stress.

Rice grain yield is strongly affected by water-deficit stress occurring during the sensitive reproductive stage. So far, breeders have improved the tolerance to water-deficit stress during the reproductive stage by introgression of QTLs identified in traditional bi-parental mapping populations of rice. Although this approach has resulted in significant progress, yet many of the QTLs/genes/alleles remain hidden in the rice genetic diversity, which cannot be explored through traditional linkage analysis. Therefore, in Chapter 4, I have explored the same indica rice diversity panel that was used in Chapter 3, to identify those hidden QTLs/genes for grain yield and its components through a GWAS under well-watered conditions and under waterdeficit stress during the reproductive stage in field experiments for two years. I have followed staggered sowing of rice genotypes to maximally synchronize their flowering and thus the phenological timing of the exposure to the water-deficit stress. One hundred two loci were detected in non-stress conditions (38 loci in 2013 and 64 loci in 2014) and 124 loci (69 loci in 2013 and 55 in 2014) in water-deficit stress. Some desynchronised flowering time strongly confounded the grain yield and its components in the data set for water-deficit stress in 2013. To minimise the confounding effect, I have carried out a statistical correction of grain yield and 
yield components using days to flowering, which helped to detect 31 additional genetic loci for grain yield, its components and harvest index in 2013. In addition, most of these QTLs were specific to treatment and year, which means that there was a high QTL $\times$ environment interaction (QEI). The QEI plays an important role in adaptation to changing environments, and is regulated by key environmental sensing genes. We identified key a priori candidate genes within the linkage disequilibrium block of grain yield loci regulating abiotic stress tolerant biological processes.

Grain yield is a complex trait determined by action and interaction of different component traits. A deeper understanding of how these component traits contribute to grain yield is a prerequisite for designing the future new plant type for improved grain yield under changing climatic conditions. Crop growth models are widely used to understand the complex grain yield under water-deficit stress. In Chapter 5, I have used the generic crop model, GECROS (Genotype-by-Environment interaction on CROp growth Simulator), to quantify grain yield in the indica rice diversity panel. The physiological component traits as model inputs included pre-flowering period $\left(m_{\mathrm{V}}\right)$, post-flowering period $\left(m_{\mathrm{R}}\right)$, photoperiod sensitivity $(\delta)$, maximum plant height $\left(H_{\max }\right)$, single-grain weight $(\mathrm{Sw})$, grain set $\left(g_{\text {set }}\right)$, grain nitrogen concentration $\left(n_{\mathrm{so}}\right)$, and total crop nitrogen uptake $\left(N_{\max }\right)$. These component traits were estimated from the control treatment in one season (2013) for an indica rice diversity panel consisting of 267 genotypes in Chapter 4 . With these component traits, the model could account for $58 \%$ of the variation in grain yield among 267 rice genotypes under control conditions and $40 \%$ under water-deficit conditions. In addition, I have identified SNP loci associated with component traits through a GWAS in randomly selected 213 genotypes as the training datasets and the remaining 54 genotypes were used as the testing datasets. SNP-based component trait values were calculated from estimated effects of the loci, and were fed into the model. The SNP-based model could account for $37 \%$ and $29 \%$ of the yield variation under control and water-deficit conditions, respectively, in the training datasets. However, the SNP-based model could account for only $10 \%$ of the yield variation in control conditions and $15 \%$ of the yield variation under water-deficit stress in the testing datasets. The performance of the model was lower, using either original or SNP-based parameter values, when the model was used to simulate yields in an independent season (2014). Model-based sensitivity analysis ranked the relative importance of the individual SNP loci identified for component traits in determining the grain yield variation. The ranking differed greatly between control and water-deficit environments. The grain yield variation in the well-watered treatment was mainly explained by 
SNP loci associated with the total crop nitrogen uptake $\left(N_{\max }\right)$, whereas the yield variation under the water-deficit stress during the reproductive stage was explained mainly by loci associated with pre-flowering time $\left(m_{\mathrm{V}}\right)$. Further, the GECROS-based dissection approach detected more SNP loci than the analysis using yield per se. Virtual ideotypes based on SNPs identified by modelling had higher yield than those based on SNPs for grain yield per se (Chapter 5), illustrating potential values of the model-based approach in supporting marker-assisted selection.

In the general discussion (Chapter 6), I have discussed the results obtained in Chapters 2-5 based on the specific objectives designed for this thesis (Chapter 1). I have also discussed the future prospects on how to improve the integration of crop growth modelling with quantitative genetics to narrow down the genotype-by-phenotype gap. The generic GECROS model needs to be tailored to include those important morphological and physiological traits identified in Chapters 3 and 4 to more effectively explain genotype-by-environment interactions exhibited in a diversity panel of rice. 


\section{Acknowledgements}

First and foremost, I should express my deepest gratitude to an anonymous private donor who provided financial support towards my $\mathrm{PhD}$ fellowship, and for most of the research work reported in this study. I am also indebted to the Federal Ministry for Economic Cooperation and Development, Germany, and the USAID and Bill and Melinda Gates Foundation for their partial financial support.

This thesis would not have been possible without my excellent multidisciplinary supervision team: Dr. Krishna Jagadish, Prof. Paul C Struik, and Dr. Xinyou Yin. I want to express my deepest gratitude to Dr. Krishna Jagadish, for selecting me as $\mathrm{PhD}$ candidate in "Growing Rice Like Wheat" project. Krishna, it has been honored to work with your heat stress group at IRRI. During my initial journey, I have always received very useful personal and professional guidance, constant encouragement, and unconditional support from you to finish my challenging experiments. I also really appreciate your habit to encourage students for writing scientific publications, which have not only improved my writing skills but also improved my communications skills. Paul, I have been so fortunate to have you as a promotor and thank you very much for giving me as an opportunity to pursue the doctoral degree in the Centre for Crop Systems analysis. As a promoter, your kindness always helped to lift my selfconfidence, whenever I was nervous and frustrated. Xinyou, being a thesis co-promotor and daily supervisors your office was always open to discuss the ideas and organize the work. I also would also like to thank you for introducing me to the QTL-based ecophysiological modeling, which is very interesting but quite new to me.

Members from the heat stress group at IRRI also immensely contributed to smoothly finish my $\mathrm{PhD}$, and without their constant support, I would not have reached this stage. So, I am greatful to all of them namely Ancio Macahia (we like to call him Alex), Joel Evangelista, Allan Malabanan, Alexander Aringo, Pablo Gupit, Siena Calibo, Reneeliza Jean, Lovely lawas, Cherryl Quinones and Jacinta Evangelista. Members of the group have been the source of friendships, and are actively collaborating and helping each other. I am especially grateful to the researcher Lovely and Cherryl, for their unconditional assistance to organize the pot weighing, and root harvesting of the greenhouse experiment. I also would like to thanks technicians team (Alex, Joel, Allan, and Siena), who constantly helped in conducting the greenhouse and field experiments. Alex, your years of experience in organizing and conducting the experiments greatly helped during my large-scale field experiments. Allan, your abilities to meticulously organize the greenhouse experiments, helped a lot to successfully finish the 
challenging greenhouse experiments. Siena, I would like to thank you for constantly supervising the most difficult task of root scanning, and processing bulk of the yield and yield component samples from the field experiments. I am also thankful to Dr. Rajeev N Bahuguna, Dr. Nicolas Mattes, Dr. David Sabella, Dr. Raju Bheemanhali, Dr. Jiang and Dr. Wanju Shi, from whom I learnt many things and very much enjoyed scientific debate during meetings. A very special thanks to Jacinta Evangelista for managing the administrative and financial matters that have made my life so easy.

My special thanks to Uttam Kumar and Umair Aslam with whom I have shared the house at IRRI for more than two years. I am very much enjoyed preparing the food and listening bollywood songs during dinner time with lots of fun.

I am grateful to all the group members of Centre for Crop Systems Analysis for their direct or indirect support. I also would also like to thanks my Ph.D. colleagues at the Centre for Crop Systems, for their love and care during my difficult times despite my shy nature: Gunfei Wenjing, Alejandro, Herman, Utah, Ioannis, Marcelo, Kailei, Dennis, Luuk, Martin, Ali, Shenghao, Ambra, Wanju, Cesar, Engida, Naznin, Preethi and many more..... I wish and pray for your PhD success and future endeavors, and good luck be with you always. I also would like to express my deepest gratitude to Sjanie, Nicole and Alex Leeuw for handling most of the administrative issues that made my life so easy in the Netherlands. Sjanie, you are very kind and always likes to know about the different cultures. Nicole, thank you very much for your support during setting up the final layout of thesis.

I am also grateful to the other members of "Growing Rice Like Wheat" (GRLW) team for their constant support and encouragement: Dr. Prem Bindraban, Prof. Harro Bouwmeester, Dr. Carolien Ruyter-Spira, Dr. M.S. Sheshashayee, Dr. Nataraja Karba and Dr. CG van der Linden Gerard. My special thanks to all the PhD students of GRWL (Wenjing, Beatriz, Giovanni, and Preethi), with whom I have actively collaborated and shared the experiments, materials and results. One of the unique features of GRLW project was a higher level of interaction between research topics of all the $\mathrm{PhD}$ students, which is not often the case in academic research. I strongly believe that we will continue our teamwork in the future to "Grow Rice Like Wheat".

I also would like to express my deepest gratitude to Jaap, Marita, Vincent and other family members for their hospitality, care and love. Jaap and Marita I had always enjoyed our Tuesday dinner together with playing the games. 
My special thanks to my two paranymphs, Alejandro M. Sierra and Manjunath Prasad for helping me to organise the defence. I am looking forward my one of the special day with you. I am also indebted to Manjunath and his family for offering me the house, and delicious homemade food during last phase of my $\mathrm{PhD}$.

Finally, I would like to thank all my family members, especially my mother (Ratnamala) and father (Narharirao), and wife (Sandhya) for their love, care and support to move forward with my $\mathrm{PhD}$. I also would like to express special thanks to my wife Sandhya for giving me the world's best gift -a baby boy! and making me as a father. I welcome my little champ (Arjun Pandit) to the most beautiful world with lots of love and happiness.

Niteen N. Kadam

Wageningen 2018, The Netherlands 



\section{List of Publications}

Mathithumilan, Balachandran ${ }^{\Psi}$, Niteen N. Kadam ${ }^{\Psi}$, Jyoti Biradar, Sowmya H. Reddy, Mahadeva Ankaiah, Madhura J. Narayanan, Udayakumar Makarla, Paramjit Khurana, and Sheshshayee M. Sreeman. Development and characterization of microsatellite markers for Morus spp. and assessment of their transferability to other closely related species. BMC Plant Biology (2013): 194. DOI:10.1186/1471-2229-13-194. $\Psi=$ First two author equal contribution.

Niteen N. Kadam, Gui Xiao, Reneeliza Jean Melgar, Rajeev N. Bahuguna, Cherryl Quinones, Anandhan Tamilselvan, and Pagadala Vara V. Prasad, Jagadish, Krishna S.V. Agronomic and Physiological Responses to high temperature, drought, and elevated $\mathbf{C O}^{2}$ interactions in cereals. Advances in Agronomy (2014): 111-156. DOI:10.1016/B978-0-12-800131-8.00003-0.

Niteen N. Kadam, Xinyou Yin, Prem S. Bindraban, Paul C. Struik, and Krishna S.V. Jagadish. Does morphological and anatomical plasticity during the vegetative stage make wheat more tolerant of water-deficit stress than rice? Plant Physiology (2015): 1389-1401. DOI:10.1104/pp.114.253328.

Niteen N. Kadam, Anandan Tamilselven, Lovely MF Lawas, Cherryl Quinones, Rajeev N Bahuguna, Michael J Thomson, Michael Dingkhun, Raveendran Muthurajan, Paul C Struik, Xinyou Yin, and Krishna SV Jagadish Genetic control of plasticity in root morphology and anatomy of rice in response to water-deficit. Plant Physiology (2017): 2302-2315. DOI: 10.1104/pp.17.00500. 



\section{PE\&RC Training and Education Statement}

With the training and education activities listed below the $\mathrm{PhD}$ candidate has complied with the requirements set by the C.T. de Wit Graduate School for Production Ecology and Resource Conservation (PE\&RC) which comprises of a minimum total of 32 ECTS (= 22 weeks of activities)

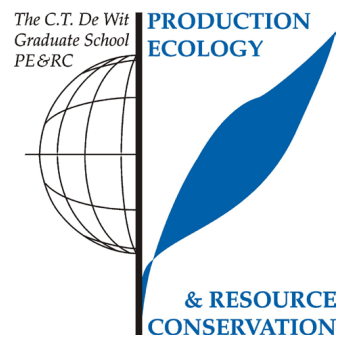

\section{Review of literature (6 ECTS)}

- Role of genomics in improvement of rice adaptation to water limited conditions

\section{Writing of project proposal (4.5 ECTS)}

- Physiological and genetic dissection of rice tolerance to water-deficit stress

\section{Post-graduate courses (6 ECTS)}

- $\quad$ Mixed model based QTL mapping; PE \& RC (2012)

- SNP data analysis; International Rice Research Institute (IRRI), Philippines (2014)

- Rice breeding; IRRI, Philippines (2015)

\section{Laboratory training and working visits ( 3 ECTS)}

- Stable 13C isotope studies for water use efficiency in crop plants; Department of Crop Physiology, University of Agricultural Sciences, GKVK, Bangalore, India (2014)

\section{Deficiency, refresh, brush-up courses (3.7 ECTS)}

- Basic statistics; PE\&RC (2015)

- Introduction to R for statistical analysis; PE\&RC (2016)

- Multivariate analysis; PE\&RC (2016)

\section{Competence strengthening / skills courses (1.6 ECTS)}

- Basic presentation skills; IRRI, Philippines (2014)

- Basic leadership skills; IRRI, Philippines (2014)

- Research data management; IRRI, Philippines (2014)

- Reviewing a scientific paper; PE\&RC (2016)

PE\&RC Annual meetings, seminars and the PE\&RC weekend (0.6 ECTS)

- PE\&RC Last year weekend (2016)

\section{Discussion groups / local seminars / other scientific meetings (6 ECTS)}

- CESD Division seminar; IRRI, Philippines (2013-2015)

- Thursday seminar series; IRRI, Philippines (2013-2015)

\section{International symposia, workshops and conferences (7.1 ECTS)}

- $\quad 4^{\text {th }}$ International rice congress, Bangkok, Thailand (2014)

- The "Growing rice like wheat" workshop at IRRI (2015)

- Merging crop modelling and genetics; University of Florida, USA (2015) 



\section{Curriculum vitae}

Niteen N. Kadam was born on $11^{\text {th }}$ April 1985 in Dharasaur, a village in Maharashtra province, India. He received his primary and secondary education in India. In 2008, he obtained his Bachelor of Science (BSc) in Agriculture from Marathwada Agriculture University, Parbhani, India. In 2009, he obtained Junior Research Fellowships from the Indian Council of Agriculture Research, to pursue Master of Science (MSc) in Agriculture with specialisation in Crop Physiology at the University of Agriculture Sciences, Bangalore, India. Immediately after completion of MSc (in August 2011), he joined as a Senior Research Fellow in Crop Physiology department. In May 2012, he joined as a sandwich PhD student at the Centre for Crop Systems Analysis (CSA) at the Wageningen University \& Research the Netherlands, in collaboration with International Rice Research Institute (IRRI), Philippines. This study represents his fiveyear of research work on physiological and genetic dissection of rice tolerance to water-deficit stress. 


\section{Funding}

This work was supported by an anonymous private donor who provided the financial support, via the Wageningen University Fund. I also thank the Federal Ministry for Economic Cooperation and Development, Germany, and the USAID and Bill and Melinda Gates Foundation for their financial support towards field work at the International Rice Research Institute (IRRI) in the Philippines. 
